\title{
Comparison and Discrimination of Aged and Laundered Fibers by UV-Vis Microspectrophotometry and Colorimetry
}

\author{
Sushana S. Wiliams \\ West Virginia University
}

Follow this and additional works at: https://researchrepository.wvu.edu/etd

\footnotetext{
Recommended Citation

Wiliams, Sushana S., "Comparison and Discrimination of Aged and Laundered Fibers by UV-Vis Microspectrophotometry and Colorimetry" (2012). Graduate Theses, Dissertations, and Problem Reports. 3526.

https://researchrepository.wvu.edu/etd/3526

This Thesis is protected by copyright and/or related rights. It has been brought to you by the The Research Repository @ WVU with permission from the rights-holder(s). You are free to use this Thesis in any way that is permitted by the copyright and related rights legislation that applies to your use. For other uses you must obtain permission from the rights-holder(s) directly, unless additional rights are indicated by a Creative Commons license in the record and/ or on the work itself. This Thesis has been accepted for inclusion in WVU Graduate Theses, Dissertations, and Problem Reports collection by an authorized administrator of The Research Repository @ WVU. For more information, please contact researchrepository@mail.wvu.edu.
} 


\title{
Comparison and Discrimination of Aged and Laundered Fibers by UV-Vis Microspectrophotometry and Colorimetry
}

Sushana S. Williams

\author{
Thesis submitted to the \\ Eberly College of Arts and Sciences \\ at West Virginia University \\ in partial fulfillment of the requirements \\ for the degree of
}

\section{Master of Science in Forensic Science}

\author{
Suzanne Bell, Ph.D., Chair \\ Patrick Buzzini, Ph.D. \\ Keith Morris, Ph.D. \\ Department of Forensic \& Investigative Science
}

Morgantown, West Virginia

July 2012

Keywords: Fiber; Color analysis; Degradation monitoring; CIELab; ANOVA; Multifactorial study; Post hoc comparisons 


\begin{abstract}
Comparison and Discrimination of Aged and Laundered Fibers by UV-Vis

Microspectrophotometry and Colorimetry
\end{abstract}

Sushana S. Williams

Fibers are commonly encountered trace evidence materials that are observed and analyzed in forensic science. The analysis of fibers currently relies upon chromatography, microscopy, spectroscopy, and mass spectrometry $(1,2)$. While most features of fibers are easily established, the determination and comparison of one important attribute, color, is complex $(3,4)$. Factors such as environmental conditions may play an important role when identifying, analyzing and comparing color between questions and known fiber samples.

While color can be evaluated subjectively, it can also be characterized instrumentally. This property may observe subtle or significant differences when fibers have been exposed to aging and laundering. These changes cannot be adequately characterized without instrumental analysis. To standardize and quantify these changes, the Commission Internationale de l'Eclairage (CIE) color characterization system was used in this project.

For the comparison of color, microscopical examination remains the key tool (5). The Microspectrophotometry (MSP) instrumentation has become a standard analytical method used for measuring color in trace evidence. It is the preferred and accepted method because it is nondestructive. In this study, ultraviolet-visible Microspectrophotometry (UV-Vis MSP or MSP) in transmittance measurement was used to analyze the kinetics of color on aged and laundered textile fibers. In transmittance microscopy, the transmittance curve of a colored sample is an objective description of its physical characteristics, free from the subjective influence that occurs with the human eye when it perceives color (6). Therefore, the purpose of this research is to objectively measure color change on aged and laundered textile fibers using colorimetry, and statistically evaluating the data obtained to determine how aging and laundering alter the colorimetric data.

Color changes were observed on fibers subjected to the process of aging and washing. Both treatments affected the degradation of color microscopically in fibers. The artificial aging of fibers was seen to affect the saturation of color more than the gloss of the color. The process treatment of washing and aging was seen to affect the gloss of fiber more than the saturation of color within the fiber. Using colorimetry, statistical methods were able to determined where the changes took place and by how much. Unfortunately, CIELab values were unequally affected between colors such that an overall pattern of degradation could not be calculated. 


\section{Dedication}

I first give honor to God who has given me life to enjoy all things. He has been my

encouragement and prosperity in completing this project. I would then like to thank my amazing and wonderful husband Matthew, who has continually supported and assisted me in unexplainable ways. And I am very grateful that he and my mom and dad, Marcia Woodhouse and Owen Fearon, believed in me and never allowed me to quit when the tasks became discouraging. The accomplishments achieved in this project are dedicated to you all. 


\section{Acknowledgement}

I would sincerely like to thank everyone who has influenced and helped me to accomplish the challenges of this project. I am also very grateful to my committee members for joining and believing in this project. My advisor, Dr. Bell assisted in designing and organizing this project and was very supportive in numerous ways. Dr. Buzinni taught me in great depth the fundamentals of my instrument which truly gave me a better understanding of its operational concept. And lastly, Dr. Morris, who was not only there to help me with the maintenance of my instrument, but also gave me statistical advice on how to approach and gather information from my obtained data.

A great honor of gratitude goes to Dr. Jim Thorne, a prestigious engineer from Craic Technologies who virtually guided my hands in fixing and maintaining the primary instrument needed for this research. I will never forget the numerous contacts made through emails which all involved repairing the Microspectrophotometry. He is a very talented technician and I appreciate the time and attention he offered me during my difficulties.

Special thanks go to Vijay Manickam from the Mechanical and Aerospace Engineering department at WVU who assisted me with the Matlab software and with how to write and implement programs to help with the analysis of the data.

Lastly, I would like to give a substantial amount gratitude to Dr. Gerry Hobbs from the Statistics department at WVU, who provided statistical support which assisted me with the major portion of this project. He showed me how to disintegrate the data in order to extract and interpret the information necessary to answer the proposed research questions. Without his dedication and work, I would have tremendously struggled with the analysis of the data.

Without the support from each and everyone one of you, I would not have been able to surmount through this great and overwhelming challenge. Thank you all very much and God bless. 
TABLE OF CONTENTS

\begin{tabular}{|c|c|}
\hline Abstract & ii \\
\hline Dedication & iii \\
\hline Acknowledgement & iv \\
\hline List of Abbreviations & vii \\
\hline List of Figures & viii \\
\hline List of Tables & $\mathrm{xi}$ \\
\hline Research Questions & 1 \\
\hline Introduction & 1 \\
\hline Literature Review & 20 \\
\hline Materials and Method & 29 \\
\hline Materials & 29 \\
\hline Method & 32 \\
\hline Instrumentation & 38 \\
\hline Statistical Analysis & 43 \\
\hline Project Approach & 65 \\
\hline Results and Discussion & 66 \\
\hline Calibration & 66 \\
\hline Validation & 69 \\
\hline Aged Results & 82 \\
\hline Aged Results Summary & 139 \\
\hline Washed Results & 141 \\
\hline Washed Results Summary & 286 \\
\hline Conclusion & 289 \\
\hline
\end{tabular}




\begin{tabular}{lc}
\hline Recommendation and Future Applications & 290 \\
Glossary & 291 \\
References & 293 \\
Appendix & 297 \\
\hline
\end{tabular}




$\begin{array}{ll}\text { List of Abbreviations } \\ \text { CCD } & \text { Charged couple device } \\ \text { CIE } & \text { Commission Internationale de l'Eclairage } \\ \text { df } & \text { degrees of freedom } \\ \text { HSD } & \text { Tukey honest significant difference test } \\ \text { IR } & \text { Infrared } \\ \text { K-S } & \text { Kolmogorov-Smirnov test } \\ \text { Matlab } & \text { Matrix laboratory } \\ \text { MSP } & \text { see UV-Vis MSP } \\ \text { NIST } & \text { National Institute of Standards } \\ \text { NOBS } & \text { nonanolyoxbenzene sulfate } \\ \text { OD } & \text { optical density } \\ \text { RGB } & \text { red, green and blue } \\ \text { RSD } & \text { relative standard deviation } \\ \text { S-W } & \text { Shapiro- Wilk test } \\ \text { UV-Vis MSP } & \text { Ultraviolet - visible Microspectrophotometry } \\ & \end{array}$




\section{List of Figures}

\begin{tabular}{lll}
\hline Figure 1 & Electron Excitation Levels & 8 \\
Figure 2 & Interaction of Light with Matter & 9 \\
Figure 3 & Absorption of Light by a Sample & 11 \\
Figure 4 & General Diagram of the Transmission MSP & 12 \\
Figure 5 & The Standard Observer Curves & 16 \\
Figure 6 & The Color Measurement Process & 18 \\
Figure 7 & The Chromaticity Diagram & 18 \\
Figure 8 & The CIELab Color space & 30 \\
Figure 9 & Photograph of the Polyester Shorts Used & 32 \\
Figure 10 & Similarity between Reference PMCL and PMJX Samples & 33 \\
Figure 11 & Photograph of the Prepped Aged Standard & 34 \\
Figure 12 & Preparation of Aged Samples for MSP Analysis \\
Figure 13a-b & Mounted Samples for Further Analysis with the MSP & 35 \\
Figure 14 & Preparation of Washed-Aged Samples for MSP Analysis & 38 \\
Figure 15 & UV lamp and box used for Artificial Aging & 39 \\
Figure 16 & Validation Results of Blue, Green and Red Fibers & 75 \\
Figure 17 & Average Validation Results of Blue, Green and Red Fibers & 70 \\
Figure 18 & Average Validation Results of Blue, Green and Red Fibers with & 71 \\
Figure 19 & Uncertainty Ellipses & 73 \\
Figure 20 & Aalidation Results of Blue Fibers & 74 \\
Figure 21 & $\begin{array}{l}\text { Average Validation Results of Blue Fibers specified with } \\
\text { different markers }\end{array}$ & Average Validation Results of Blue Fibers Uncertainty Ellipses \\
\hline
\end{tabular}




\begin{tabular}{|c|c|c|}
\hline Figure 23 & Validation Results of Green Fibers & 76 \\
\hline Figure 24 & Average Validation Results of Green Fibers & 77 \\
\hline Figure 25 & $\begin{array}{l}\text { Average Validation Results of Green Fibers specified with } \\
\text { different markers }\end{array}$ & 77 \\
\hline Figure 26 & Average Validation Results of Green Fibers Uncertainty Ellipses & 78 \\
\hline Figure 27 & Validation Results of Red Fibers & 79 \\
\hline Figure 28 & Average Validation Results of Red Fibers & 79 \\
\hline Figure 29 & $\begin{array}{l}\text { Average Validation Results of Red Fibers specified with } \\
\text { different markers }\end{array}$ & 80 \\
\hline Figure 30 & Average Validation Results of Red Fibers Uncertainty Ellipses & 81 \\
\hline Figure 31 & Normal Distribution of Sample GMP & 85 \\
\hline Figure 32 & Normal Distribution of Sample OSDP & 86 \\
\hline Figure 33 & Interaction plot of Lab variables grouped by 'Hours' & 95 \\
\hline Figure 34 & Interaction plot of variable 'L' grouped by 'Hours' & 95 \\
\hline Figure 35a-b & Interaction plot of variables ' $a$ ' and ' $b$ ' grouped by 'Hours' & 96 \\
\hline Figure 36a-c & Interaction plot for sample DPMP grouped by 'Hours' & 97 \\
\hline Figure 37a-c: & Interaction plot for sample GMP grouped by 'Hours' & 98 \\
\hline Figure 38a-c & Interaction plot for sample OSDP grouped by 'Hours' & 99 \\
\hline Figure $39 a-c$ & Interaction plot for sample PMCL grouped by 'Hours' & 100 \\
\hline Figure 40a-c & Interaction plot for sample PMP grouped by 'Hours' & 101 \\
\hline Figure $41 \mathrm{a}-\mathrm{c}$ & Interaction plot for sample RMP grouped by 'Hours' & 102 \\
\hline Figure 42a-b & $\begin{array}{l}\text { Interaction plot of Lab grouped by ‘Sample Area’ for PMP (a) } \\
\text { and OSDP (b) }\end{array}$ & 105 \\
\hline
\end{tabular}

Figure 43a-b Interaction plot of 'L' grouped by 'Sample Area' for PMP (a) 106 and OSDP (b)

Figure 44a-b Interaction plot of 'a' grouped by 'Sample Area' for PMP (a) 106 
and OSDP (b)

Figure 45a-b Interaction plot of 'b' grouped by 'Sample Area' for PMP (a) 107 and OSDP (b)

Figure $46 \quad$ Interaction Diagram of 'Swatch * Sample Area' for sample 153 DPMP

Figure $47 \quad$ Interaction Diagram of 'Sample Area' * Bleached for sample 154 DPMP

Figure $48 \quad$ Interaction Diagram of 'Bleached * 'Washing' for sample 155 DPMP

Figure $49 \quad$ Interaction Diagram of 'Swatch * Washing' for sample DPMP 156 
List of Tables

\begin{tabular}{|c|c|c|}
\hline Table 1 & Examples of natural and manufactured fibers & 2 \\
\hline Table 2 & List of dyes commonly encountered in forensic examination of fibers & 3 \\
\hline Table 3 & Color Absorbed versus Color Observed by the eye & 10 \\
\hline Table 4 & The CIE Color Space Notation & 19 \\
\hline Table 5 & Polyester shorts used in experiment & 30 \\
\hline Table 6 & Reference labels for the Polyester shorts & 31 \\
\hline Table 7 & ANOVA Summary Table & 53 \\
\hline Table 8 & Example of the Wavelength Calibration Printout & 67 \\
\hline Table 9 & Example of the Photometric Calibration Printout & 68 \\
\hline Table 10 & Summary $\mathrm{p}$-values from the $\mathrm{S}-\mathrm{W}$ test & 83 \\
\hline Table 11 & Descriptive Statistics for Sample DPMP & 89 \\
\hline Table 12 & PMP Results from Levene's Test & 90 \\
\hline Table 13 & RMP Results from Levene's Test & 90 \\
\hline Table 14 & ANOVA results of Lab variables grouped by 'Hours' & 92 \\
\hline Table 15 & ANOVA Output for Sample DPMP & 93 \\
\hline Table 16 & Welch results of Lab variables grouped by 'Hours' & 94 \\
\hline Table 17 & ANOVA results of Lab variables grouped by 'Sample Area' & 103 \\
\hline Table 18 & Welch results of Lab variables grouped by 'Sample Area' & 104 \\
\hline Table 19 & ANOVA results of Lab grouped by 'Fibers' & 108 \\
\hline Table 20 & Welch results of Lab grouped by 'Fibers' & 109 \\
\hline Table 21 & Tukey HSD test of sample DPMP at variable ' $\mathrm{L}$ ' & 111 \\
\hline Table 22 & Homogeneous Subsets: DPMP 'L & 112 \\
\hline Table 23 & Games-Howell test of sample DPMP at variable 'L' & 113 \\
\hline
\end{tabular}




\begin{tabular}{|c|c|c|}
\hline Table 24 & Games-Howell test of sample DPMP at variable 'a' & 115 \\
\hline Table 25 & Games-Howell test of sample DPMP at variable ' $b$ ' & 116 \\
\hline Table 26 & Games-Howell test of sample GMP at variable 'L' & 117 \\
\hline Table 27 & Games-Howell test of sample GMP at variable 'a' & 118 \\
\hline Table 28 & Games-Howell test of sample GMP at variable ' $b$ ' & 119 \\
\hline Table 29 & Games-Howell test of sample OSDP at variable 'L' & 121 \\
\hline Table 30 & Games-Howell test of sample OSDP at variable 'a' & 122 \\
\hline Table 31 & Games-Howell test of sample OSDP at variable ' $b$ ' & 123 \\
\hline Table 32 & Games-Howell test of sample PMCL at variable 'L' & 124 \\
\hline Table 33 & Games-Howell test of sample PMCL at variable 'a' & 126 \\
\hline Table 34 & Games-Howell test of sample PMCL at variable ' $b$ ' & 127 \\
\hline Table 35 & Games-Howell test of sample PMJX at variable 'a' & 129 \\
\hline Table 36 & Games-Howell test of sample PMJX at variable ' $b$ ' & 130 \\
\hline Table 37 & Games-Howell test of sample PMP at variable 'L' & 131 \\
\hline Table 38 & Games-Howell test of sample PMP at variable 'a' & 132 \\
\hline Table 39 & Tukey test of sample PMP at variable ' $b$ ' & 133 \\
\hline Table 40 & Homogeneous Subset of sample PMP at variable ' $b$ ' & 133 \\
\hline Table 41 & Tukey test of sample RMP at variable 'L' & 134 \\
\hline Table 42 & Homogeneous Subset of sample RMP at variable ' $\mathrm{L}$ ' & 135 \\
\hline Table 43 & Games-Howell test of sample RMP at variable 'a' & 136 \\
\hline Table 44 & Games-Howell test of sample RMP at variable ' $b$ ' & 137 \\
\hline
\end{tabular}




\section{Research Questions}

The goal of this project is to classify and discriminate fibers based on color, specifically on how aging and laundering affects color. Other research questions included:

- Does color change within fiber such that it can no longer be compared to its untreated source without concluding type I errors?

- Is it possible to differentiate fibers from different garments of similar color after the aging and laundering process?

- Are the results dependent on parameters such as the type of detergent used?

- How can statistical analysis assist in describing the characteristics of color that change as a result of aging and laundering?

\section{Introduction}

Textile fibers are natural or man-made substances which are the components of fabrics and textiles. Natural fibers are fibers derived from animals, minerals or plants. Since these are fibers in their natural state, they do not require fiber formation or reformation as manufactured or manmade fibers. Man-made fibers are those derived through a process of manufacturing from any substance that was not originally a fiber. They require the formation of fibers for synthetic materials and the reformation of fibers for polymer materials. This class is divided into categories based on the primary material used in its development. These materials include synthetic polymers, and natural polymers. 
Table 1: Examples of natural and manufactured fibers

\begin{tabular}{|l|l|l|}
\hline Type & Fiber & Example \\
\hline Natural & Animal & Wool \\
\hline Natural & Mineral & Asbestos \\
\hline Natural & Plant & Cotton \\
\hline Manufactured & Natural polymer & Rayon \\
\hline Manufactured & Synthetic polymer & Polyester \\
\hline
\end{tabular}

Almost all manufacturing industries are concerned with its' product appearance. For that reason, color is often imparted to the end product of manufactured materials. The color of a fiber is dependent upon the dyes, pigments, and surface treatments used during manufacturing. Dyes are unsaturated organic molecules that are used to impart color on a substrate with some degree of permanence during the manufacturing process. They can be physically or chemically bound to fibers using forces such as hydrogen bonding, van der Waals forces, or covalent bonding(7). Dyes are usually classified by their chemical constituents that indicate the major chromophore present in the dye. Chromophores are the unsaturated conjugated groups of the dye that give the molecule its color. Additional information about the morphology of fiber formation and dye application is summarized elsewhere(7).

Pigments are small inorganic particles that are insoluble in water and most other solvents. Pigment particles are primarily used to deluster or color fibers and are either incorporated into the fiber at the time of production or are bonded to the surface of the fiber by a resin. Since pigments are unnaturally attracted to fibers, they must be modified to increase its affinity. Some fiber types that are not easily dyed are often pigmented. 
A table is included that discusses dyes specifically encountered in the forensic examination of fibers, and is classified based on the method of application. The application and characteristics of each type of dye provides useful information about its washfastness. Fastness, i.e. the fibers resistant to fading, depends on how color changes over time under environmental conditions. Washfastness is then defined as the fibers' resiliency to retain its color during washing.

Table 2: List of dyes commonly encountered in forensic examination of fibers (8-10)

\begin{tabular}{|l|l|l|l|l|l|}
\hline Dye class & Bond type & Fiber type & $\begin{array}{l}\text { Description/ } \\
\text { application }\end{array}$ & Example & $\begin{array}{l}\text { Characteristics/ } \\
\text { Washfastness }\end{array}$ \\
\hline Acid dyes & $\begin{array}{l}\text { Ionic } \\
\text { bonding }\end{array}$ & $\begin{array}{l}\text { Wool, silk, } \\
\text { nylon, } \\
\text { polyamide, } \\
\text { protein, }\end{array}$ & $\begin{array}{l}\text { Water-soluble } \\
\text { anionic } \\
\text { compounds; ionic } \\
\text { bond between dye } \\
\text { molecules and } \\
\text { polymer. }\end{array}$ & Congo red & $\begin{array}{l}\text { Bright color. } \\
\text { May have poor } \\
\text { fastness }\end{array}$ \\
\hline $\begin{array}{l}\text { Azoic } \\
\text { dyes }\end{array}$ & $\begin{array}{l}\text { Mechanical } \\
\text { adhesion }\end{array}$ & Cotton, viscose & $\begin{array}{l}\text { Consist of a } \\
\text { coupling } \\
\text { component } \\
\text { between diazo salt } \\
\text { and the coupling } \\
\text { component such as } \\
\text { naphthol which } \\
\text { creates one large } \\
\text { insoluble molecule }\end{array}$ & Tartrazine & $\begin{array}{l}\text { Bright shades. } \\
\text { Good to } \\
\text { excellent } \\
\text { fastness }\end{array}$ \\
\hline Basic dyes & $\begin{array}{l}\text { Ionic } \\
\text { bonding }\end{array}$ & $\begin{array}{l}\text { modified } \\
\text { acrylic, } \\
\text { polyester, } \\
\text { polyamide }\end{array}$ & $\begin{array}{l}\text { Water-soluble, } \\
\text { applied in weakly } \\
\text { acidic dye baths; } \\
\text { negative charge } \\
\text { fiber draws the dye } \\
\text { cation }\end{array}$ & $\begin{array}{l}\text { Malachite } \\
\text { green }\end{array}$ & $\begin{array}{l}\text { Very bright } \\
\text { colors. Poor } \\
\text { fastness }\end{array}$ \\
\hline $\begin{array}{l}\text { Direct } \\
\text { dyes }\end{array}$ & $\begin{array}{l}\text { Surface } \\
\text { absorption }\end{array}$ & $\begin{array}{l}\text { Cotton, viscose } \\
\text { Water soluble, } \\
\text { anionic } \\
\text { compounds; } \\
\text { applied directly } \\
\text { from aqueous } \\
\text { medium that has an } \\
\text { electrolyte; }\end{array}$ & $\begin{array}{l}\text { Direct } \\
\text { yellow 12 }\end{array}$ & $\begin{array}{l}\text { Poor to good } \\
\text { fastness unless } \\
\text { further treated }\end{array}$ \\
\hline
\end{tabular}




\begin{tabular}{|c|c|c|c|c|c|}
\hline & & & $\begin{array}{l}\text { positively charged } \\
\text { ion is attracted to } \\
\text { negatively charged } \\
\text { fiber }\end{array}$ & & \\
\hline $\begin{array}{l}\text { Disperse } \\
\text { dyes }\end{array}$ & $\begin{array}{l}\text { Surface } \\
\text { absorption }\end{array}$ & $\begin{array}{l}\text { Polyester, } \\
\text { nylon, acetate, } \\
\text { acrylic }\end{array}$ & $\begin{array}{l}\text { Not water-soluble; } \\
\text { applied from a hot } \\
\text { aqueous dispersion; } \\
\text { hydrogen and van } \\
\text { der Waals forces } \\
\text { are formed that } \\
\text { hold the dye } \\
\text { molecules in fiber. }\end{array}$ & Celliton & $\begin{array}{l}\text { Fair to excellent } \\
\text { fastness }\end{array}$ \\
\hline $\begin{array}{l}\text { Metalized } \\
\text { dyes }\end{array}$ & $\begin{array}{l}\text { Ionic } \\
\text { bonding }\end{array}$ & $\begin{array}{l}\text { Wool, nylon, } \\
\text { polypropylene }\end{array}$ & $\begin{array}{l}\text { Form colored metal } \\
\text { complexes within } \\
\text { fiber through a } \\
\text { mordant (binding } \\
\text { agent) such as } \\
\text { chrome with a } \\
\text { separate dye } \\
\text { molecule }\end{array}$ & $\begin{array}{l}\text { Acid } \\
\text { violet } 56\end{array}$ & $\begin{array}{l}\text { Good to } \\
\text { excellent } \\
\text { fastness }\end{array}$ \\
\hline $\begin{array}{l}\text { Reactive } \\
\text { dyes }\end{array}$ & $\begin{array}{l}\text { Covalent } \\
\text { bonding }\end{array}$ & $\begin{array}{l}\text { Cotton, wool, } \\
\text { polyamide }\end{array}$ & $\begin{array}{l}\text { Water-soluble; } \\
\text { forms covalent } \\
\text { bonds with the } \\
\text { functional groups } \\
\text { of the dye } \\
\text { molecule; similar } \\
\text { structure to acid } \\
\text { dyes' similar } \\
\text { application to } \\
\text { direct dyes }\end{array}$ & $\begin{array}{l}\text { C. I. } \\
\text { Reactive } \\
\text { Blue } 19\end{array}$ & $\begin{array}{l}\text { Bright shades. } \\
\text { Good fastness. } \\
\text { Sensitive to } \\
\text { bleach }\end{array}$ \\
\hline $\begin{array}{l}\text { Sulphur } \\
\text { dyes }\end{array}$ & $\begin{array}{l}\text { Mechanical } \\
\text { adhesion }\end{array}$ & Cotton & $\begin{array}{l}\text { Sulphur organic } \\
\text { compound; } \\
\text { requires reducing } \\
\text { agent to make them } \\
\text { soluble then } \\
\text { undergo oxidation } \\
\text { to its original form }\end{array}$ & $\begin{array}{l}\text { CI } \\
\text { Sulphur } \\
\text { Red } 14\end{array}$ & $\begin{array}{l}\text { Dull colors. } \\
\text { Good fastness. } \\
\text { Sensitive to } \\
\text { bleach }\end{array}$ \\
\hline
\end{tabular}




\begin{tabular}{|l|l|l|l|l|l|}
\hline Vat dyes & $\begin{array}{l}\text { Mechanical } \\
\text { adhesion }\end{array}$ & Cotton & $\begin{array}{l}\text { Water-insoluble; } \\
\text { requires reducing } \\
\text { agent to make them } \\
\text { soluble then } \\
\text { undergo oxidation } \\
\text { to create an } \\
\text { insoluble dye }\end{array}$ & Indigo & $\begin{array}{l}\text { Good to } \\
\text { excellent } \\
\text { fastness }\end{array}$ \\
\hline Pigment* & $\begin{array}{l}\text { Mechanical } \\
\text { adhesion }\end{array}$ & $\begin{array}{l}\text { Cotton, } \\
\text { viscose, } \\
\text { acrylic, } \\
\text { polyamide, } \\
\text { polyester }\end{array}$ & $\begin{array}{l}\text { Pigments are finely } \\
\text { ground solids that } \\
\text { have no affinity for } \\
\text { fibers and are } \\
\text { generally added or } \\
\text { bonded with a } \\
\text { bonding agent }\end{array}$ & $\begin{array}{l}\text { Luteolin, } \\
\text { titanium } \\
\text { oxide }\end{array}$ & $\begin{array}{l}\text { Generally give } \\
\text { bright colors. } \\
\text { Good fastness }\end{array}$ \\
\hline Ingrain & $\begin{array}{l}\text { Mechanical } \\
\text { adhesion }\end{array}$ & Cotton & $\begin{array}{l}\text { Dye is synthesized } \\
\text { directly into the } \\
\text { fiber }\end{array}$ & Azo dyes & Good fastness \\
\hline
\end{tabular}

* Pigments are not dyes but are seen as colorants or delustrant in a variety of fiber types.

The application of the dye to the fiber will determine its fastness. When the dye molecules

become a part of the fiber (e.g. reactive dyes), it is less likely to be removed by washing than dye molecules that adhere by adsorption (e.g. direct dyes). The location where the dye has penetrated the fiber, its bonding mechanism, and the stability of that molecule will influence the fastness of the dye. Physical factors and chemical reagents can alter the total dye absorbed by the fiber. To evaluate this nondestructively, MSP instrument was used and the data obtained was expressed quantitatively by the CIE system.

Since microscopical examination remains the key tool for color comparison, MSP was preferred because it objectively measures the degree of color change in a material(11). MSP is a type of spectroscopy method which studies the chemical interaction between light and matter. In spectroscopy, there are two main spectral regions of interest for the typical forensic spectroscopic applications: ultraviolet and visible (UV-Vis) region, and the infrared (IR) region. 
With the MSP and color analysis, the region of interest is the UV-Vis region of the spectrum. This region is located on the electromagnetic radiation spectrum in the $200 \mathrm{~nm}-400 \mathrm{~nm}$ range for near UV and 400nm - 800nm range for visible. It is in these regions that the electron excitation can be observed when light interacts with a molecule. The energy associated with the interaction of light to produce electronic excitation is due to the external or valence electrons found on the orbital of a molecule. MSP is based on the electronic molecular absorption as it measures the absorption of the electromagnetic radiation in the ultraviolet and visible region of the spectrum.

For absorption to occur, a molecule must first be excited by the frequency of that incident electromagnetic radiation. However, absorbance only occurs if the energy of the photon closely matches the difference in energy between two electronic states. During this excitation, an electronic transition occurs when the electron has enough energy to transition to a higher orbital state, the excited state. The electrons transits to a higher state when that photon of corresponding energy is absorbed. This process of electron jumping from ground state to an excited state is called absorbance.

After absorption, the molecule may release some of that stored energy in different ways. Since the excited state is unstable, the electron transition back to its original state (ground state) and the energy that is emitted between these transitions is dependent on the emission factor. One of the most common ways an electron returns to the ground state is by the release of energy to the surrounding molecule. An electron can return to the ground state by producing lesser energy with the emission of a photon (called fluorescence) or without this emission. It can also return to its ground state by internal conversion which occurs when the molecule releases its energy through vibration. 
The electronic transitions between different energy levels of a molecule are dependent upon the electronic configuration of the molecule, the bonding of the atom, and the environment, as well as other parameters. Molecular orbital theory provides a model for the way electromagnetic radiation interacts with molecules. This interaction is based on the bonding mechanism of the electrons.

The different bonding that can occur at the external orbital of the atom requires different energy for absorption to occur. There are three types of valence electron bonding: the sigma-electrons of the molecular frame, the pi-electrons of the double and triple bonds and the non-binding pairs of electrons, also referred to as ion-pairs. These valence electrons require different energy levels in order for excitation to occur. As the electrons get excited, the atoms can rotate or vibrate with respect to each other. Absorption of UV-Vis radiation is limited to certain functional groups depending upon the valence electrons, and thus the type of bonding between the electrons. Among the three valence electron bonding, the sigma bonds are the strongest; they require the greatest amount energy for excitation to occur. The higher amount of energy required, the shorter the wavelength needed for excitation. This wavelength range is beyond the scope of the MSP. Pielectrons are weaker and the bond structures are looser, so lesser energy is required for excitation to occur. 
Figure 1: Electronic Excitation Levels

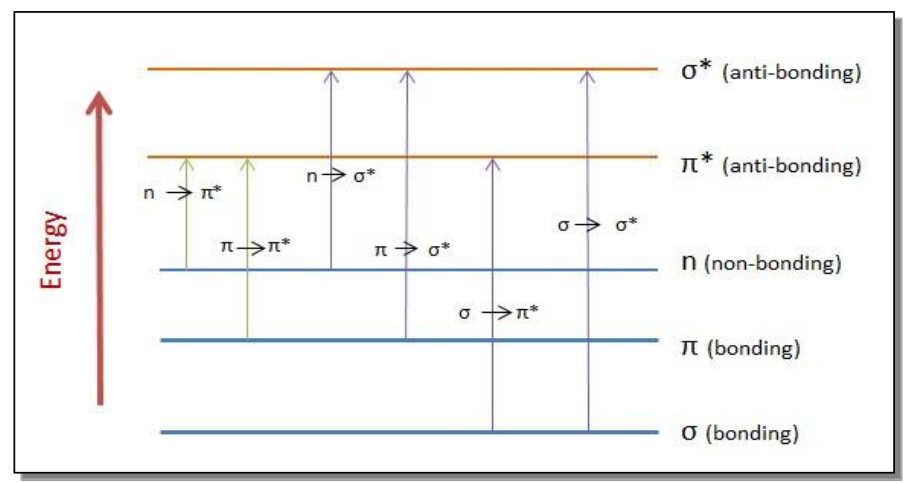

This diagram outlines the electron transitions that may occur in an organic molecule. Of the six illustrated, only the two lowest ones (left two) are achieved by the energy available in the UVVis region. Lesser energy is required to excite the pi and non-binding electrons in the visible region. These electrons are easily excited if the pi and non-binding electrons are conjugated. There is an inverse relationship between bonding and energy; the more conjugated bonds are found in a molecule, the lesser the energy necessary for those electrons to be excited. These conjugated bonding system found in the bonding of pi-electrons can absorb radiation in the visible region (the longer wavelengths) and therefore is responsible for the color that is observed.

The chemical theory of color states that absorption of radiation in the visible region of the spectrum may require the presence of some conjugated double bond groups. This will determine whether a molecule will absorb in the visible region, and describe where such absorption will occur. Knowledge of the number of double bonds and the different double bonds will help to understand the spectra of a molecule, but more importantly, to help predict the spectra if the chemical structure of the colored molecule is known. The part of the molecule responsible for absorption of UV-Vis radiation is the chromophore. Therefore, absorption of UV-Vis radiation is restricted to certain functional groups (chromophores) that contain valence electrons of low excitation energy. 
For the comparison of color, microscopic examination remains the key tool because it is able to distinguish color in which the human eye cannot perceive $(3,5,12)$. MSP has been the conventional tool for analyzing color without destroying the sample. It provides an objective method of microscopic analysis for color comparison. Microspectrophotometry uses a microscope to measure the absorption of the electromagnetic radiation from the chromophore after being excited in the visible and ultraviolet region of the spectrum (4). Since each molecule absorbs light at different wavelengths, the result is considered from the near UV to visible regions and is plotted in a graph called a spectrum. These results can be obtained in one of three modes: transmittance, reflectance and absorbance.

\section{Figure 2: Interaction of Light with Matter}

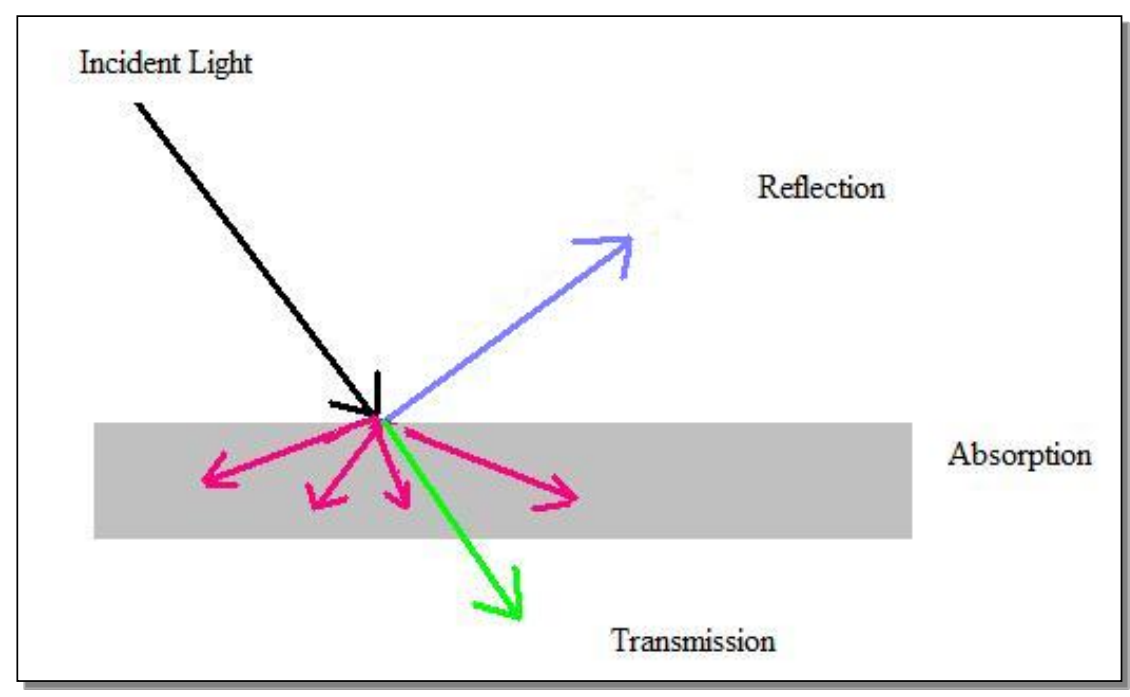

When light strikes an object, the light can react in a number of different ways (Figure 3). When a sample interacts with light, some of that light can be transmitted through, absorbed in or reflected by that sample. Each interaction provides specific details about the sample. While reflectance only measures the surface area, transmittance interrogates the whole sample. The result is a transmittance spectrum that details qualitative information about the interaction of the 
sample with the various wavelengths(13). For example, when light strikes a blue colored fiber, the red, orange and yellow wavelengths are absorbed by the fiber and the violet-blue and green wavelengths are reflected. Therefore, the color observed on the fiber is based on the wavelength being reflected or transmitted through. The color of any object is largely due to the way that object interacts with light, and how it is reflected and transmitted to the observer. These interactions are expressed in Table 2 below.

Table 3: Color absorbed versus color observed by the eye (10)

\begin{tabular}{lll}
\hline Color Absorbed & Color observed, by eye & Wavelength (nm) \\
Violet & Yellow-green & $380-430$ \\
Blue & Yellow & $430-480$ \\
Green-blue & Orange & $480-490$ \\
Blue-green & Red & $490-500$ \\
Green & Purple & $500-560$ \\
Yellow-green & Violet & $560-580$ \\
Yellow & Blue & $580-590$ \\
Orange & Green-blue & $590-610$ \\
Red & Blue-green & $610-750$ \\
\hline
\end{tabular}

In this project, transmittance mode was used for analyzing fibers because of the translucent properties of the fibers. In the transmittance mode, the light directed at the sample interacts with the sample as it passes through. The value of the transmitted light is then measured in comparison of the incident beam $\left(\mathrm{I}_{0}\right)$ and the attenuated or reduced beam (I). 
Figure 3: Absorption of Light by a Sample

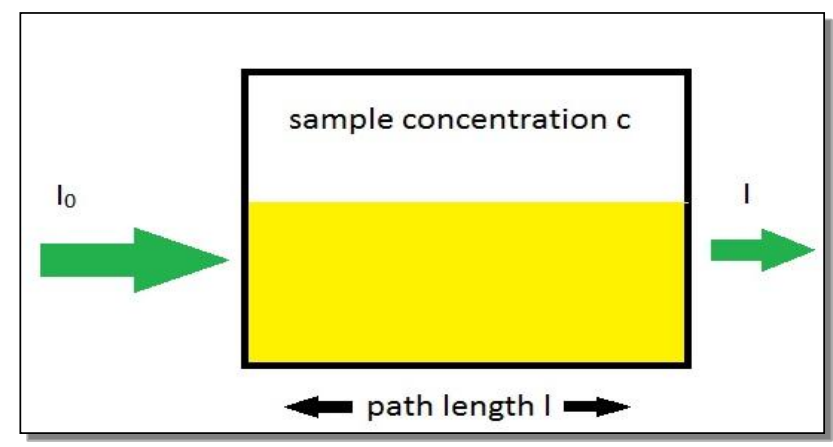

This result is also dependent on the thickness of the sample holder used (microscope slide and cover slip), the sample itself and the absorption coefficient of the sample. This absorption of energy in the visible range is governed by Beer's law given by the formula

$$
\mathrm{A}=\varepsilon_{\lambda} 1 \mathrm{c}
$$

where $\varepsilon$ is the molar absorptivity, 1 is the path length and $\mathrm{c}$ is the concentration of the sample.

For a given absorber at a given wavelength, $\mathcal{\varepsilon}$ is constant. The absorbance varies linearly with the path length and the analyte concentration. Spectrometers are typically designed with a constant path length; therefore the absorption is directly related to the concentration of the solution.

\subsection{Components of the UV-Vis MSP}

The MSP is an integration of an optical microscope and a highly sensitive spectrophotometer. The objective of the microscope is to collect light from the sample using lenses and mirrors to produce a magnified image of the sample and focus that image on the spectrophotometer 
aperture. The spectrophotometer portion is an optical instrument for measuring the intensity of light relative to its wavelength.

The MSP is composed of the following components: light source, monochromator, microscope, detector and computer. The light source necessary for ultraviolet radiation will differ from visible radiation. Therefore it is necessary that the light source is able to emit all the wavelengths of the UV-Vis region. When light is emitted, the monochromator acts as a prism and splits the light into individual wavelengths. The microscope coupled to the spectrometer, helps to visualize the microscopic materials that is being analyzed. The detector is a charged couple device (CCD) that converts light into an electrical signal. The computer, which is attached to the instrument, controls the system, and parameters, and displays the data using specific software.

Figure 4: General diagram of the transmission MSP

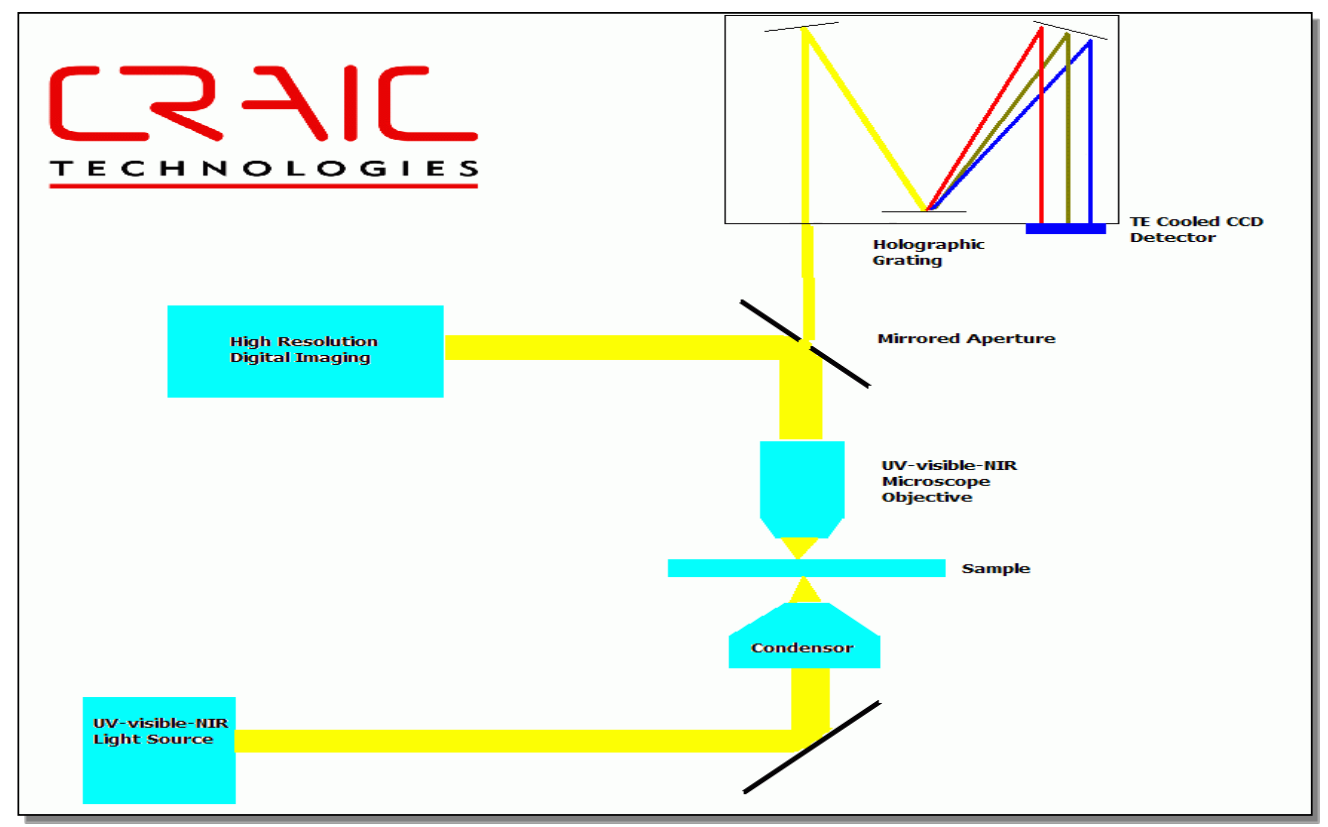

Reproduced with permission- Copyright (C) 2002-2011 CRAIC Technologies, Inc. All Rights Reserved, use of such documents from this web site is for informational and non-commercial or personal use only and will not be copied or posted on any network computer or broadcast in any media) 
The lamp emits light which is focused on the sample. The sample may absorb some of those wavelengths of light depending upon the chemical structure of the chromophore. Light that is not absorbed by the sample is collected by the MSP objective and focused on the entrance aperture of the spectrophotometer. Using a mirrored aperture, majority of the projected light is reflected to the digital imaging system. This system views the aperture and the sample simultaneously and presents the image showing what the spectrophotometer aperture is currently measuring. The remaining nonreflected light is directly sent to the head of the spectrophotometer and is separated by the monochromator into individual wavelength before reaching the detector. The CCD collects the incoming wavelengths and measures the intensity of each wavelength by the array of pixels. It then sends this information to a computer and the result is a spectrum which displays the intensity of each wavelength of light.

\subsection{Colorimetry}

Colorimetry is used to quantify descriptions of perceived color and its relationship to spectroscopic measurements(3). It may be used in trace evidence to classify objects such as fibers, paints and inks. Colorimetry was developed to replace the subjective occurrence of color vision in human observation. CIE is the standard colorimetric system used to quantify the color of objects. It is a mathematical model that attempts to express color as a linear combination of red, green and blue (RGB) receptors, which roughly stimulates how humans perceive color.

The human eye contains two types of photoreceptor cells called rods and cones where rods responds to the wavelengths of light and the cones respond to color by the reaction of light with pigments (14). The human eye can detect the light in the visible range of the spectrum. When it perceive light, the cones perceive the colors and the rods perceive tones, i.e., black, white and 
variations of gray. Within the cones, there are three sets of color receptors: red, green and blue. The red cones responds to wavelength around 560nm, the green around 530nm and the blue around $420 \mathrm{~nm}$. For this reason, the red cones are most stimulated by light in the red to yellow range, the green in the yellow to green range and the blue in the blue to violet range. Whenever an individual sees color, each cone is stimulated differently to produce that color. For instance, if a beam of light only stimulates the red cone, then the color observed is pure red; likewise for the green and blue cones. Combinations of strongly or weakly stimulated RGB cones will result in production of a variety of colors.

In principle, these three receptors are needed to describe color. Similarly, CIE is related to the sensitivity of these receptors because it is based on the response of the direct measurements of the human eye. The sensitivity of these receptors is used to calculate the tristimulus values and generate a color match. These values are the notation for the CIE system. The tristimulus system is based on the mechanism of human color vision. These cones contain three light sensitive pigment complexes that correlate with RGB of the tristimulus system. The average human response to wavelengths of light is considered the standard observer. The goal of the CIE and tristimulus system is to recreate the pattern of cone stimulation in the eye of a standard observer under controlled conditions of illumination and viewing angle.

Certain colors appear different under different illumination, a phenomena called metamerism. These metameric colors appear to match in one light setting, but differ under another. Because of this, the conditions of the illumination must be controlled to prevent metamerism. CIE also took into account that humans perceive colors most exactly in the eye if the colors impinge in the region of the fovea. Since this region deviates from the optical axis of the eye by approximately 
$2^{\circ}$, the angle under which a standard observer sees was defined to be exactly $2^{\circ}$ (illustrated in Figure 5). This angle is considered the viewing angle. However, another viewing angle of $10^{\circ}$ was created to consider the circumstances where the eye sees more distant objects under a different viewing angle. The main difference between $2^{\circ}$ and the $10^{\circ}$ viewing angle is the increase in the field of view (15).

Figure 5: The Standard Observer Curves

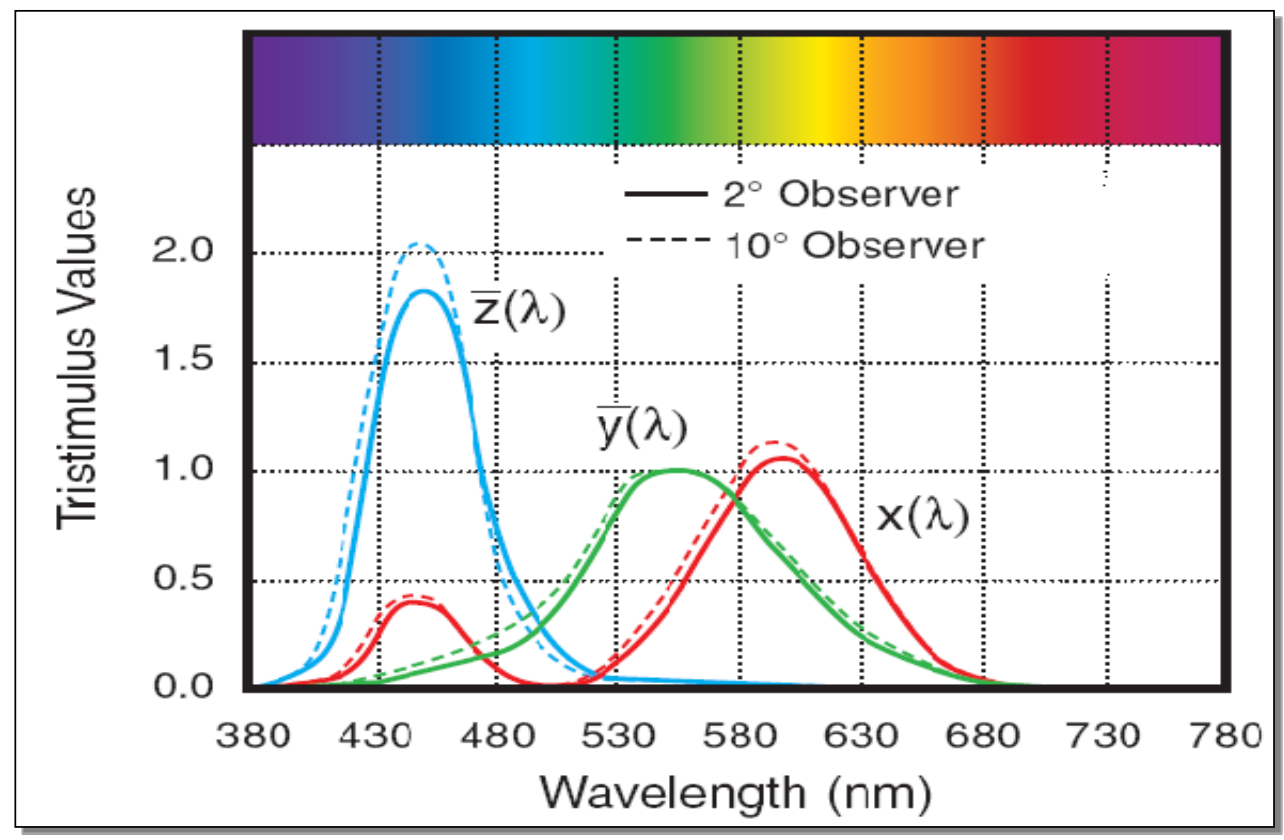

Reproduced with permission: Graphic courteous of PCI Magazine Feb 2003 by X-Rite titled 'Understanding Color Communication'

Color sensation depends on the wavelength content of the received light on the characteristics of the human observer's visual system from our three photoreceptors. While the human eye perceives color by mixtures of RGB, the CIE system employs a reflectance spectrum from the CIE's standard light source. To determine the quantity of RGB for any color, the quantities of these colors in each wavelength must be integrated(16):

$R=\int_{0}^{\infty} I(\lambda) \bar{r}(\lambda) \mathrm{d} \lambda ; \quad G=\int_{0}^{\infty} I(\lambda) \bar{g}(\lambda) \mathrm{d} \lambda ; \quad B=\int_{0}^{\infty} I(\lambda) \bar{b}(\lambda) \mathrm{d} \lambda$ 
Where $\mathrm{I}(\lambda)$ is the luminous intensity in each wavelength and $\bar{r}(\lambda), \bar{g}(\lambda)$ and $\bar{b}(\lambda)$ curves expresses the amount of red, green and blue needed for a match to be made for each wavelength of the spectrum. The color matching functions $r, g, b$ of the standard observer determines the relationship of the primary colors. If an object reflects light when irradiated, the luminous intensity is obtained by $I(\lambda)=S(\lambda) \cdot R(\lambda)$, where $S(\lambda)$ is the spectral power distribution of the standard light source, and $\mathrm{R}(\lambda)$ is the spectral reflectance curve of the object. Thus, the light source, the object reflecting the light, and the standard observer receiving the light and producing the color sensation, reflects the three elements needed in producing color (17). This is expressed in the equation below.

Figure 6: The Color Measurement process

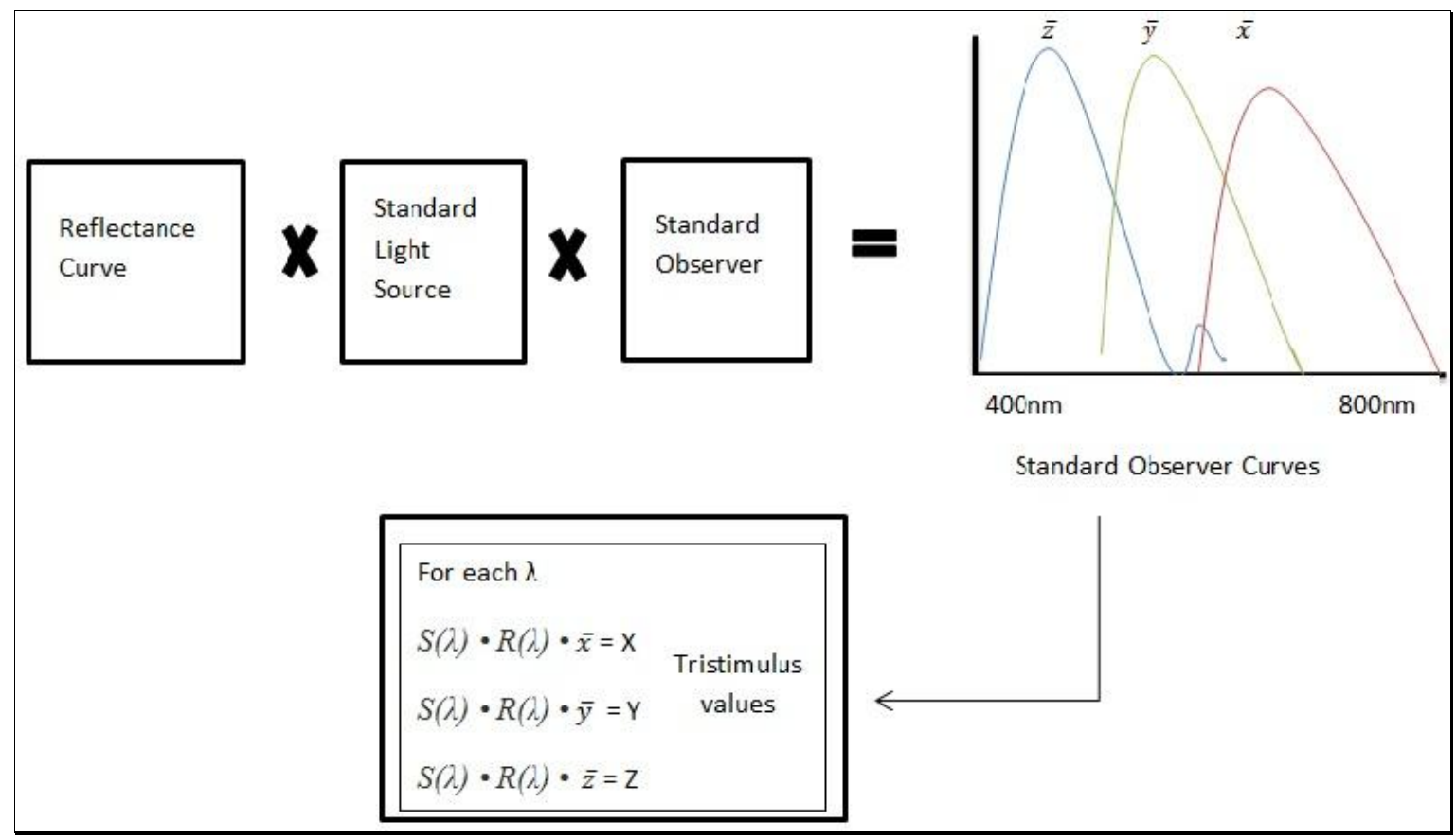

The reflection value at each wavelength in the spectrum is expressed as a weighted contribution of RGB $(\bar{z}, \bar{y}, \bar{x})$. This spectrum representing the visible region is obtained by the MSP. Once the 
illumination and degree of observation has been applied, the standard observer curves are calculated. From this, the tristimulus value X, Y, Z, which represents the total component of each color, is summed over the spectral range. The three tristimulus values $\mathrm{X}$ (red), $\mathrm{Y}$ (green), and Z (blue), can be given for any color, which will model the color perceived by an individual (18). These coordinates define the color quality of the color stimulus. Each coordinate is then calculated by summing over the entire spectral region and multiplying the amount of light by the receptor sensitivity (19).

$\mathrm{X}=\sum_{\lambda=380}^{760} S \bar{x}(\lambda) \cdot R(\lambda) ; \quad \mathrm{Y}=\sum_{\lambda=380}^{760} S \bar{y}(\lambda) \cdot R(\lambda) ; \quad \mathrm{Z}=\sum_{\lambda=380}^{760} S \bar{z}(\lambda) \cdot R(\lambda)$

The CIE tristimulus values do not directly correspond to the visual attributes of color, but are an approximation. For color to be readily understood, it must be defined in terms of hue and chroma. The hue refers to the spectral colors, and the chroma refers to the saturation or strength of the dominant wavelength or hue as the color deviates from gray. For this reason, CIE uses the XYZ tristimulus values to formulate a new set of values called the chromaticity coordinates denoted xyz:

$$
x=\frac{X}{X+Y+Z} \quad ; y=\frac{Y}{X+Y+Z} \quad ; z=\quad \frac{Z}{X+Y+Z}
$$

The sum of $\mathrm{x}, \mathrm{y}$, and $\mathrm{z}$ is always equal to one. Only the two coordinates, $\mathrm{x}$ and $\mathrm{y}$, are necessary for color determination because they specify the hue and chromaticity of the color (20). This result in a single value which is plotted in a $2 \mathrm{D}$ chromaticity diagram to observed the location of the color. The Y tristimulus value is established as a direct measure of luminance or light which 
refers to the depth of the color. By normalizing the tristimulus values, the chromaticity coordinates can be calculated into a 2-D chart illustrated below.

Figure 7: The Chromaticity diagram

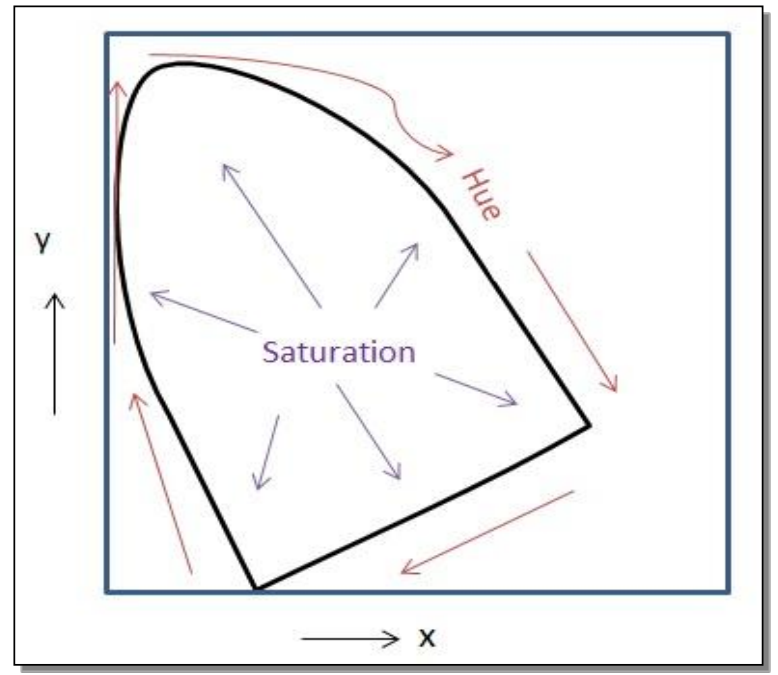

Chromaticity diagrams are limited by being asymmetric: therefore the calculations and comparisons of color differences are not uniform. To address this, a transformation must be applied. CIE later established a cyclindrical color model called Lab.

Figure 8: The CIELab Color Space

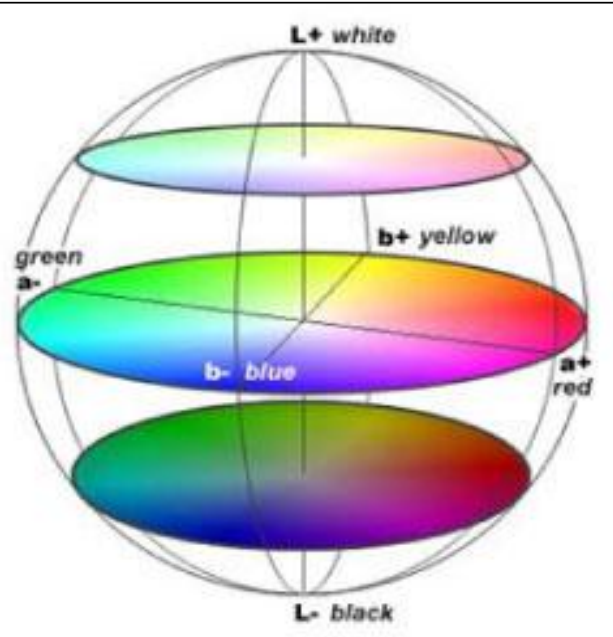

CIE LAB Color Model 
It is a uniformed color space version of CIE and has a larger color range. CIELAB describes color with three components (Table 4); 'L' for luminance or lightness, 'a' for chromatic components from green to red, and ' $b$ ' from blue to yellow (further derived from tristimulus values $\mathrm{X}, \mathrm{Y}, \mathrm{Z})$. Using this model, a color difference can be calculated. The transformation used to convert the tristimulus values to CIELab equivalent are as follows

$$
\begin{gathered}
\mathrm{L}^{*}=116(\mathrm{Y} / \mathrm{Yn})^{1 / 3}-16 \\
\mathrm{a}^{*}=500\left[(\mathrm{X} / \mathrm{Xn})^{1 / 3}-(\mathrm{Y} / \mathrm{Yn})^{1 / 3}\right] \\
\mathrm{b}^{*}=200\left[\left(\mathrm{Y} / \mathrm{Yn}_{\mathrm{n}}\right)^{1 / 3}-(\mathrm{Z} / \mathrm{Zn})^{1 / 3}\right]
\end{gathered}
$$

Hence, the CIE values are defined in terms of the ratio of the tristimulus values, $\mathrm{X}, \mathrm{Y}$ and Z (21). The difference of distance on the CIELAB plot can be stated as a single value, known as simple Euclidean distance.

There are also other color models known but CIELab was chosen as it is most frequently used to measure color $(17,21-24)$. The procedure for calculating color is based upon the illuminant used, the observer and the reflection spectral profile of the object. The recommended illuminant for forensic case work is illuminant $\mathrm{A}$, which is an incandescent tungsten lamp with a color temperature of $2856 \mathrm{~K}(25)$. For this project, the illuminant and the observer remained constant; therefore any differences in color arose from the reflectance spectra of the fiber plus any associated variables.

Table 4: The CIE Color Space Notation

\begin{tabular}{|l|l|l|l|}
\hline Notation & Description & Positive value & Negative value \\
\hline $\mathrm{L}$ & $\begin{array}{l}\text { Difference in } \\
\text { lightness/ darkness } \\
\text { value }\end{array}$ & $\begin{array}{l}\text { Lighter- if the value }= \\
\text { 0, the sample is white }\end{array}$ & $\begin{array}{l}\text { Darker- if the value }= \\
100, \text { the sample is } \\
\text { black }\end{array}$ \\
\hline
\end{tabular}




\begin{tabular}{|l|l|l|l|}
\hline a & $\begin{array}{l}\text { Difference on } \\
\text { red/green axis }\end{array}$ & $\begin{array}{l}\text { Redder or has red } \\
\text { shading }\end{array}$ & $\begin{array}{l}\text { Greener or has green } \\
\text { shading }\end{array}$ \\
\hline $\mathrm{b}$ & $\begin{array}{l}\text { Difference on } \\
\text { yellow/blue axis }\end{array}$ & $\begin{array}{l}\text { yellower or has } \\
\text { yellow shading }\end{array}$ & $\begin{array}{l}\text { Bluer or has blue } \\
\text { shading }\end{array}$ \\
\hline
\end{tabular}

\section{Literature review}

One of the most common questions asked of trace evidence examiners in court is "How common are these fibers?" (1). To determine the commonness and rareness of fibers, the properties of the fiber type, the color, the cross section as well as other parameters, must be recorded and considered. Since color has high discriminatory characteristics and can be determined objectively, further experimenting of color in fibers was approached. The majority of the studies that have been completed thus far have been directed toward color fading of multi-cycle laundering processed on single fiber type such as cotton, polyester and wool $(26,27)$.

The study performed by Phillips et al attempted to develop a method to identify colored cotton fabrics susceptible to fading (26). One goal of this study was to predict the effect of bleached detergent on cotton batches after multiple laundered cycles. The activated bleach used was hydrogen peroxide with tetra-acetylethylene diamine (TAED). This oxygenated bleaching system is commonly found in laundry detergents in Western Europe where the research was performed. The author evaluated 39 commonly used reactive, sulfur and vat dyes, which were washed using four different detergents: two commercial detergent brands containing TAED and two reference detergents with only one containing the bleach system TAED. Prior to washing, the fabrics were screened by an existing standard to eliminate any bleeding and staining that may occur if the fabrics are washed collectively. Of the 64 fabrics, 11 were removed from the experiment as they were deemed unsuitable for washing because they would have caused high 
levels of bleeding and staining to adjacent fibers. These 11 direct dyes excluded from the study were all water soluble; hence, as the fiber was submerged in the liquid, high levels of bleeding and staining occurred as the dye was not retained in the fiber. The remaining 53 fabrics were washed 20 times in a washing machine set to the 'main cotton' washing cycle. The color change was recorded using a spectrophotometer and the results were compared to the unprocessed fabrics.

The results from the study showed that certain dyed fabrics were more sensitive to bleach than others. It was further concluded that even with the absence of the bleach, certain dyes observed a high fading, therefore estimating that the mechanism behind fading can be both a physical and chemical process. This result was limited to CI Vat Yellow 46 dye; the other vat dyes exhibited very little bleach sensitivity. This experiment has been validated by another study which took place in the United Kingdom.

Philips et. al. experimented on establishing a correlation between the fading of a fiber subjected to bleach in a single cycle versus multiple wash cycles. Sixty-four cotton substrates dyed with direct, reactive, sulfur and vat dyes were washed for a total of 20 times(28). The results were categorized into two groups: the sensitivity of the dye to the bleaching system, or those considered to be resistant or robust to bleaching. With direct dyes, fading is influenced by both the chemistry of the dye and the after treatment that followed. While reactive dyes varied in its sensitivity, sulfur was extremely sensitive to bleach. In agreement to the previous study, vat dyes did not exhibit a significant amount of bleach fading. In conclusion, bleaching systems can greatly influence fading of dye during their life-time of laundering. 
Another study led to the conclusion that the degree of fading in domestic washing progressively increases with the number of wash cycles for different dyed fabrics (29). Although the study was limited to wool fibers, the change in temperature and the effect of water on wool altered the durability of the wool fiber to endure. This can also be assumed for other types of fibers under similar conditions.

There are obvious limitations observed throughout the studies performed. Since most of the research was done outside the United States, the European studies do not adequately represent the US laundry settings. In each case, the water temperature, the length cycle of washing, and the detergents, differ from the US conditions of water temperature, the purity of the water, the duration of the washing cycle and the detergents found in the US markets $(30,31)$.

Another limitation observed in the studies was the partial explanation of the dye fading in the fabrics. The conclusions did not explain the mechanism of dye fading and no studies have attempted to analyze what exactly occurs during washing. For instance, can it be assumed that only the dye fades, or does the fiber age when washed? Most studies have not adequately explained that the dye has faded because of the washing, and not that the fiber has aged. If the fiber does age after laundry, then it can be expected that the color would also look faded. To demonstrate that the dye has faded, the dye would need to be extracted from the fiber and analyzed separately before any further conclusions can be gathered.

Fortunately, the CIE coordinates for a dye solution will be the same regardless of the concentration so long as the solution obeys Beer's law. This implies that it is not possible to distinguish between fibers lightly and heavily dyed with the same dye (1). The analysis of differentiating dyes is not of greater concern when attempting to determine whether two fibers 
are common or rare. It is the specific dye that was used to color the fiber that has the greater impact. The same dye solution used to color different fibers will not change because the substrate on which it is dyed is different. However, the concentration will be dependent on that substrate as each substrate might retain the dye solution differently. Therefore the necessity to know the washfastness of the dye over time, especially over aging and washing, is very important to aid in forensic comparison of color in fibers.

A recent attempt was made by Was-Gubala to determine the mechanism involving color change(32). This study evaluated a variety of textiles treated with different detergent solutions. The goal in this experiment was to assess various color related characteristics in fibers by subjecting the fiber to repeated long term laundry using different detergent solutions. Four different types of fibers and dyes were used: acrylic, cotton, polyester, and wool, dyed with basic, direct, dispersed and acid dyes, respectively. Three different colors for each type of fiber were analyzed: blue, dark grey and red. The detergents used were based on the brand name and compositional diversity found in Poland and other European markets. The fabrics were stored in separate plastic containers based on the recommended detergent solution over a 14 day period. Each detergent solution was changed and replaced with fresh solution on a daily basis. At the same time daily, small pieces were cut from each of the laundered sample, dried and analyzed by sensory analysis and fluorescence. Because visual examination is subjective, the necessity of objective results are preferred and therefore a second part of the same experiment was done using the MSP (33).

The MSP was used and the results confirmed the previous observational findings which indicated that the first detergent resulted in the most noticeable change in color. This was likely 
due to a potent, oxygen-based chemical whitener present in this formulation. And with the aid of fluorescence microscopy, the degradation of the color change could be readily observed. The greatest amount of color change was found in the red indirect dyes, which were used to dye cotton textiles.

Although there was substantial inter-variability of different types of fibers, there was limited mention of analysis for fiber intra-variability. The authors used four types of textiles and three different colors for inter-variability purposes, but they failed to reference the intra-variability within the textile and fiber. Observing intra-variability is also useful in monitoring the range of color change within a single fiber. Another noticeable limitation was the inadequate portrayal of a normal laundered cycle. The color change in the fibers might have been different due to the mere rubbing, turning and constant moving of clothes as it is being washed in an actual washing machine. Instead, the fibers were placed in separate containers and shaken regularly to depict physical movement. Lastly, the duration of the fabric being submerged in the detergent solution is not comparable to a normal cycle. Even with a heavy duty cycle which lasts approximately 35 to 40 minutes in the laundry, the fabrics were submerged for 24 hours. Initially, a difference in color change was expected as a function of extensive detergent exposure. On the other hand, lack of physical contact of clothes in washing, could have also influenced that change to a lesser extent.

A more realistic approach of washing was done to compare dye fading due to different washing machines. Rohwer's project compared dye fading of 14 dyes in a multi-wash test between Linitest and a normal washing machine (34). Lintiest is a simple machine that rotates eight closed 500ml beakers in a temperature controlled water bath. Unlike a regular washing machine, 
Linitest is limited in its mechanical action of slipping, rubbing and tumbling of textiles through washing. For the washing cycle, three different detergents were used: non-bleach, high bleach and new bleach. The main ingredients of the bleaching systems were the same; however, the difference among them was the content of the bleach. While high bleach referred to the inclusion of TAED, new bleach referred to the addition of a catalyst with TAED. The bleach catalyst 'Clariant' was added to the high bleach solution to create the 'new bleach'. The 14 dyes were washed 20 times collectively based on the similar shade in color among the dyes to minimize any influence of dye transfer or bleed.

The results obtained were in collaboration from six independent laboratories. A poor correlation was observed between the washing machine and Linitest in the non-bleaching environment. There was significant fading in the washing machine of certain dyes than in the Linitest. This difference was only observed at lower washing temperatures. In contrast, the correlation in the high bleach system was better. A larger quantity of the dyes faded in the actual washing machine. In the new bleaching system, the only difference observed was the fading profile. Since a catalyst only increases the rate of the reaction without changing it, the fading profile of the bleaching system was unexpected. Even with a catalyst added to the system to influence the results, only one dye exhibited substantial fading. The mechanism behind this reaction requires further evaluation and study. Three factors were determined to predict dye fading in washing operations: bleach, temperature and mechanical action from the washing machine. While both instruments identified the same fading ranks of the dyes, the full extent of dye fading was more apparent in the actual washing machine than in Linitest. 
The research showed that color fading gives a descriptive result when done as realistically as possible. Although fading was observed in the laboratory setting, it denies portraying truthful results as one would obtain outside the lab. One limitation apparent in this and the previous studies is the inadequate representation of the US laundry and the bleaching condition. There was also incomplete information about the fiber dyes. Knowing the dye and its bonding mechanism to the fiber will assist in understanding the intensity of the color change that will occur.

McLean's experiment focused on the interaction of textiles and bleach under North American conditions (31). To modify these conditions, the bleach activator TAED was replaced with nonanoyloxybenzene sulfonate (NOBS). Both bleaching systems attempt to perform similar results, but both are also influenced by temperature, duration of wash cycle, volume of water and concentration of detergent for cleaning performance. Seventeen commercial dyes were washed for a total of 50 cycles under normal US conditions with both bleaching systems. It was concluded that certain dyes reacted differently or are more sensitive to one bleaching system than the other and that the recommended US conditions of activated bleaching system NOBS is more favorable for the analysis of color fading in the US.

This gateway that allows for accurate analysis of color fading under the US conditions was only peer-reviewed but not published. No validation of the results have been presented and confirmed by the author. Other peer reviewed articles have agreed that NOBS appears more robust than TAED in industrial textile bleaching applications because of its excellent solubility in water, and that the minimal need to control the $\mathrm{pH}$ of the water its gives superior bleach performance (35). 
Modern research has incorporated the modified COX conditions. This method is used to identify colored cotton fabrics susceptible to fading by laundering with detergents containing activated bleach(30). It focuses on comparing the two primary bleach activators, TAED and NOBS, and their effects on fibers. Seventeen dyes were chosen as a representative of the commercial dye class which ranged from acid, basic, direct, disperse, reactive, sulfur and vat dyes, and were subjected to 50 wash cycles using the two detergents. The results indicated that the dyes were affected by both bleaching systems; however, most were observed to be sensitive to the beach activator TAED. Thus, the modified version of COX, replacing TAED with NOBS, was recommended by the authors.

Other recent studies evaluated the reproducibility of washfastness in cotton and polyester fibers within six laboratories (36). The samples were washed under four different conditions to observe the range of washfastness of the dyes in the fabrics. These conditions varied in temperature, $\mathrm{pH}$, and additions in the base detergent used. The results of the color change were recorded by a gray scale value that was visually determined. Although there were observed differences in the absolute values of color change, the results were consistent in the sensitivities of the dyes. Successfully, the reproducibility for the fibers averaged over the participating laboratories. Other studies focused on the population of fibers recovered from washing machines and evaluated their persistence, not necessarily their color change(37).

Scientists realized that the fading of colors (or dyes) due to washing is still an important issue (38). Because of this, it is important to accurately represent the family of textile fibers by subjecting them to similar aging, washing and storing conditions as an individual would and gathering objective discriminatory data that greater influence the analysis of fibers. It is 
important to further observe color fading in fibers, normal wear and tear, and aging of the fiber under environmental conditions.

Later studies have been concerned with the degradation of color in which environmental conditions, such as light and temperature, plays a major role. Some of these studies have been directed towards monitoring the degradation of paintings over time through aging $(39,40)$. The study performed by Marengo et. al evaluated the conservation state of paintings by understanding one of the natural cause of degradation in paintings: its exposure to sunlight. To evaluate this phenomena, a cotton canvas strip painted with a natural pigment (Alizarin) was exposed to UV light $(\lambda=254 \mathrm{~nm})$ for a total of 276 hours. At every 12 hours of exposure, the irradiation was interrupted and the IR spectra were obtained using the Attenuated-TotalReflectance Fourier Transform IR (ATR-FT-IR) spectroscopy instrument. Results showed that exposure to UV light produced some changes in the loss of gloss on the sample surface and that the aggression of the UV light caused the degradation (oxidation) of the pigment and the cotton canvas.

Similar conclusion was observed in a later research performed by the same authors (41). Ten mixtures of three organic pigments (Alizarin, Permanent Red and Phtalocyanine Green) were prepared, mixed with linseed oil and spread on 10 canvas strips, to represent the typical complexity of a real painting. The canvases were irradiated with UV light $(\lambda=254 \mathrm{~nm})$ for a total of 272 hours. Regular exposure was interrupted every 16 hours for analysis using the ATR-FT-IR spectroscopy. With the aim to individuate color difference between treated and untreated samples, no color change, but only a loss of gloss on the surfaces of the paintings was observed after UV treatment. 
The authors performed another related study by using the FT-Raman instrument to investigate color change on the surface of canvases exposed to UV light (42). Raman spectroscopy was used because of its ability to monitor changes without the necessity of sample preparation and its lack of destruction towards the samples. Unlike the previous studies, the intensity of the UV exposure was lessened. Red and blue colored pigments mixed with linseed oil (1:1, w/w) were painted onto a cotton canvas and exposed to UV light $(\lambda=234 \mathrm{~nm})$ for a total of 12 hours. The radiation was interrupted every 45minutes and the Raman spectra was recorded. Macroscopic changes could be observed in two of the colored pigments; the blue pigment faded and became opaque and the red pigment became darker and opaque. It was estimated that both the samples became opaque because of the degradation of the linseed oil.

In summary, a review of the current literature shows that color changes can be observed when a sample has been exposed to UV aging for a period of time. Although the notice of color change is not always evident, the degree of fading increases as the fiber is gradually exposed to any environmental conditions.

\section{Materials and Methods}

\section{$\underline{4.1 \text { Materials }}$}

\subsubsection{Textile materials used}

The type of fiber chosen for this experiment was $100 \%$ polyester because of its popularity and widespread use. Seventeen polyester shorts were bought from local Wal-Mart stores (Morgantown, WV). These shorts were all new and were never processed or worn. The two brand names bought were Danskin Now ${ }^{\circledR}$ and Garanimals ${ }^{\circledR}$. The Danskin Now ${ }^{\circledR}$ products only consisted of polyester shorts are dyed with.., but the Garanimal ${ }^{\circledR}$ products were both polyester 
and cotton skirt-short style shorts dyed with pigment print. The Garanimal ${ }^{\circledR}$ skirts were $100 \%$ polyester and the under-shorts were $100 \%$ cotton. The portion of the cotton fabrics was not analyzed.

These two brands were chosen because of its low-cost, variability in colors and accessibility. Each item was bought in quantities of two or three. Table 5 describes in detail the information about the shorts and Table 6 describes how the shorts were labeled for this project.

Figure 9: Photograph of the Polyester shorts used
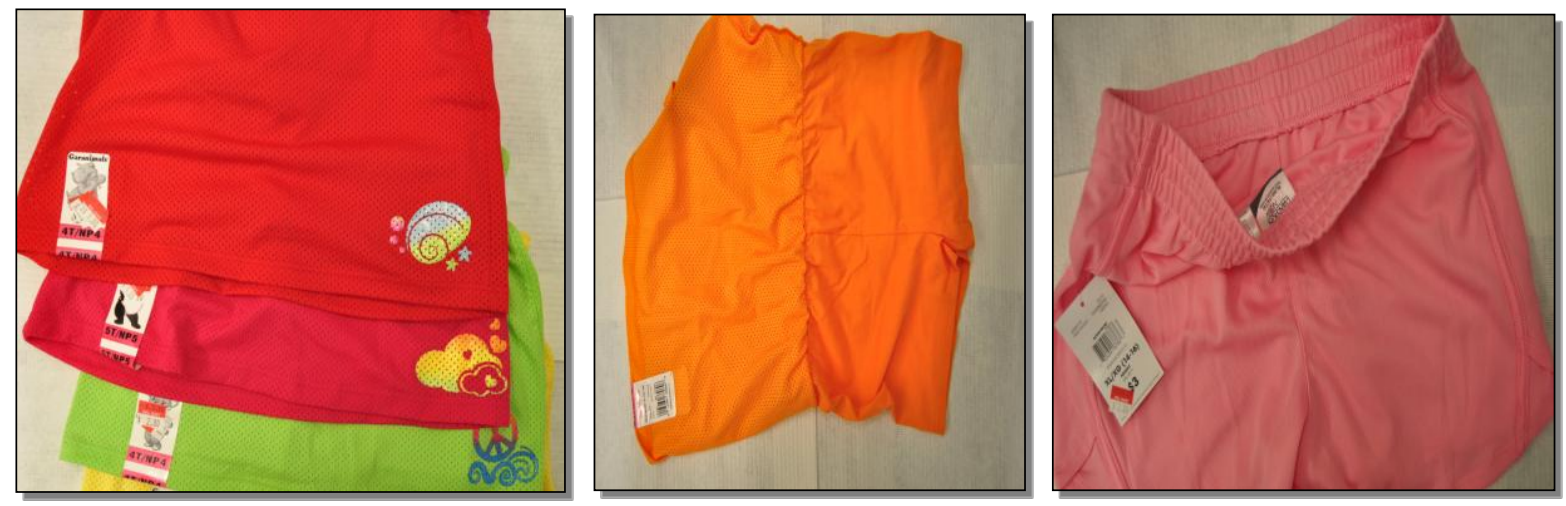

Note: Garanimals ${ }^{\circledR}$ style shorts left two pictures and Danskin Now ${ }^{\circledR}$ at right.

Table 5: Polyester shorts used in the project

\begin{tabular}{|l|l|l|l|l|l|l|l|}
\hline Brand & $\begin{array}{l}\text { Fiber } \\
\text { Type }\end{array}$ & Total & Sample name & Style & UPC & Size & $\begin{array}{l}\text { Manufactured } \\
\text { in }\end{array}$ \\
\hline G & P & 2 & $\begin{array}{l}\text { Orange solid } \\
\text { dazzle short }\end{array}$ & $\begin{array}{l}\text { GAB } \\
2103001\end{array}$ & 01326486542 & $18 \mathrm{M}$ & Honduras \\
\hline G & C \& P & 2 & $\begin{array}{l}\text { Green mesh short } \\
\text { w/ PRT WB skirt }\end{array}$ & $\begin{array}{l}\text { GAG } \\
2102031\end{array}$ & $\begin{array}{l}01326486956 \\
01326486954\end{array}$ & $\begin{array}{l}24 \mathrm{M} \\
12 \mathrm{M}\end{array}$ & Honduras \\
\hline G & C \& P & 2 & $\begin{array}{l}\text { Red mesh short } \\
\text { w/ PRT WB skirt }\end{array}$ & $\begin{array}{l}\text { GAG } \\
210203 \mathrm{~T}\end{array}$ & 01326487346 & $\begin{array}{l}4 \mathrm{~T} / \mathrm{N} \\
\text { P4 }\end{array}$ & Honduras \\
\hline G & P & 3 & $\begin{array}{l}\text { Dark pink mesh } \\
\text { short w/ PRT WB }\end{array}$ & $\begin{array}{l}\text { GAG } \\
2102031\end{array}$ & 01326486990 & $12 \mathrm{M}$ & Honduras \\
\hline G & C \& P & 2 & Purple mesh & GAG & 01326486985 & $18 \mathrm{M}$ & Honduras \\
\hline
\end{tabular}




\begin{tabular}{|l|l|l|l|l|l|l|l|}
\hline & & & short w/ PRT WB & 2102031 & 01326486984 & $12 \mathrm{M}$ & \\
\hline DN & $\mathrm{P}$ & 3 & $\begin{array}{l}\text { Pink magic } \\
\text { active short (w/ } \\
\text { gray sides) }\end{array}$ & DG20B006 & 66046913740 & $\begin{array}{l}\text { L/G } \\
10- \\
12\end{array}$ & China \\
\hline DN & $\mathrm{P}$ & 3 & $\begin{array}{l}\text { Pink magic DN } \\
\text { color block athltc } \\
\text { short (w/ gray } \\
\text { sides) }\end{array}$ & DG20B006 & 84391301744 & $\begin{array}{l}\text { XL/ } \\
14- \\
16\end{array}$ & Jordan \\
& & & & & \\
\hline
\end{tabular}

Legend: C-cotton; DN-Danskin Now, G- Garanimals, P-polyester

Table 6: Reference labels for the Polyester short.

\begin{tabular}{|l|l|}
\hline Reference name (labeled as) & Item's actual name \\
\hline GMP & Polyester shorts \\
\hline RMP & Polyester shorts \\
\hline PMP & Polyester shorts \\
\hline OSDP & Orange Solid Dazzle Polyester short \\
\hline DPMP & Dark Pink Solid Dazzle Polyester short \\
\hline PMJX & Pink Magic (Danskin Now polyester shorts )Jordan size XL/ S/ S sides \\
\hline PMCL & Pink Magic (Danskin Now polyester shorts) China size L \\
\hline
\end{tabular}

In total, five different colors were analyzed in this experiment: green, orange, pink, purple, and red. The colors were selected to represent the low, medium and high energy ranges of the visible spectrum. Three pink colors of varying shades were chosen to observe if the treatment would affect each sample similarly. Among the three, sample DPMP varied greatly in shade from the two other pink shorts. In fact, samples PMCL and PMJX were not easily differentiated. The difference between them was their manufacturing location: PMCL was made in China and PMJX in Jordan. This closeness in color was also evaluated for the discriminating power of the MSP. 
Figure 10: Similarity between reference PMCL (left) and PMJX (right) samples

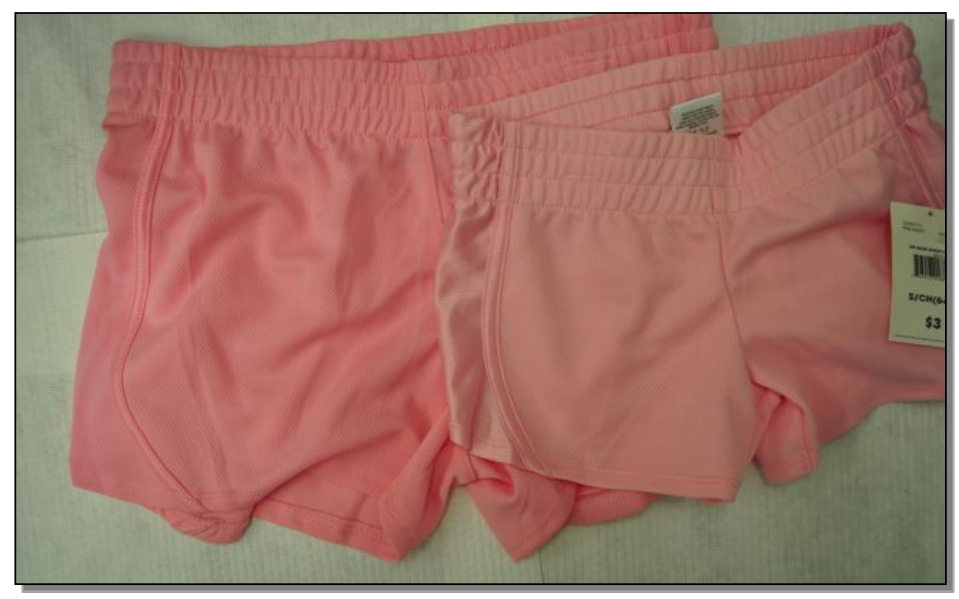

\subsubsection{Detergents tested}

The two detergents used for washing were Tide ${ }^{\circledR}$ (24 loads, original scent) and Tide ${ }^{\circledR}$ with bleach (18 loads, original scent). The oxygenated bleaching system was sodium percarbonate. This brand of detergent was chosen based on consumers' reviews of its effectiveness in cleaning (43). Both detergents were in powder form.

\subsection{Methods}

\subsubsection{Aging Treatment}

\subsection{1a Aged Standards}

For reference controls, 3 swatches of approximately $2 \mathrm{~cm}$ by $1.5 \mathrm{~cm}$ were randomly cut from each sets of shorts, and taped onto a Fisherbrand frosted microscope slide (size $25 \times 75 \times 1 \mathrm{~mm}$ ) at the sides using clear tape. The slides were labeled and numbered appropriately based on the item's reference name, and swatch number1, 2 or 3 . From each swatch, individual fibers were also extracted, then mounted with Entellan, a permanent mounting media, before being covered with 
a Fisherbrand cover glass slides (size 22x 22-2mm). These slides were also labeled in the same manner. These standards were then organized by color and stored in a microscope slide box. This box was immediately stored in the dark.

Figure 11: Photograph of the Prepped Aged Standards

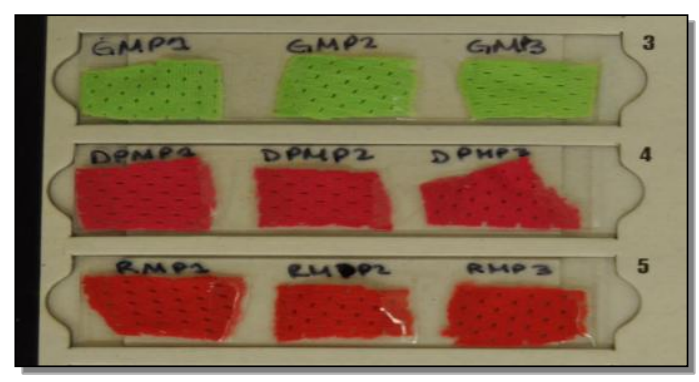

\subsection{1b Aged Samples}

One item for each color of shorts was used. From each garment, one large swatch was cut into three rectangular pieces and taped horizontally on the outer surface on the slide. To eliminate UV absorption from the glass slide, the samples were taped on Fisherbrand precleaned microscope slides (size 75 x 50 x 1mm) that were already completely covered with aluminum foil. The slide was then labeled on the bottom left corner ' $\mathrm{O}$ ' for 'outer-surface' using permanent marker. Small pieces of blank paper were taped on the opposing side in order to record the item's reference name and other information. As the samples were aged, the date and hours of aging were subsequently recorded.

\subsection{1c Aging Process}

Since seven slides were prepared where each slide had a total of three rectangular taped swatches, a total of 21 swatches of samples were aged during part one of this experiment. The source used to provide the aging treatment is discussed in section 4.3.1. These samples were aged for a total of 80 hours. At 16 hour intervals, the samples were removed, and fibers were 
extracted and preserved for MSP analysis. Three sample areas, A, B and C were chosen from every swatch. A miniscule amount of fibers were extracted from these sample areas and stored in $1.5 \mu 1$ eppendorf tubes. These tubes were appropriately labeled based on the reference name, the sample area, and the total hours aged. They were then organized by colors and hours aged and immediately stored in Ziploc ${ }^{\circledR}$ bags in the dark. Twenty-one eppendorf tubes were stored for each aged period totaling 105 eppendorf tubes with the extracted fibers for analysis.

\subsection{1d MSP Preparation and Analysis for Aged Samples}

For MSP preparation, small amounts of fibers were obtained from each eppendorf tube and mounted with Entellan on a Fisherbrand precleaned microscope slide (size 75 x 50x 1mm). Three mountings were performed on each slide to represent sample areas A, B and C. Three fibers were examined per sample area: one fiber was analyzed at two different locations on that fiber, and two fibers were analyzed at only one location (see Figure 12 below).

Figure 12: Preparation of Aged Samples for MSP analysis

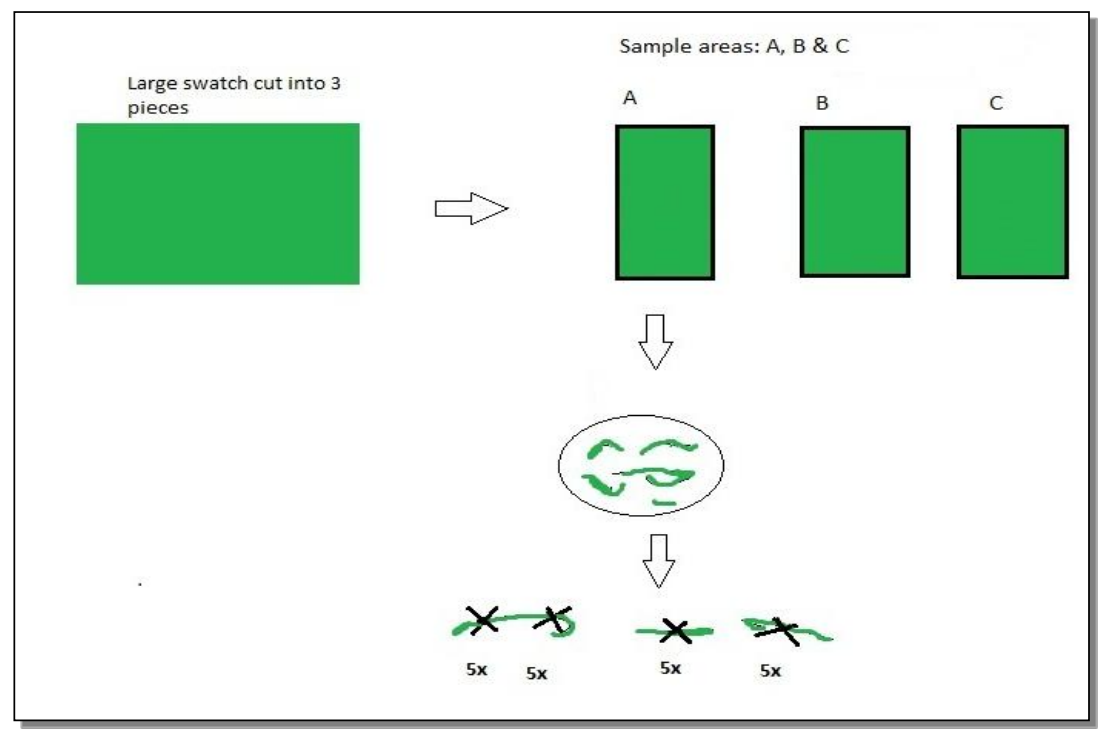

Note: Green swatch represent reference sample GMP 
The first fiber was used to evaluate fiber intra-variability and the latter two fibers were used to evaluate inter-variability among neighboring fibers. A minimal amount of fiber intra-variation is expected from manufactured fibers; therefore, only one fiber per sample area was analyzed at two different locations. Analyzing three different sample areas from the same garment enables inter-variability determination across that garment.

All analysis was done in replicates of five; the measurements were performed simultaneously without moving the stage or removing the samples between measurements. This replicate of five simultaneous measurements accounts for any instrumental variability. The sample mounting can be seen in Figure 13a below.

Figure 13a-b: The mounted samples for further analysis with the MSP

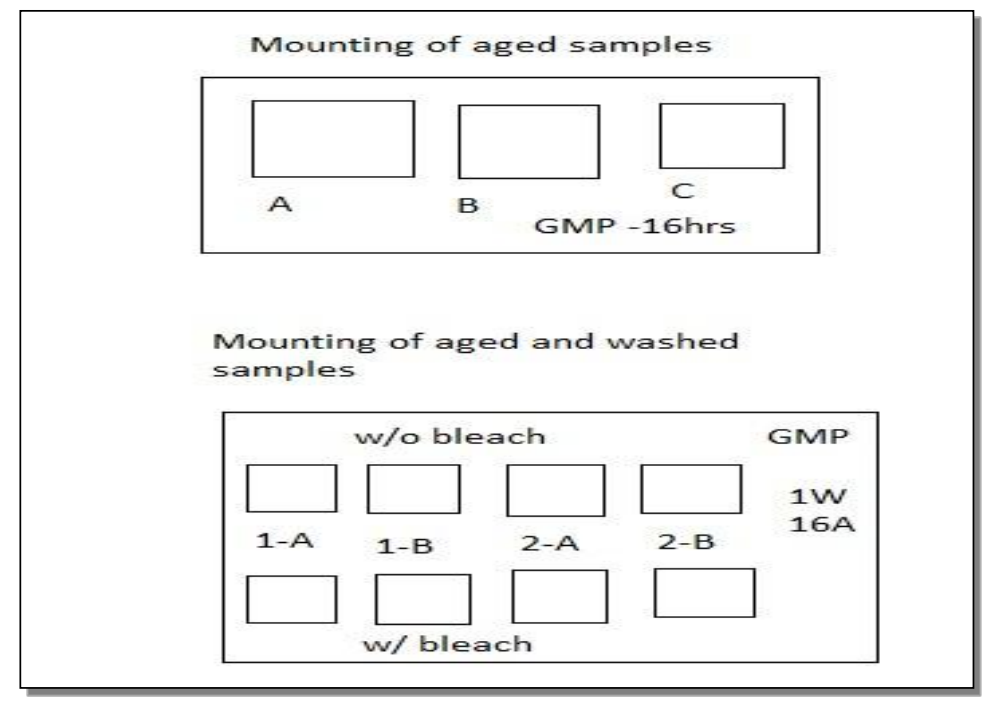

Note: The top image (a) represents the aged samples; the bottom image (b) represents the washed and aged samples. ' $W$ ' represents 'Washed' and 'A' represents 'Aged'.

\subsubsection{Washed-Aged Treatment}

\subsection{2a Washing Process for Washed Standards}


A pretrial washing was done with both detergents to determine if any color bleeding would occur among the light or dark colors being washed together. All washings were performed using a Kenmore 70 Series Heavy Duty Plus washing machine set for a normal wash cycle (cotton sturdy cycle), with the water level set to medium, and the water temperature set to warm-warm (wash-rinse). The samples were washed with approximately 15 to 20lbs of similar color laundry to incorporate the mechanical action from rubbing, spinning and tumbling of clothes during washing. Four large swatches were cut from each item; two were washed with bleach and the other two washed without bleach. Since samples PMCL and PMJX could not be easily differentiated visually, samples PMCL were cut in the middle to prevent any error and confusion. Two ounces (line 1 from the measuring scoop) were used for washing and kept constant throughout the experiment. After washing, the samples were air dried in the dark to lessen any exposure to light.

\subsection{2b Preparation and analysis for Washed standards for MSP analysis}

Once the samples were dried, fibers were extracted and mounted in the same manner as the aged samples (Figure 13b and 14). Although the aged samples analyzed fibers at three sample areas, only two were observed for the washed-aged treatment. This was done for efficiency and comparison purposes. Figure 14 below diagrams this sampling procedure. Similar to the aged analysis, three fibers from each swatch were analyzed in replicates of five: the first fiber was analyzed at two different locations, and the last two fibers were analyzed at one location each.

\subsection{2c Washing Process for Washed-Aged Samples}

From each reference garment, four medium size swatches were cut from different places and labeled swatch one and two, for each detergent. To differentiate the swatches, swatch one was 
cut diagonally at one corner, and swatch two was cut diagonally at both corners (see Figure 16). PMCL samples were also cut in the middle to differentiate it from PMJX samples. The swatches were stored in manila envelopes and grouped by detergent, and labeled either 'With bleach' or 'Without bleach'. The samples were then washed using the same parameters set during this project. Immediately after air drying, the swatches were then aged for 16 hours. Aluminum foil was placed at the bottom of the UV box and using a permanent marker, a line was drawn to use as a divider between the bleached and non-bleached detergent samples. This washed-aged cycle repeated until the total number of washing was equal to five and aging equal to 80 hours. The samples were washed and aged once per day around the same time. After analysis, the samples were stored in its manila envelope in the dark until the next day.

The labeling on the microscope slide was abbreviated as follows: 1W 0A- the sample was washed once but was not aged; 1W 16A- the sample was washed once and aged for 16 hours; 2W 32A- the sample was washed twice and aged for 32 hours...; 5W 80A- the sample was washed five times and aged for 80 hours.

\subsection{2d MSP Preparation and Analysis for Washed-Aged Samples}

Fibers were extracted from each swatch at two sample areas, A and B. These sample areas were on extreme sides of each swatch for separation purposes and remained consistent throughout most of the experiment. Note that between swatches, sample areas may have differed by location. Fibers were randomly collected from the outer surface of the swatch, mounted, and labeled according to its reference name and the number of the washed-aged cycles (see Figure 13b). Similar to the analysis of the aged samples, three fibers were analyzed. The first fiber from each sample area was analyzed at two locations on that particular fiber, whereas the last two 
fibers were only analyzed at one particular location. These measurements were performed in replicates of five, totaling 20 measurements. Thus from every cycle, a total of 40 measurements were collected per swatch.

Figure 14: Preparation of Washed-Aged Samples for MSP Analysis

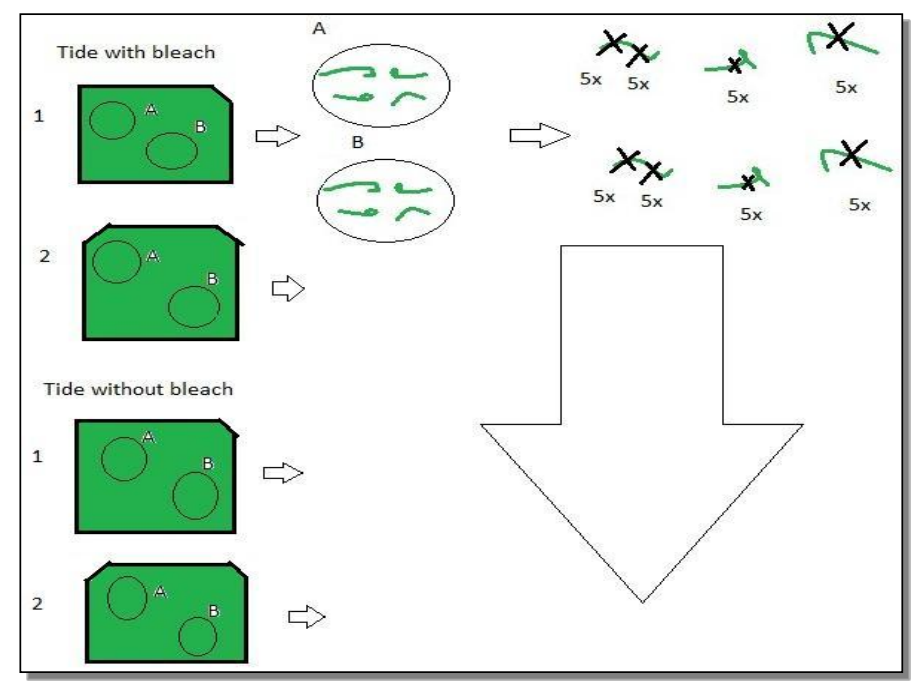

\section{$\underline{4.3 \text { Instrumentation }}$}

\subsubsection{UV lamp}

The UV lamp used for artificial lighting was manufactured through the American Ultraviolet Company (model CE-6-BL, $120 \mathrm{~V}, 60 \mathrm{~Hz}$ and 194Amps). It emits wavelengths of $405 \mathrm{~nm}$, $440 \mathrm{~nm}, 550 \mathrm{~nm}$ and $580 \mathrm{~nm}$ with a power of $23 \mathrm{~W}$. Mercury bulb was used as the light source. The UV lamp was assembled into a built wooden box with an integrated built-in timer. A $9^{1 / 2} \times 4^{1 / 2}$ in opening in the wooden cover of the UV box, sealed with black Styrofoam-like material was created for easy insertion and removal of the UV lamp. When the box was closed, the dimensions were 18 in x 14 in x 7in in length, width, and height, respectively. The inside 
dimensions were $16^{5 / 8}$ in $\times 12^{5 / 8}$ in x 6 in. The height of the UV lamp from the sample directly below was $6^{3 / 4}$ in.

Figure 15: UV lamp and box used for artificial aging
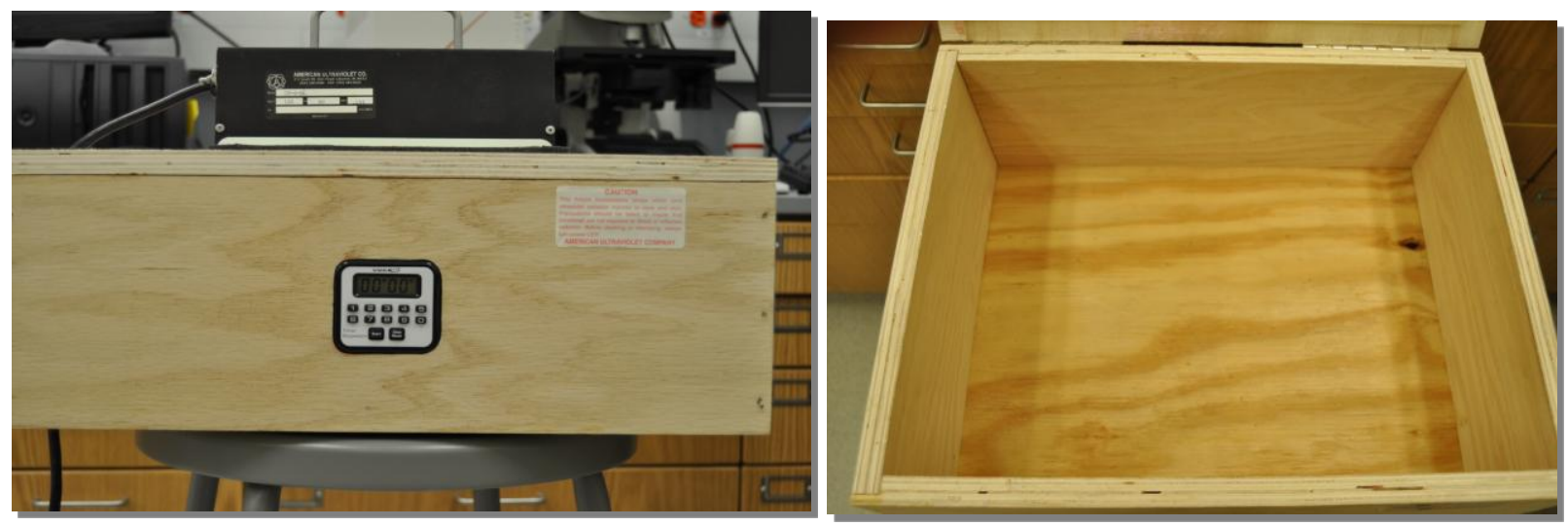

The samples were artificially aged by exposing them to UV light. The simulation of the exposure to sunlight was obtained by exposing the samples surfaces to the UV light to produce aging as it would occur through normal wearing and fading of fibers (41).

\subsubsection{Stereomicroscope}

The stereomicroscope used to initially observe and extract the fibers during mounting was the Leica EZ4 [Leica Microsystems (Schweiz) AG; with input of 100-240V, 15W and 60HZ]. Because of its ability to magnify an object between $10 \mathrm{X}$ to $40 \mathrm{X}$, the magnification was effective for fiber extraction. No further analysis was performed with this instrument other than to initially observe the samples for any contamination (especially when washed) and to extract the fibers from its respective swatches.

\subsubsection{Ultraviolet - Visible- Near Infrared Microscpectrophotometer}


The instrument used in this research experiment was the QDI 1000 ultra-violet- visible- near infrared Microspectrophotometer (serial number 20040122A1). The light source was provided by Leica DMRX (12Vmax at 100W). The transmission power supply necessary for analysis in the transmittance mode was received from a $75 \mathrm{~W}$ xenon bulb. The data acquisition software used was the CRAIC MSP created on the 14Sep 07. The image acquisition software used during this experiment was the Image Control (IC) Capture 2.0 (version 2.0.0.290).

\subsection{3a Measurement of samples using the MSP}

The MSP was operated in transmission mode (95W xenon source) using the 36x objective. Spectra were collected by taking an average of 50 scans over the spectral range of $350-850 \mathrm{~nm}$, with a bandwidth (resolution factor) of 10. The integration time was adjusted to the optimized value obtained during daily calibration and this value was recorded each day. The purpose of integrating the instrument is to maximize sensitivity of the measured signals approaching the detector. This improves the signal-to-noise ratio and the precision of the photometer. The integration period is analogous to the shutter speed of a camera. The higher the value specified, the longer the detector collects the incoming photons. Aperture size of the MSP was 10x10 microns. The UV-Vis spectra were recorded from three randomly selected fibers from each mounting. Five replicates of spectra were taken for each fiber and by using the IC Capture Image software; one image per sample was photographed and stored. CIELab results were also obtained from each spectrum using the GRAMS/AI system (version 7.02). The settings used to obtain color analysis were as follows: standard observer 10 degree, illuminant CIE A, calculation type CIE L*a*b and spectral range 360-830nm.

\section{$\underline{4.3 .3 b \text { Instrument Calibration }}$}


The instrument was daily calibrated before use. Craic Technologies issued the National Institute of Standards (NIST) traceable standard sets to be used for calibration and the expected calibration results including the uncertainty values. The calibration set used throughout this experiment was CAL2710 (Craic Technologies with recertification due Sept 25, 2011). The purpose of calibrating the MSP is to increase the productivity of the instrument, by assuring consistency and precision to guarantee that the results are valid and reliable in spectral measurements. Daily calibration ensures proper system functioning and will help to detect systematic errors that might occur. The instrument was calibrated to manufactured instructions.

The QDI Image and Data Acquisition Software instrumentation were turned on for thirty minutes before any analysis was done, to allow the lights from the system to warm up. The reference filter was placed on the stage in sharp focus, and remained in that location during the entire calibration process. The instrument was first optimized to set the instrument parameters. Once the instrument is successfully optimized, a dark and reference scans were then performed. A dark scan is taken when the light is blocked to the spectrometer head, therefore only allowing the noise of the instrument to be recorded. In the reference scan, light is allowed to the spectrometer head. The reference scan accounts the light intensity, and the blank or microscope slide that is also analyzed before any absorbance, transmittance, or reflectance spectra can be obtained. In the MSP, calibrations are required in three areas:

1. Wavelength accuracy and spectral resolution

2. Absorbance and spectral linearity

3. Colorimetry 


\section{Wavelength accuracy and spectral resolution}

Wavelength accuracy over the visible region can be checked using holmium oxide glass and didymium glass filters because these filters both have some narrow absorption bands. The spectrometer is calibrated in the spectral range from $240 \mathrm{~nm}$ to $650 \mathrm{~nm}$. Once completed the blank and reference scan is performed, the wavelength calibration can be executed. To initiate the wavelength calibration, the holmium filter is placed on the field diaphragm of the microscope stand. Once the sample is analyzed, the filters are then switched and analyzed. After the filters have been analyzed, results are stating if the instrument is within established calibrating limits.

\section{Absorbance and spectral linearity}

Standardization of absorbance is required particularly if colorimetry is to be used for recording and comparing color. Accuracy can be controlled using optical density (OD) filters OD0.1, OD0.5 and OD1.0. OD filters reduce the amount of energy transmitted through; higher OD values indicate very low transmission and lower OD values indicate high transmission. Therefore, OD 0.1 filter will have a higher transmission and OD 1.0 will have a lower transmission. This system which obeys Beer's Law can roughly indicate the spectral linearity of the system. The calibrating procedure performed was similar to the wavelength calibration procedure. The filters were placed on the field diaphragm and analyzed. Once all three filters were measured, the results of the analysis were produced, which affirmed if the instrument is within established calibrating limits.

\section{Colorimetry}


Three set of filters blue, green, and red must be used to calibrate its respective area of the spectrum for the accuracy of the tristimulus values and the chromaticity coordinates. However, this calibration was not performed because of the unavailability of these filters. For this reason, the instrument was instead validated with red, green and blue fibers.

\section{$\underline{4.3 .3 c \text { Validation }}$}

To validate the instrument, blue, green, and red color fibers were analyzed once per day for two weeks. Each sample was examined in transmittance mode in replicates of five. In total, 150 measurements were obtained for each colored fiber. CIELab values were obtained using the GRAMS/ AI under the same parameter settings. Fibers were photographed only once per analysis and stored in the same folder with its corresponding spectra. These values were stored in the hard drive of the computer and later copied to a personal computer as backup files.

\section{$\underline{4.4 \text { Statistical Analysis }}$}

The first step in any data analysis is to inspect the distribution of the data. To ensure proper analysis, the data was screened to detect missing values, outliers, and departures from assumptions on which the analyses were based.

4.4.1 Descriptive statistics- although there are many descriptive statistics available, only ones of interest were mentioned here: distribution, median, percentiles, quantiles, quartiles and variables. Distribution is the amount (spread) and pattern (shape) of variation, both characterized by the average and typical value (location). Median is the $50^{\text {th }}$ percentile, which conveys information about what a typical value is. It is informative to compare it to the mean, as these two statistics will provide information about the symmetry of the distribution. Percentiles are the division of 
the total observations into 100 equal parts. Quantiles are the division of the observations into $n$ equal parts and is the fraction of the observations that are at or below this division. These cutpoints are referred to as quartiles. Q1, Q2 and Q3 correspond to the $25^{\text {th }}, 50^{\text {th }}$ and $75^{\text {th }}$ percentiles or $.25, .50$, and .75 quantiles. Variables are attributes that can be measured and that varies between subject, place or time. When measured, a number is assigned to each of its levels.

4.4.2 Graphical summarization- Two plots were used to help assess and describe the distribution of the data:

4.4.2a Box and whisker plot: is a type of graph showing the distribution of a set of data along a number line. It describes any type of population, regardless of the data's distribution(44). The data is described in five parameters: mean, upper and lower extremes or values and upper and lower quartiles. The mean of the data is plotted as a dot; the box is delimited by quartiles of the upper and lower means and standard deviations, and the whiskers by the extremes of the ninety-five percent confidence interval. Note that the default settings for a typical box plot differ from the settings used in this experiment. With these graphs, outliers are easier to visualize because they are plotted as separate dots. Several box and whisker plots can be plotted simultaneously on the same graph for easier observation of several variables. An example is given below.

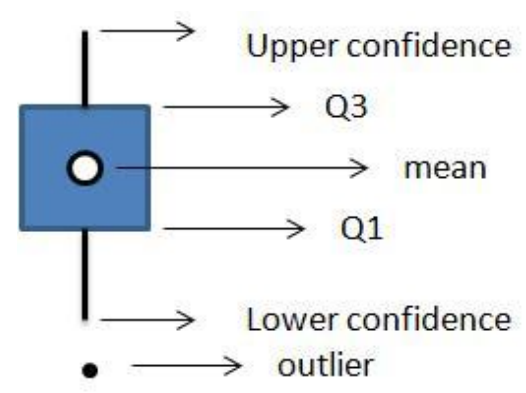


4.4.2b Histogram: is a type of bar graph representing the frequency distribution of a data. It was employed because the parameters of a distribution can be estimated from the plotted graph. This graph depends on the width of the bins, or class intervals that are based on how many dividing points there are and where they are made. The height of each bar gives the frequency in its respective interval. The histogram graphically displays the data obtained (x-axis) against the frequency of those values (y-axis).

4.4.3 Assumptions- Three assumptions were made in regards to the data obtained:

4.4.2a Independence: occurs when a random sample, as a subset of the population, is chosen in such a way that any subset of equal size is equally likely to be chosen. Any two observations in a random sample are statistically independent of each other. Since the fibers were randomly chosen from each garment for analysis, the results are then independent from each other.

4.4.2b Homogeneity of variances: variance estimation from two samples is made to infer about the two populations. This assumption states that the groups are similar in essence regardless of the independent variables and that the variances are equal. This is often referred to as homogeneity of variance. Homogeneity of variance exists when $H_{0}: \sigma_{1}{ }^{2}=\sigma_{2}{ }^{2}$. There are several test procedures that can be used to check the equal variance assumption; however, the Levene's test was employed because it is one of the most frequently used.

i. Levene's test: checks whether or not the variances of the groups being analyzed are statistically different. It has the advantage that the samples being drawn from a normal distribution is not assumed. The Levene's test evaluates the deviations around the median in each group, instead of the variances across the group. The larger the 
deviations in one group in comparison to the others, the greater the spread. Larger spreads increase the difference in variability within the population from which the samples are being drawn. If the results show a value $\leq 0.05$, then it can be concluded that the variances are significantly different, and that at least one of the samples in the test has a significantly different variance. When significant differences are observed in group variances, then the Welch test was applied.

I. Welch test: when samples have different standard deviations, there is a greater likelihood that the test will reach incorrect conclusions. The Welch correction is an approximate degree of freedom solution that not only depends on the sample size, but also on the sample variance to assess the significance of the t-statistics computed (45). For the Welch test, two hypotheses were tested:

- $\mathrm{H}_{0}: \mu_{1}=\mu_{2} \ldots=\mu_{\mathrm{k}}$

- $\mathrm{H}_{\mathrm{A}}: \mu_{\mathrm{i}} \neq \mu_{\mathrm{j}}$, for some $\mathrm{i}, \mathrm{j}$.

Consider two populations A and B which in this project could be red and green fibers. Assume that we have two independent samples: Swatch 1 with 50 observations from red, and swatch 1 with 45 observations from green. The appropriate test statistic is

$\mathrm{W}^{*}=\left(\overline{r e d}-\overline{\text { green }}=\sqrt{\left({\frac{\mathrm{S}_{\text {red }}}{50}}^{2}+\frac{\mathrm{S}_{\text {green }}}{45}\right)}\right.$

Where $\mathrm{W}^{*}$ is approximately $\mathrm{t}$-distributed with $\mathrm{F}_{\mathrm{w}}$ degrees of freedom and $\mathrm{S}^{2}$ is the sample variance of either groups: 


$$
\mathrm{F}_{\mathrm{W}}=\left({\frac{\mathrm{S}_{\mathrm{red}}}{50}}^{2}+{\frac{\mathrm{S}_{\text {green }}}{45}}^{2}\right)^{2} /\left({\frac{\mathrm{S}_{\mathrm{red}}}{50^{3}-50^{2}}}^{4}+{\frac{\mathrm{S}_{\text {green }}}{45^{3}-45^{2}}}^{4}\right)
$$

The Welch test for testing equality of the population means compares the statistic $\mathrm{W}^{*}$ to the $\mathrm{F}$ distribution. The Welch test rejects the null hypothesis if $\mathrm{W}^{*} \geq \mathrm{F}_{50-1}$, 45-1,1-a. If the population means of red fibers in swatch 1 equals that from swatch 1 of the green fibers, (although the standard deviations

4.4.2c Normal Distribution(47)- the pattern for the distribution of a dataset in which the curve is bell-shaped. When the distribution is normal, reliable and valid inference and prediction can be drawn about the population from the subset samples. The normal distribution has two parameters: the mean tells where the central point of the distribution lies, and the standard deviation tells how wide the distribution is spread. To access whether a sample comes from a normal distribution, the graphical approach of the normal quantile plot, plus two statistical tests for normality were employed.

i. Normal Quantile plot: a graphical technique for assessing normality. Each ordered observation is plotted against the value it would be expected to assume if the data came from a standard normal distribution or normal quantile $(\mu=0, \sigma=1)$. The resulting graph is two-dimensional with the property that if the random sample comes from a normal distribution, the plotted points appear to lie in a straight line (48). It is a mapping between the distribution of the data and the ideal distribution. This graph is easy to understand by just examining the plot to check linearity(49). Any non-linear plot would suggest that the data did not come from a normal distribution. An example of this plot is inserted below. 


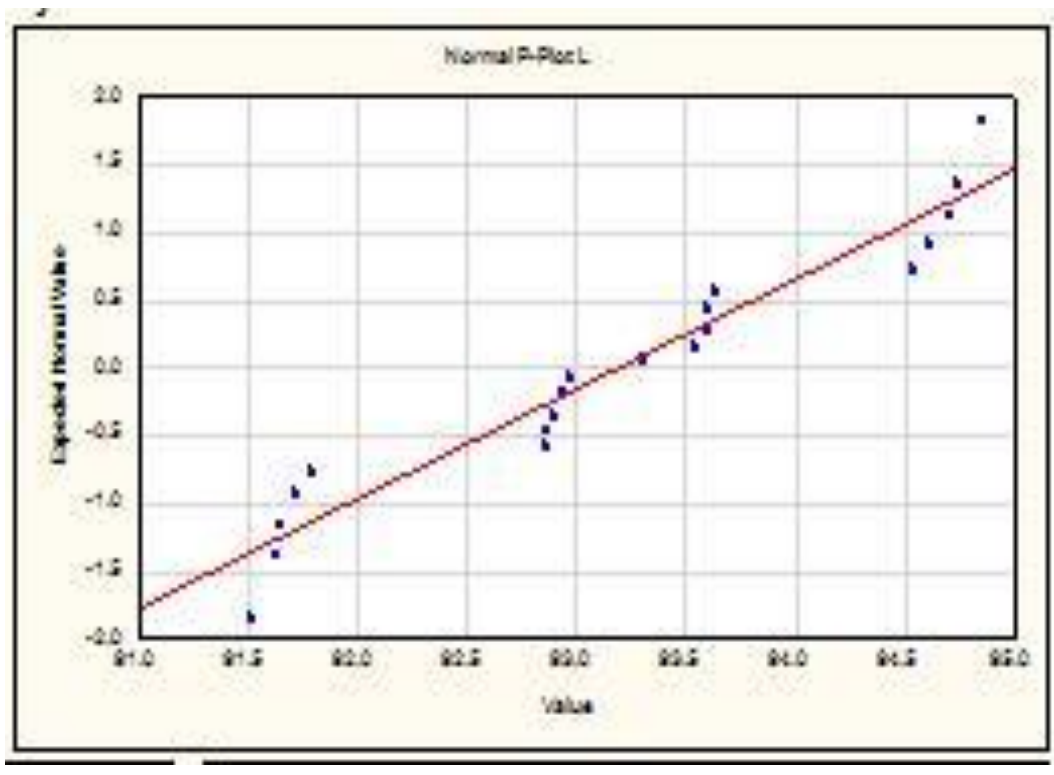

Say for my red fibers, I analyzed four different areas on swatch 1 , and for each area five measurements were taken. As these values are plotted, it first can be observed that the values between each groups varies. Also, within each group, the plotted points observed little intravariability. As the measurements approaches linearity, or that the points fall on the line, suggests that the measurements taken from the swatch comes from a normal distribution.

ii. Kolmogorov-Smirnov (K-S): a test that is often used to test the normality of a distribution. The testing statistic ' $d$ ' is the maximum difference between the empirical distribution (an estimation from the data) and the theoretical distribution (a standard normal distribution), to assess if the proportion difference is due to chance alone(50). Previous studies have reported that the $\mathrm{K}-\mathrm{S}$ test is liable in producing type II errors of not rejecting the null hypothesis when it is indeed false, when the parameters of the distribution are estimated from the same dataset (51-53). The K-S test is appropriate to use when the parameters of the hypothesized distribution is known. In other words, the hypothesized distribution is taken from a normal distribution with known mean and standard deviation. However, if this distribution is unknown, a Lillefors modification of the K-S test must be employed(54). The Lillefors test employs the same statistical method as the K-S test but the critical values are adjusted based on the estimated sample used and not affected by the estimated population 
parameters(55). The null hypothesis of a normal distribution is rejected at a given significance level when the testing statistic exceeds the critical value.

iii. The Shapiro-Wilk (S-W): a test that is another powerful alternative method used to determine normality because it can be applied against an extensive range of alternative distributions and sample sizes (54-56). It is statistically similar to the Lillefors test. The S-W test is based on the fact that the correlation coefficient of the normal probability is a measure of the strength of the linear relationship between the empirical and normal quantiles (48). The tested statistic ' $\mathrm{W}$ ' ranges from zero to one, where one is the maximum value that occurs when all the data points on the normal $\mathrm{Q}$ plot fall on a straight line. Its correlation coefficient depends upon the sample size, which must fall below the critical value in order to reject the hypothesis of normal distribution. If ' $\mathrm{W}$ ' is small, the null hypothesis is then rejected.

4.4.2d Interpretation of normality testing -to test normality, two hypotheses were created:

- $\mathrm{H}_{0}: \mu_{1}=\mu_{2}$ (the dataset came from a normal distribution)

- $\mathrm{H}_{1}: \mu_{1} \neq \mu_{2}$ (the dataset did not come from a normal distribution)

To interpret the K-S and S-W test, both methods produce a p-value, which is based on the probability or assumption that the distribution is normal. In forensic science, $\alpha=0.05$ (95\% confidence interval) is frequently cited and was used here (57). This is considered the significant or alpha level. With this confidence, you can be $95 \%$ sure that $95 \%$ of the time, the calculated data range is included in the true population value, or that the probability of rejecting the hypothesis when the means are really equal, is 0.05 . 
When the null hypothesis is rejected, this means that the results provided strong evidence against the null hypothesis, and when it is accepted, it means that the results did not provide strong evidence against it. For this purpose, the p-value is reported with the results. A test is said to be statistically significant at that alpha level if the p-value is smaller than alpha. A small p-value provides evidence against the null hypothesis. Furthermore, the null hypothesis is rejected when the $p$-value is less than or equal to the alpha value.

\section{$\underline{4.5 \text { Statistical Methods }}$}

The statistical method of choice was based on the research questions asked. In this project, comparing color before and after treatment in fibers is the primary concern. For this reason, ANOVA was chosen for such analysis.

Analysis of variance (ANOVA) tests the significant difference between means within a population by actually comparing or analyzing the variances. This significant testing is done by comparing the variance due to the between-group variability (called the mean square effect) with the within-group variability (called the mean square error) by partitioning the total variance into components due to random effects (difference of means within group) and systematic effects (difference of means between groups). The latter component is then tested for statistical significance to conclude the acceptance or rejection of the null hypothesis (58).

The types of factors influencing the different variances are due to the systematic or random effects. Systematic effects are the controlled factors being tested. In this experiment, the controlled factors being investigated are aging and washing. The random effects may arise potentially from different sources within the sample, such as the lack of homogeneity within the 
fibers themselves or from the inconsistency with dye uptake. However, it is best to consider experiments of three kinds:

1. Those in which the factors affected are systematic (Model I);

2. Those where all the factors are random (Model II);

3. Those where there is a mixture of random and systematic effects (Mixed Model Effect).

By analyzing each experiment under the three models stresses that the appropriate tests in a particular case depends on the experimental conditions (59). If one controlled factor is being examined, such as the factor aging in part 1 of this project, then one-way ANOVA was used. In one-way ANOVA, the variance due to the systematic effect is compared with the variance due to the random effect. In my aging treatment, the variance due to aging is compared to the variance due to any random effect that was not due to aging. The heterogeneity of the fibers or the heterogeneity of the dye uptake along the fiber may produce within-group variability that is significant. Consequently, one-way ANOVA is used to determine if variances occur, and if so, additional statistical test can locate where these variances occur. The statistical test used to compare two such values is called the F-test. If multiple controlled factors are being examined, such as the different detergents used and washings performed in part 2 of this project, then multifactorial ANOVA was used. Once significant results are found in both analysis, post hoc tests were employed (discussed in section 4.5.3).

\subsubsection{One-way ANOVA and the F-test}

In part A of this analysis, one-way ANOVA was used because only a single factor, aging, was varied. To test the significance of the mean between populations, two hypotheses were created: 
- $\mathrm{H}_{\mathrm{o}}: \mu_{1}=\mu_{2}=\mu_{3}=\ldots \mu_{\mathrm{k}}$ (no significant mean difference observed between population)

- $\mathrm{H}_{1}$ : at least one $\mu$ is different (at least two of the population means are different)

The purpose of ANOVA is to determine whether differences exist between two or more populations. If the population means are equal, it can be concluded that no treatment effect was observed and no variances exist among the groups. If the population means are not equal, and at least one population mean is different, then some treatment effect was observed. However, it does not assume that the entire population mean differs. For example, my fiber was aged at five intervals of 16 hours each, creating five populations. If the mean values do not differ significantly among intervals, it can then be concluded that the aging treatment had no effect on the sample. However, if two of those intervals observe significant mean differences between them, it can then be concluded that the aging treatment affected at least two of the populations, not necessarily all of them.

The F-test, named after the statistician R.A. Fisher, is used to compare the variances due to the effect under investigation with the variance due to chance.

\section{$\mathrm{F}=\quad$ Systematic effect variance (between groups) Random effect variance (within groups)}

In ANOVA, the $\mathrm{F}$ value has to reach a certain value to attain statistical significance and this value is dictated by the degrees of freedom (df). There are always two df values with a Fdistribution: $\mathrm{df}_{1}$ is associated with the numerator and $\mathrm{df}_{2}$ is associated with the denominator, of the $\mathrm{F}$ ratio. Thus, to attain significance, the $\mathrm{F}$ value must be greater than 1 . 
Consider a single factor which has $p$ different levels and suppose that $n$ observations have been made at each level, totaling $N=n p$. Let the results be represented by $x_{i j}(i=1 \ldots p, j=1 \ldots n)$. Note that the first subscript in $x_{i j}$ specifies the category group or level and the second subscript specifies the observations. Assuming for any given level, $n$ observations have a mean, which combines the overall mean plus any variation found in the levels chosen. Then the mathematical formula for ANOVA would resemble:

$$
x_{i j}=\mu+F_{j}+\varepsilon_{i j}
$$

Where:

- $\mathrm{x}_{\mathrm{ij}}$ is the single response from factor $j$,

- $\mu$ is the overall mean,

- $\mathrm{F}_{\mathrm{j}}$ is the effect due to the contribution of $j$, and

- $\varepsilon_{\mathrm{ij}}$ is the variation of the results within a particular factor level (random effects).

ANOVA assumes that observations at a fixed factor level are normally distributed about the mean value $\left(\mu+\mathrm{F}_{\mathrm{j}}\right)$ with a common variance $\sigma^{2}$. The variance estimate is calculated using the sum of squares. The variability of $n$ sample measurements about their mean can be measured using the sum of squared deviations from the grand mean: $\mathrm{S}=\sum_{\mathrm{ij}}\left(\mathrm{x}_{\mathrm{ij}}-\mathrm{x}_{\text {... }}\right)^{2}$

- The sum of square deviations variability between levels: $\mathrm{S}_{1}=\mathrm{n} \sum_{\mathrm{ij}}\left(\mathrm{x}_{\mathrm{i}} \cdot-\mathrm{x} . .\right)^{2}$

○ The sum of square deviations variability within each level: $\mathrm{S}_{2}=\sum_{\mathrm{ij}}\left(\mathrm{x}_{\mathrm{ij}}-\mathrm{x}_{\mathrm{i}}\right)^{2}$

Table 7: ANOVA Summary Table

\begin{tabular}{|l|l|l|l|l|}
\hline $\begin{array}{l}\text { Source of } \\
\text { estimate }\end{array}$ & Sums of squares (SS) & $\begin{array}{l}\text { Degrees of } \\
\text { freedom (df) }\end{array}$ & Mean squares (MS) & F-statistics \\
\hline
\end{tabular}




\begin{tabular}{|l|l|c|c|c|}
\hline Between levels & $\mathrm{S}_{1}=\mathrm{n} \sum_{\mathrm{ij}}\left(\mathrm{x}_{\mathrm{i}}-\mathrm{x} . .\right)^{2}$ & $\mathrm{p}-1$ & $\mathrm{M}_{1}=\frac{\mathrm{S}_{1}}{(\mathrm{p}-1)}$ & $\mathrm{F}_{\text {calc }}=\frac{\mathrm{S}_{1}{ }^{2}}{\mathrm{~S}_{2}{ }^{2}}$ \\
Within levels & $\mathrm{S}_{2}=\sum_{\mathrm{ij}}\left(\mathrm{x}_{\mathrm{ij}}-\mathrm{x}_{\mathrm{i}}\right)^{2}$ & $\mathrm{~N}-\mathrm{p}$ & $\mathrm{M}_{2}=\frac{\mathrm{S}_{2}}{(\mathrm{~N}-\mathrm{p})}$ & \\
\hline Total & $\mathrm{S}=\sum_{\mathrm{ij}}\left(\mathrm{x}_{\mathrm{ij}}-\mathrm{x} . .\right)^{2}$ & $\mathrm{~N}-1$ & & \\
\hline
\end{tabular}

Note: The notation of a dot in place of a suffix means that that particular suffix has been averaged out over its appropriate observations.

The table is broken down into five items: the SS, df, and MS for the between and within group levels, the f-value and the p-value. The p-value, although not included in the table above, indicates the likelihood that a given result could have occurred by chance alone. It basically tests the level of significance.

For example, the red swatch was aged for five intervals equating to six populations (with the untreated source). For each interval, three samples areas were analyzed and three fibers within each sample area were analyzed. The ANOVA summary table would look similar to this:

\begin{tabular}{|c|c|c|c|c|c|c|}
\hline $\begin{array}{l}\text { Source of } \\
\text { estimate }\end{array}$ & Sums of squares (SS) & $\begin{array}{l}\text { Degrees of } \\
\text { freedom }(\mathrm{df})\end{array}$ & \multicolumn{2}{|c|}{$\begin{array}{l}\text { Mean squares } \\
\text { (MS) }\end{array}$} & \multicolumn{2}{|c|}{ F-statistics } \\
\hline \multirow[t]{2}{*}{ Between levels } & \multirow[t]{2}{*}{$S_{1}=3 \sum_{6,54}\left(x_{6 \cdot}-x_{. .}\right)^{2}$} & \multirow[t]{2}{*}{$6-1$} & \multirow{2}{*}{$\mathrm{M}_{1}=$} & $\mathrm{S}_{1}$ & \multirow{4}{*}{$-F_{\text {calc }}=$} & $\mathrm{S}_{1}{ }^{2}$ \\
\hline & & & & $(6-1)$ & & $\mathrm{S}_{2}{ }^{2}$ \\
\hline \multirow[t]{2}{*}{ Within levels } & $S_{2}=\sum_{654}\left(x_{654}-x_{6} .\right)^{2}$ & \multirow[t]{2}{*}{$15-6$} & \multirow{2}{*}{$\mathrm{M}_{2}=$} & $\mathrm{S}_{2}$ & & \\
\hline & $S_{2}-\angle 6,54\left(x_{6,54}-x_{6} \cdot\right)$ & & & $(15-6)$ & & \\
\hline Total & $S=\sum_{6,54}\left(x_{6,54}-x_{. .}\right)^{2}$ & $15-1$ & & & & \\
\hline
\end{tabular}

Note: The notation of a dot in place of a suffix means that that particular suffix has been averaged out over its appropriate observations.

\section{$\underline{4.5 .1 \mathrm{a} \text { Interpretation of the ANOVA F- test and p-values }}$}


In any given test, the question of "How do we determine if something is significantly different" usually arises. In order to compute if the means are equal in the population from which the samples are taken, the hypothesis test called the ANOVA F-test was performed. The significance test carried out with one-way ANOVA is compared with the F-distribution such that if the hypothesis is incorrect, the statistic will tend to be greater than one. This upper tail of the fdistribution is the criteria for rejecting the null hypothesis.

There are two approaches that can be used to interpret the results for the F test. Using the same example above:

\section{Critical Value Approach}

The critical value is $\mathrm{F}_{\alpha}$ with $\mathrm{df}=(6-1,15-6)$

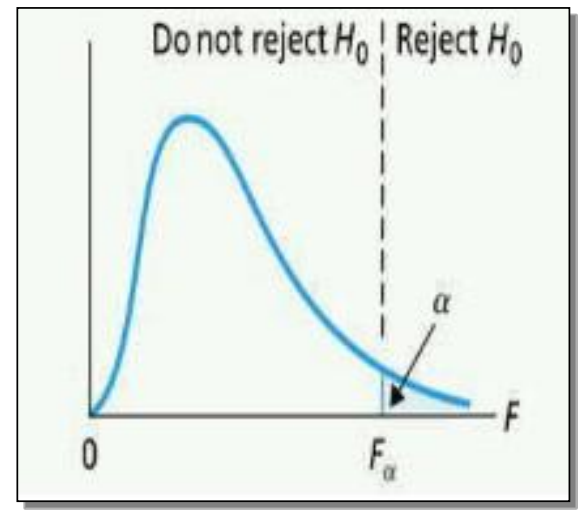

If F-statistics $\geq$ F-critical at $\alpha=0.05$, reject $\mathrm{H}_{\mathrm{o}}$; otherwise, do not reject $\mathrm{H}_{\mathrm{o}}$. or

P-Value Approach

The critical value is $\mathrm{F}_{\alpha}$ with $\mathrm{df}=(6-1,15-6)$

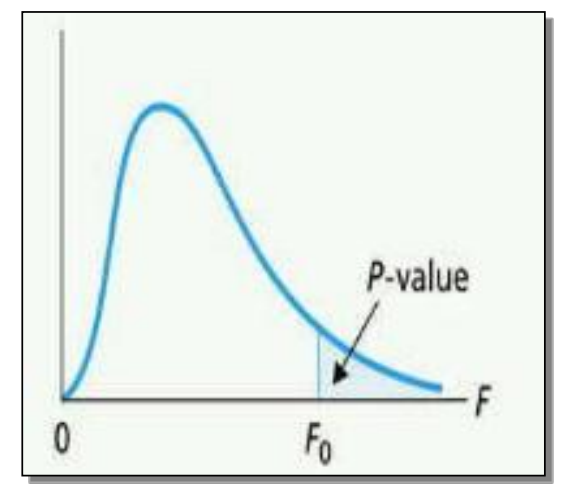

If $\mathrm{P} \leq \alpha$, reject $\mathrm{H}_{0}$, otherwise do not reject $\mathrm{H}_{0}$.

Since most software packages compute the F and p-value with the ANOVA tests, the decision was made to compare the level of significance with the p-value. The p-value provides information about how unusual the calculated F statistic is if the population means are the same. 
When the p-value is less than the critical or alpha value, the null hypothesis of equal means is rejected. When this is true, the effect is said to be significant.

The results obtained from the one-way ANOVA are presented in four headings: Descriptives, Test of Homogeneity of Variances, the ANOVA table, Post Hoc Comparisons. Each will be discussed separately below.

The first table provides descriptive statistics for each factor. For aging, the number of scores, mean, standard deviation, standard error, upper and lower bounds of the $95 \%$ confidence interval and the minimum and maximum values for each aged interval.

The second table supplies the results of the Levene's test. The results are nonsignificant when the p-value is $<0.05$, showing that there is no evidence to support that the variances of the six factors of aging are different from one another.

The third table is the standard ANOVA table observed in Table 7. An additional column with the appropriate $\mathrm{p}$-values is included.

Two tables are produced for the post hoc test because two different post hoc tests were applied. These tables show the results of the multiple pair-wise comparisons among the six intervals, representing the five aging intervals and the standard.

\subsubsection{Multifactorial Analysis of Variance}

In part B of the analysis, multifactorial ANOVA was used because the number of independent factors was greater than one. It is basically an extension of the one-way ANOVA, used to simultaneously analyze the effects of two or more factors on a dependent variable. This extension accounts for the possible interactions and the effects associated with them. In this 
method, the difference among several group means is analyzed by partitioning the total variance from the dependent variable into effects due to: each of the factors (called main effects), interactions between the factors, and the error variance (60).

From the data obtained in part $\mathrm{B}, 2^{2}$ experimental designs, a two way ANOVA was done to establish the presence or absence of significant differences in the treatment of the fibers considering the detergent and the washings performed. Thus, in this two-way ANOVA, the main effects of the detergents used and the washed-aged cycles are analyzed separately to determine of either factor affected the outcome of the results, or if any interaction between both groups are significant.

\subsection{2a Interpretation of multifactorial ANOVA}

The results obtained from the multifactorial ANOVA are presented in five general headings: Univariate Analysis of Variance, Estimated Marginal Means, Post Hoc Tests, Homogeneous Subsets, and Profile Plots. Each section will be discussed separately.

The univariate analysis of variance is broken down into two separate analyses tables: the 'between-subjects factors' and the 'test of between-subjects effects'. The first table of the output, labeled Between-Subjects Factors, summarizes the factors and shows how the factors were labeled and how many scores are in each group. The second table, labeled Test of BetweenSubjects Effects, is a standard ANOVA table with a few additions. This addition lists the sources of variation that are analyzed including the main and interaction effects of the factors. The main body of the table corresponds to Table 7 . However, a final column is added to the table along with the $\mathrm{F}$ value, listing the significance of $\mathrm{F}(\mathrm{Sig})$. 
Under the heading Estimated Marginal Means, the number of tables are produced based on the total amount of factors. In each responding table, the means, standard error and 95\% confidence interval is recorded.

Under the Post Hoc Test general heading, a table is generated with the results of the multiple pair-wise comparisons among the independent variables or groups. This result was based on the post hoc test chosen for analysis, which is discussed in section 4.5.3. Column 1 lists the representing group (listed as I) and its pair-wise counterpart (listed as J). This pair is compared to determine any significant difference at the 0.05 level. Columns 2-6 list various descriptive statistics: the mean difference between 'I' and ' $\mathrm{J}$ ', the standard error, the significance, and the lower and upper $95 \%$ confidence interval.

A final table is generated under the Homogeneous Subsets heading. Similarly to the previous table, it shows the result of the post hoc test chosen but in slightly different ways. Based on the multiple pair-wise comparisons, the independent groups are divided into homogeneous subsets. Homogeneous subsets are the factors grouped together based on significance; factors that are not significantly different from another are grouped into one homogeneous subset, and factors that are significant are grouped in separate subsets.

Lastly, under the Profile Plot heading, an interaction graph is generated. A profile plot is a type of line plot in which each point indicates the estimated mean of the dependent variable at one level of a factor. It shows whether the estimated means are increasing or decreasing across levels. The interaction effects between groups are significant to note, and through the observation of the graph, the interpretation is sometimes obvious. For two or more factors, if the lines are parallel, this indicates that there is no interaction between the factors. If the lines are 
nonparallel and intercross, this indicates that an interaction occurs between the factors, even if this interaction is not significant.

\subsubsection{Post Hoc Comparisons}

The post hoc (meaning 'after the fact' or 'after the data collection') comparisons were employed when the ANOVA F was found significant. ANOVA does not provide specific insights into what caused the null hypothesis to be rejected, thus by using a post hoc procedure, the researcher attempts to investigate the data to find out which of the possible non-null scenarios are most likely to be true. It is basically conducted once the outcome of the F-test in ANOVA yields a significant $\mathrm{F}$ to help in understanding why the ANOVA $\mathrm{H}_{\mathrm{o}}$ was rejected. The F-value in ANOVA can be significant for different reasons; for instance, there could be different possible mean patterns. For this reason the post hoc analysis helps the researcher in their effort to understand the true pattern of the population means.

The post hoc procedures will function correctly if three underlying conditions holds true for the population and samples involved in the study. These conditions being met are the same as those for the one-way ANOVA F-test: independence, normality and homogeneity of variance. Although this test is generally robust to the normality assumption, they might be affected if the equal variance assumption does not hold true, especially when the sample sizes are very dissimilar.

In the post hoc procedure, two hypotheses were analyzed:

- $\mathrm{H}_{0}=$ the observed difference in group means is entirely accounted for by inherent variability 
- $\mathrm{H}_{\mathrm{A}}=$ the observed difference in group means is not due to inherent variability alone, it is the result of experimental intervention or treatment (61)

Again, the accepted criterion for significance was set to 0.05 , such that the probability of a Type 1 error must be less than 0.05 . With this probability, there is a $5 \%$ chance or less that the difference in the data is caused by inherent variability. Two post hoc tests were applied with ANOVA based on the results of the Levene's test of equal or unequal sample variances.

\subsection{3a The Tukey Honest Significant Difference (HSD) test}

Although a wide array of statistical procedures are employed for post hoc comparison, the most frequently used for color analysis is the Tukey HSD test, and thus employed in this project (6266). As long as the assumption for homogeneity of variance is met, this test can be used. This test has also been adapted to unequal sample sizes, and uses the harmonic mean as its $n$ value (67).

The Tukey test permits complete pair-wise or pair-by-pair comparisons for all possible combinations and their contrasts. The term 'pair-wise' simply means that groups are being compared two at a time. Fifteen pair-wise comparisons were made for the analysis of the aged samples:

$$
\begin{aligned}
& \mathrm{H}_{\mathrm{o}: \mu 0}=\mu_{16} ; \quad \mathrm{H}_{\mathrm{o}: \mu 16}=\mu_{32} ; \quad \mathrm{H}_{\mathrm{o}: \mu 32}=\mu_{48} ; \quad \mathrm{H}_{\mathrm{o}: \mu 48}=\mu_{64} ; \quad \mathrm{H}_{\mathrm{o}: \mu 64}=\mu_{80} ; \\
& \mathrm{H}_{\mathrm{o}: \mu 0}=\mu_{32} ; \quad \mathrm{H}_{\mathrm{o}: \mu 16}=\mu_{48} ; \quad \mathrm{H}_{\mathrm{o}: \mu 32}=\mu_{64} ; \quad \mathrm{H}_{\mathrm{o}: \mu 48}=\mu_{80} ; \\
& \mathrm{H}_{\mathrm{o}: \mu 0}=\mu_{48} ; \quad \mathrm{H}_{\mathrm{o}: \mu 16}=\mu_{64} ; \quad \mathrm{H}_{\mathrm{o}: \mu 32}=\mu_{80} ; \\
& \mathrm{H}_{\mathrm{o}: \mu 0}=\mu_{64} ; \quad \mathrm{H}_{\mathrm{o}: \mu 16}=\mu_{80} ; \\
& \mathrm{H}_{\mathrm{o}: \mu 0}=\mu_{80} \text {; }
\end{aligned}
$$


The null hypothesis of each pair states that the means of each group are the same. If this hypothesis is true, then there is no reason to believe that the variances of the two groups are different from each other. The Tukey test is more conservative than liberal in the fact that conservative procedures provide more control over Type I errors at the expense of higher Type II error risks.

\section{i. Presentation and Interpretation of Tukey HSD test}

Two tables are produced for the Tukey test. The first table is presented in a triangular table of mean differences. In the table, each numerical entry is simply the difference between the means of the group that label the row and column where the number is located. Each of these mean differences is evaluated to observe if the value is greater than what would be expected by chance alone. At a significant level of 0.05 , the $\mathrm{p}$-value is compared to ' $\alpha$ ' to make the determination of whether this pair is significantly different. Marked differences are significant when $p \leq \alpha$, where the null hypothesis of the two groups being different is rejected. These significant differences, illustrated in bold, reveal where they are found among the comparing groups.

The second table, labeled as Homogeneous Subsets, presents the results of the Tukey pair-wise comparison differently. In this table, the groups are divided into homogeneous subsets. All the insignificant groups are placed together in one subset. When the values are significant, i.e. when the means are significantly different, those groups are then placed in its individual subset. For instance, if the mean values for the untreated fibers (only) present a significant value from the remaining pair-wise groups, then this group will be placed separately in subset 1 while the other groups are placed together in subset 2 . This differentiation in subsets accounts for the difference observed between the untreated standard and the treated fibers. 


\section{$\underline{4.5 .3 b}$ Games-Howell (G-H) test}

The Games-Howell test is another pair-wise comparison method that is employed when the data being analyzed does not meet the homogeneity of variance assumption (68). It can also be used when the total number of observation varies between groups. This test defines critical value of each pair-wise comparison by determining the variances and the number of observation in each comparing group.

\section{Presentation and Interpretation of the $G-H$ test}

The results of the G-H test is presented in table format: Column 1 lists the dependent variable; Column 2 list the pair-wise groups; Column 3 lists the mean difference between the pair-wise groups; Column 4 list the standard error associated with these groups; Column 5 list the significant p-values, and, Columns 6 and 7 lists the lower and upper bounds of the confidence interval.

The interpretation of the G-H test is consistent with the TUKEY test. Marked differences are significant when $\mathrm{p} \leq \alpha$, where the null hypothesis of the two groups being different is rejected. These significant differences, illustrated in bold, reveal where they are found among the comparing groups.

\subsection{Statistical Software Used}

\subsubsection{Matrix Laboratory (Matlab)}

Matrix Laboratory or Matlab is a numerical computation and simulation tool where its strength is observed in matrix manipulation (69). It has been used as a program that implements many 
multivariate statistics and is preferred to use during this research because of its simplicity (70, 71). The data analysis was conducted using Excel spreadsheets and graphed using Matlab (version 7.12.0.635). Matlab was used to create 3-D bubble plots for the validation results.

The validation results were transferred into an Excel file which were then organized and imported into Matlab. Three-dimensional bubble plots were graphed based on the 3-D bubble script downloads available on Matlab's website. In total, 12 plots were created of the validation results. The first plot contained all 150 measurements obtained from the blue, green and red fibers over the 10 day period. The second and third plots contained the average measurements of each day for all three colors, as well as the $95 \%$ confidence interval, respectively. Plots four to six are the breakdown of all 150 measurements plotted separately for each color. Plots seven to nine are the averaged measurements per day plotted separately for each color. And finally, plots ten to twelve are the $95 \%$ confidence interval of the averaged measurements of plots seven to nine.

\subsubsection{STATISTICA}

STATISTICA (version 10) software was used to compute one-way ANOVA of the aged samples, and the results were produced in tabulated form with the $\mathrm{F}$ and $\mathrm{p}$-values provided. This software was chosen for its accessibility. Mean plots were graphed of the overall dependent variables Lab. Three additional plots were also graphed for each dependent variable grouped by the independent variable 'hours'. These interaction plots were used for observation of the mean varying between the hours aged. If the results produced were significant, i.e. if the alpha value exceeded the p-value, then the post hoc test, Tukey HSD, was applied. Statistica software is 
limited in calculating the H-W test. The Games-Howell test was calculated using another statistical software discussed below.

The data obtained from aging was transferred into Excel, organized and imported into Statistica. To compute one-way ANOVA, from the dialog box, the following factors were chosen: dependent variables 'Lab'; categorical factors 'Hours, Swatch, Sample Area and Fibers' and the between effects were all levels for each factor; the summary table of descriptive statistics; the Welch and Levene's test; the Tukey post hoc test; and, the interaction plots of each categorical factor against the Lab variables.

\subsubsection{Statistical Procedure and Service Solutions (SPSS)}

SPSS is one of the oldest and most popular statistics computer programs that provide statistical analysis of data. It was employed in this project to evaluate the washed-aged samples using multifactorial ANOVA. This software is able to calculate both post hoc comparison tests. This software was also used to calculate the Games-Howell post hoc test for the aged samples that was not available on STATISTICA software. IBM ${ }^{\circledR}$ SPSS $^{\circledR}$ Statistics software version 20 was used.

The results obtained were transferred into Excel, appropriately organized before imported into SPSS. For the multifactorial analysis, the Univariate General Liner Model was chosen because one dependent factor was being analyzed at a time. In this case, variables Lab were analyzed separately. In the Univariate dialog box, the following factors were chosen: the dependent variable was ' $\mathrm{L}$, a or b'; the fixed factors were 'bleached, non-bleached and washing'; five plots were created for the interaction effects: 'Sample Area * Swatch', 'Sample Area * Bleached', 'Washing * Bleached', 'Washing * Swatch', 'Washing * Sample Area'; and as an options, 
Display Means for the main effects of: 'Sample Area', 'Swatch', 'Bleached'. 'Washing', as well as the interaction effects listed above.

For the one-way ANOVA, the post hoc tests were also included in the results.

\section{Project Approach}

This project was separated into two parts: Part 1 focused on understanding the degradation of color in fibers by direct exposure to UV light and Part 2 focused on understanding the degradation of color change in fibers after being laundered and aged. This project attempted to replicate the washing and aging affect in a fiber multiple times to determine if precise color comparisons between the treated and unaltered fiber is possible.

\section{$\underline{5.1 \text { Original Project Plan }}$}

In the original plan, cotton textiles were to be used and compared. Five of the eight colors of interest to be analyzed, blue, gray and yellow were not used in this project. In addition, two other detergent brands were to be used: All, and Gain. However, with limited accessibility to the MSP instrument, and for efficiency purposes, only one type of textiles, five colors, and one type of detergent brand was chosen.

\section{$\underline{5.2 \text { Budget }}$}

The resources necessary for this project was limited to the materials needed: the detergents, and garments. The costs of the Tide detergents were six dollars each and the price of the polyester garments price range from three to seven dollars. In total, the budget for this project was approximately ninety dollars. 


\section{$\underline{5.3 \text { Limitations and implications }}$}

The purpose of this project was to determine if a fiber can be accurately compared to its source after being laundered and aged for a two week duration period. To age the fiber without including unknown variables, UV-lamp was used to maintain control of the experiment.

Part one for the research tends to focus on the visual and objective expectation of aged fibers under UV exposure. The aim of the experiment will only focus on the color change as the fibers are aged and not on the components that generate the color in the dye. The UV-Vis MSP generated spectral data and computed the chromaticity coordinates to provide a means of comparing the overall color of the sample. It has been published that MSP gives no information about the individual dye component with some exceptions (72). Therefore, no attempt was made to reference the mechanism of dye degradation in the fibers.

Part two of the project created a true representation of actual washing of samples using washers found in a Laundromat. It is expected that the samples might be contaminated when washed simultaneously with other clothing. Therefore the samples were first evaluated under the stereoscope to decipher the relevant fibers from any external source. This process was performed after drying and before analysis with the MSP.

\section{Results and Discussion}

\section{$\underline{6.1 \text { Calibration }}$}

\subsection{1a Wavelength Calibration}


Holymium and Didyium Oxide filters were used to measure the wavelength accuracy and spectral resolution. Table 7 below shows an example of the calibration results: Column 1 refers to the target absorbance band of the filters, column 2 refers to the measured absorbance band of the instrument, column 3 refers to the deviation of the measured value from the true value; and Column 4 refers to the acceptance or rejection of this difference. As long as the measured results are within the uncertainty values, the wavelength calibration will be accurate within the established limits.

Table 8: Example of the Wavelength Calibration Printout

\begin{tabular}{|c|c|c|c|}
\hline \multicolumn{4}{|c|}{ 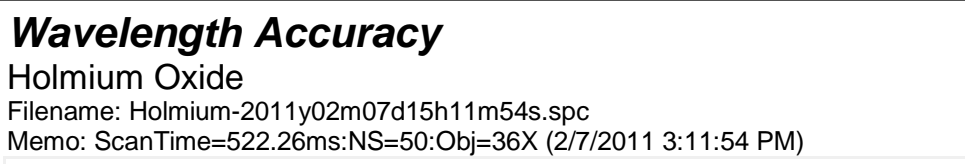 } \\
\hline NIST Peak & MSP Value & Difference & Passed? \\
\hline 360.2 & 359.4 & 0.8 & Yes \\
\hline 418.5 & 416.8 & 1.7 & Yes \\
\hline 445.8 & 445.5 & 0.3 & Yes \\
\hline 536.0 & 535.4 & 0.6 & Yes \\
\hline 637.2 & 636.5 & 0.7 & Yes \\
\hline \multicolumn{4}{|c|}{$\begin{array}{l}\text { Didymium } \\
\text { Filename: Didy-2011y02m07d15h12m39s.spc } \\
\text { Memo: ScanTime=522.26ms:NS=50:Obj=36X (2/7/2011 3:12:39 PM) }\end{array}$} \\
\hline NIST Peak & MSP Value & Difference & Passed? \\
\hline 441.2 & 440.9 & 0.3 & Yes \\
\hline 513.5 & 512.9 & 0.6 & Yes \\
\hline 684.4 & 683.5 & 0.9 & Yes \\
\hline 806.8 & 806.2 & 0.6 & Yes \\
\hline
\end{tabular}

Note the unit of measurement is in absorbance.

\subsection{1b Photometric Calibration}

The OD filters were used to calculate and control the absolute absorbance accuracy of the instrument by checking the spectral linearity of the system. Table 8 below shows an example of 
the calibration results. In addition to the results obtained in the calibration table, another column is added which specifies the wavelength range.

Spectral linearity is observed in the absorption values. As the OD filter values increase, the absorption values increase. For example, the absorbance value at 400nm increases from 0.108 (OD 0.1), to 0.529 (OD 0.05), to 0.987 (OD 1.0). At low absorption values, more light is being transmitted because the absorbance matter is quite small. In contrast, at high absorption values, lesser light is being transmitted because the absorbance matter is greater. Thus the spectral linearity of the system is observed through the use of these filters.

Table 9: Example of the Photometric Calibration Printout

\begin{tabular}{|c|c|c|c|c|}
\hline \multicolumn{5}{|c|}{$\begin{array}{l}\text { ND } 0.1 \\
\text { Filename: ND01-2011y02m07d15h13m40s.spc } \\
\text { Memo: ScanTime }=522.26 \mathrm{~ms}: \mathrm{NS}=50: \mathrm{Obj}=36 \mathrm{X} \text { (2/7/2011 3:13:40 PM) }\end{array}$} \\
\hline Wavelength & NIST & MSP & Difference & Passed? \\
\hline 400 & 0.106 & 0.108 & -0.002 & Yes \\
\hline 500 & 0.095 & 0.091 & 0.004 & Yes \\
\hline 635 & 0.085 & 0.083 & 0.002 & Yes \\
\hline \multicolumn{5}{|c|}{$\begin{array}{l}\text { ND } 0.5 \\
\text { Filename: ND05-2011 y02m07d15h14m22s.spc } \\
\text { Memo: ScanTime }=522.26 \mathrm{~ms}: \mathrm{NS}=50: \mathrm{Obj}=36 \mathrm{X}(2 / 7 / 2011 \text { 3:14:22 PM) }\end{array}$} \\
\hline Wavelength & NIST & MSP & Difference & Passed? \\
\hline 400 & 0.524 & 0.529 & -0.005 & Yes \\
\hline 500 & 0.510 & 0.499 & 0.011 & Yes \\
\hline 635 & 0.474 & 0.466 & 0.008 & Yes \\
\hline \multicolumn{5}{|c|}{$\begin{array}{l}\text { ND } 1.0 \\
\text { Filename: ND10-2011y02m07d15h15m04s.spc } \\
\text { Memo: ScanTime }=522.26 \mathrm{~ms}: \mathrm{NS}=50: \mathrm{Obj}=36 \mathrm{X}(2 / 7 / 20113: 15: 04 \mathrm{PM})\end{array}$} \\
\hline Wavelength & NIST & MSP & Difference & Passed? \\
\hline 400 & 0.977 & 0.987 & -0.010 & Yes \\
\hline 500 & 1.008 & 1.008 & -0.000 & Yes \\
\hline 635 & 1.009 & 1.016 & -0.007 & Yes \\
\hline
\end{tabular}

Note that the unit of measurement is absorbance. 


\section{$\underline{6.2 \text { Method validation }}$}

The MSP was validated to verify the proper functioning of the instrument by checking the accuracy and precision of wavelength and photometric measurements. Suggested by Craic Technologies, 50 measurements of each red, green and blue sample were analyzed over a two week period. These colors were analyzed once per day for two weeks and their CIELab coordinates were recorded. Five replicates per analysis were performed to examine variability.

Twelve charts were plotted for the validation results. These charts included the overall measurements of all the samples analyzed over the 10-day period. It also included the average measurements taken per day and the $95 \%$ confidence interval for each of these average measurements. Different magnification was used to enlarge the data points for visualization purposes. There were some instances where the uncertainty points were larger than others and blocked the visibility of the smaller uncertainties; when this occurred, the magnification was decreased to a value of 1 , so that visibility was observed for all the points.

\subsubsection{Discrimination between colors}

Figure 16: Validation Results of Blue, Green and Red Fibers

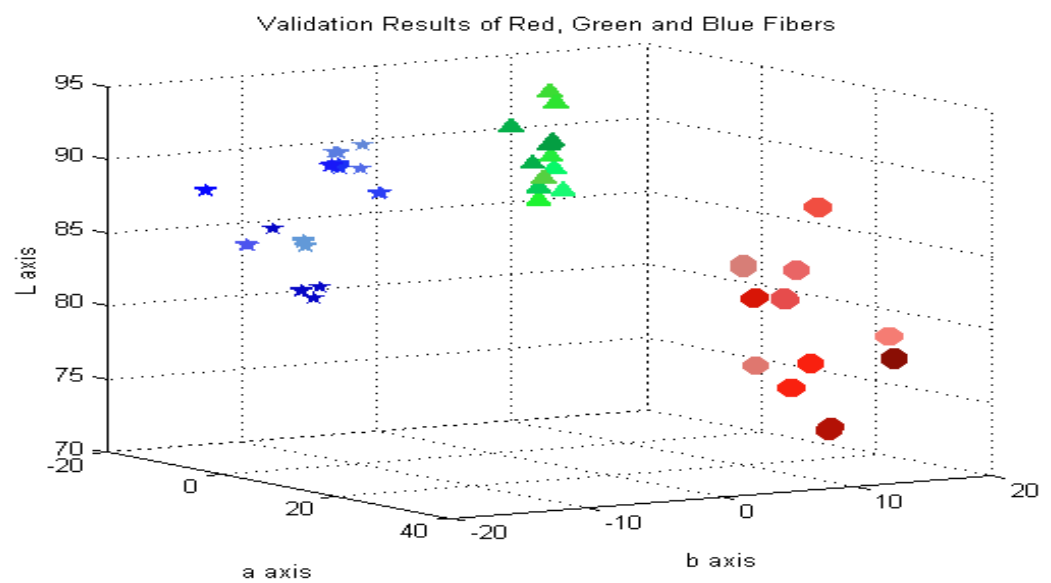


Figure 16 encompasses the total measurements collected in the 10-day validation experiment. Fifty measurements were plotted for each color fiber, with a total of 150 data points on this graph. However, some overlap each other. This was better observed as the data was plotted separately for each color.

Figure 17 below is the averaged 50 measurements for each color over the 10-days studies. Intervariability can be readily observed here. As the fibers are analyzed daily, the Lab values vary. This may be due to the variability within the fiber itself, but more so, to the fact that the same fiber was not analyzed daily as the selection day-to-day was randomly done. Another possible explanation is the variability within the instrument from day-to-day.

Figure 17: Average Validation Results of Blue, Green and Red Fibers

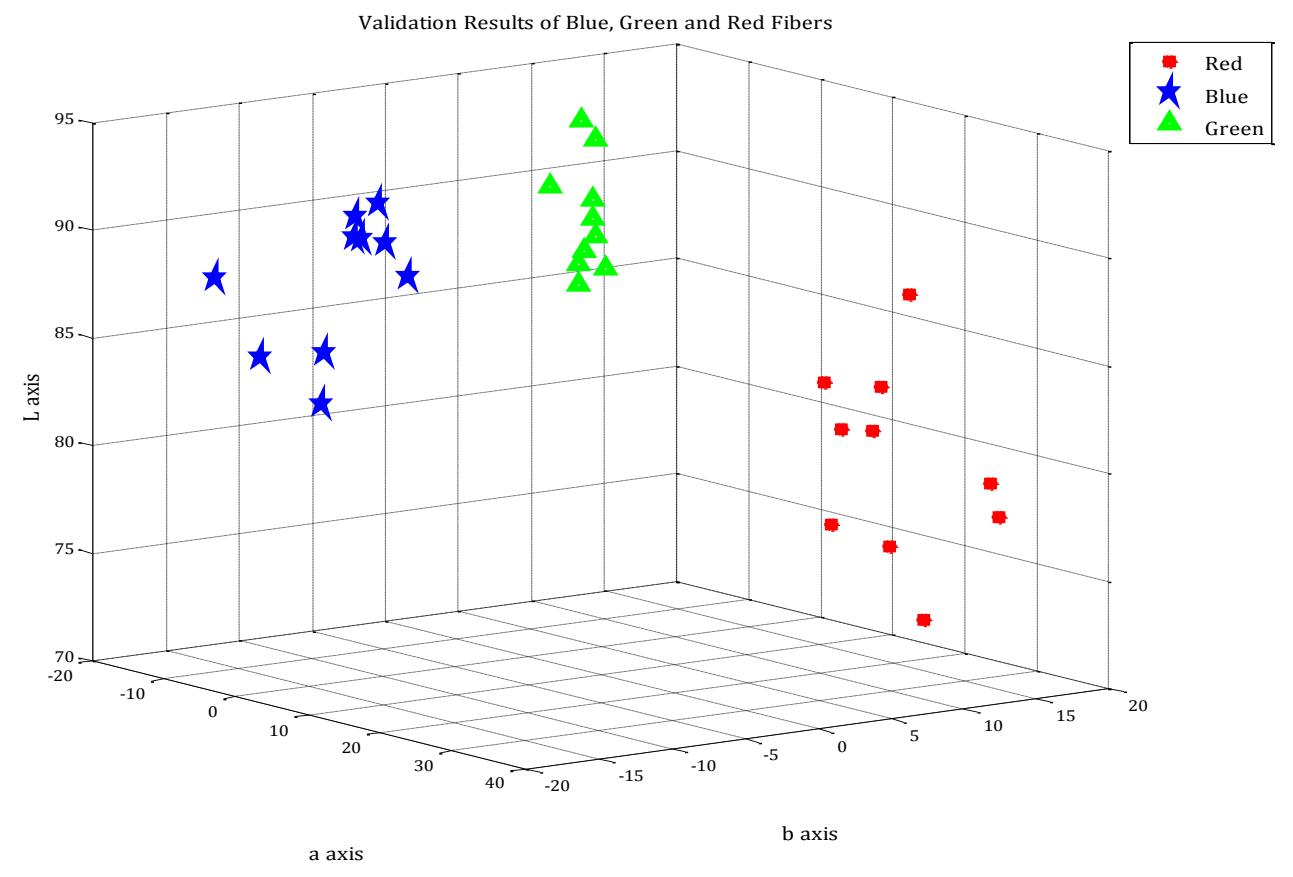

Figure 18 is the individual $95 \%$ uncertainty values plotted for the average measurements taken over the 10-day studies. The 95\% CI defines the elliptical shape for each sample on the L-, aand $b$-axes with $n=5$. It can observed that the blue, green and red fibers are clearly separated. 
Although red is clearly differentiated from blue and green, it also observes larger volumes of deviation as seen in the larger ellipses. Inter-variation is also observed greatest in the red fibers. Blue and green fibers are very closely observed although a separation can be seen. Blue fibers also observes a great amount of deviation among the 10-day study. Inter-variation is also observed here. With green fibers, which also observes some deviations, the inter-variation of the sample among the 10-day studies is minimal. Infact, the values in some days overlap others.

Figure 18: Average Validation Results of Blue, Green and Red Fibers with Uncertainty Ellipses

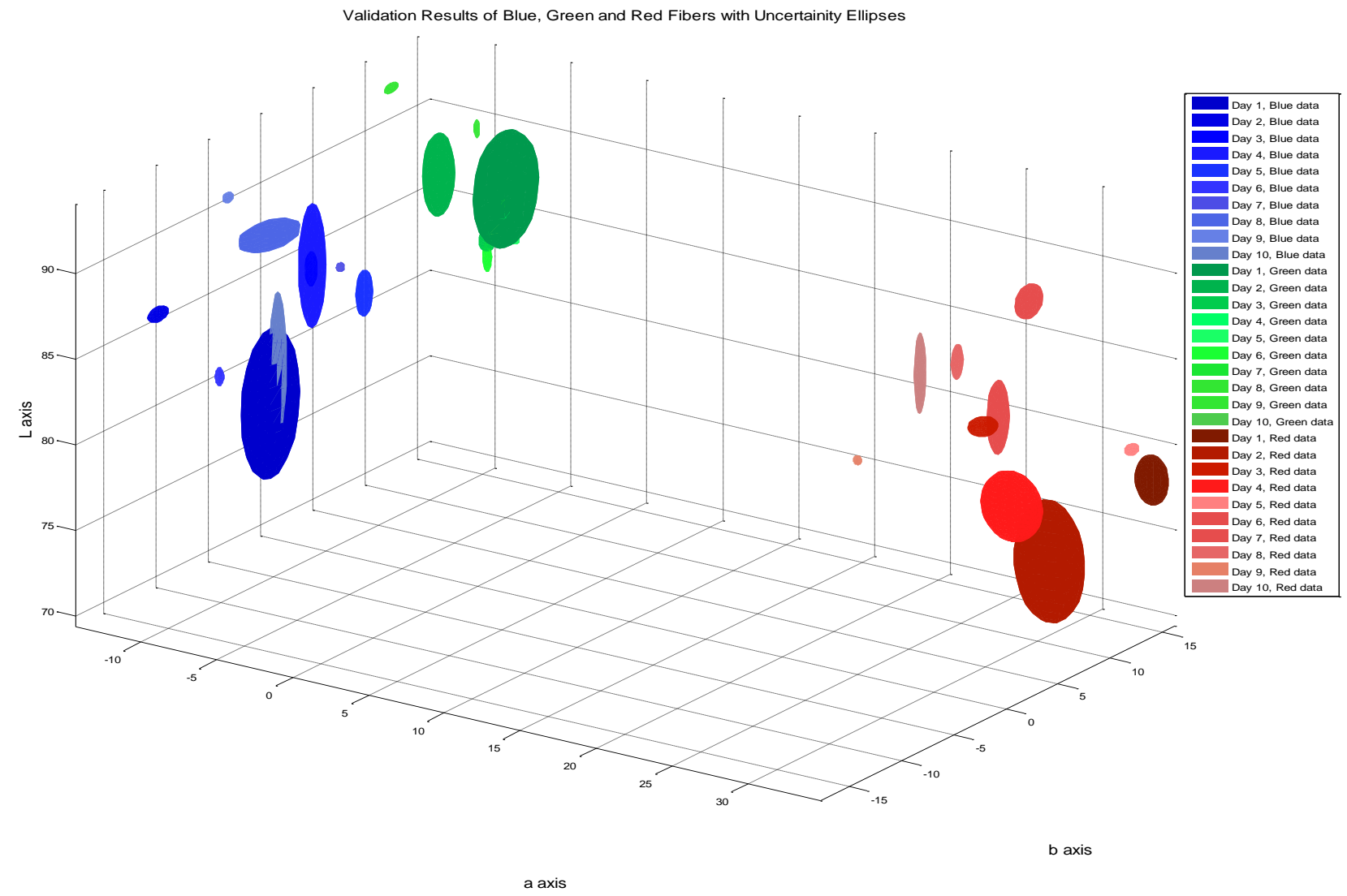

The variation that occurs for each sample is observed at specific coordinates. For the blue fibers, the greatest amount of variation seems to occur at the b-axis. This result is plausible because the results would most likely affect the yellow to blue region since the color of the fiber is blue. 
Notice this contrast with the red and green fibers. In both instances, both fibers observed the greatest amount of variation at the a-axis. For the green fibers, the 'a' values moves towards the green region as all of the values were negative, whereas for the red fibers, the 'a' values move towards the red region as all the values were positive. It can be further concluded that variation would exist greater in the coordinate at which the color is represented by, and must be taken into account for the remainder of this study.

\subsubsection{Precision}

The precision of the validation results was investigated through its repeatability. Precision is the closeness of agreement (degree of scatter) between a series of measurements obtained from multiple sampling of the same sample under a set condition. The precision of the instrument was investigated through repeatability. Repeatability is the precision estimate obtained from replicate measurements in a single batch of analysis made during a single setting by one analyst. For two weeks, the samples were analyzed by the same analyst. The $95 \%$ confidence interval was computed to describe the uncertainty associated with the samples. These results were discussed separately by color.

\subsection{2a Blue Fibers}

Of the 50 total observations, the degree of scatter in the Lab values minimal. Two clusters of values are observed in the graph below. Within one cluster, the deviation seems to be very minimal, however with the second cluster; this degree of variation is greater. Also, two potential outliers were present. It is unclear as to what may have caused these values to differ from the normal values, however, observing the averaged and uncertainty values may shed light onto this matter. 


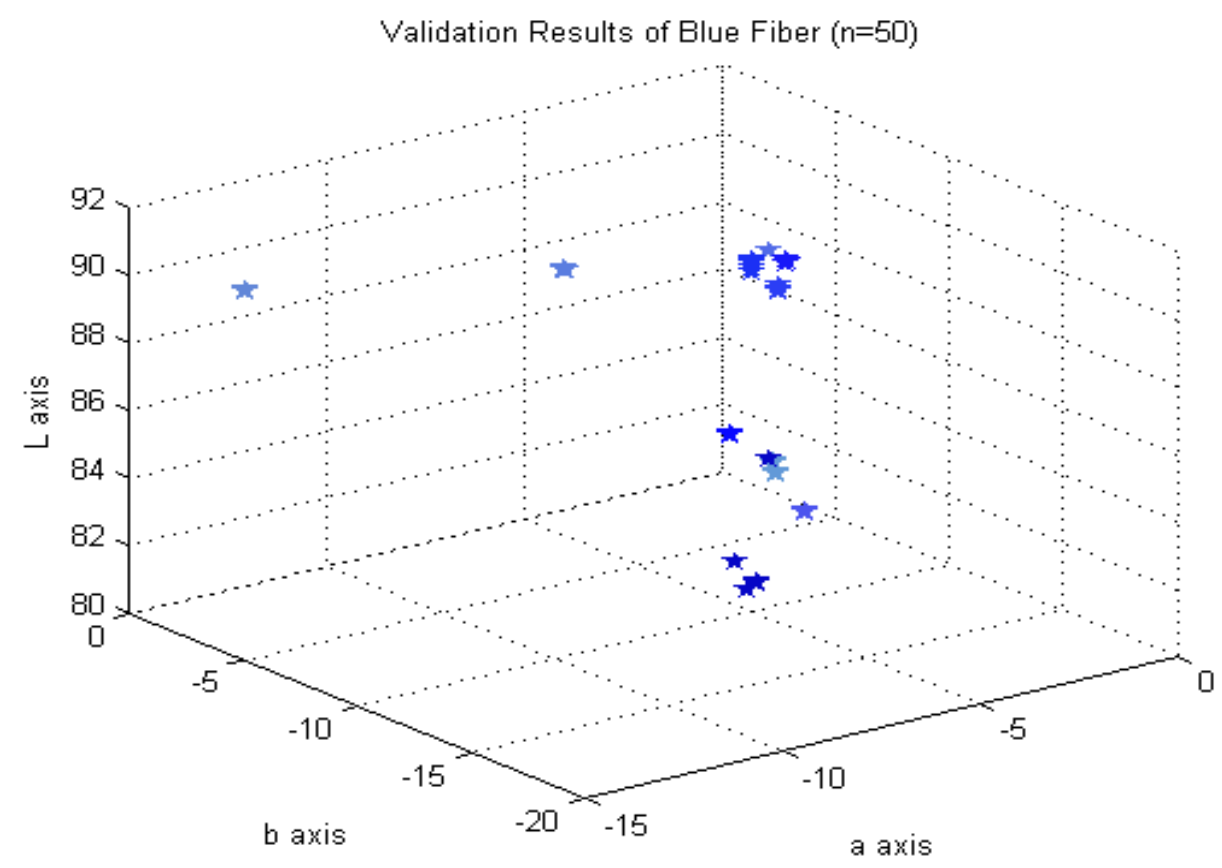

Figures 20 and 21 show the average measurements of the 10-day studies. The difference between these two figures is the markers used to illustrate the actual days. This was done for accurate interpretation. Everything else remains consistent between the graphs. As the data is averaged, fluctuations can be observed between days. However, with the averaging, the groupings of the two clusters are better observed. Cluster 1 is comprised of days 1,2, 6 and 10, and cluster 2 is comprised of days $3-5$, and 7 . The clusters themselves are random with the days they are comprised of. The samples were randomly measured daily, so the same fiber was not analyzed. This might account for the random variability observed between the days. Again, two outliers can be seen here which represents days 8 and 9 , respectively. Unless this pattern is observed for the other samples, then the variability could have been caused by the instrument's configuration. 
Figure 20: Averaged Validation Results of Blue Fibers

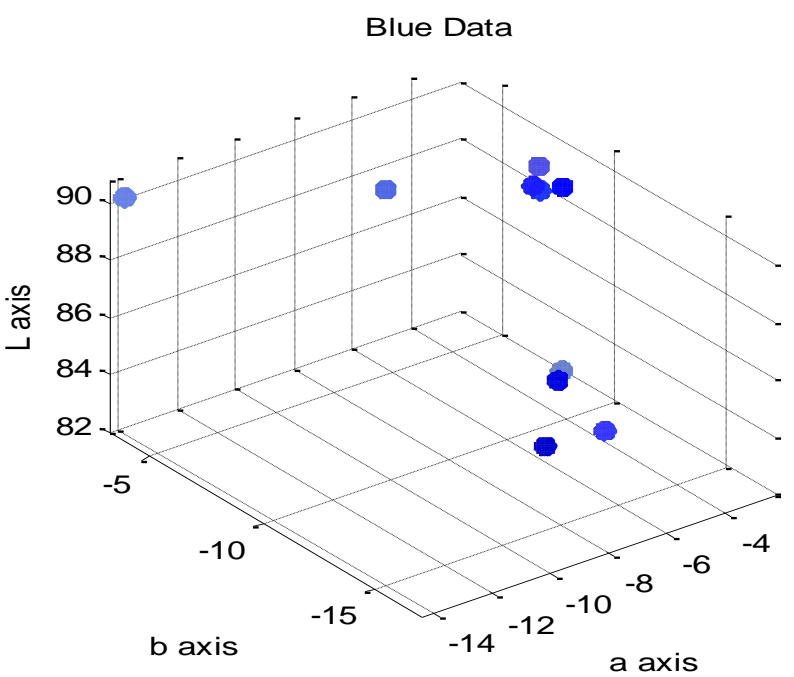

\begin{tabular}{|l}
\hline Day 1, Blue data \\
Day 2, Blue data \\
Day 3, Blue data \\
Day 4, Blue data \\
Day 5, Blue data \\
Day 6, Blue data \\
Day 7, Blue data \\
Day 8, Blue data \\
Day 9, Blue data \\
Day 10, Blue data
\end{tabular}

Figure 21: Averaged Validation Results of Blue Fibers, specified in difference markers

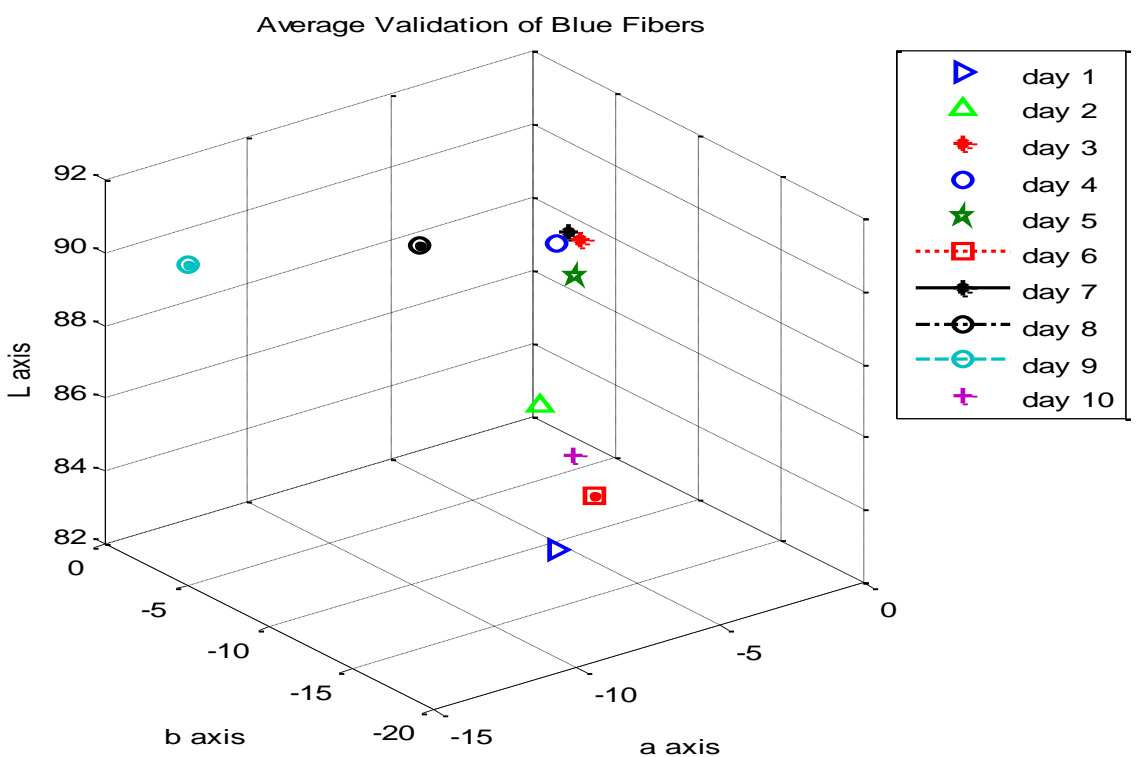




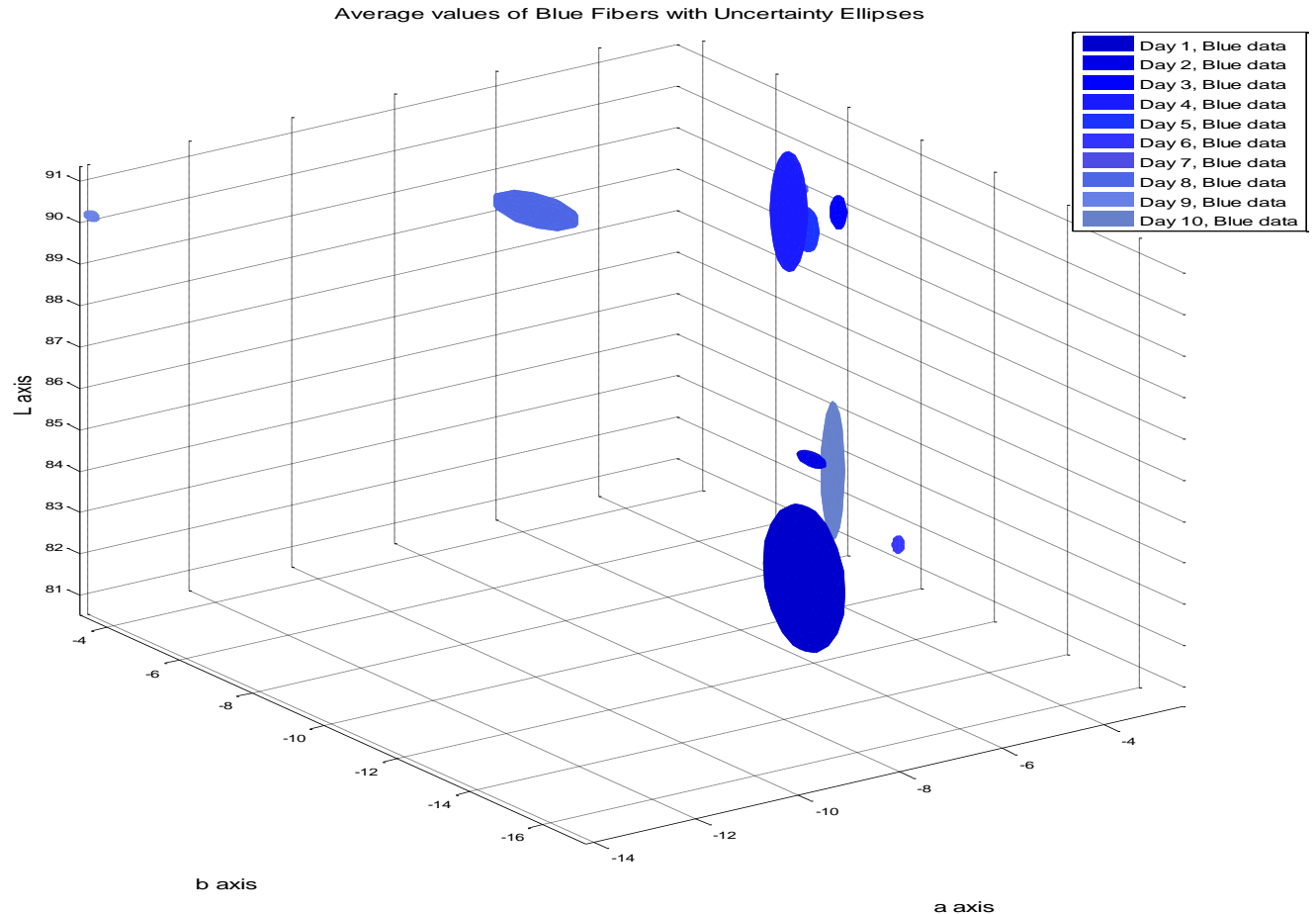

Between the 10-day repeatability studies, most of the days can be separated from each other. And even within days, the variations in the Lab values are observed by the elliptical shapes where the larger the shapes respond to greater amounts of variations. The greatest amounts of deviations are observed at days 1 and 2. So between the measurements on both days, the greatest amount of deviations occurs here.

\subsection{2b Green Fibers}

Similarly the precision of the repeatability study was investigated for the green fibers. Figure 23 below is the overall 50 measurements obtained during the 10-day repeatability study. 
Figure 23: Validation Results of Green Fibers over the 10-days period

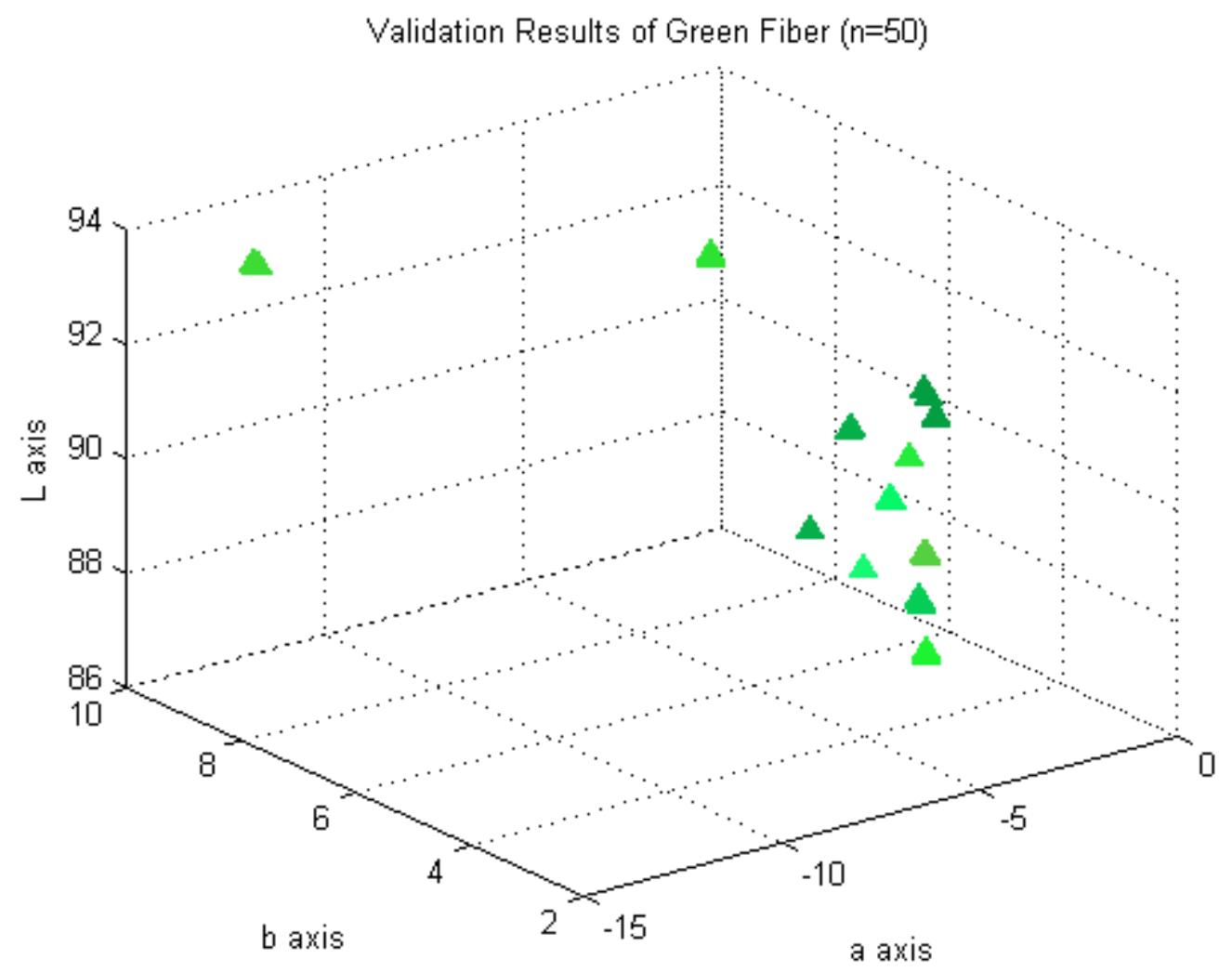

Over the 10-day period, the degree of scatter in the Lab values was more conjugated than the blue fibers. One large cluster of values is observed in the graph above. Within the cluster, the deviation from day to day measurements can be observed indicating inter-sample variation. The intra-sample variation cannot be clearly observed as most of the individual points for each data measurements are summed as one large data point. The day-to-day intra-sample variation is very minimal here. Also, two outliers are present. It is unclear at this point if the instrument or the fiber themselves contribute to the variation observed. 
Figure 24: Average Validation Results of 50 Measurements Green Fibers
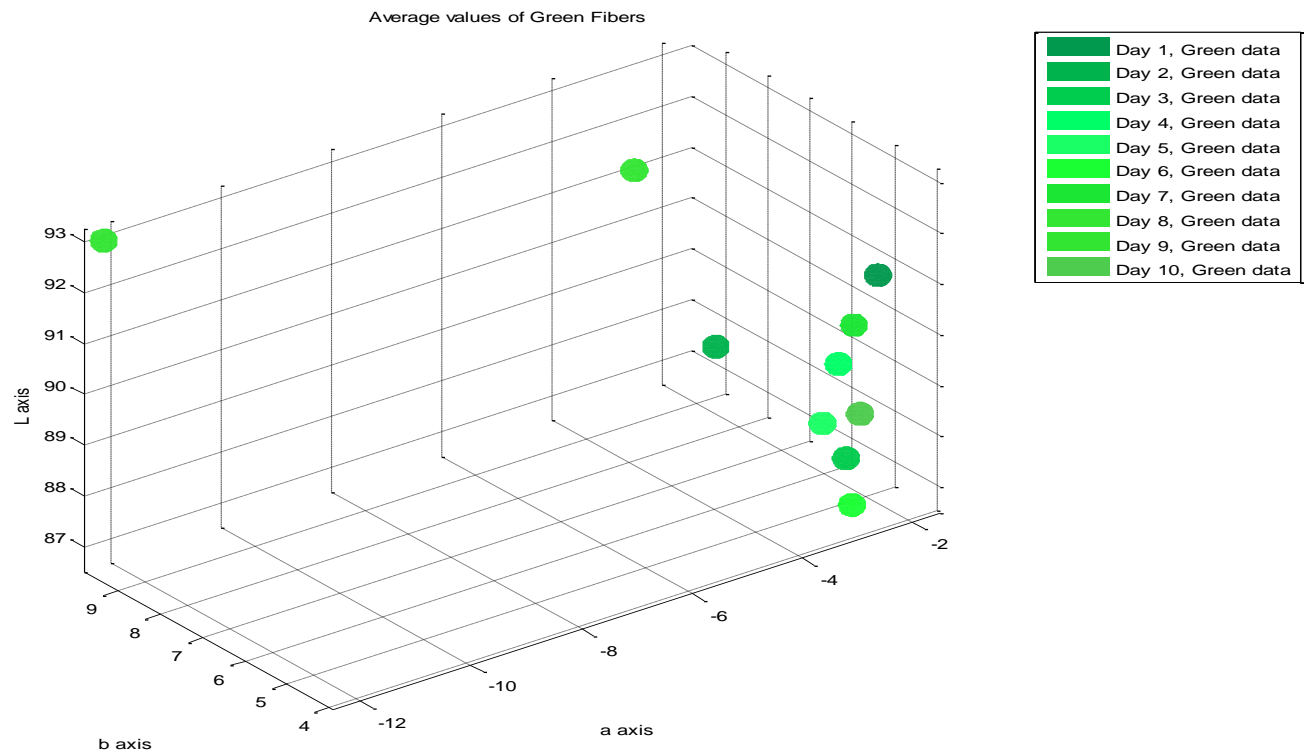

Figure 25: Average Validation Results of Green Fibers specified in difference markers

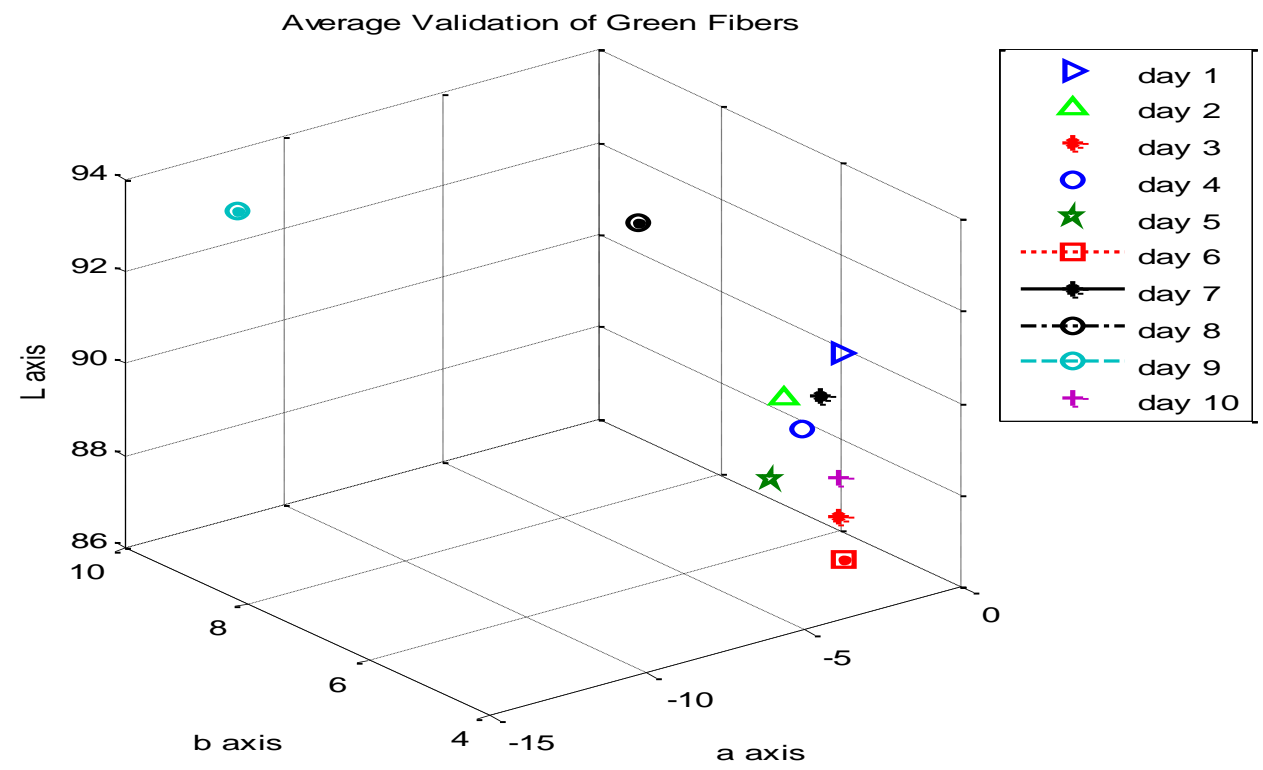

Figures 24 and 25 are the average measurements of the 10-day studies for the green fibers. As the data are averaged, fluctuations can be observed between days. However, with the averaging, one large cluster and two outliers are observed. This cluster is comprised of days 1-7 9, and 10. 
The samples were randomly measured daily, so the same fiber was not analyzed twice. This might account for the random variability observed between the days. Again, two outliers can be seen here which represents days 8 and 9 , respectively. This pattern was also observed for the blue fibers, may have been caused by any fluctuations within the instrument's configuration.

Figure 26: Average Validation Results of 50 Measurements Green Fibers with Uncertainty

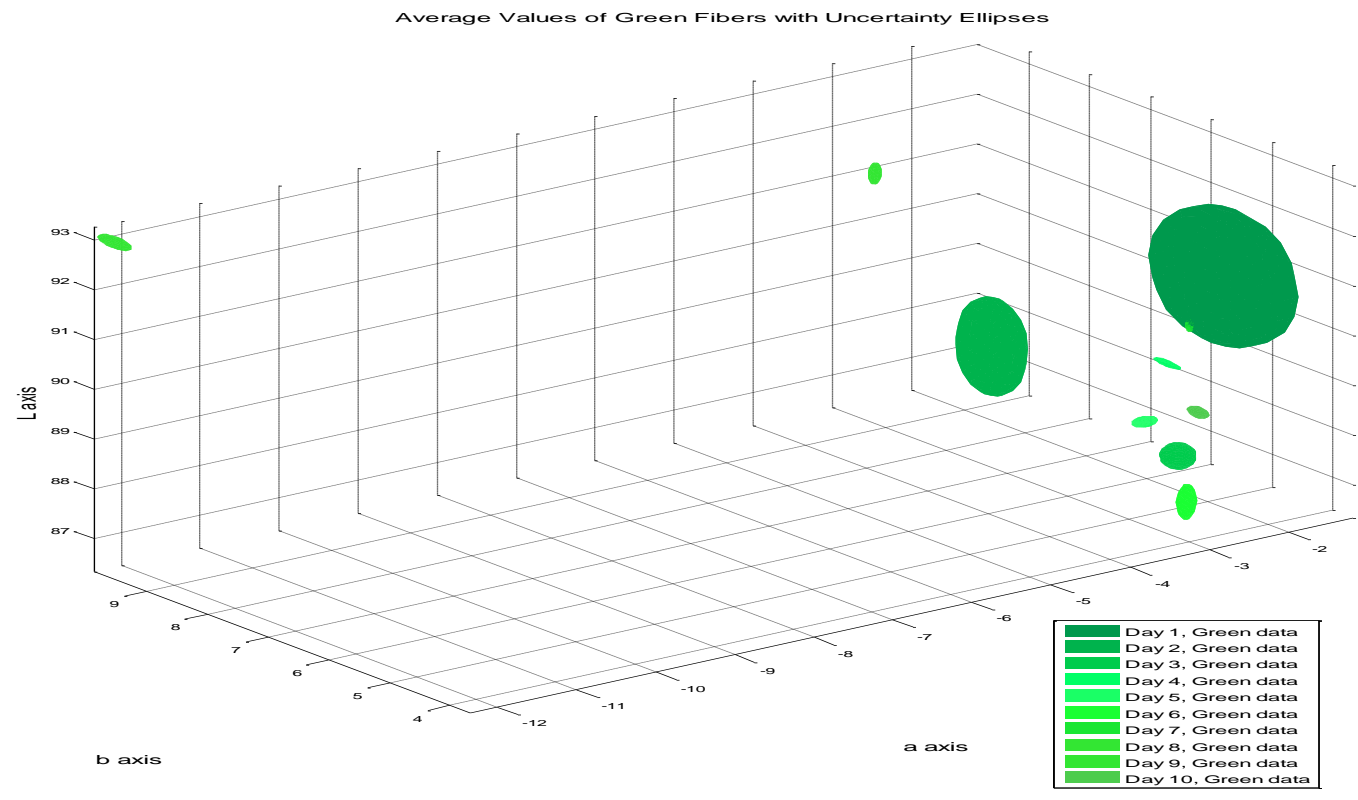

In Figure 26, two large CI can be observed which corresponds to days 1 and 2 . So between the measurements on both days, the greatest amount of deviations occurs here. Over the 10-day period, the degree of scatter in the Lab values was more conjugated than for the blue or green fibers. One large cluster of values was observed in the graph above. Within the cluster, the deviation from day to day measurements can be observed indicating inter-sample variation. The day-to-day intra-sample variation is very minimal here. Also, two outliers are present. This figure shows the variation of the instrument when five simultaneous measurements are taken for each sample. The instrument's variability and the fiber's inter-variability are observed within and 
among the day-to-day measurements, respectively. The instrument's variability, although observed, is not substantial in some analyses but is in others. This would suggest that the variation also occurs within the fibers themselves.

\subsection{2c Red Fibers}

Figure 27: Validation Results of Red Fibers

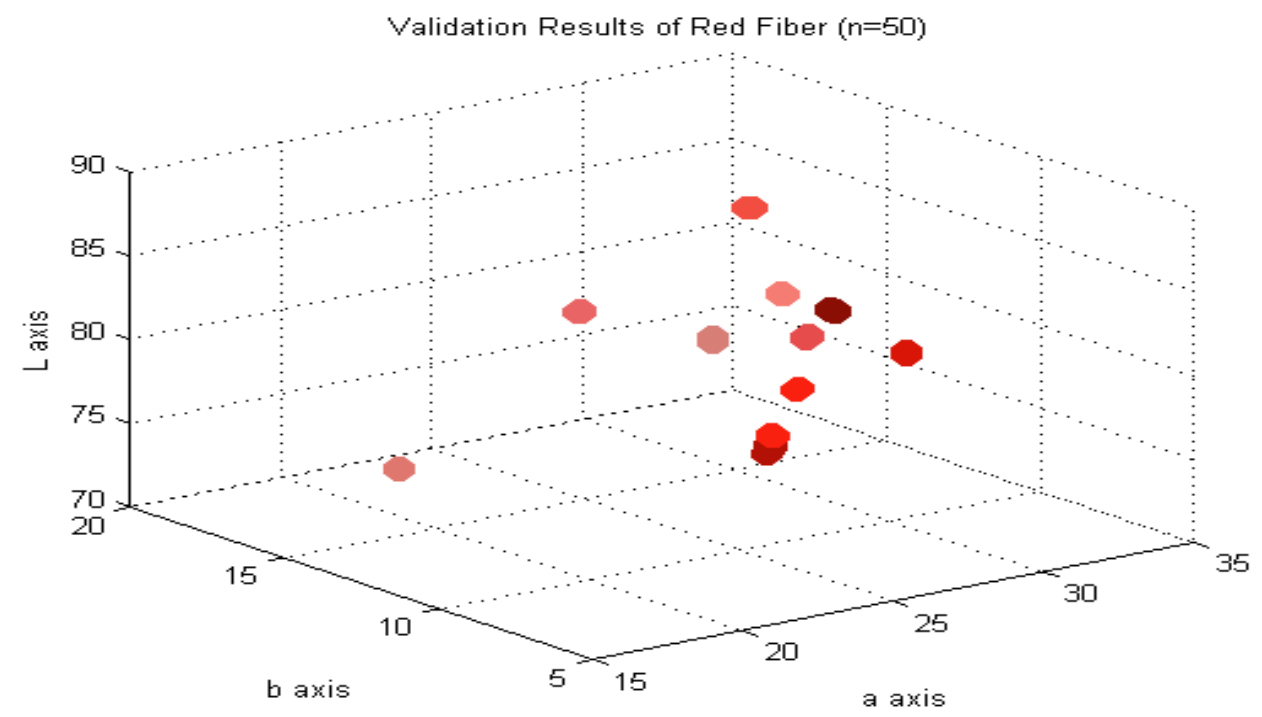

Figure 28: Average Validation Results of Red Fibers

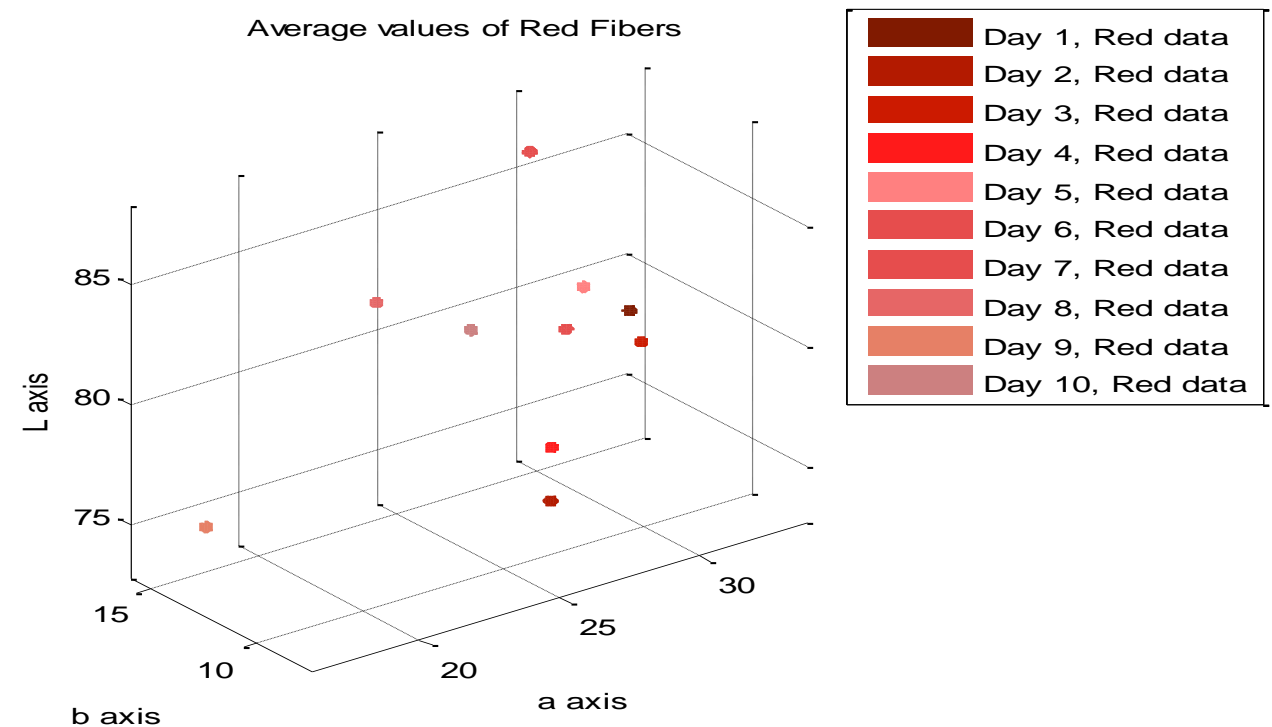


Figure 29: Average Validation Results of Red Fibers specified in different markers

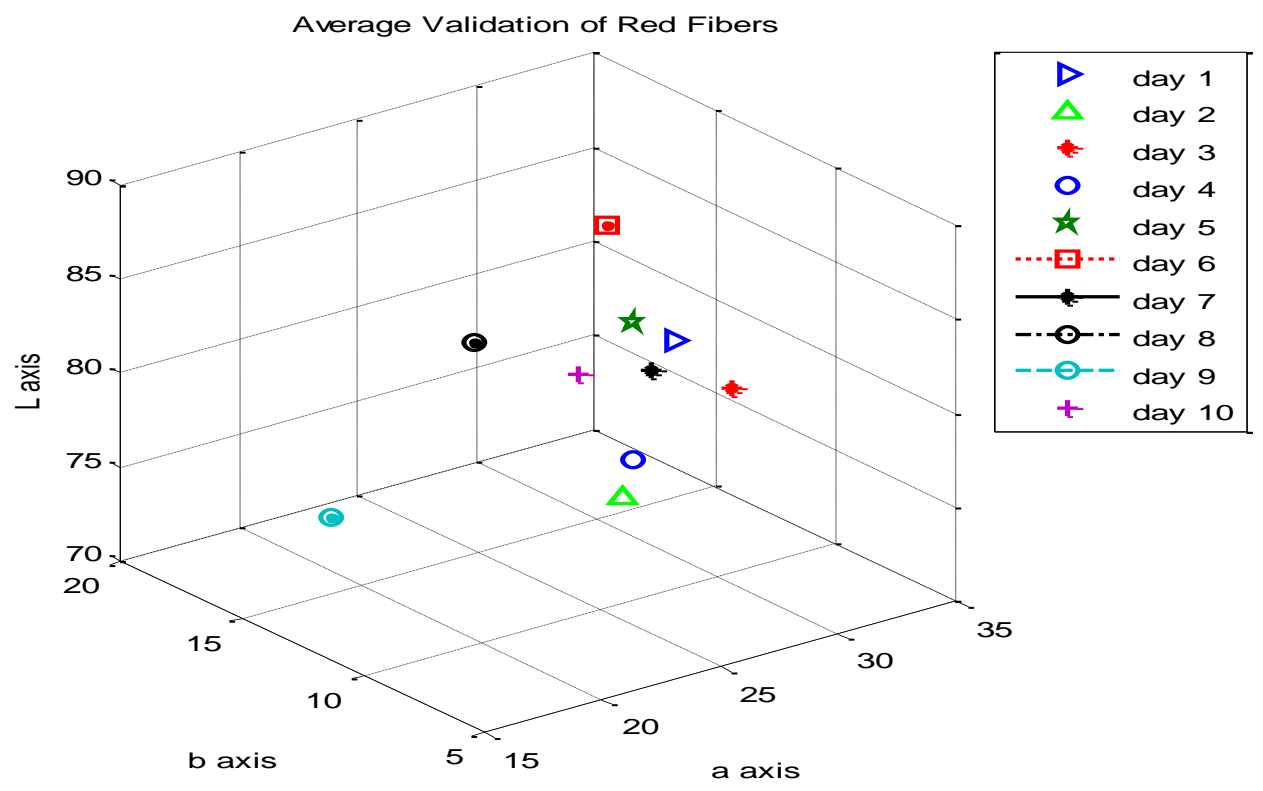

Upon further analysis, the deviations between the 10-day studies observe to be minimal as the data points are observed to be more clustered together. It is also clearly perceived that two outliers are present at days 8 and 9 . Day 6 can be considered as a potential outlier, but it also seems to be associated with the clustered data. Since both days were considered as outliers for all three colors, it can be concluded that the variations presents in these days were contributed from the instrument's configuration.

For figure 30, unlike the other colors, the elliptical shapes did not observe as much variation. These ellipses are smaller than those observed in the blue and green fibers. Although, the intervariation was greatest in this sample, the intra-variation was smallest.

It can be concluded that inter-variation and intra-variation of fibers are prominent in fibers, independent of the color being analyzed. The variation within the instrument can readily be 
observed, which negatively affected the results at days 8 and 9. Otherwise, this variation is present, but not significant in altering the outcome of the results.

Figure 30: Average Validation Results of Red Fibers specified in different markers

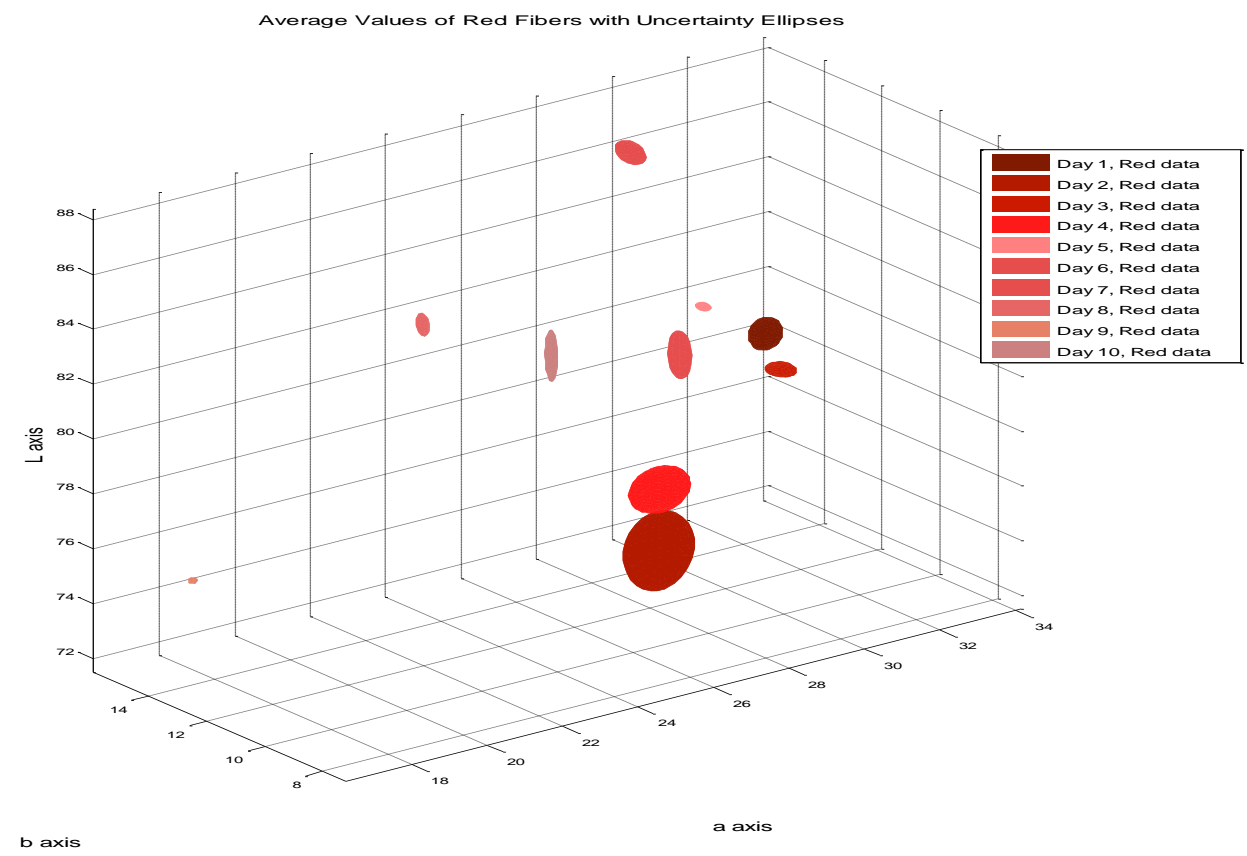

\section{$\underline{6.3 \text { Analysis of Results }}$}

The objective was to determine how color is affected through aging and washing. The questions addressed were:

- Is there intra-variability within the fiber that would significantly affect the analytical result from the treatments? In other words, does it matter where on the fiber the sample is being analyzed?

- Is there inter-variability among the fibers that would significantly affect the analytical results from the treatment? 
- Is each colored garment affected differently?

- Do the treated fibers change such that Lab values can be used to analyze these results?

To answer these questions, two hypotheses were created:

- $\mathrm{H}_{0}: \mu_{1}=\mu_{2}$ (no significant color changes were observed between fibers of the same garment)

- $\mathrm{H}_{1}: \mu_{1} \neq \mu_{2}$ (significant color changes were observed between fibers of the same garment)

6.3.1 Analysis of Aged Results- Part one of this study focused on understanding the degradation of fibers through artificial UV aging.

\subsection{1a Normality Testing of Aged Samples}

For normal distribution determination, a chart producing a histogram, a normal plot, and a box and whisker plot was graphed. Two statistical tests, K-S Lillefors and S-W test were employed for each variable to check the normality of each distribution. The data analysis was broken down into three separate steps because of the type of analysis that was performed on the samples. These steps were:

1) Normal distribution was determined for the overall Lab variables;

2) Normal distribution was determined based on the sample area and hours for each $\mathrm{L}$, a, and $b$ value; 
3) Normal distribution was determined based on the sample area, hours and fiber of each L, $a$, and $b$ variable. (Since it is impossible to analyze the same fiber aged at different time intervals, the analyzed fibers varied between hours aged).

Two hypotheses were created to test the distribution of the sample:

- $\mathrm{H}_{\mathrm{o}}: \mu_{1}=\mu_{2}$ (sample comes from a population that is normally distributed)

- $\mathrm{H}_{1}: \mu_{1} \neq \mu_{2}$ (sample does not originate from a population that is normally distributed)

Using a significance level of 0.05 , a p-value less than or equal to the significance level would result in the rejection of the null hypothesis.

\section{1) Normal distribution of all groups against variables Lab.}

The distributions for all samples were not normal. Therefore, the null hypothesis of normal distribution was rejected. Table 10 below provides the summary results taken from the S-W test.

Table 10: Summary p-values from the $S$-W test

\begin{tabular}{|l|l|l|l|l|}
\hline \multicolumn{2}{|l|}{ P-value from the Shapiro-Wilk 'W' test } \\
\hline Grouping & Ref name & L & a & b \\
\hline \multirow{4}{*}{ all groups } & DPMP & 0.00332 & 0.00057 & 0.000 \\
\cline { 2 - 5 } & GMP & 0.00927 & 0.00000 & 0.000 \\
\cline { 2 - 5 } & OSDP & 0.00646 & 0.00000 & 0.000 \\
\cline { 2 - 6 } & PMCL & 0.000 & 0.002 & 0.000 \\
\cline { 2 - 5 } & PMJX & 0.006 & 0.000 & 0.000 \\
\cline { 2 - 5 } & PMP & 0.004 & 0.011 & 0.000 \\
\cline { 2 - 5 } & RMP & 0.000 & 0.000 & 0.000 \\
\hline
\end{tabular}


At this level of interaction, it is understandable that normal distribution might not be observed on the overall sample as the inter-variability across a garment may vary greatly. With large samples, minor deviations from the normal may be flagged as very significant. A subset of the population was also tested for normality.

2) Normal distribution of 'Hours' and 'Sample Area' against variables Lab.

Three charts producing histogram, normal plot and box and whisker plot were graphed for each CIE Lab variable grouped by the sample areas A, B and C, at each hour interval. In total, 54 charts were plotted for each reference sample.

The p-values calculated from reference samples DPMP, PMCL, PMP, PMJX and RMP were all below the significant value and thus, the hypothesis of normal distribution was rejected. The summary results of the p-values taken from the S-W test can be found in Section 2 of the appendix. Only samples GMP and OSDP were found to have some data normally distributed. These samples are discussed separately below.

\section{i. $\quad G M P$}

Only one measured dataset was observed to be normally distributed and is presented below. The histogram has numerous peaks and lacks the pleasant normal bell-shape curve. However, upon observing the $S-W$-value, the value at $\mathrm{p}=0.06348$ is only slightly larger than 0.05 . There is evidence that supports the null hypothesis of normal distribution. Note that as the p-value approaches 1 , linearity and normality is better achieved. 
Figure 31: Normal Distribution of Sample GMP

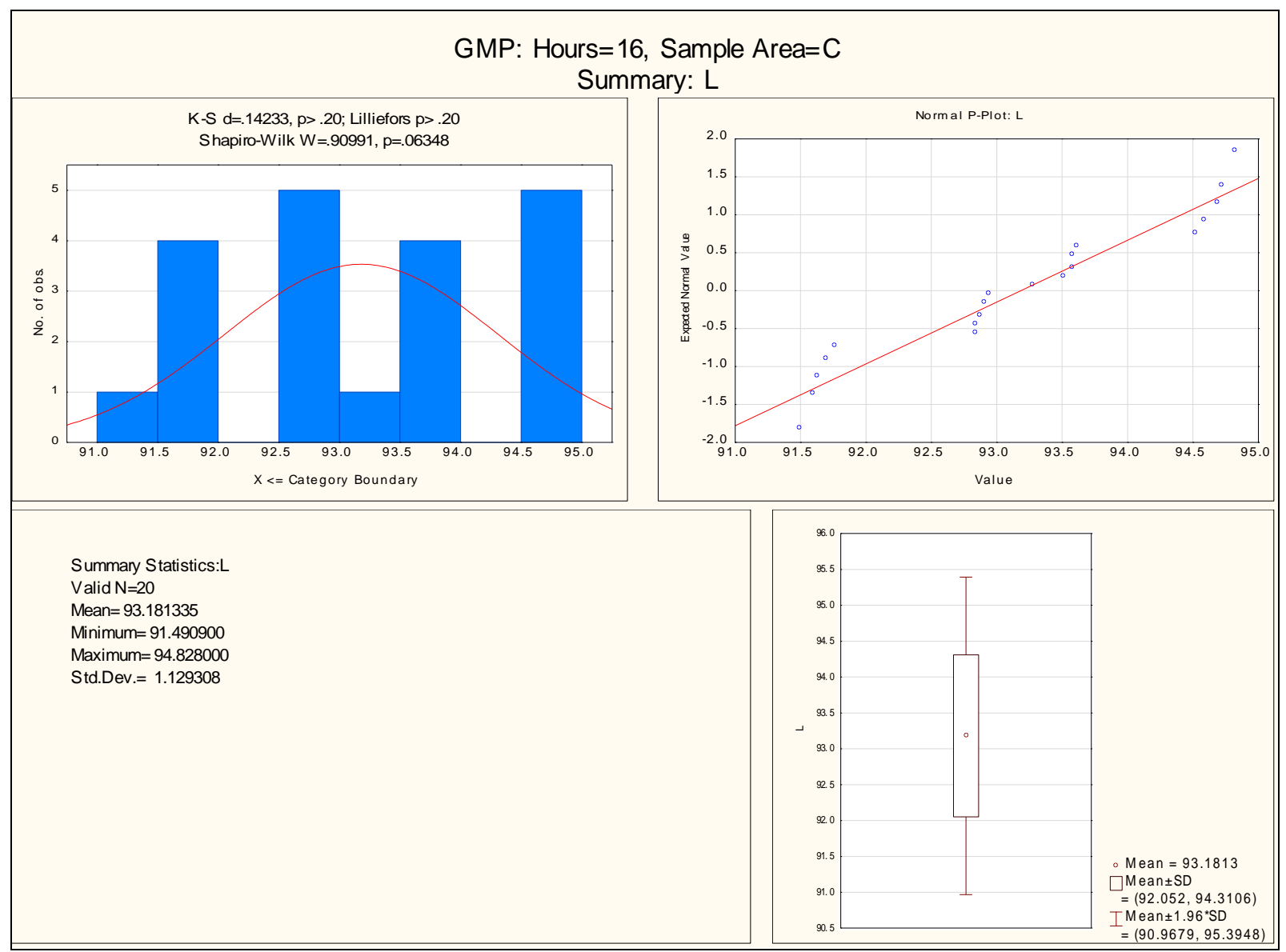

At 16 hours of aging, at sample area $C$, variable ' $L$ ' was observed to be normally distributed. Yet, normal distribution was not observed for the remainder of the data. No explanation or assumption can be inferred with regards to this random observation.

ii. $\quad$ OSDP

Three normally distributed datasets were observed in this sample: at 0 hours (untreated), at sample area A variable 'b'; and again at both 16 and 32 hours, at sample area C, variable 'a'. Only these three datasets observed a normal distribution at the p-values calculated at the given 
significant level. With such a small report of normality, again no inference can be suggested based on the outcome of these results.

Figure 32: Normal Distribution of Sample OSDP

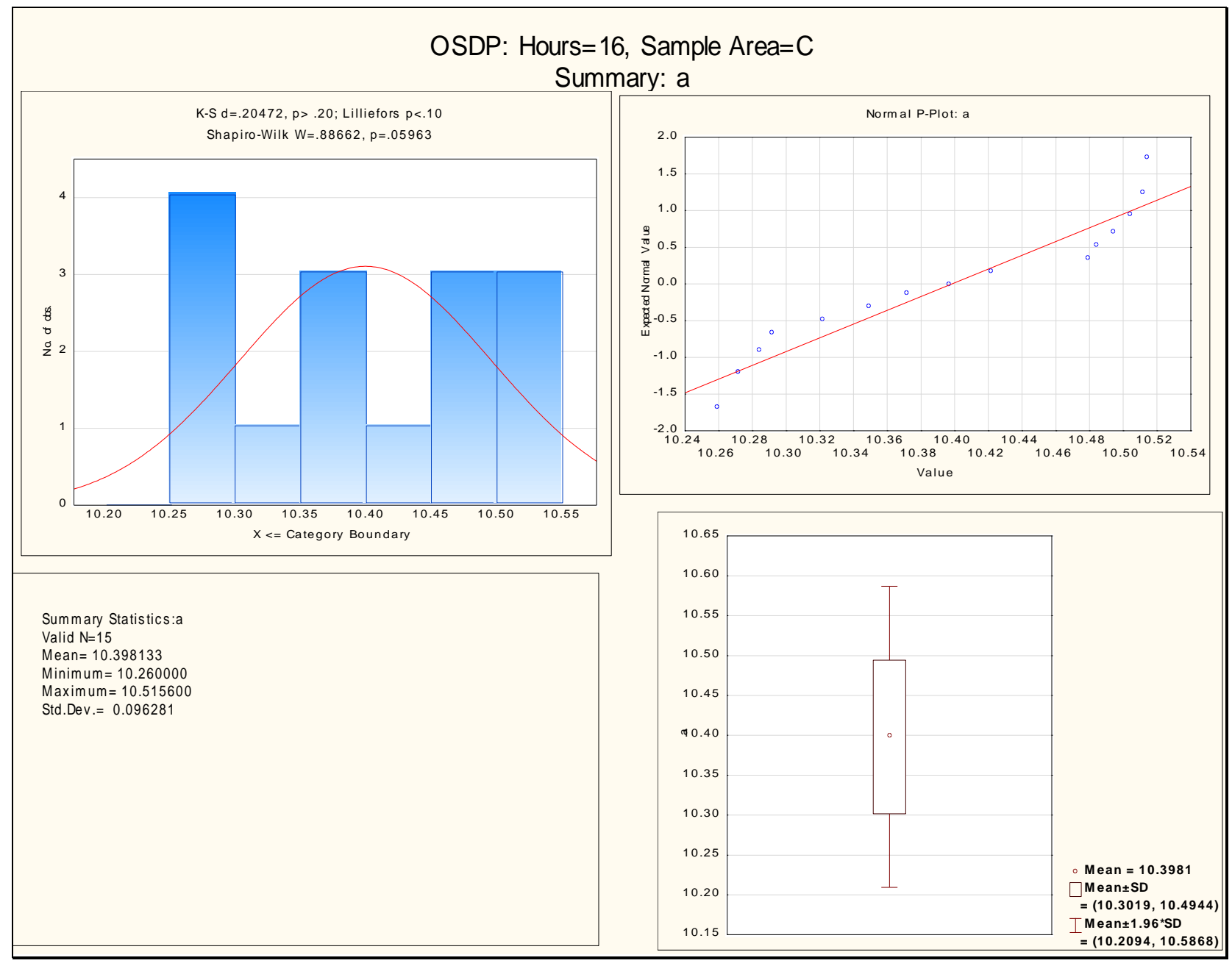

Sample Area is a subset of the population taken from the swatch. As the levels of sampling become more concentrated, inter-variability decreases and intra-variability increases. For instance, if fibers are analyzed randomly about the swatch, the inter-variability can be observed better. As the subset of the population becomes more concentrated, i.e., as the analysis is only performed on a smaller area, the inter-variability will decrease. Finally, if a single fiber is 
analyzed continually at different locations, inter-variability is not observed, but the intravariability will increase. Observing the sample area of the garment might not truly represent a subset of the population because of the intra-variability of the fiber. The analysis was further broken down to observe individual fibers from each garment.

3) Normal distribution of 'Hours', 'Sample Area' and 'Fiber' against variables Lab.

Three charts producing a histogram, normal plot and box and whisker plot were plotted for each Lab variable, grouped by hours, sample area and fiber. Nine different fibers were analyzed per sample area for every hour aged, totaling 54 different fibers. The result of the normal distribution test was tabulated in Section 2 of the appendix.

Normal distributions were observed for all samples but not for all data measurements. At this discrete level of sampling, normal distribution should be expected because the sample set taken from a larger population (in this case, the sample area) should have a smaller variance and standard deviation than from the larger population. However, small discrepancies were still present. For certain measurements, normal distribution was rejected when the analysis of the fiber was performed at two locations on that fiber. However, this was not always the case. Also, some variables observed normal distribution while others did not. For example, in reference sample DPMP at 48 hours, sample area A at fiber 29, variable 'a' was not observed to be normally distributed while the ' $L$ ' and 'b' variables were normally distributed. Such irregularities were observed throughout the reference samples.

It can be estimated that most of the fibers come from a normally distributed sample although variation within the fibers exist and sometimes affect this result. 


\section{$\underline{\text { 6.3.1b One-way ANOVA of Aged Results }}$}

One-way ANOVA analysis was performed on the samples that were aged during the experiment. The questions addressed in this part of the analysis were:

1. Are there significant changes observed in the aging process?

a. If so, what are these changes and where do they occur?

To answer these questions, two hypotheses were created:

- $\mathrm{H}_{\mathrm{o}}: \mu_{1}=\mu_{2}=\mu_{3}=\ldots \mu_{\mathrm{k}}$ (no mean differences were observed between populations);

- $\mathrm{H}_{1}$ : at least one $\mu$ is different (at least two of the population means were different).

\section{Descriptive Statistics}

The first table from the ANOVA output provides descriptive statistics for the dependent variables 'Lab' for each sample. They are presented in table format in 10 columns: Column 1 lists the name of the dependent variable; Column 2 lists the independent variable; Column 3 lists the number of observations; Column 4 to 6 list the mean, the standard deviation, and the standard error; Column 7 and 8 list the upper and lower confidence intervals; and Columns 9 and 10 list the minimum and maximum observation values. Table 11 below shows a descriptive statistics for sample DPMP. See Section 3 in appendix for the remaining values. 
Table 11: Descriptive Statistics for Sample DPMP

\begin{tabular}{|c|c|c|c|c|c|c|c|c|c|}
\hline \multirow[b]{2}{*}{ DPMP } & \multirow[b]{2}{*}{ Hours } & \multirow[b]{2}{*}{$\mathbf{N}$} & \multirow[b]{2}{*}{ Means } & \multirow[b]{2}{*}{$\begin{array}{l}\text { Std. } \\
\text { Dev }\end{array}$} & \multirow[b]{2}{*}{$\begin{array}{l}\text { Std. } \\
\text { Err. }\end{array}$} & \multicolumn{2}{|c|}{$\begin{array}{l}\text { 95\% Confidence } \\
\text { Interval for Means }\end{array}$} & \multirow[b]{2}{*}{ Minimum } & \multirow[b]{2}{*}{ Maximum } \\
\hline & & & & & & $\begin{array}{l}\text { Lower } \\
\text { Bound }\end{array}$ & $\begin{array}{l}\text { Upper } \\
\text { bound }\end{array}$ & & \\
\hline \multirow[t]{7}{*}{ L } & 0 & 45 & 84.484 & 3.922 & 0.585 & 83.306 & 85.663 & 77.915 & 89.022 \\
\hline & 16 & 60 & 86.446 & 3.638 & 0.470 & 85.506 & 87.386 & 82.267 & 94.640 \\
\hline & 32 & 60 & 83.659 & 2.694 & 0.348 & 82.963 & 84.355 & 77.552 & 86.551 \\
\hline & 48 & 60 & 83.798 & 3.064 & 0.396 & 83.006 & 84.589 & 78.376 & 88.713 \\
\hline & 64 & 60 & 86.303 & 3.465 & 0.447 & 85.408 & 87.198 & 81.939 & 94.845 \\
\hline & 80 & 60 & 83.305 & 3.815 & 0.493 & 82.319 & 84.290 & 76.581 & 90.204 \\
\hline & $\begin{array}{l}\text { All } \\
\text { Grps }\end{array}$ & & 84.674 & 3.647 & 0.196 & 84.288 & 85.060 & & 94.845 \\
\hline \multirow[t]{7}{*}{ a } & 0 & 45 & 26.830 & 2.344 & 0.349 & 26.126 & 27.534 & 23.068 & 30.920 \\
\hline & 16 & 60 & 26.928 & 2.826 & 0.365 & 26.198 & 27.658 & 21.818 & 31.669 \\
\hline & 32 & 60 & 25.791 & 4.281 & 0.553 & 24.685 & 26.897 & 19.549 & 35.793 \\
\hline & 48 & 60 & 25.035 & 3.333 & 0.430 & 24.174 & 25.896 & 21.321 & 33.077 \\
\hline & 64 & 60 & 26.935 & 3.556 & 0.459 & 26.016 & 27.853 & 21.023 & 32.374 \\
\hline & 80 & 60 & 25.384 & 2.577 & 0.333 & 24.719 & 26.050 & 20.792 & 30.484 \\
\hline & $\begin{array}{l}\text { All } \\
\text { Grps }\end{array}$ & 345 & 26.121 & 3.323 & 0.179 & 25.769 & & & 35.793 \\
\hline \multirow[t]{7}{*}{ b } & 0 & 45 & 7.307 & 2.610 & 0.389 & 6.523 & 8.091 & 0.762 & 9.900 \\
\hline & 16 & 60 & 8.467 & 1.692 & 0.218 & 8.030 & 8.904 & 5.518 & 11.350 \\
\hline & 32 & 60 & 7.599 & 1.656 & 0.214 & 7.172 & 8.027 & 4.866 & 10.948 \\
\hline & 48 & 60 & 7.161 & 1.827 & 0.236 & 6.689 & 7.633 & 4.716 & 10.067 \\
\hline & 64 & 60 & 8.070 & 2.132 & 0.275 & 7.519 & 8.620 & 4.561 & 11.151 \\
\hline & 80 & 60 & 7.652 & 1.471 & 0.190 & 7.271 & 8.032 & 5.234 & 9.894 \\
\hline & $\begin{array}{l}\text { All } \\
\text { Grps }\end{array}$ & 345 & 7.727 & 1.938 & 0.104 & 7.522 & 7.932 & 0.762 & 11.350 \\
\hline
\end{tabular}

\section{The Levene's test for Homogeneity of Variance}

To check the assumption that variances of each reference sample is equal, i.e., not significantly different, Levene's test was employed. This test is not sensitive to departures from normality (73). In order to check this assumption, two hypotheses were created:

- $\mathrm{H}_{\mathrm{o}}: \sigma_{1}{ }^{2}=\sigma_{2}{ }^{2}=\sigma_{3}{ }^{2}=\ldots \sigma_{\mathrm{k}}{ }^{2}$ (no differences in variance were observed between groups);

- $\mathrm{H}_{1}$ : at least one $\sigma_{\mathrm{k}}{ }^{2}$ is different (at least two of the group variances were different). 
Based on the results from the Levene's test, the null hypothesis was rejected for all the samples except samples PMP and RMP. The remaining reference samples produced significant results at all three variables (Section 4 in the appendix). Sample PMP and RMP did not produce significant results at variables 'b', and ' $L$ ', respectively.

Table 12: PMP Results from Levene's Test

\begin{tabular}{|c|c|c|c|c|c|c|c|c|}
\hline \multirow[b]{2}{*}{ Variable } & \multicolumn{8}{|c|}{$\begin{array}{l}\text { Levene Test of Homogeneity of Variances (PMP spreadsheet) } \\
\text { Marked effects are significant at } p<.05000\end{array}$} \\
\hline & $\begin{array}{c}\text { SS } \\
\text { Effect }\end{array}$ & $\begin{array}{c}\mathrm{df} \\
\text { Effect }\end{array}$ & $\begin{array}{c}\text { MS } \\
\text { Effect }\end{array}$ & $\begin{array}{l}\text { SS } \\
\text { Error }\end{array}$ & \begin{tabular}{c|}
$\mathrm{df}$ \\
Error
\end{tabular} & \begin{tabular}{l|} 
MS \\
Error
\end{tabular} & $\mathrm{F}$ & $p$ \\
\hline $\mathrm{L}$ & $402.9^{-1}$ & 5 & $80.5 \varepsilon$ & 1518.4 & $33 \mathrm{c}$ & $4.47 \mathrm{q}$ & $17.99^{\circ}$ & 0.000 \\
\hline a & $27.2 \varsigma$ & 5 & $5.4 €$ & 178. & $33 \mathrm{~s}$ & $0.52 \epsilon$ & $10.38^{\circ}$ & $0.00 c$ \\
\hline b & 26.51 & 5 & $5.3 \mathrm{C}$ & 1098. & $33 \mathrm{~s}$ & $3.23 \mathrm{c}$ & $1.63 i$ & $0.15 \mathrm{C}$ \\
\hline
\end{tabular}

Note: Significance are written in red and marked in bold.

The result is not significant at variable ' $b$ ', as the significance is far greater than 0.05 . Therefore, there is no reason to assume that the variance of this variable in this sample is not different.

Table 13: RMP Results from Levene's Test

\begin{tabular}{|c|c|c|c|c|c|c|c|c|}
\hline \multirow[b]{2}{*}{ Variable } & \multicolumn{8}{|c|}{$\begin{array}{l}\text { Levene Test of Homogeneity of Variances (RMP spreadsheet) } \\
\text { Marked effects are significant at } p<.05000\end{array}$} \\
\hline & $\begin{array}{c}\text { SS } \\
\text { Effect }\end{array}$ & $\begin{array}{c}d f \\
\text { Effect }\end{array}$ & $\begin{array}{c}\text { MS } \\
\text { Effect }\end{array}$ & $\begin{array}{l}\text { SS } \\
\text { Error }\end{array}$ & $\begin{array}{c}d f \\
\text { Error }\end{array}$ & $\begin{array}{l}\text { MS } \\
\text { Error }\end{array}$ & \begin{tabular}{l|l}
$\mathrm{F}$ \\
\end{tabular} & $p$ \\
\hline $\mathrm{L}$ & 70.48 & 5 & 14.05 & $2384 . \varepsilon$ & $33 \mathrm{C}$ & 7.035 & $2.00 k$ & $0.07 \varepsilon$ \\
\hline$a$ & $580.8 c$ & 5 & 116.16 & $2349 . \varepsilon$ & 339 & 6.931 & $16.75 !$ & $0.00 c$ \\
\hline b & $404.4 \varepsilon$ & 5 & 80.90 & 1310. & $33 \mathrm{C}$ & $3.86 \epsilon$ & $20.92 i$ & 0.000 \\
\hline
\end{tabular}

Note: Significance are written in red and marked in bold.

The result is not significant at variable ' $L$ ', as the significance is greater than 0.05 . It can then be assumed, that the variance at this variable is not different across the data.

III. One-way ANOVA for Aged Samples 
To test the significant difference of means between populations using one-way ANOVA, the data analysis was broken down into three separate steps to account for the independent or grouping variables. These steps were:

1) ANOVA was performed on variables Lab grouped by variable 'Hours';

2) ANOVA was performed on variables Lab grouped by 'Sample Area';

3) ANOVA was performed on variables Lab grouped by 'Fibers'.

One table and four graphs were plotted for each color sample. The table comprised of the computed results from the ANOVA calculation. Based on these results, a plot of mean and confidence interval was graphed was as summary plot that included all three dependent variables. Three additional mean plots were graphed separately for each dependent variable, and the calculated F- and p-values were recorded on its respective graph.

\section{1) ANOVA was performed on variables Lab grouped by 'Hours'}

Based on the ANOVA calculations, there was strong evidence (at $\mathrm{p}=0.05$, or the $5 \%$ level) that the means differ significantly from one another. Of the samples analyzed, all but PMJX calculated significant p-values to reject the null hypothesis. The mean values between groups were substantial in proving that there were differences greater than those that would occur by random chances alone. The 'L' value in sample PMJX was not be significantly different among the hours aged. The F-value below, yet close 1, signifies that the within-group error was much greater than the between-group effect. However, variables ' $a$ ' and ' $b$ ' produced different outcome. Based on the calculated value in variable ' $a$ ', the probability of obtaining an F-value greater than the calculated value 10.70831 when the population means are really equal, is 0.00 , 
interpreted as 0.01. It can be concluded that the aging treatment affected the outcome of the results.

Table 14: ANOVA results of Lab variables grouped by 'Hours'

\begin{tabular}{|c|c|c|c|c|c|c|c|c|}
\hline \multicolumn{9}{|c|}{ Grouped by Hours } \\
\hline DPMP & SS & df & MS & SS & df & MS & $\mathbf{F}$ & $p$ \\
\hline $\mathrm{L}$ & 569.71 & 5 & 113.94 & 4006.62 & 339 & 11.819 & 9.641 & 0.000 \\
\hline$a$ & 211.27 & 5 & 42.25 & 3587.82 & 339 & 10.584 & 3.992 & 0.002 \\
\hline$b$ & 68.42 & 5 & 13.68 & 1223.05 & 339 & 3.608 & 3.793 & 0.002 \\
\hline GMP & SS & df & MS & SS & df & MS & $F$ & $\mathbf{p}$ \\
\hline $\mathrm{L}$ & 467.35 & 5 & 93.47 & 6871.38 & 339 & 20.270 & 4.611 & 0.000 \\
\hline$a$ & 12.13 & 5 & 2.43 & 271.79 & 339 & 0.802 & 3.025 & 0.011 \\
\hline$b$ & 59.06 & 5 & 11.81 & 1636.50 & 339 & 4.827 & 2.447 & 0.034 \\
\hline OSDP & SS & df & MS & SS & df & MS & $\mathbf{F}$ & $\mathbf{p}$ \\
\hline $\mathrm{L}$ & 319.39 & 5 & 63.88 & 6404.05 & 339 & 18.891 & 3.381 & 0.005 \\
\hline$a$ & 60.05 & 5 & 12.01 & 904.65 & 339 & 2.669 & 4.500 & 0.001 \\
\hline b & 1084.60 & 5 & 216.92 & 7931.01 & 339 & 23.395 & 9.272 & 0.000 \\
\hline PMCL & SS & df & MS & SS & df & MS & $F$ & $p$ \\
\hline $\mathrm{L}$ & 584.50 & 5 & 116.90 & 7208.86 & 339 & 21.265 & 5.497 & 0.000 \\
\hline$a$ & 11.06 & 5 & 2.21 & 177.70 & 339 & 0.524 & 4.220 & 0.001 \\
\hline b & 34.09 & 5 & 6.82 & 508.53 & 339 & 1.500 & 4.545 & 0.001 \\
\hline PMJX & SS & df & MS & SS & df & MS & $F$ & $p$ \\
\hline $\mathrm{L}$ & 88.77 & 5 & 17.75 & 6060.02 & 339 & 17.876 & 0.993 & 0.422 \\
\hline$a$ & 22.01 & 5 & 4.40 & 139.34 & 339 & 0.411 & 10.708 & 0.000 \\
\hline$b$ & 144.62 & 5 & 28.92 & 505.76 & 339 & 1.492 & 19.387 & 0.000 \\
\hline PMP & SS & df & MS & SS & df & MS & $F$ & $p$ \\
\hline $\mathrm{L}$ & 6011.57 & 53 & 113.43 & 1166.09 & 291 & 4.007 & 28.306 & 0.000 \\
\hline$a$ & 659.30 & 53 & 12.44 & 104.98 & 291 & 0.361 & 34.481 & 0.000 \\
\hline$b$ & 3149.89 & 53 & 59.43 & 370.70 & 291 & 1.274 & 46.654 & 0.000 \\
\hline RMP & SS & df & MS & SS & df & MS & $F$ & $\mathbf{p}$ \\
\hline $\mathrm{L}$ & 355.70 & 5 & 71.14 & 5259.36 & 339 & 15.514 & 4.585 & 0.000 \\
\hline $\mathrm{a}$ & 317.16 & 5 & 63.43 & 6965.90 & 339 & 20.548 & 3.087 & 0.010 \\
\hline$b$ & 304.24 & 5 & 60.85 & 4095.89 & 339 & 12.082 & 5.036 & 0.000 \\
\hline
\end{tabular}

Note: Significances are marked in bold italics.

The ANOVA table listed below was computed using the SPSS software. This is a key table because it shows whether the overall F-ratio for ANOVA is significant. 
Table 15: ANOVA Output for Sample DPMP

\begin{tabular}{|ll|r|r|r|r|r|}
\hline \multicolumn{6}{|c|}{ ANOVA: DPMP } \\
\hline & \multicolumn{1}{c|}{$\begin{array}{c}\text { Sum of } \\
\text { Squares }\end{array}$} & \multicolumn{1}{c|}{ df } & \multicolumn{1}{c|}{$\begin{array}{c}\text { Sean } \\
\text { Square }\end{array}$} & \multicolumn{1}{c|}{$\mathrm{F}$} & Sig. \\
\hline L & Between Groups & 569.71 & 5 & 113.94 & 9.641 & .000 \\
& Within Groups & 4006.6 & 339 & 11.819 & & \\
& Total & 4576.3 & 344 & & & \\
& Between Groups & 211.27 & 5 & 42.253 & 3.992 & .002 \\
& Within Groups & 3587.8 & 339 & 10.584 & & \\
& Total & 3799.1 & 344 & & & \\
& Between Groups & 68.42 & 5 & 13.684 & 3.793 & .002 \\
& Within Groups & 1223.1 & 339 & 3.608 & & \\
& Total & 1291.5 & 344 & & & \\
\hline
\end{tabular}

The results show that the three means for the variables are significant. Within each variable, the means are observed to be significantly different from the other: the significance level of the $\mathrm{F}$ ratio is given as $<.001, .002$ and 0.02 for variables ' $\mathrm{L}$ ', 'a' and 'b', respectively.

For variable ' $L$ ', $F(5,339)=9.641, \mathrm{p}<0.05$. The 5 and 339 are the two df values for the between- groups effect and the within-groups error, respectively. The 9.641 is the obtained Fvalue and the $\mathrm{p}<0.05$, is the probability of obtaining that $\mathrm{F}$ value by chance alone. The results produced in this table show that significant values are observed and thus, the null hypothesis is rejected. This hypothesis of all five groups being equal is rejected. It can be concluded that at least one of the group means is significantly different from the others.

The adjusted F-test called the Welch test, was also employed because of the violation of the equal variance assumption. The F-test commonly used with ANOVA is based on the assumption of equal population variance. To address the problem of unequal variance, the Welch test was developed as an alternative to the F-test. The Welch test was used along with the ANOVA F-test 
for more accurate results. The p-values were also computed using the Welch test as a robust test for the equality of means.

Table 16: Welch results of Lab variables grouped by 'Hours'

\begin{tabular}{|c|c|c|c|c|c|c|c|c|c|}
\hline \multicolumn{10}{|c|}{ Grouped by Hours } \\
\hline DPMP & $\begin{array}{c}\text { Welch } \\
\text { df }\end{array}$ & $\begin{array}{c}\text { Welch } \\
\text { df }\end{array}$ & $\begin{array}{l}\text { Welch } \\
\text { F }\end{array}$ & $\begin{array}{c}\text { Welch } \\
\text { p }\end{array}$ & PMJX & $\begin{array}{c}\text { Welch } \\
\text { df }\end{array}$ & $\begin{array}{c}\text { Welch } \\
\text { df }\end{array}$ & Welch F & $\begin{array}{l}\text { Welch } \\
\text { p }\end{array}$ \\
\hline $\mathrm{L}$ & 5 & 154.17 & 9.486 & 0.000 & $\mathrm{~L}$ & 5 & 153.17 & 1.145 & 0.339 \\
\hline$a$ & 5 & 156.65 & 4.653 & 0.001 & $a$ & 5 & 151.36 & 21.280 & 0.000 \\
\hline$b$ & 5 & 153.29 & 4.017 & 0.002 & $b$ & 5 & 151.78 & 30.584 & 0.000 \\
\hline GMP & $\begin{array}{c}\text { Welch } \\
\text { df }\end{array}$ & $\begin{array}{c}\text { Welch } \\
\text { df }\end{array}$ & $\begin{array}{l}\text { Welch } \\
\text { F }\end{array}$ & $\begin{array}{c}\text { Welch } \\
\text { p }\end{array}$ & PMP & $\begin{array}{c}\text { Welch } \\
\text { df }\end{array}$ & $\begin{array}{c}\text { Welch } \\
\text { df }\end{array}$ & Welch F & $\begin{array}{c}\text { Welch } \\
\text { p }\end{array}$ \\
\hline $\mathrm{L}$ & 5 & 151.93 & 4.508 & 0.001 & $\mathrm{~L}$ & 53 & 88.21 & 48231.2 & 0.000 \\
\hline a & 5 & 151.15 & 6.311 & 0.000 & a & 53 & 88.24 & 30178.9 & 0.000 \\
\hline$b$ & 5 & 156.65 & 3.077 & 0.011 & $b$ & 53 & 87.08 & 84713.2 & 0.000 \\
\hline OSDP & $\begin{array}{c}\text { Welch } \\
\text { df }\end{array}$ & $\begin{array}{c}\text { Welch } \\
\text { df }\end{array}$ & $\begin{array}{l}\text { Welch } \\
\text { F }\end{array}$ & $\begin{array}{c}\text { Welch } \\
\text { p }\end{array}$ & RMP & $\begin{array}{c}\text { Welch } \\
\text { df }\end{array}$ & $\begin{array}{c}\text { Welch } \\
\text { df }\end{array}$ & Welch F & $\begin{array}{c}\text { Welch } \\
\text { p }\end{array}$ \\
\hline L & 5 & 156.54 & 4.428 & 0.001 & L & 5 & 154.37 & 3.909 & 0.002 \\
\hline a & 5 & 157.12 & 9.757 & 0.000 & $a$ & 5 & 148.30 & 6.801 & 0.000 \\
\hline$b$ & 5 & 156.33 & 31.895 & 0.000 & $b$ & 5 & 150.57 & 4.934 & 0.000 \\
\hline PMCL & $\begin{array}{c}\text { Welch } \\
\text { df }\end{array}$ & $\begin{array}{c}\text { Welch } \\
\text { df }\end{array}$ & $\begin{array}{l}\text { Welch } \\
\text { F }\end{array}$ & $\begin{array}{c}\text { Welch } \\
\text { p }\end{array}$ & & & & & \\
\hline $\mathrm{L}$ & 5 & 153.71 & 4.292 & 0.001 & & & & & \\
\hline$a$ & 5 & 153.82 & 3.879 & 0.002 & & & & & \\
\hline$b$ & 5 & 153.01 & 6.183 & 0.000 & & & & & \\
\hline
\end{tabular}

Note: Significances are marked in bold italics.

The results from the Welch test complement those from the ANOVA results. Both test produced insignificant mean values of sample PMJX at variable 'L', was not observed to be significantly different. Since the results of the two tests are similar, the results of the standard ANOVA are discussed. Charts were created better interpretation purposes. A summary mean plot of the overall Lab variables was plotted, and three additional charts were created for each variable. The results of sample PMJX are provided below. These plots were also used for greater visualization of the means of each dependent variable at each hour interval and thus accessibly note where the error bars overlap. The F-test was then employed for each dependent variable disjointedly. 
Figure 33: Mean plot of Lab variables grouped by 'Hours'

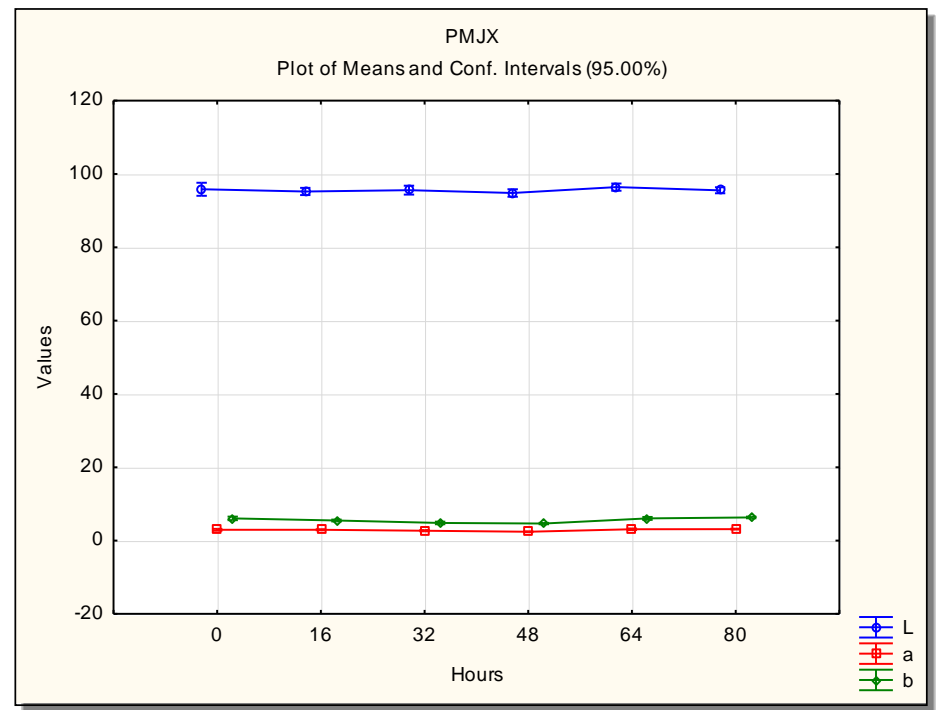

Notice that in the mean plot of variable ' $L$ ', overlapping occurs within the intervals. A sign of overlapping within the confidence interval implies that the population means are similar. Since the confidence overlaps, it means that at a $95 \%$ level of confidence, there is not sufficient evidence (at $\mathrm{p}=.4218$ ) in rejecting the null hypothesis.

Figure 34: Mean plot of variable 'L' grouped by 'Hours'

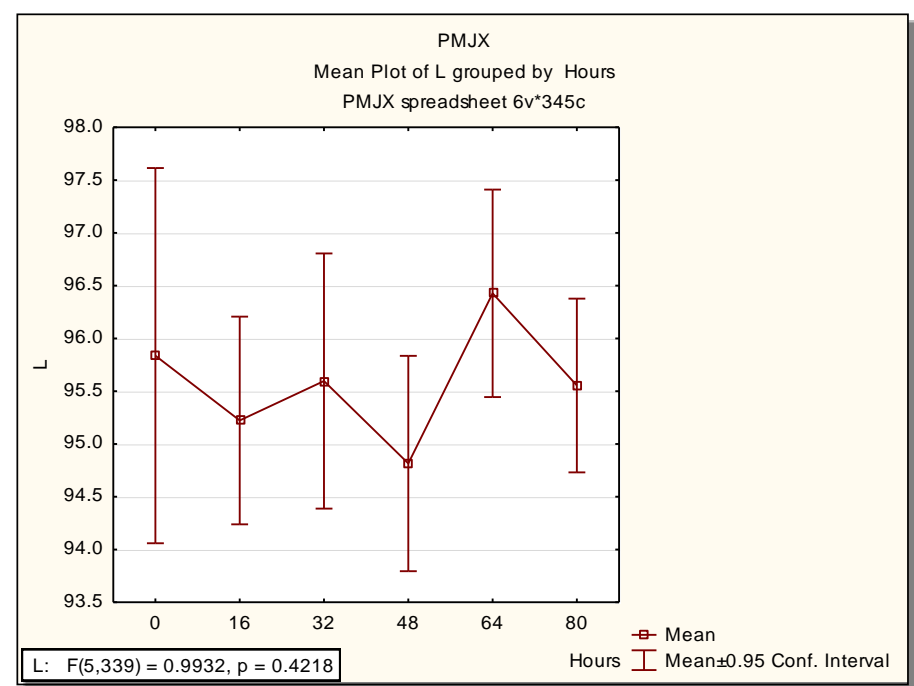


Notice the contrast among the patterns of the confidence level from variable ' $\mathrm{L}$ ' to variables ' $\mathrm{a}$ ' and ' $b$ '.

Figure 35a-b: Mean plot of variables ' $a$ ' and ' $b$ ' grouped by 'Hours'
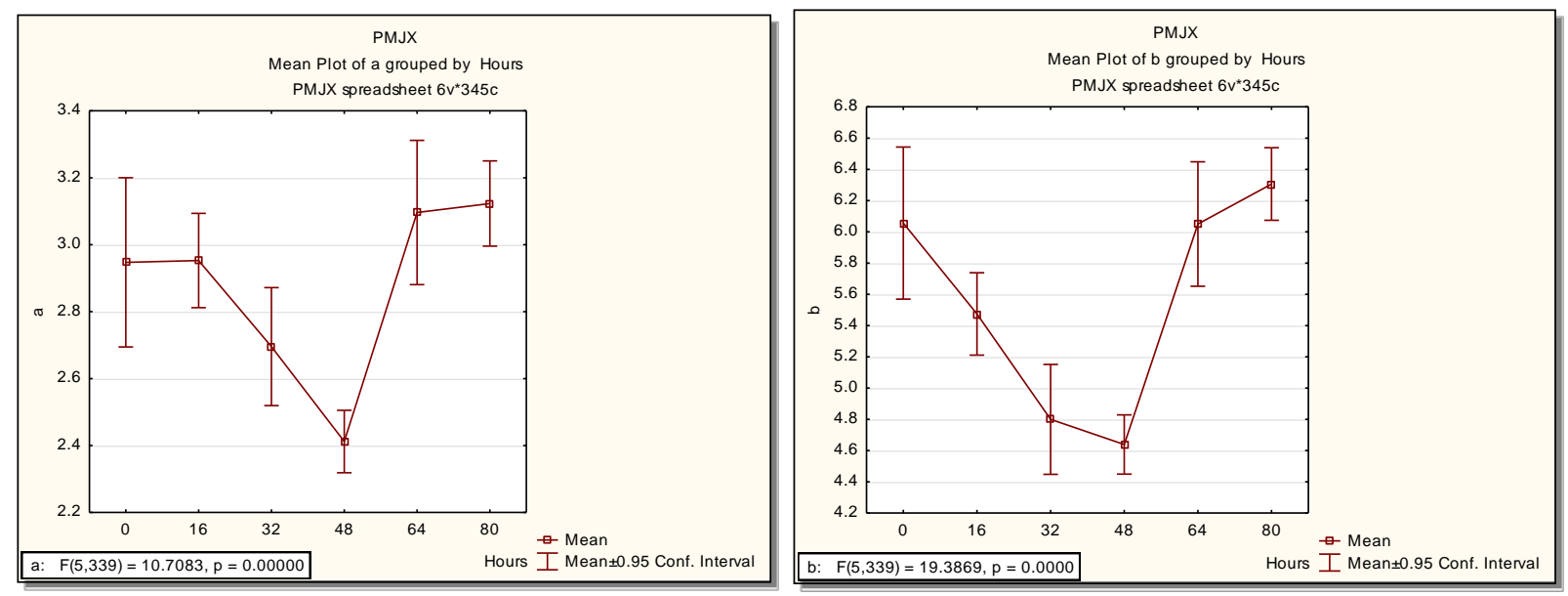

Although some overlap is seen, there are instances in the aging intervals, where the mean varies between different groups. For instance, variable 'a' at $0,16,64$, and 80 hours overlaps each other in some areas. This suggests that they were some similarities between the groups. However, at 48 hours of aging, the confidence interval does not overlap with the means of the other groups. This therefore implies that the mean is different in this group. This result is also very similar to variable ' $b$ '. The ANOVA test establishes that there is some significance among the means, but it is limited in explaining where the differences lie. Analyzing the mean plots only provides insight as to where this might occur. Again, the p-value was used in corroboration with the charts to reach the decision of rejecting the null hypothesis.

Samples DPMP, GMP, OSDP, PMCL, PMP, and RMP

Significant changes were observed throughout the aging treatment based on the calculated ANOVA F-statistics and p-values, as well as for the patterns of the means and confidence 
interval plots. As the samples were aged, the changes observed in color were greater than what would occur by random chance alone and the variances that occurred were substantially greater between-groups than within-groups. When the error bars overlap each other, they suggest that the average value is not significantly different from the other, whereas when the error bars do not overlap, they suggest significant difference. Based on the results in table 10, each sample will be summarized below. See Section 6 in the appendix for the remaining graphs.

\section{$D P M P$}

Figure 36a-c: Mean plot for sample DPMP grouped by 'Hours'
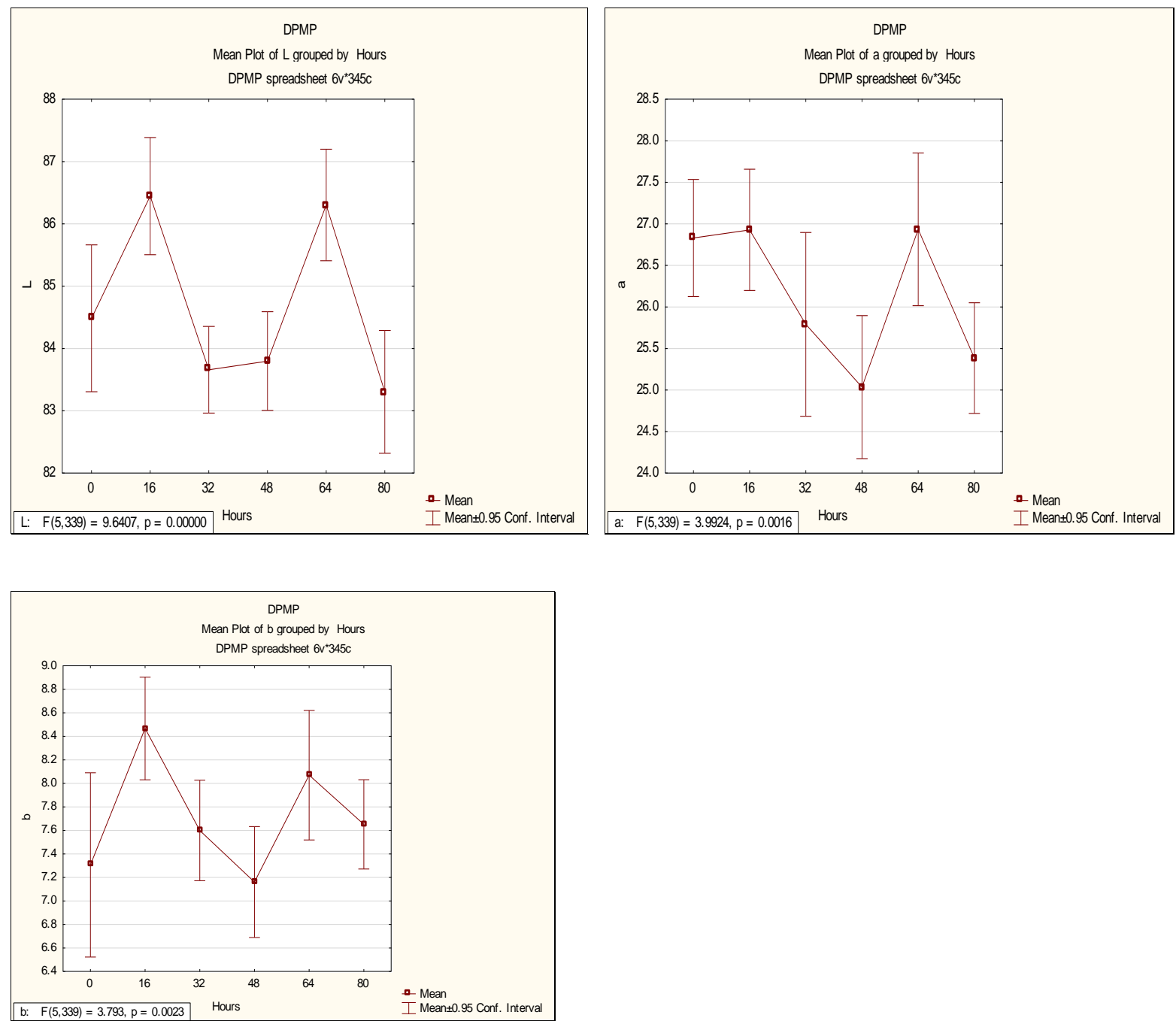
The results showed that the three means from each variable are significantly different in each group. At variable 'L', it can be observed that the means at 16 and 64 hours of aging vary significantly from the others. At variable 'a', differences can be observed at more than one group. For instance, the means of hours 0, 16 and 64 are significantly difference from 48 hours. The means of hours 32 and 48 are observed to not be significantly different from each other as their bars overlaps. And lastly at variable ' $b$ ', the mean value at 0 hours is not significantly different from the others. Some of the remaining bars do not overlap each other which would then suggest significant. Again, the p-values assist in these interpretations.

\section{GMP}

Figure 37a-c: Mean plot for Sample GMP of variables Lab grouped by 'Hours'
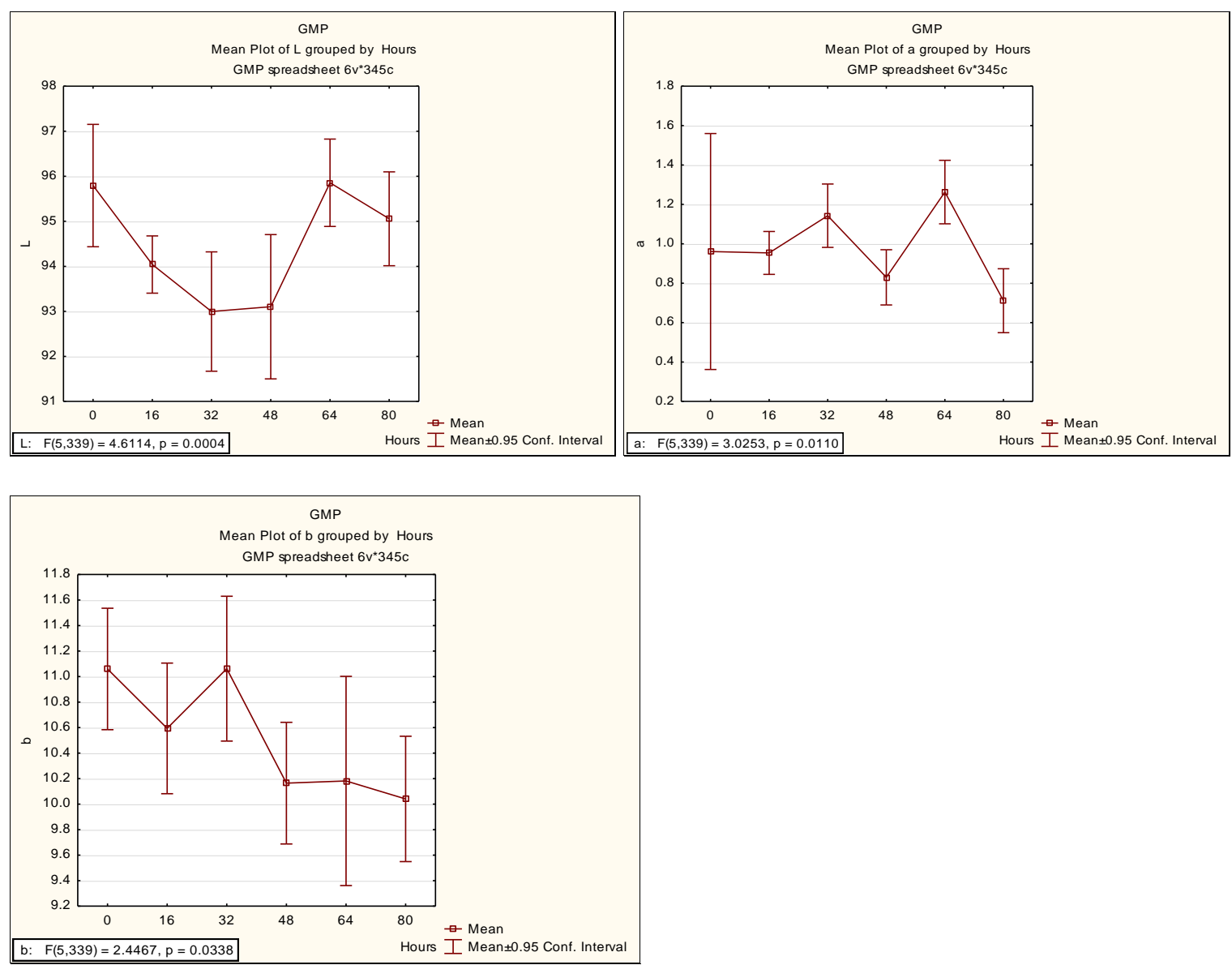
Significant differences are observed in the means of all three variables. The interesting point in these graphs is the fact that the patterns are dissimilar. The aging treatment affects each variable differently; from 0 to 48 hours, the mean values decrease for ' $L$ ' and fluctuates for ' $a$ ' and ' $b$ '. Then, from 48 to 80 hours, the mean values increase for ' $\mathrm{L}$ ', continues to fluctuate for 'a', and decrease for ' $b$ '.

\section{OSDP}

Figure 38a-c: Mean plot for sample OSDP grouped by 'Hours'
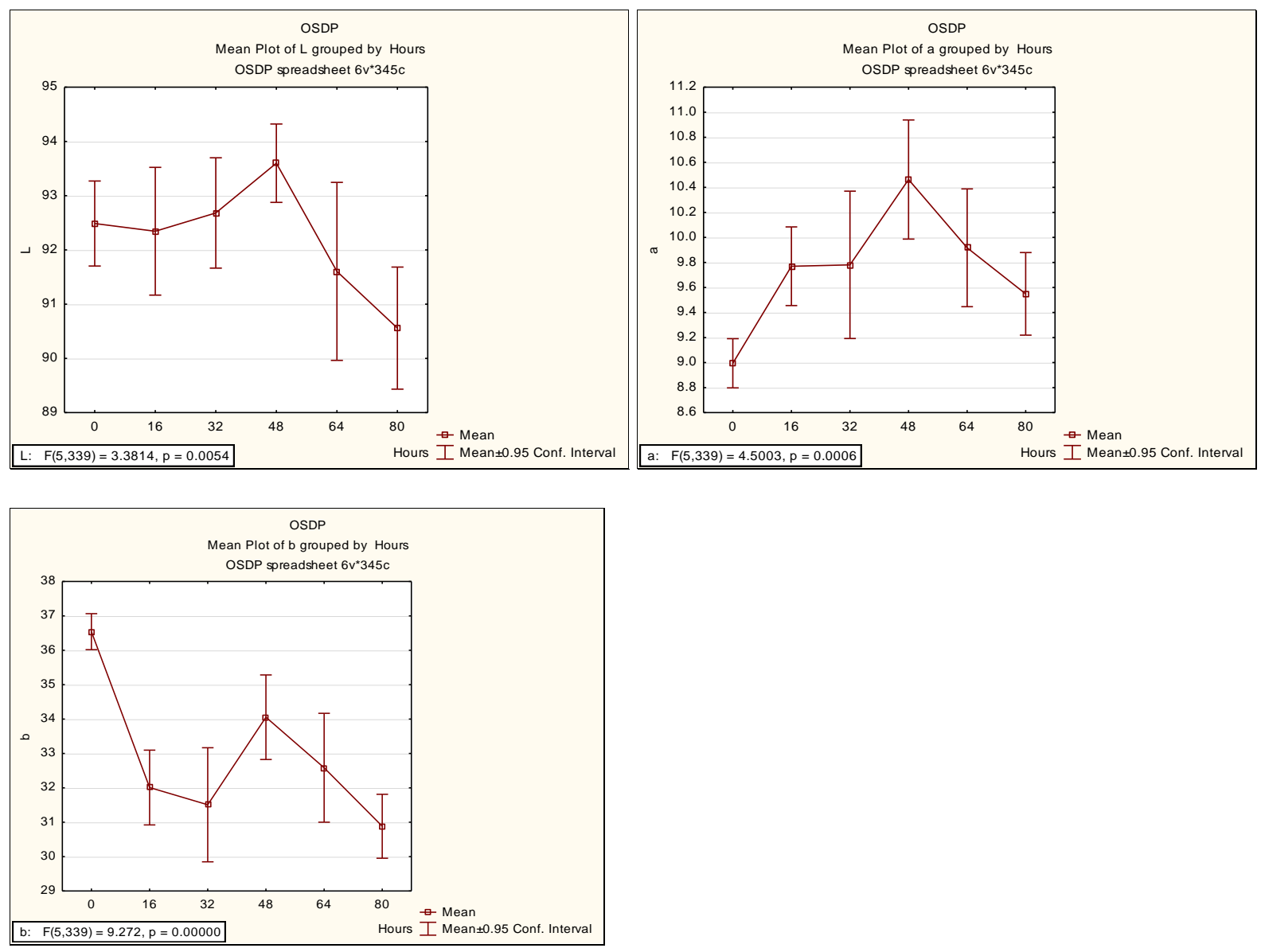

Significant differences in means were observed for all three variables. A similar pattern was observed in variables ' $a$ ' and ' $b$ ' where at 0 hours; the means of this group was significantly 
different from the remaining groups. Also notice in variable ' $\mathrm{L}$ ', a pattern was starting to develop until at 64 hours of aging. This pattern was not observed in the other samples, so no inference can be made at this stage.

\section{$P M C L$}

Figure 39a-c: Mean plot for Sample PMCL grouped by 'Hours'
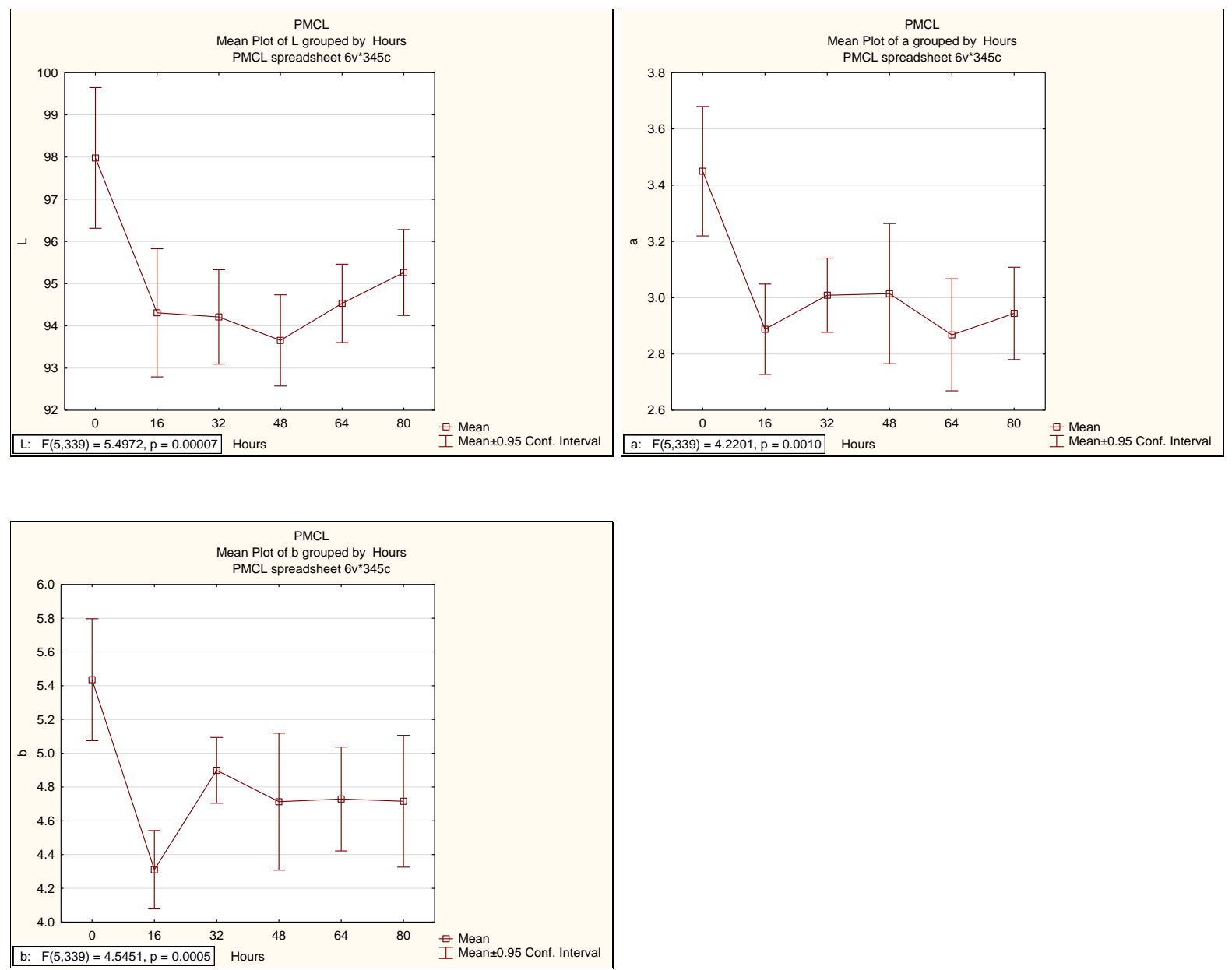

Significant differences were observed for all three variable means. A pattern can be observed for all three variables at 0 hours, where the mean value differs from most of the other groups. There is a decrease in mean value for the other groups whether significant differences occurred or not. 
Also in variable ' $b$ ', the changes in mean difference at 48,64 and 80 hours were not significant. In fact, among these hours, the changes seemed to be so minor.

\section{$P M P$}

Figure 40a-c: Mean plot for Sample PMP grouped by 'Hours'
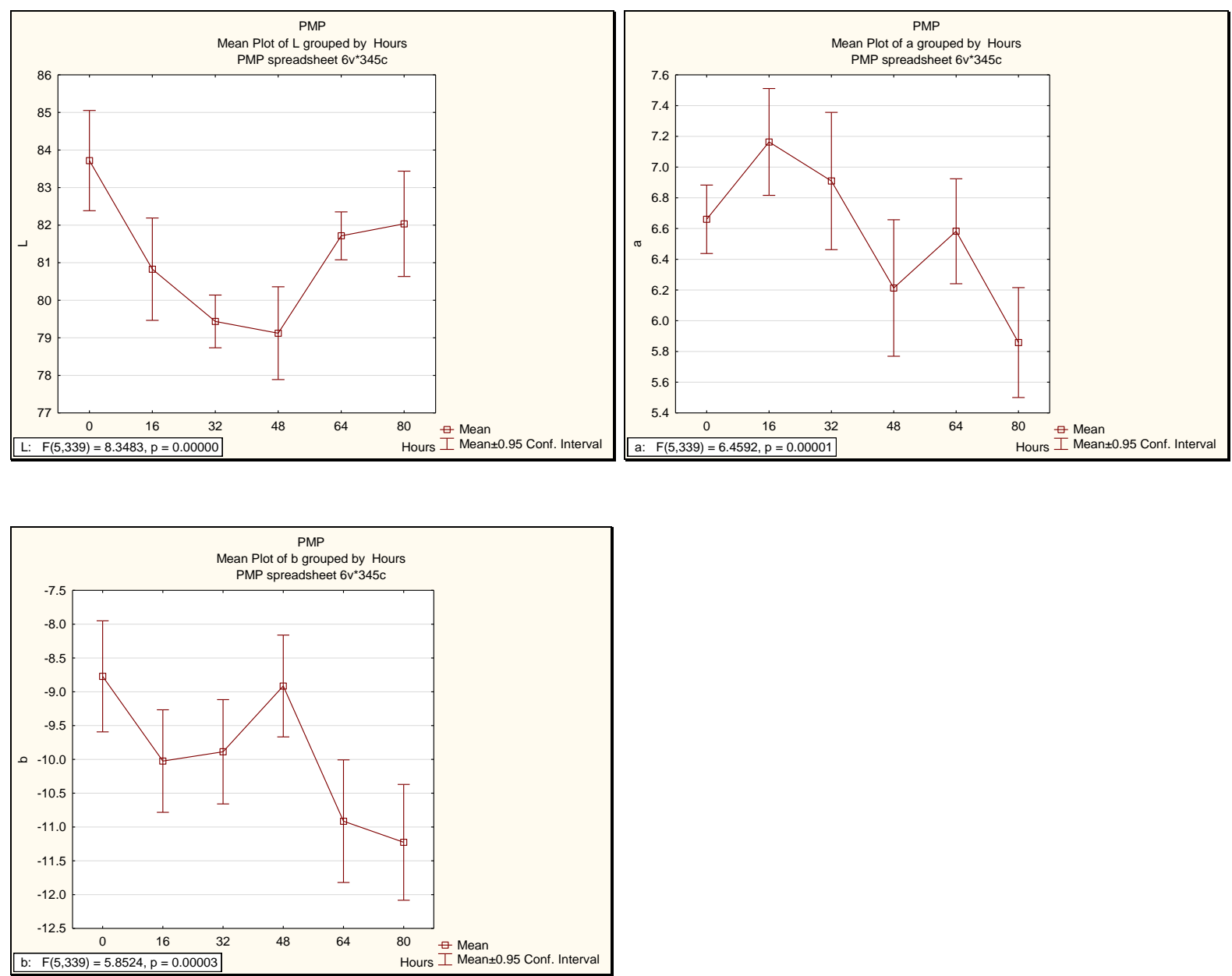

Significant differences in means are observed for all three variables. There is also a notable pattern observed in these variables; as the samples continue to age, there is a slight decrease that is observed in the mean values of each aging interval. However, at 64 hours, this decrease in values is interrupted for variables ' $\mathrm{L}$ ' and ' $\mathrm{a}$ ' but is interrupted at for variable ' $\mathrm{b}$ ' at 48 hours. 
Again, there are no consistencies in the way the Lab variables are affected by the aging treatment.

\section{Lastly, sample RMP}

Figure 4la-c: Mean plot for Sample RMP grouped by 'Hours'
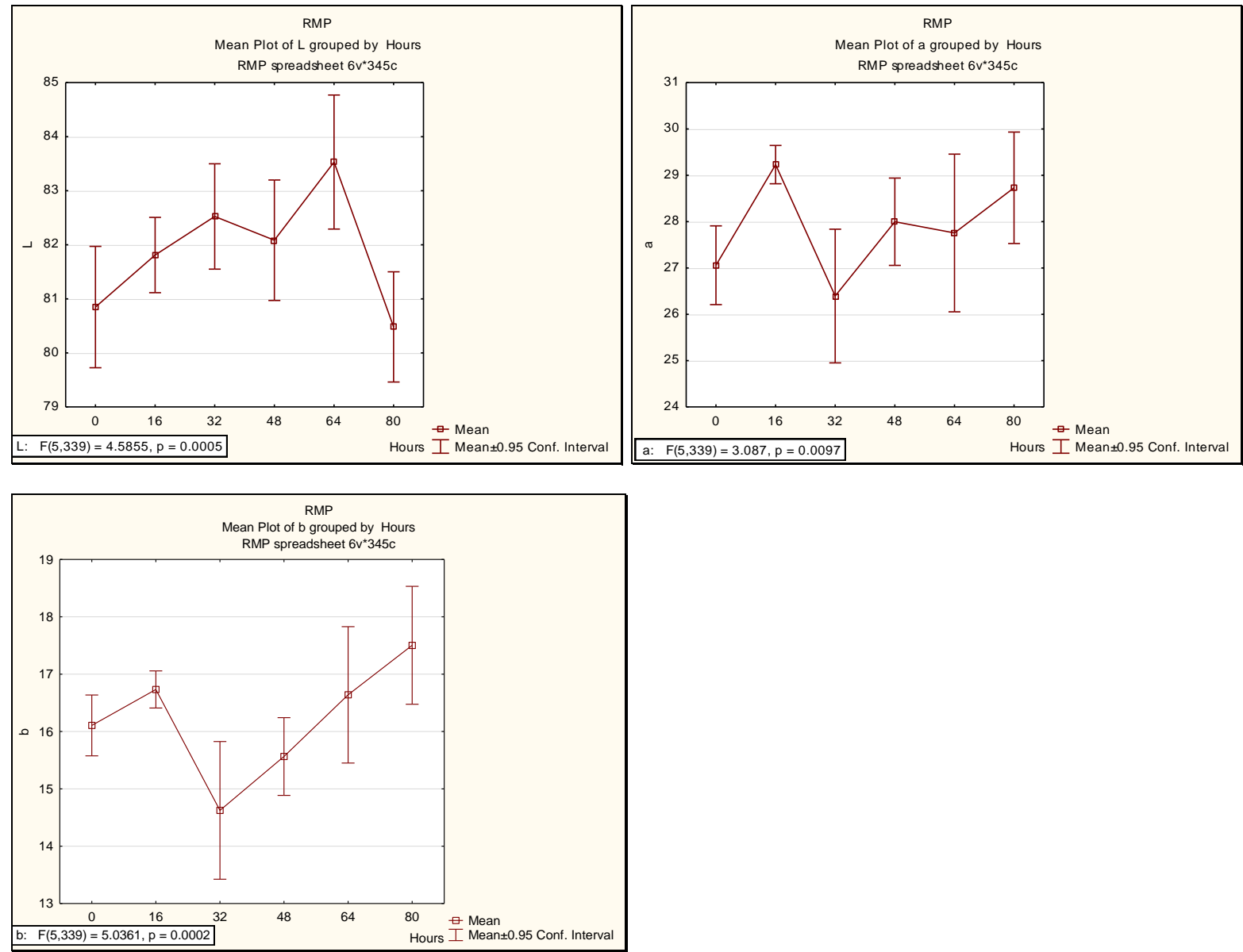

Significant differences in means are observed for all three variables. Unlike sample PMP, as the aging interval increases, the mean values also increases between groups. For example, at variable ' $\mathrm{L}$ ', the mean values continued to increase until 80 hours where it substantially decreases.

Further analysis of these results will be investigated using the post hoc tests.

2) ANOVA performed on variables L, a, b grouped 'Sample Area' 
Unlike hours, sample area was used to analyze inter-variability of the fibers for the Lab values. At $\mathrm{p}=0.05$, or the $5 \%$ level, there is strong evidence that the means differ significantly from one another. Among the sample areas, significant differences were not observed for any one reference sample at all three dependent variables.

Table 17: ANOVA results of Lab variables grouped by 'Sample Area'

\begin{tabular}{|c|c|c|c|c|c|c|c|c|}
\hline \multicolumn{9}{|c|}{ Grouped by Sample Area } \\
\hline DPMP & SS & df & MS & SS & df & MS & $\mathbf{F}$ & $\mathbf{p}$ \\
\hline $\mathrm{L}$ & 60.19 & 2 & 30.10 & 4516.14 & 342 & 13.205 & 2.279 & 0.104 \\
\hline$a$ & 192.99 & 2 & 96.49 & 3606.10 & 342 & 10.544 & 9.151 & 0.000 \\
\hline $\mathrm{b}$ & 45.95 & 2 & 22.97 & 1245.52 & 342 & 3.642 & 6.308 & 0.002 \\
\hline GMP & SS & df & MS & SS & df & MS & $\mathbf{F}$ & $\mathbf{p}$ \\
\hline $\mathrm{L}$ & 540.49 & 2 & 270.25 & 6798.24 & 342 & 19.878 & 13.595 & 0.000 \\
\hline$a$ & 9.39 & 2 & 4.69 & 274.53 & 342 & 0.803 & 5.848 & 0.003 \\
\hline $\mathrm{b}$ & 12.33 & 2 & 6.16 & 1683.22 & 342 & 4.922 & 1.253 & 0.287 \\
\hline OSDP & SS & df & MS & SS & df & MS & $F$ & $\mathbf{p}$ \\
\hline $\mathrm{L}$ & 468.82 & 2 & 234.41 & 6254.62 & 342 & 18.288 & 12.817 & 0.000 \\
\hline$a$ & 3.26 & 2 & 1.63 & 961.43 & 342 & 2.811 & 0.580 & 0.560 \\
\hline$b$ & 12.83 & 2 & 6.42 & 9002.78 & 342 & 26.324 & 0.244 & 0.784 \\
\hline PMCL & SS & df & MS & SS & df & MS & $\mathbf{F}$ & $\mathbf{p}$ \\
\hline $\mathrm{L}$ & 91.62 & 2 & 45.81 & 7701.74 & 342 & 22.520 & 2.034 & 0.132 \\
\hline$a$ & 5.66 & 2 & 2.83 & 183.10 & 342 & 0.535 & 5.284 & 0.005 \\
\hline$b$ & 37.68 & 2 & 18.84 & 504.94 & 342 & 1.476 & 12.760 & 0.000 \\
\hline PMJX & SS & df & MS & SS & df & MS & $F$ & $p$ \\
\hline L & 82.78 & 2 & 41.39 & 6066.01 & 342 & 17.737 & 2.334 & 0.098 \\
\hline$a$ & 3.63 & 2 & 1.81 & 157.72 & 342 & 0.461 & 3.931 & 0.021 \\
\hline$b$ & 12.47 & 2 & 6.23 & 637.90 & 342 & 1.865 & 3.342 & 0.037 \\
\hline PMP & SS & df & MS & SS & df & MS & $F$ & $\mathbf{p}$ \\
\hline $\mathrm{L}$ & 786.90 & 5 & 157.38 & 6390.75 & 339 & 18.852 & 8.348 & 0.000 \\
\hline$a$ & 66.48 & 5 & 13.30 & 697.80 & 339 & 2.058 & 6.459 & 0.000 \\
\hline$b$ & 279.74 & 5 & 55.95 & 3240.85 & 339 & 9.560 & 5.852 & 0.000 \\
\hline RMP & SS & df & MS & SS & df & MS & $\mathbf{F}$ & $p$ \\
\hline $\mathrm{L}$ & 28.51 & 2 & 14.25 & 5586.55 & 342 & 16.335 & 0.873 & 0.419 \\
\hline$a$ & 458.17 & 2 & 229.08 & 6824.89 & 342 & 19.956 & 11.479 & 0.000 \\
\hline$b$ & 350.72 & 2 & 175.36 & 4049.41 & 342 & 11.840 & 14.811 & 0.000 \\
\hline
\end{tabular}

Note: Significances are marked in bold italics. 
Table 18: Welch results of Lab variables grouped by 'Sample Area'

\begin{tabular}{|c|c|c|c|c|c|c|c|c|c|}
\hline \multicolumn{10}{|c|}{ Grouped by Sample Area } \\
\hline DPMP & $\begin{array}{c}\text { Welch } \\
\text { df }\end{array}$ & $\begin{array}{c}\text { Welch } \\
\text { df }\end{array}$ & $\begin{array}{l}\text { Welch } \\
\text { F }\end{array}$ & $\begin{array}{c}\text { Welch } \\
\text { p }\end{array}$ & PMJX & $\begin{array}{c}\text { Welch } \\
\text { df }\end{array}$ & $\begin{array}{c}\text { Welch } \\
\text { df }\end{array}$ & Welch F & $\begin{array}{c}\text { Welch } \\
\text { p }\end{array}$ \\
\hline $\mathrm{L}$ & 2 & 226.96 & 2.214 & 0.112 & $\mathrm{~L}$ & 2 & 227.93 & 2.299 & 0.103 \\
\hline $\mathrm{a}$ & 2 & 226.16 & 8.258 & 0.000 & $a$ & 2 & 226.96 & 3.699 & 0.026 \\
\hline$b$ & 2 & 222.50 & 5.649 & 0.004 & $b$ & 2 & 227.92 & 3.407 & 0.035 \\
\hline GMP & $\begin{array}{c}\text { Welch } \\
\text { df }\end{array}$ & $\begin{array}{c}\text { Welch } \\
\text { df }\end{array}$ & $\begin{array}{c}\text { Welch } \\
\text { F }\end{array}$ & $\begin{array}{c}\text { Welch } \\
\text { p }\end{array}$ & PMP & $\begin{array}{c}\text { Welch } \\
\text { df }\end{array}$ & $\begin{array}{c}\text { Welch } \\
\text { df }\end{array}$ & Welch F & $\begin{array}{c}\text { Welch } \\
\text { p }\end{array}$ \\
\hline $\mathrm{L}$ & 2 & 227.65 & 14.090 & 0.000 & $\mathrm{~L}$ & 5 & 151.71 & 10.412 & 0.000 \\
\hline $\mathrm{a}$ & 2 & 187.75 & 4.074 & 0.019 & $\mathrm{a}$ & 5 & 158.03 & 6.498 & 0.000 \\
\hline$b$ & 2 & 221.09 & 1.154 & 0.317 & $b$ & 5 & 156.13 & 5.745 & 0.000 \\
\hline OSDP & $\begin{array}{c}\text { Welch } \\
\text { df }\end{array}$ & $\begin{array}{c}\text { Welch } \\
\text { df }\end{array}$ & $\begin{array}{l}\text { Welch } \\
\text { F }\end{array}$ & $\begin{array}{c}\text { Welch } \\
\text { p }\end{array}$ & RMP & $\begin{array}{c}\text { Welch } \\
\text { df }\end{array}$ & $\begin{array}{c}\text { Welch } \\
\text { df }\end{array}$ & Welch F & $\begin{array}{c}\text { Welch } \\
\text { p }\end{array}$ \\
\hline $\mathrm{L}$ & 2 & 227.57 & 12.732 & 0.000 & $\mathrm{~L}$ & 2 & 226.39 & 0.886 & 0.414 \\
\hline$a$ & 2 & 223.08 & 0.504 & 0.605 & $a$ & 2 & 226.12 & 11.598 & 0.000 \\
\hline$b$ & 2 & 222.73 & 0.296 & 0.744 & $b$ & 2 & 216.91 & 18.298 & 0.000 \\
\hline PMCL & $\begin{array}{c}\text { Welch } \\
\text { df }\end{array}$ & $\begin{array}{c}\text { Welch } \\
\text { df }\end{array}$ & $\begin{array}{l}\text { Welch } \\
\text { F }\end{array}$ & $\begin{array}{c}\text { Welch } \\
\text { p }\end{array}$ & & & & & \\
\hline $\mathrm{L}$ & 2 & 227.17 & 1.887 & 0.154 & & & & & \\
\hline $\mathrm{a}$ & 2 & 221.42 & 6.391 & 0.002 & & & & & \\
\hline$b$ & 2 & 221.61 & 16.628 & 0.000 & & & & & \\
\hline
\end{tabular}

Note: Significances are marked in bold italics.

Again, the Welch test was used in corroboration with the F-test when the variances are not homogenous. The ANOVA results are discussed in two different groups below based on similar patterns of results observed in the dependent variables.

Samples DPMP, PMCL, PMJX, and RMP

A similar pattern was noticed for all four samples: variables 'a' and ' $b$ ' observed significant differences in their mean values. These four samples were the only ones that observed this pattern. Note that three of the colors were pink and one was red, which might play an important role into deciphering this pattern. This will be further investigated in the post hoc comparison test. See Section 7-11 in the appendix for the ANOVA results and the mean plots. 
Samples GMP, OSDP and PMP were separated because there was no similar pattern observed with these samples in comparison to the others listed above. Although all three samples had marked significances, only two will be discussed in greater detail below. The mean plots of sample GMP can be found in Section 11 of the appendix.

Figure 42 below shows the overall mean plot of variables Lab for samples PMP and OSDP. The changes that occur among sample areas are not readily observed in these graphs; these graphs were included to observe the overall change.

Figure 42a-b: Mean plot of Lab grouped by 'Sample Area' for PMP (a) and OSDP (b)
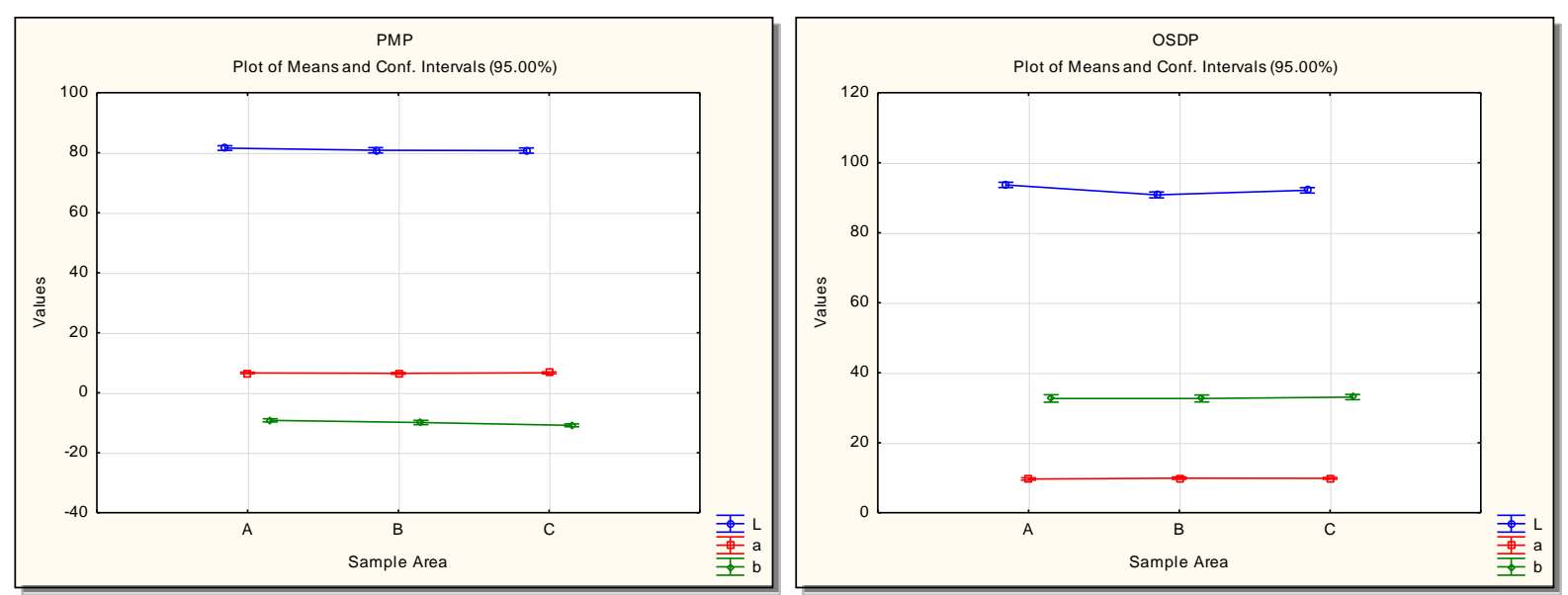

Figure 43 below shows the mean plot at variable 'L' for samples PMP and OSDP. The changes that occur among sample areas can be easily observed here. The difference in bar overlapping can be seen in samples PMP and OSDP. While all three intervals overlaps in sample PMP, sample area B overlaps with $\mathrm{C}$ for sample OSDP. Based on the associated p-values for each graph, the null hypothesis of no means differences was rejected for sample OSDP but was accepted for sample PMP at variable ' $L$ '. 
Figure 43a-b: Mean plot of 'L' grouped by 'Sample Area' for PMP (a) and OSDP (b)
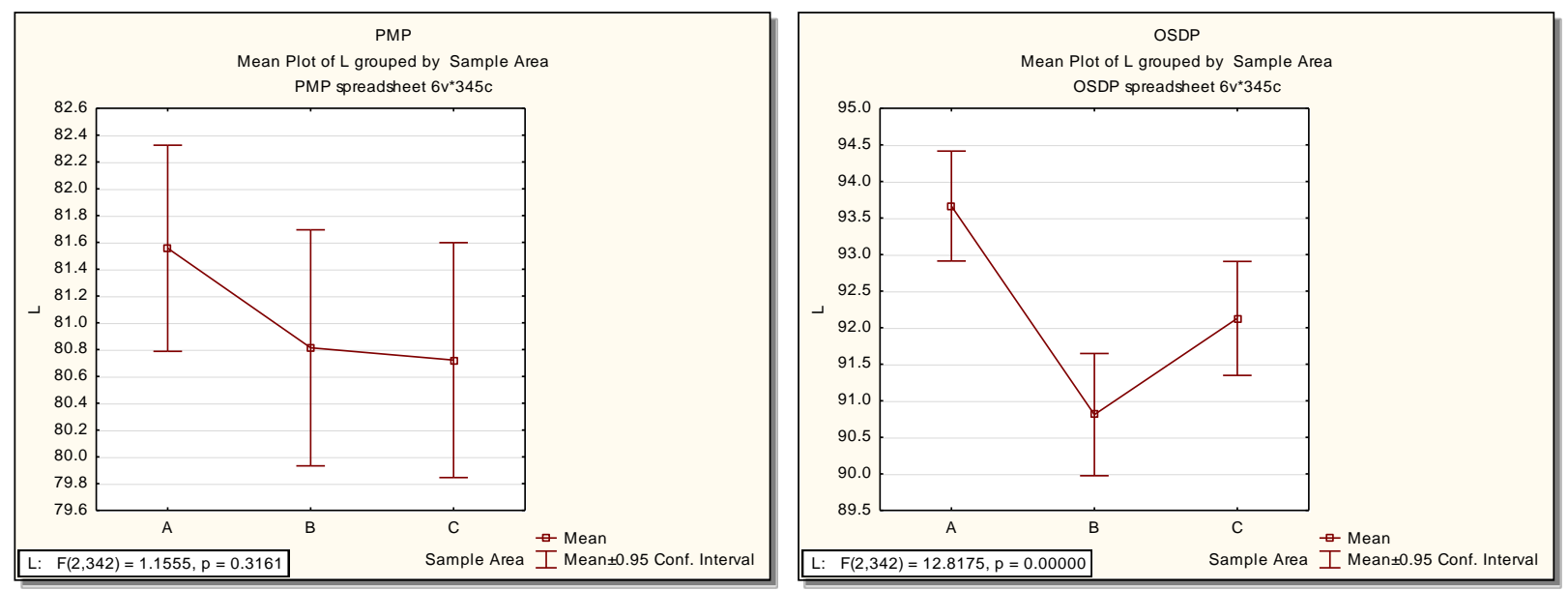

Figure 44below shows the mean plot at variable ' $a$ ' for samples PMP and OSDP.

Figure 44a-b: Interaction plot of ' $a$ ' grouped by 'Sample Area' for PMP (a) and OSDP (b)
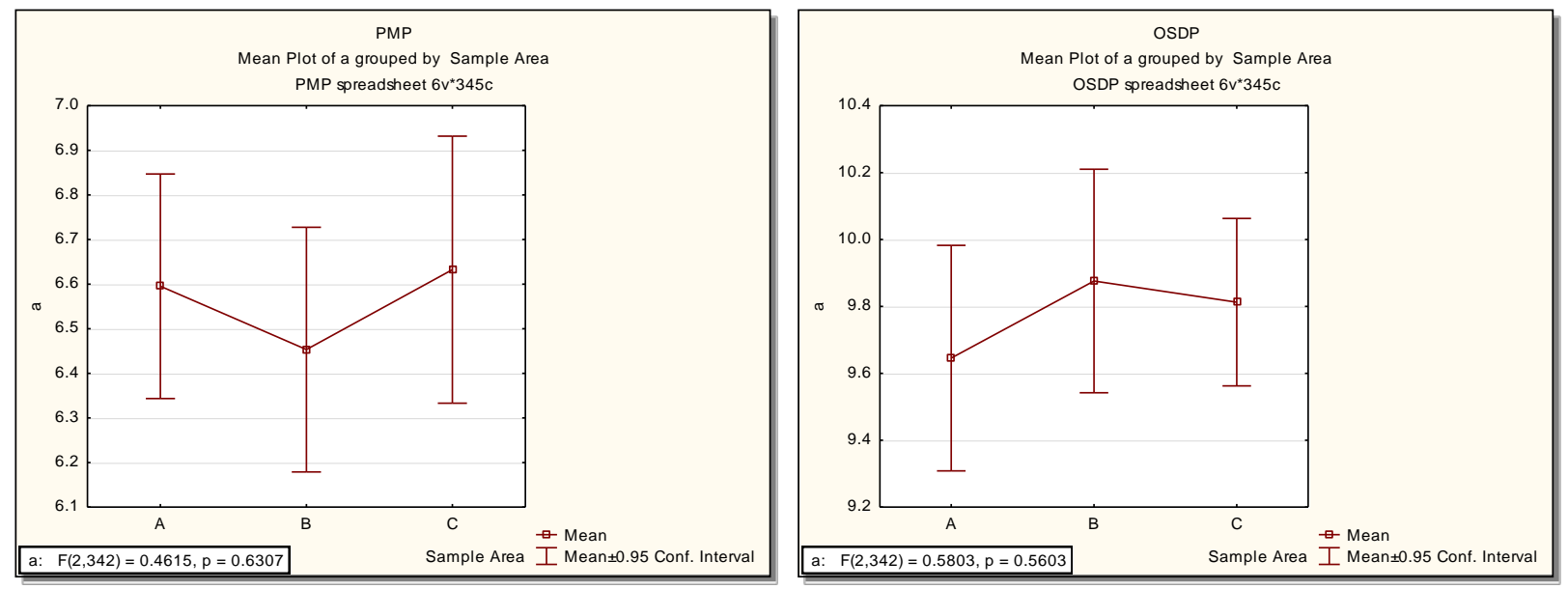

For variable ' $a$ ', both charts shows similarities as the error bars overlap among the sample areas.

Therefore, for both samples, the null hypothesis was not rejected.

Lastly, Figure 45 below shows the mean plot at variable ' $b$ ' for samples PMP and OSDP. 
Figure 45a-b: Mean plot of 'b' grouped by 'Sample Area' for PMP (a) and OSDP (b)
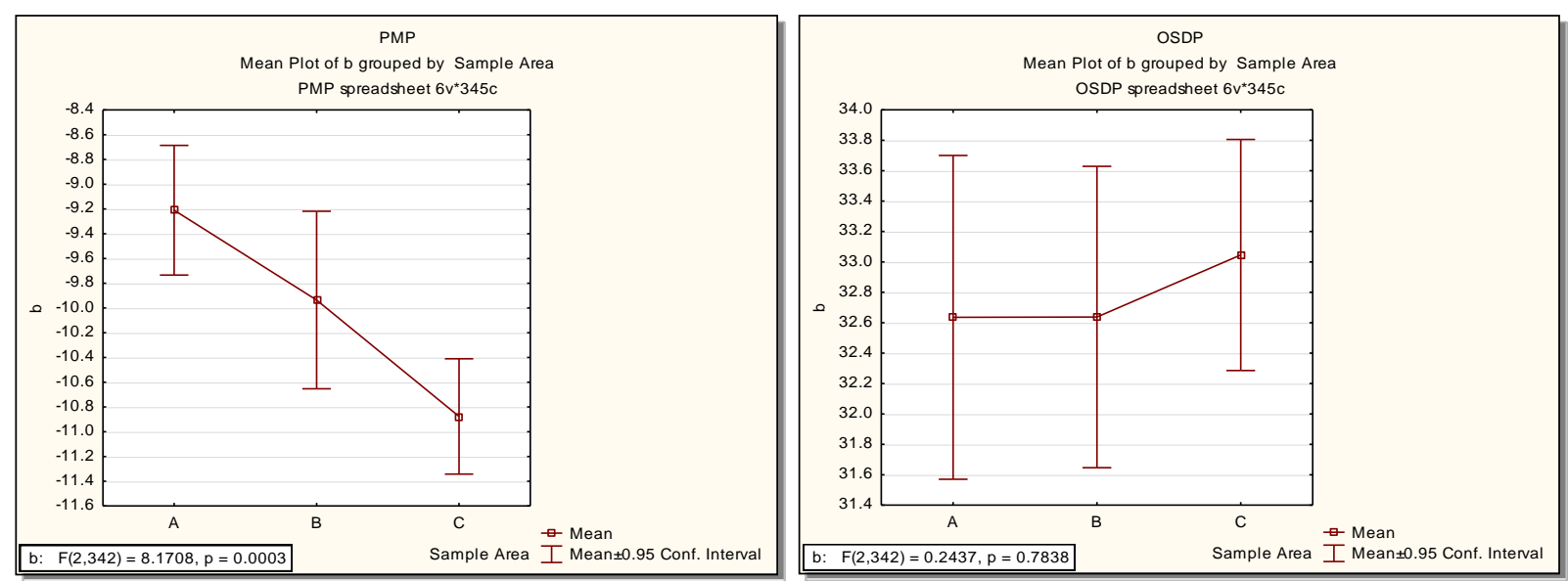

For variable 'b', again there was a difference in overlapping as was observed in variable ' $L$ '. A notable difference in group means was observed among the groups in sample PMP, whereas, this difference was not observed in OSDP. In fact, sample OSDP observes such similarity that it has a substantial p-value. As the p-value approached 1, it indicated that the samples are the same or that they were from the same population. For this reason, the null hypothesis was accepted for sample OSDP, but was rejected for sample PMP at variable 'b'.

\section{3) ANOVA was performed on variables Lab grouped by variable 'Fiber'}

Variable 'Fibers' was used to analyze intra-variability of the fibers for the Lab values. Among the fibers, significant differences were observed for all measurements. Based on the results, there was strong evidence (at $\mathrm{p}=0.05$, or the $5 \%$ level) that the means differ significantly from one another.

Significant differences were observed among the fibers. One explanation for this is the fact that inter-variability and intra-variability observed among the fibers are substantial. If the fibers 
themselves are not homogeneous, it can be stipulated that with or without treatment, differences are probable.

Table 19: ANOVA results of Lab grouped by 'Fiber'

\begin{tabular}{|c|c|c|c|c|c|c|c|c|}
\hline \multicolumn{9}{|c|}{ Grouped by fibers } \\
\hline DPMP & SS & df & MS & SS & df & MS & $\mathbf{F}$ & $p$ \\
\hline L & 3729.97 & 53 & 70.38 & 846.36 & 291 & 2.908 & 24.197 & 0.000 \\
\hline$a$ & 2590.73 & 53 & 48.88 & 1208.36 & 291 & 4.152 & 11.772 & 0.000 \\
\hline$b$ & 964.17 & 53 & 18.19 & 327.30 & 291 & 1.125 & 16.174 & 0.000 \\
\hline GMP & SS & df & MS & SS & df & MS & $\mathbf{F}$ & $\mathbf{p}$ \\
\hline $\mathrm{L}$ & 5582.56 & 53 & 105.33 & 1756.17 & 291 & 6.035 & 17.454 & 0.000 \\
\hline$a$ & 246.23 & 53 & 4.65 & 37.69 & 291 & 0.130 & 35.870 & 0.000 \\
\hline$b$ & 1270.59 & 53 & 23.97 & 424.96 & 291 & 1.460 & 16.416 & 0.000 \\
\hline OSDP & SS & df & MS & SS & df & MS & $\mathbf{F}$ & $\mathbf{p}$ \\
\hline $\mathrm{L}$ & 5694.21 & 53 & 107.44 & 1029.22 & 291 & 3.537 & 30.377 & 0.000 \\
\hline$a$ & 753.18 & 53 & 14.21 & 211.51 & 291 & 0.727 & 19.552 & 0.000 \\
\hline$b$ & 7484.85 & 53 & 141.22 & 1530.76 & 291 & 5.260 & 26.847 & 0.000 \\
\hline PMCL & SS & df & MS & SS & df & MS & $\mathbf{F}$ & $p$ \\
\hline $\mathrm{L}$ & 5972.71 & 53 & 112.69 & 1820.65 & 291 & 6.257 & 18.012 & 0.000 \\
\hline$a$ & 158.45 & 53 & 2.99 & 30.31 & 291 & 0.104 & 28.705 & 0.000 \\
\hline$b$ & 464.20 & 53 & 8.76 & 78.42 & 291 & 0.269 & 32.502 & 0.000 \\
\hline PMJX & SS & df & MS & SS & df & MS & $\mathbf{F}$ & $p$ \\
\hline $\mathrm{L}$ & 5621.41 & 53 & 106.06 & 527.39 & 291 & 1.812 & 58.524 & 0.000 \\
\hline$a$ & 130.42 & 53 & 2.46 & 30.92 & 291 & 0.106 & 23.160 & 0.000 \\
\hline$b$ & 546.36 & 53 & 10.31 & 104.02 & 291 & 0.357 & 28.840 & 0.000 \\
\hline PMP & SS & df & MS & SS & df & MS & $F$ & $p$ \\
\hline $\mathrm{L}$ & 48.17 & 2 & 24.09 & 7129.48 & 342 & 20.846 & 1.155 & 0.316 \\
\hline a & 2.06 & 2 & 1.03 & 762.22 & 342 & 2.229 & 0.461 & 0.631 \\
\hline$b$ & 160.55 & 2 & 80.28 & 3360.04 & 342 & 9.825 & 8.171 & 0.000 \\
\hline RMP & SS & df & MS & SS & df & MS & $F$ & $p$ \\
\hline $\mathrm{L}$ & 4770.90 & 53 & 90.02 & 844.16 & 291 & 2.901 & 31.031 & 0.000 \\
\hline$a$ & 6120.40 & 53 & 115.48 & 1162.66 & 291 & 3.995 & 28.903 & 0.000 \\
\hline$b$ & 3987.32 & 53 & 75.23 & 412.81 & 291 & 1.419 & 53.033 & 0.000 \\
\hline
\end{tabular}

Note: Significances are marked in bold italics.

Again, the Welch test was used to corroborate the ANOVA F-test. 
Table 20: Welch results of Lab grouped by 'Fiber'

\begin{tabular}{|c|c|c|c|c|c|c|c|c|c|}
\hline \multicolumn{9}{|c|}{ Grouped by Fibers } & \multirow[b]{2}{*}{$\begin{array}{c}\text { Welch } \\
\text { p }\end{array}$} \\
\hline DPMP & $\begin{array}{c}\text { Welch } \\
\text { df }\end{array}$ & $\begin{array}{l}\text { Welch } \\
\text { df }\end{array}$ & $\begin{array}{l}\text { Welch } \\
\text { F }\end{array}$ & $\begin{array}{c}\text { Welch } \\
p\end{array}$ & PMJX & $\begin{array}{c}\text { Welch } \\
\text { df }\end{array}$ & $\begin{array}{c}\text { Welch } \\
\text { df }\end{array}$ & $\begin{array}{c}\text { Welch } \\
\text { F } \\
\end{array}$ & \\
\hline $\mathrm{L}$ & 53 & 88.78 & 18349 & 0.000 & $\mathrm{~L}$ & 53 & 88.80 & 33611 & 0.000 \\
\hline a & 53 & 88.63 & 40029 & 0.000 & a & 53 & 88.86 & 32073 & 0.000 \\
\hline $\mathrm{b}$ & 53 & 88.87 & 39886 & 0.000 & $\mathrm{~b}$ & 53 & 88.37 & 44990 & 0.000 \\
\hline GMP & $\begin{array}{l}\text { Welch } \\
\text { df }\end{array}$ & $\begin{array}{c}\text { Welch } \\
\text { df }\end{array}$ & $\begin{array}{l}\text { Welch } \\
\text { F }\end{array}$ & $\begin{array}{c}\text { Welch } \\
\text { p }\end{array}$ & PMP & $\begin{array}{c}\text { Welch } \\
\text { df }\end{array}$ & $\begin{array}{c}\text { Welch } \\
\text { df }\end{array}$ & $\begin{array}{l}\text { Welch } \\
\text { F }\end{array}$ & $\begin{array}{c}\text { Welch } \\
\text { p }\end{array}$ \\
\hline L & 53 & 88.94 & 76705 & 0.000 & L & 2 & 227.04 & 1.257 & 0.287 \\
\hline a & 53 & 89.09 & 17043 & 0.000 & $\mathrm{a}$ & 2 & 226.88 & 0.449 & 0.639 \\
\hline b & 53 & 88.40 & 47740 & 0.000 & $b$ & 2 & 222.18 & 11.177 & 0.000 \\
\hline PMCL & $\begin{array}{c}\text { Welch } \\
\text { df }\end{array}$ & $\begin{array}{c}\text { Welch } \\
\text { df }\end{array}$ & $\begin{array}{l}\text { Welch } \\
\text { F }\end{array}$ & $\begin{array}{c}\text { Welch } \\
p\end{array}$ & RMP & $\begin{array}{l}\text { Welch } \\
\text { df }\end{array}$ & $\begin{array}{c}\text { Welch } \\
\text { df }\end{array}$ & $\begin{array}{l}\text { Welch } \\
\text { F }\end{array}$ & $\begin{array}{c}\text { Welch } \\
\text { p }\end{array}$ \\
\hline $\mathrm{L}$ & 53 & 88.65 & 100584 & 0.000 & $\mathrm{~L}$ & 53 & 88.24 & 273758 & 0.000 \\
\hline$a$ & 53 & 88.85 & 32532 & 0.000 & $a$ & 53 & 88.51 & 88586 & 0.000 \\
\hline $\mathrm{b}$ & 53 & 88.68 & 26469 & 0.000 & $b$ & 53 & 88.45 & 89798 & 0.000 \\
\hline
\end{tabular}

Note: no values were produced for sample OSDP

The Welch test cannot be performed for sample OSDP because at least one group has a variance of 0 . Two groups of measurements had missing data where either the data was not collected, or it was corrupted using the GRAIMS software. When missing data was observed in this sample, the average value for the entire measurement replaced the sets of missing data. For this reason, the variance would be zero for these two groups.

Post hoc comparisons were not applied to the ANOVA results taken from the variable 'Fiber' as this result was only used as initial observations to determine if intra-variability is found in fibers. However, for the variable 'Sample Area', only certain measurements were further analyzed. Among the pink and red samples, similarities were observed in the dependent variables over the aging treatment. To observe where these similarities might have occurred, the post hoc comparisons were applied. For the remaining samples, only intra-variability was checked and determined based on the ANOVA results. This result is sufficient to prove that intra-variability 
occurs within a garment. However, since the objective of part 1 of this project is to determine how the aging treatment affects the color in the fibers, further statistical approaches from the variable 'Hours' were investigated using the post hoc tests. By this, it can be determined where the significant differences between groups occurred.

\section{$\underline{\text { 6.3.1c Post Hoc Comparisons of Aged Results }}$}

In the post hoc test, means between groups are compared two at a time. In order to interpret the results, two hypotheses were created:

- Ho: $\mu_{1}=\mu_{2}$ (the means of this group are the same)

- $\mathrm{H}_{\mathrm{A}}: \mu_{1} \neq \mu_{2}$ (the means of this group are not the same)

Two post hoc tests, Tukey HSD and Games-Howell methods were employed. The tests are broken down into two analyses:

1) Post Hoc Comparisons of Lab grouped by 'Hours'

2) Post Hoc Comparisons of Lab grouped by 'Sample Area'

The results of these tests were broken down into three tables for each dependent variable.

i. Tukey Multiple Comparisons Results of variables Lab;

ii. Games-Howell Multiple Comparisons of variables Lab;

iii. Homogeneous Subsets Results based on Tukey of variables Lab.

Based on the previous ANOVA results in which the null hypotheses were rejected, two post hoc comparisons, Tukey HSD and Games-Howell tests, were employed. This was done to evaluate 
the null hypothesis associated with each contrast or group that is investigated. The mean differences highlights where significant differences are found among the comparing group. The results of the Tukey test are tabulated in a triangular table of mean differences, whereas the results of Games-Howell were only tabulated. Note that the Tukey results were computed using Statistica, and the Games-Howell and Homogeneous Subsets results were computed using the SPSS software.

1) Post Hoc Comparisons of Lab grouped by 'Hours'

Based on the previous ANOVA results, all the reference samples, except sample PMJX, observed significant p-values. Sample PMJX only observed significant p-values at variable 'a' and ' $b$ '. The null hypothesis of mean differences between groups at variable ' $L$ ' was accepted. These results are discussed separately below by reference samples.

\section{DPMP - Variable ' $L$ '}

In sample DPMP, significant changes were observed greatest in the ' $L$ ' value then variables ' $a$ ' and ' $b$ '. Since L refers to light or luminance, the greatest change observed during UV exposure affected the gloss of the sample.

Table 21: Tukey HSD test of sample DPMP at variable ' $L$ '

\begin{tabular}{|c|c|c|c|c|c|c|c|}
\hline \multirow{2}{*}{\multicolumn{2}{|c|}{ Hours }} & \multicolumn{6}{|c|}{$\begin{array}{l}\text { Tukey HSD test; Variable: L (DPMP spreadsheet) } \\
\text { Marked differences are significant at } p<.05000\end{array}$} \\
\hline & & $\begin{array}{c}\{1\} \\
M=84.484\end{array}$ & $\begin{array}{c}\{2\} \\
M=86.44 \epsilon\end{array}$ & $\begin{array}{c}\{3\} \\
M=83.65 \mathrm{~s}\end{array}$ & $\begin{array}{c}\{4\} \\
M=83.79 \varepsilon\end{array}$ & $\begin{array}{c}\{5\} \\
M=86.30 €\end{array}$ & $\begin{array}{c}\{6\} \\
M=83.30 \leftleftarrows\end{array}$ \\
\hline $0 \quad\{1$ & $\{1\}$ & & 0.044 & 0.8286 & $0.913 \varepsilon$ & 0.078 & 0.504 \\
\hline 16 & $\{2\}$ & 0.044 & & 0.000 & 0.000 & 0.999 s & 0.0001 \\
\hline 32 & $\{3\}$ & $0.828 \epsilon$ & 0.000 & & 0.999 s & 0.000 & 0.993 \\
\hline 48 & $\{4\}$ & 0.913\{ & 0.000 & 0.999 ؟ & & $0.000 !$ & $0.970^{\circ}$ \\
\hline 64 & $\{5\}$ & 0.078 & 0.999 s & 0.000 & $0.000 !$ & & 0.0001 \\
\hline 80 & $\{6\}$ & 0.504 ؛ & 0.0001 & 0.993 & $0.970^{\circ}$ & 0.0001 & \\
\hline
\end{tabular}


Note: Significances are marked in bold italics.

This table shows the results of multiple pair-wise comparisons among the six groups, representing the hours aged: from 0 hours for the untreated sample to 80 hours of aging. $M$ in each column is the average mean of each group. In the first column, second row shows the first pair-wise comparison; this cell shows that the mean at 0 hours is significantly different from the mean at 16 hours (where the values in bold indicates a difference at the 0.05 level). No other significant difference was observed at 0 hours (group 1).

Table 22: Homogeneous Subsets: DPMP ' $L$ '

\begin{tabular}{|c|c|c|c|c|c|}
\hline \multicolumn{6}{|c|}{ Homogeneous Subsets: Variable 'L' } \\
\hline \multirow[b]{2}{*}{ Hours } & & \multirow[b]{2}{*}{$\mathrm{N}$} & \multicolumn{3}{|c|}{ Subset for alpha $=0.05$} \\
\hline & & & 1 & 2 & 3 \\
\hline \multirow{7}{*}{$\begin{array}{l}\text { Tukey } \\
\text { HSD }^{a, b}\end{array}$} & 80 & 60 & 83.3046 & & \\
\hline & 32 & 60 & 83.6591 & & \\
\hline & 48 & 60 & 83.7976 & & \\
\hline & 0 & 45 & 84.4845 & 84.4845 & \\
\hline & 64 & 60 & & 86.3032 & 86.3032 \\
\hline & 16 & 60 & & & 86.4462 \\
\hline & Sig. & & .448 & .057 & 1.000 \\
\hline \multicolumn{6}{|c|}{$\begin{array}{l}\text { Means for groups in homogeneous subsets are displayed. } \\
\text { a. Uses Harmonic Mean Sample Size }=56.842 \text {. } \\
\text { b. The group sizes are unequal. The harmonic mean of the group sizes is used. } \\
\text { Type I error levels are not guaranteed. }\end{array}$} \\
\hline
\end{tabular}

Three subset groups were created: in group 1, the means of the $80,32,48$ and 0 hours of aging are not significantly different from each other, and forms a homogeneous subset according the Tukey HSD test. It can be observed that the means are quite close together in this group. Yet this subset differs significantly from subset 2 and 4 . It may then be concluded that the aging treatment had a significant effect at 16 hours of aging. 
Table 23: Games-Howell test of sample DPMP at variable ' $L$ '

Multiple Comparisons: DPMP

\begin{tabular}{|c|c|c|c|c|c|c|c|c|}
\hline \multirow{2}{*}{\multicolumn{2}{|c|}{ Dependent Variable }} & \multirow[b]{2}{*}{ Group (I) } & \multirow[b]{2}{*}{$\begin{array}{c}\text { Group } \\
(\mathrm{J})\end{array}$} & \multirow{2}{*}{$\begin{array}{c}\text { Mean } \\
\text { Difference } \\
(\mathrm{I}-\mathrm{J})\end{array}$} & \multirow[b]{2}{*}{$\begin{array}{l}\text { Std. } \\
\text { Error }\end{array}$} & \multirow[b]{2}{*}{ Sig. } & \multicolumn{2}{|c|}{$\begin{array}{c}95 \% \text { Confidence } \\
\text { Interval }\end{array}$} \\
\hline & & & & & & & $\begin{array}{l}\text { Lower } \\
\text { Bound }\end{array}$ & $\begin{array}{l}\text { Upper } \\
\text { Bound }\end{array}$ \\
\hline \multirow[t]{30}{*}{$\mathrm{L}$} & Games- & 0 & 16 & -1.9617 & 0.7499 & .104 & -4.1451 & 0.2217 \\
\hline & Howell & & 32 & 0.8253 & 0.6802 & .829 & -1.1650 & 2.8156 \\
\hline & & & 48 & 0.6869 & 0.7059 & .925 & -1.3736 & 2.7474 \\
\hline & & & 64 & -1.8187 & 0.7361 & .144 & -3.9633 & 0.3258 \\
\hline & & & 80 & 1.1799 & 0.7644 & .637 & -1.0444 & 3.4041 \\
\hline & & 16 & 0 & 1.9617 & 0.7499 & .104 & -0.2217 & 4.1451 \\
\hline & & & 32 & $2.7870^{*}$ & 0.5844 & .000 & 1.0915 & 4.4826 \\
\hline & & & 48 & $2.6486^{\star}$ & 0.6141 & .000 & 0.8687 & 4.4285 \\
\hline & & & 64 & 0.1430 & 0.6486 & 1.000 & -1.7361 & 2.0221 \\
\hline & & & 80 & $3.1416^{*}$ & 0.6806 & .000 & 1.1698 & 5.1134 \\
\hline & & 32 & 0 & -0.8253 & 0.6802 & .829 & -2.8156 & 1.1650 \\
\hline & & & 16 & -2.7870 & 0.5844 & .000 & -4.4826 & -1.0915 \\
\hline & & & 48 & -0.1385 & 0.5267 & 1.000 & -1.6647 & 1.3878 \\
\hline & & & 64 & -2.6440 & 0.5665 & .000 & -4.2871 & -1.0011 \\
\hline & & & 80 & 0.3545 & 0.6029 & 992 & -1.3954 & 2.1044 \\
\hline & & 48 & 0 & -0.6869 & 0.7059 & .925 & -2.7474 & 1.3736 \\
\hline & & & 16 & -2.6486 & 0.6141 & .000 & -4.4285 & -0.8687 \\
\hline & & & 32 & 0.1385 & 0.5267 & 1.000 & -1.3878 & 1.6647 \\
\hline & & & 64 & -2.5056 & 0.5971 & .001 & -4.2358 & -0.7754 \\
\hline & & & 80 & 0.4930 & 0.6317 & .970 & -1.3385 & 2.3245 \\
\hline & & 64 & 0 & 1.8187 & 0.7361 & .144 & -0.3258 & 3.9633 \\
\hline & & & 16 & -0.1430 & 0.6486 & 1.000 & -2.0221 & 1.7361 \\
\hline & & & 32 & $2.6440^{*}$ & 0.5665 & .000 & 1.0011 & 4.2871 \\
\hline & & & 48 & 2.505603 & 0.5971 & .001 & 0.7754 & 4.2358 \\
\hline & & & 80 & 2.9986 & 0.6653 & .000 & 1.0709 & 4.9263 \\
\hline & & 80 & 0 & -1.1799 & 0.7644 & .637 & -3.4041 & 1.0444 \\
\hline & & & 16 & -3.1416 & 0.6806 & .000 & -5.1134 & -1.1698 \\
\hline & & & 32 & -0.3545 & 0.6029 & .992 & -2.1044 & 1.3954 \\
\hline & & & 48 & -0.4930 & 0.6317 & .970 & -2.3245 & 1.3385 \\
\hline & & & 64 & -2.9986 & 0.6653 & .000 & -4.9263 & -1.0709 \\
\hline
\end{tabular}

*. The mean difference is significant at the 0.05 level.

Since the assumption of homogeneity of variance was not met for sample DPMP, only the results Games-Howell test was reviewed and accepted as accurate results. For the remaining dependent variables, only the Games-Howell test will be discussed. The information for the Tukey test can 
be ignored at this point. Both post hoc comparisons are in agreement with the significant values found for the samples. In this table, there are only certain pieces of data that is needed to make a conclusion. This table is broken down into 8 columns: Column 1 list the dependent variables; Column 2 list the post hoc test being used; Column 3 and 4 lists the factors being compared to each other; Column 5 list the mean difference between the two compared groups; Column 6 through 9 lists the standard error, significant p-value, and the 95\% upper and lower confidence interval. The columns of interests are columns 3, 4 and 7. Group $0(\mathrm{M}=84.484)$ is not observed to be significantly different from Group $16(\mathrm{M}=86.446)$ with a mean difference of -1.962 and a p-value of 0.104 . However, Group $16(\mathrm{M}=86.446)$ was observed to be significantly different from Group $32(\mathrm{M}=83.659)$ with a mean difference of 2.787 and a p-value of 0.001 . In total, six marked significant difference in means was observed between the comparing groups. It can therefore be concluded that the aging treatment have reasonably affected the gloss of the sample.

\section{DPMP- Variable ' $a$ '}

Only five significant differences in means are observed in the variable. From the untreated sample, Group 0, only two marked differences were observed: at Group $48(\mathrm{M}=25.035)$ with a mean difference of 1.765 and a p-value of 0.020 ; and again at Group $80(\mathrm{M}=25.384)$ with a difference of 3.142 and a p-value of 0.001 . It can therefore be concluded that the color saturation was greatly affected in the red/green region. Two possible explanations can be drawn: the aging treatment reasonably affected the color saturation in the red/green region, or, based on the validation results, variation is typically observed in this region which could have influenced the post hoc results and the aging treatment. 
Table 24: Games-Howell test of sample DPMP at variable ' $a$ '

Multiple Comparisons: DPMP

\begin{tabular}{|c|c|c|c|c|c|c|c|c|}
\hline \multirow{2}{*}{\multicolumn{2}{|c|}{ Dependent Variable }} & \multirow[b]{2}{*}{ Group (I) } & \multirow[b]{2}{*}{$\underset{(J)}{\text { Group }}$} & \multirow{2}{*}{$\begin{array}{c}\text { Mean } \\
\text { Difference } \\
(\mid-J)\end{array}$} & \multirow[b]{2}{*}{$\begin{array}{l}\text { Std. } \\
\text { Error }\end{array}$} & \multirow[b]{2}{*}{ Sig. } & \multicolumn{2}{|c|}{$\begin{array}{l}95 \% \text { Confidence } \\
\text { Interval }\end{array}$} \\
\hline & & & & & & & $\begin{array}{l}\text { Lower } \\
\text { Bound }\end{array}$ & $\begin{array}{l}\text { Upper } \\
\text { Bound }\end{array}$ \\
\hline \multirow[t]{30}{*}{$a$} & \multirow{30}{*}{$\begin{array}{l}\text { Games- } \\
\text { Howell }\end{array}$} & \multirow[t]{5}{*}{0} & 16 & -0.0977 & 0.5052 & 1.000 & -1.5651 & 1.3697 \\
\hline & & & 32 & 1.0395 & 0.6539 & .607 & -0.8625 & 2.9415 \\
\hline & & & 48 & $1.7949^{*}$ & 0.5543 & .020 & 0.1851 & 3.4048 \\
\hline & & & 64 & -0.1047 & 0.5770 & 1.000 & -1.7808 & 1.5714 \\
\hline & & & 80 & $1.4457^{*}$ & 0.4825 & .039 & 0.0435 & 2.8480 \\
\hline & & \multirow[t]{5}{*}{16} & 0 & 0.0977 & 0.5052 & 1.000 & -1.3697 & 1.5651 \\
\hline & & & 32 & 1.1371 & 0.6623 & .524 & -0.7864 & 3.0607 \\
\hline & & & 48 & $1.8926^{\pi}$ & 0.5641 & .013 & 0.2576 & 3.5276 \\
\hline & & & 64 & -0.0070 & 0.5864 & 1.000 & -1.7074 & 1.6933 \\
\hline & & & 80 & $1.5433^{*}$ & 0.4937 & .027 & 0.1128 & 2.9740 \\
\hline & & \multirow[t]{5}{*}{32} & 0 & -1.0395 & 0.6539 & .607 & -2.9415 & 0.8625 \\
\hline & & & 16 & -1.1371 & 0.6623 & .524 & -3.0607 & 0.7864 \\
\hline & & & 48 & 0.7555 & 0.7005 & .889 & -1.2758 & 2.7868 \\
\hline & & & 64 & -1.1442 & 0.7185 & .605 & -3.2270 & 0.9386 \\
\hline & & & 80 & 0.4062 & 0.6451 & .989 & -1.4695 & 2.2820 \\
\hline & & \multirow[t]{5}{*}{48} & 0 & -1.7949 & 0.5543 & .020 & -3.4048 & -0.1851 \\
\hline & & & 16 & -1.8926 & 0.5641 & .013 & -3.5276 & -0.2576 \\
\hline & & & 32 & -0.7555 & 0.7005 & .889 & -2.7868 & 1.2758 \\
\hline & & & 64 & -1.8996 & 0.6292 & .036 & -3.7227 & -0.0766 \\
\hline & & & 80 & -0.3492 & 0.5439 & .988 & -1.9266 & 1.2281 \\
\hline & & \multirow[t]{5}{*}{64} & 0 & 0.1047 & 0.5770 & 1.000 & -1.5714 & 1.7808 \\
\hline & & & 16 & 0.0070 & 0.5864 & 1.000 & -1.6933 & 1.7074 \\
\hline & & & 32 & 1.1442 & 0.7185 & .605 & -0.9386 & 3.2270 \\
\hline & & & 48 & $1.8996{ }^{*}$ & 0.6292 & .036 & 0.0766 & 3.7227 \\
\hline & & & 80 & 1.5504 & 0.5670 & .077 & -0.0948 & 3.1957 \\
\hline & & \multirow[t]{5}{*}{80} & 0 & -1.4457 & 0.4825 & .039 & -2.8480 & -0.0435 \\
\hline & & & 16 & -1.5433 & 0.4937 & .027 & -2.9740 & -0.1128 \\
\hline & & & 32 & -0.4062 & 0.6451 & .989 & -2.2820 & 1.4695 \\
\hline & & & 48 & 0.3492 & 0.5439 & .988 & -1.2281 & 1.9266 \\
\hline & & & 64 & -1.5504 & 0.5670 & .077 & -3.1957 & 0.0948 \\
\hline
\end{tabular}

*. The mean difference is significant at the 0.05 level.

\section{DPMP-Variable ' $b$ '}

Only one significant difference was observed at this variable. Group $16(\mathrm{M}=8.467)$ was observed to be significantly different from Group $48(M=7.161)$ with a mean difference of 
1.306 and a p-value of 0.001. It can be concluded that the aging treatment did not substantially affect the color saturation of this sample at this particular variable.

Table 25: Games-Howell test of sample DPMP at variable ' $b$ '

Multiple Comparisons: DPMP

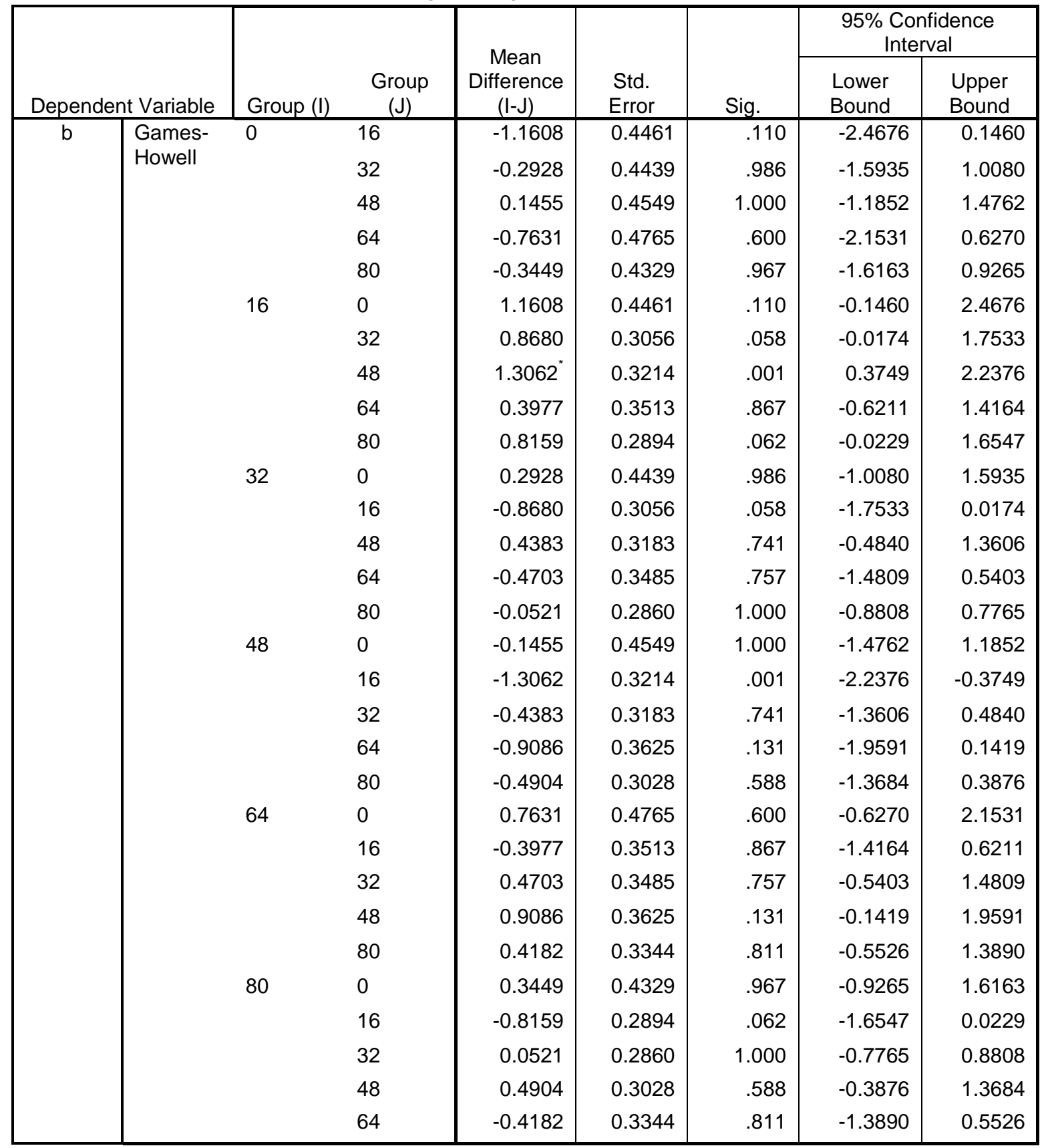

*. The mean difference is significant at the 0.05 level. 


\section{GMP}

This sample observed the greatest amount of change at variable ' $a$ '. The results from the

validation section must also be considered as variations were observed in the red/green region.

Each variable will be discussed separately below.

GMP-Variable ' $L$ '

Table 26: Games-Howell test of sample GMP at variable ' $L$ '

Multiple Comparisons: GMP

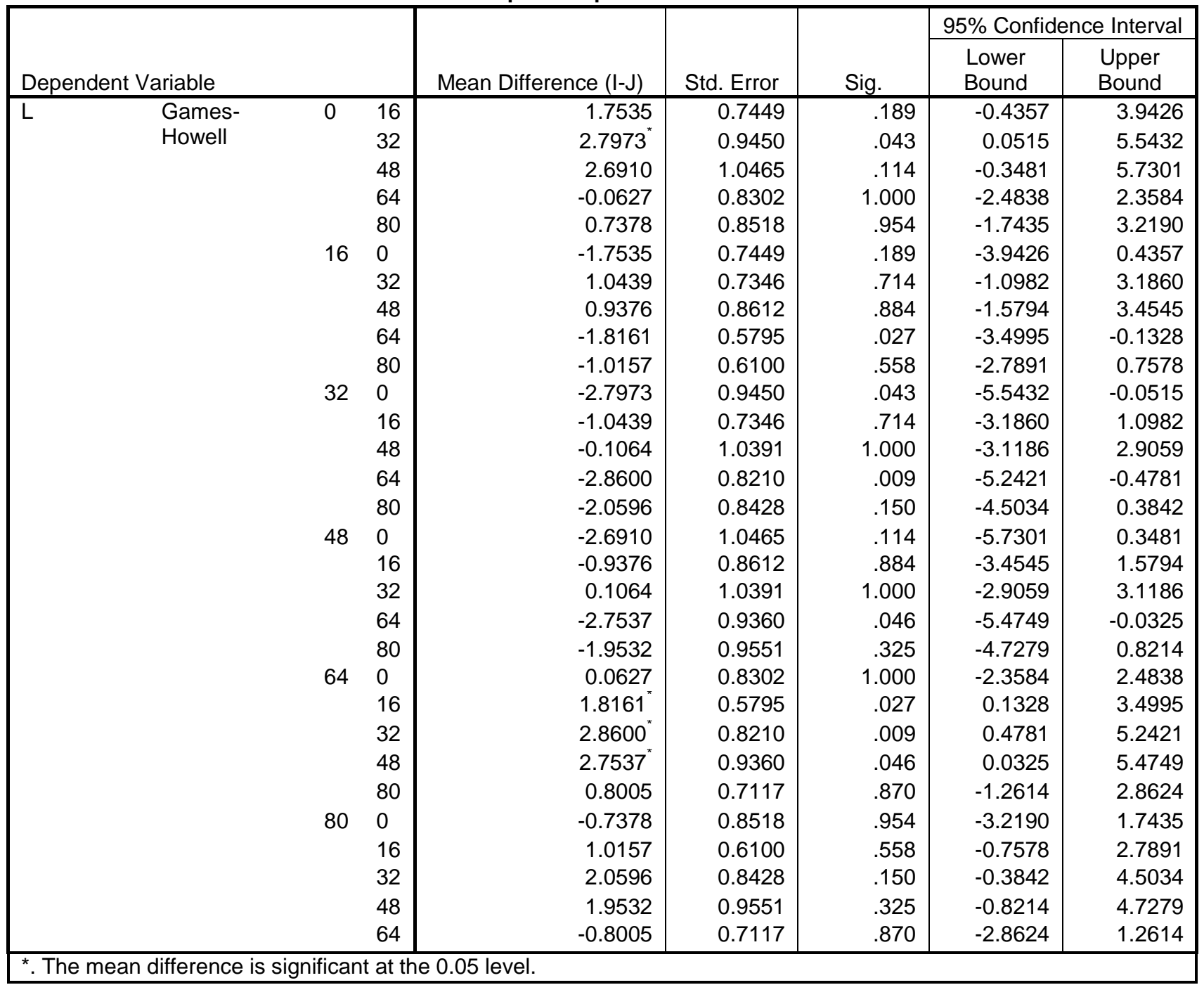


Since sample GMP did not meet the homogeneity of variance assumption, only the results taken from Games-Howell test was used. Based on the results, five marked differences were observed. At this variable, an unsubstantial amount of differences in means were observed. Out of the fifteen comparing pairs, only four groups observed to have significant mean differences suggesting that the lightness of the sample was affected but not at an extensively.

GMP-Variable ' $a$ '

Table 27: Games-Howell test of sample GMP at variable ' $a$ '

Multiple Comparisons: GMP

\begin{tabular}{|c|c|c|c|c|c|c|c|c|}
\hline & & & & Mean & & & $95 \%$ Confid & e Interval \\
\hline & ariable & & & $\begin{array}{c}\text { Difference (I- } \\
\mathrm{J})\end{array}$ & Std. Error & Sig. & $\begin{array}{l}\text { Lower } \\
\text { Bound }\end{array}$ & $\begin{array}{l}\text { Upper } \\
\text { Bound }\end{array}$ \\
\hline $\bar{a}$ & Games-Howell & 0 & 16 & 0.0071 & 0.3021 & 1.000 & -0.8904 & 0.9045 \\
\hline & & & 32 & -0.1817 & 0.3079 & .991 & -1.0936 & 0.7302 \\
\hline & & & 48 & 0.1310 & 0.3053 & .998 & -0.7744 & 1.0365 \\
\hline & & & 64 & -0.3020 & 0.3079 & .922 & -1.2139 & 0.6100 \\
\hline & & & 80 & 0.2496 & 0.3080 & .964 & -0.6627 & 1.1619 \\
\hline & & 16 & 0 & -0.0071 & 0.3021 & 1.000 & -0.9045 & 0.8904 \\
\hline & & & 32 & -0.1888 & 0.0970 & .381 & -0.4706 & 0.0930 \\
\hline & & & 48 & 0.1240 & 0.0887 & .728 & -0.1332 & 0.3811 \\
\hline & & & 64 & -0.3090 & 0.0972 & .023 & -0.5912 & -0.0269 \\
\hline & & & 80 & 0.2425 & 0.0976 & 138 & -0.0410 & 0.5260 \\
\hline & & 32 & 0 & 0.1817 & 0.3079 & .991 & -0.7302 & 1.0936 \\
\hline & & & 16 & 0.1888 & 0.0970 & .381 & -0.0930 & 0.4706 \\
\hline & & & 48 & $.3127^{*}$ & 0.1066 & .045 & 0.0038 & 0.6217 \\
\hline & & & 64 & -0.1203 & 0.1138 & .897 & -0.4499 & 0.2094 \\
\hline & & & 80 & $.4313^{x}$ & 0.1142 & .003 & 0.1005 & 0.7621 \\
\hline & & 48 & 0 & -0.1310 & 0.3053 & .998 & -1.0365 & 0.7744 \\
\hline & & & 16 & -0.1240 & 0.0887 & .728 & -0.3811 & 0.1332 \\
\hline & & & 32 & -0.3127 & 0.1066 & .045 & -0.6217 & -0.0038 \\
\hline & & & 64 & -0.4330 & 0.1067 & .001 & -0.7423 & -0.1238 \\
\hline & & & 80 & 0.1186 & 0.1071 & .878 & -0.1919 & 0.4291 \\
\hline & & 64 & 0 & 0.3020 & 0.3079 & .922 & -0.6100 & 1.2139 \\
\hline & & & 16 & $.3090^{x}$ & 0.0972 & .023 & 0.0269 & 0.5912 \\
\hline & & & 32 & 0.1203 & 0.1138 & .897 & -0.2094 & 0.4499 \\
\hline & & & 48 & $.4330^{\circ}$ & 0.1067 & .001 & 0.1238 & 0.7423 \\
\hline & & & 80 & $.5515^{x}$ & 0.1143 & .000 & 0.2205 & 0.8826 \\
\hline & & 80 & 0 & -0.2496 & 0.3080 & .964 & -1.1619 & 0.6627 \\
\hline & & & 16 & -0.2425 & 0.0976 & .138 & -0.5260 & 0.0410 \\
\hline & & & 32 & -0.4313 & 0.1142 & .003 & -0.7621 & -0.1005 \\
\hline & & & 48 & -0.1186 & 0.1071 & .878 & -0.4291 & 0.1919 \\
\hline & & & 64 & -0.5515 & 0.1143 & .000 & -0.8826 & -0.2205 \\
\hline
\end{tabular}


The greatest amount of differences in means was observed at this variable. Five mean significant differences were observed here. Although, this number is unsubstantial in comparison to the fifteen pair-wise groups being analyzed, it is still able to determine that negligible degree of color saturation occurred in the red/green region. Again two possible explanations can be drawn: the aging treatment reasonably affected the color saturation in the red/green region, or, based on the validation results, variation is typically observed in this region which could have influenced the post hoc results and the aging treatment.

\section{GMP-Variable ' $b$ '}

Table 28: Games-Howell test of sample GMP at variable ' $b$ '

\begin{tabular}{|c|c|c|c|c|c|c|c|c|}
\hline \multicolumn{9}{|c|}{ Multiple Comparisons: GMP } \\
\hline \multirow{2}{*}{\multicolumn{4}{|c|}{ Dependent Variable }} & \multirow[b]{2}{*}{ Mean Difference (I-J) } & \multirow[b]{2}{*}{ Std. Error } & \multirow[b]{2}{*}{ Sig. } & \multicolumn{2}{|c|}{$95 \%$ Confidence Interval } \\
\hline & & & & & & & $\begin{array}{l}\text { Lower } \\
\text { Bound } \\
\end{array}$ & $\begin{array}{l}\text { Upper } \\
\text { Bound } \\
\end{array}$ \\
\hline \multirow[t]{30}{*}{$\mathrm{b}$} & Games- & 0 & 16 & 0.4658 & 0.3482 & .763 & -0.5455 & 1.4771 \\
\hline & Howell & & 32 & -0.0025 & 0.3692 & 1.000 & -1.0748 & 1.0697 \\
\hline & & & 48 & 0.8951 & 0.3355 & .091 & -0.0797 & 1.8699 \\
\hline & & & 64 & 0.8779 & 0.4731 & .436 & -0.4993 & 2.2551 \\
\hline & & & 80 & $1.0188^{*}$ & 0.3407 & .040 & 0.0292 & 2.0085 \\
\hline & & 16 & 0 & -0.4658 & 0.3482 & .763 & -1.4771 & 0.5455 \\
\hline & & & 32 & -0.4684 & 0.3821 & .823 & -1.5755 & 0.6388 \\
\hline & & & 48 & 0.4293 & 0.3497 & .822 & -0.5838 & 1.4424 \\
\hline & & & 64 & 0.4121 & 0.4832 & .957 & -0.9922 & 1.8164 \\
\hline & & & 80 & 0.5530 & 0.3546 & .627 & -0.4744 & 1.5804 \\
\hline & & 32 & 0 & 0.0025 & 0.3692 & 1.000 & -1.0697 & 1.0748 \\
\hline & & & 16 & 0.4684 & 0.3821 & .823 & -0.6388 & 1.5755 \\
\hline & & & 48 & 0.8977 & 0.3706 & .157 & -0.1764 & 1.9718 \\
\hline & & & 64 & 0.8805 & 0.4985 & .492 & -0.5668 & 2.3277 \\
\hline & & & 80 & 1.0214 & 0.3753 & .079 & -0.0661 & 2.1089 \\
\hline & & 48 & 0 & -0.8951 & 0.3355 & .091 & -1.8699 & 0.0797 \\
\hline & & & 16 & -0.4293 & 0.3497 & .822 & -1.4424 & 0.5838 \\
\hline & & & 32 & -0.8977 & 0.3706 & .157 & -1.9718 & 0.1764 \\
\hline & & & 64 & -0.0172 & 0.4741 & 1.000 & -1.3964 & 1.3620 \\
\hline & & & 80 & 0.1237 & 0.3422 & .999 & -0.8676 & 1.1151 \\
\hline & & 64 & 0 & -0.8779 & 0.4731 & .436 & -2.2551 & 0.4993 \\
\hline & & & 16 & -0.4121 & 0.4832 & .957 & -1.8164 & 0.9922 \\
\hline & & & 32 & -0.8805 & 0.4985 & .492 & -2.3277 & 0.5668 \\
\hline & & & 48 & 0.0172 & 0.4741 & 1.000 & -1.3620 & 1.3964 \\
\hline & & & 80 & 0.1409 & 0.4778 & 1.000 & -1.2484 & 1.5303 \\
\hline & & 80 & 0 & -1.0188 & 0.3407 & .040 & -2.0085 & -0.0292 \\
\hline & & & 16 & -0.5530 & 0.3546 & .627 & -1.5804 & 0.4744 \\
\hline & & & 32 & -1.0214 & 0.3753 & .079 & -2.1089 & 0.0661 \\
\hline & & & 48 & -0.1237 & 0.3422 & .999 & -1.1151 & 0.8676 \\
\hline & & & 64 & -0.1409 & 0.4778 & 1.000 & -1.5303 & 1.2484 \\
\hline
\end{tabular}


At this variable, only one significant difference was observed. Group $0(M=11.05)$ was observed to be significantly different from Group $48(\mathrm{M}=10.04)$ with a mean difference of 1.019 and a $\mathrm{p}$-value of 0.04 . Notice that this $\mathrm{p}$-value is close to the alpha level, and thus this difference in means are not very dissimilar. It can be concluded that the aging treatment had trace amount of effect in color change in the yellow/blue region. However, this amount was minimal.

\section{Sample OSDP}

Sample OSDP observed the greatest amount of mean differences in the pair-wise comparing groups at variable 'b'. Each variable will be discussed separately below.

\section{OSDP-Variable ' $L$ '}

Based on the results, only one marked significant difference was observed at this variable. At Group $48(\mathrm{M}=93.56)$ was significantly different from Group $80(\mathrm{M}=90.56)$, with a mean difference of -3.041 and a p-value of .001. No other unique pair comparisons were observed. It can then be concluded that with the aging treatment, the loss of gloss in the sample was detected but minimal. 
Table 29: Games-Howell test of sample OSDP at variable ' $L$ '

Multiple Comparisons: OSDP

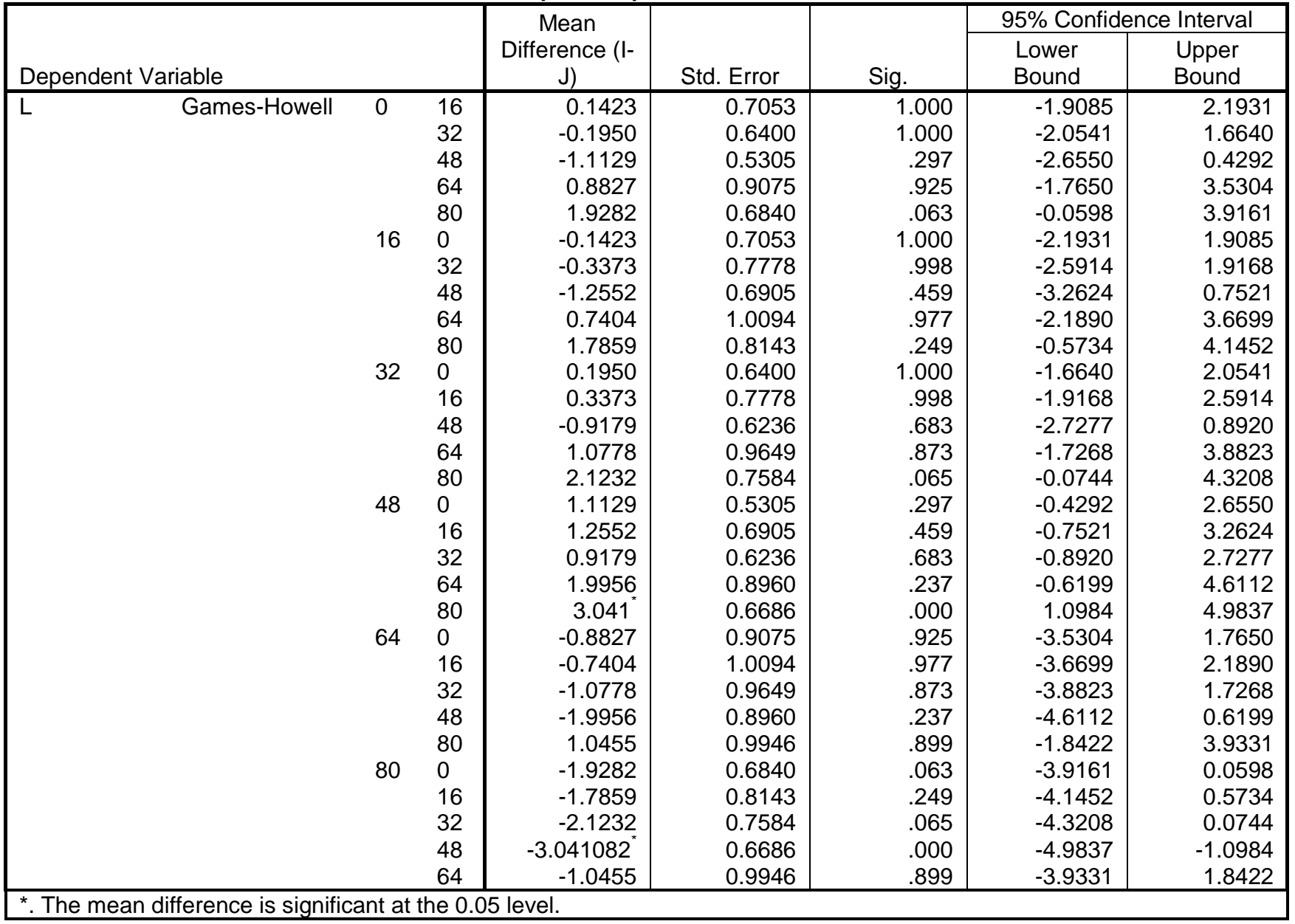

\section{OSDP-Variable ' $a$ '}

Based on the results, four marked significant differences were observed at this variable, in which three of these differences were observed at Group 0. Group $0(M=8.994)$ was significantly lower than: Group $16(\mathrm{M}=9.769)$, with a mean difference of -0.7751 and a $\mathrm{p}$-value of .001 ; Group $48(\mathrm{M}=10.462)$, with a mean difference of -1.468 and a p-value of .001; and Group 64 $(\mathrm{M}=9.917)$, with a mean difference of -0.9223 and a $\mathrm{p}$-value of .007 . It can then be concluded that with the aging treatment, color difference in the treated fibers were affected in comparison to its untreated source in the red/green region. 
Table 30: Games-Howell test of sample OSDP at variable ' $a$ '

Multiple Comparisons: OSDP

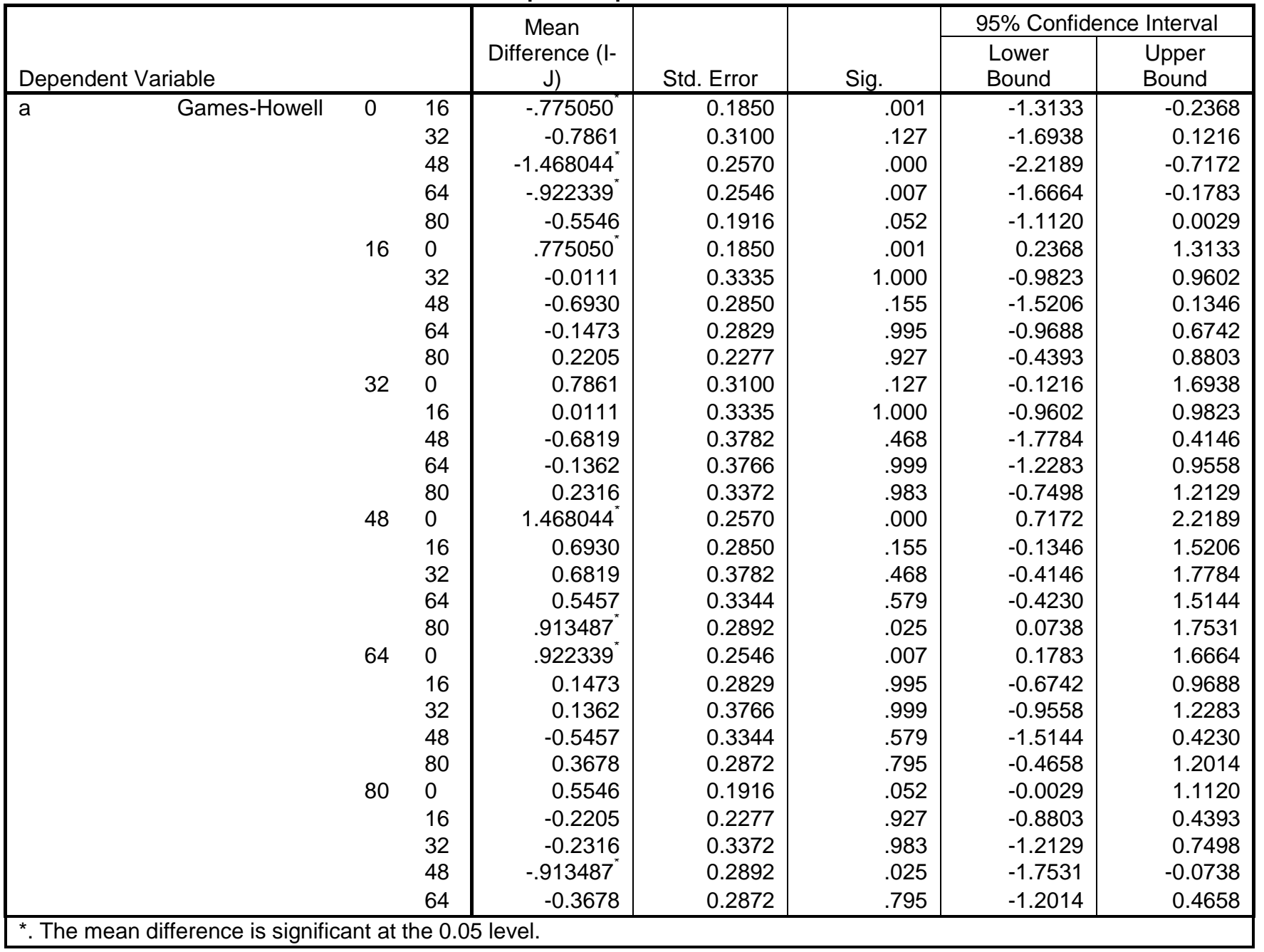

\section{OSDP-Variable ' $b$ '}

The greatest amount of change was observed at this variable, where six mean differences were noted. Five of these marked differences were observed at Group 0. Group $0(\mathrm{M}=36.54)$ was significantly higher than: Group $16(\mathrm{M}=32.01)$, with a mean difference of 4.531 and a p-value .001 ; Group $32(\mathrm{M}=31.51)$, with a mean difference of 5.033 and a p-value .001 ; Group $48(\mathrm{M}=$ 34.05), with a mean difference of 2.487 and a p-value .005; Group $64(\mathrm{M}=32.58)$, with a mean difference of 3.957 and a p-value .001; and lastly, at Group $80(\mathrm{M}=30.88)$ with a mean 
difference of 5.657 and a p-value of .001. It can then be concluded that with the aging treatment, a change in color saturation in the yellow/blue region was detectable in comparison to its untreated source.

Table 31: Games-Howell test of sample OSDP at variable 'b'

Multiple Comparisons: OSDP

\begin{tabular}{|c|c|c|c|c|c|c|c|c|}
\hline \multirow{2}{*}{\multicolumn{2}{|c|}{ Dependent Variable }} & & & \multirow{2}{*}{$\begin{array}{c}\text { Mean } \\
\text { Difference } \\
(I-J)\end{array}$} & \multirow[b]{2}{*}{ Std. Error } & \multirow[b]{2}{*}{ Sig. } & \multicolumn{2}{|c|}{ 95\% Confidence Interval } \\
\hline & & & & & & & $\begin{array}{l}\text { Lower } \\
\text { Bound }\end{array}$ & $\begin{array}{l}\text { Upper } \\
\text { Bound }\end{array}$ \\
\hline \multirow{30}{*}{\multicolumn{2}{|c|}{$\begin{array}{ll}\mathrm{b} & \text { Games- } \\
& \text { Howell }\end{array}$}} & 0 & 16 & 4.531434 & 0.6021 & .000 & 2.7751 & 6.2878 \\
\hline & & & 32 & $5.032972^{*}$ & 0.8691 & .000 & 2.4866 & 7.5794 \\
\hline & & & 48 & 2.486964 & 0.6673 & .005 & 0.5375 & 4.4364 \\
\hline & & & 64 & $3.956709^{*}$ & 0.8328 & .000 & 1.5176 & 6.3958 \\
\hline & & & 80 & $5.657093^{\pi}$ & 0.5325 & .000 & 4.1064 & 7.2078 \\
\hline & & 16 & 0 & $-4.531434^{\pi}$ & 0.6021 & .000 & -6.2878 & -2.7751 \\
\hline & & & 32 & 0.5015 & 0.9913 & .996 & -2.3781 & 3.3811 \\
\hline & & & 48 & -2.0445 & 0.8202 & .135 & -4.4213 & 0.3323 \\
\hline & & & 64 & -0.5747 & 0.9597 & .991 & -3.3610 & 2.2116 \\
\hline & & & 80 & 1.1257 & 0.7148 & .617 & -0.9460 & 3.1973 \\
\hline & & 32 & 0 & $-5.032972^{\pi}$ & 0.8691 & .000 & -7.5794 & -2.4866 \\
\hline & & & 16 & -0.5015 & 0.9913 & .996 & -3.3811 & 2.3781 \\
\hline & & & 48 & -2.5460 & 1.0323 & .143 & -5.5408 & 0.4488 \\
\hline & & & 64 & -1.0763 & 1.1463 & .936 & -4.3972 & 2.2447 \\
\hline & & & 80 & 0.6241 & 0.9507 & .986 & -2.1425 & 3.3908 \\
\hline & & 48 & 0 & $-2.486964^{\pi}$ & 0.6673 & .005 & -4.4364 & -0.5375 \\
\hline & & & 16 & 2.0445 & 0.8202 & .135 & -0.3323 & 4.4213 \\
\hline & & & 32 & 2.5460 & 1.0323 & .143 & -0.4488 & 5.5408 \\
\hline & & & 64 & 1.4697 & 1.0019 & .686 & -1.4359 & 4.3754 \\
\hline & & & 80 & $3.170128^{\star}$ & 0.7706 & .001 & 0.9350 & 5.4053 \\
\hline & & 64 & 0 & $-3.956709^{x}$ & 0.8328 & .000 & -6.3958 & -1.5176 \\
\hline & & & 16 & 0.5747 & 0.9597 & .991 & -2.2116 & 3.3610 \\
\hline & & & 32 & 1.0763 & 1.1463 & .936 & -2.2447 & 4.3972 \\
\hline & & & 48 & -1.4697 & 1.0019 & .686 & -4.3754 & 1.4359 \\
\hline & & & 80 & 1.7004 & 0.9176 & .437 & -0.9686 & 4.3693 \\
\hline & & 80 & 0 & $-5.657093^{x}$ & 0.5325 & .000 & -7.2078 & -4.1064 \\
\hline & & & 16 & -1.1257 & 0.7148 & .617 & -3.1973 & 0.9460 \\
\hline & & & 32 & -0.6241 & 0.9507 & .986 & -3.3908 & 2.1425 \\
\hline & & & 48 & $-3.170128^{\pi}$ & 0.7706 & .001 & -5.4053 & -0.9350 \\
\hline & & & 64 & -1.7004 & 0.9176 & .437 & -4.3693 & 0.9686 \\
\hline
\end{tabular}

IV. PMCL 
With this sample, the same amount of change was observed at variables ' $L$ ' and ' $a$ '. It is

expected that this sample being pink, would be affected in the red/green region. Therefore, the

result obtained could also be contributed to variation in this region. Each variable will be

discussed separately.

PMCL-Variable ' $L$ '

Table 32: Games-Howell test of sample PMCL at variable ' $L$ '

Multiple Comparisons: PMCL

\begin{tabular}{|c|c|c|c|c|c|c|c|c|}
\hline \multirow{2}{*}{\multicolumn{2}{|c|}{ Dependent Variable }} & \multirow[t]{2}{*}{ (I) Hours } & \multirow[t]{2}{*}{ (J) Hours } & \multirow{2}{*}{$\begin{array}{c}\text { Mean } \\
\text { Difference } \\
(\mathrm{I}-\mathrm{J})\end{array}$} & \multirow[t]{2}{*}{ Std. Error } & \multirow[t]{2}{*}{ Sig. } & \multicolumn{2}{|c|}{ 95\% Confidence Interval } \\
\hline & & & & & & & $\begin{array}{l}\text { Lower } \\
\text { Bound } \\
\end{array}$ & $\begin{array}{l}\text { Upper } \\
\text { Bound } \\
\end{array}$ \\
\hline \multirow{30}{*}{\multicolumn{2}{|c|}{$\begin{array}{l}\text { Games- } \\
\text { Howell }\end{array}$}} & 0 & 16 & $3.670063^{*}$ & 1.1225 & 0.018 & $.40681^{*}$ & 6.9333 \\
\hline & & & 32 & $3.766913^{*}$ & 0.9985 & 0.004 & $.85219^{*}$ & 6.6816 \\
\hline & & & 48 & $4.323544^{*}$ & 0.9879 & 0.001 & $1.43786^{*}$ & 7.2092 \\
\hline & & & 64 & $3.447446^{*}$ & 0.9487 & 0.007 & $.66857^{*}$ & 6.2263 \\
\hline & & & 80 & $2.714988^{*}$ & 0.9711 & 0.069 & $-.12473^{*}$ & 5.5547 \\
\hline & & 16 & 0 & $-3.670063^{*}$ & 1.1225 & 0.018 & -6.93332 & -0.4068 \\
\hline & & & 32 & .096850 & 0.9423 & 1 & $-2.63712^{x}$ & 2.8308 \\
\hline & & & 48 & $.653482^{*}$ & 0.9311 & 0.981 & $-2.04891^{*}$ & 3.3559 \\
\hline & & & 64 & -0.2226 & 0.8894 & 1 & -2.8082 & 2.3630 \\
\hline & & & 80 & -0.9551 & 0.9133 & 0.901 & -3.6073 & 1.6972 \\
\hline & & 32 & 0 & -3.7669 & 0.9985 & 0.004 & -6.6816 & -0.8522 \\
\hline & & & 16 & -0.0969 & 0.9423 & 1 & -2.8308 & 2.6371 \\
\hline & & & 48 & $.556632^{*}$ & 0.7771 & 0.98 & $-1.69477^{*}$ & 2.8080 \\
\hline & & & 64 & -0.3195 & 0.7266 & 0.998 & -2.4257 & 1.7868 \\
\hline & & & 80 & -1.0519 & 0.7556 & 0.732 & -3.2414 & 1.1376 \\
\hline & & 48 & 0 & -4.3235 & 0.9879 & 0.001 & -7.2092 & -1.4379 \\
\hline & & & 16 & -0.6535 & 0.9311 & 0.981 & -3.3559 & 2.0489 \\
\hline & & & 32 & $-.556632^{*}$ & 0.7771 & 0.98 & $-2.80804^{*}$ & 1.6948 \\
\hline & & & 64 & -0.8761 & 0.7120 & 0.821 & -2.9397 & 1.1875 \\
\hline & & & 80 & -1.6086 & 0.7417 & 0.26 & -3.7573 & 0.5402 \\
\hline & & 64 & 0 & -3.4474 & 0.9487 & 0.007 & -6.2263 & -0.6686 \\
\hline & & & 16 & 0.2226 & 0.8894 & 1 & -2.3630 & 2.8082 \\
\hline & & & 32 & $.319467^{*}$ & 0.7266 & 0.998 & $-1.78676^{*}$ & 2.4257 \\
\hline & & & 48 & 0.8761 & 0.7120 & 0.821 & -1.1875 & 2.9397 \\
\hline & & & 80 & -0.7325 & 0.6886 & 0.895 & -2.7276 & 1.2626 \\
\hline & & 80 & 0 & -2.7150 & 0.9711 & 0.069 & -5.5547 & 0.1247 \\
\hline & & & 16 & 0.9551 & 0.9133 & 0.901 & -1.6972 & 3.6073 \\
\hline & & & 32 & $1.051925^{*}$ & 0.7556 & 0.732 & $-1.13758^{*}$ & 3.2414 \\
\hline & & & 48 & 1.6086 & 0.7417 & 0.26 & -0.5402 & 3.7573 \\
\hline & & & 64 & 0.7325 & 0.6886 & 0.895 & -1.2626 & 2.7276 \\
\hline
\end{tabular}


Six significant mean differences were observed at this variable. Similar to sample OSDP, five of these marked differences were observed at Group 0 . Group $0(M=97.98)$ was significantly higher than: Group $16(\mathrm{M}=94.31)$, with a mean difference of 3.670 and a p-value .018 ; Group $32(\mathrm{M}=93.21)$, with a mean difference of 3.767 and a p-value .004 ; Group $48(\mathrm{M}=93.66)$, with a mean difference of 4.324 and a p-value .001; Group $64(\mathrm{M}=94.53)$, with a mean difference of 3.447 and a p-value .007 ; and lastly, at Group $80(\mathrm{M}=95.26)$ with a mean difference of 2.715 and a p-value of .069. An incorrect estimation was observed at Group 80 where it was marked significant but the p-value is actually greater than 0.05 which would conclude as a nonsignificant value. Upon observing the results from Tukey, a significant p-value of 0.036 was observed. This particular result will not be concluded as significant as the p-value is below the alpha level. It can then be concluded that with the aging treatment, the gloss of the sample was affected in comparison to its untreated source.

PMCL-Variable ' $a$ '

Unlike variable 'L' which observed six marked significant differences, these differences were observed at variable ' $a$ '. Five of these marked differences were observed at Group 16. Group 16 $(\mathrm{M}=2.888)$ was significantly higher from Group $0(\mathrm{M}=32.01)$, with a mean difference of 4.531 and a p-value .001; Group $32(\mathrm{M}=31.51)$, with a mean difference of 5.033 and a p-value .001 ; Group $48(\mathrm{M}=34.05)$, with a mean difference of 2.487 and a p-value .005; Group $64(\mathrm{M}=$ 32.58), with a mean difference of 3.957 and a p-value .001 ; and lastly, at Group $80(\mathrm{M}=30.88)$ with a mean difference of 5.657 and a p-value of .001 . It can then be concluded that with the aging treatment, the treated fibers were affected in color saturation in the red/green region in comparison to its untreated source. 
Table 33: Games-Howell test of sample PMCL at variable ' $a$ '

Multiple Comparisons: PMCL

\begin{tabular}{|c|c|c|c|c|c|c|c|}
\hline \multirow[t]{2}{*}{ Dependent Variable } & \multirow[t]{2}{*}{ (I) Hours } & \multirow[t]{2}{*}{ (J) Hours } & \multirow{2}{*}{$\begin{array}{c}\text { Mean } \\
\text { Difference (I- } \\
\text { J) }\end{array}$} & \multirow[t]{2}{*}{ Std. Error } & \multirow[t]{2}{*}{ Sig. } & \multicolumn{2}{|c|}{ 95\% Confidence Interval } \\
\hline & & & & & & Lower Bound & $\begin{array}{l}\text { Upper } \\
\text { Bound }\end{array}$ \\
\hline \multirow[t]{30}{*}{ Games-Howell } & 0 & 16 & 0.5615 & 0.1395 & 0.002 & 0.1546 & 0.9685 \\
\hline & & 32 & 0.4408 & 0.1318 & 0.016 & 0.0550 & 0.8265 \\
\hline & & 48 & 0.4351 & 0.1689 & 0.113 & -0.0554 & 0.9256 \\
\hline & & 64 & 0.5817 & 0.1514 & 0.003 & 0.1415 & 1.0219 \\
\hline & & 80 & $.50534689^{*}$ & 0.1405 & 0.007 & $.0956998^{*}$ & 0.9150 \\
\hline & 16 & 0 & -.56151839 & 0.1395 & 0.002 & $-.9684504^{*}$ & -0.1546 \\
\hline & & 32 & $-.12074983^{*}$ & 0.1039 & 0.854 & $-.4219258^{*}$ & 0.1804 \\
\hline & & 48 & $-.12640933^{*}$ & 0.1482 & 0.957 & $-.5569092^{*}$ & 0.3041 \\
\hline & & 64 & $.02019917^{\star}$ & 0.1278 & 1 & $-.3503806^{\star}$ & 0.3908 \\
\hline & & 80 & -.05617150 * & 0.1147 & 0.996 & $-.3885597^{*}$ & 0.2762 \\
\hline & 32 & 0 & $-.440768566^{*}$ & 0.1318 & 0.016 & $-.8265240^{*}$ & -0.0550 \\
\hline & & 16 & $.12074983^{*}$ & 0.1039 & 0.854 & $-.1804261^{*}$ & 0.4219 \\
\hline & & 48 & -0.0057 & 0.1409 & 1 & -0.4161 & 0.4048 \\
\hline & & 64 & 0.1409 & 0.1193 & 0.845 & -0.2057 & 0.4876 \\
\hline & & 80 & 0.0646 & 0.1052 & 0.99 & -0.2404 & 0.3696 \\
\hline & 48 & 0 & -0.4351 & 0.1689 & 0.113 & -0.9256 & 0.0554 \\
\hline & & 16 & $.12640933^{*}$ & 0.1482 & 0.957 & $-.3040905^{*}$ & 0.5569 \\
\hline & & 32 & 0.0057 & 0.1409 & 1 & -0.4048 & 0.4161 \\
\hline & & 64 & 0.1466 & 0.1594 & 0.941 & -0.3155 & 0.6088 \\
\hline & & 80 & 0.0702 & 0.1491 & 0.997 & -0.3628 & 0.5033 \\
\hline & 64 & 0 & -0.5817 & 0.1514 & 0.003 & -1.0219 & -0.1415 \\
\hline & & 16 & $-.02019917^{*}$ & 0.1278 & 1 & $-.3907789^{*}$ & 0.3504 \\
\hline & & 32 & -0.1409 & 0.1193 & 0.845 & -0.4876 & 0.2057 \\
\hline & & 48 & -0.1466 & 0.1594 & 0.941 & -0.6088 & 0.3155 \\
\hline & & 80 & -0.0764 & 0.1289 & 0.991 & -0.4500 & 0.2973 \\
\hline & 80 & 0 & -0.5053 & 0.1405 & 0.007 & -0.9150 & -0.0957 \\
\hline & & 16 & $.05617150^{*}$ & 0.1147 & 0.996 & $-.2762167^{*}$ & 0.3886 \\
\hline & & 32 & -0.0646 & 0.1052 & 0.99 & -0.3696 & 0.2404 \\
\hline & & 48 & -0.0702 & 0.1491 & 0.997 & -0.5033 & 0.3628 \\
\hline & & 64 & 0.0764 & 0.1289 & 0.991 & -0.2973 & 0.4500 \\
\hline
\end{tabular}

*. The mean difference is significant at the 0.05 level.

\section{PMCL-Variable ' $b$ '}

The greatest amount of change was observed at this variable, where six mean differences were noted. Five of these marked differences were observed at Group 0. Group $0(M=36.54)$ was significantly higher from Group $16(\mathrm{M}=32.01)$, with a mean difference of 4.531 and a p-value .001 ; Group $32(\mathrm{M}=31.51)$, with a mean difference of 5.033 and a p-value .001 ; Group $48(\mathrm{M}=$ 
34.05), with a mean difference of 2.487 and a p-value .005 ; Group $64(\mathrm{M}=32.58)$, with a mean difference of 3.957 and a p-value .001 ; and lastly, at Group $80(\mathrm{M}=30.88)$ with a mean difference of 5.657 and a p-value of .001 . It can then be concluded that with the aging treatment, the treated fibers were affected in comparison to its untreated source.

Table 34: Games-Howell test of sample PMCL at variable 'b'

Multiple Comparisons: PMCL

\begin{tabular}{|c|c|c|c|c|c|c|c|c|}
\hline \multirow{2}{*}{\multicolumn{2}{|c|}{ Dependent Variable }} & \multirow[t]{2}{*}{ (I) Hours } & \multirow[t]{2}{*}{ (J) Hours } & \multirow{2}{*}{$\begin{array}{c}\text { Mean } \\
\text { Difference } \\
(I-J)\end{array}$} & \multirow[t]{2}{*}{ Std. Error } & \multirow[t]{2}{*}{ Sig. } & \multicolumn{2}{|c|}{ 95\% Confidence Interval } \\
\hline & & & & & & & Lower Bound & $\begin{array}{l}\text { Upper } \\
\text { Bound }\end{array}$ \\
\hline \multirow{30}{*}{\multicolumn{2}{|c|}{ Games- }} & \multirow[t]{5}{*}{0} & 16 & 1.1255 & 0.2134 & 0 & 0.5020 & 1.7490 \\
\hline & & & 32 & 0.5369 & 0.2040 & 0.103 & -0.0611 & 1.1348 \\
\hline & & & 48 & 0.7225 & 0.2706 & 0.09 & -0.0633 & 1.5083 \\
\hline & & & 64 & $.7066339^{*}$ & 0.2361 & 0.04 & $.019864^{*}$ & 1.3934 \\
\hline & & & 80 & 0.7197 & 0.2649 & 0.081 & -0.0497 & 1.4891 \\
\hline & & \multirow[t]{5}{*}{16} & 0 & -1.1255 & 0.2134 & 0 & -1.7490 & -0.5020 \\
\hline & & & 32 & -0.5887 & 0.1514 & 0.002 & -1.0275 & -0.1498 \\
\hline & & & 48 & -0.4030 & 0.2335 & 0.518 & -1.0824 & 0.2763 \\
\hline & & & 64 & $-.4188787^{*}$ & 0.1924 & 0.257 & $-.977094^{*}$ & 0.1393 \\
\hline & & & 80 & $-.4057937^{*}$ & 0.2269 & 0.478 & $-1.065603^{*}$ & 0.2540 \\
\hline & & \multirow[t]{5}{*}{32} & 0 & $-.5368619^{*}$ & 0.2040 & 0.103 & $-1.134822^{*}$ & 0.0611 \\
\hline & & & 16 & $.5886507^{\star}$ & 0.1514 & 0.002 & $.149795^{*}$ & 1.0275 \\
\hline & & & 48 & $.1856385^{*}$ & 0.2249 & 0.962 & $-.470238^{*}$ & 0.8415 \\
\hline & & & 64 & $.1697720^{*}$ & 0.1820 & 0.937 & $-.358975^{*}$ & 0.6985 \\
\hline & & & 80 & $.1828570^{*}$ & 0.2181 & 0.959 & -.452694 & 0.8184 \\
\hline & & \multirow[t]{5}{*}{48} & 0 & $-.7225004^{*}$ & 0.2706 & 0.09 & $-1.508322^{*}$ & 0.0633 \\
\hline & & & 16 & 0.4030 & 0.2335 & 0.518 & -0.2763 & 1.0824 \\
\hline & & & 32 & -0.1856 & 0.2249 & 0.962 & -0.8415 & 0.4702 \\
\hline & & & 64 & -0.0159 & 0.2544 & 1 & -0.7537 & 0.7220 \\
\hline & & & 80 & -0.0028 & 0.2813 & 1 & -0.8179 & 0.8123 \\
\hline & & \multirow[t]{5}{*}{64} & 0 & $-.7066339^{*}$ & 0.2361 & 0.04 & $-1.393404^{*}$ & -0.0199 \\
\hline & & & 16 & 0.4189 & 0.1924 & 0.257 & -0.1393 & 0.9771 \\
\hline & & & 32 & -0.1698 & 0.1820 & 0.937 & -0.6985 & 0.3590 \\
\hline & & & 48 & 0.0159 & 0.2544 & 1 & -0.7220 & 0.7537 \\
\hline & & & 80 & 0.0131 & 0.2483 & 1 & -0.7070 & 0.7332 \\
\hline & & \multirow[t]{5}{*}{80} & 0 & $-.7197189^{*}$ & 0.2649 & 0.081 & $-1.489096 *$ & 0.0497 \\
\hline & & & 16 & 0.4058 & 0.2269 & 0.478 & -0.2540 & 1.0656 \\
\hline & & & 32 & -0.1829 & 0.2181 & 0.959 & -0.8184 & 0.4527 \\
\hline & & & 48 & 0.0028 & 0.2813 & 1 & -0.8123 & 0.8179 \\
\hline & & & 64 & -0.0131 & 0.2483 & 1 & -0.7332 & 0.7070 \\
\hline
\end{tabular}

\section{PMJX}


Sample PMJX, in comparison to all the reference samples, observed the greatest amount of change at variable ' $b$ ' and it was the only sample that didn't observe any changes at variable ' $L$ '. Unlike sample PMJX, sample PMJX observed greatest amount of change in the blue/yellow region. This result was not expected as this fiber is pink, and thus variation in the red/green region is more likely.

\section{PMJX-Variable ' $L$ '}

The null hypothesis of each mean group being the same was accepted for the sample at this variable. With supporting evidence, the aging treatment did not alter the gloss of the sample. PMJX-Variable ' $a$ '

Five marked significant differences were observed at this variable where four was observed at Group 48.Group $48(\mathrm{M}=2.412)$ was significantly lower than: Group $0(\mathrm{M}=2.947)$, with a mean difference of -0.5354 and a p-value .002; Group $16(\mathrm{M}=2.953)$, with a mean difference of 0.5408 and a p-value .001 ; Group $64(\mathrm{M}=3.096)$, with a mean difference of -0.6843 and a pvalue .001 ; and lastly, at Group $80(\mathrm{M}=3.123)$ with a mean difference of -0.7110 and a $\mathrm{p}$-value of .001. It can then be concluded that with the aging treatment, at 16 hours of aging, the greatest amount of change in color saturation occurred in the red/green region.

Table 35: Games-Howell test of sample PMJX at variable ' $a$ ' 
Multiple Comparisons: PMJX

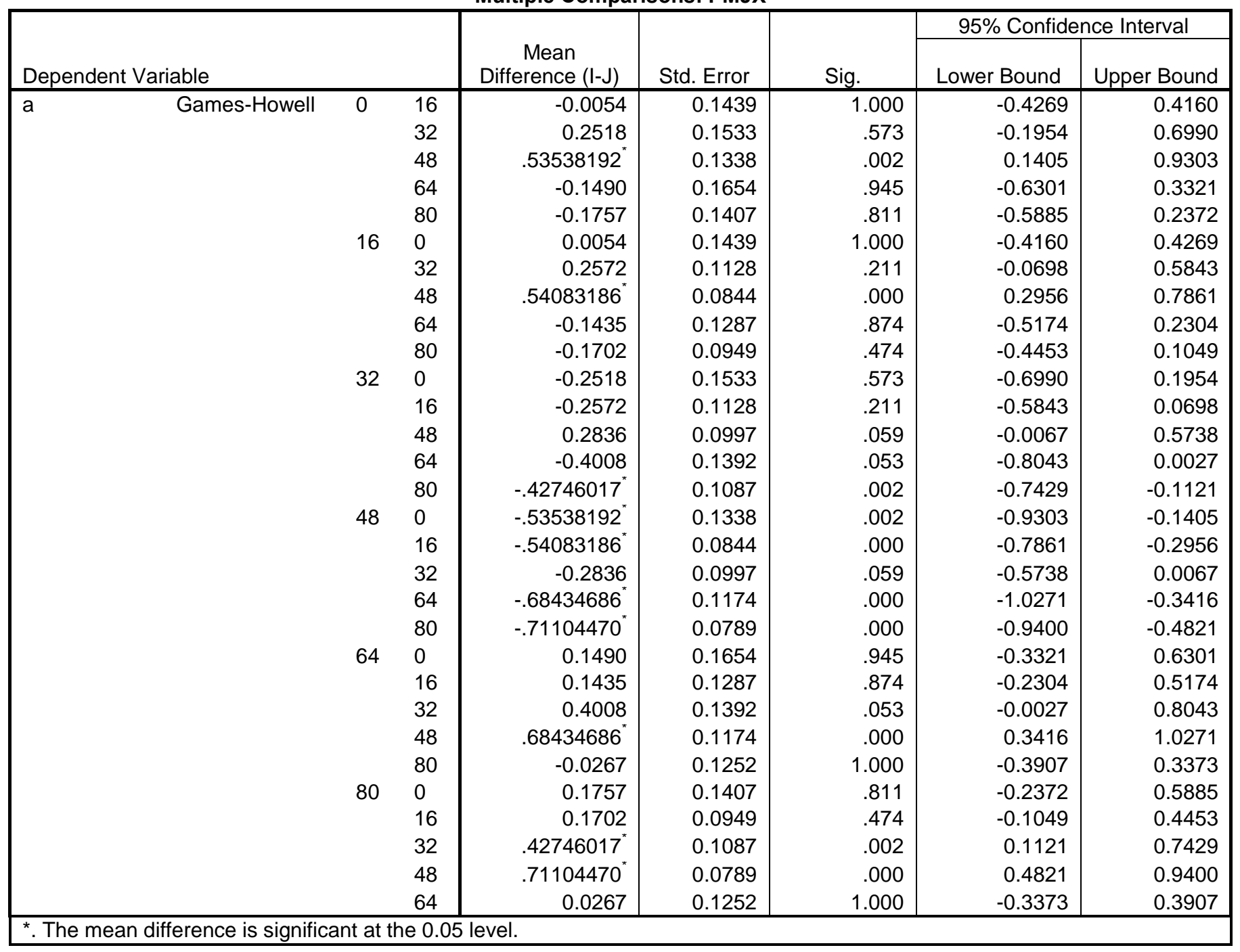

\section{PMJX-Variable ' $b$ '}

The greatest amount of change was observed at this variable, where nine marked mean differences were noted. This was the only sample that observed a substantial amount of marked mean differences in any variable. Four of these marked differences were observed at Group 32. Group $32(M=4.800)$ was significantly lower than: Group $0(M=6.056)$, with a mean difference of -1.257 and a p-value .001 ; Group $16(\mathrm{M}=5.474)$, with a mean difference of -0.675 and a p-value .031; Group $64(\mathrm{M}=6.050)$, with a mean difference of -1.250 and a p-value .001 ; 
and lastly, at Group $80(\mathrm{M}=6.306)$ with a mean difference of -1.506 and a p-value of .001 . It can then be concluded that with the aging treatment, at 16 hours of aging, the greatest amount of change in color saturation occurred in the yellow/blue region.

Table 36: Games-Howell test of sample PMJX at variable 'b'

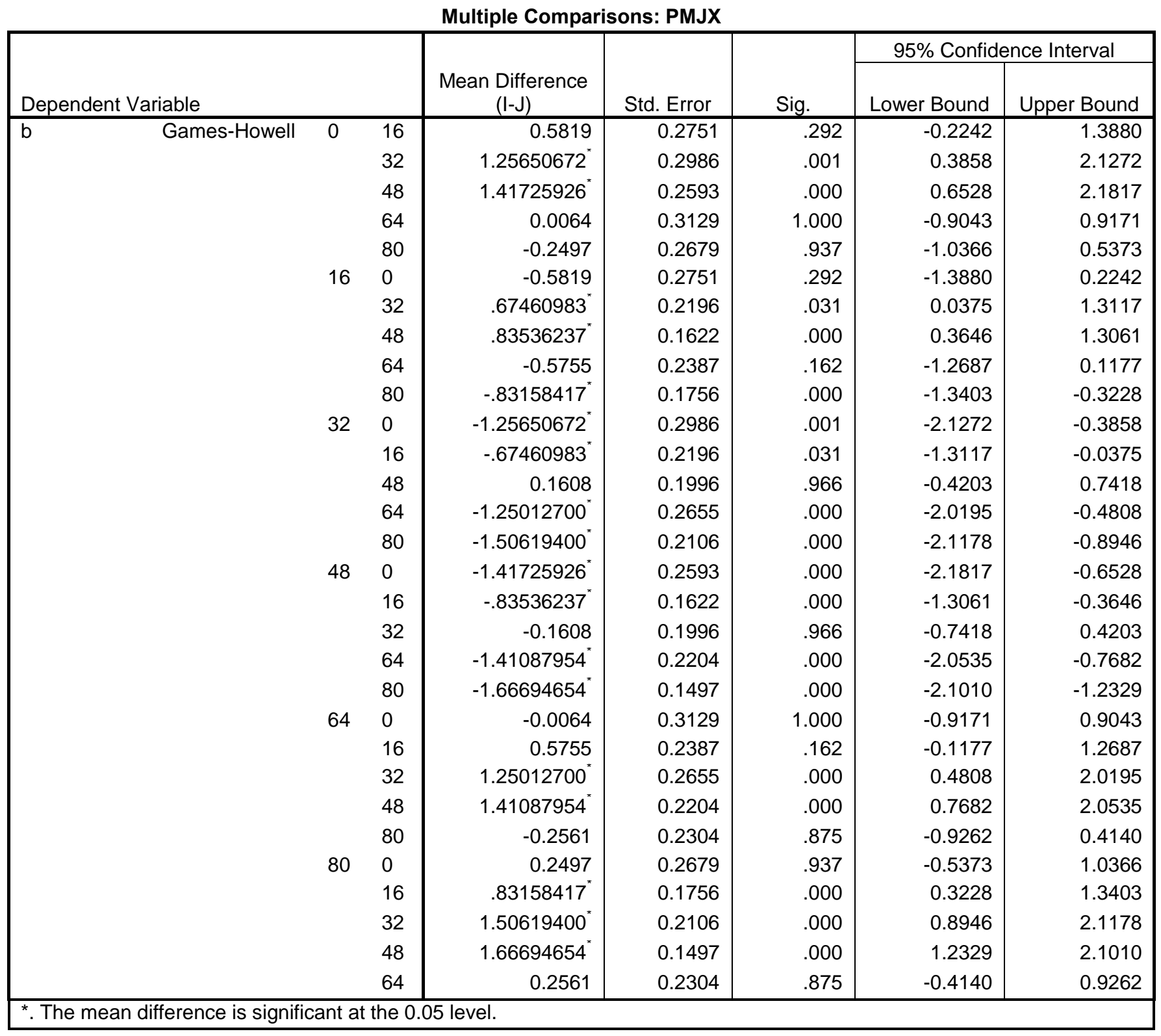

VI. PMP 
Sample PMP met the homogeneity of variance assumption only for variable ' $b$ '. The Games-

Howell test was used to calculate variables ' $L$ ' and 'a', and the Tukey test used to calculate 'b'.

\section{PMP-Variable ' $L$ '}

This sample observed the greatest amount of mean differences than any other reference samples

at this variable. It observed seven marked mean differences in total. Even with the value, there

was no one group that truly stood out from the rest. Group 0 and 32 observed three marked

differences, where the rest observed two. It can then be concluded that with the aging treatment,

loss of gloss in the sample was gradually affected as the sample was aged.

Table 37: Games-Howell test of sample PMP at variable ' $L$ '

Multiple Comparisons: PMP

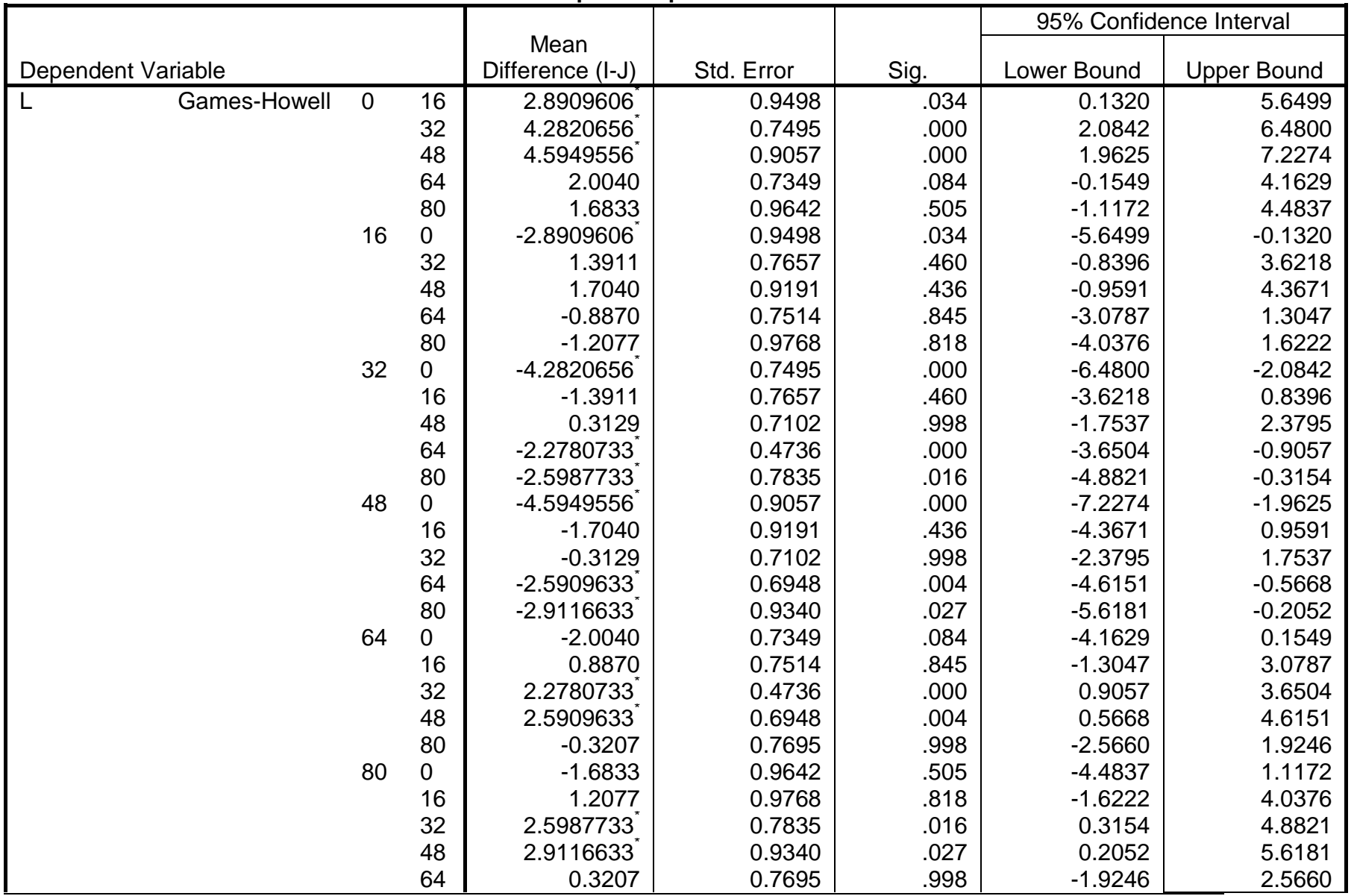

*. The mean difference is significant at the 0.05 level. 
PMP-Variable ' $a$ '

At this variable, five marked mean differences were noted. Four of these marked differences were observed at Group 80 . Group $80(\mathrm{M}=4.800)$ was significantly lower than: Group $0(\mathrm{M}=$ 6.660), with a mean difference of -0.8022 and a p-value .03 ; Group $16(\mathrm{M}=7.164)$, with a mean difference of -1.306 and a p-value .001 ; Group $32(\mathrm{M}=6.909)$, with a mean difference of -1.052 and a p-value .005 ; and lastly, at Group $64(\mathrm{M}=6.582)$ with a mean difference of -0.725 and a pvalue of .045. Although Group 64 was marked as significantly different, based on the p-values, this result is rejected as being significant. At 80 hours of aging, the greatest amount of change in color saturation occurred in the red/green region.

Table 38: Games-Howell test of sample PMP at variable ' $a$ '

Multiple Comparisons: PMP

\begin{tabular}{|c|c|c|c|c|c|c|c|c|}
\hline \multirow{2}{*}{\multicolumn{2}{|c|}{ Dependent Variable }} & & & \multirow{2}{*}{$\begin{array}{c}\text { Mean } \\
\text { Difference (I-J) } \\
\end{array}$} & \multirow[b]{2}{*}{ Std. Error } & \multirow[b]{2}{*}{ Sig. } & \multicolumn{2}{|c|}{ 95\% Confidence Interval } \\
\hline & & & & & & & Lower Bound & Upper Bound \\
\hline \multirow{30}{*}{\multicolumn{2}{|c|}{ Games-Howell }} & 0 & 16 & -0.5037 & 0.2058 & .151 & -1.1022 & 0.0949 \\
\hline & & & 32 & -0.2494 & 0.2490 & .916 & -0.9757 & 0.4768 \\
\hline & & & 48 & 0.4470 & 0.2479 & .469 & -0.2759 & 1.1699 \\
\hline & & & 64 & 0.0775 & 0.2033 & .999 & -0.5137 & 0.6688 \\
\hline & & & 80 & $.80218850^{*}$ & 0.2101 & .003 & 0.1910 & 1.4134 \\
\hline & & 16 & 0 & 0.5037 & 0.2058 & .151 & -0.0949 & 1.1022 \\
\hline & & & 32 & 0.2542 & 0.2828 & .946 & -0.5658 & 1.0743 \\
\hline & & & 48 & $.95063450^{\star}$ & 0.2818 & .013 & 0.1335 & 1.7678 \\
\hline & & & 64 & 0.5812 & 0.2435 & .169 & -0.1242 & 1.2866 \\
\hline & & & 80 & $1.30585317^{\star}$ & 0.2492 & .000 & 0.5840 & 2.0277 \\
\hline & & 32 & 0 & 0.2494 & 0.2490 & .916 & -0.4768 & 0.9757 \\
\hline & & & 16 & -0.2542 & 0.2828 & .946 & -1.0743 & 0.5658 \\
\hline & & & 48 & 0.6964 & 0.3148 & .240 & -0.2155 & 1.6083 \\
\hline & & & 64 & 0.3270 & 0.2810 & .853 & -0.4880 & 1.1419 \\
\hline & & & 80 & $1.05162583^{*}$ & 0.2859 & .005 & 0.2226 & 1.8806 \\
\hline & & 48 & 0 & -0.4470 & 0.2479 & .469 & -1.1699 & 0.2759 \\
\hline & & & 16 & -.95063450 & 0.2818 & .013 & -1.7678 & -0.1335 \\
\hline & & & 32 & -0.6964 & 0.3148 & .240 & -1.6083 & 0.2155 \\
\hline & & & 64 & -0.3694 & 0.2800 & .774 & -1.1815 & 0.4426 \\
\hline & & & 80 & 0.3552 & 0.2849 & .813 & -0.4709 & 1.1814 \\
\hline & & 64 & 0 & -0.0775 & 0.2033 & .999 & -0.6688 & 0.5137 \\
\hline & & & 16 & -0.5812 & 0.2435 & .169 & -1.2866 & 0.1242 \\
\hline & & & 32 & -0.3270 & 0.2810 & .853 & -1.1419 & 0.4880 \\
\hline & & & 48 & 0.3694 & 0.2800 & .774 & -0.4426 & 1.1815 \\
\hline & & & 80 & $.72464433^{\star}$ & 0.2471 & .045 & 0.0087 & 1.4406 \\
\hline & & 80 & 0 & -.80218850 & 0.2101 & .003 & -1.4134 & -0.1910 \\
\hline & & & 16 & $-1.30585317^{\star}$ & 0.2492 & .000 & -2.0277 & -0.5840 \\
\hline & & & 32 & $-1.05162583^{*}$ & 0.2859 & .005 & -1.8806 & -0.2226 \\
\hline & & & 48 & -0.3552 & 0.2849 & .813 & -1.1814 & 0.4709 \\
\hline & & & 64 & $-.72464433^{x}$ & 0.2471 & .045 & -1.4406 & -0.0087 \\
\hline
\end{tabular}


PMP-Variable ' $b$ '

Table 39: Tukey test of sample PMP at variable ' $b$ '

\begin{tabular}{|c|c|c|c|c|c|c|}
\hline \multirow[b]{2}{*}{ Hours } & \multicolumn{6}{|c|}{$\begin{array}{l}\text { Tukey HSD test; Variable: } b \text { (PMP spreadsheet) } \\
\text { Marked differences are significant at } p<.05000\end{array}$} \\
\hline & $\stackrel{\{1\}}{M=-8.771}$ & $\begin{array}{c}\{2\} \\
M=-10.02\end{array}$ & $\begin{array}{c}\{3\} \\
M=-9.887\end{array}$ & $\begin{array}{c}\{4\} \\
M=-8.914\end{array}$ & $\begin{array}{c}\{5\} \\
M=-10.91\end{array}$ & $\begin{array}{c}\{6\} \\
M=-11.23\end{array}$ \\
\hline $\begin{array}{ll}0 & \{1\} \\
\end{array}$ & & 0.31114 & 0.44623 & 0.99990 & 0.00592 & 0.00081 \\
\hline 16 & 0.31114 & & 0.99988 & $0.36140:$ & 0.61524 & 0.27234 \\
\hline 32 & 0.44623 & 0.99988 & & 0.51584 & 0.45394 & 0.16612 \\
\hline 48 & 0.99990 & 0.36140 & $0.51584 i$ & & 0.00534 & 0.00061 \\
\hline 64 & 0.00592 & 0.61524 & 0.453941 & 0.00534 & & 0.99383 \\
\hline 80 & 0.00081 & 0.27234 & 0.16612 & 0.00061 & 0.99383 & \\
\hline
\end{tabular}

Table 40: Homogeneous Subset of sample PMP at variable ' $b$ '

Homogeneous Subsets PMP Variable
'b'
\begin{tabular}{|ll|r|r|r|}
\hline & & & \multicolumn{3}{|c|}{ Subset for alpha $=0.05$} \\
\cline { 4 - 5 } Hours & & N & \multicolumn{1}{c|}{1} & \multicolumn{1}{c|}{2} \\
\hline Tukey & 80 & 60 & -11.226 & \\
HSD & 64 & 60 & -10.913 & \\
& 16 & 60 & -10.024 & -10.024 \\
& 32 & 60 & -9.887 & -9.887 \\
& 48 & 60 & & -8.914 \\
& 0 & & & -8.771 \\
& Sig. & & .193 & .259 \\
\hline
\end{tabular}

Means for groups in homogeneous subsets are displayed.

a. Uses Harmonic Mean Sample Size = 56.842.

b. The group sizes are unequal. The harmonic mean of the group sizes is used. Type I error levels are not guaranteed.

Both tables will be used to analyze and interpret the Tukey results. Tukey table above was generated using the Statistica software, and the Homogeneous table was generated using the SPSS software. Both tables were used in conjunction because they both show the results of the multiple pair-wise comparisons among the groups but in slightly different ways.

Four marked differences were noted: Group 1 and 4 ( 0 and 48 hours, respectively), observed significant differences with Groups 5 and 6 (64 and 80 hours, respectively). It can also be observed that the means of Group 1 and 4 are significantly smaller than Group 5 and 6 but are 
not significantly different within each other. This is better explained using the homogeneous table. Two subsets were created for this variable, meaning that these two subsets were significantly different from each other but not different within each other. The commonalities between these groups are at hours 16 and 32; there is no difference in means in comparison to the remaining hours. However, since hours 64 and 80 hours in subset 1 does not share its values in subset 2, means that these group means are significantly different. And likewise for hours 0 and 48. With such low significant mean differences, it can be concluded that the aging treatment caused detectable, yet trace amounts of change in color saturation in the yellow/blue region.

VII. RMP

Similarly to sample PMP, sample RMP meet the homogeneity of variance assumption for variable ' $L$ '.

RMP-Variable ' $L$ '

Table 41: Tukey test of sample RMP at variable ' $L$ '

\begin{tabular}{|c|c|c|c|c|c|c|}
\hline \multirow[b]{2}{*}{ Hours } & \multicolumn{6}{|c|}{$\begin{array}{l}\text { Tukey HSD test; Variable: } L \text { (RMP spreadsheet) } \\
\text { Marked differences are significant at } p<.05000\end{array}$} \\
\hline & $\begin{array}{c}\{1\} \\
M=80.84 \varepsilon\end{array}$ & $\begin{array}{c}\{2\} \\
M=81.81 C \\
\end{array}$ & $\begin{array}{c}\{3\} \\
M=82.524 \\
\end{array}$ & $\begin{array}{c}\{4\} \\
M=82.084\end{array}$ & $\begin{array}{c}\{5\} \\
M=83.53 C \\
\end{array}$ & $\begin{array}{c}\{6\} \\
M=80.481\end{array}$ \\
\hline$\{1\}$ & & $0.817 \varepsilon$ & 0.257 s & 0.603 s & 0.007: & 0.997 \\
\hline 16 & $0.817 \varepsilon$ & & 0.920 ! & 0.999 & 0.158 s & 0.434\{ \\
\hline 32 & 0.257 ؛ & 0.920 ! & & $0.990 \AA$ & 0.7274 & 0.0514 \\
\hline 48 & 0.603 ؛ & 0.999( & $0.990 i$ & & $0.336 \varsigma$ & 0.224 \\
\hline 64 & 0.007 & 0.158 ? & 0.7274 & 0.336 ؛ & & 0.000 \\
\hline 80 & $0.997^{\circ}$ & $0.434 \varepsilon$ & $0.051<$ & $0.224^{\prime}$ & 0.000 & \\
\hline
\end{tabular}

Two marked differences were noted: Group 1 ( 0 hours, $M=80.85)$, observed significant differences with Groups 5 (64 hours, $M=83.54$ ), which also observed significant differences with Group 6 ( 80 hours, $M=80.48$ ). The mean value of Group 5 is significantly greater than Group 1 and 6; hence, Group 5 was placed in a separate subset as observed in the homogeneous table. Two subsets were created for this variable, meaning that these two subsets were 
significantly different from each other but not different within each other. The commonalities between these groups are at hours 16, 32 and 48; these values share no significant difference in means in comparison to the remaining hours. However, since subset 1 at 0 and 80 hours does not share its values in subset 2 , means that these group means are significantly different from 64 hours in subset 2. It can be concluded that trace amount of loss of gloss occurred.

Table 42: Homogeneous Subset of sample RMP at variable ' $L$ ' Homogeneous Subsets: RMP Variable 'L'

\begin{tabular}{|c|c|c|c|c|}
\hline \multirow[b]{2}{*}{ Hours } & & \multirow[b]{2}{*}{$\mathrm{N}$} & \multicolumn{2}{|c|}{$\begin{array}{l}\text { Subset for alpha = } \\
0.05\end{array}$} \\
\hline & & & 1 & 2 \\
\hline \multirow{7}{*}{$\begin{array}{l}\text { Tukey } \\
\text { HSD }^{a, b}\end{array}$} & 80 & 60 & 80.481 & \\
\hline & 0 & 45 & 80.848 & \\
\hline & 16 & 60 & 81.810 & 81.810 \\
\hline & 48 & 60 & 82.084 & 82.084 \\
\hline & 32 & 60 & 82.524 & 82.524 \\
\hline & 64 & 60 & & 83.537 \\
\hline & Sig. & & .066 & .182 \\
\hline
\end{tabular}

Means for groups in homogeneous subsets are displayed.

a. Uses Harmonic Mean Sample Size $=56.842$.

b. The group sizes are unequal. The harmonic mean of the group sizes is used. Type I error levels are not guaranteed.

\section{$R M P$-Variable ' $a$ '}

Only two marked significant mean differences were observed at this variable. At some groups, for example at Group 80, no significant mean differences were observed. There were not one group that was distinctive than the rest. With such low significant mean differences, it can be concluded that the aging treatment caused detectable, yet trace amounts of change in color saturation in the red/green region. 
Table 43: Games-Howell test of sample RMP at variable ' $a$ '

Multiple Comparisons: RMP

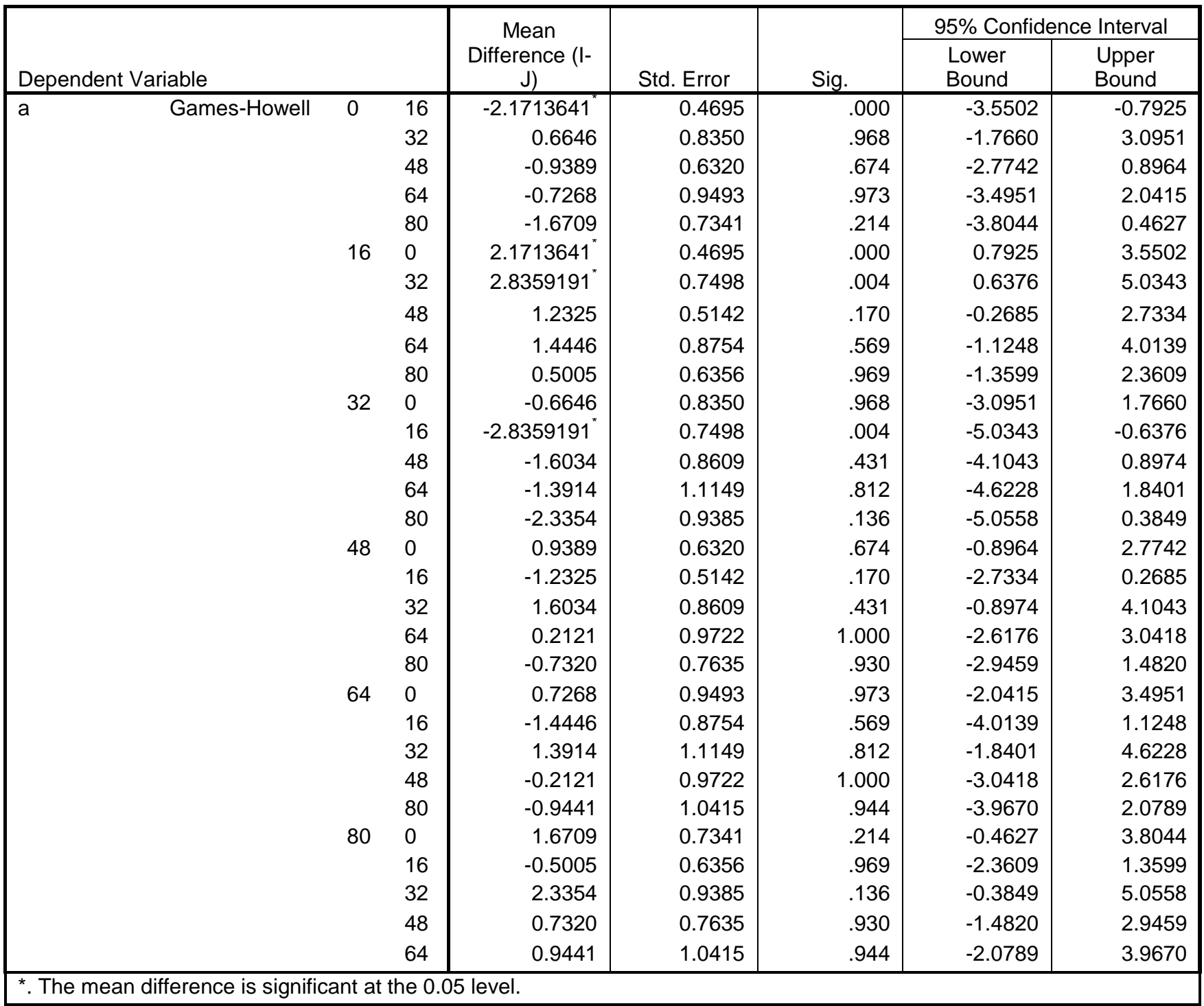

RMP-Variable ' $b$ '

Only four marked significant mean differences were observed at this variable. At some groups,

for example, at Group 64, no significant mean differences were observed. No one group was

distinctive than the other. With such low significant mean differences, it can be concluded that 
the aging treatment caused detectable, yet trace amounts of change in color saturation in the yellow/blue region.

Table 44: Games-Howell test of sample RMP at variable ' $b$ '

Multiple Comparisons: RMP

\begin{tabular}{|c|c|c|c|c|c|c|c|}
\hline \multirow{2}{*}{\multicolumn{3}{|c|}{ Dependent Variable }} & \multirow[b]{2}{*}{ Mean Difference (I-J) } & \multirow[b]{2}{*}{ Std. Error } & \multirow[b]{2}{*}{ Sig. } & \multicolumn{2}{|c|}{ 95\% Confidence Interval } \\
\hline & & & & & & $\begin{array}{l}\text { Lower } \\
\text { Bound }\end{array}$ & $\begin{array}{l}\text { Upper } \\
\text { Bound }\end{array}$ \\
\hline \multirow[t]{30}{*}{$b$} & Games- & 16 & -0.6269 & 0.3088 & .335 & -1.5299 & 0.2761 \\
\hline & Howell & 32 & 1.4819 & 0.6551 & .222 & -0.4311 & 3.3949 \\
\hline & & 48 & 0.5421 & 0.4290 & .804 & -0.7039 & 1.7881 \\
\hline & & 64 & -0.6096 & 0.6500 & .935 & -2.5074 & 1.2882 \\
\hline & & 80 & -1.3973 & 0.5775 & .161 & -3.0808 & 0.2862 \\
\hline & 16 & 0 & 0.6269 & 0.3088 & .335 & -0.2761 & 1.5299 \\
\hline & & 32 & $2.1088410^{*}$ & 0.6213 & .014 & 0.2865 & 3.9312 \\
\hline & & 48 & $1.1690590^{*}$ & 0.3752 & .029 & 0.0748 & 2.2633 \\
\hline & & 64 & 0.0173 & 0.6159 & 1.000 & -1.7890 & 1.8236 \\
\hline & & 80 & -0.7703 & 0.5388 & .709 & -2.3489 & 0.8082 \\
\hline & 32 & 0 & -1.4819 & 0.6551 & .222 & -3.3949 & 0.4311 \\
\hline & & 16 & $-2.1088410^{\pi}$ & 0.6213 & .014 & -3.9312 & -0.2865 \\
\hline & & 48 & -0.9398 & 0.6890 & .748 & -2.9446 & 1.0650 \\
\hline & & 64 & -2.0915 & 0.8445 & .140 & -4.5380 & 0.3550 \\
\hline & & 80 & $-2.8791803^{*}$ & 0.7901 & .005 & -5.1689 & -0.5894 \\
\hline & 48 & 0 & -0.5421 & 0.4290 & .804 & -1.7881 & 0.7039 \\
\hline & & 16 & -1.1690590 & 0.3752 & .029 & -2.2633 & -0.0748 \\
\hline & & 32 & 0.9398 & 0.6890 & .748 & -1.0650 & 2.9446 \\
\hline & & 64 & -1.1517 & 0.6841 & .546 & -3.1421 & 0.8386 \\
\hline & & 80 & $-1.9393983^{*}$ & 0.6156 & .025 & -3.7276 & -0.1512 \\
\hline & 64 & 0 & 0.6096 & 0.6500 & .935 & -1.2882 & 2.5074 \\
\hline & & 16 & -0.0173 & 0.6159 & 1.000 & -1.8236 & 1.7890 \\
\hline & & 32 & 2.0915 & 0.8445 & .140 & -0.3550 & 4.5380 \\
\hline & & 48 & 1.1517 & 0.6841 & .546 & -0.8386 & 3.1421 \\
\hline & & 80 & -0.7877 & 0.7858 & .916 & -3.0650 & 1.4897 \\
\hline & 80 & 0 & 1.3973 & 0.5775 & .161 & -0.2862 & 3.0808 \\
\hline & & 16 & 0.7703 & 0.5388 & .709 & -0.8082 & 2.3489 \\
\hline & & 32 & $2.8791803^{*}$ & 0.7901 & .005 & 0.5894 & 5.1689 \\
\hline & & 48 & $1.9393983^{*}$ & 0.6156 & .025 & 0.1512 & 3.7276 \\
\hline & & 64 & 0.7877 & 0.7858 & .916 & -1.4897 & 3.0650 \\
\hline
\end{tabular}

2) Post Hoc Comparisons of Lab grouped by 'Sample Area' 
Based on the previous ANOVA results, the pink and red colors observed similarities in mean values for variables ' $a$ ' and ' $b$ '. The post hoc tests were applied to acknowledge and locate the similarities. Both the Tukey and G-H test was applied depending upon the homogeneity of variance result. The results are discussed separately below by reference samples. The descriptives, and post hoc comparison results can be found in Section 7-11 in the appendix.

\section{$D P M P$}

No significant values were observed at the ' $L$ ' variable. Sample DPMP observed significant values at sample area A. As the areas are paired; only at area A was significant difference in means was observed. This was observed at both dependent variables. No further mean differences were observed to be significant.

\section{$P M C L$}

No significant values were observed at the ' $L$ ' variable. Mean significant difference was only observed between the pair-wise groups at sample area $\mathrm{B}^{*} \mathrm{C}$ for variable ' $\mathrm{a}$ '. This concludes that between these two areas, the mean differences are significant different. For variable 'b', marked significant mean differences were observed for any pair-wise groups that contained sample area C.

\section{PMJX}

No significant values were observed at the 'L' variable. At variable 'a', only pair-wise A*B group observed significant mean difference. It can be concluded that at these sample areas, the mean differences between the two groups are significantly different. At variable 'b', only pairwise $\mathrm{B} * \mathrm{C}$ group observed significant mean difference.

\section{$R M P$}


No significant values were observed at the 'L' variable. Significant values were observed at area $\mathrm{B}$ and $\mathrm{C}$ for all their pair-wise groups, for variable ' $\mathrm{a}$ ' and ' $\mathrm{b}$ ' respectively.

\subsection{Summary of Aged Results}

\section{Hours}

In summary, post hoc comparison tests were applied because these tests are able to determine which groups differ from each other based on the ANOVA results. The majority of the results were interpreted from the G-H test. The results of the G-H test shows that the treatment effect of aging influenced the mean values of the dependent variables. Through the aging study, there were limited recognizable patterns that would assist in understanding how fibers are affected UV exposure.

The variable mostly affected by this treatment was variable ' $a$ '. Thirty two marked significant differences were observed overall at this variable, 29 was observed for variable ' $b$ ' and 27 for variable ' $L$ '. For each sample, the affect varied among the variables. The five samples that are similar in hue produced different results: sample DPMP was equally affected at variables 'L' and 'a' suggesting that not only was the gloss of the sample affected, the red/green region was also affected. One marked difference was observed at ' $b$ ', suggesting that the yellow/blue region was not extensively affected by this treatment.

Samples PMCL and PMJX observed differences in how ' $L$ ' and 'b' variables were affected. PMCL observed six marked significant differences at both variables implying that both variables were equally affected by this treatment. Not only was the lightness of the sample affected, the red/green region was also affected. Variable 'b' observed four significant differences. Unlike 
sample PMCL, PMJX did not observe significant difference at variable 'L'. It can be concluded that the lightness of the sample was not affected by the treatment. Variable ' $b$ ' observed nine marked significant differences. This sample observed the greatest amount of change in the yellow/blue region. Therefore, at this region, the two samples can be differentiated.

Samples PMP and RMP which are adjacent on the CIELab color space, only observed similarities at the 'b' variable where both observed four marked differences. They were equally affected in the yellow/blue region. The opposite is true for the remaining variables; at variable ' $L$ ', seven and three significant differences were observed, and at variable ' $a$ ', five and two significant differences were observed for samples PMP and RMP, respectively. The aging treatment had a greater impact on sample PMP.

Sample GMP observed equal effects at variables ' $L$ ' and ' $a$ ' concluding that both the lightness of the sample and the red/green region of the color were affected by the treatment. Similar to sample DPMP, only one marked significant difference was observed at variable ' $b$ '.

Lastly, sample OSDP observed the greatest amount of change at variable ' $b$ ' where six marked differences was observed. Variable 'a', which had four marked differences, was also affected in the red/green region by the aging treatment. Variable ' $\mathrm{L}$ ', which only observed a single difference, was not extensively affected by this treatment.

It is understandable that the greatest amount of variation would occur at variable 'a' as most of the colors correspond to the red/green region of the color space. With this in mind, changes observed from the treatment could have been influenced by the variations in the chroma and hue of the colors being analyzed. 


\section{Sample Area}

Similarity was observed for all the samples at the ' $\mathrm{L}$ ' variable as the values were all insignificantly different. Upon further evaluation of the sample area, it can be estimated that at variable ' $a$ ', sample area B produced the greatest amount of significant differences in means among the samples. At variable ' $\mathrm{b}$ ', sample area $\mathrm{C}$ produced the greatest amount of significant differences in means among the samples. For this reason, even though sample area was used to evaluate inter-variability among the garments, the gloss of the sample was not affected by this variation. On the contrary, the red/green and yellow/blue regions are greatly affected by variations found within a garment.

6.5.1 Analysis of the Washed-Aged Results- Part two of this study focused on understanding the degradation of fibers through a process of washing and aging as one would expect of a garment that is worn and washed repeatedly.

\subsection{1 a Normality Testing of the Washed-Aged Samples}

For normal distribution determination, a chart producing a histogram, normal plot and a box and whisker plot was graphed. The two statistical tests, K-S Lillefors and S-W tests were employed for each variable to check normality. The data analysis was broken down into four separate steps because of the type of analysis that was performed on the samples. These steps were:

1) Normal distribution was calculated for the overall Lab variables;

2) Normal distribution was calculated of 'Hours' and 'Swatch' for each Lab value; 
3) Normal distribution was calculated of 'Hours', 'Swatch', and 'Sample Area' for each Lab value;

4) Normal distribution was calculated of 'Hours', 'Swatch', 'Sample Area' and 'Fiber' for each Lab value. (Since it is impossible to analyze the same fiber aged at different time interval, the analyzed fiber varied between hours aged);

1) Normal distribution with all groups against variables Lab.

A histogram, normal plot and box and whisker plot was plotted for each CIE value. Based on the calculated p-value, only reference sample OSDP observed normal distribution, however, only at variable 'L'. No other reference samples were observed to be normally distributed at this level of interaction. It is understandable that normal distribution may not occur at this level of testing.

2) Normal distribution of 'Hours' and 'Swatch' for each Lab value.

Six reference samples observed normal distribution at one to three places of the analysis.

Reference sample GMP observed two significant p-values: first wash and 16 hours aged, swatch 1 at variable ' $L$ '; fifth wash and 64 hours aged, swatch 1 at variable 'b'.

3) Normal distribution of 'Hours', 'Swatch' and 'Sample Area' for each Lab value.

On each swatch, two areas were separated and assigned as sample areas A and B. From these areas, different fibers were extracted and analyzed during the wash-aged cycle. In total, 108 charts were plotted with these results. From the results, only six reference samples were observed to be normally distribution. 


\section{4) Normal distribution of 'Hours', 'Swatch', 'Sample Area' and 'Fiber' for each Lab}

value.

In total, 69 fibers were analyzed over the washed and aged cycle. Since the same fiber cannot be washed and analyzed because of the difficulty in washing a single fiber, the same fiber was not studied between cycles. Similar to the aged results, normal distribution was observed for all the reference samples but not for all data measurements. Again, at this discrete level, a normal distribution should be expected because the sample set taken from a larger population (in this case, the sample area) should have a smaller variance and standard deviation than from the larger population. However, small discrepancies were still present; normal distribution was rejected for certain measurements when the analysis of the fiber was performed at two locations on that fiber. However, this was not always the case; some variables still observed a normal distribution while others did not.

Based on the results, it can be estimated that the fibers comes from a normally distributed sample although variation within the fibers exist and sometimes will affect this result.

\subsubsection{Multifactorial ANOVA of Washed-Aged Results}

Multifactorial ANOVA analysis was performed on the samples that were washed and aged during the experiment. The questions addressed in this part of the analysis were:

- Are there significant changes observed in the washed-aged process?

If so, what are these changes and where do they occur?

To answer these questions, two hypotheses were created: 
- $\mathrm{H}_{\mathrm{o}}: \mu_{1}=\mu_{2}=\mu_{3}=\ldots \mu_{\mathrm{k}}$ (no mean difference observed between population);

- $\mathrm{H}_{1}$ : at least one $\mu$ is different (at least two of the population means are different).

The analysis was broken down into nine steps:

1) The main effects of 'Sample Area' against the Lab variables;

2) The main effects of 'Swatch' against the Lab variables;

3) The main effects of 'Bleached' against the Lab variables;

4) The main effects of 'Washing' against the Lab variables;

5) The interaction effects of 'Sample Area * Swatch' against the Lab variables;

6) The interaction effects of 'Sample Area * Bleached' against the Lab variables;

7) The interaction effects of 'Washing * Bleached' against the Lab variables;

8) The interaction effects of 'Washing * Swatch' against the Lab variables;

The results were tabulated into four tables; the first table of the output, labeled Between-Subjects Factors, summarized the factors, showing how they were labeled and how many scores were in each group; the second table, labeled Test of Between-Subjects Effects, is a standard ANOVA table that includes an additional column for the sources of variations analyzed. This included the main and interaction effects of the factors chosen for analysis; the third output table generates specific under the heading Estimated Marginal Means. These provide the means of the main effect factors and the pair-wise comparisons for these effects separately, as well as the groups that were chosen to be examined for the interaction effects. In this project, 12 tables were 
generated; and the last item of the output was the interaction diagrams for each groups of interaction that were analyzed. As an option, the Descriptive Statistics table, analogous to the one produced in the one-way ANOVA method, was also generated and is located in the appendix. The results of these analyses will be independently discussed for specific reference samples of interest. The results and discussion of the remaining samples are located in the appendix.

\section{Sample DPMP}

\section{DPMP-Variable ' $L$ '}

This table summarizes the selected groups, the levels associated within each group or their between-subject factors, their corresponding labels and the total amount of values or scores in each factor. This table can be used as a reference for the samples as all the reference samples shared the same groups, between-subject factors, labels and scores.

Between-Subjects Factors: DPMP Variable L

\begin{tabular}{|c|c|c|c|}
\hline & & Value Label & $\mathrm{N}$ \\
\hline \multirow[t]{2}{*}{ Sample Area } & A & & 440 \\
\hline & B & & 440 \\
\hline \multirow[t]{4}{*}{ Swatch } & 1 & $1 \mathrm{w} /$ bleach & 220 \\
\hline & 2 & $2 \mathrm{w} /$ bleach & 220 \\
\hline & 3 & 3 w/o bleach & 220 \\
\hline & 4 & 4 w/o bleach & 220 \\
\hline \multirow[t]{2}{*}{ bleached } & 1 & w/ bleach & 440 \\
\hline & 2 & w/o bleach & 440 \\
\hline \multirow[t]{6}{*}{ washings } & 1 & $1 \mathrm{~W} 0 \mathrm{~A}$ & 80 \\
\hline & 2 & $1 \mathrm{~W} 16 \mathrm{~A}$ & 160 \\
\hline & 3 & $2 W 32 A$ & 160 \\
\hline & 4 & $3 W 48 A$ & 160 \\
\hline & 5 & $4 \mathrm{~W} 64 \mathrm{~A}$ & 160 \\
\hline & 6 & $5 \mathrm{~W} 80 \mathrm{~A}$ & 160 \\
\hline
\end{tabular}

In the Test of Between-Subject Effects table below, the first column identifies the sources being used for analysis. This includes the factors investigated for their main and interaction effects. 
The Corrected Model takes into account the summarizations of the sum of squares main effects and the sum of squares interaction effects. It accounts for the deviation of the cell means for each main effect plus any deviation present in the form of interactions. In other words, it corresponds to the within- between-group values. The Intercept is the grand mean of all the data squared and multiplied by the total observations. It tests the null hypothesis that the grand mean of all the data is equal to zero. A statistical significant intercept is when the grand mean of the data is not equal to zero. This term, although useful, will not be further explored as it is more meaningful when applied to advanced statistical models. There are four sources of main effects evaluated and 11 potential interaction effects, seven of which produces a result. These potential interactions have a value of 0 in columns 2 and 3, and no values in the remaining columns. This will be discussed further below.

Dependent Variable: L

Tests of Between-Subjects Effects: DPMP

\begin{tabular}{|c|c|c|c|c|c|}
\hline Source & $\begin{array}{l}\text { Type III Sum } \\
\text { of Squares }\end{array}$ & df & $\begin{array}{l}\text { Mean } \\
\text { Square }\end{array}$ & $\mathrm{F}$ & Sig. \\
\hline Corrected Model & $3353.870^{\mathrm{a}}$ & 43 & 77.997 & 7.235 & 0.000 \\
\hline Intercept & 6044243 & 1 & 6044243 & 560625.5 & 0.000 \\
\hline SampleArea & 0.006 & 1 & 0.006 & 0.001 & 0.982 \\
\hline Swatch & 241.432 & 2 & 120.716 & 11.197 & 0.000 \\
\hline Bleached & 0 & 0 & & & \\
\hline Washing & 693.601 & 5 & 138.72 & 12.867 & 0.000 \\
\hline SampleArea * Swatch & 202.673 & 2 & 101.337 & 9.399 & 0.000 \\
\hline SampleArea * Bleached & 0 & 0 & & & \\
\hline SampleArea * Washing & 185.185 & 4 & 46.296 & 4.294 & 0.002 \\
\hline Swatch * Bleached & 0 & 0 & & & \\
\hline Swatch * Washing & 612.796 & 8 & 76.6 & 7.105 & 0.000 \\
\hline Bleached * Washing & 0 & 0 & & & \\
\hline SampleArea * Swatch * Bleached & 0 & 0 & & & \\
\hline SampleArea * Swatch * Washing & 394.137 & 8 & 49.267 & 4.57 & 0.000 \\
\hline SampleArea * Bleached * Washing & 0 & 0 & & 1.01 & \\
\hline Swatch * Bleached * Washing & 0 & 0 & & . & \\
\hline SampleArea * Swatch * Bleached * & 0 & 0 & & . & \\
\hline Washing & & & & & \\
\hline Error & 9013.125 & 836 & 10.781 & & \\
\hline Total & 6458403 & 880 & & & \\
\hline Corrected Total & 12367 & 879 & & & \\
\hline
\end{tabular}

a. R Squared $=.271$ (Adjusted R Squared $=.234$ ) 
The Error noted in column 1 corresponds to the within-group values. The Total and the Corrected Total equals to sum of square values for the main effects, interaction effects and errors, and the summarization of the Corrected model and the Error, respectively. Also note that Type III Sum of Squares is calculated here. Type III gives the SS for each variable as if it was entered last in the model. The effect of each variable is evaluated after the previous factors have been accounted for, and is considered a partial SS. the SS is calculated for all the main and interaction effects being evaluated. The SS value for bleach was calculated as 0.000 . Upon observation of the main effects $p$-values, Sample Area was not significant at $p=.982$. In other words, there was no significant difference between sample areas A and B. This also suggests that the location of where the fiber was extracted from within the sample area is irrelevant. It is also clear that both the main effect of Swatch were significant at $p=0.001$, and that the four interaction effects were also significant at $\mathrm{p}=.002$.

\section{Sample Area Estimates: DPMP}

\begin{tabular}{|c|c|c|c|c|}
\hline \multirow[b]{2}{*}{$\begin{array}{l}\text { Sample } \\
\text { Area }\end{array}$} & \multirow[b]{2}{*}{ Mean } & \multirow[b]{2}{*}{$\begin{array}{l}\text { Std. } \\
\text { Error }\end{array}$} & \multicolumn{2}{|c|}{$\begin{array}{c}95 \% \text { Confidence } \\
\text { Interval }\end{array}$} \\
\hline & & & $\begin{array}{l}\text { Lower } \\
\text { Bound }\end{array}$ & $\begin{array}{l}\text { Upper } \\
\text { Bound }\end{array}$ \\
\hline$A$ & $85.656^{a}$ & 0.157 & 85.349 & 85.963 \\
\hline$B$ & $85.517^{a}$ & 0.157 & 85.21 & 85.824 \\
\hline
\end{tabular}

a. Based on modified population marginal mean.

This table shows the means for the levels within the sample area', together with their standard errors and the $95 \%$ confidence intervals. The main effects for this group did not yield significant results. The table below shows the interaction effects of this group. Again, it can be concluded that no significant differences was observed in this pair-wise comparing groups or in the main effects of this group. 
Dependent Variable:L

\begin{tabular}{|c|c|c|c|c|c|c|}
\hline \multicolumn{5}{|c|}{ Dependent Variable:L } & \multicolumn{2}{|c|}{$\begin{array}{l}95 \% \text { Confidence Interval } \\
\text { for Difference }\end{array}$} \\
\hline $\begin{array}{l}\text { (I) Sample } \\
\text { Area }\end{array}$ & $\begin{array}{l}\text { Sample } \\
\text { Area }\end{array}$ & $\begin{array}{l}\text { Difference } \\
(\mathrm{I}-\mathrm{J})\end{array}$ & Std. Error & Sig. ${ }^{\mathrm{C}}$ & $\begin{array}{l}\text { Lower } \\
\text { Bound }\end{array}$ & $\begin{array}{l}\text { Upper } \\
\text { Bound }\end{array}$ \\
\hline $\mathrm{A}$ & $\mathrm{B}$ & $.139^{\mathrm{a}, \mathrm{b}}$ & 0.221 & 0.53 & -0.296 & 0.573 \\
\hline$B$ & $\bar{A}$ & $-.139^{a, b}$ & 0.221 & 0.53 & -0.573 & 0.296 \\
\hline
\end{tabular}

Based on estimated marginal means

a. An estimate of the modified population marginal mean (I).

b. An estimate of the modified population marginal mean $(\mathrm{J})$.

c. Adjustment for multiple comparisons: Least Significant

Difference (equivalent to no adjustments).

\section{Swatch Estimates: DPMP}

Dependent Variable: L

\begin{tabular}{|c|c|c|c|c|}
\hline \multirow[b]{2}{*}{ Swatch } & \multirow[b]{2}{*}{ Mean } & \multirow[b]{2}{*}{ Std. Error } & \multicolumn{2}{|c|}{ 95\% Confidence Interval } \\
\hline & & & Lower Bound & Upper Bound \\
\hline $1 \mathrm{w} /$ bleach & $85.887^{a}$ & 0.221 & 85.453 & 86.322 \\
\hline $2 \mathrm{w} /$ bleach & $84.908^{\mathrm{a}}$ & 0.221 & 84.474 & 85.343 \\
\hline 3 w/o bleach & $85.475^{a}$ & 0.221 & 85.041 & 85.91 \\
\hline 4 w/o bleach & $86.076^{a}$ & 0.221 & 85.641 & 86.51 \\
\hline
\end{tabular}

a. Based on modified population marginal mean.

Dependent Variable: L

Pairwise Comparisons

\begin{tabular}{|c|c|c|c|c|c|c|}
\hline \multirow[b]{2}{*}{ (I) Swatch } & \multirow[b]{2}{*}{ (J) Swatch } & \multirow{2}{*}{$\begin{array}{c}\text { Mean } \\
\text { Difference (I- } \\
\mathrm{J})\end{array}$} & \multirow[b]{2}{*}{ Std. Error } & \multirow[b]{2}{*}{ Sig. $^{c}$} & \multicolumn{2}{|c|}{$\begin{array}{l}95 \% \text { Confidence Interval } \\
\text { for Difference }{ }^{c}\end{array}$} \\
\hline & & & & & $\begin{array}{l}\text { Lower } \\
\text { Bound }\end{array}$ & Upper Bound \\
\hline \multirow[t]{3}{*}{$1 \mathrm{w} /$ bleach } & $2 \mathrm{w} /$ bleach & $.979^{\circ, \mathrm{a}, \mathrm{b}}$ & 0.313 & 0.002 & 0.365 & 1.594 \\
\hline & 3 w/o bleach & $.412^{\mathrm{a}, \mathrm{b}}$ & 0.313 & 0.188 & -0.202 & 1.027 \\
\hline & 4 w/o bleach & $-.188^{a, b}$ & 0.313 & 0.547 & -0.803 & 0.426 \\
\hline \multirow[t]{3}{*}{$2 \mathrm{w} /$ bleach } & $1 \mathrm{w} /$ bleach & $-.979^{*, a, b}$ & 0.313 & 0.002 & -1.594 & -0.365 \\
\hline & 3 w/o bleach & $-.567^{a, b}$ & 0.313 & 0.07 & -1.182 & 0.047 \\
\hline & 4 w/o bleach & $-1.168^{\circ, a, b}$ & 0.313 & 0.00 & -1.782 & -0.553 \\
\hline \multirow[t]{3}{*}{3 w/o bleach } & $1 \mathrm{w} /$ bleach & $-.412^{a, b}$ & 0.313 & 0.188 & -1.027 & 0.202 \\
\hline & $2 \mathrm{w} /$ bleach & $.567^{\mathrm{a}, \mathrm{b}}$ & 0.313 & 0.07 & -0.047 & 1.182 \\
\hline & 4 w/o bleach & $-.600^{\mathrm{a}, \mathrm{b}}$ & 0.313 & 0.055 & -1.215 & 0.014 \\
\hline \multirow[t]{3}{*}{4 w/o bleach } & $1 \mathrm{w} /$ bleach & $.188^{\mathrm{a}, \mathrm{b}}$ & 0.313 & 0.547 & -0.426 & 0.803 \\
\hline & 2 w/ bleach & $1.168^{\circ, a, b}$ & 0.313 & 0.000 & 0.553 & 1.782 \\
\hline & 3 w/o bleach & $.600^{\mathrm{a}, \mathrm{b}}$ & 0.313 & 0.055 & -0.014 & 1.215 \\
\hline
\end{tabular}

Based on estimated marginal means

*. The mean difference is significant at the .05 level.

a. An estimate of the modified population marginal mean (I).

b. An estimate of the modified population marginal mean (J).

c. Adjustment for multiple comparisons: Least Significant Difference (equivalent to no adjustments). 
The first table above shows the means for the 'w/ bleach' and 'w/o bleach' swatches factors, together with their standard errors and the $95 \%$ confidence intervals. The second table shows the pair-wise comparison between the groups and their significance values. Swatch 1 was observed to be significant with swatch 2 , but not with swatches 3 and 4 . This is surprising results as one would expect insignificant values to be observed with swatch 2, since both swatches were washed with bleach. A significant difference was expected in the cases where the detergent was consistent between the swatches. Swatch 2, on the other hand, observed a significant difference with swatch 4 , but not with swatch 3 . Swatch 3 was the only group that did not produce any significant values with the remaining swatches. The results do not produce a recognizable pattern to distinguish whether the detergent used would affect the outcome of the results differently.

\section{Bleach Estimates: DPMP}

Dependent Variable: L

\begin{tabular}{|l|r|r|r|r|}
\hline \multirow{2}{*}{ Bleached } & & \multicolumn{2}{|c|}{$95 \%$ Confidence Interval } \\
\cline { 3 - 5 } & Mean & Std. Error & \multicolumn{1}{|c|}{$\begin{array}{l}\text { Lower } \\
\text { Bound }\end{array}$} & $\begin{array}{c}\text { Upper } \\
\text { Bound }\end{array}$ \\
\hline w/ bleach & $85.398^{\mathrm{a}}$ & 0.157 & 85.09 & 85.705 \\
w/o bleach & $85.775^{\mathrm{a}}$ & 0.157 & 85.468 & 86.083 \\
\hline
\end{tabular}

a. Based on modified population marginal mean.

Pairwise Comparisons: DPMP

Dependent Variable: L

\begin{tabular}{|c|c|c|c|c|c|c|}
\hline \multirow[b]{2}{*}{ (I) Bleached } & \multirow[b]{2}{*}{ (J) Bleached } & \multirow[b]{2}{*}{$\begin{array}{c}\text { Mean } \\
\text { Difference (I-J) }\end{array}$} & \multirow[b]{2}{*}{$\begin{array}{l}\text { Std. } \\
\text { Error }\end{array}$} & \multirow[b]{2}{*}{ Sig. ${ }^{\mathrm{C}}$} & \multicolumn{2}{|c|}{$\begin{array}{l}95 \% \text { Confidence Interval } \\
\text { for Difference }\end{array}$} \\
\hline & & & & & $\begin{array}{l}\text { Lower } \\
\text { Bound }\end{array}$ & $\begin{array}{l}\text { Upper } \\
\text { Bound }\end{array}$ \\
\hline w/ bleach & w/o bleach & $-.378^{a, b}$ & 0.221 & 0.088 & -0.812 & 0.057 \\
\hline w/o bleach & w/ bleach & $.378^{\mathrm{a}, \mathrm{b}}$ & 0.221 & 0.088 & -0.057 & 0.812 \\
\hline
\end{tabular}

Based on estimated marginal means

a. An estimate of the modified population marginal mean (I).

b. An estimate of the modified population marginal mean $(\mathrm{J})$.

c. Adjustment for multiple comparisons: Least Significant Difference (equivalent to no adjustments).

The first table above shows the means for the 'w/ bleach' and 'w/o bleach' factors, together with their standard errors and the $95 \%$ confidence intervals. The second table shows the pair-wise comparison between the groups and their significance values. It is clear that the groups being 
investigated do not significantly differ from each other. So, whether the samples were washed with or without bleach, are not significant in affecting the ' $L$ ' variable.

The first table below shows the means for the 'Washing' factors, together with their standard errors and the $95 \%$ confidence intervals. The second table provides the pair-wise comparisons for this group. The main effects for this group observed significant values at some levels of interaction. Four marked significant values occurred mostly at levels $1 \mathrm{~W} 16 \mathrm{~A}$ and again at 5W 80A. At $1 \mathrm{~W} 0 \mathrm{~A}$, significant values were seen against the next four cycles. The significant interaction values between the two levels convey that the levels changed and that the means explains the nature of this difference.

\section{Washing Estimates: DPMP}

Dependent Variable: $\mathrm{L}$

\begin{tabular}{|c|c|c|c|c|}
\hline \multirow[b]{2}{*}{ Washing } & \multirow[b]{2}{*}{ Mean } & \multirow[b]{2}{*}{ Std. Error } & \multicolumn{2}{|c|}{ 95\% Confidence Interval } \\
\hline & & & $\begin{array}{l}\text { Lower } \\
\text { Bound }\end{array}$ & $\begin{array}{l}\text { Upper } \\
\text { Bound }\end{array}$ \\
\hline $1 \mathrm{~W} 0 \mathrm{~A}$ & $85.119^{a}$ & 0.367 & 84.398 & 85.84 \\
\hline $1 \mathrm{~W} 16 \mathrm{~A}$ & $86.946^{a}$ & 0.26 & 86.436 & 87.455 \\
\hline $2 W 32 A$ & $84.440^{\mathrm{a}}$ & 0.26 & 83.93 & 84.949 \\
\hline $3 W 48 A$ & $85.383^{a}$ & 0.26 & 84.874 & 85.893 \\
\hline $4 \mathrm{~W} 64 \mathrm{~A}$ & $84.958^{a}$ & 0.26 & 84.449 & 85.468 \\
\hline $5 W 80 A$ & $86.439^{a}$ & 0.26 & 85.929 & 86.948 \\
\hline
\end{tabular}

a. Based on modified population marginal mean. 
Pairwise Comparisons: DPMP

Dependent Variable: L

\begin{tabular}{|c|c|c|c|c|c|c|}
\hline \multirow{2}{*}{ (I) Washing } & \multirow[b]{2}{*}{ (J) Washing } & \multirow{2}{*}{$\begin{array}{c}\text { Mean } \\
\text { Difference (I- } \\
\text { J) }\end{array}$} & \multirow[b]{2}{*}{ Std. Error } & \multirow[b]{2}{*}{ Sig. ${ }^{\mathrm{c}}$} & \multicolumn{2}{|c|}{$\begin{array}{c}95 \% \text { Confidence Interval for } \\
\text { Difference }^{c}\end{array}$} \\
\hline & & & & & $\begin{array}{l}\text { Lower } \\
\text { Bound }\end{array}$ & $\begin{array}{l}\text { Upper } \\
\text { Bound } \\
\end{array}$ \\
\hline \multirow[t]{5}{*}{$1 \mathrm{~W} 0 \mathrm{~A}$} & $1 \mathrm{~W} 16 \mathrm{~A}$ & $-1.827^{, a, b}$ & 0.45 & 0.000 & -2.709 & -0.944 \\
\hline & $2 W 32 A$ & $.679^{\mathrm{a}, \mathrm{b}}$ & 0.45 & 0.131 & -0.203 & 1.562 \\
\hline & $3 W 48 A$ & $-.264^{\mathrm{a}, \mathrm{b}}$ & 0.45 & 0.557 & -1.147 & 0.618 \\
\hline & $4 \mathrm{~W} 64 \mathrm{~A}$ & $.160^{\mathrm{a}, \mathrm{b}}$ & 0.45 & 0.721 & -0.722 & 1.043 \\
\hline & $5 W 80 A$ & $-1.320^{*}, a, b$ & 0.45 & 0.003 & -2.202 & -0.437 \\
\hline \multirow[t]{5}{*}{$1 \mathrm{~W} 16 \mathrm{~A}$} & $1 \mathrm{~W} 0 \mathrm{~A}$ & $1.827^{, \mathrm{a}, \mathrm{b}}$ & 0.45 & 0.000 & 0.944 & 2.709 \\
\hline & $2 \mathrm{~W} 32 \mathrm{~A}$ & $2.506^{\star, a, b}$ & 0.367 & 0.000 & 1.786 & 3.227 \\
\hline & $3 W 48 A$ & $1.563^{\pi, a, b}$ & 0.367 & 0.000 & 0.842 & 2.283 \\
\hline & $4 \mathrm{~W} 64 \mathrm{~A}$ & $1.987^{*}, a, b$ & 0.367 & 0.000 & 1.267 & 2.708 \\
\hline & $5 W 80 A$ & $.507^{\mathrm{a}, \mathrm{b}}$ & 0.367 & 0.167 & -0.213 & 1.228 \\
\hline \multirow[t]{5}{*}{$2 \mathrm{~W} 32 \mathrm{~A}$} & $1 \mathrm{~W} \mathrm{OA}$ & $-.679^{\mathrm{a}, \mathrm{b}}$ & 0.45 & 0.131 & -1.562 & 0.203 \\
\hline & $1 \mathrm{~W} 16 \mathrm{~A}$ & $-2.506^{x, a, b}$ & 0.367 & 0.000 & -3.227 & -1.786 \\
\hline & $3 W 48 A$ & $-.943^{x, a, b}$ & 0.367 & 0.010 & -1.664 & -0.223 \\
\hline & $4 \mathrm{~W} 64 \mathrm{~A}$ & $-.519^{a, b}$ & 0.367 & 0.158 & -1.239 & 0.202 \\
\hline & $5 W 80 A$ & $-1.999^{*}, \mathrm{a}, \mathrm{b}$ & 0.367 & 0.000 & -2.719 & -1.278 \\
\hline \multirow[t]{5}{*}{$3 \mathrm{~W} 48 \mathrm{~A}$} & $1 \mathrm{~W} 0 \mathrm{~A}$ & $.264^{\mathrm{a}, \mathrm{b}}$ & 0.45 & 0.557 & -0.618 & 1.147 \\
\hline & $1 \mathrm{~W} 16 \mathrm{~A}$ & $-1.563^{\star, a, b}$ & 0.367 & 0.000 & -2.283 & -0.842 \\
\hline & $2 W 32 A$ & $.943^{\star, a, b}$ & 0.367 & 0.010 & 0.223 & 1.664 \\
\hline & $4 \mathrm{~W} 64 \mathrm{~A}$ & $.425^{\mathrm{a}, \mathrm{b}}$ & 0.367 & 0.248 & -0.296 & 1.145 \\
\hline & $5 W 80 A$ & $-1.056^{x, a, b}$ & 0.367 & 0.004 & -1.776 & -0.335 \\
\hline \multirow[t]{5}{*}{$4 \mathrm{~W} 64 \mathrm{~A}$} & $1 \mathrm{~W} 0 \mathrm{~A}$ & $-.160^{\mathrm{a}, \mathrm{b}}$ & 0.45 & 0.721 & -1.043 & 0.722 \\
\hline & $1 \mathrm{~W} 16 \mathrm{~A}$ & $-1.987^{*, a, b}$ & 0.367 & 0.000 & -2.708 & -1.267 \\
\hline & $2 W 32 A$ & $.519^{\mathrm{a}, \mathrm{b}}$ & 0.367 & 0.158 & -0.202 & 1.239 \\
\hline & $3 W 48 A$ & $-.425^{\mathrm{a}, \mathrm{b}}$ & 0.367 & 0.248 & -1.145 & 0.296 \\
\hline & $5 W 80 A$ & $-1.480^{\circ}, a, b$ & 0.367 & 0.000 & -2.201 & -0.76 \\
\hline \multirow[t]{5}{*}{$5 \mathrm{~W} 80 \mathrm{~A}$} & $1 \mathrm{~W} 0 \mathrm{~A}$ & $1.320^{\circ, a, b}$ & 0.45 & 0.003 & 0.437 & 2.202 \\
\hline & $1 \mathrm{~W} 16 \mathrm{~A}$ & $-.507^{\mathrm{a}, \mathrm{b}}$ & 0.367 & 0.167 & -1.228 & 0.213 \\
\hline & $2 W 32 A$ & $1.999^{*}, \mathrm{a}, \mathrm{b}$ & 0.367 & 0.000 & 1.278 & 2.719 \\
\hline & $3 W 48 A$ & $1.056^{\pi, a, b}$ & 0.367 & 0.004 & 0.335 & 1.776 \\
\hline & $4 \mathrm{~W} 64 \mathrm{~A}$ & $1.480^{*}, \mathrm{a}, \mathrm{b}$ & 0.367 & 0.000 & 0.76 & 2.201 \\
\hline
\end{tabular}

Based on estimated marginal means

*. The mean difference is significant at the .05 level.

a. An estimate of the modified population marginal mean (I).

b. An estimate of the modified population marginal mean $(\mathrm{J})$.

c. Adjustment for multiple comparisons: Least Significant Difference (equivalent to no adjustments). 


\section{Sample Area * Swatch: DPMP}

Dependent Variable: $\mathrm{L}$

\begin{tabular}{|ll|r|r|r|r|}
\hline \multirow{2}{*}{ Sample Area } & Swatch & & \multicolumn{2}{|c|}{ 95\% Confidence Interval } \\
\cline { 4 - 6 } & Mean & Std. Error & Lower Bound & Upper Bound \\
\hline A & 1 w/ bleach & $85.368^{\mathrm{a}}$ & 0.3 & 84.78 & 85.956 \\
& $2 \mathrm{w} /$ bleach & $85.824^{\mathrm{a}}$ & 0.328 & 85.18 & 86.469 \\
& 3 w/o bleach & $85.546^{\mathrm{a}}$ & 0.3 & 84.958 & 86.134 \\
& $4 \mathrm{w} / \mathrm{o}$ bleach & $85.965^{\mathrm{a}}$ & 0.328 & 85.32 & 86.609 \\
\hline B & 1 w/ bleach & $86.510^{\mathrm{a}}$ & 0.328 & 85.865 & 87.154 \\
& $2 \mathrm{w} /$ bleach & $84.144^{\mathrm{a}}$ & 0.3 & 83.556 & 84.733 \\
& 3 w/o bleach & $85.390^{\mathrm{a}}$ & 0.328 & 84.745 & 86.034 \\
& $4 \mathrm{w} / \mathrm{o}$ bleach & $86.168^{\mathrm{a}}$ & 0.3 & 85.58 & 86.756 \\
\hline
\end{tabular}

a. Based on modified population marginal mean.

This table shows the interaction that was examined between the sample area and the swatch. The interaction between sample area and swatch yielded a significant result suggesting that the interaction effects of these two groups are significant. Although, the main effects for the swatches were not significant, a significant interaction is observed here. The effect of all levels in the group 'Sample Area' was not different, whereas, the effect for group 'Swatch' was. It can be concluded that interaction takes places between these groups as their means do not overlap or are similar. In fact, in sample area $B$, the mean values of ' $L$ ' are clearly different, although this difference might not a significant one.

Below is the interaction plot of these interactions. Although the main effects of the sample area did not produce significant results, the interaction between sample area and swatch did. At this point, it can be assumed that changes in both variables must have an effect on the outcome, regardless of the main effect p-values. The null hypothesis for the interaction is that there is no relationship between the two variables in their effects on the outcome. The alternative hypothesis then is that there is a 
relationship between the two variables that affects the outcome. Once the p-value is significant, then there is evidence of significant interactions, and not what would be due to random chance or error.

Figure 46: Interaction Diagram of 'Swatch * Sample Area' for sample DPMP

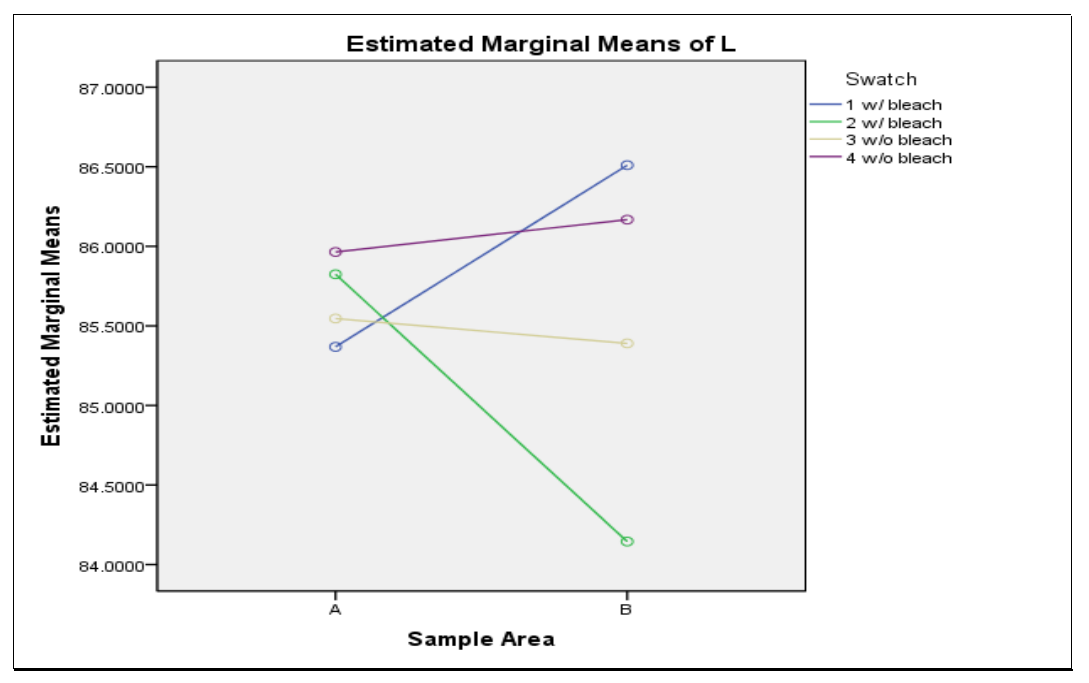

The points on this plot show the estimated population means. The lines in the interaction diagram connect the means of each pair of treatment. When the lines are parallel, suggests that no interaction takes place. The opposite is not true but very likely to be significant when the lines intersect. As soon as the lines depart from being parallel, there is indeed and interaction, but not necessarily a significant one. In this graph, only two parallel lines are observed: at swatches 3 and 4 . Although these lines slightly deviate from being parallel, it can be concluded with the p-values that there are no significant interactions between these groups. Three intersecting lines are also observed. Swatch 1 intersects with 4 , however the results are not produced to be significant with a p-value of 0.055 . Swatch 1 , also intersects with swatches 2 and 3, but only a significant result was observed within this interaction at swatch 2 . And swatch 2 
and 3, which intersected each other, also observes a significant p-value.

\section{Sample Area * Bleached: DPMP}

Dependent Variable: $\mathrm{L}$

\begin{tabular}{|c|c|c|c|c|c|}
\hline \multirow[b]{2}{*}{ Sample Area } & \multirow[b]{2}{*}{ Bleached } & \multirow[b]{2}{*}{ Mean } & \multirow[b]{2}{*}{ Std. Error } & \multicolumn{2}{|c|}{$95 \%$ Confidence Interval } \\
\hline & & & & Lower Bound & Upper Bound \\
\hline \multirow[t]{2}{*}{$A$} & w/ bleach & $85.575^{\mathrm{a}}$ & 0.221 & 85.141 & 86.01 \\
\hline & w/o bleach & $85.736^{\mathrm{a}}$ & 0.221 & 85.302 & 86.171 \\
\hline \multirow[t]{2}{*}{$B$} & w/ bleach & $85.220^{\mathrm{a}}$ & 0.221 & 84.785 & 85.654 \\
\hline & w/o bleach & $85.814^{\mathrm{a}}$ & 0.221 & 85.38 & 86.249 \\
\hline
\end{tabular}

a. Based on modified population marginal mean.

Figure 47: Interaction Diagram of 'Sample Area' * Bleached for sample DPMP

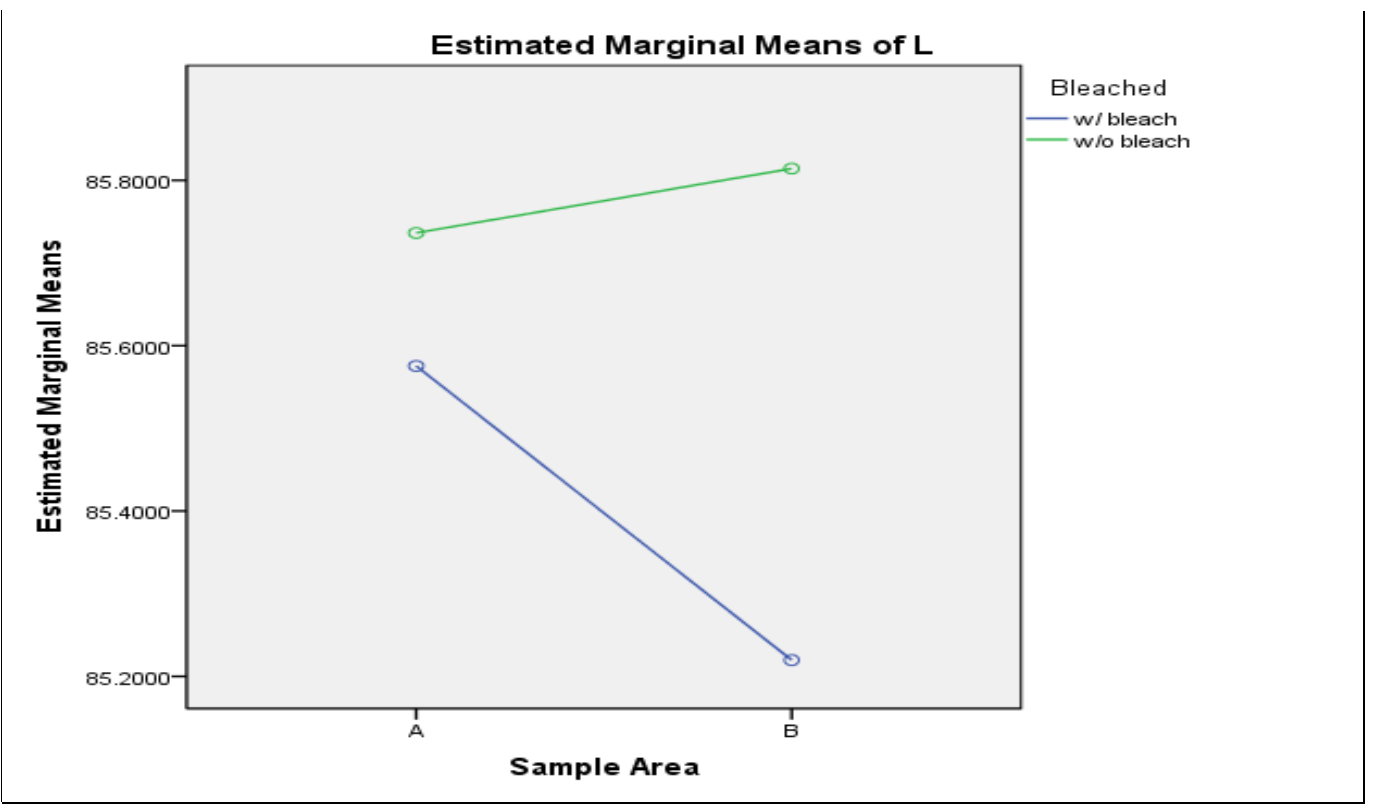

Based on the interaction plot, the mean values differ greatly at area B. 


\section{Bleached * Washing: DPMP}

Dependent Variable: L

\begin{tabular}{|cl|r|r|r|r|}
\hline & & & & \multicolumn{2}{|c|}{$95 \%$ Confidence Interval } \\
\cline { 5 - 6 } Bleached & Washing & Mean & Std. Error & \multicolumn{1}{c|}{$\begin{array}{c}\text { Lower } \\
\text { Bound }\end{array}$} & $\begin{array}{r}\text { Upper } \\
\text { Bound }\end{array}$ \\
\hline w/ bleach & 1W 0A & $84.713^{\mathrm{a}}$ & 0.519 & 83.694 & 85.732 \\
& 1W 16A & $87.342^{\mathrm{a}}$ & 0.367 & 86.622 & 88.063 \\
& 2W 32A & $85.396^{\mathrm{a}}$ & 0.367 & 84.676 & 86.117 \\
& 3W 48A & $84.501^{\mathrm{a}}$ & 0.367 & 83.781 & 85.222 \\
& 4W 64A & $83.754^{\mathrm{a}}$ & 0.367 & 83.033 & 84.474 \\
& 5W 80A & $86.337^{\mathrm{a}}$ & 0.367 & 85.616 & 87.057 \\
\hline w/o bleach & 1W 0A & $85.525^{\mathrm{a}}$ & 0.519 & 84.506 & 86.544 \\
& 1W 16A & $86.549^{\mathrm{a}}$ & 0.367 & 85.829 & 87.27 \\
& 2W 32A & $83.483^{\mathrm{a}}$ & 0.367 & 82.763 & 84.204 \\
& 3W 48A & $86.265^{\mathrm{a}}$ & 0.367 & 85.545 & 86.986 \\
& 4W 64A & $86.163^{\mathrm{a}}$ & 0.367 & 85.443 & 86.884 \\
& 5W 80A & $86.541^{\mathrm{a}}$ & 0.367 & 85.82 & 87.261 \\
\hline
\end{tabular}

a. Based on modified population marginal mean.

The table above shows the pair-wise comparisons for these two groups.

Figure 48: Interaction Diagram of 'Bleached * 'Washing' for sample DPMP

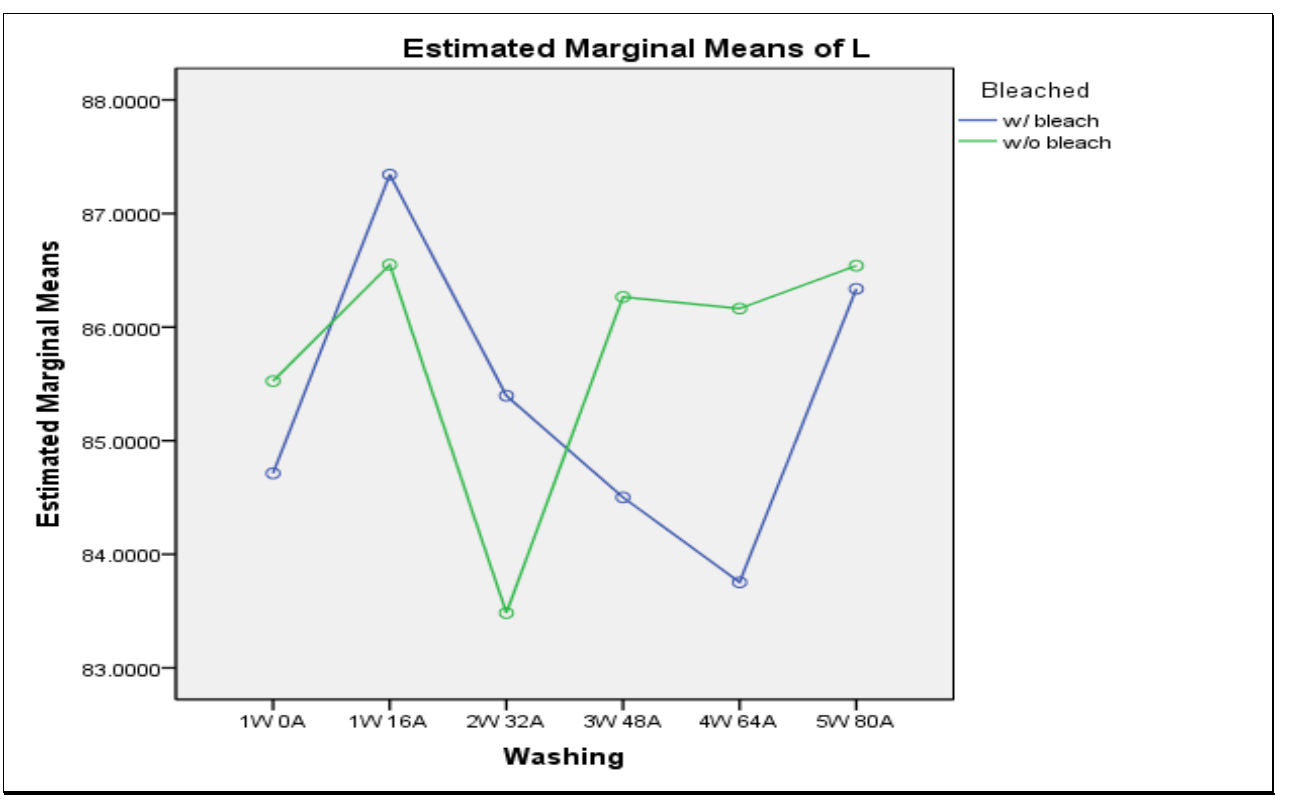

This chart shows interaction between these two groups. No further interpretation can be deferred from this chart without corresponding values to help confirm the results.

\section{Swatch * Washing: DPMP}


Dependent Variable: L

\begin{tabular}{|c|c|c|c|c|c|}
\hline \multirow[b]{2}{*}{ Swatch } & \multirow[b]{2}{*}{ Washing } & \multirow[b]{2}{*}{ Mean } & \multirow[b]{2}{*}{ Std. Error } & \multicolumn{2}{|c|}{ 95\% Confidence Interval } \\
\hline & & & & $\begin{array}{l}\text { Lower } \\
\text { Bound }\end{array}$ & $\begin{array}{l}\text { Upper } \\
\text { Bound }\end{array}$ \\
\hline \multirow{6}{*}{$\begin{array}{l}1 \mathrm{w} / \\
\text { bleach }\end{array}$} & $1 \mathrm{~W} 0 \mathrm{~A}$ & $84.360^{\mathrm{a}}$ & 0.734 & 82.919 & 85.801 \\
\hline & $1 \mathrm{~W} 16 \mathrm{~A}$ & $88.431^{a}$ & 0.519 & 87.412 & 89.45 \\
\hline & $2 W 32 A$ & $86.924^{a}$ & 0.519 & 85.905 & 87.943 \\
\hline & $3 W 48 A$ & $83.319^{a}$ & 0.519 & 82.3 & 84.338 \\
\hline & $4 \mathrm{~W} 64 \mathrm{~A}$ & $84.503^{a}$ & 0.519 & 83.484 & 85.522 \\
\hline & $5 W$ 80A & $87.022^{a}$ & 0.519 & 86.003 & 88.041 \\
\hline \multirow{6}{*}{$\begin{array}{l}2 \mathrm{w} / \\
\text { bleach }\end{array}$} & $1 \mathrm{~W} 0 \mathrm{~A}$ & $85.066^{a}$ & 0.734 & 83.625 & 86.507 \\
\hline & $1 \mathrm{~W} 16 \mathrm{~A}$ & $86.254^{a}$ & 0.519 & 85.235 & 87.273 \\
\hline & $2 W 32 A$ & $83.869^{a}$ & 0.519 & 82.85 & 84.888 \\
\hline & $3 W 48 A$ & $85.683^{a}$ & 0.519 & 84.664 & 86.702 \\
\hline & $4 \mathrm{~W} 64 \mathrm{~A}$ & $83.004^{a}$ & 0.519 & 81.985 & 84.023 \\
\hline & $5 W 80 A$ & $85.651^{a}$ & 0.519 & 84.632 & 86.67 \\
\hline \multirow{6}{*}{$\begin{array}{l}3 \mathrm{w} / 0 \\
\text { bleach }\end{array}$} & $1 \mathrm{~W} 0 \mathrm{~A}$ & $87.461^{a}$ & 0.734 & 86.019 & 88.902 \\
\hline & $1 \mathrm{~W} 16 \mathrm{~A}$ & $85.512^{a}$ & 0.519 & 84.493 & 86.531 \\
\hline & $2 W 32 A$ & $82.801^{a}$ & 0.519 & 81.782 & 83.82 \\
\hline & $3 W 48 A$ & $85.761^{a}$ & 0.519 & 84.742 & 86.78 \\
\hline & $4 W 64 A$ & $84.770^{a}$ & 0.519 & 83.751 & 85.789 \\
\hline & $5 \mathrm{~W} 80 \mathrm{~A}$ & $87.539^{a}$ & 0.519 & 86.52 & 88.558 \\
\hline \multirow{6}{*}{$\begin{array}{l}4 \mathrm{w} / 0 \\
\text { bleach }\end{array}$} & $1 \mathrm{~W} 0 \mathrm{~A}$ & $83.590^{a}$ & 0.734 & 82.149 & 85.031 \\
\hline & $1 \mathrm{~W} 16 \mathrm{~A}$ & $87.587^{a}$ & 0.519 & 86.568 & 88.606 \\
\hline & $2 W 32 A$ & $84.166^{a}$ & 0.519 & 83.147 & 85.185 \\
\hline & $3 W 48 A$ & $86.769^{a}$ & 0.519 & 85.75 & 87.788 \\
\hline & $4 W 64 A$ & $87.556^{a}$ & 0.519 & 86.537 & 88.575 \\
\hline & $5 W$ & $85.543^{a}$ & 0.519 & 84.524 & 86.562 \\
\hline
\end{tabular}

a. Based on modified population marginal mean.

Figure 49: Interaction Diagram of 'Swatch * Washing' for sample DPMP

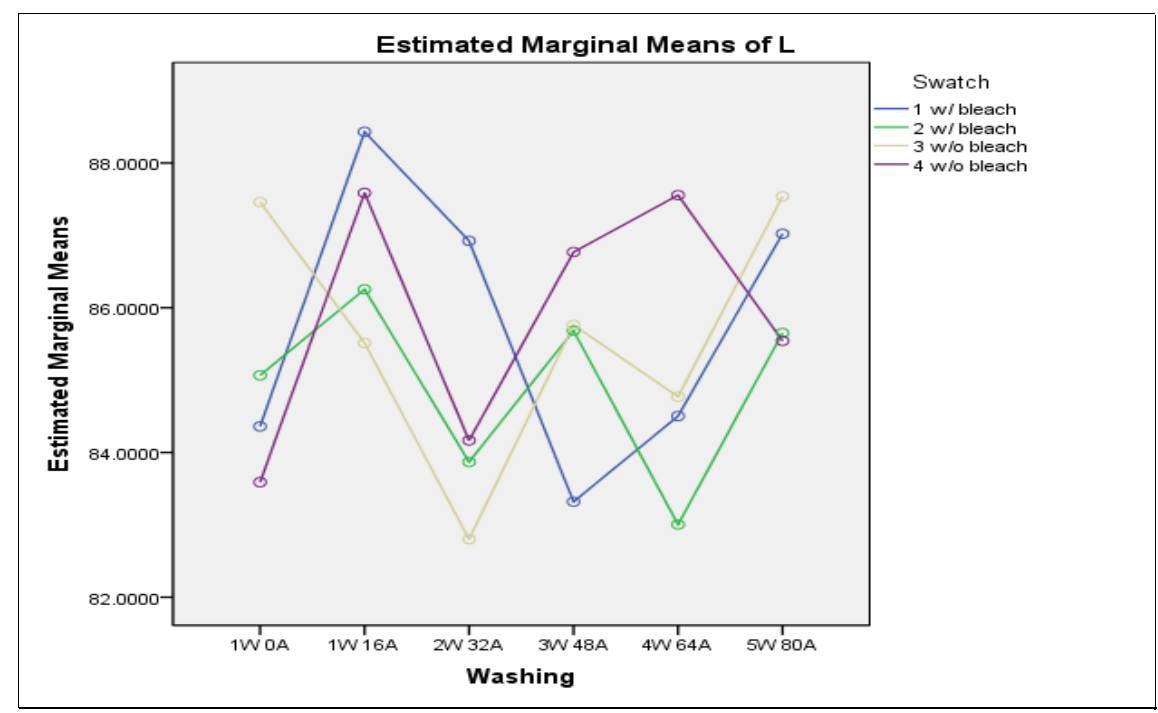


The table above shows the pair-wise comparisons for these two groups. The interaction effects for these two groups seem to be substantial. The overall patterns for swatch 1, 2 and 4 started out consistently, but after the third washing, this pattern started to deteriorate. Swatches 1 and 2 continued to have a similar pattern as their mean values decreased, while, swatch 4 increased in mean values. Swatch 3 started to follow this pattern after the first washed-aged cycle. This difference in means between groups could have created the significant interaction effects that are observed for these groups.

\section{DPMP-Variable ' $a$ '}

The same analysis was performed for variables ' $a$ ' and ' $b$ '. These variables will not be discussed in such great details as was done for variable ' $L$ ' as half of the discussion referred to the understanding and interpretation of the tables and graphs.

Dependent Variable: a

Tests of Between-Subjects Effects: DPMP

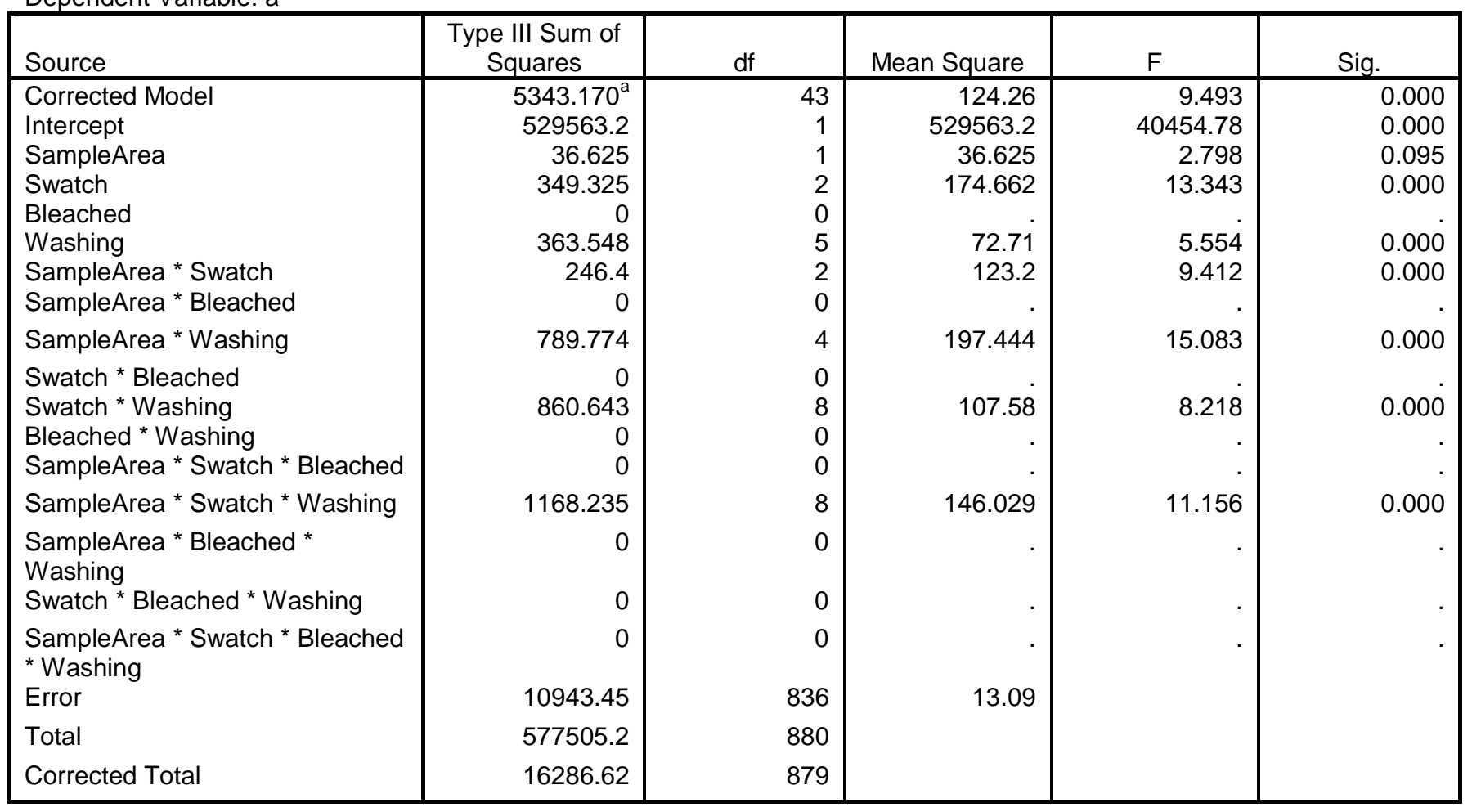


a. $\mathrm{R}$ Squared $=.328$ (Adjusted R Squared $=.294)$

The main effects of Swatch and Washing observed significant values, whereas Sample Area did not. The estimated marginal means and pair-wise comparison tables of sample area can be found in Section 15 of the appendix.

\section{Swatch Estimates: DPMP}

Dependent Variable: a

\begin{tabular}{|l|r|r|r|r|}
\hline \multirow{2}{*}{ Swatch } & & \multicolumn{2}{|c|}{$95 \%$ Confidence Interval } \\
\cline { 4 - 5 } & Mean & Std. Error & $\begin{array}{c}\text { Lower } \\
\text { Bound }\end{array}$ & $\begin{array}{c}\text { Upper } \\
\text { Bound }\end{array}$ \\
\hline $1 \mathrm{w} /$ bleach & $26.438^{\mathrm{a}}$ & 0.244 & 25.959 & 26.917 \\
$2 \mathrm{w} /$ bleach & $24.504^{\mathrm{a}}$ & 0.244 & 24.025 & 24.983 \\
3 w/o bleach & $24.733^{\mathrm{a}}$ & 0.244 & 24.255 & 25.212 \\
4 w/o bleach & $25.339^{\mathrm{a}}$ & 0.244 & 24.86 & 25.818 \\
\hline
\end{tabular}

a. Based on modified population marginal mean.

\section{Pairwise Comparisons: DPMP}

Dependent Variable: a

\begin{tabular}{|c|c|c|c|c|c|c|}
\hline \multirow[b]{2}{*}{ (I) Swatch } & \multirow[b]{2}{*}{ (J) Swatch } & \multirow{2}{*}{$\begin{array}{c}\text { Mean } \\
\text { Difference } \\
(I-J)\end{array}$} & \multirow[b]{2}{*}{ Std. Error } & \multirow[b]{2}{*}{ Sig. ${ }^{c}$} & \multicolumn{2}{|c|}{$\begin{array}{c}\text { 95\% Confidence } \\
\text { Interval for Difference }\end{array}$} \\
\hline & & & & & $\begin{array}{l}\text { Lower } \\
\text { Bound }\end{array}$ & $\begin{array}{l}\text { Upper } \\
\text { Bound }\end{array}$ \\
\hline \multirow[t]{3}{*}{$1 \mathrm{w} /$ bleach } & $2 \mathrm{w} /$ bleach & $1.934^{\circ, \mathrm{a}, \mathrm{b}}$ & 0.345 & 0.000 & 1.257 & 2.611 \\
\hline & 3 w/o bleach & $1.705^{x, a, b}$ & 0.345 & 0.000 & 1.028 & 2.382 \\
\hline & 4 w/o bleach & $1.099^{\pi}, a, b$ & 0.345 & 0.001 & 0.422 & 1.776 \\
\hline \multirow[t]{3}{*}{$2 \mathrm{w} /$ bleach } & $1 \mathrm{w} /$ bleach & $-1.934^{, a, b}$ & 0.345 & 0.000 & -2.611 & -1.257 \\
\hline & 3 w/o bleach & $-.229^{a, b}$ & 0.345 & 0.507 & -0.906 & 0.448 \\
\hline & 4 w/o bleach & $-.835^{\star}, \mathrm{a}, \mathrm{b}$ & 0.345 & 0.016 & -1.512 & -0.158 \\
\hline \multirow[t]{3}{*}{3 w/o bleach } & $1 \mathrm{w} /$ bleach & $-1.705^{\circ, a, b}$ & 0.345 & 0.000 & -2.382 & -1.028 \\
\hline & 2 w/ bleach & $.229^{a, b}$ & 0.345 & 0.507 & -0.448 & 0.906 \\
\hline & 4 w/o bleach & $-.606^{a, b}$ & 0.345 & 0.080 & -1.283 & 0.072 \\
\hline \multirow[t]{3}{*}{4 w/o bleach } & $1 \mathrm{w} /$ bleach & $-1.099^{\circ, a, b}$ & 0.345 & 0.001 & -1.776 & -0.422 \\
\hline & $2 \mathrm{w} /$ bleach & $.835^{\star, a, b}$ & 0.345 & 0.016 & 0.158 & 1.512 \\
\hline & 3 w/o bleach & $.606^{\mathrm{a}, \mathrm{b}}$ & 0.345 & 0.080 & -0.072 & 1.283 \\
\hline
\end{tabular}

Based on estimated marginal means

*. The mean difference is significant at the .05 level.

a. An estimate of the modified population marginal mean (I).

b. An estimate of the modified population marginal mean $(\mathrm{J})$.

c. Adjustment for multiple comparisons: Least Significant Difference (equivalent to no adjustments).

Marked significant differences were observed for all levels of group Swatch. The greatest

amount of significances was observed at swatch 1 which observed significant mean differences 
with all swatches. Notice that with the bleached swatches, significant values were observed between them, whereas with the unbleached swatches, no significant values were observed.

\section{Bleach Estimates: DPMP}

Dependent Variable: a

\begin{tabular}{|l|r|r|r|r|}
\hline \multirow{2}{*}{ Bleached } & & \multicolumn{2}{|c|}{$95 \%$ Confidence Interval } \\
\cline { 3 - 5 } & Mean & Std. Error & $\begin{array}{c}\text { Lower } \\
\text { Bound }\end{array}$ & $\begin{array}{c}\text { Upper } \\
\text { Bound }\end{array}$ \\
\hline w/ bleach & $25.471^{\text {a }}$ & 0.172 & 25.133 & 25.81 \\
w/o bleach & $25.036^{\text {a }}$ & 0.172 & 24.698 & 25.375 \\
\hline
\end{tabular}

a. Based on modified population marginal mean.

Pairwise Comparisons: DPMP

Dependent Variable: a

\begin{tabular}{|c|c|c|c|c|c|c|}
\hline \multirow[b]{2}{*}{$\begin{array}{l}\text { (I) } \\
\text { Bleached }\end{array}$} & \multirow[b]{2}{*}{$\begin{array}{l}(\mathrm{J}) \\
\text { Bleached }\end{array}$} & \multirow{2}{*}{$\begin{array}{c}\text { Mean } \\
\text { Difference } \\
(\mathrm{I}-\mathrm{J})\end{array}$} & \multirow[b]{2}{*}{ Std. Error } & \multirow[b]{2}{*}{ Sig. ${ }^{\mathrm{C}}$} & \multicolumn{2}{|c|}{$\begin{array}{c}95 \% \text { Confidence Interval } \\
\text { for Difference }\end{array}$} \\
\hline & & & & & $\begin{array}{l}\text { Lower } \\
\text { Bound }\end{array}$ & $\begin{array}{l}\text { Upper } \\
\text { Bound }\end{array}$ \\
\hline w/ bleach & w/o bleach & $.435^{\mathrm{a}, \mathrm{b}}$ & 0.244 & 0.075 & -0.044 & 0.914 \\
\hline w/o bleach & w/ bleach & $-.435^{a, b}$ & 0.244 & 0.075 & -0.914 & 0.044 \\
\hline
\end{tabular}

Based on estimated marginal means

a. An estimate of the modified population marginal mean (I).

b. An estimate of the modified population marginal mean (J).

c. Adjustment for multiple comparisons: Least Significant Difference (equivalent to no adjustments).

Although interaction effects cannot be observed with this group, the effects within this group can be investigated. Based on the results, there is no evidence that 'w/ bleach' and 'w/o bleach' differed in their performance. The p-value clearly shows that there is no significant difference at the $5 \%$ level.

\section{Washing Estimates: DPMP}

Dependent Variable: a

\begin{tabular}{|l|r|r|r|r|}
\hline \multirow{2}{*}{ Washing } & & & \multicolumn{2}{|c|}{$95 \%$ Confidence Interval } \\
\cline { 3 - 5 } & Mean & Std. Error & $\begin{array}{c}\text { Lower } \\
\text { Bound }\end{array}$ & $\begin{array}{c}\text { Upper } \\
\text { Bound }\end{array}$ \\
\hline 1W 0A & $25.582^{\mathrm{a}}$ & 0.405 & 24.788 & 26.376 \\
1W 16A & $25.625^{\mathrm{a}}$ & 0.286 & 25.064 & 26.186 \\
2W 32A & $24.617^{\mathrm{a}}$ & 0.286 & 24.056 & 25.179 \\
3W 48A & $26.090^{\mathrm{a}}$ & 0.286 & 25.529 & 26.652 \\
4W 64A & $24.392^{\mathrm{a}}$ & 0.286 & 23.83 & 24.953 \\
5W 80A & $25.380^{\mathrm{a}}$ & 0.286 & 24.818 & 25.941 \\
\hline
\end{tabular}

a. Based on modified population marginal mean.

Pairwise Comparisons: DPMP 
Dependent Variable: a

\begin{tabular}{|c|c|c|c|c|c|c|}
\hline \multirow[b]{2}{*}{$\begin{array}{l}\text { (I) } \\
\text { Washing }\end{array}$} & \multirow[b]{2}{*}{$\begin{array}{l}(\mathrm{J}) \\
\text { Washing }\end{array}$} & \multirow{2}{*}{$\begin{array}{c}\text { Mean } \\
\text { Difference } \\
(\mathrm{I}-\mathrm{J})\end{array}$} & \multirow[b]{2}{*}{ Std. Error } & \multirow[b]{2}{*}{ Sig. ${ }^{\mathrm{C}}$} & \multicolumn{2}{|c|}{$\begin{array}{c}\text { 95\% Confidence } \\
\text { Interval for Difference }\end{array}$} \\
\hline & & & & & $\begin{array}{l}\text { Lower } \\
\text { Bound }\end{array}$ & $\begin{array}{l}\text { Upper } \\
\text { Bound }\end{array}$ \\
\hline \multirow[t]{5}{*}{$1 \mathrm{~W} 0 \mathrm{~A}$} & $1 \mathrm{~W} 16 \mathrm{~A}$ & $-.043^{\mathrm{a}, \mathrm{b}}$ & 0.495 & 0.931 & -1.015 & 0.93 \\
\hline & $2 W 32 A$ & $.965^{\mathrm{a}, \mathrm{b}}$ & 0.495 & 0.052 & -0.007 & 1.938 \\
\hline & $3 W 48 A$ & $-.508^{a, b}$ & 0.495 & 0.306 & -1.48 & 0.465 \\
\hline & $4 W 64 A$ & $1.190^{\mathrm{a}, \mathrm{b},{ }^{*}}$ & 0.495 & 0.016 & 0.218 & 2.163 \\
\hline & $5 W$ & $.202^{\mathrm{a}, \mathrm{b}}$ & 0.495 & 0.683 & -0.77 & 1.175 \\
\hline \multirow[t]{5}{*}{$1 \mathrm{~W} 16 \mathrm{~A}$} & $1 \mathrm{~W} 0 \mathrm{~A}$ & $.043^{\mathrm{a}, \mathrm{b}}$ & 0.495 & 0.931 & -0.93 & 1.015 \\
\hline & $2 W 32 A$ & $1.008^{\mathrm{a}, \mathrm{b},{ }^{*}}$ & 0.405 & 0.013 & 0.214 & 1.802 \\
\hline & $3 W$ 48A & $-.465^{a, b}$ & 0.405 & 0.251 & -1.259 & 0.329 \\
\hline & $4 W 64 A$ & $1.233^{\mathrm{a}, \mathrm{b},{ }^{*}}$ & 0.405 & 0.002 & 0.439 & 2.027 \\
\hline & $5 W$ 80A & $.245^{\mathrm{a}, \mathrm{b}}$ & 0.405 & 0.545 & -0.549 & 1.039 \\
\hline \multirow[t]{5}{*}{$2 W 32 A$} & $1 \mathrm{~W} 0 \mathrm{~A}$ & $-.965^{a, b}$ & 0.495 & 0.052 & -1.938 & 0.007 \\
\hline & $1 \mathrm{~W} 16 \mathrm{~A}$ & $-1.008^{a, b,{ }^{*}}$ & 0.405 & 0.013 & -1.802 & -0.214 \\
\hline & $3 W$ 48A & $-1.473^{\mathrm{a}, \mathrm{b},{ }^{*}}$ & 0.405 & 0.000 & -2.267 & -0.679 \\
\hline & $4 W 64 A$ & $.225^{\mathrm{a}, \mathrm{b}}$ & 0.405 & 0.578 & -0.569 & 1.019 \\
\hline & $5 W$ 80A & $-.763^{a, b}$ & 0.405 & 0.060 & -1.557 & 0.031 \\
\hline \multirow[t]{5}{*}{$3 W 48 A$} & $1 \mathrm{~W} 0 \mathrm{~A}$ & $.508^{\mathrm{a}, \mathrm{b}}$ & 0.495 & 0.306 & -0.465 & 1.48 \\
\hline & $1 \mathrm{~W} 16 \mathrm{~A}$ & $.465^{\mathrm{a}, \mathrm{b}}$ & 0.405 & 0.251 & -0.329 & 1.259 \\
\hline & $2 W 32 A$ & $1.473^{\mathrm{a}, \mathrm{b},{ }^{*}}$ & 0.405 & 0.000 & 0.679 & 2.267 \\
\hline & $4 W 64 A$ & $1.698^{\mathrm{a}, \mathrm{b},{ }^{*}}$ & 0.405 & 0.000 & 0.904 & 2.492 \\
\hline & $5 W$ 80A & $.710^{\mathrm{a}, \mathrm{b}}$ & 0.405 & 0.079 & -0.084 & 1.504 \\
\hline \multirow[t]{5}{*}{$4 W 64 A$} & $1 \mathrm{~W} \mathrm{OA}$ & $-1.190^{\mathrm{a}, \mathrm{b},}$ & 0.495 & 0.016 & -2.163 & -0.218 \\
\hline & $1 \mathrm{~W} 16 \mathrm{~A}$ & $-1.233^{\mathrm{a}, \mathrm{b},{ }^{\star}}$ & 0.405 & 0.002 & -2.027 & -0.439 \\
\hline & $2 W 32 A$ & $-.225^{\mathrm{a}, \mathrm{b}}$ & 0.405 & 0.578 & -1.019 & 0.569 \\
\hline & $3 W$ 48A & $-1.698^{\mathrm{a}, \mathrm{b},}$ & 0.405 & 0.000 & -2.492 & -0.904 \\
\hline & $5 W$ 80A & $-.988^{\mathrm{a}, \mathrm{b},{ }^{\star}}$ & 0.405 & 0.015 & -1.782 & -0.194 \\
\hline \multirow[t]{5}{*}{$5 W 80 A$} & $1 \mathrm{~W} 0 \mathrm{~A}$ & $-.202^{a, b}$ & 0.495 & 0.683 & -1.175 & 0.77 \\
\hline & $1 \mathrm{~W} 16 \mathrm{~A}$ & $-.245^{a, b}$ & 0.405 & 0.545 & -1.039 & 0.549 \\
\hline & $2 W 32 A$ & $.763^{a, b}$ & 0.405 & 0.060 & -0.031 & 1.557 \\
\hline & $3 W 48 A$ & $-.710^{\mathrm{a}, \mathrm{b}}$ & 0.405 & 0.079 & -1.504 & 0.084 \\
\hline & $4 W 64 A$ & $.988^{\mathrm{a}, \mathrm{b},{ }^{\pi}}$ & 0.405 & 0.015 & 0.194 & 1.782 \\
\hline
\end{tabular}

Based on estimated marginal means

a. An estimate of the modified population marginal mean (I).

b. An estimate of the modified population marginal mean $(\mathrm{J})$.

c. Adjustment for multiple comparisons: Least Significant Difference (equivalent to no adjustments).

*. The mean difference is significant at the .05 level.

Marked significant differences were observed within levels of this group. Except for 5W 80A which did not observe any significant mean differences, no one level was more prominent than the other.

\section{Sample Area * Swatch}


Dependent Variable: a

\begin{tabular}{|c|c|c|c|c|c|}
\hline \multirow[b]{2}{*}{ Sample Area } & \multirow[b]{2}{*}{ Swatch } & \multirow[b]{2}{*}{ Mean } & \multirow[b]{2}{*}{ Std. Error } & \multicolumn{2}{|c|}{$\begin{array}{c}\text { 95\% Confidence } \\
\text { Interval } \\
\end{array}$} \\
\hline & & & & $\begin{array}{l}\text { Lower } \\
\text { Bound }\end{array}$ & $\begin{array}{l}\text { Upper } \\
\text { Bound }\end{array}$ \\
\hline \multirow[t]{4}{*}{$A$} & $1 \mathrm{w} /$ bleach & $25.831^{a}$ & 0.33 & 25.182 & 26.479 \\
\hline & $2 \mathrm{w} /$ bleach & $25.111^{a}$ & 0.362 & 24.401 & 25.822 \\
\hline & 3 w/o bleach & $25.108^{a}$ & 0.33 & 24.459 & 25.756 \\
\hline & 4 w/o bleach & $26.132^{a}$ & 0.362 & 25.421 & 26.842 \\
\hline \multirow[t]{4}{*}{$\mathrm{B}$} & $1 \mathrm{w} /$ bleach & $27.167^{\mathrm{a}}$ & 0.362 & 26.457 & 27.877 \\
\hline & $2 \mathrm{w} /$ bleach & $23.998^{a}$ & 0.33 & 23.35 & 24.646 \\
\hline & 3 w/o bleach & $24.284^{a}$ & 0.362 & 23.574 & 24.995 \\
\hline & 4 w/o bleach & $24.678^{a}$ & 0.33 & 24.03 & 25.327 \\
\hline
\end{tabular}

a. Based on modified population marginal mean.

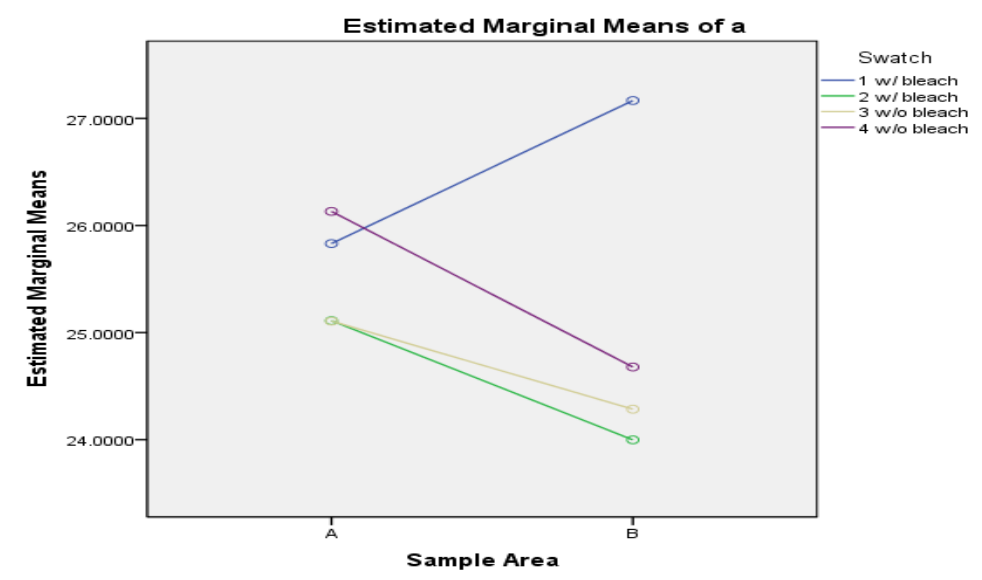

Parallel lines are observed with swatches 2-4 which would suggest that the effect of the mean outcome of a change in one factor is independent on the level of the other factor. The means values for swatches 2 and 3 are roughly the same. On the other hand, the mean for swatch 1 at area B varies greatly with the remaining swatches which possibly contributed to the significant values obtained.

Dependent Variable: a

\section{Sample Area * Bleached}

\begin{tabular}{|ll|r|r|r|r|}
\hline \multirow{2nnnyy}{*}{ Sample Area } & \multirow{2}{*}{} & \multirow{2}{*}{$95 \%$ Confidence Interval } \\
\cline { 5 - 6 } & Bleached & Mean & Std. Error & \multicolumn{1}{c|}{$\begin{array}{c}\text { Lower } \\
\text { Bound }\end{array}$} & $\begin{array}{c}\text { Upper } \\
\text { Bound }\end{array}$ \\
\hline A & w/ bleach & $25.504^{\mathrm{a}}$ & 0.244 & 25.025 & 25.983 \\
& w/o bleach & $25.573^{\mathrm{a}}$ & 0.244 & 25.094 & 26.052 \\
\hline B & w/ bleach & $25.439^{\mathrm{a}}$ & 0.244 & 24.96 & 25.917 \\
& w/o bleach & $24.499^{\mathrm{a}}$ & 0.244 & 24.02 & 24.978 \\
\hline
\end{tabular}




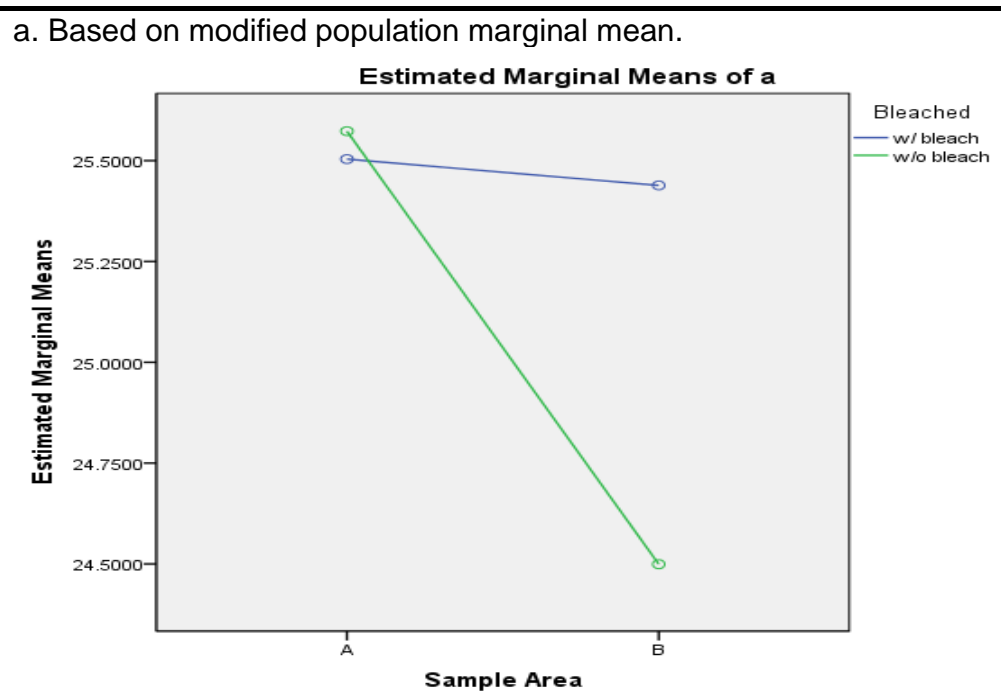

The interactions between these groups seem to be substantial at sample area B as the mean differences between the bleached and unbleached samples varies more than at area A.

\section{Bleached * Washing}

Dependent Variable: a

\begin{tabular}{|c|c|c|c|c|c|}
\hline \multirow[b]{2}{*}{ Bleached } & \multirow[b]{2}{*}{ Washing } & \multirow[b]{2}{*}{ Mean } & \multirow[b]{2}{*}{ Std. Error } & \multicolumn{2}{|c|}{$95 \%$ Confidence Interval } \\
\hline & & & & $\begin{array}{l}\text { Lower } \\
\text { Bound }\end{array}$ & $\begin{array}{l}\text { Upper } \\
\text { Bound }\end{array}$ \\
\hline \multirow[t]{6}{*}{ w/ bleach } & $1 \mathrm{~W} 0 \mathrm{~A}$ & $26.527^{\mathrm{a}}$ & 0.572 & 25.404 & 27.65 \\
\hline & $1 \mathrm{~W} 16 \mathrm{~A}$ & $27.396^{\mathrm{a}}$ & 0.405 & 26.602 & 28.19 \\
\hline & $2 W 32 A$ & $25.023^{a}$ & 0.405 & 24.229 & 25.817 \\
\hline & $3 W$ 48A & $25.352^{\mathrm{a}}$ & 0.405 & 24.558 & 26.146 \\
\hline & $4 W 64 A$ & $23.255^{\mathrm{a}}$ & 0.405 & 22.461 & 24.048 \\
\hline & $5 W$ & $25.802^{a}$ & 0.405 & 25.008 & 26.596 \\
\hline \multirow[t]{6}{*}{ w/o bleach } & $1 \mathrm{~W} 0 \mathrm{~A}$ & $24.638^{\mathrm{a}}$ & 0.572 & 23.515 & 25.76 \\
\hline & $1 \mathrm{~W} 16 \mathrm{~A}$ & $23.854^{\mathrm{a}}$ & 0.405 & 23.06 & 24.648 \\
\hline & $2 W 32 A$ & $24.211^{a}$ & 0.405 & 23.417 & 25.005 \\
\hline & $3 W 48 A$ & $26.828^{a}$ & 0.405 & 26.034 & 27.622 \\
\hline & $4 \mathrm{~W} 64 \mathrm{~A}$ & $25.529^{\mathrm{a}}$ & 0.405 & 24.735 & 26.323 \\
\hline & $5 W$ 80A & $24.958^{a}$ & 0.405 & 24.164 & 25.752 \\
\hline
\end{tabular}

a. Based on modified population marginal mean. 


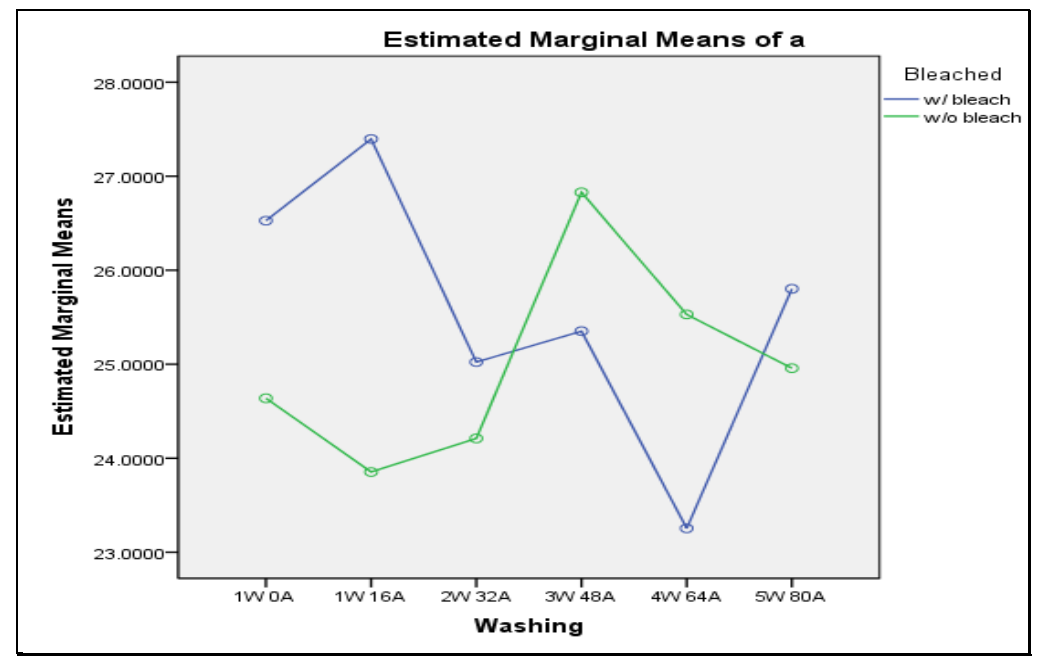

The overall patterns between these two groups are very dissimilar. In fact the mean values decreases as for samples washed with bleach, but increases for the samples washed without bleach. It is evident both groups affect the outcome of the interaction.

Dependent Variable: a

\section{Swatch * Washing: DPMP}

\begin{tabular}{|c|c|c|c|c|c|}
\hline \multirow[b]{2}{*}{ Swatch } & \multirow[b]{2}{*}{ Washing } & \multirow[b]{2}{*}{ Mean } & \multirow[b]{2}{*}{ Std. Error } & \multicolumn{2}{|c|}{ 95\% Confidence Interval } \\
\hline & & & & Lower Bound & Upper Bound \\
\hline \multirow[t]{6}{*}{$1 \mathrm{w} /$ bleach } & $1 \mathrm{~W} 0 \mathrm{~A}$ & $28.455^{\mathrm{a}}$ & 0.809 & 26.867 & 30.043 \\
\hline & $1 \mathrm{~W} 16 \mathrm{~A}$ & $29.193^{\mathrm{a}}$ & 0.572 & 28.07 & 30.316 \\
\hline & $2 W 32 A$ & $25.289^{\mathrm{a}}$ & 0.572 & 24.166 & 26.412 \\
\hline & $3 W 48 A$ & $26.804^{a}$ & 0.572 & 25.681 & 27.927 \\
\hline & $4 W 64 A$ & $24.850^{\mathrm{a}}$ & 0.572 & 23.727 & 25.972 \\
\hline & $5 \mathrm{~W} 80 \mathrm{~A}$ & $25.047^{\mathrm{a}}$ & 0.572 & 23.924 & 26.17 \\
\hline \multirow[t]{6}{*}{2 w/ bleach } & $1 \mathrm{~W} 0 \mathrm{~A}$ & $24.599^{a}$ & 0.809 & 23.012 & 26.187 \\
\hline & $1 \mathrm{~W} 16 \mathrm{~A}$ & $25.599^{a}$ & 0.572 & 24.476 & 26.722 \\
\hline & $2 W 32 A$ & $24.757^{\mathrm{a}}$ & 0.572 & 23.634 & 25.88 \\
\hline & $3 W$ 48A & $23.900^{\mathrm{a}}$ & 0.572 & 22.777 & 25.023 \\
\hline & $4 W 64 A$ & $21.659^{a}$ & 0.572 & 20.537 & 22.782 \\
\hline & $5 W$ 80A & $26.557^{\mathrm{a}}$ & 0.572 & 25.434 & 27.68 \\
\hline \multirow[t]{6}{*}{$3 \mathrm{w} / \mathrm{o}$ bleach } & $1 \mathrm{~W} 0 \mathrm{~A}$ & $24.695^{\mathrm{a}}$ & 0.809 & 23.107 & 26.283 \\
\hline & $1 \mathrm{~W} 16 \mathrm{~A}$ & $25.385^{\mathrm{a}}$ & 0.572 & 24.262 & 26.508 \\
\hline & $2 W 32 A$ & $23.783^{a}$ & 0.572 & 22.66 & 24.906 \\
\hline & $3 W 48 A$ & $26.275^{a}$ & 0.572 & 25.152 & 27.398 \\
\hline & $4 W 64 A$ & $25.199^{a}$ & 0.572 & 24.076 & 26.322 \\
\hline & $5 W$ 80A & $23.045^{a}$ & 0.572 & 21.922 & 24.168 \\
\hline \multirow[t]{6}{*}{$4 \mathrm{w} / \mathrm{o}$ bleach } & $1 \mathrm{~W} 0 \mathrm{~A}$ & $24.580^{\mathrm{a}}$ & 0.809 & 22.992 & 26.168 \\
\hline & $1 \mathrm{~W} 16 \mathrm{~A}$ & $22.323^{\mathrm{a}}$ & 0.572 & 21.2 & 23.446 \\
\hline & $2 W 32 A$ & $24.639^{a}$ & 0.572 & 23.516 & 25.762 \\
\hline & $3 W 48 A$ & $27.382^{\mathrm{a}}$ & 0.572 & 26.259 & 28.505 \\
\hline & $4 W 64 A$ & $25.859^{a}$ & 0.572 & 24.737 & 26.982 \\
\hline & $5 W$ & $26.871^{a}$ & 0.572 & 25.748 & 27.994 \\
\hline
\end{tabular}

a. Based on modified population marginal mean. 


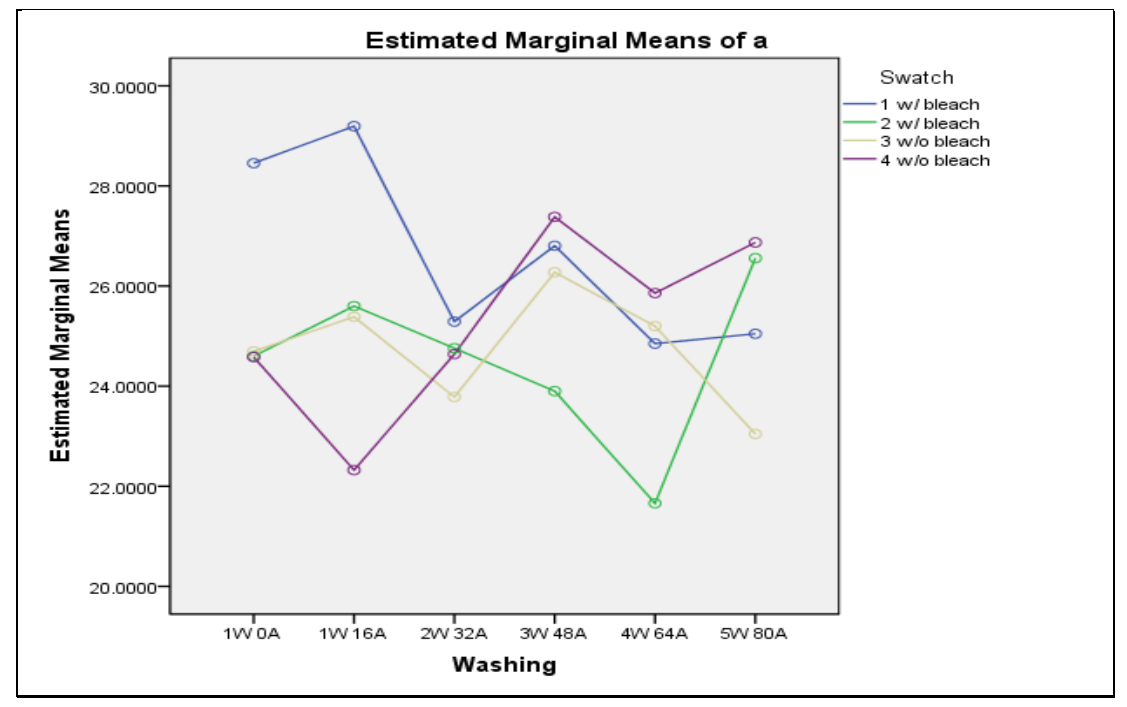

The interaction effects for these two groups observe to be substantial. The overall patterns for swatch 1, 2 and 3 started out in a consistent manner, but, after the fourth washing, swatch 4 continued on a different pattern. It was expected that the swatches washed with similar detergent would produce similar results; yet this has not been observed at either dependent variables.

DPMP-Variable ' $b$ '

Dependent Variable: $b$

Tests of Between-Subjects Effects: DPMP

\begin{tabular}{|c|c|c|c|c|c|}
\hline Source & $\begin{array}{l}\text { Type III Sum of } \\
\text { Squares }\end{array}$ & df & $\begin{array}{l}\text { Mean } \\
\text { Square }\end{array}$ & $\mathrm{F}$ & Sig. \\
\hline Corrected Model & $1677.875^{\mathrm{a}}$ & 43 & 39.02 & 9.746 & 0.000 \\
\hline Intercept & 44729.07 & 1 & 44729.07 & 11172.26 & 0.000 \\
\hline SampleArea & 6.892 & 1 & 6.892 & 1.721 & 0.190 \\
\hline Swatch & 22.682 & 2 & 11.341 & 2.833 & 0.059 \\
\hline Bleached & 0 & 0 & & & \\
\hline Washing & 115.882 & 5 & 23.176 & 5.789 & 0.000 \\
\hline SampleArea * Swatch & 69.245 & 2 & 34.622 & 8.648 & 0.000 \\
\hline SampleArea * Bleached & 0 & 0 & & & \\
\hline SampleArea * Washing & 425.388 & 4 & 106.347 & 26.563 & 0.000 \\
\hline Swatch * Bleached & 0 & 0 & & & \\
\hline Swatch * Washing & 239.837 & 8 & 29.98 & 7.488 & 0.000 \\
\hline Bleached * Washing & 0 & 0 & . & 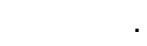 & \\
\hline SampleArea * Swatch * Bleached & 0 & 0 & . & & \\
\hline SampleArea * Swatch * Washing & 311.369 & 8 & 38.921 & 9.722 & 0.000 \\
\hline SampleArea * Bleached * Washing & 0 & 0 & 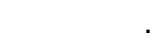 & 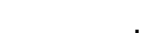 & \\
\hline Swatch * Bleached * Washing & 0 & 0 & & & \\
\hline SampleArea * Swatch * Bleached * Washing & 0 & 0 & & . & . \\
\hline Error & 3346.996 & 836 & 4.004 & & \\
\hline Total & 52144.15 & 880 & & & \\
\hline Corrected Total & 5024.871 & 879 & & & \\
\hline
\end{tabular}


a. R Squared $=.334$ (Adjusted R Squared $=.300)$

Based on the results, the main effects of the group 'Swatch' and 'Sample Area' did not produce significant results, but the main effects of 'Washing' did. Therefore, only the interaction effects of these two groups will be discussed below. The marginal means and pair-wise comparisons tables are located in the appendix.

\section{Bleach Estimates: DPMP}

Dependent Variable: b

\begin{tabular}{|l|r|r|r|r|}
\hline \multirow{2}{*}{} & & \multirow{2}{*}{} & \multicolumn{2}{|c|}{$95 \%$ Confidence Interval } \\
\cline { 4 - 5 } Bleached & Mean & Std. Error & $\begin{array}{c}\text { Lower } \\
\text { Bound }\end{array}$ & $\begin{array}{c}\text { Upper } \\
\text { Bound }\end{array}$ \\
\hline w/ bleach & $7.456^{\mathrm{a}}$ & 0.095 & 7.269 & 7.643 \\
w/o bleach & $7.179^{\mathrm{a}}$ & 0.095 & 6.991 & 7.366 \\
\hline
\end{tabular}

a. Based on modified population marginal mean.

\section{Pairwise Comparisons: DPMP}

Dependent Variable: b

\begin{tabular}{|c|c|c|c|c|c|c|}
\hline \multirow[b]{2}{*}{ (I) Bleached } & \multirow[b]{2}{*}{ (J) Bleached } & \multirow{2}{*}{$\begin{array}{c}\text { Mean } \\
\text { Difference (I- } \\
\mathrm{J})\end{array}$} & \multirow[b]{2}{*}{ Std. Error } & \multirow[b]{2}{*}{ Sig. $^{c}$} & \multicolumn{2}{|c|}{$\begin{array}{l}\text { 95\% Confidence Interval } \\
\text { for Difference }\end{array}$} \\
\hline & & & & & $\begin{array}{l}\text { Lower } \\
\text { Bound }\end{array}$ & $\begin{array}{l}\text { Upper } \\
\text { Bound }\end{array}$ \\
\hline w/ bleach & w/o bleach & $.277^{\star, a, b}$ & 0.135 & 0.04 & 0.013 & 0.542 \\
\hline w/o bleach & w/ bleach & $-.277^{\star, a, b}$ & 0.135 & 0.04 & -0.542 & -0.013 \\
\hline
\end{tabular}

Based on estimated marginal means

*. The mean difference is significant at the .05 level.

a. An estimate of the modified population marginal mean (I).

b. An estimate of the modified population marginal mean (J).

c. Adjustment for multiple comparisons: Least Significant Difference (equivalent to no adjustments).

At the 5\% level, significant mean differences were observed between these two groups.

Bleaching samples creates a significant difference in means in comparison to the unbleached samples. Unlike variables ' $L$ ' and ' $a$ ', the outcome of the means of variable ' $b$ ' was affected by the detergents used. 


\section{Washing Estimates: DPMP}

\begin{tabular}{|l|r|r|r|r|}
\hline & & & \multicolumn{2}{|c|}{$95 \%$ Confidence Interval } \\
\cline { 4 - 5 } & & & & \\
Washing & Mean & Std. Error & Lower Bound & Upper Bound \\
\hline 1W 0A & $7.509^{\mathrm{a}}$ & 0.224 & 7.069 & 7.948 \\
1W 16A & $7.136^{\mathrm{a}}$ & 0.158 & 6.826 & 7.447 \\
2W 32A & $7.394^{\mathrm{a}}$ & 0.158 & 7.084 & 7.705 \\
3W 48A & $7.921^{\mathrm{a}}$ & 0.158 & 7.61 & 8.231 \\
4W 64A & $6.850^{\mathrm{a}}$ & 0.158 & 6.539 & 7.16 \\
5W 80A & $7.191^{\mathrm{a}}$ & 0.158 & 6.88 & 7.501 \\
\hline
\end{tabular}

a. Based on modified population marginal mean.

Pairwise Comparisons: DPMP

Dependent Variable: $b$

\begin{tabular}{|c|c|c|c|c|c|c|}
\hline \multirow{2}{*}{ (I) Washing } & \multirow[b]{2}{*}{ (J) Washing } & \multirow{2}{*}{$\begin{array}{c}\text { Mean } \\
\text { Difference (I- } \\
\text { J) }\end{array}$} & \multirow[b]{2}{*}{ Std. Error } & \multirow[b]{2}{*}{ Sig. ${ }^{c}$} & \multicolumn{2}{|c|}{$\begin{array}{l}95 \% \text { Confidence Interval for } \\
\text { Difference }^{\mathrm{c}}\end{array}$} \\
\hline & & & & & Lower Bound & Upper Bound \\
\hline \multirow[t]{5}{*}{$1 \mathrm{~W} 0 \mathrm{~A}$} & $1 \mathrm{~W} 16 \mathrm{~A}$ & $.372^{\mathrm{a}, \mathrm{b}}$ & 0.274 & 0.175 & -0.166 & 0.91 \\
\hline & $2 W 32 A$ & $.114^{\mathrm{a}, \mathrm{b}}$ & 0.274 & 0.677 & -0.423 & 0.652 \\
\hline & $3 W 48 A$ & $-.412^{\mathrm{a}, \mathrm{b}}$ & 0.274 & 0.133 & -0.95 & 0.126 \\
\hline & $4 \mathrm{~W} 64 \mathrm{~A}$ & $.659^{\mathrm{a}, \mathrm{b},{ }^{*}}$ & 0.274 & 0.016 & 0.121 & 1.197 \\
\hline & $5 W 80 A$ & $.318^{a, b}$ & 0.274 & 0.246 & -0.22 & 0.856 \\
\hline \multirow[t]{5}{*}{$1 \mathrm{~W} 16 \mathrm{~A}$} & $1 \mathrm{~W} 0 \mathrm{~A}$ & $-.372^{\mathrm{a}, \mathrm{o}}$ & 0.274 & 0.175 & -0.91 & 0.166 \\
\hline & $2 W 32 A$ & $-.258^{a, b}$ & 0.224 & 0.250 & -0.697 & 0.181 \\
\hline & $3 W 48 A$ & $-.784^{\mathrm{a}, \mathrm{b},{ }^{\star}}$ & 0.224 & 0.000 & -1.223 & -0.345 \\
\hline & $4 \mathrm{~W} 64 \mathrm{~A}$ & $.287^{\mathrm{a}, \mathrm{b}}$ & 0.224 & 0.200 & -0.152 & 0.726 \\
\hline & $5 \mathrm{~W} 80 \mathrm{~A}$ & $-.054^{\mathrm{a}, \mathrm{b}}$ & 0.224 & 0.809 & -0.493 & 0.385 \\
\hline \multirow[t]{5}{*}{$2 \mathrm{~W} 32 \mathrm{~A}$} & $1 \mathrm{~W} 0 \mathrm{~A}$ & $-.114^{\mathrm{a}, \mathrm{b}}$ & 0.274 & 0.677 & -0.652 & 0.423 \\
\hline & $1 \mathrm{~W} 16 \mathrm{~A}$ & $.258^{\mathrm{a}, \mathrm{b}}$ & 0.224 & 0.250 & -0.181 & 0.697 \\
\hline & $3 W 48 A$ & $-.526^{a, b, x}$ & 0.224 & 0.019 & -0.966 & -0.087 \\
\hline & $4 \mathrm{~W} 64 \mathrm{~A}$ & $.545^{\mathrm{a}, \mathrm{b},{ }^{*}}$ & 0.224 & 0.015 & 0.105 & 0.984 \\
\hline & $5 W 80 A$ & $.204^{\mathrm{a}, \mathrm{b}}$ & 0.224 & 0.363 & -0.235 & 0.643 \\
\hline \multirow[t]{5}{*}{$3 W 48 A$} & $1 \mathrm{~W} 0 \mathrm{~A}$ & $.412^{\mathrm{a}, \mathrm{b}}$ & 0.274 & 0.133 & -0.126 & 0.95 \\
\hline & $1 \mathrm{~W} 16 \mathrm{~A}$ & $.784^{\mathrm{a}, \mathrm{b},{ }^{*}}$ & 0.224 & 0.000 & 0.345 & 1.223 \\
\hline & $2 W 32 A$ & $.526^{\mathrm{a}, \mathrm{b},}$ & 0.224 & 0.019 & 0.087 & 0.966 \\
\hline & $4 \mathrm{~W} 64 \mathrm{~A}$ & $1.071^{\mathrm{a}, \mathrm{b},{ }^{\star}}$ & 0.224 & 0.000 & 0.632 & 1.51 \\
\hline & $5 \mathrm{~W} 80 \mathrm{~A}$ & $.730^{\mathrm{a}, \mathrm{b},{ }^{*}}$ & 0.224 & 0.001 & 0.291 & 1.169 \\
\hline \multirow[t]{5}{*}{$4 \mathrm{~W} 64 \mathrm{~A}$} & $1 \mathrm{~W} 0 \mathrm{~A}$ & $-.659^{\mathrm{a}, \mathrm{b},-}$ & 0.274 & 0.016 & -1.197 & -0.121 \\
\hline & $1 \mathrm{~W} 16 \mathrm{~A}$ & $-.287^{\mathrm{a}, \mathrm{b}}$ & 0.224 & 0.200 & -0.726 & 0.152 \\
\hline & $2 W 32 A$ & $-.545^{\mathrm{a}, \mathrm{b},{ }^{*}}$ & 0.224 & 0.015 & -0.984 & -0.105 \\
\hline & $3 W 48 A$ & $-1.071^{\mathrm{a}, \mathrm{b},{ }^{\pi}}$ & 0.224 & 0.000 & -1.51 & -0.632 \\
\hline & $5 W 80 A$ & $-.341^{a, b}$ & 0.224 & 0.128 & -0.78 & 0.098 \\
\hline \multirow[t]{5}{*}{$5 \mathrm{~W} 80 \mathrm{~A}$} & $1 \mathrm{~W} 0 \mathrm{~A}$ & $-.318^{\mathrm{a}, \mathrm{b}}$ & 0.274 & 0.246 & -0.856 & 0.22 \\
\hline & $1 \mathrm{~W} 16 \mathrm{~A}$ & $.054^{\mathrm{a}, \mathrm{b}}$ & 0.224 & 0.809 & -0.385 & 0.493 \\
\hline & $2 \mathrm{~W} 32 \mathrm{~A}$ & $-.204^{\mathrm{a}, \mathrm{b}}$ & 0.224 & 0.363 & -0.643 & 0.235 \\
\hline & $3 W 48 A$ & $-.730^{\mathrm{a}, \mathrm{b},}$ & 0.224 & 0.001 & -1.169 & -0.291 \\
\hline & $4 W 64 A$ & $.341^{a, b}$ & 0.224 & 0.128 & -0.098 & 0.78 \\
\hline
\end{tabular}

Based on estimated marginal means

a. An estimate of the modified population marginal mean (I). 
b. An estimate of the modified population marginal mean (J).

c. Adjustment for multiple comparisons: Least Significant Difference (equivalent to no adjustments).

*. The mean difference is significant at the .05 level.

Marked significant differences were observed the greatest at 3W 48A. note that among the three variables, each was significantly affected at the various cycles of treatment.

\section{Sample Area * Swatch}

\begin{tabular}{|c|c|c|c|c|c|}
\hline \multirow[b]{2}{*}{ Sample Area } & \multirow[b]{2}{*}{ Swatch } & \multirow[b]{2}{*}{ Mean } & \multirow[b]{2}{*}{ Std. Error } & \multicolumn{2}{|c|}{ 95\% Confidence Interval } \\
\hline & & & & $\begin{array}{l}\text { Lower } \\
\text { Bound } \\
\end{array}$ & $\begin{array}{l}\text { Upper } \\
\text { Bound }\end{array}$ \\
\hline \multirow[t]{4}{*}{$A$} & $1 \mathrm{w} /$ bleach & $7.530^{\mathrm{a}}$ & 0.183 & 7.171 & 7.888 \\
\hline & $2 \mathrm{w} /$ bleach & $7.493^{\mathrm{a}}$ & 0.2 & 7.1 & 7.886 \\
\hline & $3 \mathrm{w} / \mathrm{o}$ bleach & $7.331^{a}$ & 0.183 & 6.973 & 7.69 \\
\hline & $4 \mathrm{w} / \mathrm{o}$ bleach & $7.623^{\mathrm{a}}$ & 0.2 & 7.23 & 8.015 \\
\hline \multirow[t]{4}{*}{ B } & $1 \mathrm{w} /$ bleach & $8.110^{a}$ & 0.2 & 7.717 & 8.502 \\
\hline & $2 \mathrm{w} /$ bleach & $6.807^{\mathrm{a}}$ & 0.183 & 6.449 & 7.166 \\
\hline & 3 w/o bleach & $6.972^{a}$ & 0.2 & 6.579 & 7.365 \\
\hline & 4 w/o bleach & $6.829^{a}$ & 0.183 & 6.47 & 7.187 \\
\hline
\end{tabular}

a. Based on modified population marginal mean.

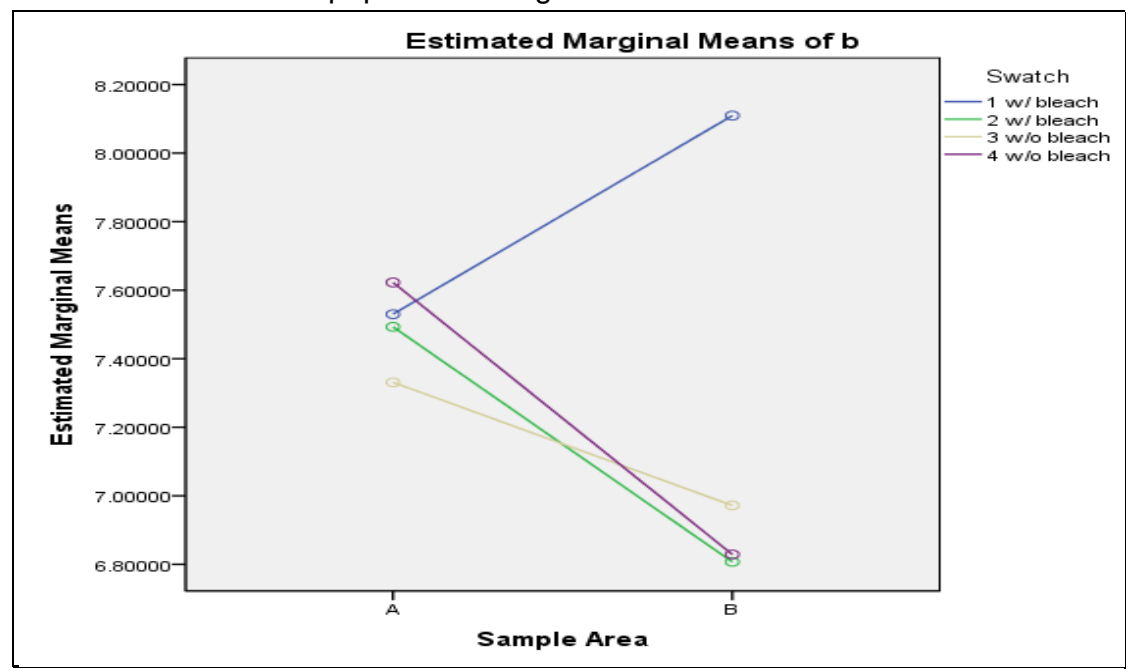

The overall pattern for interaction was similarly observed for variable ' $a$ ' where the mean difference was greatest with swatch 1 at area B to the remaining swatches. The consistencies observed between these variables suggest that it is more likely that significance is observed at swatch 1 and at sample area B.

\section{Sample Area * Bleached}


Dependent Variable: $b$

\begin{tabular}{|ll|r|r|r|r|}
\hline & & \multirow{2}{*}{} & & \multicolumn{2}{|c|}{ 95\% Confidence Interval } \\
\cline { 5 - 6 } Sample Area & Bleached & Mean & Std. Error & \multicolumn{1}{c|}{$\begin{array}{c}\text { Lower } \\
\text { Bound }\end{array}$} & $\begin{array}{c}\text { Upper } \\
\text { Bound }\end{array}$ \\
\hline A & w/ bleach & $7.513^{\mathrm{a}}$ & 0.135 & 7.248 & 7.778 \\
& w/o bleach & $7.464^{\mathrm{a}}$ & 0.135 & 7.199 & 7.728 \\
\hline B & $7.399^{\mathrm{a}}$ & 0.135 & 7.134 & 7.664 \\
& w/ bleach & $6.894^{\mathrm{a}}$ & 0.135 & 6.629 & 7.159 \\
\hline
\end{tabular}

a. Based on modified population marginal mean.

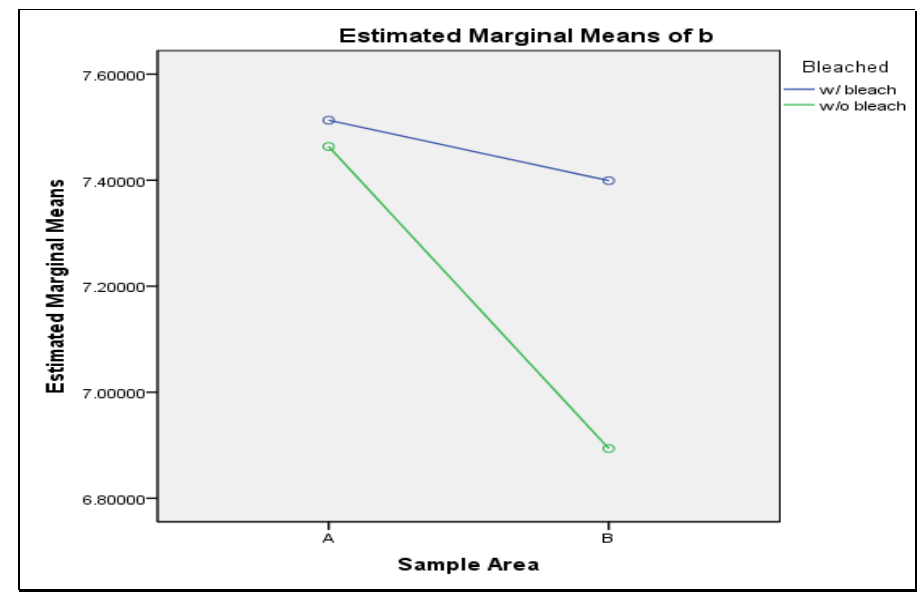

It can be concluded that interaction takes place between these groups as their means do not overlap or are neither similar. In fact, sample area B varies in a greater amount than area A. In addition, the interaction effects of sample area and swatch was also affected at this area.

Dependent Variable: $b$

\section{Bleached * Washing}

\begin{tabular}{|c|c|c|c|c|c|}
\hline \multirow[b]{2}{*}{ Bleached } & \multirow[b]{2}{*}{ Washing } & \multirow[b]{2}{*}{ Mean } & \multirow[b]{2}{*}{ Std. Error } & \multicolumn{2}{|c|}{$95 \%$ Confidence Interval } \\
\hline & & & & $\begin{array}{l}\text { Lower } \\
\text { Bound }\end{array}$ & $\begin{array}{l}\text { Upper } \\
\text { Bound }\end{array}$ \\
\hline \multirow[t]{6}{*}{ w/ bleach } & $1 \mathrm{~W} 0 \mathrm{~A}$ & $8.051^{\mathrm{a}}$ & 0.316 & 7.43 & 8.671 \\
\hline & $1 \mathrm{~W} 16 \mathrm{~A}$ & $7.935^{\mathrm{a}}$ & 0.224 & 7.496 & 8.374 \\
\hline & $2 W 32 A$ & $7.599^{\mathrm{a}}$ & 0.224 & 7.16 & 8.038 \\
\hline & $3 W 48 A$ & $7.340^{\mathrm{a}}$ & 0.224 & 6.901 & 7.779 \\
\hline & $4 \mathrm{~W} 64 \mathrm{~A}$ & $6.608^{a}$ & 0.224 & 6.169 & 7.047 \\
\hline & $5 W 80 A$ & $7.501^{\mathrm{a}}$ & 0.224 & 7.062 & 7.94 \\
\hline \multirow[t]{6}{*}{ w/o bleach } & $1 \mathrm{~W} 0 \mathrm{~A}$ & $6.967^{\mathrm{a}}$ & 0.316 & 6.346 & 7.588 \\
\hline & $1 \mathrm{~W} 16 \mathrm{~A}$ & $6.338^{\mathrm{a}}$ & 0.224 & 5.899 & 6.777 \\
\hline & $2 W 32 A$ & $7.189^{\mathrm{a}}$ & 0.224 & 6.75 & 7.628 \\
\hline & $3 W 48 A$ & $8.501^{a}$ & 0.224 & 8.062 & 8.94 \\
\hline & $4 \mathrm{~W} 64 \mathrm{~A}$ & $7.091^{\mathrm{a}}$ & 0.224 & 6.652 & 7.53 \\
\hline & $5 \mathrm{~W} 80 \mathrm{~A}$ & $6.880^{\mathrm{a}}$ & 0.224 & 6.441 & 7.319 \\
\hline
\end{tabular}

a. Based on modified population marginal mean. 


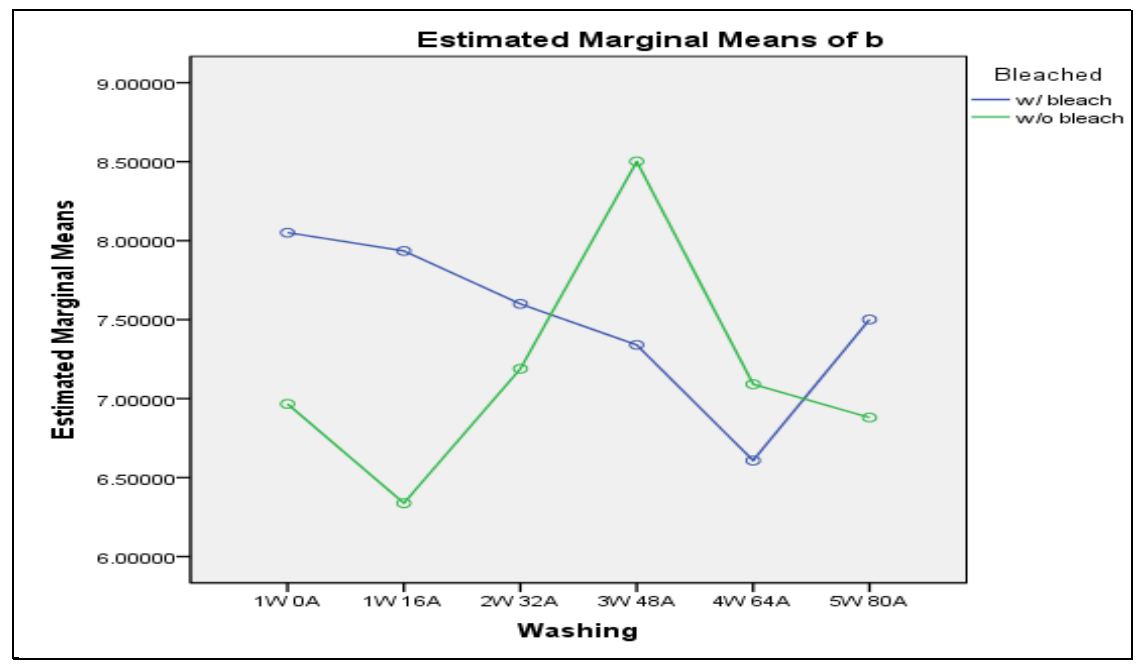

A consistent pattern is observed for the bleached samples. As the samples are further treated, the mean values decreases. This occurs until the fourth cycle in which the mean values then begin to increase. This pattern is a vast contrast from the unbleached samples.

Dependent Variable: $b$

\section{Swatch * Washing: DPMP}

\begin{tabular}{|c|c|c|c|c|c|}
\hline \multirow[b]{2}{*}{ Swatch } & \multirow[b]{2}{*}{ Washing } & \multirow[b]{2}{*}{ Mean } & \multirow[b]{2}{*}{ Std. Error } & \multicolumn{2}{|c|}{$95 \%$ Confidence Interval } \\
\hline & & & & Lower Bound & Upper Bound \\
\hline \multirow[t]{6}{*}{$1 \mathrm{w} /$ bleach } & $1 \mathrm{~W} 0 \mathrm{~A}$ & $9.460^{\mathrm{a}}$ & 0.447 & 8.582 & 10.338 \\
\hline & $1 \mathrm{~W} 16 \mathrm{~A}$ & $8.430^{\mathrm{a}}$ & 0.316 & 7.809 & 9.051 \\
\hline & $2 W 32 A$ & $7.633^{\mathrm{a}}$ & 0.316 & 7.012 & 8.254 \\
\hline & $3 W$ 48A & $7.898^{\mathrm{a}}$ & 0.316 & 7.277 & 8.519 \\
\hline & $4 W 64 A$ & $7.145^{\mathrm{a}}$ & 0.316 & 6.524 & 7.765 \\
\hline & $5 W$ 80A & $7.028^{\mathrm{a}}$ & 0.316 & 6.407 & 7.649 \\
\hline \multirow[t]{6}{*}{$2 \mathrm{w} / \mathrm{bleach}$} & $1 \mathrm{~W} \mathrm{OA}$ & $6.641^{a}$ & 0.447 & 5.763 & 7.519 \\
\hline & $1 \mathrm{~W} 16 \mathrm{~A}$ & $7.440^{\mathrm{a}}$ & 0.316 & 6.819 & 8.061 \\
\hline & $2 W 32 A$ & $7.565^{\mathrm{a}}$ & 0.316 & 6.944 & 8.186 \\
\hline & $3 W 48 A$ & $6.782^{\mathrm{a}}$ & 0.316 & 6.161 & 7.403 \\
\hline & $4 \mathrm{~W} 64 \mathrm{~A}$ & $6.071^{a}$ & 0.316 & 5.45 & 6.692 \\
\hline & $5 \mathrm{~W} 80 \mathrm{~A}$ & $7.974^{\mathrm{a}}$ & 0.316 & 7.353 & 8.595 \\
\hline \multirow[t]{6}{*}{$3 \mathrm{w} / \mathrm{o}$ bleach } & $1 \mathrm{~W} 0 \mathrm{~A}$ & $7.461^{a}$ & 0.447 & 6.583 & 8.339 \\
\hline & $1 \mathrm{~W} 16 \mathrm{~A}$ & $7.392^{\mathrm{a}}$ & 0.316 & 6.771 & 8.013 \\
\hline & $2 W 32 A$ & $7.223^{\mathrm{a}}$ & 0.316 & 6.602 & 7.844 \\
\hline & $3 W 48 A$ & $7.954^{\mathrm{a}}$ & 0.316 & 7.333 & 8.575 \\
\hline & $4 W 64 A$ & $7.136^{\mathrm{a}}$ & 0.316 & 6.515 & 7.757 \\
\hline & $5 W 80 A$ & $5.987^{\mathrm{a}}$ & 0.316 & 5.366 & 6.608 \\
\hline \multirow[t]{6}{*}{4 w/o bleach } & $1 \mathrm{~W} 0 \mathrm{~A}$ & $6.472^{\mathrm{a}}$ & 0.447 & 5.594 & 7.35 \\
\hline & $1 \mathrm{~W} 16 \mathrm{~A}$ & $5.283^{a}$ & 0.316 & 4.662 & 5.904 \\
\hline & $2 W 32 A$ & $7.155^{\mathrm{a}}$ & 0.316 & 6.534 & 7.776 \\
\hline & $3 W 48 A$ & $9.049^{a}$ & 0.316 & 8.428 & 9.67 \\
\hline & $4 W 64 A$ & $7.046^{\mathrm{a}}$ & 0.316 & 6.425 & 7.667 \\
\hline & $5 W$ & $7.773^{\mathrm{a}}$ & 0.316 & 7.152 & 8.394 \\
\hline
\end{tabular}

a. Based on modified population marginal mean. 


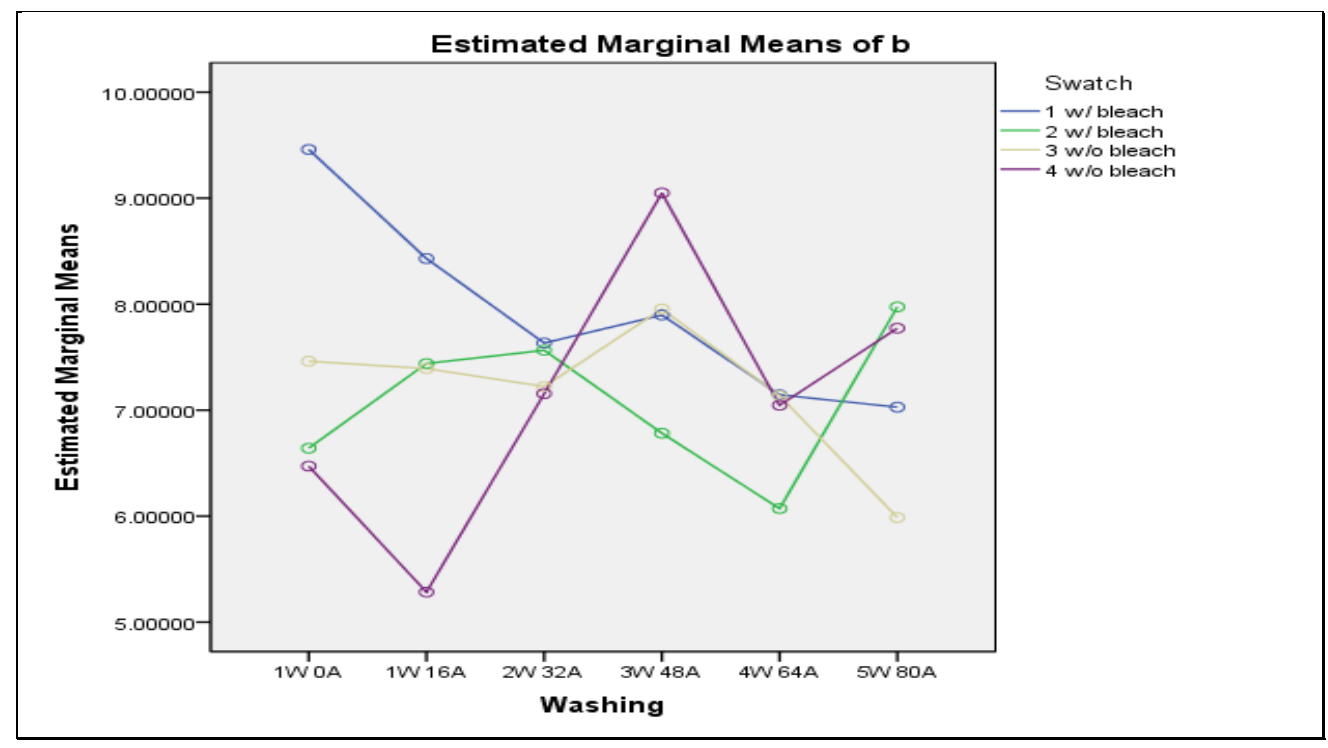

There is no consistent pattern observed at this level of interaction. In fact, all lines intersect each other at one point. It can be concluded that significant mean differences are observed at this level of interaction.

\section{Sample GMP}

\section{Variable ' $L$ '}

Dependent Variable: $\mathrm{L}$

Tests of Between-Subjects Effects: GMP

\begin{tabular}{|c|c|c|c|c|c|}
\hline Source & $\begin{array}{l}\text { Type III Sum } \\
\text { of Squares }\end{array}$ & df & $\begin{array}{c}\text { Mean } \\
\text { Square }\end{array}$ & $\mathrm{F}$ & Sig. \\
\hline Corrected Model & $5004.387^{a}$ & 43 & 116.381 & 8.122 & 0 \\
\hline Intercept & 7313681.6 & 1 & 7313682 & 510403 & 0 \\
\hline SampleArea & 254.644 & 1 & 254.644 & 17.771 & 0 \\
\hline Swatch & 229.485 & 2 & 114.742 & 8.008 & 0 \\
\hline bleached & 0 & 0 & & & \\
\hline washings & 969.01 & 5 & 193.802 & 13.525 & 0 \\
\hline SampleArea * Swatch & 164.809 & 2 & 82.404 & 5.751 & 0.003 \\
\hline SampleArea * bleached & 0 & 0 & & & \\
\hline SampleArea * washings & 610.451 & 4 & 152.613 & 10.65 & 0 \\
\hline Swatch * bleached & 0 & 0 & & & \\
\hline Swatch * washings & 408.084 & 8 & 51.01 & 3.56 & 0 \\
\hline bleached * washings & 0 & 0 & & & \\
\hline SampleArea * Swatch * bleached & 0 & 0 & & & \\
\hline SampleArea * Swatch * washings & 1345.946 & 8 & 168.243 & 11.741 & 0 \\
\hline SampleArea * bleached * washings & 0 & 0 & & & \\
\hline Swatch * bleached * washings & 0 & 0 & & & \\
\hline SampleArea * Swatch * bleached * washings & 0 & 0 & & & \\
\hline Error & 11979.238 & 836 & 14.329 & & \\
\hline Total & 7848825 & 880 & & & \\
\hline Corrected Total & 16983.625 & 879 & & & \\
\hline
\end{tabular}


a. R Squared $=.295$ (Adjusted R Squared $=.258$ )

b. Computed using alpha $=.05$

Based on the results, the main effects and interaction effects are all observed to be significant.

Dependent Variable: $\mathrm{L}$

\section{Sample Area Estimates: GMP}

\begin{tabular}{|c|c|c|c|c|}
\hline \multirow[b]{2}{*}{ Sample Area } & \multirow[b]{2}{*}{ Mean } & \multirow[b]{2}{*}{$\begin{array}{l}\text { Std. } \\
\text { Error }\end{array}$} & \multicolumn{2}{|c|}{ 95\% Confidence Interval } \\
\hline & & & $\begin{array}{l}\text { Lower } \\
\text { Bound }\end{array}$ & Upper Bound \\
\hline $\begin{array}{l}A \\
B\end{array}$ & $\begin{array}{l}93.939^{\mathrm{a}} \\
94.739^{\mathrm{a}}\end{array}$ & $\begin{array}{l}.180 \\
.180\end{array}$ & $\begin{array}{l}93.585 \\
94.385\end{array}$ & $\begin{array}{r}94.293 \\
95.093\end{array}$ \\
\hline
\end{tabular}

a. Based on modified population marginal mean.

\section{Pairwise Comparisons}

Dependent Variable: L

\begin{tabular}{|c|c|c|c|c|c|}
\hline \multirow[b]{2}{*}{ (I) Sample Area } & \multirow{2}{*}{$\begin{array}{c}\text { Mean } \\
\text { Difference } \\
(\mathrm{I}-\mathrm{J})\end{array}$} & \multirow{2}{*}{$\begin{array}{l}\text { Std. } \\
\text { Error }\end{array}$} & \multirow[b]{2}{*}{ Sig. ${ }^{d}$} & \multicolumn{2}{|c|}{$\begin{array}{l}\text { 95\% Confidence Interval for } \\
\text { Difference }^{d}\end{array}$} \\
\hline & & & & Lower Bound & Upper Bound \\
\hline $\begin{array}{ll}A & B \\
B & A\end{array}$ & $\begin{array}{r}-.800^{\circ, \mathrm{D}, \mathrm{c}} \\
.800^{\pi, \mathrm{b}, \mathrm{c}}\end{array}$ & $\begin{array}{l}.255 \\
.255\end{array}$ & $\begin{array}{l}.002 \\
.002\end{array}$ & $\begin{array}{r}-1.301 \\
.299\end{array}$ & $\begin{array}{l}-.299 \\
1.301\end{array}$ \\
\hline
\end{tabular}

Based on estimated marginal means

*. The mean difference is significant at the .05 level.

b. An estimate of the modified population marginal mean (I).

c. An estimate of the modified population marginal mean (J).

d. Adjustment for multiple comparisons: Least Significant Difference (equivalent to no adjustments).

Not only is the main effect for the sample area significant, the effects between levels are also significant.

Based on the swatch estimates and pair-wise table below, the greatest amount of marked significances is found at swatch 3. In contrast, swatch 4 which was also washed with the same detergent as swatch 3 , only observed a significant difference with swatch 3 . Swatch 1 and 2, both washed under the same conditions, did not observe marked significance differences.

\section{Swatch Estimates: GMP}

Dependent Variable: $\mathrm{L}$
\begin{tabular}{|l|r|r|r|r|}
\hline & & & \multicolumn{2}{|c|}{$95 \%$ Confidence Interval } \\
\cline { 4 - 5 } Swatch & Mean & Std. Error & Lower Bound & \multicolumn{1}{|c|}{ Upper Bound } \\
\hline 1 w/ bleach & $93.636^{\mathrm{a}}$ & 0.255 & 93.135 & 94.137 \\
2 w/ bleach & $93.596^{\mathrm{a}}$ & 0.255 & 93.095 & 94.097 \\
3 w/o bleach & $95.784^{\mathrm{a}}$ & 0.255 & 95.283 & 96.285 \\
4 w/o bleach & $94.339^{\mathrm{a}}$ & 0.255 & 93.838 & 94.84 \\
\hline
\end{tabular}

a. Based on modified population marginal mean. 
Dependent Variable: L

\begin{tabular}{|c|c|c|c|c|c|c|}
\hline \multirow[b]{2}{*}{ (I) Swatch } & & \multirow{2}{*}{$\begin{array}{c}\text { Mean } \\
\text { Difference (I- } \\
\mathrm{J})\end{array}$} & \multirow[b]{2}{*}{ Std. Error } & \multirow[b]{2}{*}{ Sig. ${ }^{d}$} & \multicolumn{2}{|c|}{$\begin{array}{c}\text { 95\% Confidence } \\
\text { Interval for Difference }^{d}\end{array}$} \\
\hline & & & & & $\begin{array}{l}\text { Lower } \\
\text { Bound }\end{array}$ & $\begin{array}{l}\text { Upper } \\
\text { Bound }\end{array}$ \\
\hline \multirow[t]{3}{*}{$1 \mathrm{w} /$ bleach } & 2 w/ bleach & $.040^{\mathrm{a}, \mathrm{b}}$ & .361 & .913 & -.669 & .748 \\
\hline & 3 w/o bleach & $-2.148^{\mathrm{a}, \mathrm{b},{ }^{*}}$ & .361 & .000 & -2.856 & -1.440 \\
\hline & 4 w/o bleach & $-.704^{\mathrm{a}, \mathrm{b}}$ & .361 & .052 & -1.412 & .005 \\
\hline \multirow[t]{3}{*}{$2 \mathrm{w} /$ bleach } & $1 \mathrm{w} /$ bleach & $-.040^{a, b}$ & .361 & .913 & -.748 & .669 \\
\hline & 3 w/o bleach & $-2.188^{a, b,{ }^{\star}}$ & .361 & .000 & -2.896 & -1.479 \\
\hline & 4 w/o bleach & $-.743^{\mathrm{a}, \mathrm{b},{ }^{*}}$ & .361 & .040 & -1.452 & -.035 \\
\hline \multirow[t]{3}{*}{3 w/o bleach } & 1 w/ bleach & $2.148^{\mathrm{a}, \mathrm{b},{ }^{\star}}$ & .361 & .000 & 1.440 & 2.856 \\
\hline & 2 w/ bleach & $2.188^{\mathrm{a}, \mathrm{b},{ }^{*}}$ & .361 & .000 & 1.479 & 2.896 \\
\hline & 4 w/o bleach & $1.444^{\mathrm{a}, \mathrm{b},{ }^{\pi}}$ & .361 & .000 & .736 & 2.153 \\
\hline \multirow[t]{3}{*}{4 w/o bleach } & 1 w/ bleach & $.704^{\mathrm{a}, \mathrm{b}}$ & .361 & .052 & -.005 & 1.412 \\
\hline & 2 w/ bleach & $.743^{\mathrm{a}, \mathrm{b},{ }^{*}}$ & .361 & .040 & .035 & 1.452 \\
\hline & 3 w/o bleach & $-1.444^{\mathrm{a}, \mathrm{b},{ }^{\star}}$ & .361 & .000 & -2.153 & -.736 \\
\hline
\end{tabular}

Based on estimated marginal means

*. The mean difference is significant at the .05 level.

a. An estimate of the modified population marginal mean (I).

b. An estimate of the modified population marginal mean $(\mathrm{J})$.

d. Adjustment for multiple comparisons: Least Significant Difference (equivalent to no adjustments).

\section{Bleach Estimates: GMP}

Dependent Variable: $\mathrm{L}$

\begin{tabular}{|l|r|r|r|r|}
\hline \multirow{2}{*}{ bleached } & & \multicolumn{2}{|c|}{$95 \%$ Confidence Interval } \\
\cline { 4 - 5 } & Mean & Std. Error & $\begin{array}{c}\text { Lower } \\
\text { Bound }\end{array}$ & $\begin{array}{c}\text { Upper } \\
\text { Bound }\end{array}$ \\
\hline w/ bleach & $93.616^{\mathrm{a}}$ & .180 & 93.262 & 93.970 \\
w/o bleach & $95.062^{\mathrm{a}}$ & .180 & 94.707 & 95.416 \\
\hline
\end{tabular}

a. Based on modified population marginal mean.

Pairwise Comparisons: GMP

Dependent Variable: $\mathrm{L}$

\begin{tabular}{|c|c|c|c|c|c|}
\hline \multirow[b]{2}{*}{ (I) bleached } & \multirow{2}{*}{$\begin{array}{c}\text { Mean } \\
\text { Difference (I- } \\
\mathrm{J})\end{array}$} & \multirow{2}{*}{$\begin{array}{l}\text { Std. } \\
\text { Error }\end{array}$} & \multirow[b]{2}{*}{ Sig. $^{d}$} & \multicolumn{2}{|c|}{$\begin{array}{l}\text { 95\% Confidence Interval for } \\
\text { Difference }\end{array}$} \\
\hline & & & & Lower Bound & Upper Bound \\
\hline $\begin{array}{ll}\text { w/ bleach } & \text { w/o bleach } \\
\text { w/o bleach } & \text { w/ bleach }\end{array}$ & $\begin{array}{r}-1.446^{*, \mathrm{~b}, \mathrm{c}} \\
1.446^{*, \mathrm{~b}, \mathrm{c}}\end{array}$ & $\begin{array}{l}.255 \\
.255\end{array}$ & $\begin{array}{l}.000 \\
.000 \\
\end{array}$ & $\begin{array}{r}-1.947 \\
.945\end{array}$ & $\begin{array}{r}-.945 \\
1.947 \\
\end{array}$ \\
\hline
\end{tabular}

Based on estimated marginal means

*. The mean difference is significant at the .05 level.

b. An estimate of the modified population marginal mean (I).

c. An estimate of the modified population marginal mean $(\mathrm{J})$.

d. Adjustment for multiple comparisons: Least Significant Difference (equivalent to no adjustments).

Both the main effects of this group, and the interacting levels observed significant mean

differences. The effects of the detergents affect the outcome of this variable. 


\section{Washing Estimates: GMP}

Dependent Variable: L

\begin{tabular}{|l|r|r|r|r|}
\hline & & & \multicolumn{2}{|c|}{$95 \%$ Confidence Interval } \\
\cline { 4 - 5 } washings & Mean & Std. Error & \multicolumn{1}{|c|}{ Lower Bound } & Upper Bound \\
\hline 1W 0A & $92.379^{\mathrm{a}}$ & .423 & 91.548 & 93.210 \\
1W 16A & $95.734^{\mathrm{a}}$ & .299 & 95.146 & 96.321 \\
2W 32A & $93.366^{\mathrm{a}}$ & .299 & 92.779 & 93.954 \\
3W 48A & $94.085^{\mathrm{a}}$ & .299 & 93.497 & 94.672 \\
4W 64A & $94.398^{\mathrm{a}}$ & .299 & 93.811 & 94.985 \\
5W 80A & $95.092^{\mathrm{a}}$ & .299 & 94.504 & 95.679 \\
\hline
\end{tabular}

a. Based on modified population marginal mean.

\section{Pairwise Comparisons: GMP}

Dependent Variable: L

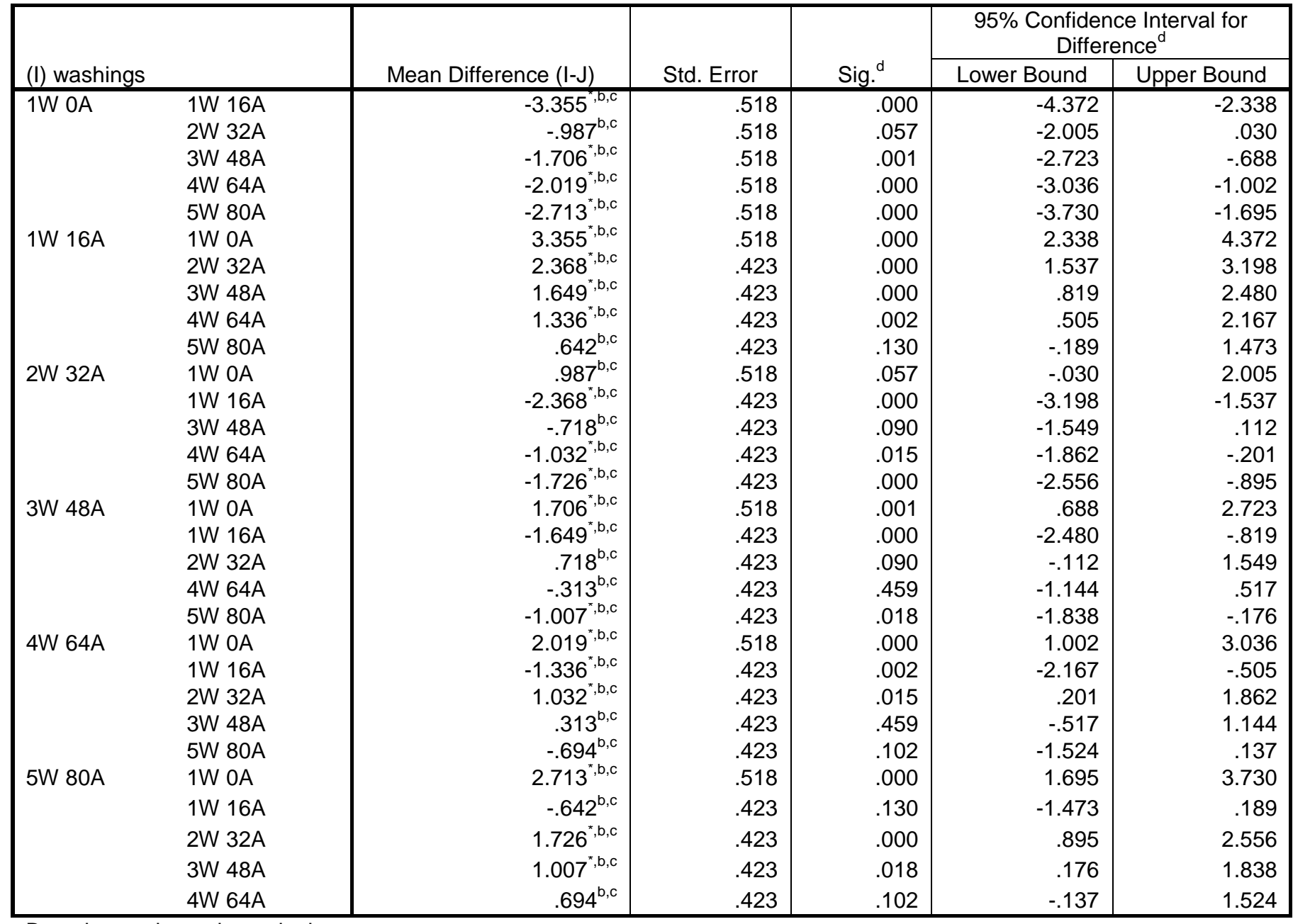

Based on estimated marginal means

*. The mean difference is significant at the .05 level.

b. An estimate of the modified population marginal mean (I).

c. An estimate of the modified population marginal mean $(\mathrm{J})$.

d. Adjustment for multiple comparisons: Least Significant Difference (equivalent to no adjustments).

Both the main effects of this group, and the interacting levels observed significant mean

differences, although not at all levels of the pair-wise comparing groups. 


\section{Sample Area * Swatch: GMP}

Dependent Variable: $\mathrm{L}$

\begin{tabular}{|c|c|c|c|c|c|}
\hline \multirow{2}{*}{\multicolumn{2}{|c|}{ Sample Area }} & \multirow[b]{2}{*}{ Mean } & \multirow[b]{2}{*}{$\begin{array}{l}\text { Std. } \\
\text { Error }\end{array}$} & \multicolumn{2}{|c|}{ 95\% Confidence Interval } \\
\hline & & & & $\begin{array}{l}\text { Lower } \\
\text { Bound }\end{array}$ & Upper Bound \\
\hline \multirow[t]{4}{*}{$\bar{A}$} & $1 \mathrm{w} /$ bleach & $93.843^{a}$ & .346 & 93.165 & 94.521 \\
\hline & $2 \mathrm{w} /$ bleach & $92.891^{\mathrm{a}}$ & .379 & 92.148 & 93.634 \\
\hline & $3 \mathrm{w} / \mathrm{o}$ bleach & $95.121^{a}$ & .346 & 94.443 & 95.799 \\
\hline & $4 \mathrm{w} / \mathrm{o}$ bleach & $93.683^{a}$ & .379 & 92.940 & 94.426 \\
\hline \multirow[t]{4}{*}{ B } & $1 \mathrm{w} /$ bleach & $93.387^{\mathrm{a}}$ & .379 & 92.644 & 94.130 \\
\hline & $2 \mathrm{w} /$ bleach & $94.184^{\mathrm{a}}$ & .346 & 93.505 & 94.862 \\
\hline & $3 \mathrm{w} / \mathrm{o}$ bleach & $96.579^{\mathrm{a}}$ & .379 & 95.836 & 97.322 \\
\hline & $4 \mathrm{w} / \mathrm{o}$ bleach & $94.887^{\mathrm{a}}$ & .346 & 94.209 & 95.565 \\
\hline
\end{tabular}

a. Based on modified population marginal mean.

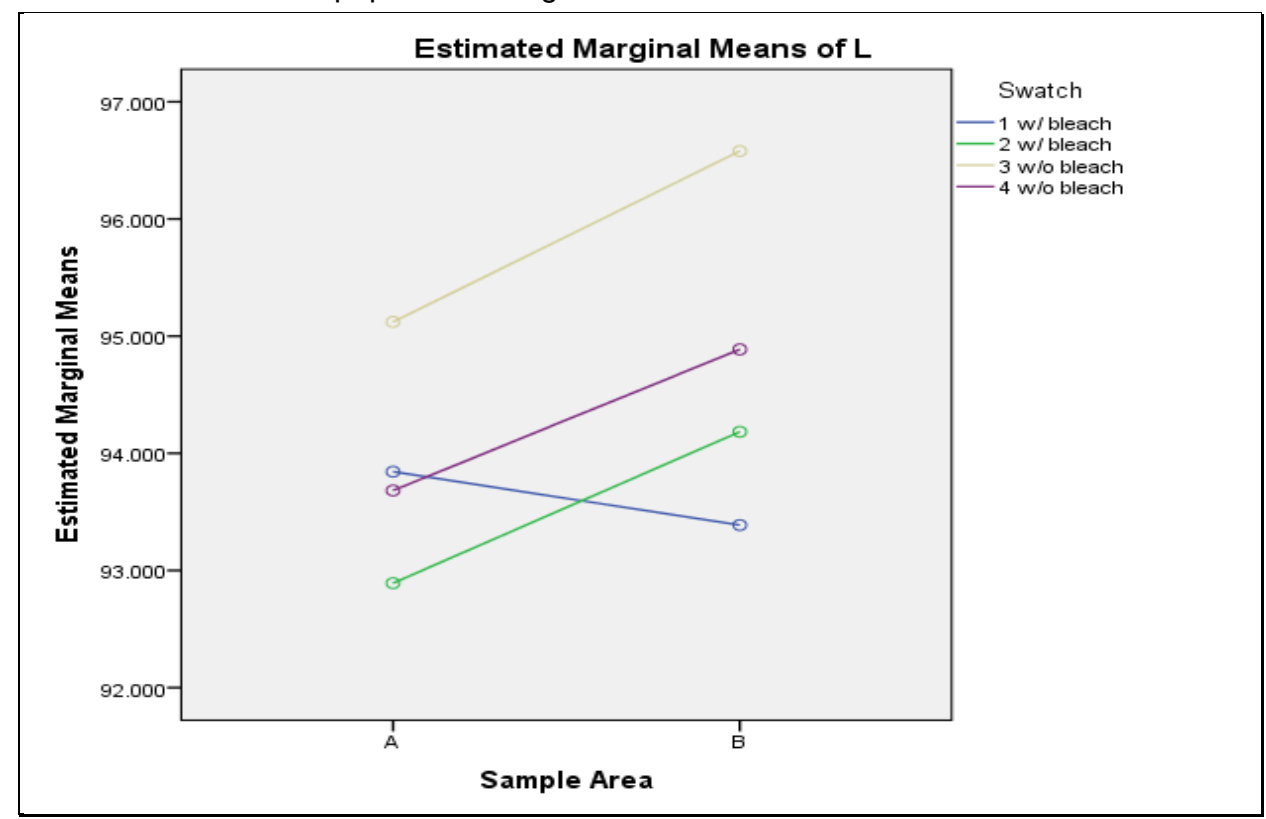

It can be observed that the difference in means is found in swatch 3 and these values increases even more between sample areas. The mean differences between swatches 2 and 4 are similar in that they both increase between the sample areas. Although these values are significant at $\mathrm{p}=0.04$, with this value so close to 0.05 , it can be observed that the significance was there but not as abundant. Swatch 1 which intersects swatches 2 and 4, did not observe significant values. 


\section{Sample Area * bleached: GMP}

Dependent Variable: L

\begin{tabular}{|ll|r|r|r|r|}
\hline \multirow{2nnnyy}{*}{ Sample Area } & & \multirow{2}{*}{ Std. Error } & \multicolumn{2}{|c|}{ 95\% Confidence Interval } \\
\cline { 5 - 6 } & & Mean & \multicolumn{1}{|c|}{ Lower Bound } & Upper Bound \\
\hline A & w/ bleach & $93.410^{\mathrm{a}}$ & .255 & 92.910 & 93.911 \\
& w/o bleach & $94.467^{\mathrm{a}}$ & .255 & 93.966 & 94.968 \\
B & w/ bleach & $93.822^{\mathrm{a}}$ & .255 & 93.321 & 94.323 \\
& w/o bleach & $95.656^{\mathrm{a}}$ & .255 & 95.155 & 96.157 \\
\hline
\end{tabular}

a. Based on modified population marginal mean.

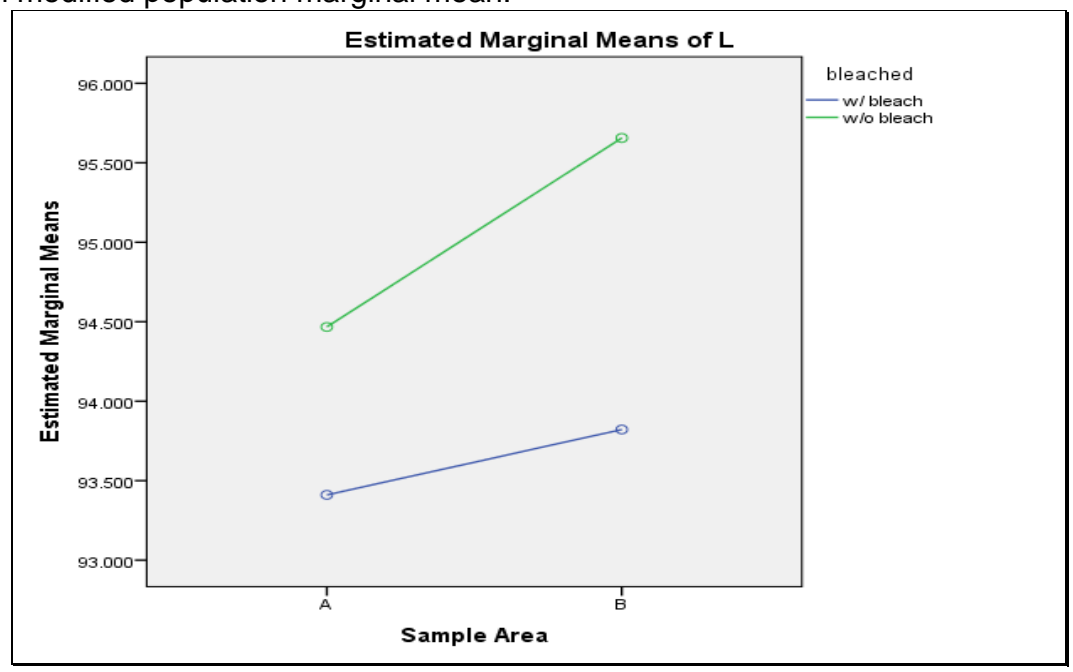

Again a difference is observed between samples at area B.

\section{7. bleached * washings: GMP}

Dependent Variable: L

\begin{tabular}{|ll|r|r|r|r|}
\hline \multirow{2}{*}{ bleached } & & & \multicolumn{2}{|c|}{ 95\% Confidence Interval } \\
\cline { 5 - 6 } & & Mean & Std. Error & Lower Bound & Upper Bound \\
\hline w/ bleach & 1W 0A & $92.235^{\mathrm{a}}$ & .599 & 91.060 & 93.410 \\
& 1W 16A & $93.815^{\mathrm{a}}$ & .423 & 92.984 & 94.646 \\
& 2W 32A & $92.582^{\mathrm{a}}$ & .423 & 91.751 & 93.412 \\
& 3W 48A & $93.104^{\mathrm{a}}$ & .423 & 92.273 & 93.934 \\
& 4W 64A & $94.021^{\mathrm{a}}$ & .423 & 93.190 & 94.852 \\
w/o bleach & 5W 80A & $95.250^{\mathrm{a}}$ & .423 & 94.419 & 96.080 \\
& 1W 0A & $92.523^{\mathrm{a}}$ & .599 & 91.348 & 93.697 \\
& 1W 16A & $97.653^{\mathrm{a}}$ & .423 & 96.822 & 98.484 \\
& 2W 32A & $94.151^{\mathrm{a}}$ & .423 & 93.320 & 94.981 \\
& 3W 48A & $95.066^{\mathrm{a}}$ & .423 & 94.235 & 95.896 \\
& 4W 64A & $94.775^{\mathrm{a}}$ & .423 & 93.944 & 95.606 \\
& 5W 80A & $94.934^{\mathrm{a}}$ & .423 & 94.103 & 95.764 \\
\hline
\end{tabular}

a. Based on modified population marginal mean. 


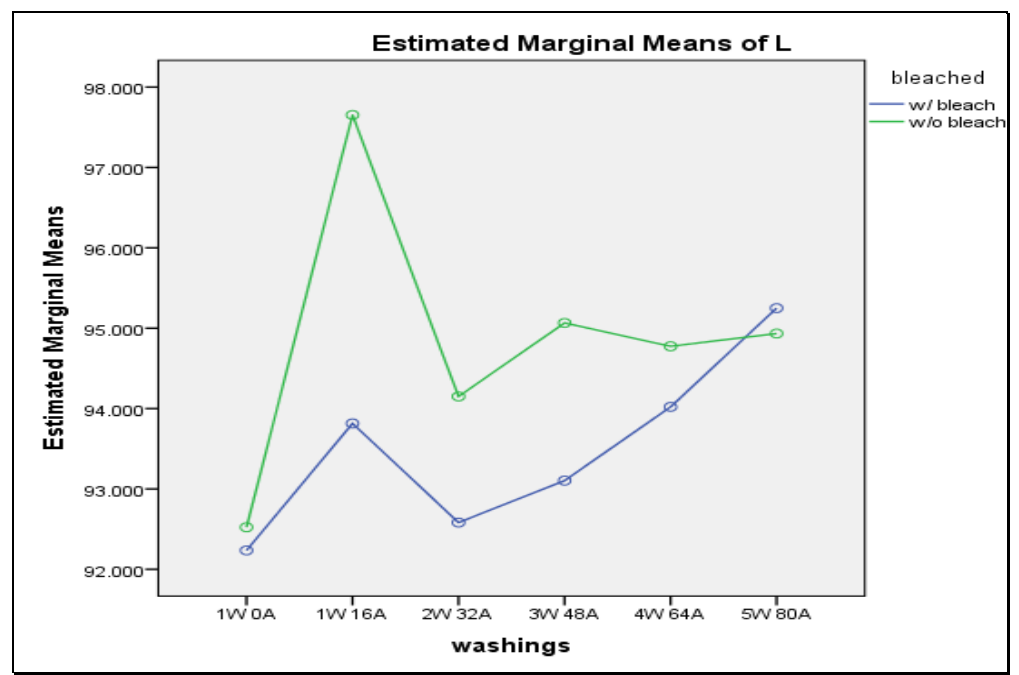

The patterns between these two samples remained consistently until the fifth cycle.

\section{Swatch * Washings: GMP}

Dependent Variable: $\mathrm{L}$

\begin{tabular}{|c|c|c|c|c|c|}
\hline \multirow{2}{*}{ Swatch } & & \multirow[b]{2}{*}{ Mean } & \multirow[b]{2}{*}{ Std. Error } & \multicolumn{2}{|c|}{ 95\% Confidence Interval } \\
\hline & & & & Lower Bound & Upper Bound \\
\hline \multirow{6}{*}{$\begin{array}{l}1 \mathrm{w} / \\
\text { bleach }\end{array}$} & $1 \mathrm{~W} 0 \mathrm{~A}$ & $94.155^{\mathrm{a}}$ & .846 & 92.493 & 95.816 \\
\hline & $1 \mathrm{~W} 16 \mathrm{~A}$ & $94.184^{\mathrm{a}}$ & .599 & 93.009 & 95.359 \\
\hline & $2 W 32 A$ & $92.298^{\mathrm{a}}$ & .599 & 91.123 & 93.473 \\
\hline & $3 W 48 A$ & $94.066^{\mathrm{a}}$ & .599 & 92.891 & 95.241 \\
\hline & $4 W 64 A$ & $94.040^{\mathrm{a}}$ & .599 & 92.865 & 95.214 \\
\hline & $5 W$ 80A & $93.332^{\mathrm{a}}$ & .599 & 92.157 & 94.507 \\
\hline \multirow{6}{*}{$\begin{array}{l}2 \text { w/ } \\
\text { bleach }\end{array}$} & $1 \mathrm{~W} 0 \mathrm{~A}$ & $90.315^{\mathrm{a}}$ & .846 & 88.654 & 91.977 \\
\hline & $1 \mathrm{~W} 16 \mathrm{~A}$ & $93.445^{\mathrm{a}}$ & .599 & 92.271 & 94.620 \\
\hline & $2 W 32 A$ & $92.865^{\mathrm{a}}$ & .599 & 91.691 & 94.040 \\
\hline & $3 W 48 A$ & $92.141^{\mathrm{a}}$ & .599 & 90.966 & 93.316 \\
\hline & $4 \mathrm{~W} 64 \mathrm{~A}$ & $94.002^{\mathrm{a}}$ & .599 & 92.827 & 95.177 \\
\hline & $5 \mathrm{~W} 80 \mathrm{~A}$ & $97.168^{\mathrm{a}}$ & .599 & 95.993 & 98.343 \\
\hline \multirow{6}{*}{$\begin{array}{l}3 \mathrm{w} / \mathrm{o} \\
\text { bleach }\end{array}$} & $1 \mathrm{~W} 0 \mathrm{~A}$ & $93.086^{\mathrm{a}}$ & .846 & 91.425 & 94.748 \\
\hline & $1 \mathrm{~W} 16 \mathrm{~A}$ & $98.150^{\mathrm{a}}$ & .599 & 96.975 & 99.325 \\
\hline & $2 W 32 A$ & $94.458^{\mathrm{a}}$ & .599 & 93.283 & 95.633 \\
\hline & $3 W 48 A$ & $95.887^{\mathrm{a}}$ & .599 & 94.713 & 97.062 \\
\hline & $4 W 64 A$ & $95.814^{\mathrm{a}}$ & .599 & 94.640 & 96.989 \\
\hline & $5 \mathrm{~W} 80 \mathrm{~A}$ & $95.958^{\mathrm{a}}$ & .599 & 94.783 & 97.133 \\
\hline \multirow{6}{*}{$\begin{array}{l}4 \mathrm{w} / 0 \\
\text { bleach }\end{array}$} & $1 \mathrm{~W} 0 \mathrm{~A}$ & $91.959^{\mathrm{a}}$ & .846 & 90.298 & 93.620 \\
\hline & $1 \mathrm{~W} 16 \mathrm{~A}$ & $97.156^{\mathrm{a}}$ & .599 & 95.981 & 98.331 \\
\hline & $2 W 32 A$ & $93.843^{a}$ & .599 & 92.668 & 95.018 \\
\hline & $3 W 48 A$ & $94.244^{\mathrm{a}}$ & .599 & 93.069 & 95.419 \\
\hline & $4 W 64 A$ & $93.736^{\mathrm{a}}$ & .599 & 92.561 & 94.910 \\
\hline & $5 W 80 A$ & $93.909^{a}$ & .599 & 92.734 & 95.084 \\
\hline
\end{tabular}

a. Based on modified population marginal mean. 


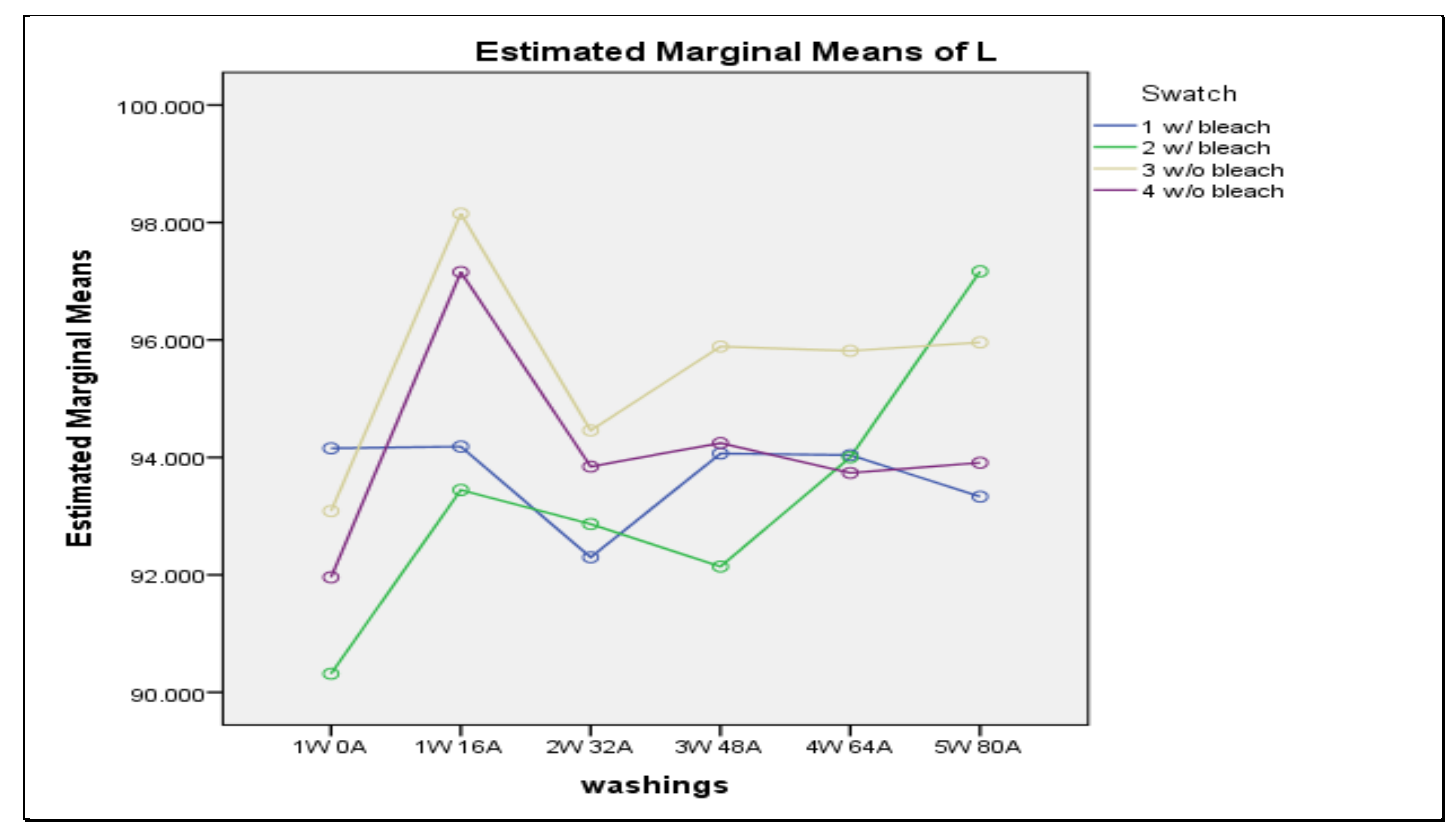

Swatches 2 and 3 follow a consistent pattern which slowly levels off between the fourth to six cycles. Swatches 1 and especially 2, varies greatly in this overall pattern.

\section{Variable ' $a$ '}

Tests of Between-Subjects Effects: GMP

Dependent Variable: a

\begin{tabular}{|c|c|c|c|c|c|}
\hline Source & Type III Sum of Squares & df & Mean Square & $\mathrm{F}$ & Sig. \\
\hline Corrected Model & $77.766^{\mathrm{a}}$ & 43 & 1.809 & 4.665 & .000 \\
\hline Intercept & 553.273 & 1 & 553.273 & 1427.056 & .000 \\
\hline SampleArea & .725 & 1 & .725 & 1.871 & .172 \\
\hline Swatch & 1.569 & 2 & .785 & 2.024 & .133 \\
\hline bleached & 0.000 & 0 & & & \\
\hline washings & 13.901 & 5 & 2.780 & 7.171 & .000 \\
\hline SampleArea * Swatch & .143 & 2 & .072 & .185 & .831 \\
\hline SampleArea * bleached & 0.000 & 0 & & & \\
\hline SampleArea * washings & 8.461 & 4 & 2.115 & 5.456 & .000 \\
\hline Swatch * bleached & 0.000 & 0 & & & \\
\hline Swatch * washings & 5.534 & 8 & .692 & 1.784 & .077 \\
\hline bleached * washings & 0.000 & 0 & & & \\
\hline SampleArea * Swatch * bleached & 0.000 & 0 & & & \\
\hline SampleArea * Swatch * washings & 7.155 & 8 & .894 & 2.307 & .019 \\
\hline SampleArea * bleached * washings & 0.000 & 0 & & & \\
\hline Swatch * bleached * washings & 0.000 & 0 & & & \\
\hline SampleArea * Swatch * bleached * washings & 0.000 & 0 & & & \\
\hline Error & 324.119 & 836 & .388 & & \\
\hline Total & 968.101 & 880 & & & \\
\hline Corrected Total & 401.885 & 879 & & & \\
\hline
\end{tabular}

a. $R$ Squared $=.194$ (Adjusted R Squared $=.152$ )

b. Computed using alpha $=.05$ 
The main effects for sample area and swatch, and the interaction effects of sample area versus

swatch and swatch versus washing did not observe significant values.

Dependent Variable: a

\section{Sample Area Estimates: GMP}

\begin{tabular}{|l|r|r|r|r|}
\hline & & \multicolumn{2}{|c|}{ Std. } & \multicolumn{2}{|c|}{$95 \%$ Confidence Interval } \\
\cline { 4 - 5 } Sample Area & Mean & Error & Lower Bound & Upper Bound \\
\hline A & $.791^{\mathrm{a}}$ & .030 & .733 & .850 \\
$\mathrm{~B}$ & $.813^{\mathrm{a}}$ & .030 & .755 & .871 \\
\hline
\end{tabular}

a. Based on modified population marginal mean.

Pairwise Comparisons: GMP

Dependent Variable: a

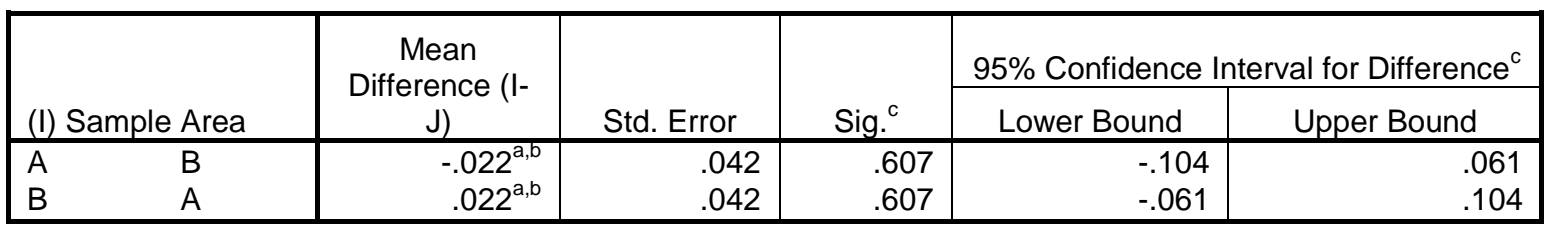

Based on estimated marginal means

a. An estimate of the modified population marginal mean (I).

b. An estimate of the modified population marginal mean (J).

c. Adjustment for multiple comparisons: Least Significant Difference (equivalent to no adjustments).

The main effects of sample as well as the variances within each level were not significant.

\section{Swatch Estimates: GMP}

Dependent Variable: a

\begin{tabular}{|c|c|c|c|c|}
\hline \multirow[b]{2}{*}{ Swatch } & \multirow[b]{2}{*}{ Mean } & \multirow[b]{2}{*}{ Std. Error } & \multicolumn{2}{|c|}{$95 \%$ Confidence Interval } \\
\hline & & & Lower Bound & Upper Bound \\
\hline $1 \mathrm{w} /$ bleach & $.950^{\mathrm{a}}$ & .042 & .867 & 1.032 \\
\hline 2 w/ bleach & $.826^{\mathrm{a}}$ & .042 & .744 & .909 \\
\hline 3 w/o bleach & $.719^{\mathrm{a}}$ & .042 & .637 & .801 \\
\hline 4 w/o bleach & $.714^{\mathrm{a}}$ & .042 & .631 & .796 \\
\hline
\end{tabular}

a. Based on modified population marginal mean.

Pairwise Comparisons: GMP

Dependent Variable: a

\begin{tabular}{|c|c|c|c|c|c|c|}
\hline & & \multirow{2}{*}{$\begin{array}{c}\text { Mean } \\
\text { Difference (I- } \\
\mathrm{J})\end{array}$} & \multirow[b]{2}{*}{ Std. Error } & \multirow[b]{2}{*}{ Sig. $^{d}$} & \multicolumn{2}{|c|}{$\begin{array}{c}\text { 95\% Confidence Interval for } \\
\text { Difference }^{d}\end{array}$} \\
\hline \multicolumn{2}{|l|}{ (I) Swatch } & & & & Lower Bound & Upper Bound \\
\hline \multirow[t]{3}{*}{$1 \mathrm{w} /$ bleach } & 2 w/ bleach & $.123^{*, b, c}$ & .059 & .038 & .007 & .240 \\
\hline & 3 w/o bleach & $.231^{\mathrm{x}, \mathrm{b}, \mathrm{c}}$ & .059 & .000 & .114 & .347 \\
\hline & 4 w/o bleach & $.236^{\pi, b, c}$ & .059 & .000 & .119 & .352 \\
\hline \multirow[t]{3}{*}{2 w/ bleach } & 1 w/ bleach & $-.123^{*, b, c}$ & .059 & .038 & -.240 & -.007 \\
\hline & 3 w/o bleach & $.107^{\mathrm{b}, \mathrm{c}}$ & .059 & .071 & -.009 & .224 \\
\hline & 4 w/o bleach & $.113^{\mathrm{b}, \mathrm{c}}$ & .059 & .058 & -.004 & .229 \\
\hline \multirow[t]{3}{*}{3 w/o bleach } & $1 \mathrm{w} /$ bleach & $-.231^{\pi, b, c}$ & .059 & .000 & -.347 & -.114 \\
\hline & 2 w/ bleach & $-.107^{\mathrm{b}, \mathrm{c}}$ & .059 & .071 & -.224 & .009 \\
\hline & 4 w/o bleach & $.005^{\mathrm{b}, \mathrm{c}}$ & .059 & .930 & -.111 & .122 \\
\hline \multirow[t]{3}{*}{4 w/o bleach } & 1 w/ bleach & $-.236^{\pi, b, c}$ & .059 & .000 & -.352 & -.119 \\
\hline & 2 w/ bleach & $-.113^{\mathrm{b}, \mathrm{c}}$ & .059 & .058 & -.229 & .004 \\
\hline & 3 w/o bleach & $-.005^{\mathrm{b}, \mathrm{c}}$ & .059 & .930 & -.122 & .111 \\
\hline
\end{tabular}

Based on estimated marginal means 
*. The mean difference is significant at the .05 level.

b. An estimate of the modified population marginal mean (I).

c. An estimate of the modified population marginal mean $(\mathrm{J})$.

d. Adjustment for multiple comparisons: Least Significant Difference (equivalent to no adjustments).

Swatches 2 and 4 and swatches 1 and 2 did not produce significant values, as this was expected

since both samples were washed under the same conditions. Of course, discrepancies observed

between the samples washed with bleach.

\section{Bleach Estimates: GMP}

Dependent Variable: a

\begin{tabular}{|c|c|c|c|c|}
\hline \multirow[b]{2}{*}{ bleached } & \multirow[b]{2}{*}{ Mean } & \multirow[b]{2}{*}{ Std. Error } & \multicolumn{2}{|c|}{ 95\% Confidence Interval } \\
\hline & & & $\begin{array}{l}\text { Lower } \\
\text { Bound }\end{array}$ & $\begin{array}{l}\text { Upper } \\
\text { Bound }\end{array}$ \\
\hline $\begin{array}{l}\text { w/ bleach } \\
\text { w/o bleach }\end{array}$ & $\begin{array}{l}.888^{\mathrm{a}} \\
.716^{\mathrm{a}}\end{array}$ & $\begin{array}{l}.030 \\
.030\end{array}$ & $\begin{array}{l}.830 \\
.658\end{array}$ & $\begin{array}{l}.946 \\
.775\end{array}$ \\
\hline
\end{tabular}

a. Based on modified population marginal mean.

Pairwise Comparisons: GMP

Dependent Variable: a

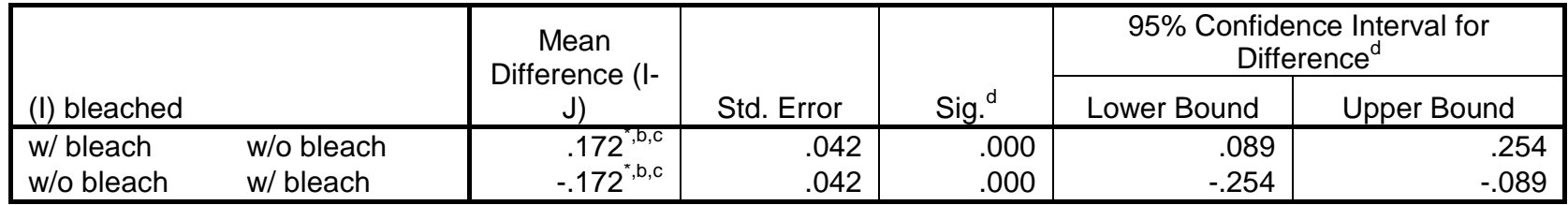

Based on estimated marginal means

*. The mean difference is significant at the .05 level.

b. An estimate of the modified population marginal mean (I).

c. An estimate of the modified population marginal mean $(\mathrm{J})$.

d. Adjustment for multiple comparisons: Least Significant Difference (equivalent to no adjustments).

Within this group, significant differences were observed between levels of pair-wise groups.

\section{Washing Estimates: GMP}

Dependent Variable: a

\begin{tabular}{|c|c|c|c|c|}
\hline \multirow[b]{2}{*}{ washings } & \multirow[b]{2}{*}{ Mean } & \multirow[b]{2}{*}{ Std. Error } & \multicolumn{2}{|c|}{$95 \%$ Confidence Interval } \\
\hline & & & Lower Bound & Upper Bound \\
\hline $1 \mathrm{~W} 0 \mathrm{~A}$ & $1.018^{\mathrm{a}}$ & .070 & .882 & 1.155 \\
\hline $1 \mathrm{~W} 16 \mathrm{~A}$ & $.925^{\mathrm{a}}$ & .049 & .828 & 1.021 \\
\hline $2 W 32 A$ & $.828^{\mathrm{a}}$ & .049 & .731 & .925 \\
\hline $3 W 48 A$ & $.846^{\mathrm{a}}$ & .049 & .750 & .943 \\
\hline $4 W 64 A$ & $.656^{\mathrm{a}}$ & .049 & .560 & .753 \\
\hline $5 W$ 80A & $.647^{\mathrm{a}}$ & .049 & .550 & .744 \\
\hline
\end{tabular}

a. Based on modified population marginal mean. 
Pairwise Comparisons: GMP

Dependent Variable: a

\begin{tabular}{|c|c|c|c|c|c|c|}
\hline \multirow{2}{*}{ (I) washings } & & \multirow{2}{*}{$\begin{array}{l}\text { Mean Difference } \\
(I-J)\end{array}$} & \multirow[b]{2}{*}{ Std. Error } & \multirow[b]{2}{*}{ Sig. ${ }^{d}$} & \multicolumn{2}{|c|}{$\begin{array}{c}\text { 95\% Confidence Interval for } \\
\text { Difference }^{d}\end{array}$} \\
\hline & & & & & Lower Bound & Upper Bound \\
\hline \multirow[t]{5}{*}{$1 \mathrm{~W} 0 \mathrm{~A}$} & $1 \mathrm{~W} 16 \mathrm{~A}$ & $.093^{\mathrm{a}, \mathrm{b}}$ & .085 & .273 & -.074 & .261 \\
\hline & $2 W 32 A$ & $.190^{\mathrm{a}, \mathrm{b},{ }^{*}}$ & .085 & .026 & .023 & .358 \\
\hline & $3 W 48 A$ & $.172^{\mathrm{a}, \mathrm{b},{ }^{*}}$ & .085 & .044 & .005 & .339 \\
\hline & $4 \mathrm{~W} 64 \mathrm{~A}$ & $.362^{\mathrm{a}, \mathrm{b},{ }^{*}}$ & .085 & .000 & .195 & .529 \\
\hline & $5 \mathrm{~W} 80 \mathrm{~A}$ & $.371^{\mathrm{a}, \mathrm{b},{ }^{*}}$ & .085 & .000 & .204 & .539 \\
\hline \multirow[t]{5}{*}{$1 \mathrm{~W} 16 \mathrm{~A}$} & $1 \mathrm{~W} 0 \mathrm{~A}$ & $-.093^{a, b}$ & .085 & .273 & -.261 & .074 \\
\hline & $2 \mathrm{~W} 32 \mathrm{~A}$ & $.097^{\mathrm{a}, \mathrm{b}}$ & .070 & .164 & -.040 & .234 \\
\hline & $3 W 48 A$ & $.078^{\mathrm{a}, \mathrm{b}}$ & .070 & .260 & -.058 & .215 \\
\hline & $4 \mathrm{~W} 64 \mathrm{~A}$ & $.268^{\mathrm{a}, \mathrm{b},{ }^{*}}$ & .070 & .000 & .132 & .405 \\
\hline & $5 \mathrm{~W} 80 \mathrm{~A}$ & $.278^{\mathrm{a}, \mathrm{b},{ }^{*}}$ & .070 & .000 & .141 & .414 \\
\hline \multirow[t]{5}{*}{$2 \mathrm{~W} 32 \mathrm{~A}$} & $1 \mathrm{~W} 0 \mathrm{~A}$ & $-.190^{\mathrm{a}, \mathrm{b},{ }^{*}}$ & .085 & .026 & -.358 & -.023 \\
\hline & $1 \mathrm{~W} 16 \mathrm{~A}$ & $-.097^{\mathrm{a}, \mathrm{b}}$ & .070 & .164 & -.234 & .040 \\
\hline & $3 W 48 A$ & $-.018^{\mathrm{a}, \mathrm{b}}$ & .070 & .791 & -.155 & .118 \\
\hline & $4 \mathrm{~W} 64 \mathrm{~A}$ & $.171^{\mathrm{a}, \mathrm{b},{ }^{*}}$ & .070 & .014 & .035 & .308 \\
\hline & $5 W 80 A$ & $.181^{\mathrm{a}, \mathrm{b},{ }^{*}}$ & .070 & .010 & .044 & .317 \\
\hline \multirow[t]{5}{*}{$3 \mathrm{~W} 48 \mathrm{~A}$} & $1 \mathrm{~W} 0 \mathrm{~A}$ & $-.172^{\mathrm{a}, \mathrm{b},{ }^{*}}$ & .085 & .044 & -.339 & -.005 \\
\hline & $1 \mathrm{~W} 16 \mathrm{~A}$ & $-.078^{a, b}$ & .070 & .260 & -.215 & .058 \\
\hline & $2 W 32 A$ & $.018^{a, b}$ & .070 & .791 & -.118 & .155 \\
\hline & $4 \mathrm{~W} 64 \mathrm{~A}$ & $.190^{\mathrm{a}, \mathrm{b},{ }^{*}}$ & .070 & .006 & .053 & .327 \\
\hline & $5 \mathrm{~W} 80 \mathrm{~A}$ & $.199^{\mathrm{a}, \mathrm{b},{ }^{*}}$ & .070 & .004 & .063 & .336 \\
\hline \multirow[t]{5}{*}{$4 \mathrm{~W} 64 \mathrm{~A}$} & $1 \mathrm{~W} 0 \mathrm{~A}$ & $-.362^{\mathrm{a}, \mathrm{b},{ }^{*}}$ & .085 & .000 & -.529 & -.195 \\
\hline & $1 \mathrm{~W} 16 \mathrm{~A}$ & $-.268^{a, b,{ }^{*}}$ & .070 & .000 & -.405 & -.132 \\
\hline & $2 W 32 A$ & $-.171^{\mathrm{a}, \mathrm{b},{ }^{\prime}}$ & .070 & .014 & -.308 & -.035 \\
\hline & $3 W 48 A$ & $-.190^{\mathrm{a}, \mathrm{b},{ }^{*}}$ & .070 & .006 & -.327 & -.053 \\
\hline & $5 \mathrm{~W} 80 \mathrm{~A}$ & $.009^{a, b}$ & .070 & .893 & -.127 & .146 \\
\hline \multirow[t]{5}{*}{$5 \mathrm{~W} 80 \mathrm{~A}$} & $1 \mathrm{~W} 0 \mathrm{~A}$ & $-.371^{\mathrm{a}, \mathrm{b}, *}$ & .085 & .000 & -.539 & -.204 \\
\hline & $1 \mathrm{~W} 16 \mathrm{~A}$ & $-.278^{\mathrm{a}, \mathrm{b},{ }^{*}}$ & .070 & .000 & -.414 & -.141 \\
\hline & $2 W 32 A$ & $-.181^{a, b,{ }^{*}}$ & .070 & .010 & -.317 & -.044 \\
\hline & $3 \mathrm{~W} 48 \mathrm{~A}$ & $-.199^{\mathrm{a}, \mathrm{b},{ }^{*}}$ & .070 & .004 & -.336 & -.063 \\
\hline & $4 \mathrm{~W} 64 \mathrm{~A}$ & $-.009^{\mathrm{a}, \mathrm{b}}$ & .070 & .893 & -.146 & .127 \\
\hline
\end{tabular}

Based on estimated marginal means

*. The mean difference is significant at the .05 level.

a. An estimate of the modified population marginal mean (I).

b. An estimate of the modified population marginal mean $(\mathrm{J})$.

d. Adjustment for multiple comparisons: Least Significant Difference (equivalent to no adjustments).

Within this group, significant differences were observed between levels of pair-wise groups.

None of the pair-wise group stood out above the other. 
Dependent Variable: a

\section{Sample Area * Swatch: GMP}

\begin{tabular}{|c|c|c|c|c|c|}
\hline \multirow{2}{*}{ Sample Area } & & \multirow[b]{2}{*}{ Mean } & \multirow[b]{2}{*}{ Std. Error } & \multicolumn{2}{|c|}{ 95\% Confidence Interval } \\
\hline & & & & $\begin{array}{l}\text { Lower } \\
\text { Bound }\end{array}$ & Upper Bound \\
\hline \multirow[t]{4}{*}{$\bar{A}$} & $1 \mathrm{w} /$ bleach & $.843^{\mathrm{a}}$ & .057 & .732 & .955 \\
\hline & $2 \mathrm{w} /$ bleach & $.720^{\mathrm{a}}$ & .062 & .598 & .842 \\
\hline & 3 w/o bleach & $.814^{\mathrm{a}}$ & .057 & .702 & .925 \\
\hline & 4 w/o bleach & $.773^{\mathrm{a}}$ & .062 & .651 & .896 \\
\hline \multirow[t]{4}{*}{$B$} & $1 \mathrm{w} /$ bleach & $1.077^{\mathrm{a}}$ & .062 & .955 & 1.199 \\
\hline & $2 \mathrm{w} /$ bleach & $.915^{\mathrm{a}}$ & .057 & .804 & 1.027 \\
\hline & 3 w/o bleach & $.605^{\mathrm{a}}$ & .062 & .483 & .727 \\
\hline & 4 w/o bleach & $.664^{\mathrm{a}}$ & .057 & .552 & .775 \\
\hline
\end{tabular}

a. Based on modified population marginal mean.

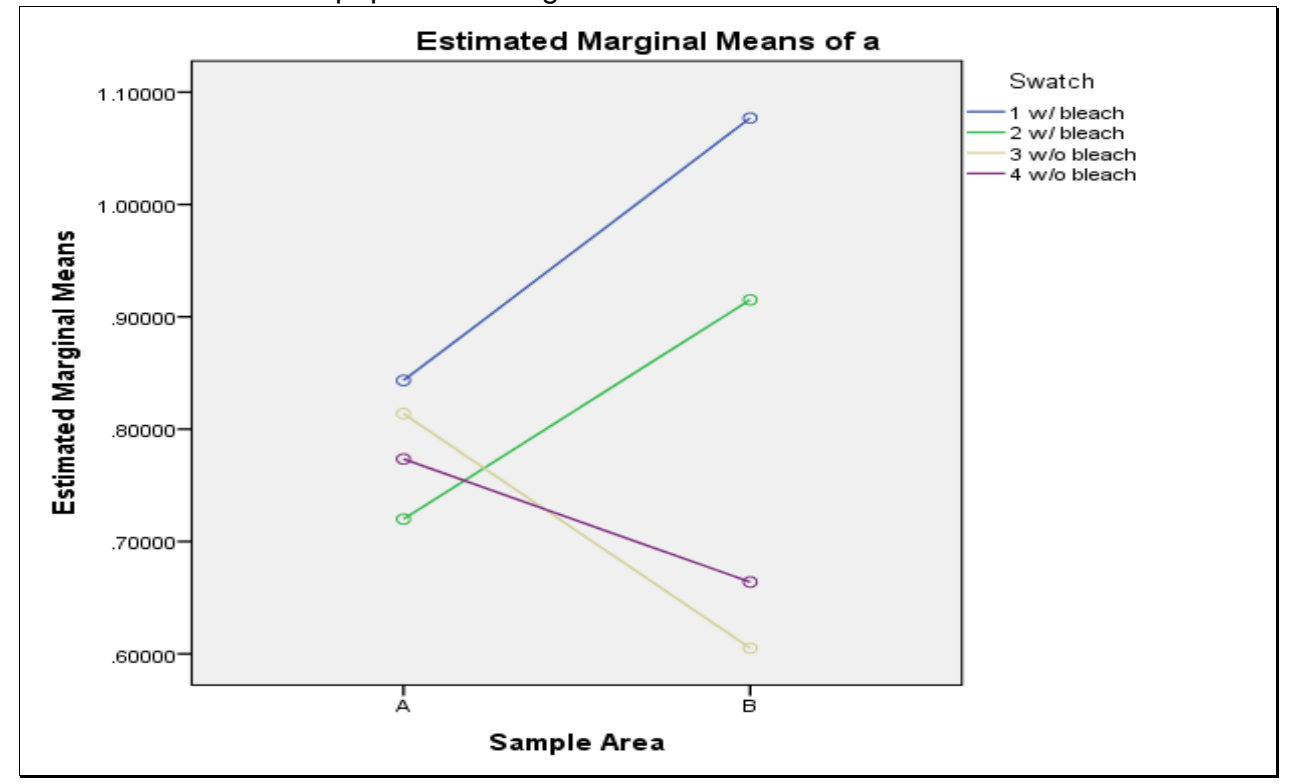

It is obvious that the mean difference between swatch 1 and 3 at sample area A is small but very large at sample area B. It can be expected that at this area, interaction occurs although it is not significant. And the same can be said for swatch 4 at this area.

\section{Sample Area * Bleached: GMP}

Dependent Variable: a

\begin{tabular}{|c|c|c|c|c|c|}
\hline \multirow{2}{*}{ Sample Area } & & \multirow[b]{2}{*}{ Mean } & \multirow[b]{2}{*}{ Std. Error } & \multicolumn{2}{|c|}{ 95\% Confidence Interval } \\
\hline & & & & $\begin{array}{l}\text { Lower } \\
\text { Bound }\end{array}$ & Upper Bound \\
\hline $\mathrm{A}$ & w/ bleach & $.787^{\mathrm{a}}$ & .042 & .705 & .870 \\
\hline & w/o bleach & $.795^{\mathrm{a}}$ & .042 & .713 & .878 \\
\hline \multirow[t]{2}{*}{ B } & w/ bleach & $.989^{\mathrm{a}}$ & .042 & .906 & 1.071 \\
\hline & w/o bleach & $.637^{\mathrm{a}}$ & .042 & .555 & .720 \\
\hline
\end{tabular}

a. Based on modified population marginal mean. 


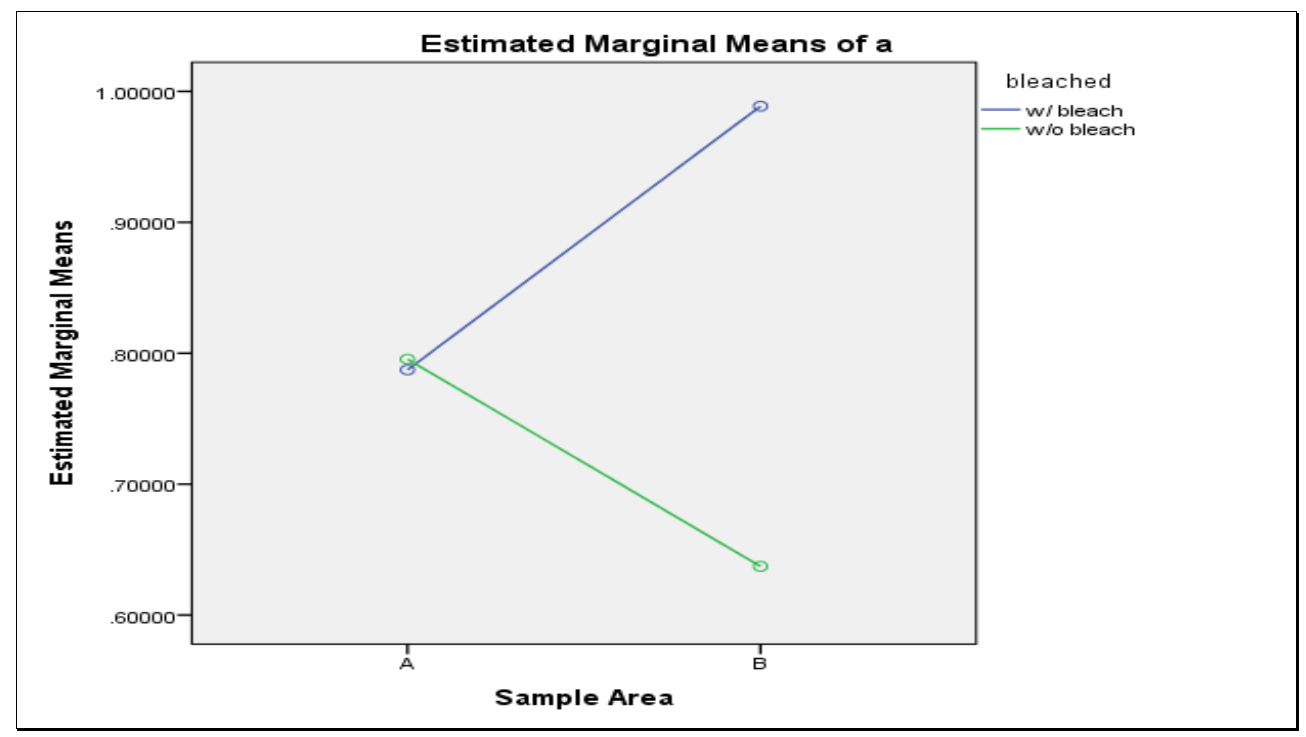

For sample area A, the means are similar between bleached samples. However, for sample area $\mathrm{B}$, this is opposite. It can then be expected that the significant interaction occurs here.

\section{Bleached * Washings: GMP}

Dependent Variable: a

\begin{tabular}{|ll|r|r|r|r|}
\hline \multirow{2}{*}{ bleached } & & & \multicolumn{2}{c|}{ 95\% Confidence Interval } \\
\cline { 4 - 5 } & & Mean & Std. Error & \multicolumn{1}{c|}{$\begin{array}{c}\text { Lower } \\
\text { Bound }\end{array}$} & Upper Bound \\
\hline w/ bleach & 1W 0A & $.929^{\mathrm{a}}$ & .098 & .736 & 1.123 \\
& 1W 16A & $1.129^{\mathrm{a}}$ & .070 & .992 & 1.265 \\
& 2W 32A & $.816^{\mathrm{a}}$ & .070 & .679 & .953 \\
& 3W 48A & $.929^{\mathrm{a}}$ & .070 & .792 & 1.065 \\
& 4W 64A & $.606^{\mathrm{a}}$ & .070 & .470 & .743 \\
w/o bleach & $.940^{\mathrm{a}}$ & .070 & .803 & 1.076 \\
& 5W 80A & $1.107^{\mathrm{a}}$ & .098 & .914 & 1.300 \\
& 1W 0A & $.721^{\mathrm{a}}$ & .070 & .584 & .858 \\
& 1W 16A & $.840^{\mathrm{a}}$ & .070 & .703 & .977 \\
& 2W 32A & $.764^{\mathrm{a}}$ & .070 & .627 & .901 \\
& 3W 48A & $.707^{\mathrm{a}}$ & .070 & .570 & .843 \\
& 4W 64A & $.355^{\mathrm{a}}$ & .070 & .218 & .491 \\
\hline
\end{tabular}

a. Based on modified population marginal mean. 


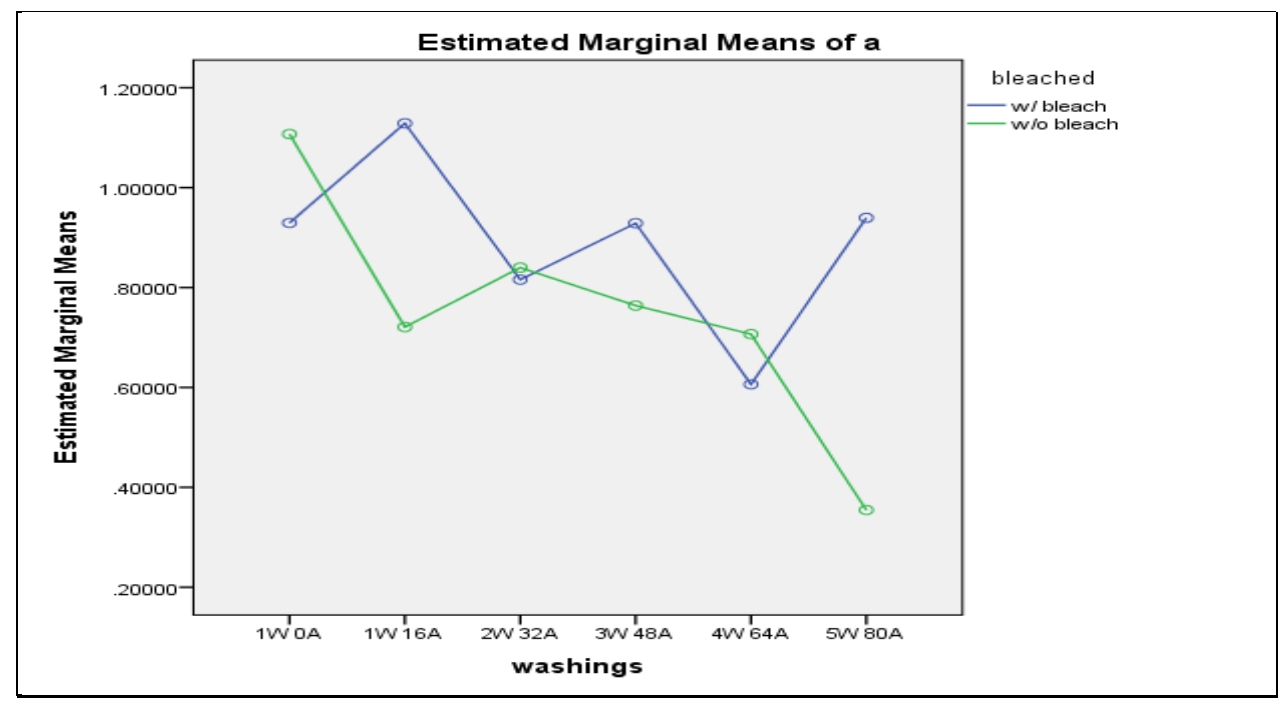

Where the means are similar, as observed at 2W 32A, no significant interaction is expected here.

However, where the means differ moderately, significant interaction is expected.

\section{Swatch * Washings: GMP}

Dependent Variable: a

\begin{tabular}{|c|c|c|c|c|c|}
\hline \multirow{2}{*}{ Swatch } & & \multirow[b]{2}{*}{ Mean } & \multirow[b]{2}{*}{ Std. Error } & \multicolumn{2}{|c|}{ 95\% Confidence Interval } \\
\hline & & & & $\begin{array}{l}\text { Lower } \\
\text { Bound }\end{array}$ & Upper Bound \\
\hline \multirow[t]{6}{*}{$1 \mathrm{w} /$ bleach } & $1 \mathrm{~W} 0 \mathrm{~A}$ & $1.027^{\mathrm{a}}$ & .139 & .754 & 1.301 \\
\hline & $1 \mathrm{~W} 16 \mathrm{~A}$ & $1.277^{\mathrm{a}}$ & .098 & 1.083 & 1.470 \\
\hline & $2 W 32 A$ & $.964^{\mathrm{a}}$ & .098 & .771 & 1.157 \\
\hline & $3 W 48 A$ & $.949^{a}$ & .098 & .755 & 1.142 \\
\hline & $4 \mathrm{~W} 64 \mathrm{~A}$ & $.652^{\mathrm{a}}$ & .098 & .458 & .845 \\
\hline & $5 W$ 80A & $.868^{a}$ & .098 & .675 & 1.061 \\
\hline \multirow[t]{6}{*}{2 w/ bleach } & $1 \mathrm{~W} 0 \mathrm{~A}$ & $.831^{\mathrm{a}}$ & .139 & .558 & 1.105 \\
\hline & $1 \mathrm{~W} 16 \mathrm{~A}$ & $.981^{\mathrm{a}}$ & .098 & .788 & 1.174 \\
\hline & $2 W 32 A$ & $.668^{\mathrm{a}}$ & .098 & .474 & .861 \\
\hline & $3 W 48 A$ & $.909^{a}$ & .098 & .716 & 1.102 \\
\hline & $4 \mathrm{~W} 64 \mathrm{~A}$ & $.561^{\mathrm{a}}$ & .098 & .367 & .754 \\
\hline & $5 W$ 80A & $1.011^{\mathrm{a}}$ & .098 & .818 & 1.205 \\
\hline \multirow[t]{6}{*}{3 w/o bleach } & $1 \mathrm{~W} 0 \mathrm{~A}$ & $1.374^{\mathrm{a}}$ & .139 & 1.101 & 1.647 \\
\hline & $1 \mathrm{~W} 16 \mathrm{~A}$ & $.707^{\mathrm{a}}$ & .098 & .514 & .900 \\
\hline & $2 W 32 A$ & $.901^{a}$ & .098 & .708 & 1.094 \\
\hline & $3 W 48 A$ & $.585^{\mathrm{a}}$ & .098 & .392 & .778 \\
\hline & $4 \mathrm{~W} 64 \mathrm{~A}$ & $.739^{a}$ & .098 & .546 & .932 \\
\hline & $5 W$ 80A & $.335^{a}$ & .098 & .142 & .528 \\
\hline \multirow[t]{6}{*}{4 w/o bleach } & $1 \mathrm{~W} 0 \mathrm{~A}$ & $.840^{\mathrm{a}}$ & .139 & .567 & 1.114 \\
\hline & $1 \mathrm{~W} 16 \mathrm{~A}$ & $.735^{\mathrm{a}}$ & .098 & .541 & .928 \\
\hline & $2 W 32 A$ & $.779^{a}$ & .098 & .586 & .972 \\
\hline & $3 W 48 A$ & $.943^{\mathrm{a}}$ & .098 & .750 & 1.136 \\
\hline & $4 W 64 A$ & $.675^{\mathrm{a}}$ & .098 & .481 & .868 \\
\hline & $5 W$ 80A & $.374^{a}$ & .098 & .181 & .567 \\
\hline
\end{tabular}

a. Based on modified population marginal mean. 


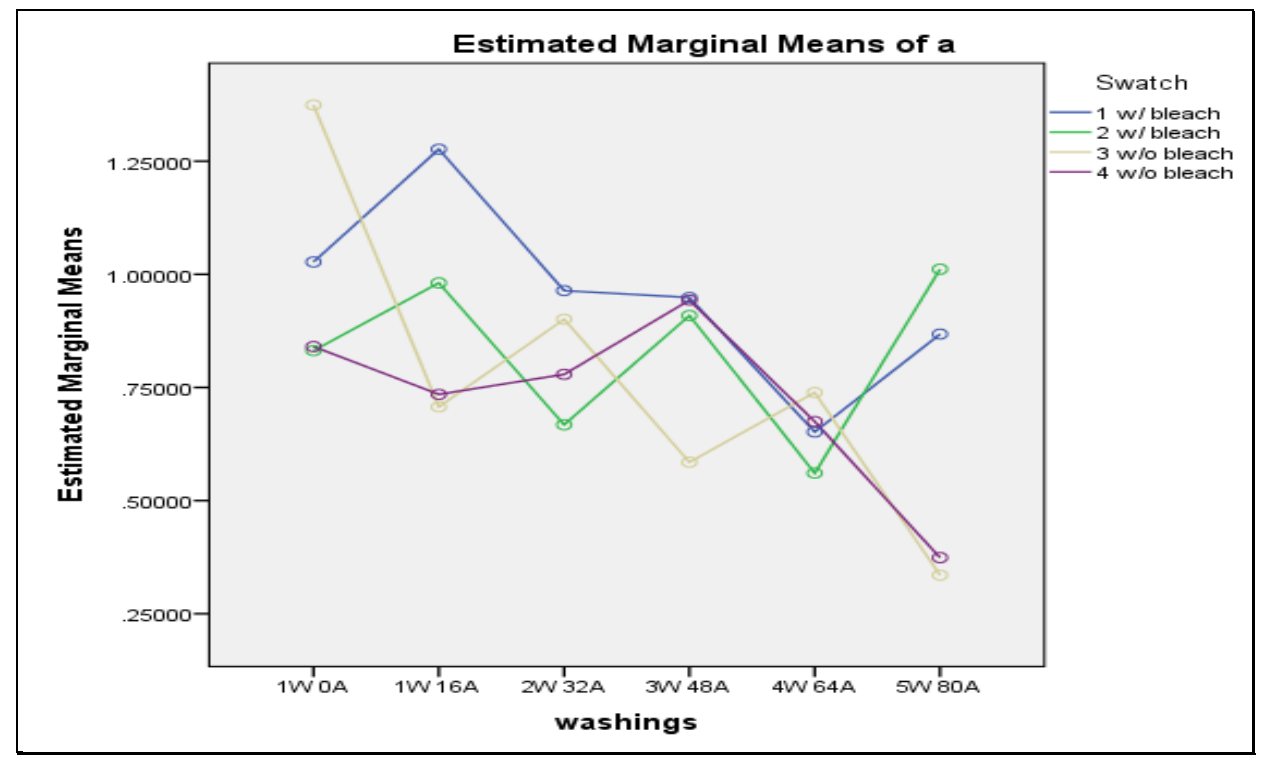

The pattern observed for swatch 1 and 2 is fairly consistent. Swatch 3 follows this pattern after the first complete washed-aged cycle. Although swatches 3 and 4 were washed under the same conditions, they had no recognizable patterns.

Variable ' $b$ '

Dependent Variable: $b$

Tests of Between-Subjects Effects: GMP

\begin{tabular}{|c|c|c|c|c|c|}
\hline Source & Type III Sum of Squares & $\mathrm{df}$ & Mean Square & $\mathrm{F}$ & Sig. \\
\hline Corrected Model & $1138.094^{\mathrm{a}}$ & 43 & 26.467 & 5.630 & .000 \\
\hline Intercept & 88995.007 & 1 & 88995.007 & 18932.141 & 0.000 \\
\hline SampleArea & 67.677 & 1 & 67.677 & 14.397 & .000 \\
\hline Swatch & 14.243 & 2 & 7.121 & 1.515 & .220 \\
\hline bleached & 0.000 & 0 & & & \\
\hline washings & 208.983 & 5 & 41.797 & 8.892 & .000 \\
\hline SampleArea * Swatch & 1.074 & 2 & .537 & .114 & .892 \\
\hline SampleArea * bleached & 0.000 & 0 & & & \\
\hline SampleArea * washings & 83.644 & 4 & 20.911 & 4.448 & .001 \\
\hline Swatch * bleached & 0.000 & 0 & & & \\
\hline Swatch * washings & 244.618 & 8 & 30.577 & 6.505 & .000 \\
\hline bleached * washings & 0.000 & 0 & & & \\
\hline SampleArea * Swatch * bleached & 0.000 & 0 & & & \\
\hline SampleArea * Swatch * washings & 123.662 & 8 & 15.458 & 3.288 & .001 \\
\hline SampleArea * bleached * washings & 0.000 & 0 & & & \\
\hline Swatch * bleached * washings & 0.000 & 0 & & & \\
\hline SampleArea * Swatch * bleached * washings & 0.000 & 0 & & & \\
\hline Error & 3929.816 & 836 & 4.701 & & \\
\hline Total & 98882.747 & 880 & & & \\
\hline Corrected Total & 5067.909 & 879 & & & \\
\hline
\end{tabular}

a. $\mathrm{R}$ Squared $=.225$ (Adjusted R Squared $=.185$ )

b. Computed using alpha $=.05$ 
The main effects of group 'Swatch' did not observe any significant results.

\section{Sample Area Estimates: GMP}

Dependent Variable: $b$

\begin{tabular}{|c|c|c|c|c|}
\hline \multirow[b]{2}{*}{ Sample Area } & \multirow[b]{2}{*}{ Mean } & \multirow[b]{2}{*}{ Std. Error } & \multicolumn{2}{|c|}{ 95\% Confidence Interval } \\
\hline & & & Lower Bound & Upper Bound \\
\hline $\mathrm{A}$ & $10.097^{a}$ & .103 & 9.894 & 10.300 \\
\hline B & $10.553^{a}$ & .103 & 10.350 & 10.756 \\
\hline
\end{tabular}

a. Based on modified population marginal mean.

Pairwise Comparisons: GMP

Dependent Variable: $b$

\begin{tabular}{|c|c|c|c|c|c|}
\hline \multirow[b]{2}{*}{ (I) Sample Area } & \multirow{2}{*}{$\begin{array}{c}\text { Mean } \\
\text { Difference (I- } \\
\mathrm{J})\end{array}$} & \multirow[b]{2}{*}{ Std. Error } & \multirow[b]{2}{*}{ Sig. ${ }^{d}$} & \multicolumn{2}{|c|}{$\begin{array}{l}\text { 95\% Confidence Interval for } \\
\text { Difference }^{d}\end{array}$} \\
\hline & & & & Lower Bound & Upper Bound \\
\hline$A \quad B$ & $-.456^{, 0, \mathrm{C}}$ & .146 & .002 & -.742 & -.169 \\
\hline$A$ & $.456^{\pi, b, c}$ & .146 & .002 & .169 & .742 \\
\hline
\end{tabular}

Based on estimated marginal means

*. The mean difference is significant at the .05 level.

b. An estimate of the modified population marginal mean (I).

c. An estimate of the modified population marginal mean $(\mathrm{J})$.

d. Adjustment for multiple comparisons: Least Significant Difference (equivalent to no adjustments).

Although the main effect was not significant, the within group effect was significant.

\section{Swatch Estimates: GMP}

Dependent Variable: $b$

\begin{tabular}{|l|r|r|r|r|}
\hline & & & \multicolumn{2}{|c|}{$95 \%$ Confidence Interval } \\
\cline { 4 - 5 } Swatch & Mean & Std. Error & Lower Bound & \multicolumn{1}{c|}{ Upper Bound } \\
\hline 1 w/ bleach & $10.578^{\mathrm{a}}$ & .146 & 10.291 & 10.865 \\
2 w/ bleach & $10.297^{\mathrm{a}}$ & .146 & 10.010 & 10.584 \\
3 w/o bleach & $10.384^{\mathrm{a}}$ & .146 & 10.097 & 10.670 \\
4 w/o bleach & $10.042^{\mathrm{a}}$ & .146 & 9.756 & 10.329 \\
\hline
\end{tabular}

a. Based on modified population marginal mean.

Pairwise Comparisons: GMP

Dependent Variable: $b$

\begin{tabular}{|c|c|c|c|c|c|c|}
\hline \multirow[b]{2}{*}{ (I) Swatch } & & \multirow{2}{*}{$\begin{array}{c}\text { Mean } \\
\text { Difference (I- } \\
\mathrm{J})\end{array}$} & \multirow[b]{2}{*}{ Std. Error } & \multirow[b]{2}{*}{ Sig. ${ }^{d}$} & \multicolumn{2}{|c|}{$95 \%$ Confidence Interval for Difference ${ }^{d}$} \\
\hline & & & & & Lower Bound & Upper Bound \\
\hline \multirow[t]{3}{*}{$1 \mathrm{w} /$ bleach } & $2 \mathrm{w} /$ bleach & $.281^{\mathrm{a}, \mathrm{b}}$ & .207 & .174 & -.125 & .687 \\
\hline & 3 w/o bleach & $.194^{\mathrm{a}, \mathrm{b}}$ & .207 & .348 & -.212 & .600 \\
\hline & 4 w/o bleach & $.535^{\mathrm{a}, \mathrm{b},{ }^{*}}$ & .207 & .010 & .129 & .941 \\
\hline \multirow[t]{3}{*}{$2 \mathrm{w} /$ bleach } & $1 \mathrm{w} /$ bleach & $-.281^{\mathrm{a}, \mathrm{b}}$ & .207 & .174 & -.687 & .125 \\
\hline & 3 w/o bleach & $-.087^{\mathrm{a}, \mathrm{b}}$ & .207 & .674 & -.493 & .319 \\
\hline & $4 \mathrm{w} / \mathrm{o}$ bleach & $.254^{\mathrm{a}, \mathrm{b}}$ & .207 & .219 & -.152 & .660 \\
\hline \multirow[t]{3}{*}{3 w/o bleach } & $1 \mathrm{w} /$ bleach & $-.194^{\mathrm{a}, \mathrm{b}}$ & .207 & .348 & -.600 & .212 \\
\hline & $2 \mathrm{w} /$ bleach & $.087^{\mathrm{a}, \mathrm{b}}$ & .207 & .674 & -.319 & .493 \\
\hline & 4 w/o bleach & $.341^{a, b}$ & .207 & .099 & -.065 & .747 \\
\hline \multirow[t]{3}{*}{4 w/o bleach } & $1 \mathrm{w} /$ bleach & $-.535^{\mathrm{a}, \mathrm{b},{ }^{*}}$ & .207 & .010 & -.941 & -.129 \\
\hline & $2 \mathrm{w} /$ bleach & $-.254^{\mathrm{a}, \mathrm{b}}$ & .207 & .219 & -.660 & .152 \\
\hline & 3 w/o bleach & $-.341^{a, b}$ & .207 & .099 & -.747 & .065 \\
\hline
\end{tabular}

Based on estimated marginal means

*. The mean difference is significant at the .05 level. 
a. An estimate of the modified population marginal mean (I).

b. An estimate of the modified population marginal mean (J).

d. Adjustment for multiple comparisons: Least Significant Difference (equivalent to no adjustments).

Swatch 2 observed to be the only group that was not significantly difference from the others.

There were no consistent patterns between what was washed with bleach versus what was

washed without.

\section{Bleach Estimates: GMP}

Dependent Variable: $b$

\begin{tabular}{|l|c|r|r|r|}
\hline & & \multirow{2}{*}{ bleached } & \multicolumn{3}{|c|}{$95 \%$ Confidence Interval } \\
\cline { 4 - 5 } & Mean & Std. Error & Lower Bound & Upper Bound \\
\hline w/ bleach & $10.437^{\mathrm{a}}$ & .103 & 10.234 & 10.640 \\
w/o bleach & $10.213^{\mathrm{a}}$ & .103 & 10.010 & 10.416 \\
\hline
\end{tabular}

a. Based on modified population marginal mean.

Pairwise Comparisons: GMP

Dependent Variable: $b$

\begin{tabular}{|c|c|c|c|c|c|c|}
\hline \multirow[b]{2}{*}{ (I) bleached } & & \multirow{2}{*}{$\begin{array}{c}\text { Mean } \\
\text { Difference } \\
(I-J)\end{array}$} & \multirow[b]{2}{*}{ Std. Error } & \multirow[b]{2}{*}{ Sig. ${ }^{c}$} & \multicolumn{2}{|c|}{$\begin{array}{c}\text { 95\% Confidence Interval for } \\
\text { Difference }^{\text {c }}\end{array}$} \\
\hline & & & & & Lower Bound & Upper Bound \\
\hline $\begin{array}{l}\text { w/ bleach } \\
\text { w/o bleach }\end{array}$ & $\begin{array}{l}\text { w/o bleach } \\
\text { w/ bleach }\end{array}$ & $\begin{array}{r}.224^{a, b} \\
-.224^{a, b}\end{array}$ & $\begin{array}{l}.146 \\
.146\end{array}$ & $\begin{array}{l}.126 \\
.126\end{array}$ & $\begin{array}{r}-.063 \\
-.511\end{array}$ & $\begin{array}{r}.511 \\
.063\end{array}$ \\
\hline
\end{tabular}

Based on estimated marginal means

a. An estimate of the modified population marginal mean (I).

b. An estimate of the modified population marginal mean (J).

c. Adjustment for multiple comparisons: Least Significant Difference (equivalent to no adjustments).

No significant difference was observed between the bleached and unbleached samples. This

gives clarity to the previous interpretation where no pattern could be observed among the

swatches washed with and without bleach.

\section{Washing Estimates: GMP}

Dependent Variable: $b$

\begin{tabular}{|l|r|r|r|r|}
\hline & & \multirow{2}{*}{\begin{tabular}{c} 
Std. \\
\cline { 4 - 5 } washings
\end{tabular}} & \multicolumn{1}{|c|}{ Mean } & \multicolumn{2}{|c|}{$\begin{array}{c}\text { Low Confidence Interval } \\
\text { Error }\end{array}$} & $\begin{array}{l}\text { Lound } \\
\text { Bound }\end{array}$ & Upper Bound \\
\hline 1W 0A & $11.265^{\mathrm{a}}$ & .242 & 10.789 & 11.741 \\
1W 16A & $10.794^{\mathrm{a}}$ & .171 & 10.457 & 11.130 \\
2W 32A & $9.621^{\mathrm{a}}$ & .171 & 9.284 & 9.957 \\
3W 48A & $10.539^{\mathrm{a}}$ & .171 & 10.203 & 10.876 \\
4W 64A & $9.909^{\mathrm{a}}$ & .171 & 9.572 & 10.245 \\
5W 80A & $10.293^{\mathrm{a}}$ & .171 & 9.957 & 10.630 \\
\hline
\end{tabular}

a. Based on modified population marginal mean. 
Pairwise Comparisons: GMP

Dependent Variable: $\mathrm{b}$

\begin{tabular}{|c|c|c|c|c|c|c|}
\hline & & \multirow{2}{*}{$\begin{array}{c}\text { Mean } \\
\text { Difference } \\
(I-J)\end{array}$} & \multirow[b]{2}{*}{ Std. Error } & \multirow[b]{2}{*}{ Sig. ${ }^{d}$} & \multicolumn{2}{|c|}{$\begin{array}{c}\text { 95\% Confidence Interval for } \\
\text { Difference }^{\mathrm{d}}\end{array}$} \\
\hline \multicolumn{2}{|c|}{ (I) washings } & & & & $\begin{array}{l}\text { Lower } \\
\text { Bound }\end{array}$ & Upper Bound \\
\hline \multirow[t]{5}{*}{$1 \mathrm{~W} 0 \mathrm{~A}$} & $1 \mathrm{~W} 16 \mathrm{~A}$ & $.471^{\mathrm{a}, \mathrm{b}}$ & .297 & .113 & -.112 & 1.054 \\
\hline & $2 W 32 A$ & $1.644^{\mathrm{a}, \mathrm{b},{ }^{,}}$ & .297 & .000 & 1.061 & 2.227 \\
\hline & $3 W 48 A$ & $.726^{\mathrm{a}, \mathrm{b},{ }^{*}}$ & 297 & .015 & .143 & 1.309 \\
\hline & $4 \mathrm{~W} 64 \mathrm{~A}$ & $1.356^{\mathrm{a}, \mathrm{b},{ }^{*}}$ & .297 & .000 & .773 & 1.939 \\
\hline & $5 W 80 A$ & $.972^{\mathrm{a}, \mathrm{b},{ }^{*}}$ & .297 & .001 & .389 & 1.555 \\
\hline \multirow[t]{5}{*}{$1 \mathrm{~W} 16 \mathrm{~A}$} & $1 \mathrm{~W} 0 \mathrm{~A}$ & $-.471^{a, b}$ & .297 & .113 & -1.054 & .112 \\
\hline & $2 W 32 A$ & $1.173^{\mathrm{a}, \mathrm{b},{ }^{\star}}$ & .242 & .000 & .697 & 1.649 \\
\hline & $3 W 48 A$ & $.255^{\mathrm{a}, \mathrm{b}}$ & .242 & .293 & -.221 & .731 \\
\hline & $4 \mathrm{~W} 64 \mathrm{~A}$ & $.885^{\mathrm{a}, \mathrm{b},{ }^{*}}$ & .242 & .000 & .409 & 1.361 \\
\hline & $5 W 80 A$ & $.501^{\mathrm{a}, \mathrm{b},{ }^{*}}$ & .242 & .039 & .025 & .977 \\
\hline \multirow[t]{5}{*}{$2 W 32 A$} & $1 \mathrm{~W} 0 \mathrm{~A}$ & $-1.644^{a, b,{ }^{*}}$ & .297 & .000 & -2.227 & -1.061 \\
\hline & $1 \mathrm{~W} 16 \mathrm{~A}$ & $-1.173^{\mathrm{a}, \mathrm{b},{ }^{*}}$ & .242 & .000 & -1.649 & -.697 \\
\hline & $3 W 48 A$ & $-.918^{\mathrm{a}, \mathrm{b},{ }^{x}}$ & .242 & .000 & -1.394 & -.443 \\
\hline & $4 \mathrm{~W} 64 \mathrm{~A}$ & $-.288^{a, b}$ & .242 & 235 & -.764 & .188 \\
\hline & $5 W 80 A$ & $-.672^{\mathrm{a}, \mathrm{b},{ }^{*}}$ & .242 & .006 & -1.148 & -.197 \\
\hline \multirow[t]{5}{*}{$3 W 48 A$} & $1 \mathrm{~W} 0 \mathrm{~A}$ & $-.726^{\mathrm{a}, \mathrm{b},{ }^{*}}$ & .297 & .015 & -1.309 & -.143 \\
\hline & $1 \mathrm{~W} 16 \mathrm{~A}$ & $-.255^{a, b}$ & .242 & .293 & -.731 & .221 \\
\hline & $2 W 32 A$ & $.918^{\mathrm{a}, \mathrm{b},{ }^{*}}$ & .242 & .000 & .443 & 1.394 \\
\hline & $4 \mathrm{~W} 64 \mathrm{~A}$ & $.630^{\mathrm{a}, \mathrm{b},{ }^{*}}$ & .242 & .009 & .154 & 1.106 \\
\hline & $5 W 80 A$ & $.246^{\mathrm{a}, \mathrm{b}}$ & .242 & .310 & -.230 & .722 \\
\hline \multirow[t]{5}{*}{$4 \mathrm{~W} 64 \mathrm{~A}$} & $1 \mathrm{~W} 0 \mathrm{~A}$ & $-1.356^{\mathrm{a}, \mathrm{b},{ }^{*}}$ & .297 & .000 & -1.939 & -.773 \\
\hline & $1 \mathrm{~W} 16 \mathrm{~A}$ & $-.885^{\mathrm{a}, \mathrm{b},{ }^{*}}$ & .242 & .000 & -1.361 & -.409 \\
\hline & $2 \mathrm{~W} 32 \mathrm{~A}$ & $.288^{\mathrm{a}, \mathrm{b}}$ & .242 & .235 & -.188 & .764 \\
\hline & $3 W 48 A$ & $-.630^{\mathrm{a}, \mathrm{b},{ }^{*}}$ & .242 & .009 & -1.106 & -.154 \\
\hline & $5 W 80 A$ & $-.384^{a, b}$ & .242 & .113 & -.860 & .091 \\
\hline \multirow[t]{5}{*}{$5 W 80 A$} & $1 \mathrm{~W} 0 \mathrm{~A}$ & $-.972^{\mathrm{a}, \mathrm{b},{ }^{*}}$ & .297 & .001 & -1.555 & -.389 \\
\hline & $1 \mathrm{~W} 16 \mathrm{~A}$ & $-.501^{\mathrm{a}, \mathrm{b},{ }^{*}}$ & .242 & .039 & -.977 & -.025 \\
\hline & $2 W 32 A$ & $.672^{\mathrm{a}, \mathrm{b},{ }^{*}}$ & .242 & .006 & .197 & 1.148 \\
\hline & $3 W 48 A$ & $-.246^{\mathrm{a}, \mathrm{b}}$ & .242 & .310 & -.722 & .230 \\
\hline & $4 \mathrm{~W} 64 \mathrm{~A}$ & $.384^{a, b}$ & .242 & .113 & -.091 & .860 \\
\hline
\end{tabular}

Based on estimated marginal means

*. The mean difference is significant at the .05 level.

a. An estimate of the modified population marginal mean (I).

b. An estimate of the modified population marginal mean $(\mathrm{J})$.

d. Adjustment for multiple comparisons: Least Significant Difference (equivalent to no adjustments). 
Marked significant differences were observed at all the comparing groups, however not for all measurements. No one pair-wise group stood out from the rest.

Dependent Variable: $b$

\section{Sample Area * Swatch: GMP}

\begin{tabular}{|c|c|c|c|c|c|}
\hline \multirow{2}{*}{ Sample Area } & & \multirow[b]{2}{*}{ Mean } & \multirow[b]{2}{*}{ Std. Error } & \multicolumn{2}{|c|}{$95 \%$ Confidence Interval } \\
\hline & & & & $\begin{array}{l}\text { Lower } \\
\text { Bound }\end{array}$ & Upper Bound \\
\hline \multirow[t]{4}{*}{$\bar{A}$} & $1 \mathrm{w} /$ bleach & $10.291^{a}$ & .198 & 9.903 & 10.680 \\
\hline & 2 w/ bleach & $9.637^{a}$ & .217 & 9.211 & 10.063 \\
\hline & $3 \mathrm{w} / 0$ bleach & $10.444^{a}$ & .198 & 10.056 & 10.833 \\
\hline & $4 \mathrm{w} / \mathrm{o}$ bleach & $9.909^{a}$ & .217 & 9.483 & 10.334 \\
\hline \multirow[t]{4}{*}{ B } & $1 \mathrm{w} /$ bleach & $10.921^{a}$ & .217 & 10.496 & 11.347 \\
\hline & $2 \mathrm{w} /$ bleach & $10.846^{a}$ & .198 & 10.458 & 11.235 \\
\hline & 3 w/o bleach & $10.311^{\mathrm{a}}$ & .217 & 9.885 & 10.736 \\
\hline & $4 \mathrm{w} / \mathrm{o}$ bleach & $10.154^{\mathrm{a}}$ & .198 & 9.765 & 10.542 \\
\hline
\end{tabular}

a. Based on modified population marginal mean.

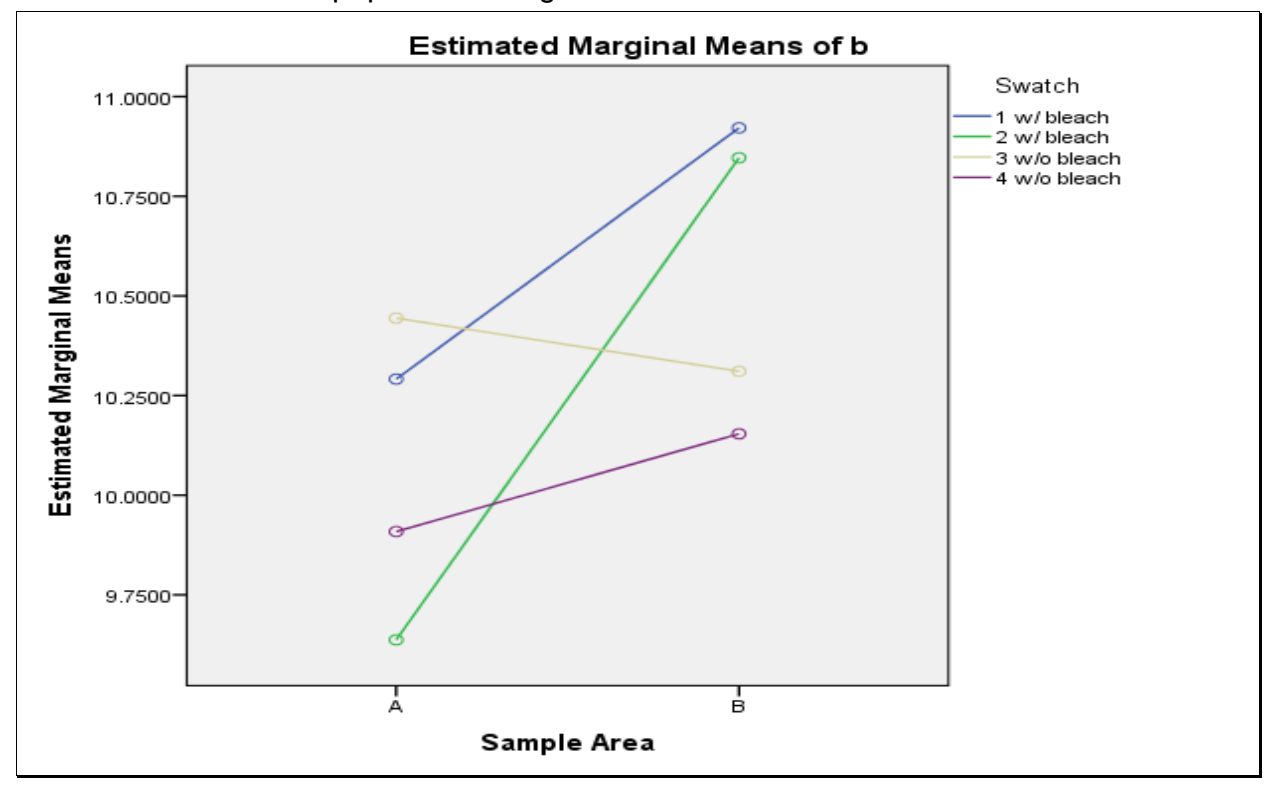

The mean values between swatches 1 and 2 , and swatches 3 and 4 at sample area B are close to each other, thus significant interaction is not expected here. However the mean values between swatches 1 and 4 at area B and 1 and 2 at area A, observes to be substantial. Even though the main effects of swatch was not significant, some changes in both factors produced significant results through their interaction. 
Dependent Variable: $b$

\section{Sample Area * bleached: GMP}

\begin{tabular}{|ll|r|r|r|r|}
\hline & & & & \multicolumn{2}{c|}{$95 \%$ Confidence Interval } \\
\cline { 4 - 6 } Sample Area & & & \multicolumn{2}{c|}{$\begin{array}{l}\text { Lower } \\
\text { Bound }\end{array}$} & Upper Bound \\
\hline A & w/ bleach & $9.994^{\mathrm{a}}$ & .146 & 9.707 & 10.281 \\
& w/o bleach & $10.201^{\mathrm{a}}$ & .146 & 9.914 & 10.488 \\
B & w/ bleach & $10.881^{\mathrm{a}}$ & .146 & 10.594 & 11.167 \\
& w/o bleach & $10.225^{\mathrm{a}}$ & .146 & 9.938 & 10.512 \\
\hline
\end{tabular}

a. Based on modified population marginal mean.

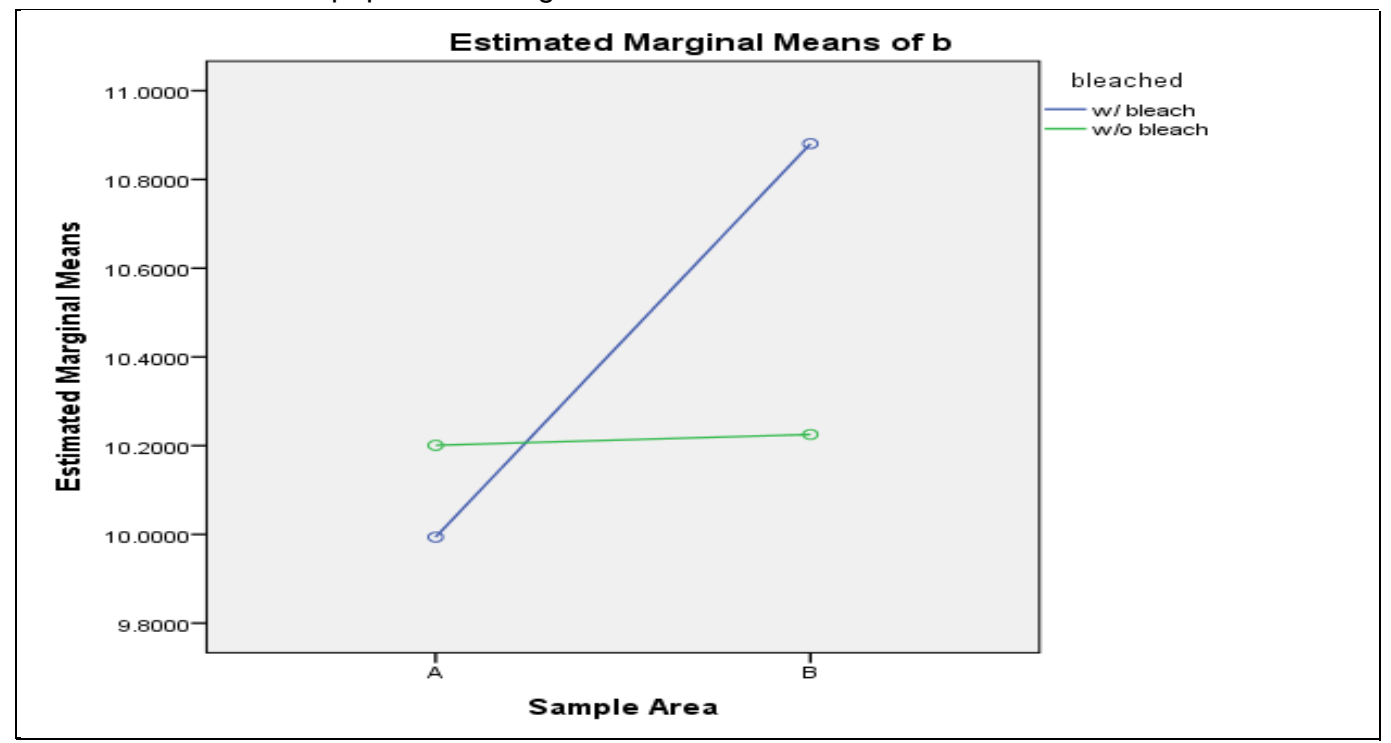

The significant interaction is possibly observed at sample area B as the mean values vary to a greater extent than at sample area A.

Dependent Variable: $\mathrm{b}$

\section{7. bleached * washings: GMP}

\begin{tabular}{|c|c|c|c|c|c|}
\hline \multirow[b]{2}{*}{ bleached } & & \multirow[b]{2}{*}{ Mean } & \multirow[b]{2}{*}{ Std. Error } & \multicolumn{2}{|c|}{ 95\% Confidence Interval } \\
\hline & & & & $\begin{array}{l}\text { Lower } \\
\text { Bound }\end{array}$ & Upper Bound \\
\hline \multirow[t]{6}{*}{ w/ bleach } & $1 \mathrm{~W} 0 \mathrm{~A}$ & $11.664^{\mathrm{a}}$ & .343 & 10.992 & 12.337 \\
\hline & $1 \mathrm{~W} 16 \mathrm{~A}$ & $10.867^{a}$ & .242 & 10.392 & 11.343 \\
\hline & $2 W 32 A$ & $8.905^{a}$ & .242 & 8.429 & 9.381 \\
\hline & 3W 48A & $10.473^{\mathrm{a}}$ & .242 & 9.997 & 10.949 \\
\hline & $4 \mathrm{~W} 64 \mathrm{~A}$ & $10.078^{a}$ & .242 & 9.602 & 10.554 \\
\hline & $5 \mathrm{~W} 80 \mathrm{~A}$ & $11.249^{a}$ & .242 & 10.773 & 11.725 \\
\hline \multirow[t]{6}{*}{ w/o bleach } & $1 \mathrm{~W} 0 \mathrm{~A}$ & $10.865^{\mathrm{a}}$ & .343 & 10.193 & 11.538 \\
\hline & $1 \mathrm{~W} 16 \mathrm{~A}$ & $10.720^{\mathrm{a}}$ & .242 & 10.245 & 11.196 \\
\hline & $2 W 32 A$ & $10.336^{a}$ & .242 & 9.861 & 10.812 \\
\hline & $3 W 48 A$ & $10.605^{a}$ & .242 & 10.130 & 11.081 \\
\hline & $4 \mathrm{~W} 64 \mathrm{~A}$ & $9.740^{\mathrm{a}}$ & .242 & 9.264 & 10.216 \\
\hline & $5 \mathrm{~W} 80 \mathrm{~A}$ & $9.337^{\mathrm{a}}$ & .242 & 8.861 & 9.813 \\
\hline
\end{tabular}

a. Based on modified population marginal mean. 


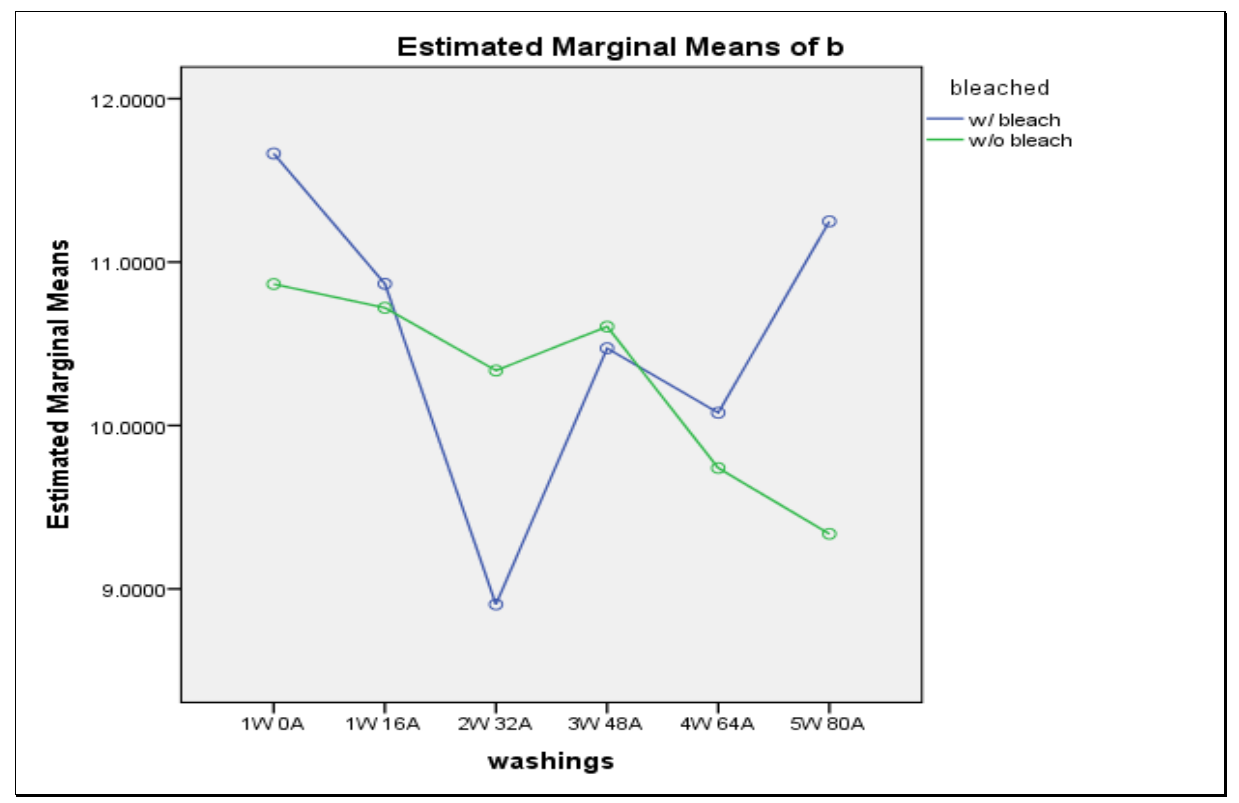

The significance in interactions can be obviously observed both at $2 \mathrm{~W} 32 \mathrm{~A}$ and $5 \mathrm{~W} 80 \mathrm{~A}$ where the mean values differ greatly between the bleached and unbleached samples.

\section{Swatch * washings: GMP}

Dependent Variable: $b$

\begin{tabular}{|c|c|c|c|c|c|}
\hline \multirow[b]{2}{*}{ Swatch } & & \multirow[b]{2}{*}{ Mean } & \multirow[b]{2}{*}{ Std. Error } & \multicolumn{2}{|c|}{ 95\% Confidence Interval } \\
\hline & & & & Lower Bound & Upper Bound \\
\hline \multirow[t]{6}{*}{$1 \mathrm{w} /$ bleach } & $1 \mathrm{~W} 0 \mathrm{~A}$ & $11.638^{\mathrm{a}}$ & .485 & 10.687 & 12.590 \\
\hline & $1 \mathrm{~W} 16 \mathrm{~A}$ & $11.234^{a}$ & .343 & 10.561 & 11.907 \\
\hline & $2 W 32 A$ & $9.455^{\mathrm{a}}$ & .343 & 8.782 & 10.128 \\
\hline & $3 W 48 A$ & $10.224^{a}$ & .343 & 9.551 & 10.897 \\
\hline & $4 \mathrm{~W} 64 \mathrm{~A}$ & $10.906^{a}$ & .343 & 10.233 & 11.579 \\
\hline & $5 W$ 80A & $10.540^{\mathrm{a}}$ & .343 & 9.867 & 11.212 \\
\hline \multirow[t]{6}{*}{2 w/ bleach } & $1 \mathrm{~W} 0 \mathrm{~A}$ & $11.691^{\mathrm{a}}$ & .485 & 10.739 & 12.642 \\
\hline & $1 \mathrm{~W} 16 \mathrm{~A}$ & $10.501^{\mathrm{a}}$ & .343 & 9.828 & 11.174 \\
\hline & $2 W 32 A$ & $8.355^{a}$ & .343 & 7.682 & 9.028 \\
\hline & $3 W 48 A$ & $10.722^{a}$ & .343 & 10.049 & 11.395 \\
\hline & $4 W 64 \mathrm{~A}$ & $9.250^{\mathrm{a}}$ & .343 & 8.577 & 9.923 \\
\hline & $5 W 80 A$ & $11.959^{a}$ & .343 & 11.286 & 12.632 \\
\hline \multirow[t]{6}{*}{3 w/o bleach } & $1 \mathrm{~W} 0 \mathrm{~A}$ & $11.698^{\mathrm{a}}$ & .485 & 10.746 & 12.649 \\
\hline & $1 \mathrm{~W} 16 \mathrm{~A}$ & $11.139^{a}$ & .343 & 10.466 & 11.811 \\
\hline & $2 W 32 A$ & $11.349^{a}$ & .343 & 10.676 & 12.022 \\
\hline & $3 W 48 A$ & $10.142^{a}$ & .343 & 9.469 & 10.814 \\
\hline & $4 \mathrm{~W} 64 \mathrm{~A}$ & $9.647^{a}$ & .343 & 8.974 & 10.320 \\
\hline & $5 W 80 A$ & $8.985^{a}$ & .343 & 8.312 & 9.657 \\
\hline \multirow[t]{6}{*}{4 w/o bleach } & $1 \mathrm{~W} 0 \mathrm{~A}$ & $10.033^{a}$ & .485 & 9.082 & 10.985 \\
\hline & $1 \mathrm{~W} 16 \mathrm{~A}$ & $10.302^{a}$ & .343 & 9.629 & 10.975 \\
\hline & $2 W 32 A$ & $9.324^{a}$ & .343 & 8.651 & 9.997 \\
\hline & $3 W 48 A$ & $11.069^{\mathrm{a}}$ & .343 & 10.396 & 11.742 \\
\hline & $4 W 64 A$ & $9.832^{a}$ & .343 & 9.159 & 10.505 \\
\hline & $5 W$ 80A & $9.689^{a}$ & .343 & 9.017 & 10.362 \\
\hline
\end{tabular}

a. Based on modified population marginal mean. 


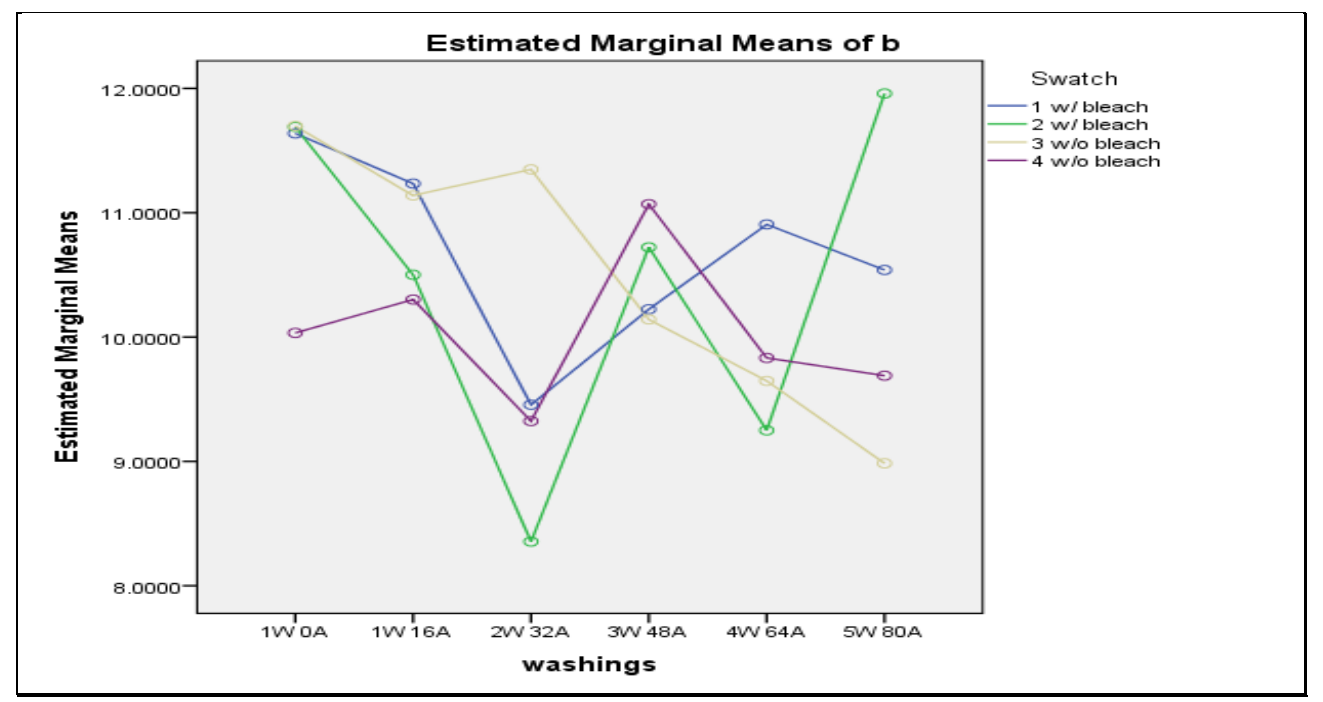

The main effect of swatch was calculated as an insignificant $p$-value. This was ignored since the interaction is significant here mainly because there is evidence supporting that changes in the exploring factors had an effect on the outcome, regardless of the main effect p-values.

\section{OSDP}

\section{Variable ' $L$ '}

Dependent Variable: L

Tests of Between-Subjects Effects: OSDP

\begin{tabular}{|c|c|c|c|c|c|}
\hline Source & Type III Sum of Squares & $\mathrm{df}$ & Mean Square & $F$ & Sig. \\
\hline Corrected Model & $4410.649^{a}$ & 43 & 102.573 & 10.173 & .000 \\
\hline Intercept & 6892059.036 & 1 & 6892059.036 & 683550.724 & 0.000 \\
\hline SampleArea & 5.842 & 1 & 5.842 & .579 & .447 \\
\hline Swatch & 193.787 & 2 & 96.893 & 9.610 & .000 \\
\hline Bleached & 0.000 & 0 & & & \\
\hline Washing & 453.649 & 5 & 90.730 & 8.999 & .000 \\
\hline SampleArea * Swatch & 23.518 & 2 & 11.759 & 1.166 & .312 \\
\hline SampleArea * Bleached & 0.000 & 0 & & & \\
\hline SampleArea * Washing & 225.039 & 4 & 56.260 & 5.580 & .000 \\
\hline Swatch * Bleached & 0.000 & 0 & & & \\
\hline Swatch * Washing & 354.222 & 8 & 44.278 & 4.391 & .000 \\
\hline Bleached * Washing & 0.000 & 0 & & & \\
\hline SampleArea * Swatch * Bleached & 0.000 & 0 & & & \\
\hline SampleArea * Swatch * Washing & 1666.408 & 8 & 208.301 & 20.659 & .000 \\
\hline SampleArea * Bleached * Washing & 0.000 & 0 & & & \\
\hline Swatch * Bleached * Washing & 0.000 & 0 & & & \\
\hline SampleArea * Swatch * Bleached * Washing & 0.000 & 0 & & & \\
\hline Error & 8429.164 & 836 & 10.083 & & \\
\hline Total & 7351867.921 & 880 & & & \\
\hline Corrected Total & 12839.813 & 879 & & & \\
\hline
\end{tabular}

a. $\mathrm{R}$ Squared $=.344$ (Adjusted R Squared $=.310$ ) 
b. Computed using alpha $=.05$

The main effects of sample area, and the interaction effects of Sample Area* Swatch did not produce significant results. The main effects were not significantly different between two or more means, and that this two-way interaction was not significantly difference between two or more differences between two or more means.

\section{Sample Area Estimates: OSDP}

Dependent Variable: $\mathrm{L}$

\begin{tabular}{|l|r|r|r|r|}
\hline & & \multirow{2}{*}{$\begin{array}{c}\text { Std. } \\
\text { Sample Area }\end{array}$} & \multicolumn{2}{|c|}{ 95\% Confidence Interval } \\
\cline { 4 - 5 } & Mean & Error & \multicolumn{1}{|c|}{ Lower Bound } & Upper Bound \\
\hline A & $91.373^{\mathrm{a}}$ & .151 & 91.076 & 91.670 \\
B & $91.272^{\mathrm{a}}$ & .151 & 90.975 & 91.569 \\
\hline
\end{tabular}

a. Based on modified population marginal mean.

Pairwise Comparisons: OSDP

Dependent Variable: L

\begin{tabular}{|c|c|c|c|c|c|}
\hline \multirow[b]{2}{*}{ (I) Sample Area } & \multirow{2}{*}{$\begin{array}{c}\text { Mean } \\
\text { Difference (I- } \\
\mathrm{J})\end{array}$} & \multirow[b]{2}{*}{ Std. Error } & \multirow[b]{2}{*}{ Sig. $^{c}$} & \multicolumn{2}{|c|}{$95 \%$ Confidence Interval for Difference } \\
\hline & & & & Lower Bound & Upper Bound \\
\hline $\begin{array}{ll}A & B \\
B & A\end{array}$ & $\begin{array}{r}.101^{\mathrm{a}, \mathrm{b}} \\
-.101^{\mathrm{a}, \mathrm{b}}\end{array}$ & $\begin{array}{l}.214 \\
.214\end{array}$ & $\begin{array}{l}.637 \\
.637\end{array}$ & $\begin{array}{r}-.319 \\
-.521\end{array}$ & $\begin{array}{r}.521 \\
.319\end{array}$ \\
\hline
\end{tabular}

Based on estimated marginal means

a. An estimate of the modified population marginal mean (I).

b. An estimate of the modified population marginal mean $(\mathrm{J})$.

c. Adjustment for multiple comparisons: Least Significant Difference (equivalent to no adjustments).

It can be observed that the mean values between these two sample areas are only a difference of

0.101 as the mean values are very similar.

\section{Swatch Estimates: OSDP}

Dependent Variable: $\mathrm{L}$

\begin{tabular}{|c|c|c|c|c|}
\hline \multirow[b]{2}{*}{ Swatch } & \multirow[b]{2}{*}{ Mean } & \multirow[b]{2}{*}{ Std. Error } & \multicolumn{2}{|c|}{ 95\% Confidence Interval } \\
\hline & & & Lower Bound & Upper Bound \\
\hline $1 \mathrm{w} /$ bleach & $92.093^{\mathrm{a}}$ & .214 & 91.673 & 92.514 \\
\hline $2 \mathrm{w} /$ bleach & $91.062^{\mathrm{a}}$ & .214 & 90.642 & 91.482 \\
\hline 3 w/o bleach & $90.749^{a}$ & .214 & 90.329 & 91.169 \\
\hline 4 w/o bleach & $91.386^{\mathrm{a}}$ & .214 & 90.965 & 91.806 \\
\hline
\end{tabular}

a. Based on modified population marginal mean. 
Pairwise Comparisons: OSDP

Dependent Variable: $\mathrm{L}$

\begin{tabular}{|c|c|c|c|c|c|c|}
\hline \multirow[b]{2}{*}{ (I) Swatch } & & \multirow{2}{*}{$\begin{array}{c}\text { Mean } \\
\text { Difference } \\
(I-J)\end{array}$} & \multirow{2}{*}{$\begin{array}{l}\text { Std. } \\
\text { Error }\end{array}$} & \multirow[b]{2}{*}{ Sig. ${ }^{d}$} & \multicolumn{2}{|c|}{$\begin{array}{c}95 \% \text { Confidence Interval for } \\
\text { Difference }^{\mathrm{d}}\end{array}$} \\
\hline & & & & & Lower Bound & Upper Bound \\
\hline \multirow[t]{3}{*}{$1 \mathrm{w} /$ bleach } & $2 \mathrm{w} /$ bleach & $1.031^{\prime, 0, C}$ & .303 & .001 & .437 & 1.626 \\
\hline & 3 w/o bleach & $1.344^{x, b, c}$ & .303 & .000 & .750 & 1.939 \\
\hline & $4 \mathrm{w} / \mathrm{o}$ bleach & $.708^{*}, \mathrm{~b}, \mathrm{c}$ & .303 & .020 & .114 & 1.302 \\
\hline \multirow[t]{3}{*}{$2 \mathrm{w} /$ bleach } & $1 \mathrm{w} /$ bleach & $-1.031^{\star}{ }^{\mathrm{x}, \mathrm{b}, \mathrm{c}}$ & .303 & .001 & -1.626 & -.437 \\
\hline & 3 w/o bleach & $.313^{\mathrm{b}, \mathrm{c}}$ & .303 & .302 & -.281 & .907 \\
\hline & 4 w/o bleach & $-.324^{\mathrm{b}, \mathrm{c}}$ & .303 & .285 & -.918 & .271 \\
\hline \multirow[t]{3}{*}{3 w/o bleach } & $1 \mathrm{w} /$ bleach & $-1.344^{x, b, c}$ & .303 & .000 & -1.939 & -.750 \\
\hline & 2 w/ bleach & $-.313^{\mathrm{b}, \mathrm{c}}$ & .303 & .302 & -.907 & .281 \\
\hline & 4 w/o bleach & $-.637^{,, \mathrm{b}, \mathrm{c}}$ & .303 & .036 & -1.231 & -.042 \\
\hline \multirow[t]{3}{*}{4 w/o bleach } & $1 \mathrm{w} /$ bleach & $-.708^{*, b, c}$ & .303 & .020 & -1.302 & -.114 \\
\hline & $2 \mathrm{w} /$ bleach & $.324^{\mathrm{b}, \mathrm{c}}$ & .303 & .285 & -.271 & .918 \\
\hline & 3 w/o bleach & $.637^{*, \mathrm{~b}, \mathrm{c}}$ & .303 & .036 & .042 & 1.231 \\
\hline
\end{tabular}

Based on estimated marginal means

*. The mean difference is significant at the .05 level.

b. An estimate of the modified population marginal mean (I).

c. An estimate of the modified population marginal mean (J).

d. Adjustment for multiple comparisons: Least Significant Difference (equivalent to no adjustments).

Swatch 1 observed marked significant differences at all levels of comparisons. No pattern was

otherwise observed among the treated swatches.

\section{Bleach Estimates: OSDP}

Dependent Variable: $\mathrm{L}$

\begin{tabular}{|c|c|c|c|c|}
\hline \multirow[b]{2}{*}{ Bleached } & \multirow[b]{2}{*}{ Mean } & \multirow[b]{2}{*}{ Std. Error } & \multicolumn{2}{|c|}{ 95\% Confidence Interval } \\
\hline & & & Lower Bound & Upper Bound \\
\hline w/ bleach & $91.578^{\mathrm{a}}$ & .151 & 91.281 & 91.875 \\
\hline & & .151 & 90.770 & 91.364 \\
\hline
\end{tabular}

a. Based on modified population marginal mean.

Pairwise Comparisons: OSDP

Dependent Variable: $\mathrm{L}$

\begin{tabular}{|c|c|c|c|c|c|c|}
\hline \multirow[b]{2}{*}{ (I) Bleacheo } & & \multirow{2}{*}{$\begin{array}{c}\text { Mean } \\
\text { Difference (I- } \\
\mathrm{J})\end{array}$} & \multirow[b]{2}{*}{ Std. Error } & \multirow[b]{2}{*}{ Sig. ${ }^{d}$} & \multicolumn{2}{|c|}{$\begin{array}{c}95 \% \text { Confidence Interval for } \\
\text { Difference }\end{array}$} \\
\hline & & & & & Lower Bound & Upper Bound \\
\hline $\begin{array}{l}\text { w/ bleach } \\
\text { w/o bleach }\end{array}$ & $\begin{array}{l}\text { w/o bleach } \\
\text { w/ bleach }\end{array}$ & $\begin{array}{r}.510^{\circ, \mathrm{b}, \mathrm{c}} \\
-.510^{\mathrm{x}, \mathrm{b}, \mathrm{c}}\end{array}$ & $\begin{array}{l}.214 \\
.214\end{array}$ & $\begin{array}{l}.017 \\
.017\end{array}$ & $\begin{array}{r}.090 \\
-.931\end{array}$ & $\begin{array}{r}.931 \\
-.090\end{array}$ \\
\hline
\end{tabular}

Based on estimated marginal means

*. The mean difference is significant at the .05 level.

b. An estimate of the modified population marginal mean (I).

c. An estimate of the modified population marginal mean (J).

d. Adjustment for multiple comparisons: Least Significant Difference (equivalent to no adjustments).

Significant values were observed for this level of comparison. 


\section{Washing Estimates: OSDP}

Dependent Variable: $\mathrm{L}$

\begin{tabular}{|l|r|r|r|r|}
\hline & & & \multicolumn{2}{|c|}{$95 \%$ Confidence Interval } \\
\cline { 4 - 5 } Washing & Mean & Std. Error & Lower Bound & $\begin{array}{c}\text { Upper } \\
\text { Bound }\end{array}$ \\
\hline 1W 0A & $92.366^{\mathrm{a}}$ & .355 & 91.669 & 93.063 \\
1W 16A & $92.093^{\mathrm{a}}$ & .251 & 91.600 & 92.585 \\
2W 32A & $90.411^{\mathrm{a}}$ & .251 & 89.918 & 90.904 \\
3W 48A & $91.907^{\mathrm{a}}$ & .251 & 91.414 & 92.400 \\
4W 64A & $91.060^{\mathrm{a}}$ & .251 & 90.567 & 91.553 \\
5W 80A & $90.621^{\mathrm{a}}$ & .251 & 90.128 & 91.113 \\
\hline
\end{tabular}

a. Based on modified population marginal mean.

Pairwise Comparisons: OSDP

Dependent Variable: L

\begin{tabular}{|c|c|c|c|c|c|c|}
\hline \multirow{2}{*}{\multicolumn{2}{|c|}{ (I) Washing }} & \multirow{2}{*}{$\begin{array}{c}\text { Mean } \\
\text { Difference (I- } \\
\mathrm{J})\end{array}$} & \multirow[b]{2}{*}{ Std. Error } & \multirow[b]{2}{*}{ Sig. ${ }^{d}$} & \multicolumn{2}{|c|}{$\begin{array}{c}\text { 95\% Confidence Interval for } \\
\text { Difference }^{d}\end{array}$} \\
\hline & & & & & Lower Bound & Upper Bound \\
\hline \multirow[t]{5}{*}{$1 \mathrm{~W} 0 \mathrm{~A}$} & $1 \mathrm{~W} 16 \mathrm{~A}$ & $.273^{\mathrm{a}, \mathrm{b}}$ & .435 & .530 & -.580 & 1.127 \\
\hline & $2 W 32 A$ & $1.955^{\mathrm{a}, \mathrm{b},{ }^{*}}$ & .435 & .000 & 1.101 & 2.808 \\
\hline & $3 W$ 48A & $.459^{a, b}$ & .435 & .291 & -.394 & 1.312 \\
\hline & $4 \mathrm{~W} 64 \mathrm{~A}$ & $1.306^{\mathrm{a}, \mathrm{b},{ }^{*}}$ & .435 & .003 & .452 & 2.159 \\
\hline & $5 W 80 A$ & $1.745^{\mathrm{a}, \mathrm{b},{ }^{*}}$ & .435 & .000 & .892 & 2.599 \\
\hline \multirow[t]{5}{*}{$1 \mathrm{~W} 16 \mathrm{~A}$} & $1 \mathrm{~W} 0 \mathrm{~A}$ & $-.273^{a, b}$ & .435 & .530 & -1.127 & .580 \\
\hline & $2 W 32 A$ & $1.682^{\mathrm{a}, \mathrm{b},{ }^{*}}$ & .355 & .000 & .985 & 2.379 \\
\hline & $3 W 48 A$ & $.186^{\mathrm{a}, \mathrm{b}}$ & .355 & .601 & -.511 & .883 \\
\hline & $4 W 64 \mathrm{~A}$ & $1.033^{\mathrm{a}, \mathrm{b},{ }^{*}}$ & .355 & .004 & .336 & 1.730 \\
\hline & $5 W 80 A$ & $1.472^{\mathrm{a}, \mathrm{b},{ }^{\star}}$ & .355 & .000 & .775 & 2.169 \\
\hline \multirow[t]{5}{*}{$2 \mathrm{~W} 32 \mathrm{~A}$} & $1 \mathrm{~W} 0 \mathrm{~A}$ & $-1.955^{\mathrm{a}, \mathrm{b},{ }^{*}}$ & .435 & .000 & -2.808 & -1.101 \\
\hline & $1 \mathrm{~W} 16 \mathrm{~A}$ & $-1.682^{\mathrm{a}, \mathrm{b},{ }^{*}}$ & .355 & .000 & -2.379 & -.985 \\
\hline & $3 W 48 A$ & $-1.496^{a, b, *}$ & .355 & .000 & -2.193 & -.799 \\
\hline & $4 W 64 A$ & $-.649^{a, b}$ & .355 & .068 & -1.346 & .048 \\
\hline & $5 W 80 A$ & $-.210^{a, b}$ & .355 & .555 & -.906 & .487 \\
\hline \multirow[t]{5}{*}{$3 W$ 48A } & $1 \mathrm{~W} 0 \mathrm{~A}$ & $-.459^{a, b}$ & .435 & .291 & -1.312 & .394 \\
\hline & $1 \mathrm{~W} 16 \mathrm{~A}$ & $-.186^{a, b}$ & .355 & .601 & -.883 & .511 \\
\hline & $2 W 32 A$ & $1.496^{\mathrm{a}, \mathrm{b},{ }^{*}}$ & .355 & .000 & .799 & 2.193 \\
\hline & $4 \mathrm{~W} 64 \mathrm{~A}$ & $.847^{\mathrm{a}, \mathrm{b},{ }^{*}}$ & .355 & .017 & .150 & 1.544 \\
\hline & $5 W 80 A$ & $1.286^{\mathrm{a}, \mathrm{b},{ }^{\star}}$ & .355 & .000 & .589 & 1.983 \\
\hline \multirow[t]{5}{*}{$4 W 64 A$} & $1 \mathrm{~W} 0 \mathrm{~A}$ & $-1.306^{\mathrm{a}, \mathrm{b},{ }^{\pi}}$ & .435 & .003 & -2.159 & -.452 \\
\hline & $1 \mathrm{~W} 16 \mathrm{~A}$ & $-1.033^{a, b, *}$ & .355 & .004 & -1.730 & -.336 \\
\hline & $2 W 32 A$ & $.649^{\mathrm{a}, \mathrm{b}}$ & .355 & .068 & -.048 & 1.346 \\
\hline & $3 W 48 A$ & $-.847^{\mathrm{a}, \mathrm{b},{ }^{*}}$ & .355 & .017 & -1.544 & -.150 \\
\hline & $5 W 80 A$ & $.439^{a, b}$ & .355 & .216 & -.258 & 1.136 \\
\hline \multirow[t]{5}{*}{$5 \mathrm{~W} 80 \mathrm{~A}$} & $1 \mathrm{~W} 0 \mathrm{~A}$ & $-1.745^{\mathrm{a}, \mathrm{b},{ }^{*}}$ & .435 & .000 & -2.599 & -.892 \\
\hline & $1 \mathrm{~W} 16 \mathrm{~A}$ & $-1.472^{\mathrm{a}, \mathrm{b},{ }^{\star}}$ & .355 & .000 & -2.169 & -.775 \\
\hline & $2 W 32 A$ & $.210^{a, b}$ & .355 & .555 & -.487 & .906 \\
\hline & $3 W 48 A$ & $-1.286^{a, b,{ }^{*}}$ & .355 & .000 & -1.983 & -.589 \\
\hline & $4 W 64 A$ & $-.439^{a, b}$ & .355 & .216 & -1.136 & .258 \\
\hline
\end{tabular}

Based on estimated marginal means

*. The mean difference is significant at the .05 level.

a. An estimate of the modified population marginal mean (I).

b. An estimate of the modified population marginal mean (J).

d. Adjustment for multiple comparisons: Least Significant Difference (equivalent to no adjustments).

Significant values were observed for all groups but not for all pair-wise levels of this factor.

\section{Sample Area * Bleached: OSDP}


Dependent Variable: $\mathrm{L}$

\begin{tabular}{|c|c|c|c|c|c|}
\hline \multirow[b]{2}{*}{ Sample Area } & & \multirow[b]{2}{*}{ Mean } & \multirow[b]{2}{*}{ Std. Error } & \multicolumn{2}{|c|}{ 95\% Confidence Interval } \\
\hline & & & & $\begin{array}{l}\text { Lower } \\
\text { Bound }\end{array}$ & Upper Bound \\
\hline \multirow{4}{*}{$\begin{array}{l}A \\
B\end{array}$} & w/ bleach & $91.560^{\mathrm{a}}$ & .214 & 91.140 & 91.981 \\
\hline & w/o bleach & $91.186^{\mathrm{a}}$ & .214 & 90.765 & 91.606 \\
\hline & w/ bleach & $91.595^{\mathrm{a}}$ & .214 & 91.175 & 92.015 \\
\hline & w/o bleach & $90.949^{\mathrm{a}}$ & .214 & 90.529 & 91.369 \\
\hline
\end{tabular}

a. Based on modified population marginal mean.

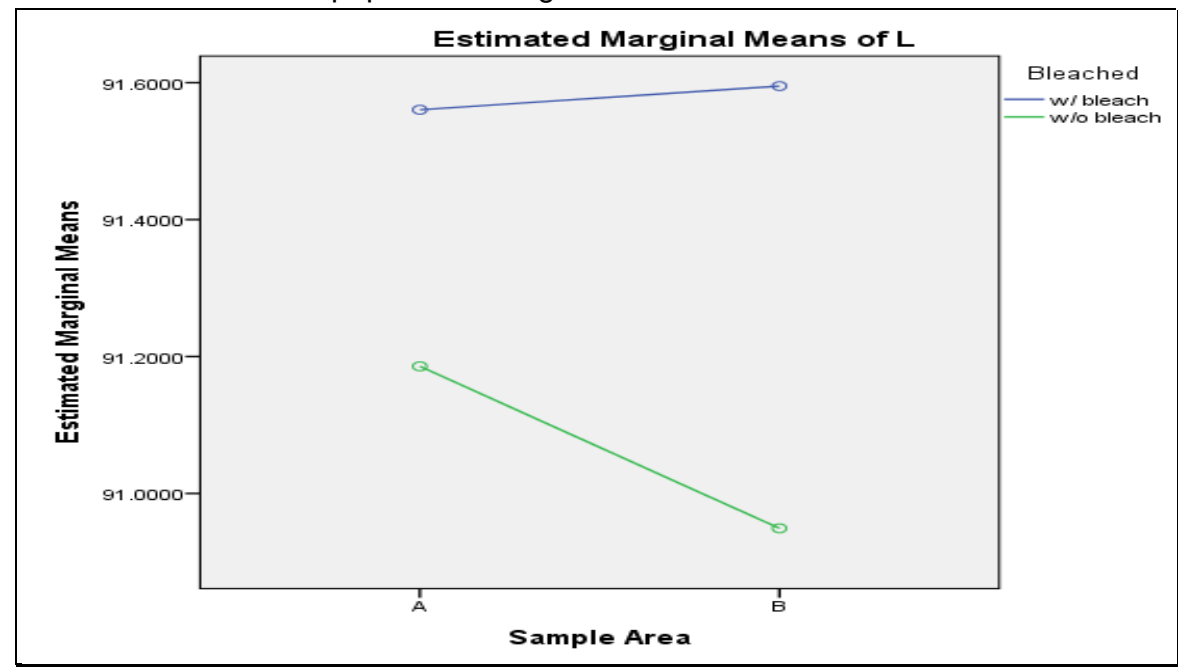

The mean difference for the bleached samples is approximately 0.035 and 0.237 for the unbleached samples. Even though a difference is noticed, without the calculation of the p-values, a significant difference cannot be assumed.

Dependent Variable: L

\section{Bleached * Washing: OSDP}

\begin{tabular}{|ll|r|r|r|r|}
\hline \multirow{2}{*}{ Bleached } & & & \multicolumn{2}{c|}{$95 \%$ Confidence Interval } \\
\cline { 4 - 5 } & & Mean & Std. Error & $\begin{array}{c}\text { Lower } \\
\text { Bound }\end{array}$ & Upper Bound \\
\hline W/ bleach & 1W 0A & $91.285^{\mathrm{a}}$ & .502 & 90.299 & 92.270 \\
& 1W 16A & $92.526^{\mathrm{a}}$ & .355 & 91.829 & 93.222 \\
& 2W 32A & $91.935^{\mathrm{a}}$ & .355 & 91.238 & 92.632 \\
& 3W 48A & $92.845^{\mathrm{a}}$ & .355 & 92.148 & 93.541 \\
& 4W 64A & $89.780^{\mathrm{a}}$ & .355 & 89.083 & 90.476 \\
& 5W 80A & $90.951^{\mathrm{a}}$ & .355 & 90.254 & 91.647 \\
W/o bleach & $93.447^{\mathrm{a}}$ & .502 & 92.461 & 94.432 \\
& 1W 0A & $91.660^{\mathrm{a}}$ & .355 & 90.963 & 92.357 \\
& 1W 16A & $88.887^{\mathrm{a}}$ & .355 & 88.190 & 89.584 \\
& 2W 32A & $90.969^{\mathrm{a}}$ & .355 & 90.272 & 91.666 \\
& 3W 48A & $92.340^{\mathrm{a}}$ & .355 & 91.643 & 93.037 \\
& 4W 64A & $90.291^{\mathrm{a}}$ & .355 & 89.594 & 90.987 \\
\hline
\end{tabular}


a. Based on modified population marginal mean.

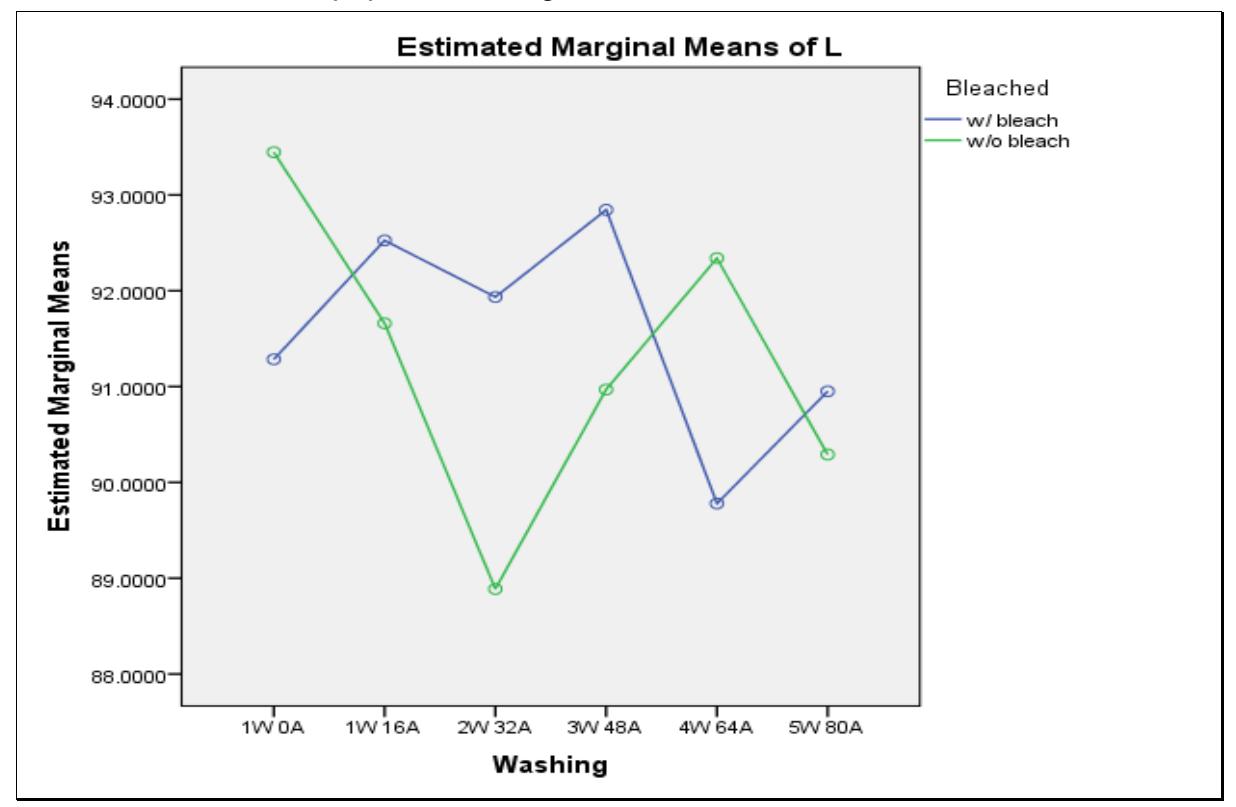

At $2 \mathrm{~W} 32 \mathrm{~A}$, the greatest difference in means are observed here for bleached and unbleached samples. It can be assumed that a significant interaction effect occurs at this level of interaction.

\section{Swatch * Washing: OSDP}

\begin{tabular}{|c|c|c|c|c|c|}
\hline \multirow[b]{2}{*}{ Swatch } & & \multirow[b]{2}{*}{ Mean } & \multirow[b]{2}{*}{ Std. Error } & \multicolumn{2}{|c|}{ 95\% Confidence Interval } \\
\hline & & & & Lower Bound & Upper Bound \\
\hline \multirow[t]{6}{*}{$1 \mathrm{w} /$ bleach } & $1 \mathrm{~W} 0 \mathrm{~A}$ & $92.371^{\mathrm{a}}$ & .710 & 90.977 & 93.764 \\
\hline & $1 \mathrm{~W} 16 \mathrm{~A}$ & $92.080^{a}$ & .502 & 91.094 & 93.065 \\
\hline & $2 W 32 A$ & $91.405^{\mathrm{a}}$ & .502 & 90.420 & 92.391 \\
\hline & $3 W 48 A$ & $94.487^{\mathrm{a}}$ & .502 & 93.502 & 95.473 \\
\hline & $4 \mathrm{~W} 64 \mathrm{~A}$ & $90.311^{a}$ & .502 & 89.325 & 91.296 \\
\hline & $5 W$ 80A & $92.046^{\mathrm{a}}$ & .502 & 91.060 & 93.031 \\
\hline \multirow[t]{6}{*}{2 w/ bleach } & $1 \mathrm{~W} 0 \mathrm{~A}$ & $90.199^{a}$ & .710 & 88.805 & 91.593 \\
\hline & $1 \mathrm{~W} 16 \mathrm{~A}$ & $92.971^{\mathrm{a}}$ & .502 & 91.986 & 93.957 \\
\hline & $2 W 32 A$ & $92.464^{a}$ & .502 & 91.479 & 93.450 \\
\hline & $3 W 48 A$ & $91.202^{\mathrm{a}}$ & .502 & 90.217 & 92.187 \\
\hline & $4 W 64 A$ & $89.248^{a}$ & .502 & 88.263 & 90.234 \\
\hline & $5 W 80 A$ & $89.856^{a}$ & .502 & 88.870 & 90.841 \\
\hline \multirow[t]{6}{*}{3 w/o bleach } & $1 \mathrm{~W} 0 \mathrm{~A}$ & $95.181^{a}$ & .710 & 93.788 & 96.575 \\
\hline & $1 \mathrm{~W} 16 \mathrm{~A}$ & $91.015^{\mathrm{a}}$ & .502 & 90.030 & 92.001 \\
\hline & $2 W 32 A$ & $89.018^{a}$ & .502 & 88.032 & 90.003 \\
\hline & $3 W 48 A$ & $90.639^{a}$ & .502 & 89.653 & 91.624 \\
\hline & $4 W 64 A$ & $91.651^{a}$ & .502 & 90.666 & 92.637 \\
\hline & $5 W 80 A$ & $89.207^{a}$ & .502 & 88.221 & 90.192 \\
\hline \multirow[t]{6}{*}{4 w/o bleach } & $1 \mathrm{~W} 0 \mathrm{~A}$ & $91.712^{\mathrm{a}}$ & .710 & 90.319 & 93.106 \\
\hline & $1 \mathrm{~W} 16 \mathrm{~A}$ & $92.304^{a}$ & .502 & 91.319 & 93.290 \\
\hline & $2 W 32 A$ & $88.757^{a}$ & .502 & 87.771 & 89.742 \\
\hline & $3 W 48 A$ & $91.300^{a}$ & .502 & 90.314 & 92.285 \\
\hline & $4 W 64 A$ & $93.029^{a}$ & .502 & 92.044 & 94.015 \\
\hline & $5 \mathrm{~W} 80 \mathrm{~A}$ & $91.375^{\mathrm{a}}$ & .502 & 90.389 & 92.360 \\
\hline
\end{tabular}


a. Based on modified population marginal mean.

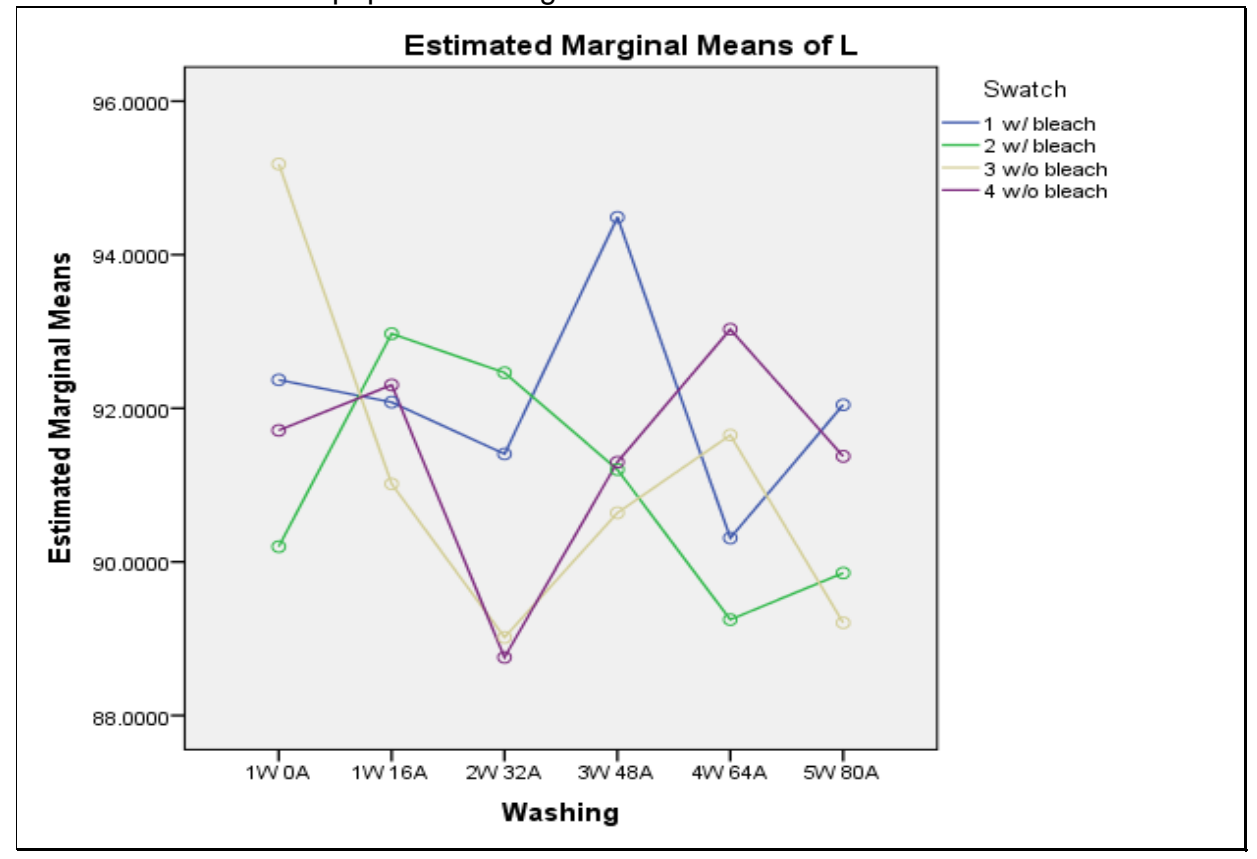

All four swatches observed different patterns suggesting that the conditions of the detergent had no impact among the swatches. The washed* swatch interaction was also significant at $\mathrm{p}=.001$.

Variable ' $a$ '

Dependent Variable: a

Tests of Between-Subjects Effects: OSDP

\begin{tabular}{|c|c|c|c|c|c|}
\hline Source & Type III Sum of Squares & df & $\begin{array}{c}\text { Mean } \\
\text { Square }\end{array}$ & $\mathrm{F}$ & Sig. \\
\hline Corrected Model & $642.81^{a}$ & 43 & 14.949 & 6.392 & .000 \\
\hline Intercept & 77883 & 1 & 77883 & 33300 & 0.000 \\
\hline SampleArea & 10.255 & 1 & 10.255 & 4.385 & .037 \\
\hline Swatch & 4.728 & 2 & 2.364 & 1.011 & .364 \\
\hline Bleached & 0.000 & 0 & & & \\
\hline Washing & 122.766 & 5 & 24.553 & 10.498 & .000 \\
\hline SampleArea * Swatch & 1.420 & 2 & .710 & .304 & .738 \\
\hline SampleArea * Bleached & 0.000 & 0 & & & \\
\hline SampleArea * Washing & 73.682 & 4 & 18.420 & 7.876 & .000 \\
\hline Swatch * Bleached & 0.000 & 0 & & & \\
\hline Swatch * Washing & 56.166 & 8 & 7.021 & 3.002 & .003 \\
\hline Bleached * Washing & 0.000 & 0 & & & \\
\hline SampleArea * Swatch * Bleached & 0.000 & 0 & & & \\
\hline SampleArea * Swatch * Washing & 77.445 & 8 & 9.681 & 4.139 & .000 \\
\hline SampleArea * Bleached * Washing & 0.000 & 0 & & & \\
\hline Swatch * Bleached * Washing & 0.000 & 0 & & & \\
\hline SampleArea * Swatch * Bleached * Washing & 0.000 & 0 & & & \\
\hline Error & 1955.2 & 836 & 2.339 & & \\
\hline Total & 86875 & 880 & & & \\
\hline Corrected Total & 2598 & 879 & & & \\
\hline
\end{tabular}

a. R Squared $=.247$ (Adjusted R Squared $=.209$ ) 
b. Computed using alpha $=.05$

The main effect of group 'Swatch' and the interaction effects of Swatch* Sample Area were not significant.

\section{Sample Area Estimates: OSDP}

Dependent Variable: a

\begin{tabular}{|c|c|c|c|c|}
\hline \multirow{2}{*}{$\begin{array}{l}\text { Sample } \\
\text { Area }\end{array}$} & \multirow[b]{2}{*}{ Mean } & \multirow[b]{2}{*}{ Std. Error } & \multicolumn{2}{|c|}{ 95\% Confidence Interval } \\
\hline & & & Lower Bound & Upper Bound \\
\hline A & $9.710^{\mathrm{a}}$ & .073 & 9.567 & 9.853 \\
\hline
\end{tabular}

a. Based on modified population marginal mean.

\section{Pairwise Comparisons: OSDP}

Dependent Variable: a

\begin{tabular}{|c|c|c|c|c|c|}
\hline \multirow[b]{2}{*}{ (I) Sample Area } & \multirow{2}{*}{$\begin{array}{c}\text { Mean } \\
\text { Difference (I- } \\
\mathrm{J})\end{array}$} & \multirow[b]{2}{*}{ Std. Error } & \multirow[b]{2}{*}{ Sig. ${ }^{\mathrm{C}}$} & \multicolumn{2}{|c|}{$95 \%$ Confidence Interval for Difference ${ }^{c}$} \\
\hline & & & & Lower Bound & Upper Bound \\
\hline $\begin{array}{ll}A & B\end{array}$ & $-.152^{a, b}$ & .103 & .140 & -.355 & .050 \\
\hline A & $.152^{\mathrm{a}, \mathrm{b}}$ & .103 & .140 & -.050 & .355 \\
\hline
\end{tabular}

Based on estimated marginal means

a. An estimate of the modified population marginal mean (I).

b. An estimate of the modified population marginal mean $(\mathrm{J})$.

c. Adjustment for multiple comparisons: Least Significant Difference (equivalent to no adjustments).

No significant difference between the two differences between the two differences in means was

observed although the main effect was found to be significantly different between the two

means. In other words, differences between the means were observed, but differences between

these differences (of the means) were not observed.

\section{Swatch Estimates: OSDP}

Dependent Variable: a

\begin{tabular}{|c|c|c|c|c|}
\hline \multirow[b]{2}{*}{ Swatch } & \multirow[b]{2}{*}{ Mean } & \multirow[b]{2}{*}{ Std. Error } & \multicolumn{2}{|c|}{ 95\% Confidence Interval } \\
\hline & & & Lower Bound & Upper Bound \\
\hline $1 \mathrm{w} /$ bleach & $9.835^{\mathrm{a}}$ & .103 & 9.633 & 10.038 \\
\hline 2 w/ bleach & $9.827^{\mathrm{a}}$ & .103 & 9.624 & 10.029 \\
\hline 3 w/o bleach & $9.926^{\mathrm{a}}$ & .103 & 9.724 & 10.128 \\
\hline 4 w/o bleach & $9.557^{\mathrm{a}}$ & .103 & 9.355 & 9.759 \\
\hline
\end{tabular}

a. Based on modified population marginal mean. 
Pairwise Comparisons: OSDP

Dependent Variable: a

\begin{tabular}{|c|c|c|c|c|c|c|}
\hline \multirow[b]{2}{*}{ (I) Swatch } & & \multirow{2}{*}{$\begin{array}{c}\text { Mean } \\
\text { Difference } \\
(\mathrm{I}-\mathrm{J})\end{array}$} & \multirow[b]{2}{*}{ Std. Error } & \multirow[b]{2}{*}{ Sig. ${ }^{d}$} & \multicolumn{2}{|c|}{$\begin{array}{c}\text { 95\% Confidence Interval for } \\
\text { Difference }\end{array}$} \\
\hline & & & & & Lower Bound & Upper Bound \\
\hline \multirow[t]{3}{*}{$1 \mathrm{w} /$ bleach } & 2 w/ bleach & $.009^{a, b}$ & .146 & .952 & -.277 & .295 \\
\hline & 3 w/o bleach & $-.091^{a, b}$ & .146 & .534 & -.377 & .195 \\
\hline & 4 w/o bleach & $.278^{a, b}$ & .146 & .057 & -.008 & .565 \\
\hline \multirow[t]{3}{*}{$2 \mathrm{w} /$ bleach } & 1 w/ bleach & $-.009^{a, b}$ & .146 & .952 & -.295 & .277 \\
\hline & 3 w/o bleach & $-.099^{a, b}$ & .146 & .495 & -.386 & .187 \\
\hline & 4 w/o bleach & $.270^{\mathrm{a}, \mathrm{b}}$ & .146 & .065 & -.017 & .556 \\
\hline \multirow[t]{3}{*}{3 w/o bleach } & 1 w/ bleach & $.091^{\mathrm{a}, \mathrm{b}}$ & .146 & .534 & -.195 & .377 \\
\hline & 2 w/ bleach & $.099^{a, b}$ & .146 & .495 & -.187 & .386 \\
\hline & 4 w/o bleach & $.369^{\mathrm{a}, \mathrm{b},{ }^{*}}$ & .146 & .012 & .083 & .655 \\
\hline \multirow[t]{3}{*}{4 w/o bleach } & 1 w/ bleach & $-.278^{\mathrm{a}, \mathrm{b}}$ & .146 & .057 & -.565 & .008 \\
\hline & 2 w/ bleach & $-.270^{\mathrm{a}, \mathrm{b}}$ & .146 & .065 & -.556 & .017 \\
\hline & 3 w/o bleach & $-.369^{\mathrm{a}, \mathrm{b},{ }^{*}}$ & .146 & .012 & -.655 & -.083 \\
\hline
\end{tabular}

Based on estimated marginal means

*. The mean difference is significant at the .05 level.

a. An estimate of the modified population marginal mean (I).

b. An estimate of the modified population marginal mean $(\mathrm{J})$.

d. Adjustment for multiple comparisons: Least Significant Difference (equivalent to no adjustments).

Swatches 1 and 2 did not observe any significant differences, whereas swatch 3 observed a

difference with swatch 4 .

\section{Bleach Estimates: OSDP}

Dependent Variable: a

\begin{tabular}{|l|r|r|r|r|}
\hline & & & \multicolumn{2}{|c|}{$95 \%$ Confidence Interval } \\
\cline { 4 - 5 } Bleached & Mean & Std. Error & Lower Bound & Upper Bound \\
\hline w/ bleach & $9.831^{\mathrm{a}}$ & .073 & 9.688 & 9.974 \\
w/o bleach & $9.741^{\mathrm{a}}$ & .073 & 9.598 & 9.885 \\
\hline
\end{tabular}

a. Based on modified population marginal mean.

\section{Pairwise Comparisons: OSDP}

Dependent Variable: a

\begin{tabular}{|c|c|c|c|c|c|c|}
\hline \multirow[b]{2}{*}{ (I) Bleached } & & \multirow{2}{*}{$\begin{array}{c}\text { Mean } \\
\text { Difference (I- } \\
\mathrm{J})\end{array}$} & \multirow[b]{2}{*}{ Std. Error } & \multirow[b]{2}{*}{ Sig. ${ }^{c}$} & \multicolumn{2}{|c|}{$\begin{array}{c}\text { 95\% Confidence Interval for } \\
\text { Difference }^{c}\end{array}$} \\
\hline & & & & & Lower Bound & Upper Bound \\
\hline $\begin{array}{l}\text { w/ bleach } \\
\text { w/o bleach }\end{array}$ & $\begin{array}{l}\text { w/o bleach } \\
\text { w/ bleach }\end{array}$ & $\begin{array}{r}.089^{\mathrm{a}, \mathrm{b}} \\
-.089^{\mathrm{a}, \mathrm{b}}\end{array}$ & $\begin{array}{l}.103 \\
.103\end{array}$ & $\begin{array}{l}.386 \\
.386\end{array}$ & $\begin{array}{l}-.113 \\
-.292\end{array}$ & $\begin{array}{l}.292 \\
.113\end{array}$ \\
\hline
\end{tabular}

Based on estimated marginal means

a. An estimate of the modified population marginal mean (I).

b. An estimate of the modified population marginal mean $(\mathrm{J})$.

c. Adjustment for multiple comparisons: Least Significant Difference (equivalent to no adjustments).

At this level of interaction, no significant differences were observed between the bleached and

unbleached samples. 


\section{Washing Estimates: OSDP}

Dependent Variable: a

\begin{tabular}{|l|r|r|r|r|}
\hline & & & \multicolumn{2}{|c|}{ 95\% Confidence Interval } \\
\cline { 4 - 5 } Washing & Mean & Std. Error & Lower Bound & Upper Bound \\
\hline 1W 0A & $8.697^{\mathrm{a}}$ & .171 & 8.361 & 9.033 \\
1W 16A & $10.198^{\mathrm{a}}$ & .121 & 9.961 & 10.436 \\
2W 32A & $9.922^{\mathrm{a}}$ & .121 & 9.685 & 10.160 \\
3W 48A & $9.872^{\mathrm{a}}$ & .121 & 9.635 & 10.109 \\
4W 64A & $9.929^{\mathrm{a}}$ & .121 & 9.691 & 10.166 \\
5W 80A & $9.554^{\mathrm{a}}$ & .121 & 9.317 & 9.792 \\
\hline
\end{tabular}

a. Based on modified population marginal mean.

Pairwise Comparisons: OSDP

Dependent Variable: a

\begin{tabular}{|c|c|c|c|c|c|c|}
\hline & & \multirow{2}{*}{$\begin{array}{c}\text { Mean } \\
\text { Difference (I- } \\
\mathrm{J})\end{array}$} & \multirow[b]{2}{*}{ Std. Error } & \multirow[b]{2}{*}{ Sig. ${ }^{d}$} & \multicolumn{2}{|c|}{$\begin{array}{l}\text { 95\% Confidence Interval for } \\
\text { Difference }^{d}\end{array}$} \\
\hline \multicolumn{2}{|c|}{ (I) Washing } & & & & Lower Bound & Upper Bound \\
\hline \multirow[t]{5}{*}{$1 \mathrm{~W} \mathrm{OA}$} & $1 \mathrm{~W} 16 \mathrm{~A}$ & $-1.501^{, b, c}$ & .209 & .000 & -1.912 & -1.090 \\
\hline & $2 W 32 A$ & $-1.225^{*, b, c}$ & .209 & .000 & -1.636 & -.814 \\
\hline & $3 W 48 A$ & $-1.175^{\star, b, c}$ & .209 & .000 & -1.586 & -.764 \\
\hline & $4 W 64 A$ & $-1.232^{*, b, c}$ & .209 & .000 & -1.643 & -.821 \\
\hline & $5 W 80 A$ & $-.857^{\pi, b, c}$ & .209 & .000 & -1.268 & -.446 \\
\hline \multirow[t]{5}{*}{$1 \mathrm{~W} 16 \mathrm{~A}$} & $1 \mathrm{~W} 0 \mathrm{~A}$ & $1.501^{\pi, b, c}$ & .209 & .000 & 1.090 & 1.912 \\
\hline & $2 W 32 A$ & $.276^{\mathrm{b}, \mathrm{c}}$ & .171 & .107 & -.060 & .611 \\
\hline & $3 W 48 A$ & $.326^{\mathrm{b}, \mathrm{c}}$ & .171 & .057 & -.009 & .662 \\
\hline & $4 W 64 A$ & $.270^{\mathrm{b}, \mathrm{c}}$ & .171 & .115 & -.066 & .605 \\
\hline & $5 W 80 A$ & $.644^{\pi, b, c}$ & .171 & .000 & .308 & .979 \\
\hline \multirow[t]{5}{*}{$2 W 32 A$} & $1 \mathrm{~W} 0 \mathrm{~A}$ & $1.225^{*}, \mathrm{~b}, \mathrm{c}$ & .209 & .000 & .814 & 1.636 \\
\hline & $1 \mathrm{~W} 16 \mathrm{~A}$ & $-.276^{\mathrm{b}, \mathrm{c}}$ & .171 & .107 & -.611 & .060 \\
\hline & $3 W 48 A$ & $.050^{\mathrm{b}, \mathrm{c}}$ & .171 & .769 & -.285 & .386 \\
\hline & $4 W 64 A$ & $-.006^{b, c}$ & .171 & .971 & -.342 & .329 \\
\hline & $5 W 80 A$ & $.368^{*, b, c}$ & .171 & .032 & .032 & .704 \\
\hline \multirow[t]{5}{*}{$3 W 48 A$} & $1 \mathrm{~W} 0 \mathrm{~A}$ & $1.175^{\pi, b, c}$ & .209 & .000 & .764 & 1.586 \\
\hline & $1 \mathrm{~W} 16 \mathrm{~A}$ & $-.326^{\mathrm{b}, \mathrm{c}}$ & .171 & .057 & -.662 & .009 \\
\hline & $2 W 32 A$ & $-.050^{b, c}$ & .171 & .769 & -.386 & .285 \\
\hline & $4 W 64 A$ & $-.057^{\mathrm{b}, \mathrm{c}}$ & .171 & .741 & -.392 & .279 \\
\hline & $5 W 80 A$ & $.318^{b, c}$ & .171 & .064 & -.018 & .653 \\
\hline \multirow[t]{5}{*}{$4 W 64 A$} & $1 \mathrm{~W} 0 \mathrm{~A}$ & $1.232^{\pi, b, c}$ & .209 & .000 & .821 & 1.643 \\
\hline & $1 \mathrm{~W} 16 \mathrm{~A}$ & $-.270^{b, c}$ & .171 & .115 & -.605 & .066 \\
\hline & $2 W 32 A$ & $.006^{\mathrm{b}, \mathrm{c}}$ & .171 & .971 & -.329 & .342 \\
\hline & $3 W$ 48A & $.057^{\mathrm{b}, \mathrm{c}}$ & .171 & .741 & -.279 & .392 \\
\hline & $5 W 80 A$ & $.374^{\pi, b, c}$ & .171 & .029 & .039 & .710 \\
\hline \multirow[t]{5}{*}{$5 \mathrm{~W} 80 \mathrm{~A}$} & $1 \mathrm{~W} 0 \mathrm{~A}$ & $.857^{x, \mathrm{~b}, \mathrm{c}}$ & .209 & .000 & .446 & 1.268 \\
\hline & $1 \mathrm{~W} 16 \mathrm{~A}$ & $-.644^{\pi}, \mathrm{b}, \mathrm{c}$ & .171 & .000 & -.979 & -.308 \\
\hline & $2 W 32 A$ & $-.368^{*}, \mathrm{~b}, \mathrm{c}$ & .171 & .032 & -.704 & -.032 \\
\hline & $3 W 48 A$ & $-.318^{\mathrm{b}, \mathrm{c}}$ & .171 & .064 & -.653 & .018 \\
\hline & $4 W 64 A$ & $-.374^{*, b, c}$ & .171 & .029 & -.710 & -.039 \\
\hline \multicolumn{7}{|c|}{$\begin{array}{l}\text { Based on estimated marginal means } \\
\star \text {. The mean difference is significant at the } .05 \text { level. }\end{array}$} \\
\hline \multicolumn{7}{|c|}{ b. An estimate of the modified population marginal mean (I). } \\
\hline \multicolumn{7}{|c|}{ c. An estimate of the modified population marginal mean (J). } \\
\hline
\end{tabular}


Marked significant differences were observed for all groups but not for all levels of pair-wise comparisons. In fact, group 1W 0A observed significant values for all pair-wise comparisons at a constant p-value at.001.

\section{Sample Area * Bleached: OSDP}

Dependent Variable: a

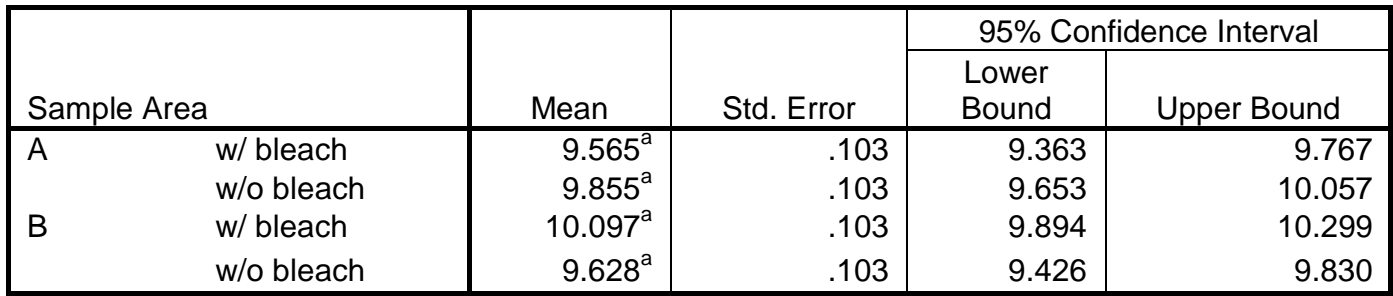

a. Based on modified population marginal mean.

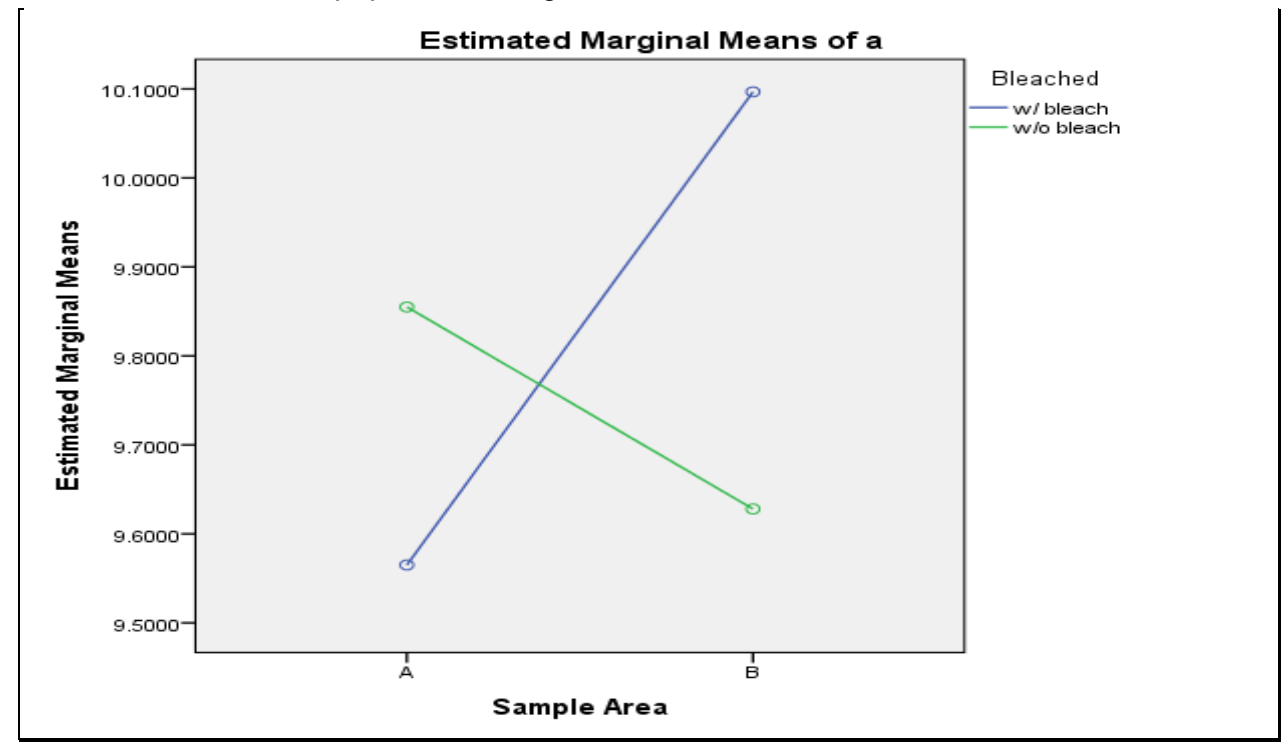

It can be observed that the mean values differed greatly at sample area B, however, since pvalues were not calculated for this interaction, no further interpretation can be concluded without proper statistical evidence. 
Dependent Variable: a

\section{Bleached * Washing: OSDP}

\begin{tabular}{|c|c|c|c|c|c|}
\hline \multirow[b]{2}{*}{ Bleached } & & \multirow[b]{2}{*}{ Mean } & \multirow[b]{2}{*}{ Std. Error } & \multicolumn{2}{|c|}{ 95\% Confidence Interval } \\
\hline & & & & $\begin{array}{l}\text { Lower } \\
\text { Bound }\end{array}$ & Upper Bound \\
\hline \multirow[t]{7}{*}{ w/ bleach } & $1 \mathrm{~W} 0 \mathrm{~A}$ & $8.890^{\mathrm{a}}$ & .242 & 8.415 & 9.364 \\
\hline & $1 \mathrm{~W} 16 \mathrm{~A}$ & $9.919^{a}$ & .171 & 9.583 & 10.255 \\
\hline & $2 W 32 A$ & $10.411^{a}$ & .171 & 10.075 & 10.747 \\
\hline & $3 W 48 A$ & $10.197^{a}$ & .171 & 9.862 & 10.533 \\
\hline & $4 W 64 \mathrm{~A}$ & $9.399^{a}$ & .171 & 9.064 & 9.735 \\
\hline & $5 W 80 A$ & $9.699^{a}$ & .171 & 9.363 & 10.034 \\
\hline & $1 \mathrm{~W} O A$ & $8.504^{a}$ & .242 & 8.030 & 8.979 \\
\hline \multirow{5}{*}{ w/o bleach } & $1 \mathrm{~W} 16 \mathrm{~A}$ & $10.477^{\mathrm{a}}$ & .171 & 10.142 & 10.813 \\
\hline & $2 W 32 A$ & $9.434^{a}$ & .171 & 9.098 & 9.769 \\
\hline & $3 W 48 A$ & $9.547^{\mathrm{a}}$ & .171 & 9.211 & 9.882 \\
\hline & $4 W 64 A$ & $10.458^{a}$ & .171 & 10.122 & 10.794 \\
\hline & $5 W 80 A$ & $9.410^{\mathrm{a}}$ & .171 & 9.075 & 9.746 \\
\hline
\end{tabular}

a. Based on modified population marginal mean.

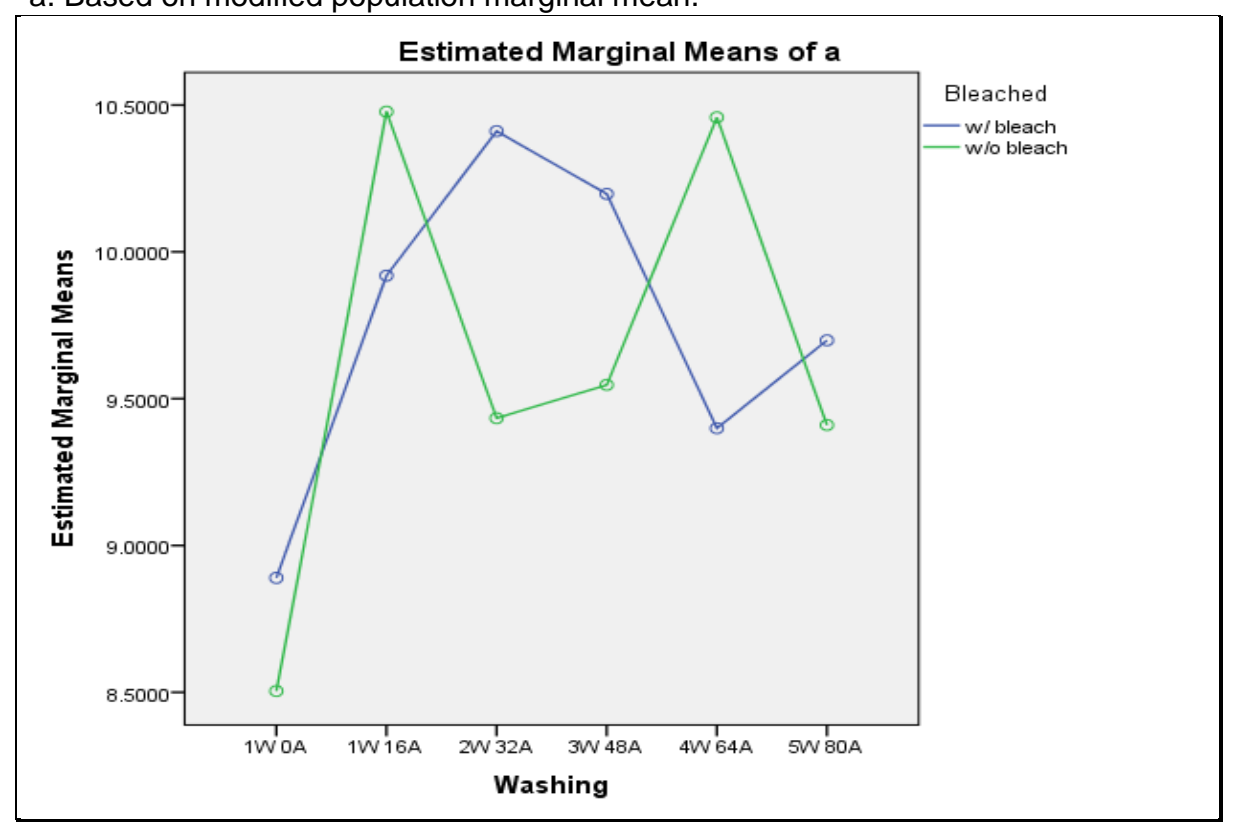

No consistent pattern can be observed for these samples. The mean values between the bleached and unbleached samples differ the greatest at 2W 32 and 4W 64. Significant interaction effects can be assumed.

For the swatch* washing interaction, the main effect p-value was ignored for swatch because the interaction was found to be significant. This means that the outcome of the interaction was affected by both factors. 


\section{Swatch * Washing: OSDP}

Dependent Variable: a

\begin{tabular}{|c|c|c|c|c|c|}
\hline \multirow{2}{*}{ Swatch } & & \multirow[b]{2}{*}{ Mean } & \multirow[b]{2}{*}{ Std. Error } & \multicolumn{2}{|c|}{ 95\% Confidence Interval } \\
\hline & & & & $\begin{array}{l}\text { Lower } \\
\text { Bound }\end{array}$ & Upper Bound \\
\hline \multirow[t]{6}{*}{$1 \mathrm{w} /$ bleach } & $1 \mathrm{~W} 0 \mathrm{~A}$ & $8.382^{\mathrm{a}}$ & .342 & 7.711 & 9.054 \\
\hline & $1 \mathrm{~W} 16 \mathrm{~A}$ & $9.926^{a}$ & .242 & 9.452 & 10.401 \\
\hline & $2 W 32 A$ & $10.539^{a}$ & .242 & 10.064 & 11.013 \\
\hline & $3 W 48 \mathrm{~A}$ & $9.696^{\mathrm{a}}$ & .242 & 9.221 & 10.170 \\
\hline & $4 \mathrm{~W} 64 \mathrm{~A}$ & $9.890^{\mathrm{a}}$ & .242 & 9.416 & 10.365 \\
\hline & $5 \mathrm{~W} 80 \mathrm{~A}$ & $9.852^{\mathrm{a}}$ & .242 & 9.377 & 10.326 \\
\hline \multirow[t]{6}{*}{$2 \mathrm{w} /$ bleach } & $1 \mathrm{~W} 0 \mathrm{~A}$ & $9.397^{\mathrm{a}}$ & .342 & 8.726 & 10.068 \\
\hline & $1 \mathrm{~W} 16 \mathrm{~A}$ & $9.912^{\mathrm{a}}$ & .242 & 9.437 & 10.386 \\
\hline & $2 W 32 A$ & $10.283^{a}$ & .242 & 9.809 & 10.758 \\
\hline & $3 W 48 A$ & $10.699^{a}$ & .242 & 10.224 & 11.173 \\
\hline & $4 \mathrm{~W} 64 \mathrm{~A}$ & $8.908^{a}$ & .242 & 8.433 & 9.383 \\
\hline & $5 \mathrm{~W} 80 \mathrm{~A}$ & $9.545^{\mathrm{a}}$ & .242 & 9.071 & 10.020 \\
\hline \multirow[t]{6}{*}{3 w/o bleach } & $1 \mathrm{~W} 0 \mathrm{~A}$ & $9.600^{\mathrm{a}}$ & .342 & 8.928 & 10.271 \\
\hline & $1 \mathrm{~W} 16 \mathrm{~A}$ & $10.791^{a}$ & .242 & 10.316 & 11.266 \\
\hline & $2 W 32 A$ & $9.673^{\mathrm{a}}$ & .242 & 9.199 & 10.148 \\
\hline & $3 W 48 A$ & $9.386^{a}$ & .242 & 8.912 & 9.861 \\
\hline & $4 \mathrm{~W} 64 \mathrm{~A}$ & $10.644^{\mathrm{a}}$ & .242 & 10.169 & 11.118 \\
\hline & $5 \mathrm{~W} 80 \mathrm{~A}$ & $9.299^{\mathrm{a}}$ & .242 & 8.824 & 9.773 \\
\hline \multirow[t]{6}{*}{4 w/o bleach } & $1 \mathrm{~W} 0 \mathrm{~A}$ & $7.409^{a}$ & .342 & 6.738 & 8.080 \\
\hline & $1 \mathrm{~W} 16 \mathrm{~A}$ & $10.164^{\mathrm{a}}$ & .242 & 9.689 & 10.638 \\
\hline & $2 W 32 A$ & $9.194^{\mathrm{a}}$ & .242 & 8.719 & 9.668 \\
\hline & $3 W 48 A$ & $9.707^{\mathrm{a}}$ & .242 & 9.233 & 10.182 \\
\hline & $4 \mathrm{~W} 64 \mathrm{~A}$ & $10.272^{\mathrm{a}}$ & .242 & 9.798 & 10.747 \\
\hline & $5 \mathrm{~W} 80 \mathrm{~A}$ & $9.522^{\mathrm{a}}$ & .242 & 9.047 & 9.996 \\
\hline
\end{tabular}

a. Based on modified population marginal mean.

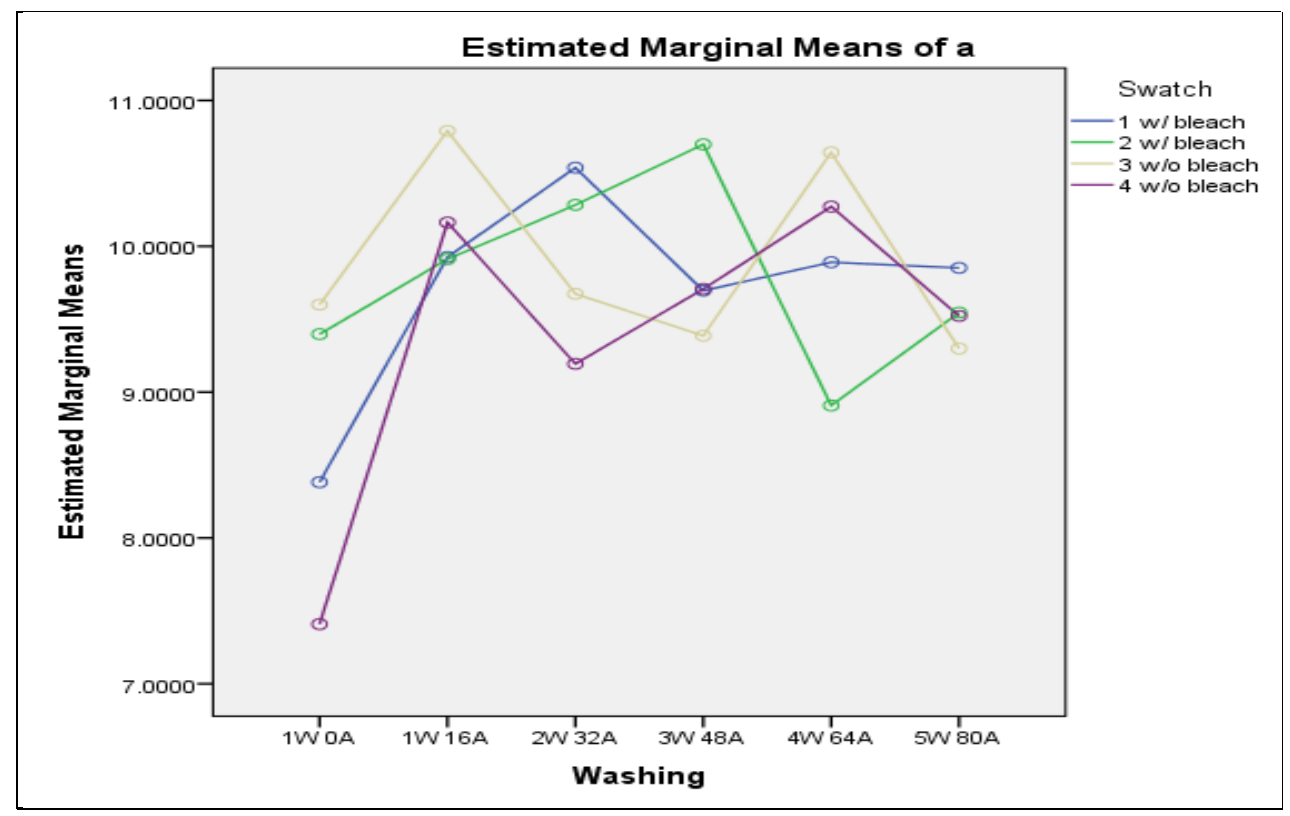


Variable ' $b$ '

Dependent Variable: $b$

Tests of Between-Subjects Effects: OSDP

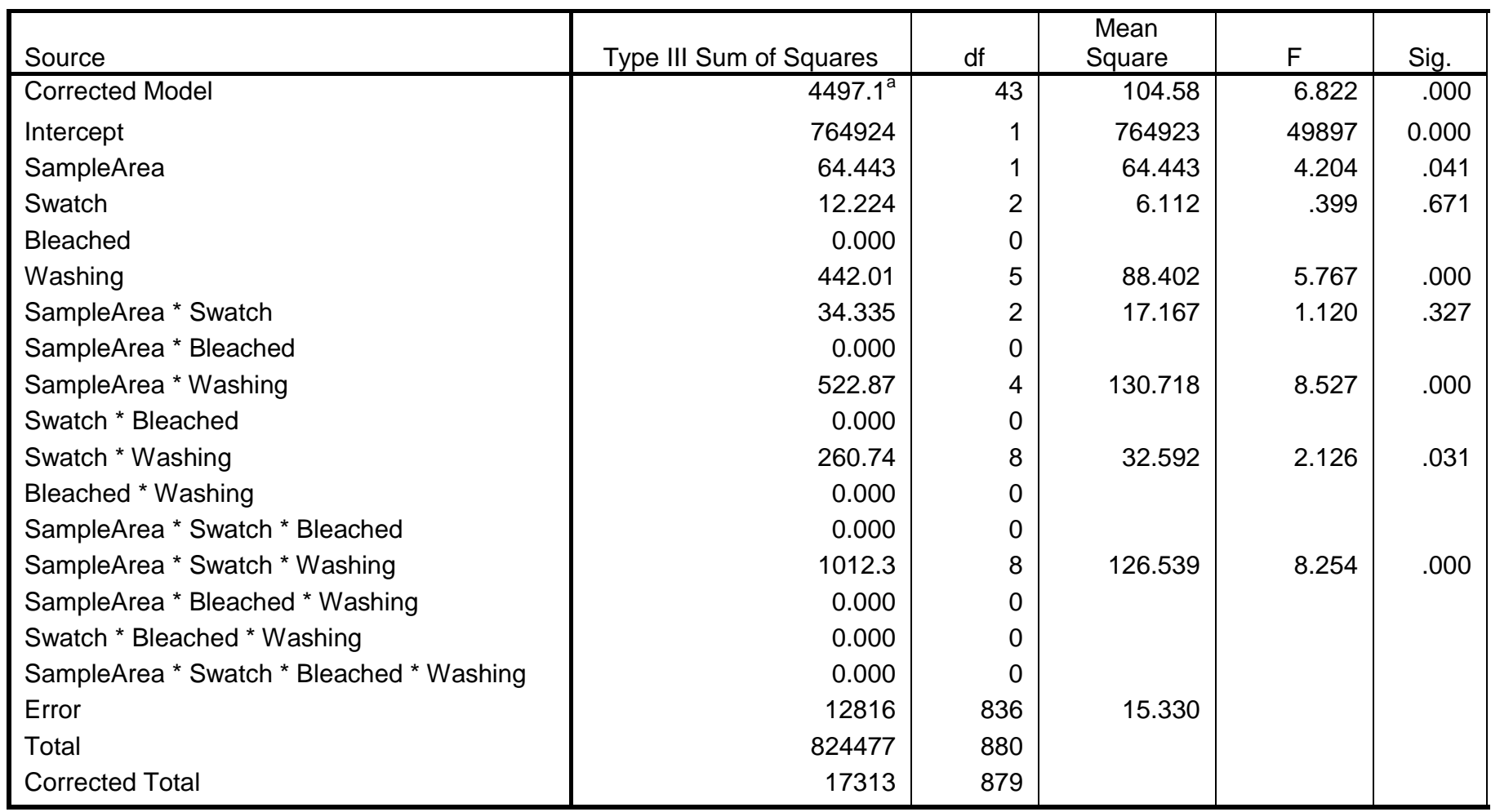

a. $\mathrm{R}$ Squared $=.260$ (Adjusted R Squared $=.222$ )

b. Computed using alpha $=.05$

The main effect of the swatch and the interaction effects of the Swatch* Sample Area were not

significant.

\section{Sample Area Estimates: OSDP}

Dependent Variable: $b$

\begin{tabular}{|l|r|r|r|r|}
\hline Sample & & \multirow{2}{*}{ 95\% Confidence Interval } \\
\cline { 4 - 5 } Area & Mean & Std. Error & Lower Bound & Upper Bound \\
\hline A & $30.133^{\mathrm{a}}$ & .187 & 29.767 & 30.500 \\
B & $30.438^{\mathrm{a}}$ & .187 & 30.072 & 30.805 \\
\hline
\end{tabular}

a. Based on modified population marginal mean.

Pairwise Comparisons: OSDP

Dependent Variable: $b$

\begin{tabular}{|c|c|c|c|c|c|}
\hline \multirow[b]{2}{*}{ (I) Sample Area } & \multirow{2}{*}{$\begin{array}{c}\text { Mean } \\
\text { Difference (I- } \\
\mathrm{J})\end{array}$} & \multirow[b]{2}{*}{ Std. Error } & \multirow[b]{2}{*}{ Sig. ${ }^{c}$} & \multicolumn{2}{|c|}{$95 \%$ Confidence Interval for Difference ${ }^{c}$} \\
\hline & & & & Lower Bound & Upper Bound \\
\hline A $\quad B$ & $-.305^{\mathrm{a}, \mathrm{b}}$ & .264 & .249 & -.823 & .213 \\
\hline$A$ & $.305^{a, b}$ & .264 & .249 & -.213 & .823 \\
\hline
\end{tabular}

Based on estimated marginal means

a. An estimate of the modified population marginal mean (I). 
b. An estimate of the modified population marginal mean (J).

c. Adjustment for multiple comparisons: Least Significant Difference (equivalent to no adjustments).

Although the main effects of sample area were significant, the differences between the levels of

pair-wise comparison were not significant. This means that the effects on the outcome of a

particular level (A), does not depend on the other level (B).

\section{Swatch Estimates: OSDP}

Dependent Variable: $b$

\begin{tabular}{|c|c|c|c|c|}
\hline \multirow[b]{2}{*}{ Swatch } & \multirow[b]{2}{*}{ Mean } & \multirow[b]{2}{*}{ Std. Error } & \multicolumn{2}{|c|}{ 95\% Confidence Interval } \\
\hline & & & Lower Bound & Upper Bound \\
\hline $1 \mathrm{w} /$ bleach & $30.449^{a}$ & .264 & 29.931 & 30.967 \\
\hline $2 \mathrm{w} /$ bleach & $30.379^{\mathrm{a}}$ & .264 & 29.861 & 30.897 \\
\hline 3 w/o bleach & $30.557^{\mathrm{a}}$ & .264 & 30.039 & 31.075 \\
\hline 4 w/o bleach & $29.758^{a}$ & .264 & 29.240 & 30.276 \\
\hline
\end{tabular}

a. Based on modified population marginal mean.

Pairwise Comparisons: OSDP

Dependent Variable: $b$

\begin{tabular}{|c|c|c|c|c|c|c|}
\hline \multirow[b]{2}{*}{ (I) Swatch } & & \multirow{2}{*}{$\begin{array}{c}\text { Mean } \\
\text { Difference } \\
(\mathrm{I}-\mathrm{J})\end{array}$} & \multirow[b]{2}{*}{ Std. Error } & \multirow[b]{2}{*}{ Sig. ${ }^{d}$} & \multicolumn{2}{|c|}{$\begin{array}{c}95 \% \text { Confidence Interval for } \\
\text { Difference }\end{array}$} \\
\hline & & & & & Lower Bound & Upper Bound \\
\hline \multirow[t]{3}{*}{$1 \mathrm{w} /$ bleach } & 2 w/ bleach & $.070^{\mathrm{a}, \mathrm{b}}$ & .373 & .851 & -.663 & .803 \\
\hline & 3 w/o bleach & $-.108^{a, b}$ & .373 & .772 & -.841 & .625 \\
\hline & 4 w/o bleach & $.691^{a, b}$ & .373 & .064 & -.042 & 1.424 \\
\hline \multirow[t]{3}{*}{$2 \mathrm{w} /$ bleach } & $1 \mathrm{w} /$ bleach & $-.070^{a, b}$ & .373 & .851 & -.803 & .663 \\
\hline & 3 w/o bleach & $-.178^{\mathrm{a}, \mathrm{b}}$ & .373 & .633 & -.911 & .554 \\
\hline & 4 w/o bleach & $.621^{\mathrm{a}, \mathrm{b}}$ & .373 & .096 & -.112 & 1.354 \\
\hline \multirow[t]{3}{*}{3 w/o bleach } & 1 w/ bleach & $.108^{\mathrm{a}, \mathrm{b}}$ & .373 & .772 & -.625 & .841 \\
\hline & 2 w/ bleach & $.178^{a, b}$ & .373 & .633 & -.554 & .911 \\
\hline & 4 w/o bleach & $.799^{\mathrm{a}, \mathrm{b},{ }^{*}}$ & .373 & .033 & .067 & 1.532 \\
\hline \multirow[t]{3}{*}{4 w/o bleach } & $1 \mathrm{w} /$ bleach & $-.691^{a, b}$ & .373 & .064 & -1.424 & .042 \\
\hline & 2 w/ bleach & $-.621^{a, b}$ & .373 & .096 & -1.354 & .112 \\
\hline & 3 w/o bleach & $-.799^{\mathrm{a}, \mathrm{b},{ }^{*}}$ & .373 & .033 & -1.532 & -.067 \\
\hline
\end{tabular}

Based on estimated marginal means

*. The mean difference is significant at the .05 level.

a. An estimate of the modified population marginal mean (I).

b. An estimate of the modified population marginal mean $(\mathrm{J})$.

d. Adjustment for multiple comparisons: Least Significant Difference (equivalent to no adjustments).

The bleached swatches did not observe significant values, however, significant values were

observed between the unbleached swatches. 


\section{Bleach Estimates: OSDP}

Dependent Variable: $b$

\begin{tabular}{|l|r|r|r|r|}
\hline & & \multirow{2}{*}{ Bleached } & \multicolumn{2}{|c|}{ 95\% Confidence Interval } \\
\cline { 4 - 5 } w/ bleach & Mean & Std. Error & Lower Bound & Upper Bound \\
\hline w/o bleach & $30.414^{\mathrm{a}}$ & .187 & 30.048 & 30.780 \\
& $30.158^{\mathrm{a}}$ & .187 & 29.791 & 30.524 \\
\hline
\end{tabular}

a. Based on modified population marginal mean.

Pairwise Comparisons: OSDP

Dependent Variable: $b$

\begin{tabular}{|ll|r|r|r|r|r|}
\hline & & \multirow{2}{*}{$\begin{array}{c}\text { Mean } \\
\text { Difference }(\mathrm{I}-\end{array}$} & & & \multicolumn{2}{|c|}{$\begin{array}{c}\text { 95\% Confidence Interval for } \\
\text { Difference }\end{array}$} \\
\cline { 5 - 7 } & & $\mathrm{J})$ & Std. Error & Sig. $^{\mathrm{c}}$ & Lower Bound & Upper Bound \\
\hline w/ bleach & w/o bleach & $.256^{\mathrm{a}, \mathrm{b}}$ & .264 & .332 & -.262 & .775 \\
w/o bleach & w/ bleach & $-.256^{\mathrm{a}, \mathrm{b}}$ & .264 & .332 & -.775 & .262 \\
\hline
\end{tabular}

Based on estimated marginal means

a. An estimate of the modified population marginal mean (I).

b. An estimate of the modified population marginal mean (J).

c. Adjustment for multiple comparisons: Least Significant Difference (equivalent to no adjustments).

No marked significances were observed between the bleached and unbleached samples.

\section{Washing Estimates: OSDP}

Dependent Variable: $b$

\begin{tabular}{|c|c|c|c|c|}
\hline \multirow[b]{2}{*}{ Washing } & \multirow[b]{2}{*}{ Mean } & \multirow[b]{2}{*}{ Std. Error } & \multicolumn{2}{|c|}{$95 \%$ Confidence Interval } \\
\hline & & & Lower Bound & Upper Bound \\
\hline $1 \mathrm{~W} 0 \mathrm{~A}$ & $32.242^{\mathrm{a}}$ & .438 & 31.383 & 33.101 \\
\hline $1 \mathrm{~W} 16 \mathrm{~A}$ & $30.489^{a}$ & .310 & 29.881 & 31.096 \\
\hline $2 W 32 A$ & $30.081^{a}$ & .310 & 29.474 & 30.689 \\
\hline $3 W 48 A$ & $30.167^{a}$ & .310 & 29.559 & 30.774 \\
\hline $4 \mathrm{~W} 64 \mathrm{~A}$ & $30.150^{a}$ & .310 & 29.543 & 30.758 \\
\hline $5 W 80 A$ & $29.564^{\mathrm{a}}$ & .310 & 28.957 & 30.172 \\
\hline
\end{tabular}

a. Based on modified population marginal mean.

At 0W 0A, marked significant differences were observed for all the pair-wise comparing groups.

Only one other significant values was observed between $1 \mathrm{~W} 16 \mathrm{~A}$ and $5 \mathrm{~W} 80 \mathrm{~A}$. It can be

concluded the washing treatment did not affect the saturation of the color considerably. 
Dependent Variable: $b$

Pairwise Comparisons: OSDP

\begin{tabular}{|c|c|c|c|c|c|c|}
\hline \multirow{2}{*}{ (I) Washing } & & \multirow[b]{2}{*}{$\begin{array}{c}\text { Mean Difference (I- } \\
\mathrm{J})\end{array}$} & \multirow[b]{2}{*}{ Std. Error } & \multirow[b]{2}{*}{ Sig. ${ }^{d}$} & \multicolumn{2}{|c|}{$\begin{array}{l}\text { 95\% Confidence Interval for } \\
\text { Difference }^{d}\end{array}$} \\
\hline & & & & & Lower Bound & $\begin{array}{l}\text { Upper } \\
\text { Bound }\end{array}$ \\
\hline \multirow[t]{5}{*}{$1 \mathrm{~W} 0 \mathrm{~A}$} & $1 \mathrm{~W} 16 \mathrm{~A}$ & $1.753^{, b, c}$ & .536 & .001 & .701 & 2.806 \\
\hline & $2 W 32 A$ & $2.161^{\pi, b, c}$ & .536 & .000 & 1.108 & 3.213 \\
\hline & $3 W 48 A$ & $2.075^{\star}, \mathrm{b}, \mathrm{c}$ & .536 & .000 & 1.023 & 3.128 \\
\hline & $4 \mathrm{~W} 64 \mathrm{~A}$ & $2.092^{*}, \mathrm{~b}, \mathrm{c}$ & .536 & .000 & 1.039 & 3.144 \\
\hline & $5 W 80 A$ & $2.677^{*, \mathrm{~b}, \mathrm{c}}$ & .536 & .000 & 1.625 & 3.730 \\
\hline \multirow[t]{5}{*}{$1 \mathrm{~W} 16 \mathrm{~A}$} & $1 \mathrm{~W} 0 \mathrm{~A}$ & $-1.753^{\pi, \mathrm{b}, \mathrm{c}}$ & .536 & .001 & -2.806 & -.701 \\
\hline & $2 W 32 A$ & $.408^{\mathrm{b}, \mathrm{c}}$ & .438 & .352 & -.452 & 1.267 \\
\hline & $3 W 48 A$ & $.322^{\mathrm{b}, \mathrm{c}}$ & .438 & .462 & -.537 & 1.181 \\
\hline & $4 \mathrm{~W} 64 \mathrm{~A}$ & $.339^{\mathrm{b}, \mathrm{c}}$ & .438 & .439 & -.521 & 1.198 \\
\hline & $5 W 80 A$ & $.924^{\pi, b, c}$ & .438 & .035 & .065 & 1.783 \\
\hline \multirow[t]{5}{*}{$2 W 32 A$} & $1 \mathrm{~W} 0 \mathrm{~A}$ & $-2.161^{*}, \mathrm{~b}, \mathrm{c}$ & .536 & .000 & -3.213 & -1.108 \\
\hline & $1 \mathrm{~W} 16 \mathrm{~A}$ & $-.408^{\mathrm{b}, \mathrm{c}}$ & .438 & .352 & -1.267 & .452 \\
\hline & $3 W 48 A$ & $-.085^{b, c}$ & .438 & .845 & -.945 & .774 \\
\hline & $4 W 64 A$ & $-.069^{b, c}$ & .438 & .875 & -.928 & .790 \\
\hline & $5 W 80 A$ & $.517^{\mathrm{b}, \mathrm{c}}$ & .438 & .238 & -.343 & 1.376 \\
\hline \multirow[t]{5}{*}{$3 W 48 A$} & $1 \mathrm{~W} 0 \mathrm{~A}$ & $-2.075^{\pi, b, c}$ & .536 & .000 & -3.128 & -1.023 \\
\hline & $1 \mathrm{~W} 16 \mathrm{~A}$ & $-.322^{b, c}$ & .438 & .462 & -1.181 & .537 \\
\hline & $2 W 32 A$ & $.085^{\mathrm{b}, \mathrm{c}}$ & .438 & .845 & -.774 & .945 \\
\hline & $4 W 64 A$ & $.016^{b, c}$ & .438 & .970 & -.843 & .876 \\
\hline & $5 W 80 A$ & $.602^{\mathrm{b}, \mathrm{c}}$ & .438 & .169 & -.257 & 1.461 \\
\hline \multirow[t]{5}{*}{$4 W 64 A$} & $1 \mathrm{~W} 0 \mathrm{~A}$ & $-2.092^{\pi, b, c}$ & .536 & .000 & -3.144 & -1.039 \\
\hline & $1 \mathrm{~W} 16 \mathrm{~A}$ & $-.339^{b, c}$ & .438 & .439 & -1.198 & .521 \\
\hline & $2 W 32 A$ & $.069^{b, c}$ & .438 & .875 & -.790 & .928 \\
\hline & $3 W$ 48A & $-.016^{b, c}$ & .438 & .970 & -.876 & .843 \\
\hline & $5 W 80 A$ & $.586^{\mathrm{b}, \mathrm{c}}$ & .438 & .181 & -.274 & 1.445 \\
\hline \multirow[t]{5}{*}{$5 W$} & $1 \mathrm{~W} 0 \mathrm{~A}$ & $-2.677^{\pi, b, c}$ & .536 & .000 & -3.730 & -1.625 \\
\hline & $1 \mathrm{~W} 16 \mathrm{~A}$ & $-.924^{\pi, b, c}$ & .438 & .035 & -1.783 & -.065 \\
\hline & $2 W 32 A$ & $-.517^{\mathrm{b}, \mathrm{c}}$ & .438 & .238 & -1.376 & .343 \\
\hline & $3 W 48 A$ & $-.602^{b, c}$ & .438 & .169 & -1.461 & .257 \\
\hline & $4 \mathrm{~W} 64 \mathrm{~A}$ & $-.586^{b, c}$ & .438 & .181 & -1.445 & .274 \\
\hline
\end{tabular}

Based on estimated marginal means

*. The mean difference is significant at the .05 level.

b. An estimate of the modified population marginal mean (I).

c. An estimate of the modified population marginal mean $(\mathrm{J})$.

d. Adjustment for multiple comparisons: Least Significant Difference (equivalent to no adjustments). 


\section{Sample Area * Bleached: OSDP}

Dependent Variable: $b$

\begin{tabular}{|c|c|c|c|c|c|}
\hline \multirow{2}{*}{\multicolumn{2}{|c|}{ Sample Area }} & \multirow[b]{2}{*}{ Mean } & \multirow[b]{2}{*}{ Std. Error } & \multicolumn{2}{|c|}{$95 \%$ Confidence Interval } \\
\hline & & & & Lower Bound & Upper Bound \\
\hline \multirow[t]{4}{*}{$\bar{A}$} & w/ bleach & $29.966^{\mathrm{a}}$ & .264 & 29.448 & 30.484 \\
\hline & w/o bleach & $30.301^{a}$ & .264 & 29.783 & 30.819 \\
\hline & w/ bleach & $30.862^{a}$ & .264 & 30.344 & 31.380 \\
\hline & w/o bleach & $30.014^{a}$ & .264 & 29.496 & 30.532 \\
\hline
\end{tabular}

a. Based on modified population marginal mean.

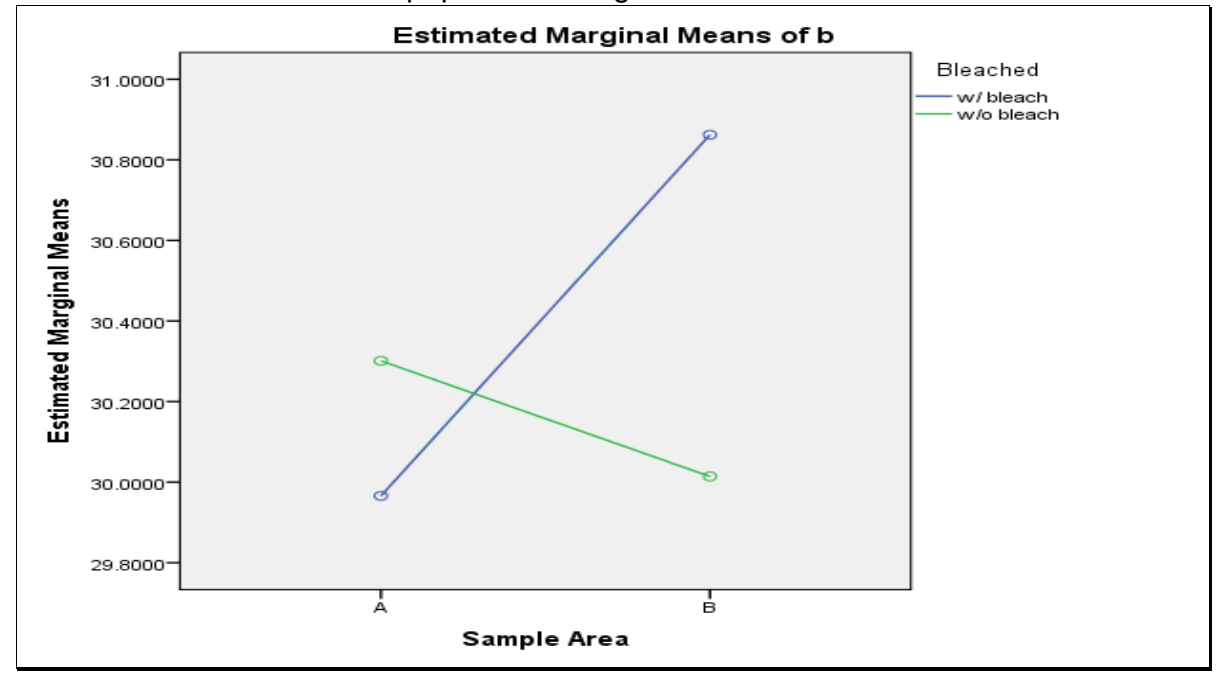

Although it can be observed that the differences in means between the bleached and unbleached sample is evident, without statistical results, only an implication can be made to suggest that the interaction effects here are significant.

Dependent Variable: $b$

\section{Bleached * Washing: OSDP}

\begin{tabular}{|c|c|c|c|c|c|}
\hline \multirow[b]{2}{*}{ Bleached } & & \multirow[b]{2}{*}{ Mean } & \multirow[b]{2}{*}{ Std. Error } & \multicolumn{2}{|c|}{ 95\% Confidence Interval } \\
\hline & & & & Lower Bound & Upper Bound \\
\hline \multirow[t]{6}{*}{ w/ bleach } & $1 \mathrm{~W} 0 \mathrm{~A}$ & $33.091^{\mathrm{a}}$ & .619 & 31.875 & 34.306 \\
\hline & $1 \mathrm{~W} 16 \mathrm{~A}$ & $29.710^{a}$ & .438 & 28.851 & 30.570 \\
\hline & $2 W 32 A$ & $31.674^{\mathrm{a}}$ & .438 & 30.814 & 32.533 \\
\hline & $3 W 48 A$ & $31.148^{\mathrm{a}}$ & .438 & 30.289 & 32.007 \\
\hline & $4 W 64 A$ & $28.331^{a}$ & .438 & 27.472 & 29.190 \\
\hline & $5 W 80 A$ & $29.869^{a}$ & .438 & 29.010 & 30.729 \\
\hline \multirow[t]{6}{*}{ w/o bleach } & $1 \mathrm{~W} 0 \mathrm{~A}$ & $31.393^{a}$ & .619 & 30.178 & 32.608 \\
\hline & $1 \mathrm{~W} 16 \mathrm{~A}$ & $31.267^{a}$ & .438 & 30.408 & 32.126 \\
\hline & $2 W 32 A$ & $28.489^{a}$ & .438 & 27.630 & 29.348 \\
\hline & $3 W 48 A$ & $29.185^{a}$ & .438 & 28.326 & 30.044 \\
\hline & $4 \mathrm{~W} 64 \mathrm{~A}$ & $31.969^{\mathrm{a}}$ & .438 & 31.110 & 32.829 \\
\hline & $5 \mathrm{~W} 80 \mathrm{~A}$ & $29.260^{a}$ & .438 & 28.400 & 30.119 \\
\hline
\end{tabular}

a. Based on modified population marginal mean. 


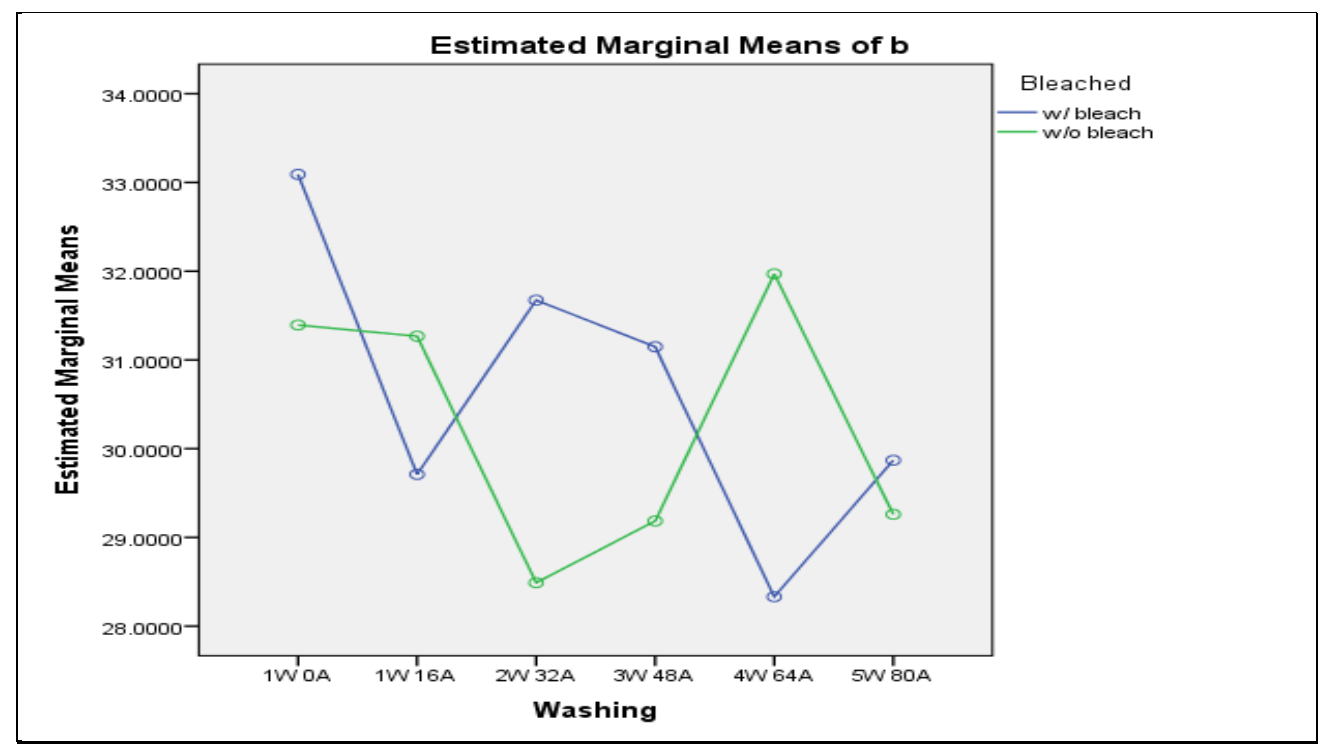

Similar to the above implication, although differences in means can be observed between the samples, without a calculated p-value, this assumption cannot be confirmed.

Dependent Variable: $b$

\section{Swatch * Washing: OSDP}

\begin{tabular}{|c|c|c|c|c|c|}
\hline \multirow{2}{*}{ Swatch } & & \multirow[b]{2}{*}{ Mean } & \multirow[b]{2}{*}{ Std. Error } & \multicolumn{2}{|c|}{ 95\% Confidence Interval } \\
\hline & & & & Lower Bound & Upper Bound \\
\hline \multirow[t]{6}{*}{$1 \mathrm{w} /$ bleach } & $1 \mathrm{~W} 0 \mathrm{~A}$ & $32.382^{a}$ & .876 & 30.663 & 34.100 \\
\hline & $1 \mathrm{~W} 16 \mathrm{~A}$ & $29.686^{a}$ & .619 & 28.471 & 30.901 \\
\hline & $2 W 32 A$ & $31.505^{\mathrm{a}}$ & .619 & 30.290 & 32.720 \\
\hline & $3 W 48 A$ & $30.244^{a}$ & .619 & 29.029 & 31.459 \\
\hline & $4 W 64 A$ & $29.258^{a}$ & .619 & 28.043 & 30.474 \\
\hline & $5 W 80 A$ & $30.586^{a}$ & .619 & 29.371 & 31.802 \\
\hline \multirow[t]{6}{*}{2 w/ bleach } & $1 \mathrm{~W} 0 \mathrm{~A}$ & $33.800^{a}$ & .876 & 32.081 & 35.518 \\
\hline & $1 \mathrm{~W} 16 \mathrm{~A}$ & $29.735^{a}$ & .619 & 28.520 & 30.950 \\
\hline & $2 W 32 A$ & $31.842^{\mathrm{a}}$ & .619 & 30.627 & 33.057 \\
\hline & $3 W 48 A$ & $32.052^{\mathrm{a}}$ & .619 & 30.837 & 33.267 \\
\hline & $4 W 64 A$ & $27.403^{\mathrm{a}}$ & .619 & 26.188 & 28.618 \\
\hline & $5 W 80 A$ & $29.152^{a}$ & .619 & 27.937 & 30.367 \\
\hline \multirow[t]{6}{*}{3 w/o bleach } & $1 \mathrm{~W} 0 \mathrm{~A}$ & $34.427^{\mathrm{a}}$ & .876 & 32.709 & 36.145 \\
\hline & $1 \mathrm{~W} 16 \mathrm{~A}$ & $31.485^{\mathrm{a}}$ & .619 & 30.270 & 32.700 \\
\hline & $2 W 32 A$ & $28.671^{a}$ & .619 & 27.456 & 29.886 \\
\hline & $3 W 48 A$ & $28.801^{a}$ & .619 & 27.586 & 30.016 \\
\hline & $4 W 64 A$ & $32.911^{a}$ & .619 & 31.696 & 34.126 \\
\hline & $5 W 80 A$ & $28.984^{\mathrm{a}}$ & .619 & 27.769 & 30.199 \\
\hline \multirow[t]{6}{*}{4 w/o bleach } & $1 \mathrm{~W} 0 \mathrm{~A}$ & $28.360^{\mathrm{a}}$ & .876 & 26.641 & 30.078 \\
\hline & $1 \mathrm{~W} 16 \mathrm{~A}$ & $31.049^{a}$ & .619 & 29.834 & 32.264 \\
\hline & $2 W 32 A$ & $28.306^{a}$ & .619 & 27.091 & 29.522 \\
\hline & $3 W$ 48A & $29.570^{a}$ & .619 & 28.355 & 30.785 \\
\hline & $4 W 64 A$ & $31.028^{a}$ & .619 & 29.813 & 32.243 \\
\hline & $5 W 80 A$ & $29.535^{a}$ & .619 & 28.320 & 30.750 \\
\hline
\end{tabular}


a. Based on modified population marginal mean.

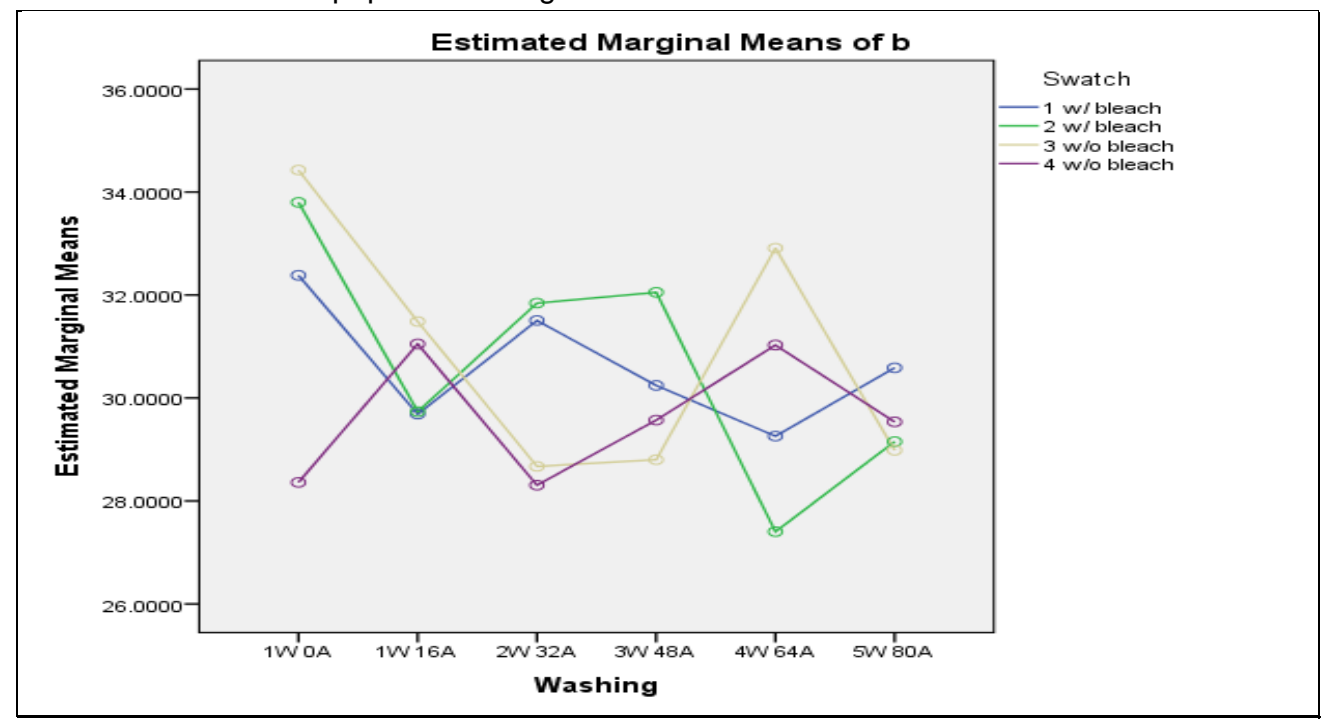

The interaction effect was deemed significant although the main effect of swatch was not.

IV and V. Samples PMCL and PMJX (discussed simultaneously because of their similarity in color)

\section{Variable ' $L$ '}

Dependent Variable: L

Tests of Between-Subjects Effects: PMCL

\begin{tabular}{|c|c|c|c|c|c|}
\hline Source & Type III Sum of Squares & $\mathrm{df}$ & Mean Square & $\mathrm{F}$ & Sig. \\
\hline Corrected Model & $6764.5^{a}$ & 43 & 157.313 & 12.097 & .000 \\
\hline Intercept & 7401034 & 1 & 7401034 & 569100 & 0.000 \\
\hline SampleArea & 30.645 & 1 & 30.645 & 2.356 & .125 \\
\hline Swatch & 49.137 & 2 & 24.568 & 1.889 & .152 \\
\hline Bleached & 0.000 & 0 & & & \\
\hline Washing & 305.35 & 5 & 61.069 & 4.696 & .000 \\
\hline SampleArea * Swatch & 364.81 & 2 & 182.40 & 14.026 & .000 \\
\hline SampleArea * Bleached & 0.000 & 0 & & & \\
\hline SampleArea * Washing & 490.21 & 4 & 122.55 & 9.424 & .000 \\
\hline Swatch * Bleached & 0.000 & 0 & & & \\
\hline Swatch * Washing & 2452.3 & 8 & 306.54 & 23.571 & .000 \\
\hline Bleached * Washing & 0.000 & 0 & & & \\
\hline SampleArea * Swatch * Bleached & 0.000 & 0 & & & \\
\hline SampleArea * Swatch * Washing & 1103.0 & 8 & 137.89 & 10.601 & .000 \\
\hline SampleArea * Bleached * Washing & 0.000 & 0 & & & \\
\hline Swatch * Bleached * Washing & 0.000 & 0 & & & \\
\hline SampleArea * Swatch * Bleached * Washing & 0.000 & 0 & & & \\
\hline Error & 10872 & 836 & 13.005 & & \\
\hline Total & 7912172 & 880 & & & \\
\hline Corrected Total & 17636 & 879 & & & \\
\hline
\end{tabular}

a. $\mathrm{R}$ Squared $=.384$ (Adjusted R Squared $=.352$ )

b. Computed using alpha $=.05$ 
Dependent Variable: L

Tests of Between-Subjects Effects: PMJX

\begin{tabular}{|c|c|c|c|c|c|}
\hline Source & Type III Sum of Squares & df & Mean Square & $\mathrm{F}$ & Sig. \\
\hline Corrected Model & $4037.9^{a}$ & 43 & 93.904 & 7.303 & .000 \\
\hline Intercept & 7383481 & 1 & 7383481 & 574238 & 0.000 \\
\hline SampleArea & 78.063 & 1 & 78.063 & 6.071 & .014 \\
\hline Swatch & 119.60 & 2 & 59.801 & 4.651 & .010 \\
\hline bleached & 0.000 & 0 & & & \\
\hline washings & 954.92 & 5 & 190.99 & 14.854 & .000 \\
\hline SampleArea * Swatch & 228.45 & 2 & 114.22 & 8.884 & .000 \\
\hline SampleArea * bleached & 0.000 & 0 & & & \\
\hline SampleArea * washings & 75.62 & 4 & 18.90 & 1.470 & .209 \\
\hline Swatch * bleached & 0.000 & 0 & & & \\
\hline Swatch * washings & 302.23 & 8 & 37.778 & 2.938 & .003 \\
\hline bleached * washings & 0.000 & 0 & & & \\
\hline SampleArea * Swatch * bleached & 0.000 & 0 & & & \\
\hline SampleArea * Swatch * washings & 774.23 & 8 & 96.778 & 7.527 & .000 \\
\hline SampleArea * bleached * washings & 0.000 & 0 & & & \\
\hline Swatch * bleached * washings & 0.000 & 0 & & & \\
\hline SampleArea * Swatch * bleached * washings & 0.000 & 0 & & & \\
\hline Error & 10749 & 836 & 12.858 & & \\
\hline Total & 7902539 & 880 & & & \\
\hline Corrected Total & 14787 & 879 & & & \\
\hline
\end{tabular}

a. $\mathrm{R}$ Squared $=.273$ (Adjusted R Squared $=.236$ )

b. Computed using alpha $=.05$

The main effects of swatch and sample area were observed insignificant at sample PMCL. The

interaction effects for sample PMJX was observed to be insignificant at the Sample Area*

Washing level. No similarity was observed with these samples at this variable.

\section{Sample Area Estimates: PMCL}

Dependent Variable: L

\begin{tabular}{|c|c|c|c|c|}
\hline \multirow{2}{*}{$\begin{array}{l}\text { Sample } \\
\text { Area }\end{array}$} & \multirow[b]{2}{*}{ Mean } & \multirow[b]{2}{*}{ Std. Error } & \multicolumn{2}{|c|}{$95 \%$ Confidence Interval } \\
\hline & & & Lower Bound & Upper Bound \\
\hline$A$ & $94.572^{a}$ & .172 & 94.234 & 94.909 \\
\hline B & $94.860^{\mathrm{a}}$ & .172 & 94.522 & 95.197 \\
\hline
\end{tabular}

a. Based on modified population marginal mean.

Pairwise Comparisons: PMCL

Dependent Variable: L

\begin{tabular}{|c|c|c|c|c|c|c|}
\hline \multirow{2}{*}{\multicolumn{2}{|c|}{ (I) Sample Area }} & \multirow{2}{*}{$\begin{array}{c}\text { Mean } \\
\text { Difference (I- } \\
\mathrm{J})\end{array}$} & \multirow{2}{*}{$\begin{array}{l}\text { Std. } \\
\text { Error }\end{array}$} & \multirow[b]{2}{*}{ Sig. ${ }^{c}$} & \multicolumn{2}{|c|}{$95 \%$ Confidence Interval for Difference ${ }^{c}$} \\
\hline & & & & & Lower Bound & Upper Bound \\
\hline$A$ & $\mathrm{~B}$ & $-.288^{\mathrm{a}, \mathrm{b}}$ & .243 & .237 & -.765 & .189 \\
\hline$B$ & $A$ & $.288^{\mathrm{a}, \mathrm{b}}$ & .243 & .237 & -.189 & .765 \\
\hline
\end{tabular}

Based on estimated marginal means 
a. An estimate of the modified population marginal mean (I).

b. An estimate of the modified population marginal mean $(\mathrm{J})$.

c. Adjustment for multiple comparisons: Least Significant Difference (equivalent to no adjustments).

Dependent Variable: L

1. Sample Area Estimates: PMJX

\begin{tabular}{|c|c|c|c|c|}
\hline \multirow{2}{*}{$\begin{array}{l}\text { Sample } \\
\text { Area }\end{array}$} & \multirow[b]{2}{*}{ Mean } & \multirow{2}{*}{$\begin{array}{l}\text { Std. } \\
\text { Error }\end{array}$} & \multicolumn{2}{|c|}{$95 \%$ Confidence Interval } \\
\hline & & & Lower Bound & Upper Bound \\
\hline $\mathrm{A}$ & $95.087^{\mathrm{a}}$ & .171 & 94.752 & 95.423 \\
\hline B & $94.263^{\mathrm{a}}$ & .171 & 93.927 & 94.598 \\
\hline
\end{tabular}

a. Based on modified population marginal mean.

Pairwise Comparisons: PMJX

Dependent Variable: $L$

\begin{tabular}{|c|c|c|c|c|c|}
\hline \multirow[b]{2}{*}{ (I) Sample Area } & \multirow{2}{*}{$\begin{array}{c}\text { Mean } \\
\text { Difference (I- } \\
\mathrm{J})\end{array}$} & \multirow[b]{2}{*}{ Std. Error } & \multirow[b]{2}{*}{ Sig. ${ }^{d}$} & \multicolumn{2}{|c|}{$\begin{array}{l}\text { 95\% Confidence Interval for } \\
\text { Difference }\end{array}$} \\
\hline & & & & Lower Bound & Upper Bound \\
\hline $\begin{array}{ll}A & B\end{array}$ & $.824^{\circ, b, c}$ & .242 & .001 & .350 & 1.299 \\
\hline$A$ & $-.824^{\pi, b, c}$ & .242 & .001 & -1.299 & -.350 \\
\hline
\end{tabular}

Based on estimated marginal means

*. The mean difference is significant at the .05 level.

b. An estimate of the modified population marginal mean (I).

C. An estimate of the modified population marginal mean $(\mathrm{J})$.

d. Adjustment for multiple comparisons: Least Significant Difference (equivalent to no adjustments).

It can be observed between the samples, that while PMCL did not obtain significant values,

PMJX did. At this interaction level, difference between two or more main effects was not

observed for sample PMCL but for sample PMJX.

\section{Swatch Estimates: PMCL}

Dependent Variable: L

\begin{tabular}{|c|c|c|c|c|}
\hline \multirow[b]{2}{*}{ Swatch } & \multirow[b]{2}{*}{ Mean } & \multirow[b]{2}{*}{$\begin{array}{l}\text { Std. } \\
\text { Error }\end{array}$} & \multicolumn{2}{|c|}{ 95\% Confidence Interval } \\
\hline & & & $\begin{array}{l}\text { Lower } \\
\text { Bound }\end{array}$ & $\begin{array}{l}\text { Upper } \\
\text { Bound }\end{array}$ \\
\hline $1 \mathrm{w} /$ bleach & $95.292^{a}$ & .243 & 94.815 & 95.769 \\
\hline $2 \mathrm{w} /$ bleach & $95.105^{a}$ & .243 & 94.628 & 95.582 \\
\hline $3 \mathrm{w} /$ bleach & $93.817^{\mathrm{a}}$ & .243 & 93.340 & 94.294 \\
\hline $4 \mathrm{w} /$ bleach & $94.649^{a}$ & .243 & 94.171 & 95.126 \\
\hline
\end{tabular}

a. Based on modified population marginal mean. 
Pairwise Comparisons: PMCL

Dependent Variable: L

\begin{tabular}{|c|c|c|c|c|c|c|}
\hline \multirow[b]{2}{*}{ (I) Swatch } & & \multirow{2}{*}{$\begin{array}{c}\text { Mean } \\
\text { Difference } \\
(\mathrm{I}-\mathrm{J})\end{array}$} & \multirow[b]{2}{*}{$\begin{array}{l}\text { Std. } \\
\text { Error }\end{array}$} & \multirow[b]{2}{*}{ Sig. ${ }^{d}$} & \multicolumn{2}{|c|}{$\begin{array}{l}\text { 95\% Confidence Interval for } \\
\text { Difference } \\
\end{array}$} \\
\hline & & & & & $\begin{array}{l}\text { Lower } \\
\text { Bound }\end{array}$ & Upper Bound \\
\hline \multirow[t]{3}{*}{$1 \mathrm{w} /$ bleach } & 2 w/ bleach' & $.187^{\mathrm{a}, \mathrm{b}}$ & .344 & .586 & -.488 & .862 \\
\hline & 3 w/o bleach & $1.475^{\mathrm{a}, \mathrm{b},{ }^{*}}$ & .344 & .000 & .800 & 2.150 \\
\hline & 4 w/o bleach & $.643^{\mathrm{a}, \mathrm{b}}$ & .344 & .062 & -.031 & 1.318 \\
\hline \multirow[t]{3}{*}{2 w/ bleach' } & 1 w/ bleach & $-.187^{a, b}$ & .344 & .586 & -.862 & .488 \\
\hline & 3 w/o bleach & $1.288^{\mathrm{a}, \mathrm{b},{ }^{*}}$ & .344 & .000 & .613 & 1.963 \\
\hline & 4 w/o bleach & $.456^{\mathrm{a}, \mathrm{b}}$ & .344 & .185 & -.219 & 1.131 \\
\hline \multirow[t]{3}{*}{3 w/o bleach } & $1 \mathrm{w} /$ bleach & $-1.475^{\mathrm{a}, \mathrm{b},{ }^{*}}$ & .344 & .000 & -2.150 & -.800 \\
\hline & 2 w/ bleach' & $-1.288^{a, b,{ }^{*}}$ & .344 & .000 & -1.963 & -.613 \\
\hline & 4 w/o bleach & $-.832^{\mathrm{a}, \mathrm{b},{ }^{*}}$ & .344 & .016 & -1.506 & -.157 \\
\hline \multirow[t]{3}{*}{4 w/o bleach } & $1 \mathrm{w} /$ bleach & $-.643^{a, b}$ & .344 & .062 & -1.318 & .031 \\
\hline & 2 w/ bleach' & $-.456^{\mathrm{a}, \mathrm{b}}$ & .344 & .185 & -1.131 & .219 \\
\hline & 3 w/o bleach & $.832^{\mathrm{a}, \mathrm{b},{ }^{*}}$ & .344 & .016 & .157 & 1.506 \\
\hline
\end{tabular}

Based on estimated marginal means

*. The mean difference is significant at the .05 level.

a. An estimate of the modified population marginal mean (I).

b. An estimate of the modified population marginal mean $(\mathrm{J})$.

\section{Swatch Estimates: PMJX}

Dependent Variable: L

\begin{tabular}{|c|c|c|c|c|}
\hline \multirow[b]{2}{*}{ Swatch } & \multirow[b]{2}{*}{ Mean } & \multirow[b]{2}{*}{ Std. Error } & \multicolumn{2}{|c|}{ 95\% Confidence Interval } \\
\hline & & & Lower Bound & $\begin{array}{l}\text { Upper } \\
\text { Bound }\end{array}$ \\
\hline $1 \mathrm{w} /$ bleach & $95.372^{a}$ & .242 & 94.897 & 95.846 \\
\hline 2 w/ bleach & $94.008^{a}$ & .242 & 93.533 & 94.482 \\
\hline $3 \mathrm{w} / \mathrm{o}$ bleach & $94.901^{a}$ & .242 & 94.426 & 95.375 \\
\hline 4 w/o bleach & $94.419^{a}$ & .242 & 93.945 & 94.894 \\
\hline
\end{tabular}

a. Based on modified population marginal mean.

Dependent Variable: $\mathrm{L}$

\section{Pairwise Comparisons: PMJX}

\begin{tabular}{|c|c|c|c|c|c|c|}
\hline \multirow[b]{2}{*}{ (I) Swatch } & & \multirow{2}{*}{$\begin{array}{c}\text { Mean } \\
\text { Difference } \\
(\mathrm{I}-\mathrm{J})\end{array}$} & \multirow[b]{2}{*}{$\begin{array}{l}\text { Std. } \\
\text { Error }\end{array}$} & \multirow[b]{2}{*}{ Sig. ${ }^{d}$} & \multicolumn{2}{|c|}{$\begin{array}{l}\text { 95\% Confidence Interval for } \\
\text { Difference }^{d}\end{array}$} \\
\hline & & & & & $\begin{array}{l}\text { Lower } \\
\text { Bound }\end{array}$ & Upper Bound \\
\hline \multirow[t]{3}{*}{$1 \mathrm{w} /$ bleach } & $2 \mathrm{w} /$ bleach & $1.364^{\circ, D, C}$ & .342 & .000 & .693 & 2.035 \\
\hline & 3 w/o bleach & $.471^{\mathrm{b}, \mathrm{c}}$ & .342 & .169 & -.200 & 1.142 \\
\hline & $4 \mathrm{w} / \mathrm{o}$ bleach & $.953^{\star}, \mathrm{b}, \mathrm{c}$ & .342 & .005 & .282 & 1.624 \\
\hline \multirow[t]{3}{*}{2 w/ bleach } & $1 \mathrm{w} /$ bleach & $-1.364^{\pi, b, c}$ & .342 & .000 & -2.035 & -.693 \\
\hline & $3 \mathrm{w} / 0$ bleach & $-.893^{*, b, c}$ & .342 & .009 & -1.564 & -.222 \\
\hline & $4 \mathrm{w} / \mathrm{o}$ bleach & $-.411^{\mathrm{b}, \mathrm{c}}$ & .342 & .229 & -1.082 & .260 \\
\hline \multirow[t]{3}{*}{3 w/o bleach } & $1 \mathrm{w} /$ bleach & $-.471^{b, c}$ & .342 & .169 & -1.142 & .200 \\
\hline & 2 w/ bleach & $.893^{*}, \mathrm{~b}, \mathrm{c}$ & .342 & .009 & .222 & 1.564 \\
\hline & 4 w/o bleach & $.482^{\mathrm{b}, \mathrm{c}}$ & .342 & .159 & -.189 & 1.153 \\
\hline \multirow[t]{3}{*}{4 w/o bleach } & $1 \mathrm{w} /$ bleach & $-.953^{\pi, b, c}$ & .342 & .005 & -1.624 & -.282 \\
\hline & 2 w/ bleach & $.411^{\mathrm{b}, \mathrm{c}}$ & .342 & .229 & -.260 & 1.082 \\
\hline & 3 w/o bleach & $-.482^{\mathrm{b}, \mathrm{c}}$ & .342 & .159 & -1.153 & .189 \\
\hline
\end{tabular}

Based on estimated marginal means

*. The mean difference is significant at the .05 level.

b. An estimate of the modified population marginal mean (I).

c. An estimate of the modified population marginal mean (J). 
d. Adjustment for multiple comparisons: Least Significant Difference (equivalent to no adjustments).

For sample PMCL, the bleached swatches were not significantly different from each other;

though, the unbleached samples varied. With sample PMJX, no pattern was observed between

the bleached and unbleached samples.

\section{Bleach Estimates: PMCL}

Dependent Variable: L

\begin{tabular}{|l|r|r|r|r|}
\hline & & & \multicolumn{2}{|c|}{$95 \%$ Confidence Interval } \\
\cline { 4 - 5 } Bleached & Mean & Std. Error & $\begin{array}{c}\text { Lower } \\
\text { Bound }\end{array}$ & $\begin{array}{c}\text { Upper } \\
\text { Bound }\end{array}$ \\
\hline w/ bleach & $95.198^{\mathrm{a}}$ & .172 & 94.861 & 95.536 \\
w/o bleach & $94.233^{\mathrm{a}}$ & .172 & 93.895 & 94.570 \\
\hline
\end{tabular}

a. Based on modified population marginal mean.

Dependent Variable: L

Pairwise Comparisons: PMCL

\begin{tabular}{|c|c|c|c|c|c|c|}
\hline \multirow[b]{2}{*}{ (I) Bleached } & \multirow[b]{2}{*}{ (J) Bleached } & \multirow[b]{2}{*}{$\begin{array}{c}\text { Mean } \\
\text { Difference (I-J) }\end{array}$} & \multirow[b]{2}{*}{ Std. Error } & \multirow[b]{2}{*}{ Sig. ${ }^{c}$} & \multicolumn{2}{|c|}{$\begin{array}{l}\text { 95\% Confidence Interval } \\
\text { for Difference }\end{array}$} \\
\hline & & & & & $\begin{array}{l}\text { Lower } \\
\text { Bound }\end{array}$ & $\begin{array}{l}\text { Upper } \\
\text { Bound }\end{array}$ \\
\hline w/ bleach & w/o bleach & $.966, a, b$ & .243 & .000 & .488 & 1.443 \\
\hline w/o bleach & w/ bleach & $-.966^{*}, a, b$ & .243 & .000 & -1.443 & -.488 \\
\hline
\end{tabular}

Based on estimated marginal means

*. The mean difference is significant at the .05 level.

a. An estimate of the modified population marginal mean (I).

b. An estimate of the modified population marginal mean (J).

c. Adjustment for multiple comparisons: Least Significant Difference (equivalent to no adjustments).

\section{Bleach Estimates: PMJX}

Dependent Variable: L

\begin{tabular}{|l|r|r|r|r|}
\hline & & & \multicolumn{2}{|c|}{$95 \%$ Confidence Interval } \\
\cline { 4 - 5 } bleached & Mean & Std. Error & $\begin{array}{l}\text { Lower } \\
\text { Bound }\end{array}$ & $\begin{array}{c}\text { Upper } \\
\text { Bound }\end{array}$ \\
\hline w/ bleach & $94.690^{\mathrm{a}}$ & .171 & 94.354 & 95.025 \\
w/o bleach & $94.660^{\mathrm{a}}$ & .171 & 94.325 & 94.996 \\
\hline
\end{tabular}

a. Based on modified population marginal mean.

Pairwise Comparisons: PMJX

Dependent Variable: $\mathrm{L}$

\begin{tabular}{|c|c|c|c|c|c|c|}
\hline \multirow[b]{2}{*}{ (I) bleached } & & \multirow{2}{*}{$\begin{array}{c}\text { Mean } \\
\text { Difference } \\
(I-J)\end{array}$} & \multirow[b]{2}{*}{ Std. Error } & \multirow[b]{2}{*}{ Sig. $^{c}$} & \multicolumn{2}{|c|}{$\begin{array}{l}\text { 95\% Confidence Interval for } \\
\text { Difference }^{c}\end{array}$} \\
\hline & & & & & Lower Bound & Upper Bound \\
\hline $\begin{array}{l}\text { w/ bleach } \\
\text { w/o bleach }\end{array}$ & $\begin{array}{l}\text { w/o bleach } \\
\text { w/ bleach }\end{array}$ & $\begin{array}{r}.030^{\mathrm{a}, \mathrm{b}} \\
-.030^{\mathrm{a}, \mathrm{b}}\end{array}$ & $\begin{array}{l}.242 \\
.242\end{array}$ & $\begin{array}{l}.902 \\
.902\end{array}$ & $\begin{array}{l}-.445 \\
-.504\end{array}$ & $\begin{array}{r}.504 \\
.445\end{array}$ \\
\hline
\end{tabular}

Based on estimated marginal means

a. An estimate of the modified population marginal mean (I).

b. An estimate of the modified population marginal mean $(\mathrm{J})$.

c. Adjustment for multiple comparisons: Least Significant Difference (equivalent to no adjustments).

The type of detergent only mattered for sample PMCL. 


\section{Washing Estimates: PMCL}

Dependent Variable: $\mathrm{L}$

\begin{tabular}{|l|r|r|r|r|}
\hline & & & \multicolumn{2}{|c|}{$95 \%$ Confidence Interval } \\
\cline { 4 - 5 } Washing & Mean & Std. Error & Lower Bound & Upper Bound \\
\hline 1W 0A & $95.802^{\mathrm{a}}$ & .403 & 95.011 & 96.593 \\
1W 16A & $94.625^{\mathrm{a}}$ & .285 & 94.065 & 95.185 \\
2W 32A & $94.739^{\mathrm{a}}$ & .285 & 94.180 & 95.299 \\
3W 48A & $93.773^{\mathrm{a}}$ & .285 & 93.214 & 94.333 \\
4W 64A & $95.591^{\mathrm{a}}$ & .285 & 95.031 & 96.150 \\
5W 80A & $94.307^{\mathrm{a}}$ & .285 & 93.747 & 94.867 \\
\hline
\end{tabular}

a. Based on modified population marginal mean.

Pairwise Comparisons: PMCL

Dependent Variable: L

\begin{tabular}{|c|c|c|c|c|c|c|}
\hline \multirow[b]{2}{*}{ (I) Washin } & & \multirow{2}{*}{$\begin{array}{c}\text { Mean } \\
\text { Difference } \\
(I-J)\end{array}$} & \multirow[b]{2}{*}{ Std. Error } & \multirow[b]{2}{*}{ Sig. ${ }^{d}$} & \multicolumn{2}{|c|}{$\begin{array}{l}\text { 95\% Confidence Interval for } \\
\text { Difference }^{d}\end{array}$} \\
\hline & & & & & Lower Bound & Upper Bound \\
\hline \multirow[t]{5}{*}{$1 \mathrm{~W} \mathrm{OA}$} & $1 \mathrm{~W} 16 \mathrm{~A}$ & $1.177^{, \mathrm{b,c}}$ & .494 & .017 & .208 & 2.146 \\
\hline & $2 W 32 A$ & $1.063^{\mathrm{*}, \mathrm{b}, \mathrm{c}}$ & .494 & .032 & .094 & 2.032 \\
\hline & $3 W 48 A$ & $2.029^{\pi, b, c}$ & .494 & .000 & 1.059 & 2.998 \\
\hline & $4 W 64 A$ & $.211^{\mathrm{b}, \mathrm{c}}$ & .494 & .669 & -.758 & 1.181 \\
\hline & $5 W$ 80A & $1.495^{\pi, b, c}$ & .494 & .003 & .526 & 2.464 \\
\hline \multirow[t]{5}{*}{$1 \mathrm{~W} 16 \mathrm{~A}$} & $1 \mathrm{~W} O \mathrm{~A}$ & $-1.177^{\pi, b, c}$ & .494 & .017 & -2.146 & -.208 \\
\hline & $2 W 32 A$ & $-.114^{b, c}$ & .403 & .777 & -.905 & .677 \\
\hline & $3 W 48 A$ & $.852^{\pi, b, c}$ & .403 & .035 & .060 & 1.643 \\
\hline & $4 W 64 A$ & $-.966^{\pi, b, c}$ & .403 & .017 & -1.757 & -.174 \\
\hline & $5 W$ 80A & $.318^{b, c}$ & .403 & .430 & -.473 & 1.110 \\
\hline \multirow[t]{5}{*}{$2 W 32 A$} & $1 \mathrm{~W} 0 \mathrm{~A}$ & $-1.063^{*, b, c}$ & .494 & .032 & -2.032 & -.094 \\
\hline & $1 \mathrm{~W} 16 \mathrm{~A}$ & $.114^{\mathrm{b}, \mathrm{c}}$ & .403 & .777 & -.677 & .905 \\
\hline & $3 W 48 A$ & $.966^{\pi}, \mathrm{b}, \mathrm{c}$ & .403 & .017 & .174 & 1.757 \\
\hline & $4 \mathrm{~W} 64 \mathrm{~A}$ & $-.852^{*, b, c}$ & .403 & .035 & -1.643 & -.060 \\
\hline & $5 W$ 80A & $.432^{b, c}$ & .403 & .284 & -.359 & 1.224 \\
\hline \multirow[t]{5}{*}{$3 W 48 A$} & $1 \mathrm{~W} O \mathrm{~A}$ & $-2.029^{\pi}, \mathrm{b}, \mathrm{c}$ & .494 & .000 & -2.998 & -1.059 \\
\hline & $1 \mathrm{~W} 16 \mathrm{~A}$ & $-.852^{\pi, b, c}$ & .403 & .035 & -1.643 & -.060 \\
\hline & $2 W 32 A$ & $-.966^{\pi, b, c}$ & .403 & .017 & -1.757 & -.174 \\
\hline & $4 W 64 A$ & $-1.817^{\pi, b, c}$ & .403 & .000 & -2.609 & -1.026 \\
\hline & $5 W 80 A$ & $-.533^{\mathrm{b}, \mathrm{c}}$ & .403 & .186 & -1.325 & .258 \\
\hline \multirow[t]{5}{*}{$4 W 64 A$} & $1 \mathrm{~W} 0 \mathrm{~A}$ & $-.211^{\mathrm{b}, \mathrm{c}}$ & .494 & .669 & -1.181 & .758 \\
\hline & $1 \mathrm{~W} 16 \mathrm{~A}$ & $.966^{\circ, \mathrm{b}, \mathrm{c}}$ & .403 & .017 & .174 & 1.757 \\
\hline & $2 W 32 A$ & $.852^{\pi, b, c}$ & .403 & .035 & .060 & 1.643 \\
\hline & $3 W \quad 48 A$ & $1.817^{\pi, \mathrm{b}, \mathrm{c}}$ & .403 & .000 & 1.026 & 2.609 \\
\hline & $5 W$ 80A & $1.284^{*, b, c}$ & .403 & .002 & .492 & 2.075 \\
\hline \multirow[t]{5}{*}{$5 \mathrm{~W} 80 \mathrm{~A}$} & $1 \mathrm{~W} 0 \mathrm{~A}$ & $-1.495^{\star, b, c}$ & .494 & .003 & -2.464 & -.526 \\
\hline & $1 \mathrm{~W} 16 \mathrm{~A}$ & $-.318^{b, c}$ & .403 & .430 & -1.110 & .473 \\
\hline & $2 W 32 A$ & $-.432^{b, c}$ & .403 & .284 & -1.224 & .359 \\
\hline & $3 W$ 48A & $.533^{b, c}$ & .403 & .186 & -.258 & 1.325 \\
\hline & $4 W 64 A$ & $-1.284^{*, \mathrm{~b}, \mathrm{c}}$ & .403 & .002 & -2.075 & -.492 \\
\hline
\end{tabular}

Based on estimated marginal means

*. The mean difference is significant at the .05 level.

b. An estimate of the modified population marginal mean (I).

c. An estimate of the modified population marginal mean $(J)$.

d. Adjustment for multiple comparisons: Least Significant Difference (equivalent to no adjustments).

For the washing estimates, both reference samples observed marked significant differences for at

least at two levels of interactions for sample PMCL, and at least three levels of interactions for sample PMJX. 


\section{Washing Estimates: PMJX}

Dependent Variable: L

\begin{tabular}{|c|c|c|c|c|}
\hline \multirow[b]{2}{*}{ washings } & \multirow[b]{2}{*}{ Mean } & \multirow[b]{2}{*}{ Std. Error } & \multicolumn{2}{|c|}{$95 \%$ Confidence Interval } \\
\hline & & & $\begin{array}{l}\text { Lower } \\
\text { Bound }\end{array}$ & $\begin{array}{l}\text { Upper } \\
\text { Bound }\end{array}$ \\
\hline $1 \mathrm{~W} 0 \mathrm{~A}$ & $92.656^{a}$ & .401 & 91.869 & 93.443 \\
\hline $1 \mathrm{~W} 16 \mathrm{~A}$ & $96.299^{a}$ & .283 & 95.742 & 96.855 \\
\hline $2 W 32 A$ & $95.313^{a}$ & .283 & 94.757 & 95.869 \\
\hline $3 W 48 A$ & $95.140^{\mathrm{a}}$ & .283 & 94.584 & 95.696 \\
\hline $4 W 64 A$ & $93.493^{\mathrm{a}}$ & .283 & 92.936 & 94.049 \\
\hline $5 W 80 A$ & $94.140^{a}$ & .283 & 93.584 & 94.696 \\
\hline
\end{tabular}

a. Based on modified population marginal mean.

Pairwise Comparisons: PMJX

Dependent Variable: L

\begin{tabular}{|c|c|c|c|c|c|c|}
\hline & & Mean & & & $\begin{array}{r}\text { 95\% Confide } \\
\text { Diffe }\end{array}$ & $\begin{array}{l}\text { Ice Interval for } \\
\text { ence }\end{array}$ \\
\hline (I) washir & & $\begin{array}{c}\text { Difference } \\
(\mathrm{I}-\mathrm{J})\end{array}$ & $\begin{array}{l}\text { Std. } \\
\text { Error }\end{array}$ & Sig. ${ }^{d}$ & $\begin{array}{l}\text { Lower } \\
\text { Bound }\end{array}$ & Upper Bound \\
\hline $1 \mathrm{~W} 0 \mathrm{~A}$ & $1 \mathrm{~W} 16 \mathrm{~A}$ & $-3.643^{, b, c}$ & .491 & .000 & -4.606 & -2.679 \\
\hline & $2 W 32 A$ & $-2.657^{\pi, b, c}$ & .491 & .000 & -3.621 & -1.693 \\
\hline & $3 W$ 48A & $-2.484^{\pi, b, c}$ & .491 & .000 & -3.448 & -1.520 \\
\hline & $4 W 64 A$ & $-.836^{b, c}$ & .491 & .089 & -1.800 & .127 \\
\hline & $5 W 80 A$ & $-1.484^{\pi, b, c}$ & .491 & .003 & -2.448 & -.520 \\
\hline $1 \mathrm{~W} 16 \mathrm{~A}$ & $1 \mathrm{~W} 0 \mathrm{~A}$ & $3.643^{x, b, c}$ & .491 & .000 & 2.679 & 4.606 \\
\hline & $2 W 32 A$ & $.986^{*}, \mathrm{~b}, \mathrm{c}$ & .401 & .014 & .199 & 1.773 \\
\hline & $3 W 48 A$ & $1.159^{\pi, b, c}$ & .401 & .004 & .372 & 1.946 \\
\hline & $4 W 64 A$ & $2.806^{*, \mathrm{~b}, \mathrm{c}}$ & .401 & .000 & 2.019 & 3.593 \\
\hline & $5 W 80 A$ & $2.159^{*}, \mathrm{~b}, \mathrm{c}$ & .401 & .000 & 1.372 & 2.946 \\
\hline $2 W 32 A$ & $1 \mathrm{~W} 0 \mathrm{~A}$ & $2.657^{\pi, b, c}$ & .491 & .000 & 1.693 & 3.621 \\
\hline & $1 \mathrm{~W} 16 \mathrm{~A}$ & $-.986^{\pi, b, c}$ & .401 & .014 & -1.773 & -.199 \\
\hline & $3 W 48 A$ & $.173^{b, c}$ & .401 & .666 & -.614 & .960 \\
\hline & $4 W 64 A$ & $1.821^{*}, \mathrm{~b}, \mathrm{c}$ & .401 & .000 & 1.034 & 2.607 \\
\hline & $5 W 80 A$ & $1.173^{\pi, \mathrm{b}, \mathrm{c}}$ & .401 & .004 & .386 & 1.960 \\
\hline 3W 48A & $1 \mathrm{~W} 0 \mathrm{~A}$ & $2.484^{*}, \mathrm{~b}, \mathrm{c}$ & .491 & .000 & 1.520 & 3.448 \\
\hline & $1 \mathrm{~W} 16 \mathrm{~A}$ & $-1.159^{\circ}, \mathrm{b}, \mathrm{c}$ & .401 & .004 & -1.946 & -.372 \\
\hline & $2 W 32 A$ & $-.173^{\mathrm{b}, \mathrm{c}}$ & .401 & .666 & -.960 & .614 \\
\hline & $4 \mathrm{~W} 64 \mathrm{~A}$ & $1.647^{\pi, b, c}$ & .401 & .000 & .861 & 2.434 \\
\hline & $5 W 80 A$ & $1.000^{\pi, b, c}$ & .401 & .013 & .213 & 1.787 \\
\hline $4 W 64 A$ & $1 \mathrm{~W} 0 \mathrm{~A}$ & $.836^{\mathrm{b}, \mathrm{c}}$ & .491 & .089 & -.127 & 1.800 \\
\hline & $1 \mathrm{~W} 16 \mathrm{~A}$ & $-2.806^{*}, \mathrm{~b}, \mathrm{c}$ & .401 & .000 & -3.593 & -2.019 \\
\hline & $2 W 32 A$ & $-1.821^{\pi, b, c}$ & .401 & .000 & -2.607 & -1.034 \\
\hline & $3 W 48 A$ & $-1.647^{\pi, b, c}$ & .401 & .000 & -2.434 & -.861 \\
\hline & $5 W 80 A$ & $-.648^{b, c}$ & .401 & .107 & -1.434 & .139 \\
\hline $5 W 80 A$ & $1 \mathrm{~W} 0 \mathrm{~A}$ & $1.484^{*}, \mathrm{~b}, \mathrm{c}$ & .491 & .003 & .520 & 2.448 \\
\hline & $1 \mathrm{~W} 16 \mathrm{~A}$ & $-2.159^{\pi}, \mathrm{b}, \mathrm{c}$ & .401 & .000 & -2.946 & -1.372 \\
\hline & $2 W 32 A$ & $-1.173^{\pi, b, c}$ & .401 & .004 & -1.960 & -.386 \\
\hline & $3 W 48 A$ & $-1.000^{\pi}, \mathrm{b}, \mathrm{c}$ & .401 & .013 & -1.787 & -.213 \\
\hline & $4 W 64 A$ & $.648^{\mathrm{b}, \mathrm{c}}$ & .401 & .107 & -.139 & 1.434 \\
\hline
\end{tabular}

Based on estimated marginal means

*. The mean difference is significant at the .05 level.

b. An estimate of the modified population marginal mean (I).

c. An estimate of the modified population marginal mean (J).

d. Adjustment for multiple comparisons: Least Significant Difference (equivalent to no adjustments). 


\section{Sample Area * Swatch: PMCL}

Dependent Variable: $\mathrm{L}$

\begin{tabular}{|c|c|c|c|c|c|}
\hline \multirow{2}{*}{ Sample Area } & & \multirow[b]{2}{*}{ Mean } & \multirow[b]{2}{*}{ Std. Error } & \multicolumn{2}{|c|}{ 95\% Confidence Interval } \\
\hline & & & & Lower Bound & Upper Bound \\
\hline \multirow[t]{4}{*}{ A } & $1 \mathrm{w} /$ bleach & $95.186^{a}$ & .329 & 94.540 & 95.832 \\
\hline & $2 \mathrm{w} /$ bleach & $93.156^{a}$ & .361 & 92.448 & 93.864 \\
\hline & $3 \mathrm{w} /$ bleach & $94.900^{\mathrm{a}}$ & .329 & 94.254 & 95.546 \\
\hline & $4 \mathrm{w} /$ bleach & $94.856^{a}$ & .361 & 94.148 & 95.564 \\
\hline \multirow[t]{4}{*}{ B } & $1 \mathrm{w} /$ bleach & $95.419^{a}$ & .361 & 94.711 & 96.127 \\
\hline & $2 \mathrm{w} / \mathrm{bleach}$ & $96.729^{a}$ & .329 & 96.082 & 97.375 \\
\hline & 3 w/ bleach & $92.518^{a}$ & .361 & 91.810 & 93.226 \\
\hline & $4 \mathrm{w} /$ bleach & $94.476^{\mathrm{a}}$ & .329 & 93.830 & 95.122 \\
\hline
\end{tabular}

a. Based on modified population marginal mean.

Dependent Variable: L

\section{Sample Area * Swatch: PMJX}

\begin{tabular}{|c|c|c|c|c|c|}
\hline \multirow{2}{*}{\multicolumn{2}{|c|}{ Sample Area }} & \multirow[b]{2}{*}{ Mean } & \multirow[b]{2}{*}{ Std. Error } & \multicolumn{2}{|c|}{ 95\% Confidence Interval } \\
\hline & & & & Lower Bound & Upper Bound \\
\hline \multirow[t]{4}{*}{$\bar{A}$} & $1 \mathrm{w} /$ bleach & $96.069^{\mathrm{a}}$ & .327 & 95.426 & 96.711 \\
\hline & 2 w/ bleach & $95.975^{\mathrm{a}}$ & .359 & 95.271 & 96.679 \\
\hline & 3 w/o bleach & $93.706^{\mathrm{a}}$ & .327 & 93.063 & 94.348 \\
\hline & 4 w/o bleach & $94.679^{\mathrm{a}}$ & .359 & 93.976 & 95.383 \\
\hline \multirow[t]{4}{*}{ B } & $1 \mathrm{w} /$ bleach & $94.536^{\mathrm{a}}$ & .359 & 93.832 & 95.240 \\
\hline & $2 \mathrm{w} /$ bleach & $92.369^{a}$ & .327 & 91.726 & 93.011 \\
\hline & 3 w/o bleach & $96.335^{\mathrm{a}}$ & .359 & 95.631 & 97.039 \\
\hline & $4 \mathrm{w} / \mathrm{o}$ bleach & $94.202^{\mathrm{a}}$ & .327 & 93.560 & 94.845 \\
\hline
\end{tabular}

a. Based on modified population marginal mean.
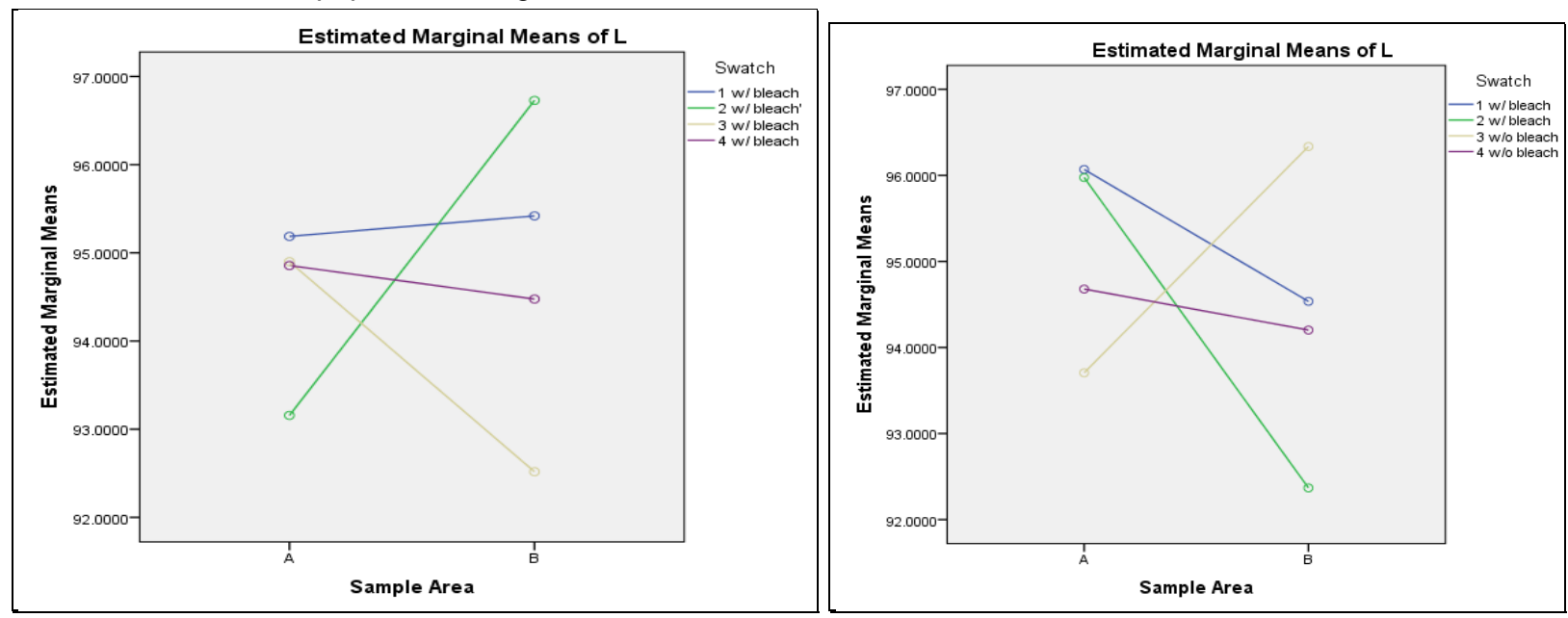

The plots above correspond to sample PMCL and PMJX, from left to right, respectively. The patterns between these two charts are different: swatch 4 not only remained consistent observed the lowest difference in means. The greatest difference in mean can be observed at swatch 2 in sample PMCL and swatches 2 and 3 in sample PMJX. 


\section{Sample Area * Bleached: PMCL}

Dependent Variable: L

\begin{tabular}{|ll|r|r|r|r|}
\hline & & & & \multicolumn{2}{|c|}{$95 \%$ Confidence Interval } \\
\cline { 5 - 6 } Sample Area & & Mean & Std. Error & Lower Bound & Upper Bound \\
\hline A & w/ bleach & $94.264^{\mathrm{a}}$ & .243 & 93.786 & 94.741 \\
& w/o bleach & $94.880^{\mathrm{a}}$ & .243 & 94.403 & 95.357 \\
B & w/ bleach & $96.133^{\mathrm{a}}$ & .243 & 95.656 & 96.611 \\
& w/o bleach & $93.586^{\mathrm{a}}$ & .243 & 93.109 & 94.063 \\
\hline
\end{tabular}

a. Based on modified population marginal mean.

\section{Sample Area * Beached: PMJX}

Dependent Variable: L

\begin{tabular}{|ll|r|r|r|r|}
\hline \multirow{2}{*}{ Sample Area } & & & \multicolumn{2}{|c|}{$95 \%$ Confidence Interval } \\
\cline { 5 - 6 } A & & Mean & Std. Error & Lower Bound & \multicolumn{1}{c|}{ Upper Bound } \\
& w/ bleach & $96.026^{\mathrm{a}}$ & .242 & 95.552 & 96.501 \\
B & w/o bleach & $94.148^{\mathrm{a}}$ & .242 & 93.674 & 94.623 \\
& w/ bleach & $93.354^{\mathrm{a}}$ & .242 & 92.879 & 93.828 \\
& w/o bleach & $95.172^{\mathrm{a}}$ & .242 & 94.697 & 95.646 \\
\hline
\end{tabular}

a. Based on modified population marginal mean.
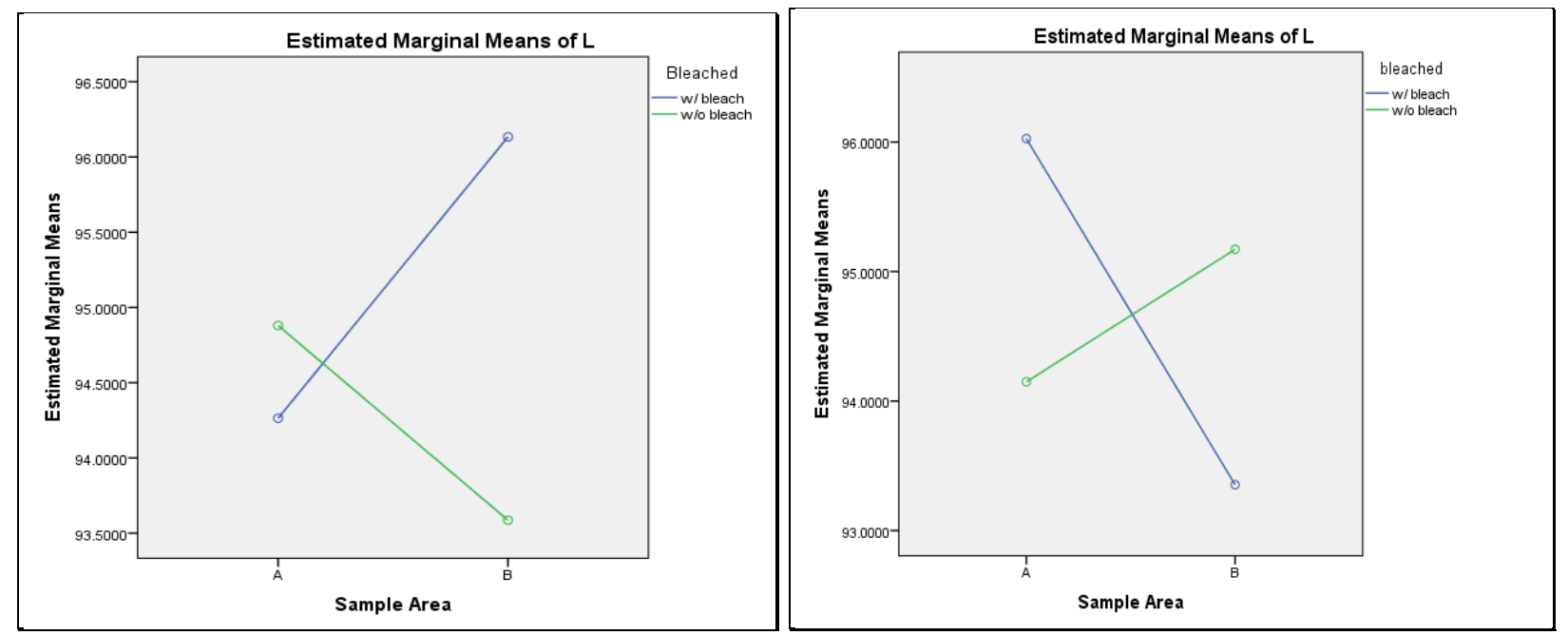

It can be observed that sample PMCL had a considerable mean difference between bleached and

unbleached at sample area $\mathrm{B}$, in comparison to A; whereas, for sample PMJX, this difference seemed to be moderately the same between sample areas.

For the bleached* washing interaction below, it is very obvious that the patterns between these two reference samples are different where the type of detergent affected the outcome of the results for sample PMCL. 


\section{Bleached * Washing: PMCL}

Dependent Variable: $\mathrm{L}$

\begin{tabular}{|ll|r|r|r|r|}
\hline & & & \multicolumn{2}{|c|}{ 95\% Confidence Interval } \\
\cline { 5 - 6 } Bleached & & Mean & Std. Error & Lower Bound & Upper Bound \\
\hline w/ bleach & 1W 0A & $95.425^{\mathrm{a}}$ & .570 & 94.306 & 96.544 \\
& 1W 16A & $96.474^{\mathrm{a}}$ & .403 & 95.682 & 97.265 \\
& 2W 32A & $95.499^{\mathrm{a}}$ & .403 & 94.707 & 96.290 \\
& 3W 48A & $93.551^{\mathrm{a}}$ & .403 & 92.760 & 94.343 \\
& 4W 64A & $96.124^{\mathrm{a}}$ & .403 & 95.332 & 96.915 \\
& 5W 80A & $94.232^{\mathrm{a}}$ & .403 & 93.440 & 95.023 \\
w/o bleach & 1W 0A & $96.179^{\mathrm{a}}$ & .570 & 95.060 & 97.298 \\
& 1W 16A & $92.776^{\mathrm{a}}$ & .403 & 91.985 & 93.568 \\
& 2W 32A & $93.980^{\mathrm{a}}$ & .403 & 93.188 & 94.771 \\
& 3W 48A & $93.995^{\mathrm{a}}$ & .403 & 93.204 & 94.787 \\
& 4W 64A & $95.058^{\mathrm{a}}$ & .403 & 94.267 & 95.849 \\
& 5W 80A & $94.382^{\mathrm{a}}$ & .403 & 93.591 & 95.173 \\
\hline
\end{tabular}

a. Based on modified population marginal mean.

\section{Bleached * Washings: PMJX}

Dependent Variable: L

\begin{tabular}{|c|c|c|c|c|c|}
\hline \multirow{2}{*}{ bleached } & & \multirow[b]{2}{*}{ Mean } & \multirow{2}{*}{$\begin{array}{l}\text { Std. } \\
\text { Error }\end{array}$} & \multicolumn{2}{|c|}{$95 \%$ Confidence Interval } \\
\hline & & & & Lower Bound & Upper Bound \\
\hline \multirow[t]{6}{*}{ w/ bleach } & $1 \mathrm{~W} 0 \mathrm{~A}$ & $92.315^{\mathrm{a}}$ & .567 & 91.202 & 93.428 \\
\hline & $1 \mathrm{~W} 16 \mathrm{~A}$ & $95.904^{a}$ & .401 & 95.117 & 96.691 \\
\hline & $2 W 32 A$ & $96.553^{\mathrm{a}}$ & .401 & 95.766 & 97.339 \\
\hline & $3 W 48 A$ & $95.081^{a}$ & .401 & 94.294 & 95.868 \\
\hline & $4 W 64 A$ & $93.357^{\mathrm{a}}$ & .401 & 92.570 & 94.144 \\
\hline & $5 W 80 A$ & $93.743^{a}$ & .401 & 92.956 & 94.530 \\
\hline \multirow[t]{6}{*}{ w/o bleach } & $1 \mathrm{~W} 0 \mathrm{~A}$ & $92.997^{\mathrm{a}}$ & .567 & 91.884 & 94.110 \\
\hline & $1 \mathrm{~W} 16 \mathrm{~A}$ & $96.694^{a}$ & .401 & 95.907 & 97.481 \\
\hline & $2 W 32 A$ & $94.074^{\mathrm{a}}$ & .401 & 93.287 & 94.860 \\
\hline & $3 W 48 A$ & $95.199^{a}$ & .401 & 94.412 & 95.986 \\
\hline & $4 W 64 A$ & $93.628^{a}$ & .401 & 92.841 & 94.415 \\
\hline & $5 W 80 A$ & $94.537^{\mathrm{a}}$ & .401 & 93.751 & 95.324 \\
\hline
\end{tabular}

a. Based on modified population marginal mean.
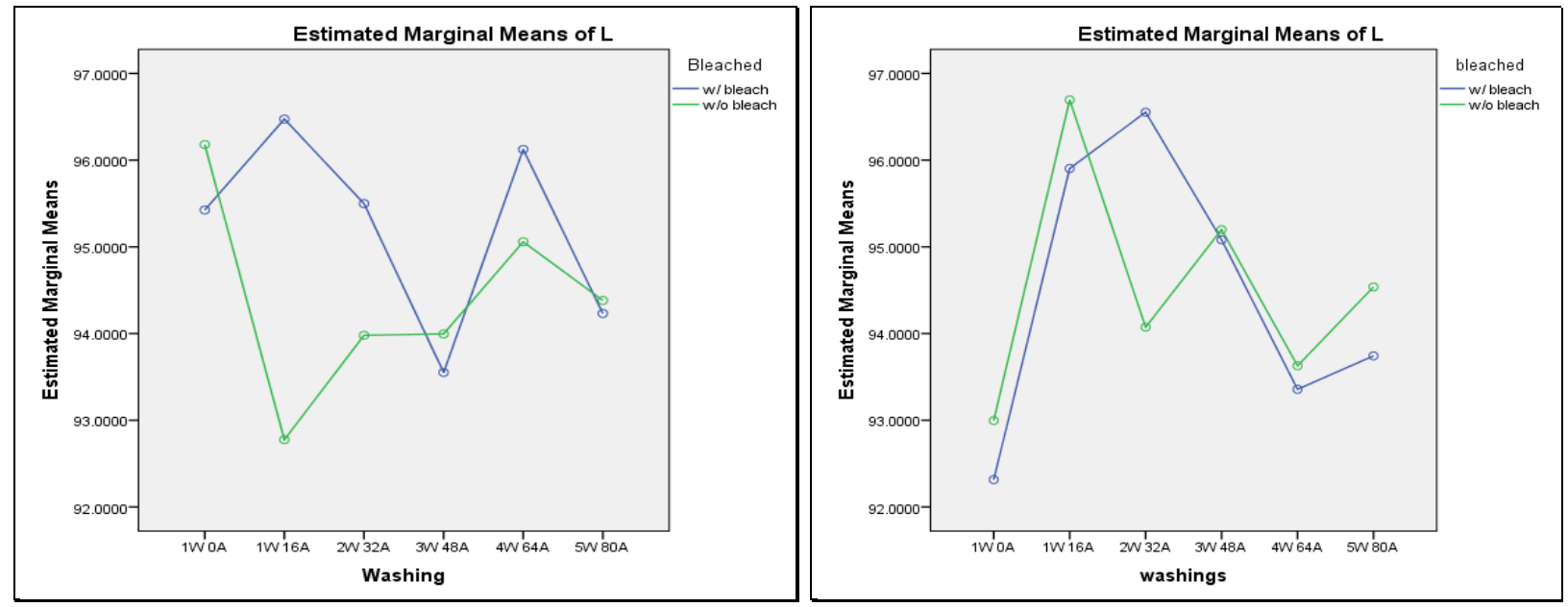


\section{Swatch * Washing: PMCL}

Dependent Variable: L

\begin{tabular}{|ll|r|r|r|r|}
\hline \multirow{3}{*}{ Swatch } & & & \multicolumn{2}{|c|}{$95 \%$ Confidence Interval } \\
\cline { 4 - 6 } 1 w/ bleach & Mean & Std. Error & Lower Bound & Upper Bound \\
& 1W 0A & $97.359^{\mathrm{a}}$ & .806 & 95.777 & 98.942 \\
& 1W 16A & $97.301^{\mathrm{a}}$ & .570 & 96.182 & 98.420 \\
& 2W 32A & $94.971^{\mathrm{a}}$ & .570 & 93.852 & 96.090 \\
& 3W 48A & $94.363^{\mathrm{a}}$ & .570 & 93.243 & 95.482 \\
& 4W 64A & $95.699^{\mathrm{a}}$ & .570 & 94.580 & 96.818 \\
2 w/ bleach' & 5W 80A & $93.094^{\mathrm{a}}$ & .570 & 91.975 & 94.213 \\
& 1W 0A & $93.490^{\mathrm{a}}$ & .806 & 91.908 & 95.073 \\
& 1W 16A & $95.646^{\mathrm{a}}$ & .570 & 94.527 & 96.765 \\
& 2W 32A & $96.027^{\mathrm{a}}$ & .570 & 94.907 & 97.146 \\
& 3W 48A & $92.740^{\mathrm{a}}$ & .570 & 91.621 & 93.860 \\
& 4W 64A & $96.548^{\mathrm{a}}$ & .570 & 95.429 & 97.668 \\
3 w/o bleach & 5W 80A & $95.370^{\mathrm{a}}$ & .570 & 94.251 & 96.489 \\
& 1W 0A & $94.992^{\mathrm{a}}$ & .806 & 93.409 & 96.575 \\
& 1W 16A & $95.410^{\mathrm{a}}$ & .570 & 94.290 & 96.529 \\
& 2W 32A & $94.948^{\mathrm{a}}$ & .570 & 93.829 & 96.067 \\
& 3W 48A & $89.699^{\mathrm{a}}$ & .570 & 88.580 & 90.819 \\
& 4W 64A & $93.738^{\mathrm{a}}$ & .570 & 92.619 & 94.857 \\
& 5W 80A & $94.703^{\mathrm{a}}$ & .570 & 93.584 & 95.822 \\
4 w/o bleach & 1W 0A & $97.367^{\mathrm{a}}$ & .806 & 95.784 & 98.949 \\
& 1W 16A & $90.143^{\mathrm{a}}$ & .570 & 89.024 & 91.263 \\
& 2W 32A & $93.011^{\mathrm{a}}$ & .570 & 91.892 & 94.130 \\
& 3W 48A & $98.291^{\mathrm{a}}$ & .570 & 97.172 & 99.411 \\
& 4W 64A & $96.378^{\mathrm{a}}$ & .570 & 95.258 & 97.497 \\
& 5W 80A & $94.061^{\mathrm{a}}$ & .570 & 92.942 & 95.180 \\
\hline
\end{tabular}

a. Based on modified population marginal mean.

\section{Swatch * Washings: PMJX}

Dependent Variable: $\mathrm{L}$

\begin{tabular}{|c|c|c|c|c|c|}
\hline \multirow{2}{*}{ Swatch } & & \multirow[b]{2}{*}{ Mean } & \multirow[b]{2}{*}{ Std. Error } & \multicolumn{2}{|c|}{ 95\% Confidence Interval } \\
\hline & & & & Lower Bound & Upper Bound \\
\hline \multirow[t]{6}{*}{$1 \mathrm{w} /$ bleach } & $1 \mathrm{~W} 0 \mathrm{~A}$ & $94.919^{\mathrm{a}}$ & .802 & 93.345 & 96.493 \\
\hline & $1 \mathrm{~W} 16 \mathrm{~A}$ & $95.945^{\mathrm{a}}$ & .567 & 94.832 & 97.058 \\
\hline & $2 W 32 A$ & $97.241^{a}$ & .567 & 96.128 & 98.354 \\
\hline & $3 W 48 A$ & $95.709^{a}$ & .567 & 94.597 & 96.822 \\
\hline & $4 \mathrm{~W} 64 \mathrm{~A}$ & $92.963^{a}$ & .567 & 91.850 & 94.076 \\
\hline & $5 W$ 80A & $95.227^{\mathrm{a}}$ & .567 & 94.114 & 96.340 \\
\hline \multirow[t]{6}{*}{$2 \mathrm{w} /$ bleach } & $1 \mathrm{~W} 0 \mathrm{~A}$ & $89.711^{a}$ & .802 & 88.137 & 91.285 \\
\hline & $1 \mathrm{~W} 16 \mathrm{~A}$ & $95.863^{\mathrm{a}}$ & .567 & 94.750 & 96.976 \\
\hline & $2 W 32 A$ & $95.864^{a}$ & .567 & 94.751 & 96.977 \\
\hline & $3 W 48 A$ & $94.452^{\mathrm{a}}$ & .567 & 93.340 & 95.565 \\
\hline & $4 \mathrm{~W} 64 \mathrm{~A}$ & $93.751^{\mathrm{a}}$ & .567 & 92.638 & 94.864 \\
\hline & $5 W 80 A$ & $92.258^{\mathrm{a}}$ & .567 & 91.145 & 93.371 \\
\hline \multirow[t]{6}{*}{$3 \mathrm{w} / \mathrm{o}$ bleach } & $1 \mathrm{~W} 0 \mathrm{~A}$ & $93.214^{a}$ & .802 & 91.641 & 94.788 \\
\hline & $1 \mathrm{~W} 16 \mathrm{~A}$ & $97.493^{\mathrm{a}}$ & .567 & 96.380 & 98.606 \\
\hline & $2 W 32 A$ & $93.922^{a}$ & .567 & 92.809 & 95.035 \\
\hline & $3 W 48 A$ & $96.308^{a}$ & .567 & 95.196 & 97.421 \\
\hline & $4 W 64 A$ & $93.406^{\mathrm{a}}$ & .567 & 92.293 & 94.518 \\
\hline & $5 W 80 A$ & $94.219^{a}$ & .567 & 93.107 & 95.332 \\
\hline \multirow[t]{6}{*}{4 w/o bleach } & $1 \mathrm{~W} 0 \mathrm{~A}$ & $92.780^{\mathrm{a}}$ & .802 & 91.206 & 94.354 \\
\hline & $1 \mathrm{~W} 16 \mathrm{~A}$ & $95.895^{\mathrm{a}}$ & .567 & 94.782 & 97.008 \\
\hline & $2 W 32 A$ & $94.225^{\mathrm{a}}$ & .567 & 93.112 & 95.338 \\
\hline & $3 W 48 A$ & $94.090^{\mathrm{a}}$ & .567 & 92.977 & 95.203 \\
\hline & $4 W 64 A$ & $93.851^{a}$ & .567 & 92.738 & 94.964 \\
\hline & $5 W 80 A$ & $94.855^{\mathrm{a}}$ & .567 & 93.743 & 95.968 \\
\hline
\end{tabular}

a. Based on modified population marginal mean. 

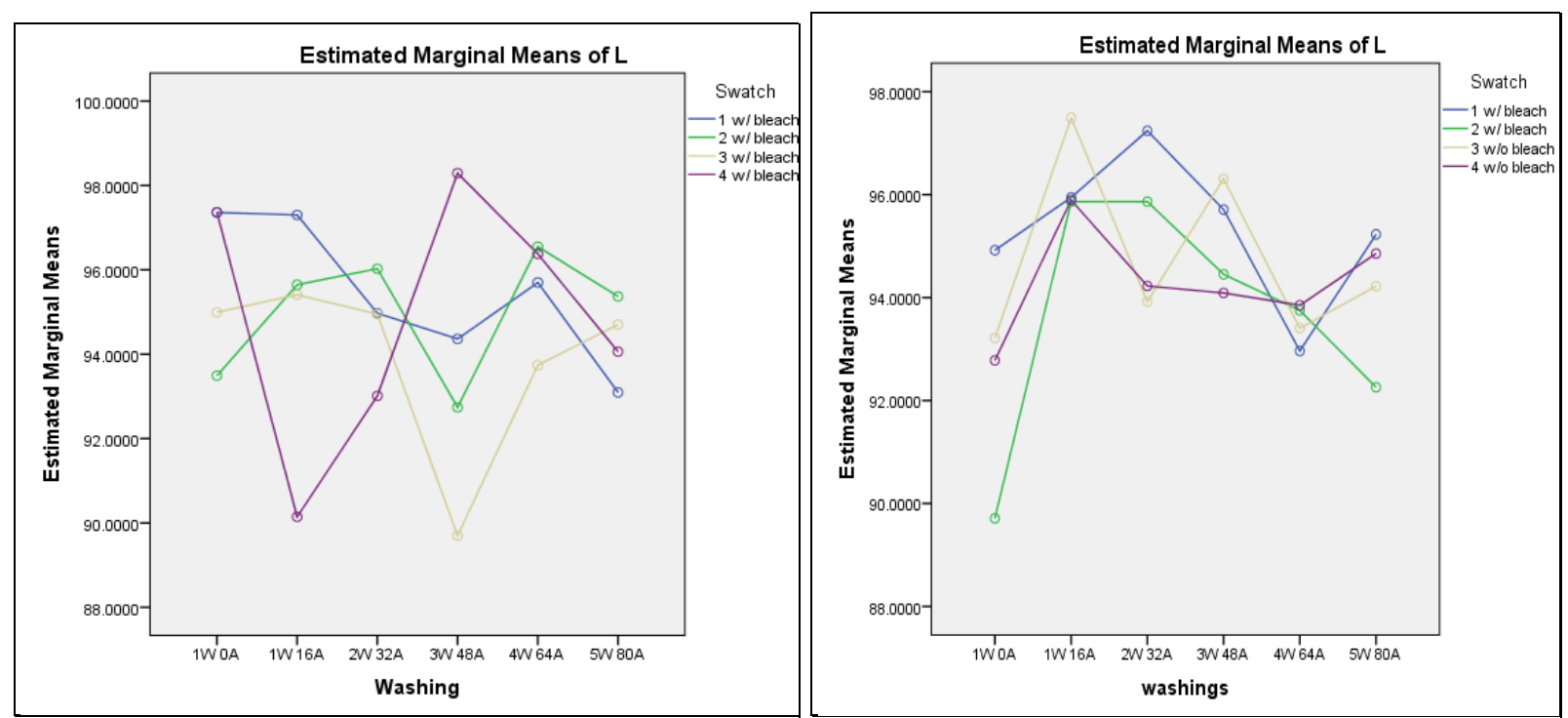

While large differences in means can be observed for sample PMCL, these differences are not as obvious in sample PMJX. And based on the calculated p-values, the Swatch * Washing interaction is significant at .001 for sample PMCL and at .003 for sample PMJX.

Variable ' $a$ '

Dependent Variable: a

Tests of Between-Subjects Effects: PMCL

\begin{tabular}{|c|c|c|c|c|c|}
\hline Source & Type III Sum of Squares & df & Mean Square & $\bar{F}$ & Sig. \\
\hline SampleArea & 2.763 & 1 & 2.763 & 5.512 & .019 \\
\hline Bleached & 0.000 & 0 & & & \\
\hline Washing & 13.786 & 5 & 2.757 & 5.500 & .000 \\
\hline SampleArea * Swatch & 25.559 & 2 & 12.779 & 25.493 & .000 \\
\hline Swatch * Bleached & 0.000 & 0 & & & \\
\hline Swatch * Washing & 32.74 & 8 & 4.092 & 8.163 & .000 \\
\hline Bleached * Washing & 0.000 & 0 & & & \\
\hline SampleArea * Swatch * Bleached & 0.000 & 0 & & & \\
\hline SampleArea * Swatch * Washing & 31.70 & 8 & 3.962 & 7.903 & .000 \\
\hline SampleArea * Bleached * Washing & 0.000 & 0 & & & \\
\hline Corrected Total & 628.79 & 879 & & & \\
\hline
\end{tabular}

a. R Squared $=.334$ (Adjusted R Squared $=.299$ )

b. Computed using alpha $=.05$

Tests of Between-Subjects Effects: PMJX 
Dependent Variable: a

\begin{tabular}{|c|c|c|c|c|c|}
\hline Source & Type III Sum of Squares & $d f$ & Mean Square & $\mathrm{F}$ & Sig. \\
\hline Corrected Model & $132.37^{\mathrm{a}}$ & 43 & 3.078 & 7.467 & .000 \\
\hline Intercept & 5763.8 & 1 & 5763.8 & 13980 & 0.000 \\
\hline SampleArea & .022 & 1 & .022 & .054 & .816 \\
\hline Swatch & 6.742 & 2 & 3.371 & 8.177 & .000 \\
\hline bleached & 0.000 & 0 & & & \\
\hline washings & 22.75 & 5 & 4.551 & 11.037 & .000 \\
\hline SampleArea * Swatch & 1.243 & 2 & .622 & 1.508 & .222 \\
\hline SampleArea * bleached & 0.000 & 0 & & & \\
\hline SampleArea * washings & 8.698 & 4 & 2.175 & 5.274 & .000 \\
\hline Swatch * bleached & 0.000 & 0 & & & \\
\hline Swatch * washings & 12.70 & 8 & 1.588 & 3.852 & .000 \\
\hline bleached * washings & 0.000 & 0 & & & \\
\hline SampleArea * Swatch * bleached & 0.000 & 0 & & & \\
\hline SampleArea * Swatch * washings & 22.065 & 8 & 2.758 & 6.690 & .000 \\
\hline SampleArea * bleached * washings & 0.000 & 0 & & & \\
\hline Swatch * bleached * washings & 0.000 & 0 & & & \\
\hline SampleArea * Swatch * bleached * washings & 0.000 & 0 & & & \\
\hline Error & 344.68 & 836 & .412 & & \\
\hline Total & 6671 & 880 & & & \\
\hline Corrected Total & 477.05 & 879 & & & \\
\hline
\end{tabular}

a. $R$ Squared $=.277$ (Adjusted R Squared $=.240$ )

b. Computed using alpha $=.05$

While the main and interaction effects were all significant for sample PMCL, sample PMJX

observed insignificant main effect values for sample area, and its interaction effects Sample

Area* Swatch.

\section{Sample Area Estimates: PMCL}

Dependent Variable: a

\begin{tabular}{|l|r|r|r|r|}
\hline & & \multirow{2}{*}{ Std. } & \multicolumn{2}{|c|}{$95 \%$ Confidence Interval } \\
\cline { 4 - 5 } Sample Area & Mean & Error & Lower Bound & \multicolumn{1}{c|}{ Upper Bound } \\
\hline A & $2.686^{\mathrm{a}}$ & .034 & 2.619 & 2.752 \\
B & $2.856^{\mathrm{a}}$ & .034 & 2.790 & 2.923 \\
\hline
\end{tabular}

a. Based on modified population marginal mean.

Dependent Variable: a

\section{Pairwise Comparisons: PMCL}

\begin{tabular}{|c|c|c|c|c|c|}
\hline \multirow[b]{2}{*}{ (I) Sample Area } & \multirow[b]{2}{*}{ Mean Difference (I-J) } & \multirow[b]{2}{*}{ Std. Error } & \multirow[b]{2}{*}{ Sig. ${ }^{d}$} & \multicolumn{2}{|c|}{$\begin{array}{l}\text { 95\% Confidence Interval for } \\
\text { Difference }\end{array}$} \\
\hline & & & & Lower Bound & Upper Bound \\
\hline $\begin{array}{ll}A & B \\
B & A\end{array}$ & $\begin{array}{r}-.171^{{ }^{*}, \mathrm{~b}, \mathrm{c}} \\
.171^{\mathrm{x}, \mathrm{b}, \mathrm{c}}\end{array}$ & $\begin{array}{l}.048 \\
.048\end{array}$ & $\begin{array}{l}.000 \\
.000\end{array}$ & $\begin{array}{r}-.265 \\
.077\end{array}$ & $\begin{array}{r}-.077 \\
.265\end{array}$ \\
\hline
\end{tabular}

Based on estimated marginal means

*. The mean difference is significant at the .05 level.

b. An estimate of the modified population marginal mean (I).

c. An estimate of the modified population marginal mean $(\mathrm{J})$. 
d. Adjustment for multiple comparisons: Least Significant Difference (equivalent to no adjustments).

\section{Sample Area Estimates: PMJX}

Dependent Variable: a

\begin{tabular}{|l|r|r|r|r|}
\hline \multirow{2}{*}{ Sample Area } & Mean & Std. Error & \multicolumn{2}{|c|}{ 95\% Confidence Interval } \\
\cline { 4 - 5 } & $2.656^{\mathrm{a}}$ & .031 & 2.596 & Upper Bound \\
\hline A & $2.650^{\mathrm{a}}$ & .031 & 2.590 & 2.716 \\
$\mathrm{~B}$ & & .710 \\
\hline
\end{tabular}

a. Based on modified population marginal mean.

Dependent Variable: a

Pairwise Comparisons: PMJX

\begin{tabular}{|c|c|c|c|c|c|}
\hline \multirow[b]{2}{*}{ (I) Sample Area } & \multirow{2}{*}{$\begin{array}{c}\text { Mean Difference (I- } \\
\mathrm{J})\end{array}$} & \multirow[b]{2}{*}{ Std. Error } & \multirow[b]{2}{*}{ Sig. $^{\mathrm{C}}$} & \multicolumn{2}{|c|}{$\begin{array}{c}\text { 95\% Confidence Interval for } \\
\text { Difference }^{c}\end{array}$} \\
\hline & & & & Lower Bound & Upper Bound \\
\hline $\begin{array}{ll} & B\end{array}$ & $.006^{\mathrm{a}, \mathrm{b}}$ & .043 & .883 & -.079 & .091 \\
\hline$A$ & $-.006^{a, b}$ & .043 & .883 & -.091 & .079 \\
\hline
\end{tabular}

Based on estimated marginal means
a. An estimate of the modified population marginal mean (I).
b. An estimate of the modified population marginal mean $(\mathrm{J})$.
c. Adjustment for multiple comparisons: Least Significant Difference (equivalent to no adjustments).

Significant values were observed for sample PMCL but not for sample PMJX. With this result,

it is irrelevant as to what area of the sample is being analyzed.

\section{Swatch Estimates: PMCL}

Dependent Variable: a

\begin{tabular}{|l|r|r|r|r|}
\hline & & & \multicolumn{2}{|c|}{$95 \%$ Confidence Interval } \\
\cline { 4 - 5 } Swatch & Mean & Std. Error & Lower Bound & Upper Bound \\
\hline 1 w/ bleach & $2.323^{\mathrm{a}}$ & .048 & 2.230 & 2.417 \\
2 w/ bleach & $2.892^{\mathrm{a}}$ & .048 & 2.798 & 2.985 \\
3 w/o bleach & $2.867^{\mathrm{a}}$ & .048 & 2.773 & 2.960 \\
4 w/o bleach & $3.002^{\mathrm{a}}$ & .048 & 2.909 & 3.096 \\
\hline
\end{tabular}

a. Based on modified population marginal mean.

Dependent Variable: a

Pairwise Comparisons: PMCL

\begin{tabular}{|c|c|c|c|c|c|c|}
\hline \multirow[b]{2}{*}{ (I) Swatch } & & \multirow{2}{*}{$\begin{array}{c}\text { Mean } \\
\text { Difference } \\
(\mathrm{I}-\mathrm{J})\end{array}$} & \multirow[b]{2}{*}{ Std. Error } & \multirow[b]{2}{*}{ Sig. ${ }^{d}$} & \multicolumn{2}{|c|}{$\begin{array}{c}\text { 95\% Confidence Interval for } \\
\text { Difference } \\
\end{array}$} \\
\hline & & & & & Lower Bound & Upper Bound \\
\hline \multirow[t]{3}{*}{$1 \mathrm{w} / \mathrm{bleach}$} & 2 w/ bleach & $-.568, \mathrm{D,C}$ & .068 & .000 & -.701 & -.436 \\
\hline & 3 w/o bleach & $-.543^{*}, \mathrm{~b}, \mathrm{c}$ & .068 & .000 & -.676 & -.411 \\
\hline & 4 w/o bleach & $-.679^{\pi, b, c}$ & .068 & .000 & -.812 & -.546 \\
\hline \multirow[t]{3}{*}{2 w/ bleach } & 1 w/ bleach & $.568^{x, \mathrm{~b}, \mathrm{c}}$ & .068 & .000 & .436 & .701 \\
\hline & 3 w/o bleach & $.025^{\mathrm{b}, \mathrm{c}}$ & .068 & .710 & -.107 & .158 \\
\hline & 4 w/o bleach & $-.111^{\mathrm{b}, \mathrm{c}}$ & .068 & .102 & -.243 & .022 \\
\hline \multirow[t]{3}{*}{3 w/o bleach } & $1 \mathrm{w} /$ bleach & $.543^{\pi, b, c}$ & .068 & .000 & .411 & .676 \\
\hline & 2 w/ bleach & $-.025^{\mathrm{b}, \mathrm{c}}$ & .068 & .710 & -.158 & .107 \\
\hline & 4 w/o bleach & $-.136^{\pi, b, c}$ & .068 & .045 & -.268 & -.003 \\
\hline \multirow[t]{3}{*}{4 w/o bleach } & 1 w/ bleach & $.679^{\pi}, \mathrm{b}, \mathrm{c}$ & .068 & .000 & .546 & .812 \\
\hline & 2 w/ bleach & $.111^{\mathrm{b}, \mathrm{c}}$ & .068 & .102 & -.022 & .243 \\
\hline & 3 w/o bleach & $.136^{\pi, b, c}$ & .068 & .045 & .003 & .268 \\
\hline
\end{tabular}

Based on estimated marginal means 
*. The mean difference is significant at the .05 level.

b. An estimate of the modified population marginal mean (I).

c. An estimate of the modified population marginal mean (J).

d. Adjustment for multiple comparisons: Least Significant Difference (equivalent to no adjustments).

\section{Swatch Estimates: PMJX}

Dependent Variable: a

\begin{tabular}{|l|r|r|r|r|}
\hline & & & \multicolumn{2}{|c|}{$95 \%$ Confidence Interval } \\
\cline { 3 - 5 } Swatch & Mean & Std. Error & $\begin{array}{c}\text { Lower } \\
\text { Bound }\end{array}$ & $\begin{array}{c}\text { Upper } \\
\text { Bound }\end{array}$ \\
\hline $1 \mathrm{w} /$ bleach & $2.500^{\mathrm{a}}$ & .043 & 2.415 & 2.585 \\
2 w/ bleach & $2.787^{\mathrm{a}}$ & .043 & 2.702 & 2.872 \\
3 w/o bleach & $2.749^{\mathrm{a}}$ & .043 & 2.664 & 2.834 \\
4 w/o bleach & $2.577^{\mathrm{a}}$ & .043 & 2.492 & 2.662 \\
\hline
\end{tabular}

a. Based on modified population marginal mean.

Pairwise Comparisons: PMJX

Dependent Variable: a

\begin{tabular}{|c|c|c|c|c|c|c|}
\hline \multirow[b]{2}{*}{ (I) Swatch } & & \multirow{2}{*}{$\begin{array}{c}\text { Mean } \\
\text { Difference } \\
(\mathrm{I}-\mathrm{J}) \\
\end{array}$} & \multirow[b]{2}{*}{ Std. Error } & \multirow[b]{2}{*}{ Sig. ${ }^{d}$} & \multicolumn{2}{|c|}{$\begin{array}{c}\text { 95\% Confidence Interval for } \\
\text { Difference }\end{array}$} \\
\hline & & & & & Lower Bound & Upper Bound \\
\hline \multirow[t]{3}{*}{$1 \mathrm{w} /$ bleach } & 2 w/ bleach & $-.287^{, b, c}$ & .061 & .000 & -.407 & -.167 \\
\hline & 3 w/o bleach & $-.249^{\pi, b, c}$ & .061 & .000 & -.369 & -.129 \\
\hline & $4 \mathrm{w} / \mathrm{o}$ bleach & $-.077^{\mathrm{b}, \mathrm{c}}$ & .061 & 207 & -.197 & .043 \\
\hline \multirow[t]{3}{*}{2 w/ bleach } & 1 w/ bleach & $.287^{\pi}, \mathrm{b}, \mathrm{c}$ & .061 & .000 & .167 & .407 \\
\hline & 3 w/o bleach & $.038^{\mathrm{b}, \mathrm{c}}$ & .061 & .535 & -.082 & .158 \\
\hline & 4 w/o bleach & $.210^{*}, \mathrm{~b}, \mathrm{c}$ & .061 & .001 & .090 & .330 \\
\hline \multirow[t]{3}{*}{3 w/o bleach } & $1 \mathrm{w} /$ bleach & $249^{\pi, b, c}$ & .061 & .000 & .129 & .369 \\
\hline & 2 w/ bleach & $-.038^{b, c}$ & .061 & .535 & -.158 & .082 \\
\hline & 4 w/o bleach & $.172^{*}, \mathrm{~b}, \mathrm{c}$ & .061 & .005 & .052 & .292 \\
\hline \multirow[t]{3}{*}{4 w/o bleach } & $1 \mathrm{w} /$ bleach & $.077^{\mathrm{b}, \mathrm{c}}$ & .061 & .207 & -.043 & .197 \\
\hline & $2 \mathrm{w} /$ bleach & $-.210^{*}, \mathrm{~b}, \mathrm{c}$ & .061 & .001 & -.330 & -.090 \\
\hline & 3 w/o bleach & $-.172^{\mathrm{x}, \mathrm{b}, \mathrm{c}}$ & .061 & .005 & -.292 & -.052 \\
\hline
\end{tabular}

Based on estimated marginal means

*. The mean difference is significant at the .05 level.

b. An estimate of the modified population marginal mean (I).

c. An estimate of the modified population marginal mean $(\mathrm{J})$.

d. Adjustment for multiple comparisons: Least Significant Difference (equivalent to no adjustments).

Swatch 1 did observe all significant values for sample PMCL. No patterns were observed

between the reference samples for the bleached or unbleached samples.

\section{Bleach Estimates: PMCL}

Dependent Variable:a

\begin{tabular}{|l|r|r|r|r|}
\hline & & \multirow{2}{*}{ Mleached } & \multicolumn{2}{|c|}{$95 \%$ Confidence Interval } \\
\cline { 4 - 5 } & Mean & Std. Error & Lower Bound & \multicolumn{1}{c|}{ Upper Bound } \\
\hline w/ bleach & $2.608^{\mathrm{a}}$ & .034 & 2.541 & 2.674 \\
w/o bleach & $2.934^{\mathrm{a}}$ & .034 & 2.868 & 3.001 \\
\hline
\end{tabular}

a. Based on modified population marginal mean.

Dependent Variable:a

Pairwise Comparisons: PMCL

\begin{tabular}{|c|c|c|c|c|c|c|}
\hline \multirow[b]{2}{*}{ (I) Bleached } & \multirow[b]{2}{*}{ (J) Bleached } & \multirow[b]{2}{*}{ Mean Difference (I-J) } & \multirow[b]{2}{*}{ Std. Error } & \multirow[b]{2}{*}{ Sig. $^{c}$} & \multicolumn{2}{|c|}{$\begin{array}{c}\text { 95\% Confidence Interval for } \\
\text { Difference }\end{array}$} \\
\hline & & & & & Lower Bound & Upper Bound \\
\hline w/ bleach & w/o bleach & $-.327^{*}, \mathrm{a}, \mathrm{b}$ & .048 & .000 & -.421 & -.233 \\
\hline w/o bleach & w/ bleach & $.327^{*}, \mathrm{a}, \mathrm{b}$ & .048 & .000 & .233 & .421 \\
\hline
\end{tabular}

Based on estimated marginal means 
*. The mean difference is significant at the .05 level.

a. An estimate of the modified population marginal mean (I).

b. An estimate of the modified population marginal mean (J).

c. Adjustment for multiple comparisons: Least Significant Difference (equivalent to no adjustments).

\section{Bleach Estimates: PMJX}

Dependent Variable: a

\begin{tabular}{|c|c|c|c|c|}
\hline \multirow[b]{2}{*}{ bleached } & \multirow[b]{2}{*}{ Mean } & \multirow[b]{2}{*}{ Std. Error } & \multicolumn{2}{|c|}{ 95\% Confidence Interval } \\
\hline & & & Lower Bound & Upper Bound \\
\hline w/ bleach & $2.643^{\mathrm{a}}$ & .031 & 2.583 & 2.703 \\
\hline w/o bleach & $2.663^{a}$ & .031 & 2.603 & 2.723 \\
\hline
\end{tabular}

a. Based on modified population marginal mean.

Pairwise Comparisons: PMJX

Dependent Variable: a

\begin{tabular}{|c|c|c|c|c|c|c|}
\hline \multirow[b]{2}{*}{ (I) bleached } & & \multirow[b]{2}{*}{ Mean Difference (I-J) } & \multirow[b]{2}{*}{ Std. Error } & \multirow[b]{2}{*}{ Sig. $^{c}$} & \multicolumn{2}{|c|}{$\begin{array}{c}95 \% \text { Confidence Interval for } \\
\text { Difference }^{c}\end{array}$} \\
\hline & & & & & Lower Bound & Upper Bound \\
\hline w/ bleach & w/o bleach & $-.020^{\mathrm{a}, \mathrm{b}}$ & .043 & .650 & -.105 & .065 \\
\hline w/o bleach & w/ bleach & $.020^{\mathrm{a}, \mathrm{b}}$ & .043 & .650 & -.065 & .105 \\
\hline
\end{tabular}

Based on estimated marginal means

a. An estimate of the modified population marginal mean (I).

b. An estimate of the modified population marginal mean (J).

c. Adjustment for multiple comparisons: Least Significant Difference (equivalent to no adjustments).

Significant values were observed for both samples.

\section{Washing Estimates: PMCL}

Dependent Variable:a

\begin{tabular}{|l|r|r|r|r|}
\hline & & & \multicolumn{2}{|c|}{$95 \%$ Confidence Interval } \\
\cline { 4 - 5 } Washing & Mean & Std. Error & \multicolumn{1}{|c|}{ Lower Bound } & \multicolumn{1}{c|}{ Upper Bound } \\
\hline 1W 0A & $2.896^{\mathrm{a}}$ & .079 & 2.741 & 3.051 \\
1W 16A & $2.727^{\mathrm{a}}$ & .056 & 2.617 & 2.837 \\
2W 32A & $2.606^{\mathrm{a}}$ & .056 & 2.496 & 2.716 \\
3W 48A & $2.731^{\mathrm{a}}$ & .056 & 2.622 & 2.841 \\
4W 64A & $3.003^{\mathrm{a}}$ & .056 & 2.893 & 3.113 \\
5W 80A & $2.725^{\mathrm{a}}$ & .056 & 2.615 & 2.835 \\
\hline
\end{tabular}

a. Based on modified population marginal mean. 
Dependent Variable:a

Pairwise Comparisons: PMCL

\begin{tabular}{|c|c|c|c|c|c|c|}
\hline \multirow[b]{2}{*}{ (I) Washing } & \multirow[b]{2}{*}{ (J) Washing } & \multirow[b]{2}{*}{ Mean Difference (I-J) } & \multirow[b]{2}{*}{ Std. Error } & \multirow[b]{2}{*}{ Sig. ${ }^{c}$} & \multicolumn{2}{|c|}{$\begin{array}{l}\text { 95\% Confidence Interval for } \\
\text { Difference }\end{array}$} \\
\hline & & & & & Lower Bound & Upper Bound \\
\hline \multirow[t]{5}{*}{$1 \mathrm{~W} 0 \mathrm{~A}$} & $1 \mathrm{~W} 16 \mathrm{~A}$ & $.169^{\mathrm{a}}, \mathrm{b}$ & .097 & .082 & -.021 & .359 \\
\hline & $2 W 32 A$ & $.290^{\mathrm{a}}, \mathrm{b},{ }^{*}$ & .097 & .003 & .100 & .480 \\
\hline & $3 W 48 A$ & $.165^{a}, b$ & .097 & .090 & -.026 & .355 \\
\hline & $4 W 64 A$ & $-.107^{a}, b$ & .097 & .268 & -.298 & .083 \\
\hline & $5 W 80 A$ & $.171^{a}, b$ & .097 & .078 & -.019 & .361 \\
\hline \multirow[t]{5}{*}{$1 \mathrm{~W} 16 \mathrm{~A}$} & $1 \mathrm{~W} 0 \mathrm{~A}$ & $-.169^{a}, b$ & .097 & .082 & -.359 & .021 \\
\hline & $2 W 32 A$ & $.121^{a}, b$ & .079 & .127 & -.034 & .276 \\
\hline & $3 W 48 A$ & $-.004^{a}, b$ & .079 & .955 & -.160 & .151 \\
\hline & $4 W 64 A$ & $-.276^{\mathrm{a}}, \mathrm{b},{ }^{*}$ & .079 & .001 & -.432 & -.121 \\
\hline & $5 W 80 A$ & $.002^{a}, b$ & .079 & .980 & -.153 & .157 \\
\hline \multirow[t]{5}{*}{$2 W 32 A$} & $1 \mathrm{~W} \mathrm{0A}$ & $-.290^{\mathrm{a}}, \mathrm{b},{ }^{*}$ & .097 & .003 & -.480 & -.100 \\
\hline & $1 \mathrm{~W} 16 \mathrm{~A}$ & $-.121^{a}, b$ & .079 & .127 & -.276 & .034 \\
\hline & $3 W 48 A$ & $-.125^{a}, b$ & .079 & .113 & -.281 & .030 \\
\hline & $4 W 64 A$ & $-.397^{\mathrm{a}}, \mathrm{b},{ }^{*}$ & .079 & .000 & -.553 & -.242 \\
\hline & $5 W 80 A$ & $-.119^{a}, b$ & .079 & .133 & -.274 & .036 \\
\hline \multirow[t]{5}{*}{$3 W$ 48A } & $1 \mathrm{~W} 0 \mathrm{~A}$ & $-.165^{a}, b$ & .097 & .090 & -.355 & .026 \\
\hline & $1 \mathrm{~W} 16 \mathrm{~A}$ & $.004^{a}, b$ & .079 & .955 & -.151 & .160 \\
\hline & $2 W 32 A$ & $.125^{\mathrm{a}}, \mathrm{b}$ & .079 & .113 & -.030 & .281 \\
\hline & $4 W 64 A$ & $-.272^{\mathrm{a}}, \mathrm{b},{ }^{*}$ & .079 & .001 & -.427 & -.117 \\
\hline & $5 \mathrm{~W} 80 \mathrm{~A}$ & $.006^{\mathrm{a}}, \mathrm{b}$ & .079 & .936 & -.149 & .162 \\
\hline \multirow[t]{5}{*}{$4 \mathrm{~W} 64 \mathrm{~A}$} & $1 \mathrm{~W} 0 \mathrm{~A}$ & $.107^{a}, b$ & .097 & .268 & -.083 & .298 \\
\hline & $1 \mathrm{~W} 16 \mathrm{~A}$ & $.276^{\mathrm{a}}, \mathrm{b},{ }^{*}$ & .079 & .001 & .121 & .432 \\
\hline & $2 W 32 A$ & $.397^{a}, b,{ }^{*}$ & .079 & .000 & .242 & .553 \\
\hline & $3 W 48 A$ & $.272^{\mathrm{a}}, \mathrm{b},{ }^{*}$ & .079 & .001 & .117 & .427 \\
\hline & $5 \mathrm{~W} 80 \mathrm{~A}$ & $.278^{\mathrm{a}}, \mathrm{b},{ }^{*}$ & .079 & .000 & .123 & .434 \\
\hline \multirow[t]{5}{*}{$5 W 80 A$} & $1 \mathrm{~W} 0 \mathrm{~A}$ & $-.171^{a}, b$ & .097 & .078 & -.361 & .019 \\
\hline & $1 \mathrm{~W} 16 \mathrm{~A}$ & $-.002^{a}, b$ & .079 & .980 & -.157 & .153 \\
\hline & $2 W 32 A$ & $.119^{a}, \mathrm{~b}$ & .079 & .133 & -.036 & .274 \\
\hline & $3 W 48 A$ & $-.006^{a}, b$ & .079 & .936 & -.162 & .149 \\
\hline & $4 W 64 A$ & $-.278^{a}, b,{ }^{*}$ & .079 & .000 & -.434 & -.123 \\
\hline
\end{tabular}

Based on estimated marginal means

a. An estimate of the modified population marginal mean (I).

b. An estimate of the modified population marginal mean $(\mathrm{J})$.

c. Adjustment for multiple comparisons: Least Significant Difference (equivalent to no adjustments).

${ }^{*}$. The mean difference is significant at the .05 level.

\section{Washing Estimates: PMJX}

Dependent Variable: a

\begin{tabular}{|c|c|c|c|c|}
\hline \multirow[b]{2}{*}{ washings } & \multirow[b]{2}{*}{ Mean } & \multirow[b]{2}{*}{ Std. Error } & \multicolumn{2}{|c|}{ 95\% Confidence Interval } \\
\hline & & & Lower Bound & Upper Bound \\
\hline $1 \mathrm{~W} \mathrm{0A}$ & $2.471^{\mathrm{a}}$ & .072 & 2.331 & 2.612 \\
\hline $1 \mathrm{~W} 16 \mathrm{~A}$ & $2.857^{\mathrm{a}}$ & .051 & 2.757 & 2.956 \\
\hline $2 W 32 A$ & $2.800^{\mathrm{a}}$ & .051 & 2.701 & 2.900 \\
\hline $3 W 48 A$ & $2.618^{a}$ & .051 & 2.519 & 2.718 \\
\hline $4 \mathrm{~W} 64 \mathrm{~A}$ & $2.683^{a}$ & .051 & 2.584 & 2.783 \\
\hline $5 \mathrm{~W} 80 \mathrm{~A}$ & $2.398^{a}$ & .051 & 2.298 & 2.497 \\
\hline
\end{tabular}

a. Based on modified population marginal mean. 
Pairwise Comparisons: PMJX

Dependent Variable: a

\begin{tabular}{|c|c|c|c|c|c|c|}
\hline \multirow{2}{*}{\multicolumn{2}{|c|}{ (I) washings }} & \multirow[b]{2}{*}{ Mean Difference (I-J) } & \multirow[b]{2}{*}{ Std. Error } & \multirow[b]{2}{*}{ Sig. ${ }^{d}$} & \multicolumn{2}{|c|}{$\begin{array}{c}\text { 95\% Confidence Interval for } \\
\text { Difference }^{d}\end{array}$} \\
\hline & & & & & Lower Bound & Upper Bound \\
\hline \multirow[t]{5}{*}{$1 \mathrm{~W} 0 \mathrm{~A}$} & $1 \mathrm{~W} 16 \mathrm{~A}$ & $-.385^{, b, c}$ & .088 & .000 & -.558 & -.213 \\
\hline & $2 W 32 A$ & $-.329^{\pi}, \mathrm{b}, \mathrm{c}$ & .088 & .000 & -.501 & -.156 \\
\hline & $3 W 48 A$ & $-.147^{\mathrm{b}, \mathrm{c}}$ & .088 & .095 & -.319 & .026 \\
\hline & $4 W 64 A$ & $-.212^{x, b, c}$ & .088 & .016 & -.384 & -.039 \\
\hline & $5 W 80 A$ & $.074^{\mathrm{b}, \mathrm{c}}$ & .088 & .401 & -.099 & .246 \\
\hline \multirow[t]{5}{*}{$1 \mathrm{~W} 16 \mathrm{~A}$} & $1 \mathrm{~W} 0 \mathrm{~A}$ & $.385^{\pi, b, c}$ & .088 & .000 & .213 & .558 \\
\hline & $2 W 32 A$ & $.057^{\mathrm{b}, \mathrm{c}}$ & .072 & .430 & -.084 & .198 \\
\hline & $3 W 48 A$ & $.239^{\star}, \mathrm{b}, \mathrm{c}$ & .072 & .001 & .098 & .380 \\
\hline & $4 W 64 A$ & $.174^{\pi, b, c}$ & .072 & .016 & .033 & .315 \\
\hline & $5 W 80 A$ & $.459^{\circ}, \mathrm{b}, \mathrm{c}$ & .072 & .000 & .318 & .600 \\
\hline \multirow[t]{5}{*}{$2 W 32 A$} & $1 \mathrm{~W} 0 \mathrm{~A}$ & $.329^{*}, \mathrm{~b}, \mathrm{c}$ & .088 & .000 & .156 & .501 \\
\hline & $1 \mathrm{~W} 16 \mathrm{~A}$ & $-.057^{\mathrm{b}, \mathrm{c}}$ & .072 & .430 & -.198 & .084 \\
\hline & $3 W 48 A$ & $.182^{*}, \mathrm{~b}, \mathrm{c}$ & .072 & .011 & .041 & .323 \\
\hline & $4 W 64 A$ & $.117^{\mathrm{b}, \mathrm{c}}$ & .072 & .104 & -.024 & .258 \\
\hline & $5 W 80 A$ & $.403^{\pi, b, c}$ & .072 & .000 & .262 & .543 \\
\hline \multirow[t]{5}{*}{$3 W 48 A$} & $1 \mathrm{~W} 0 \mathrm{~A}$ & $.147^{\mathrm{b}, \mathrm{c}}$ & .088 & .095 & -.026 & .319 \\
\hline & $1 \mathrm{~W} 16 \mathrm{~A}$ & $-.239^{\pi, b, c}$ & .072 & .001 & -.380 & -.098 \\
\hline & $2 W 32 A$ & $-.182^{x, b, c}$ & .072 & .011 & -.323 & -.041 \\
\hline & $4 W 64 A$ & $-.065^{b, c}$ & .072 & .366 & -.206 & .076 \\
\hline & $5 W$ 80A & $.221^{\pi, b, c}$ & .072 & .002 & .080 & .362 \\
\hline \multirow[t]{5}{*}{$4 \mathrm{~W} 64 \mathrm{~A}$} & $1 \mathrm{~W} 0 \mathrm{~A}$ & $.212^{*}, \mathrm{~b}, \mathrm{c}$ & .088 & .016 & .039 & .384 \\
\hline & $1 \mathrm{~W} 16 \mathrm{~A}$ & $-.174^{*}, \mathrm{~b}, \mathrm{c}$ & .072 & .016 & -.315 & -.033 \\
\hline & $2 W 32 A$ & $-.117^{b, c}$ & .072 & .104 & -.258 & .024 \\
\hline & $3 W$ 48A & $.065^{b, c}$ & .072 & .366 & -.076 & .206 \\
\hline & $5 W$ 80A & $.286^{*, b, c}$ & .072 & .000 & .145 & .427 \\
\hline \multirow[t]{5}{*}{$5 W$} & $1 \mathrm{~W} 0 \mathrm{~A}$ & $-.074^{b, c}$ & .088 & .401 & -.246 & .099 \\
\hline & $1 \mathrm{~W} 16 \mathrm{~A}$ & $-.459^{*}, b, c$ & .072 & .000 & -.600 & -.318 \\
\hline & $2 W 32 A$ & $-.403^{x, b, c}$ & .072 & .000 & -.543 & -.262 \\
\hline & $3 W$ 48A & $-.221^{\mathrm{x}, \mathrm{b}, \mathrm{c}}$ & .072 & .002 & -.362 & -.080 \\
\hline & $4 W 64 A$ & $-.286^{*}, \mathrm{~b}, \mathrm{c}$ & .072 & .000 & -.427 & -.145 \\
\hline
\end{tabular}

Based on estimated marginal means

*. The mean difference is significant at the .05 level.

b. An estimate of the modified population marginal mean (I).

c. An estimate of the modified population marginal mean (J).

d. Adjustment for multiple comparisons: Least Significant Difference (equivalent to no adjustments).

Marked significant differences were observed in greater amounts in sample PMJX than PMCL. 


\section{Sample Area * Swatch: PMCL}

Dependent Variable: a

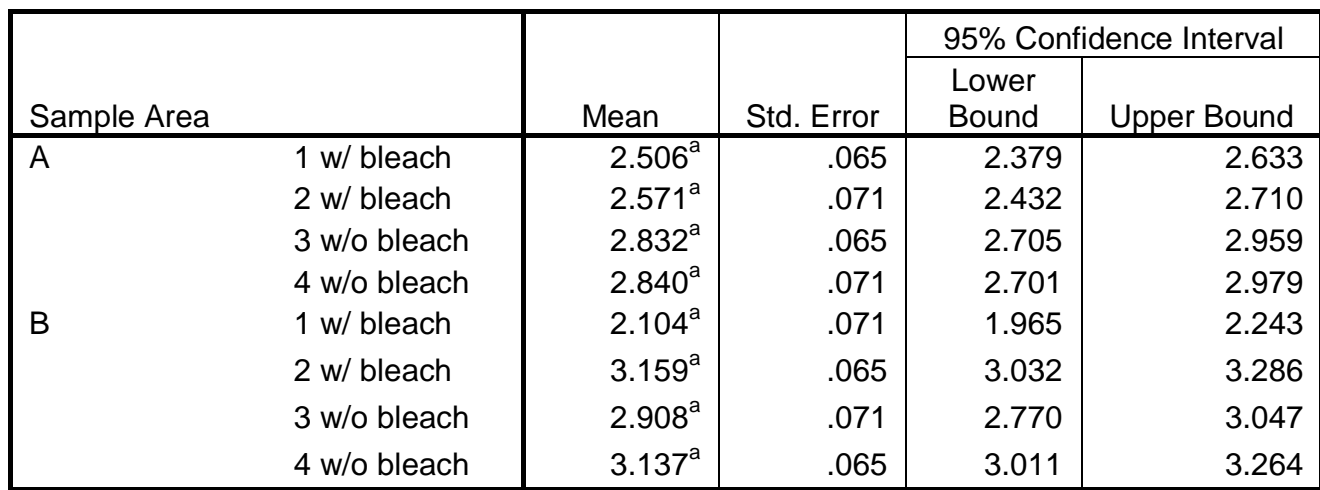

a. Based on modified population marginal mean.

\section{Sample Area * Swatch: PMJX}

Dependent Variable: a

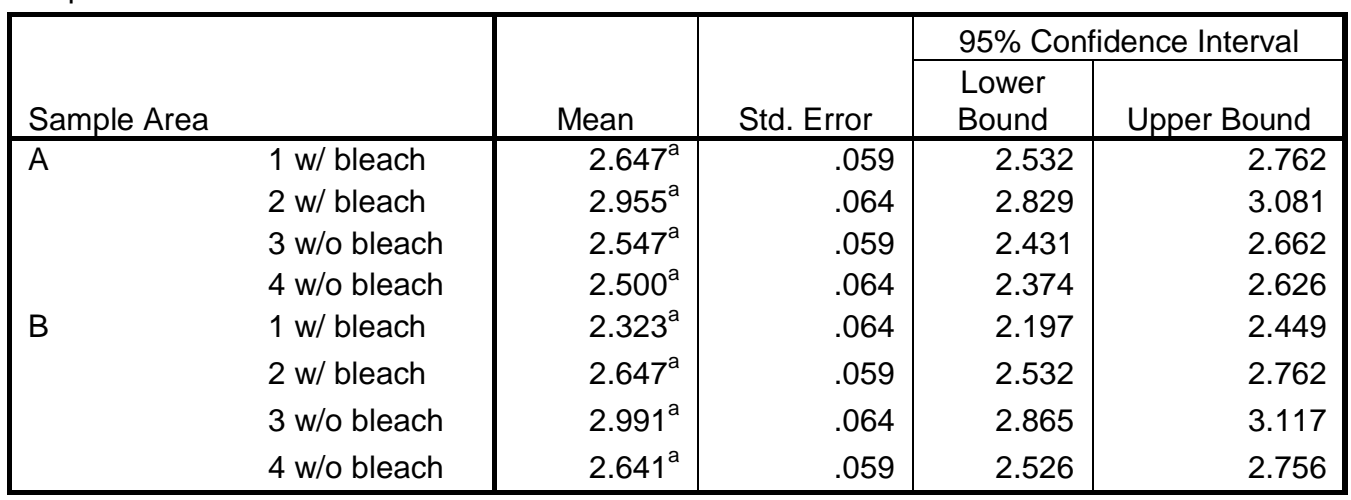

a. Based on modified population marginal mean.
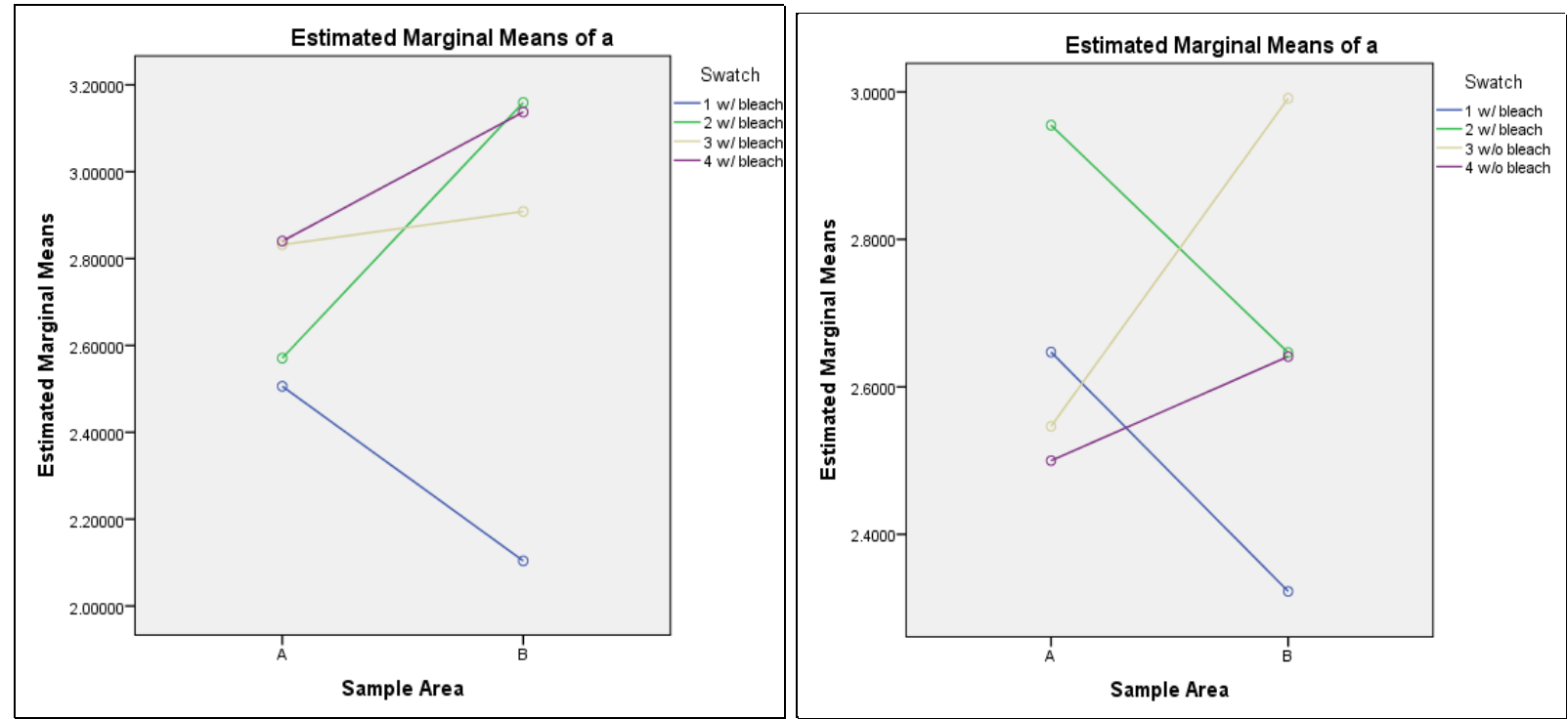

The patterns are obviously different between both reference samples. Swatch 1 observes to be consistent between the two diagrams, and closely follows is swatch 3, however, the values differ. 
The mean values are slighter greater in sample PMCL. The Swatch* Sample Area interaction is significant at $p=.001$ for sample PMCL and is insignificant at $p=.222$ for sample PMJX.

Dependent Variable: a

\section{Sample Area * Bleached: PMCL}

\begin{tabular}{|c|c|c|c|c|c|}
\hline \multirow{2}{*}{ Sample Area } & & \multirow[b]{2}{*}{ Mean } & \multirow[b]{2}{*}{ Std. Error } & \multicolumn{2}{|c|}{ 95\% Confidence Interval } \\
\hline & & & & $\begin{array}{l}\text { Lower } \\
\text { Bound }\end{array}$ & Upper Bound \\
\hline \multirow[t]{2}{*}{$\bar{A}$} & w/ bleach & $2.535^{\mathrm{a}}$ & .048 & 2.442 & 2.629 \\
\hline & w/o bleach & $2.836^{a}$ & .048 & 2.742 & 2.929 \\
\hline \multirow[t]{2}{*}{$B$} & w/ bleach & $2.680^{\mathrm{a}}$ & .048 & 2.586 & 2.773 \\
\hline & w/o bleach & $3.033^{a}$ & .048 & 2.940 & 3.127 \\
\hline
\end{tabular}

a. Based on modified population marginal mean.

Dependent Variable: a

\section{Sample Area * Bleached: PMJX}

\begin{tabular}{|c|c|c|c|c|c|}
\hline \multirow{2}{*}{\multicolumn{2}{|c|}{ Sample Area }} & \multirow[b]{2}{*}{ Mean } & \multirow[b]{2}{*}{ Std. Error } & \multicolumn{2}{|c|}{$\begin{array}{c}95 \% \text { Confidence } \\
\text { Interval }\end{array}$} \\
\hline & & & & $\begin{array}{l}\text { Lower } \\
\text { Bound }\end{array}$ & $\begin{array}{l}\text { Upper } \\
\text { Bound }\end{array}$ \\
\hline \multirow{4}{*}{$\begin{array}{l}A \\
B\end{array}$} & w/ bleach & $2.787^{a}$ & .043 & 2.702 & 2.872 \\
\hline & w/o bleach & $2.525^{\mathrm{a}}$ & .043 & 2.440 & 2.610 \\
\hline & w/ bleach & $2.499^{a}$ & .043 & 2.414 & 2.584 \\
\hline & w/o bleach & $2.800^{\mathrm{a}}$ & .043 & 2.715 & 2.885 \\
\hline
\end{tabular}

a. Based on modified population marginal mean.
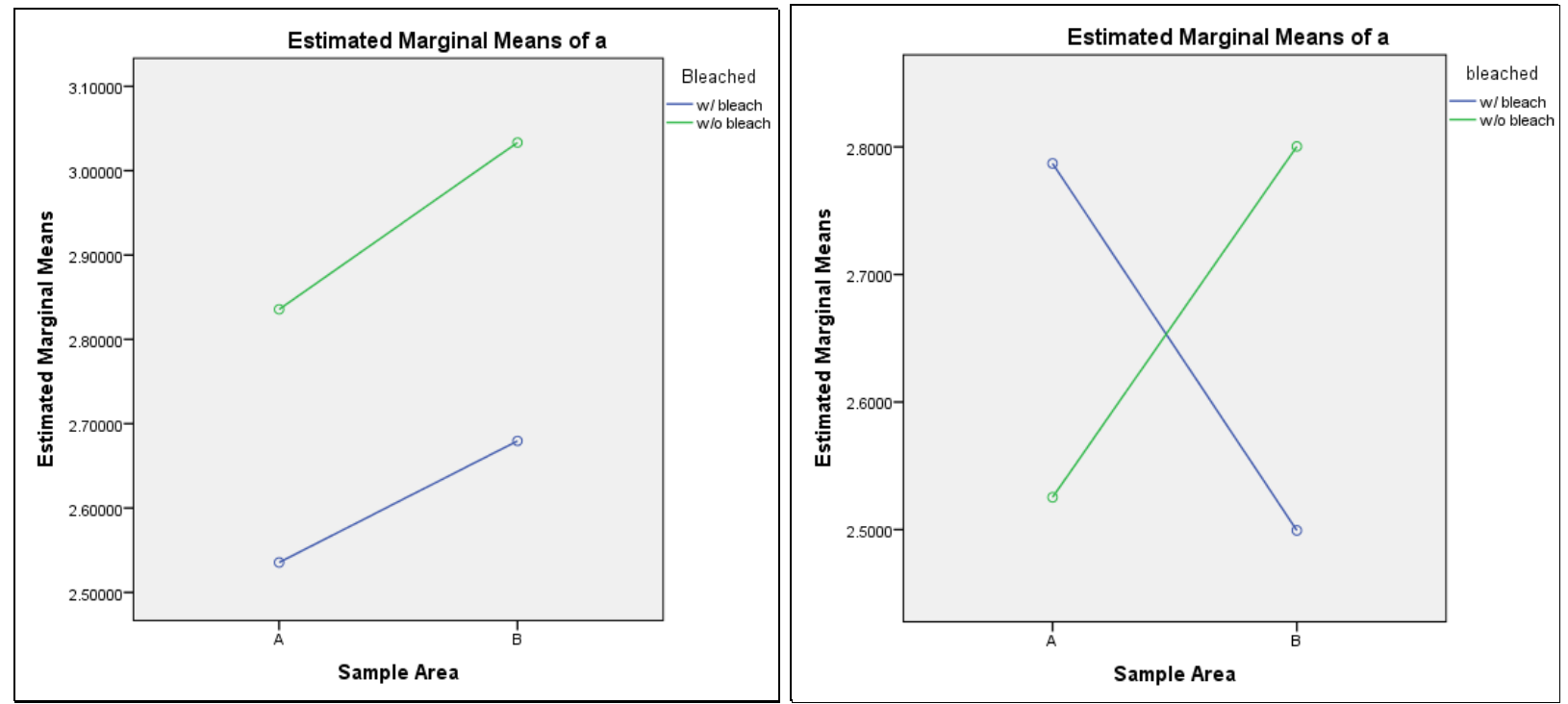

Again, the difference between the two patterns are obvious.

For the bleached * washing interaction below, the pattern for sample PMCL is more similar than for sample PMJX. 


\section{Bleached * Washing: PMCL}

Dependent Variable: a

\begin{tabular}{|ll|r|r|r|r|}
\hline & & & \multicolumn{2}{|c|}{$95 \%$ Confidence Interval } \\
\cline { 4 - 5 } Bleached & & Mean & Std. Error & Lower Bound & Upper Bound \\
\hline w/ bleach & 1W 0A & $2.852^{\mathrm{a}}$ & .112 & 2.632 & 3.072 \\
& 1W 16A & $2.646^{\mathrm{a}}$ & .079 & 2.491 & 2.802 \\
& 2W 32A & $2.512^{\mathrm{a}}$ & .079 & 2.357 & 2.667 \\
& 3W 48A & $2.440^{\mathrm{a}}$ & .079 & 2.285 & 2.596 \\
& 4W 64A & $2.916^{\mathrm{a}}$ & .079 & 2.761 & 3.071 \\
w/o bleach & 5W 80A & $2.401^{\mathrm{a}}$ & .079 & 2.245 & 2.556 \\
& 1W 0A & $2.940^{\mathrm{a}}$ & .112 & 2.720 & 3.160 \\
& 1W 16A & $2.807^{\mathrm{a}}$ & .079 & 2.652 & 2.963 \\
& 2W 32A & $2.700^{\mathrm{a}}$ & .079 & 2.545 & 2.855 \\
& 3W 48A & $3.023^{\mathrm{a}}$ & .079 & 2.867 & 3.178 \\
& 4W 64A & $3.091^{\mathrm{a}}$ & .079 & 2.935 & 3.246 \\
& 5W 80A & $3.049^{\mathrm{a}}$ & .079 & 2.894 & 3.204 \\
\hline
\end{tabular}

a. Based on modified population marginal mean.

\section{Bleached * Washings: PMJX}

Dependent Variable: a

\begin{tabular}{|ll|r|r|r|r|}
\hline & & & \multicolumn{2}{|c|}{$95 \%$ Confidence Interval } \\
\cline { 4 - 5 } bleached & & Mean & Std. Error & Lower Bound & Upper Bound \\
\hline W/ bleach & 1W 0A & $2.564^{\mathrm{a}}$ & .102 & 2.365 & 2.764 \\
& 1W 16A & $2.851^{\mathrm{a}}$ & .072 & 2.710 & 2.992 \\
& 2W 32A & $2.856^{\mathrm{a}}$ & .072 & 2.715 & 2.997 \\
& 3W 48A & $2.540^{\mathrm{a}}$ & .072 & 2.399 & 2.681 \\
& 4W 64A & $2.552^{\mathrm{a}}$ & .072 & 2.411 & 2.693 \\
w/o bleach & $2.456^{\mathrm{a}}$ & .072 & 2.315 & 2.597 \\
& 5W 80A & $2.378^{\mathrm{a}}$ & .102 & 2.179 & 2.578 \\
& 1W 0A & $2.863^{\mathrm{a}}$ & .072 & 2.722 & 3.003 \\
& 1W 16A & $2.744^{\mathrm{a}}$ & .072 & 2.603 & 2.885 \\
& 2W 32A & $2.696^{\mathrm{a}}$ & .072 & 2.555 & 2.837 \\
& 3W 48A & $2.815^{\mathrm{a}}$ & .072 & 2.674 & 2.956 \\
& 4W 64A & $2.339^{\mathrm{a}}$ & .072 & 2.198 & 2.480 \\
\hline
\end{tabular}

a. Based on modified population marginal mean.
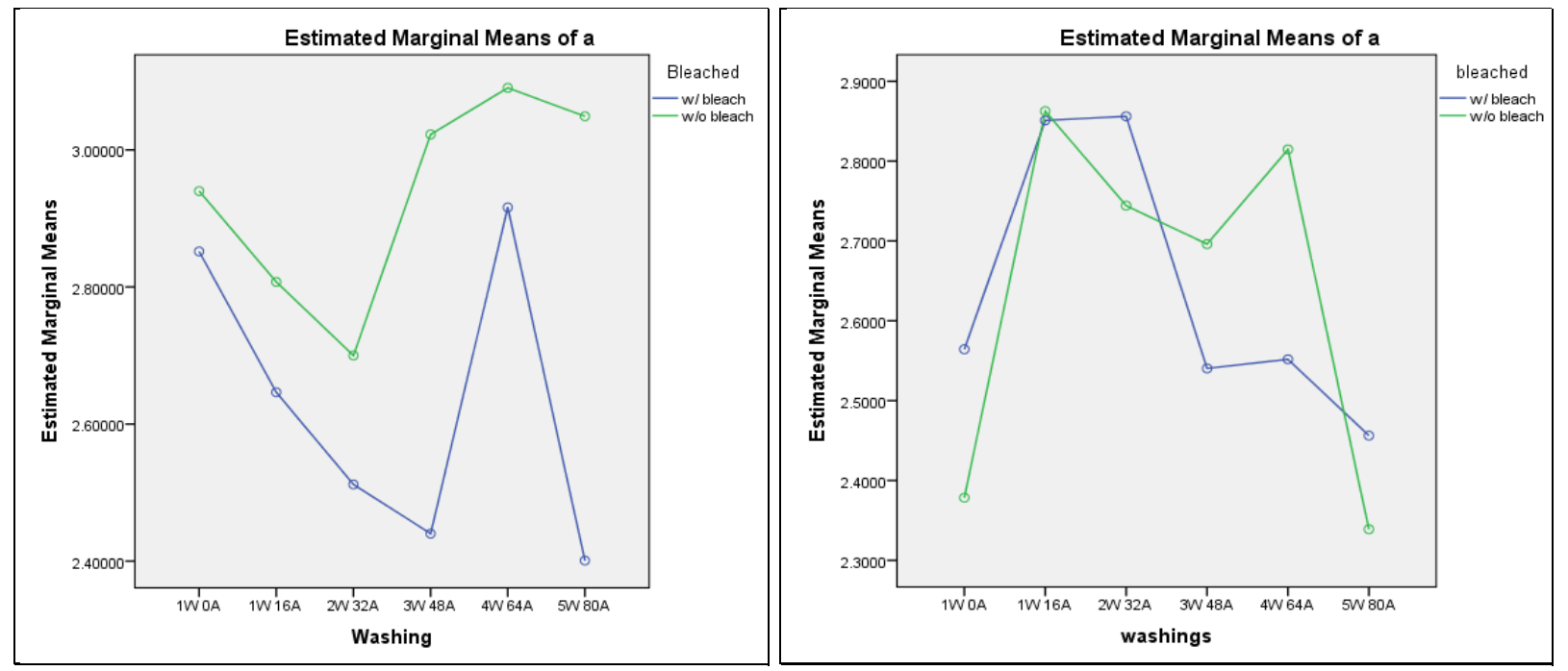


\section{Swatch * Washing: PMCL}

Dependent Variable: a

\begin{tabular}{|c|c|c|c|c|c|}
\hline \multirow[b]{2}{*}{ Swatch } & & \multirow[b]{2}{*}{ Mean } & \multirow[b]{2}{*}{ Std. Error } & \multicolumn{2}{|c|}{$95 \%$ Confidence Interval } \\
\hline & & & & Lower Bound & Upper Bound \\
\hline \multirow[t]{6}{*}{$1 \mathrm{w} /$ bleach } & $1 \mathrm{~W} 0 \mathrm{~A}$ & $2.422^{a}$ & .158 & 2.111 & 2.733 \\
\hline & $1 \mathrm{~W} 16 \mathrm{~A}$ & $2.303^{a}$ & .112 & 2.084 & 2.523 \\
\hline & $2 W 32 A$ & $2.125^{\mathrm{a}}$ & .112 & 1.905 & 2.344 \\
\hline & $3 W 48 A$ & $2.174^{a}$ & .112 & 1.955 & 2.394 \\
\hline & $4 \mathrm{~W} 64 \mathrm{~A}$ & $2.763^{\mathrm{a}}$ & .112 & 2.544 & 2.983 \\
\hline & $5 \mathrm{~W} 80 \mathrm{~A}$ & $2.202^{\mathrm{a}}$ & .112 & 1.982 & 2.421 \\
\hline \multirow[t]{6}{*}{2 w/ bleach } & $1 \mathrm{~W} 0 \mathrm{~A}$ & $3.282^{\mathrm{a}}$ & .158 & 2.971 & 3.593 \\
\hline & $1 \mathrm{~W} 16 \mathrm{~A}$ & $2.989^{\mathrm{a}}$ & .112 & 2.770 & 3.209 \\
\hline & $2 W 32 A$ & $2.899^{a}$ & .112 & 2.679 & 3.119 \\
\hline & $3 W 48 A$ & $2.706^{\mathrm{a}}$ & .112 & 2.486 & 2.926 \\
\hline & $4 \mathrm{~W} 64 \mathrm{~A}$ & $3.069^{a}$ & .112 & 2.849 & 3.289 \\
\hline & $5 W$ 80A & $2.600^{\mathrm{a}}$ & .112 & 2.380 & 2.820 \\
\hline \multirow[t]{6}{*}{3 w/o bleach } & $1 \mathrm{~W} 0 \mathrm{~A}$ & $2.665^{\mathrm{a}}$ & .158 & 2.354 & 2.976 \\
\hline & $1 \mathrm{~W} 16 \mathrm{~A}$ & $2.954^{\mathrm{a}}$ & .112 & 2.734 & 3.174 \\
\hline & $2 W 32 A$ & $3.017^{\mathrm{a}}$ & .112 & 2.797 & 3.237 \\
\hline & $3 W 48 A$ & $2.679^{a}$ & .112 & 2.459 & 2.899 \\
\hline & $4 W 64 A$ & $3.112^{\mathrm{a}}$ & .112 & 2.892 & 3.331 \\
\hline & $5 W 80 A$ & $2.672^{\mathrm{a}}$ & .112 & 2.453 & 2.892 \\
\hline \multirow[t]{6}{*}{4 w/o bleach } & $1 \mathrm{~W} 0 \mathrm{~A}$ & $3.215^{\mathrm{a}}$ & .158 & 2.904 & 3.526 \\
\hline & $1 \mathrm{~W} 16 \mathrm{~A}$ & $2.661^{a}$ & .112 & 2.441 & 2.881 \\
\hline & $2 W 32 A$ & $2.383^{\mathrm{a}}$ & .112 & 2.163 & 2.603 \\
\hline & $3 W 48 A$ & $3.366^{a}$ & .112 & 3.146 & 3.586 \\
\hline & $4 W 64 A$ & $3.069^{a}$ & .112 & 2.850 & 3.289 \\
\hline & $5 \mathrm{~W} 80 \mathrm{~A}$ & $3.426^{a}$ & .112 & 3.206 & 3.646 \\
\hline
\end{tabular}

a. Based on modified population marginal mean.

Dependent Variable: a

\section{Swatch * washings: PMJX}

\begin{tabular}{|c|c|c|c|c|c|}
\hline \multirow{2}{*}{ Swatch } & & \multirow[b]{2}{*}{ Mean } & \multirow[b]{2}{*}{ Std. Error } & \multicolumn{2}{|c|}{$95 \%$ Confidence Interval } \\
\hline & & & & Lower Bound & Upper Bound \\
\hline \multirow[t]{6}{*}{$1 \mathrm{w} /$ bleach } & $1 \mathrm{~W} 0 \mathrm{~A}$ & $2.153^{\mathrm{a}}$ & .144 & 1.871 & 2.435 \\
\hline & $1 \mathrm{~W} 16 \mathrm{~A}$ & $2.635^{\mathrm{a}}$ & .102 & 2.436 & 2.834 \\
\hline & $2 W 32 A$ & $2.830^{\mathrm{a}}$ & .102 & 2.631 & 3.029 \\
\hline & $3 W$ 48A & $2.233^{\mathrm{a}}$ & .102 & 2.034 & 2.432 \\
\hline & $4 W 64 A$ & $2.708^{a}$ & .102 & 2.509 & 2.908 \\
\hline & $5 W$ 80A & $2.266^{\mathrm{a}}$ & .102 & 2.066 & 2.465 \\
\hline \multirow[t]{6}{*}{2 w/ bleach } & $1 \mathrm{~W} 0 \mathrm{~A}$ & $2.976^{a}$ & .144 & 2.694 & 3.258 \\
\hline & $1 \mathrm{~W} 16 \mathrm{~A}$ & $3.067^{\mathrm{a}}$ & .102 & 2.868 & 3.266 \\
\hline & $2 W 32 A$ & $2.882^{\mathrm{a}}$ & .102 & 2.683 & 3.082 \\
\hline & $3 W 48 A$ & $2.848^{\mathrm{a}}$ & .102 & 2.648 & 3.047 \\
\hline & $4 W 64 A$ & $2.395^{\mathrm{a}}$ & .102 & 2.196 & 2.594 \\
\hline & $5 W$ 80A & $2.647^{\mathrm{a}}$ & .102 & 2.448 & 2.846 \\
\hline \multirow[t]{6}{*}{3 w/o bleach } & $1 \mathrm{~W} 0 \mathrm{~A}$ & $2.754^{\mathrm{a}}$ & .144 & 2.472 & 3.036 \\
\hline & $1 \mathrm{~W} 16 \mathrm{~A}$ & $2.956^{a}$ & .102 & 2.757 & 3.155 \\
\hline & $2 W 32 A$ & $2.664^{a}$ & .102 & 2.465 & 2.863 \\
\hline & $3 W$ 48A & $2.804^{\mathrm{a}}$ & .102 & 2.605 & 3.004 \\
\hline & $4 W 64 A$ & $2.890^{\mathrm{a}}$ & .102 & 2.691 & 3.089 \\
\hline & $5 W 80 A$ & $2.426^{a}$ & .102 & 2.227 & 2.626 \\
\hline \multirow[t]{6}{*}{4 w/o bleach } & $1 \mathrm{~W} 0 \mathrm{~A}$ & $2.003^{a}$ & .144 & 1.721 & 2.284 \\
\hline & $1 \mathrm{~W} 16 \mathrm{~A}$ & $2.769^{\mathrm{a}}$ & .102 & 2.570 & 2.968 \\
\hline & $2 W 32 A$ & $2.825^{\mathrm{a}}$ & .102 & 2.625 & 3.024 \\
\hline & $3 W$ 48A & $2.588^{a}$ & .102 & 2.388 & 2.787 \\
\hline & $4 W 64 A$ & $2.739^{a}$ & .102 & 2.540 & 2.938 \\
\hline & $5 \mathrm{~W} 80 \mathrm{~A}$ & $2.251^{a}$ & .102 & 2.052 & 2.451 \\
\hline
\end{tabular}

a. Based on modified population marginal mean. 

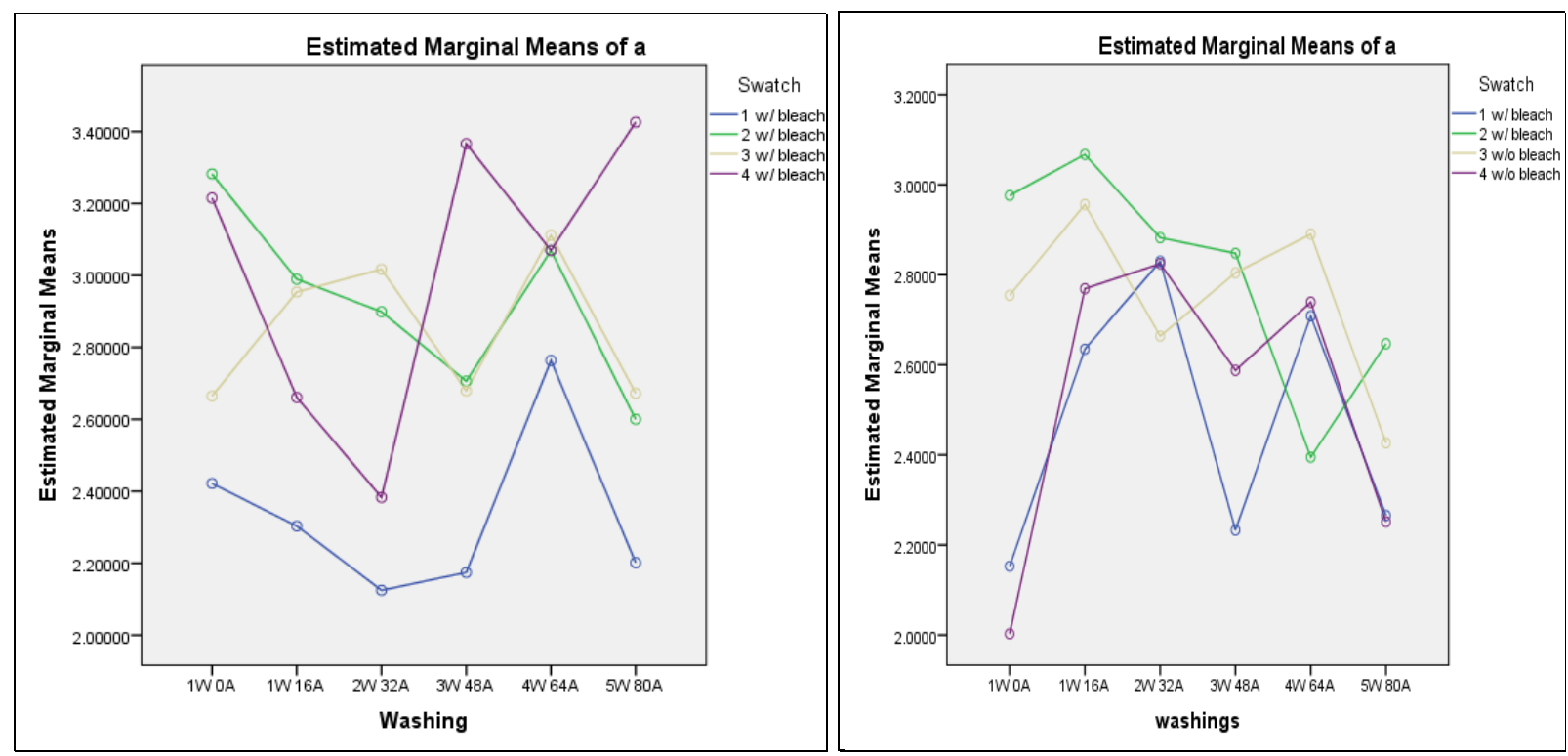

Swatches 2 and 4 follow a similar pattern in sample PMCL, and swatches 1, 2 and 4 follows their own pattern. In both cases, swatch 3 observed its own individual pattern between both the samples.

Variable ' $b$ '

Dependent Variable: $b$

Tests of Between-Subjects Effects: PMCL

\begin{tabular}{|c|c|c|c|c|c|}
\hline Source & Type III Sum of Squares & df & Mean Square & $\mathrm{F}$ & Sig. \\
\hline Corrected Model & $687.42^{\mathrm{a}}$ & 43 & 15.987 & 9.669 & .000 \\
\hline Intercept & 15946 & 1 & 15946 & 9644.5 & .000 \\
\hline Swatch & 108.59 & 2 & 54.294 & 32.838 & .000 \\
\hline SampleArea & 7.673 & 1 & 7.673 & 4.641 & .032 \\
\hline Bleached & .000 & 0 & & & \\
\hline Washing & 66.962 & 5 & 13.392 & 8.100 & .000 \\
\hline Swatch * SampleArea & 68.975 & 2 & 34.488 & 20.859 & .000 \\
\hline Swatch * Bleached & .000 & 0 & & & \\
\hline Swatch * Washing & 91.231 & 8 & 11.404 & 6.897 & .000 \\
\hline SampleArea * Bleached & .000 & 0 & & & \\
\hline SampleArea * Washing & 78.406 & 4 & 19.601 & 11.855 & .000 \\
\hline Bleached* Washing & .000 & 0 & . & & \\
\hline Swatch * SampleArea * Bleached & .000 & 0 & & & \\
\hline Swatch * SampleArea * Washing & 134.02 & 8 & 16.752 & 10.132 & .000 \\
\hline Swatch * Bleached * Washing & .000 & 0 & & & \\
\hline SampleArea * Bleached * Washing & .000 & 0 & & & \\
\hline Swatch * SampleArea * Bleached * Washing & .000 & 0 & & . & . \\
\hline Error & 1382.2 & 836 & 1.653 & & \\
\hline Total & 19329 & 880 & & & \\
\hline Corrected Total & 2069.7 & 879 & & & \\
\hline
\end{tabular}

a. R Squared $=.332$ (Adjusted R Squared $=.298$ ) 
Dependent Variable: $b$

Tests of Between-Subjects Effects: PMJX

\begin{tabular}{|c|c|c|c|c|c|}
\hline Source & Type III Sum of Squares & df & Mean Square & $\mathrm{F}$ & Sig. \\
\hline Corrected Model & $472.78^{\mathrm{a}}$ & 43 & 10.995 & 6.641 & .000 \\
\hline Intercept & 21506 & 1 & 21506 & 12990 & 0.000 \\
\hline SampleArea & .586 & 1 & .586 & .354 & .552 \\
\hline Swatch & 26.126 & 2 & 13.063 & 7.890 & .000 \\
\hline bleached & 0.000 & 0 & & & \\
\hline washings & 92.907 & 5 & 18.581 & 11.223 & .000 \\
\hline SampleArea * Swatch & 2.054 & 2 & 1.027 & .620 & .538 \\
\hline SampleArea * bleached & 0.000 & 0 & & & \\
\hline SampleArea * washings & 15.132 & 4 & 3.783 & 2.285 & .059 \\
\hline Swatch * bleached & 0.000 & 0 & & & \\
\hline Swatch * washings & 30.702 & 8 & 3.838 & 2.318 & .018 \\
\hline bleached * washings & 0.000 & 0 & & & \\
\hline SampleArea * Swatch * bleached & 0.000 & 0 & & & \\
\hline SampleArea * Swatch * washings & 98.557 & 8 & 12.320 & 7.441 & .000 \\
\hline SampleArea * bleached * washings & 0.000 & 0 & & & \\
\hline Swatch * bleached * washings & 0.000 & 0 & & & \\
\hline SampleArea * Swatch * bleached * washings & 0.000 & 0 & & & \\
\hline Error & 1384.1 & 836 & 1.656 & & \\
\hline Total & 25135 & 880 & & & \\
\hline Corrected Total & 1856.9 & 879 & & & \\
\hline
\end{tabular}

a. $\mathrm{R}$ Squared $=.255$ (Adjusted R Squared $=.216$ )

b. Computed using alpha $=.05$

Both samples produced insignificant values for the main effect of the sample area. In addition, sample PMJX also produced insignificant results for Sample Area* Swatch and Sample Area*

Washing interactions at p-values of .538 and .059 , respectively.

\section{Swatch Estimates: PMCL}

Dependent Variable: $b$

\begin{tabular}{|l|r|r|r|r|}
\hline & & \multicolumn{2}{|c|}{$95 \%$ Confidence Interval } \\
\cline { 4 - 5 } Swatch & Mean & Std. Error & Lower Bound & Upper Bound \\
\hline $1 \mathrm{w} /$ bleach & $3.618^{\mathrm{a}}$ & .087 & 3.447 & 3.788 \\
$2 \mathrm{w}$ bleach & $4.713^{\mathrm{a}}$ & .087 & 4.543 & 4.883 \\
$3 \mathrm{w} / \mathrm{o}$ bleach & $4.618^{\mathrm{a}}$ & .087 & 4.447 & 4.788 \\
$4 \mathrm{w} / \mathrm{o}$ bleach & $4.767^{\mathrm{a}}$ & .087 & 4.597 & 4.937 \\
\hline
\end{tabular}

a. Based on modified population marginal mean. 
Pairwise Comparisons: PMCL

Dependent Variable: $b$

\begin{tabular}{|c|c|c|c|c|c|c|}
\hline \multirow[b]{2}{*}{ (I) Swatch } & & \multirow[b]{2}{*}{ Mean Difference (I-J) } & \multirow[b]{2}{*}{ Std. Error } & \multirow[b]{2}{*}{ Sig. $^{d}$} & \multicolumn{2}{|c|}{$\begin{array}{l}\text { 95\% Confidence Interval for } \\
\text { Difference }\end{array}$} \\
\hline & & & & & Lower Bound & Upper Bound \\
\hline \multirow[t]{3}{*}{$1 \mathrm{w} /$ bleach } & $2 \mathrm{w} /$ bleach & $-1.095^{, b, \mathrm{c}}$ & .123 & .000 & -1.336 & -.855 \\
\hline & $3 \mathrm{w} / 0$ bleach & $-1.000^{\pi, b, c}$ & .123 & .000 & -1.241 & -.759 \\
\hline & $4 \mathrm{w} / \mathrm{o}$ bleach & $-1.149^{\mathrm{x}, \mathrm{b}, \mathrm{c}}$ & .123 & .000 & -1.390 & -.909 \\
\hline \multirow[t]{3}{*}{2 w/ bleach } & $1 \mathrm{w} /$ bleach & $1.095^{*, \mathrm{~b}, \mathrm{c}}$ & .123 & .000 & .855 & 1.336 \\
\hline & 3 w/o bleach & $.095^{\mathrm{b}, \mathrm{c}}$ & .123 & .438 & -.146 & .336 \\
\hline & 4 w/o bleach & $-.054^{\mathrm{b}, \mathrm{c}}$ & .123 & .659 & -.295 & .187 \\
\hline \multirow[t]{3}{*}{3 w/o bleach } & $1 \mathrm{w} /$ bleach & $1.000^{*}, \mathrm{~b}, \mathrm{c}$ & .123 & .000 & .759 & 1.241 \\
\hline & 2 w/ bleach & $-.095^{\mathrm{b}, \mathrm{c}}$ & .123 & .438 & -.336 & .146 \\
\hline & 4 w/o bleach & $-.149^{\mathrm{b}, \mathrm{c}}$ & .123 & .224 & -.390 & .091 \\
\hline \multirow[t]{3}{*}{4 w/o bleach } & $1 \mathrm{w} /$ bleach & $1.149^{*}, \mathrm{~b}, \mathrm{c}$ & .123 & .000 & .909 & 1.390 \\
\hline & $2 \mathrm{w} /$ bleach & $.054^{\mathrm{b}, \mathrm{c}}$ & .123 & .659 & -.187 & .295 \\
\hline & 3 w/o bleach & $.149^{\mathrm{b}, \mathrm{c}}$ & .123 & .224 & -.091 & .390 \\
\hline
\end{tabular}

Based on estimated marginal means

*. The mean difference is significant at the .05 level.

b. An estimate of the modified population marginal mean (I).

c. An estimate of the modified population marginal mean $(\mathrm{J})$.

d. Adjustment for multiple comparisons: Least Significant Difference (equivalent to no adjustments).

\section{Swatch Estimates: PMJX}

Dependent Variable: b

\begin{tabular}{|l|r|r|r|r|}
\hline & & & \multicolumn{2}{|c|}{ 95\% Confidence Interval } \\
\cline { 3 - 4 } Swatch & Mean & Std. Error & Lower Bound & Upper Bound \\
\hline 1 w/ bleach & $4.842^{\mathrm{a}}$ & .087 & 4.672 & 5.012 \\
2 w/ bleach & $5.435^{\mathrm{a}}$ & .087 & 5.265 & 5.606 \\
3 w/o bleach & $5.260^{\mathrm{a}}$ & .087 & 5.090 & 5.430 \\
4 w/o bleach & $5.035^{\mathrm{a}}$ & .087 & 4.865 & 5.206 \\
\hline
\end{tabular}

a. Based on modified population marginal mean.

Dependent Variable: $b$

Pairwise Comparisons: PMJX

\begin{tabular}{|c|c|c|c|c|c|c|}
\hline \multirow[b]{2}{*}{ (I) Swatch } & & \multirow[b]{2}{*}{ Mean Difference (I-J) } & \multirow[b]{2}{*}{ Std. Error } & \multirow[b]{2}{*}{ Sig. ${ }^{d}$} & \multicolumn{2}{|c|}{$\begin{array}{l}\text { 95\% Confidence Interval for } \\
\text { Difference }\end{array}$} \\
\hline & & & & & Lower Bound & Upper Bound \\
\hline \multirow[t]{3}{*}{$1 \mathrm{w} /$ bleach } & 2 w/ bleach & $-.593^{, 6, c}$ & .123 & .000 & -.834 & -.353 \\
\hline & 3 w/o bleach & $-.418^{x, b, c}$ & .123 & .001 & -.659 & -.177 \\
\hline & 4 w/o bleach & $-.193^{b, c}$ & .123 & .115 & -.434 & .047 \\
\hline \multirow[t]{3}{*}{$2 \mathrm{w} / \mathrm{bleach}$} & $1 \mathrm{w} /$ bleach & $.593^{*}, \mathrm{~b}, \mathrm{c}$ & .123 & .000 & .353 & .834 \\
\hline & 3 w/o bleach & $.175^{\mathrm{b}, \mathrm{c}}$ & .123 & .153 & -.065 & .416 \\
\hline & 4 w/o bleach & $.400^{*}, \mathrm{~b}, \mathrm{c}$ & .123 & .001 & .159 & .641 \\
\hline \multirow[t]{3}{*}{3 w/o bleach } & 1 w/ bleach & $.418^{\pi, b, c}$ & .123 & .001 & .177 & .659 \\
\hline & 2 w/ bleach & $-.175^{b, c}$ & .123 & .153 & -.416 & .065 \\
\hline & 4 w/o bleach & $.225^{\mathrm{b}, \mathrm{c}}$ & .123 & .067 & -.016 & .465 \\
\hline \multirow[t]{3}{*}{4 w/o bleach } & $1 \mathrm{w} /$ bleach & $.193^{\mathrm{b}, \mathrm{c}}$ & .123 & .115 & -.047 & .434 \\
\hline & 2 w/ bleach & $-.400^{*}, \mathrm{~b}, \mathrm{c}$ & .123 & .001 & -.641 & -.159 \\
\hline & $3 \mathrm{w} / \mathrm{o}$ bleach & $-.225^{\mathrm{b}, \mathrm{c}}$ & .123 & .067 & -.465 & .016 \\
\hline
\end{tabular}

Based on estimated marginal means

*. The mean difference is significant at the .05 level.

b. An estimate of the modified population marginal mean (I).

c. An estimate of the modified population marginal mean $(\mathrm{J})$.

d. Adjustment for multiple comparisons: Least Significant Difference (equivalent to no adjustments).

Similar to variable 'a', no observed pattern was developed.

\section{Bleach Estimates: PMCL}


Dependent Variable: $b$

\begin{tabular}{|l|r|r|r|r|}
\hline & & & \multicolumn{2}{|c|}{$95 \%$ Confidence Interval } \\
\cline { 3 - 5 } Bleached & Mean & Std. Error & Lower & Bound \\
\hline w/ bleach & $4.165^{\mathrm{a}}$ & .061 & 4.045 & Upper Bound \\
w/o bleach & $4.692^{\mathrm{a}}$ & .061 & 4.572 & 4.285 \\
\hline
\end{tabular}

a. Based on modified population marginal mean.

Pairwise Comparisons: PMCL

Dependent Variable: b

\begin{tabular}{|c|c|c|c|c|c|c|}
\hline \multirow[b]{2}{*}{ (I) Bleached } & \multirow[b]{2}{*}{ (J) Bleached } & \multirow[b]{2}{*}{$\begin{array}{c}\text { Mean } \\
\text { Difference (I-J) }\end{array}$} & \multirow[b]{2}{*}{ Std. Error } & \multirow[b]{2}{*}{ Sig. ${ }^{\mathrm{C}}$} & \multicolumn{2}{|c|}{$\begin{array}{l}\text { 95\% Confidence Interval for } \\
\text { Difference }\end{array}$} \\
\hline & & & & & $\begin{array}{l}\text { Lower } \\
\text { Bound }\end{array}$ & $\begin{array}{l}\text { Upper } \\
\text { Bound }\end{array}$ \\
\hline w/ bleach & w/o bleach & $-.527^{*}, \mathrm{a}, \mathrm{b}$ & .087 & .000 & -.697 & -.357 \\
\hline w/o bleach & w/ bleach & $.527^{*}, a, b$ & .087 & .000 & .357 & .697 \\
\hline
\end{tabular}

Based on estimated marginal means

*. The mean difference is significant at the .05 level.

a. An estimate of the modified population marginal mean (I).

b. An estimate of the modified population marginal mean $(\mathrm{J})$.

c. Adjustment for multiple comparisons: Least Significant Difference (equivalent to no adjustments).

\section{Bleach Estimates: PMJX}

Dependent Variable: $b$

\begin{tabular}{|l|r|r|r|r|}
\hline & & & \multicolumn{2}{|c|}{ 95\% Confidence Interval } \\
\cline { 4 - 5 } bleached & Mean & Std. Error & Lower Bound & Upper Bound \\
\hline w/ bleach & $5.139^{\mathrm{a}}$ & .061 & 5.018 & 5.259 \\
w/o bleach & $5.148^{\mathrm{a}}$ & .061 & 5.027 & 5.268 \\
\hline
\end{tabular}

a. Based on modified population marginal mean.

Pairwise Comparisons: PMJX

Dependent Variable: $b$

\begin{tabular}{|c|c|c|c|c|c|c|}
\hline \multirow[b]{2}{*}{ (I) bleached } & & \multirow[b]{2}{*}{ Mean Difference (I-J) } & \multirow[b]{2}{*}{ Std. Error } & \multirow[b]{2}{*}{ Sig. ${ }^{c}$} & \multicolumn{2}{|c|}{$\begin{array}{c}\text { 95\% Confidence Interval for } \\
\text { Difference }^{c}\end{array}$} \\
\hline & & & & & Lower Bound & Upper Bound \\
\hline w/ bleach & w/o bleach & $-.009^{\mathrm{a}, \mathrm{b}}$ & .087 & .917 & -.179 & .161 \\
\hline w/o bleach & w/ bleach & $.009^{a, b}$ & .087 & .917 & -.161 & .179 \\
\hline
\end{tabular}

Based on estimated marginal means

a. An estimate of the modified population marginal mean (I).

b. An estimate of the modified population marginal mean $(\mathrm{J})$.

c. Adjustment for multiple comparisons: Least Significant Difference (equivalent to no adjustments).

Based on the significant values, the type of detergent used affected the outcome of PMCL samples.

\section{Washing Estimates: PMCL}

Dependent Variable: $\mathrm{b}$

\begin{tabular}{|l|r|r|r|r|}
\hline \multirow{2}{*}{ Washing } & & \multirow{2}{*}{\begin{tabular}{l} 
Std. \\
\cline { 4 - 5 }
\end{tabular}} & Mean & \multicolumn{2}{|c|}{ 95\% Confidence Interval } \\
\hline 1W 0A & $4.397^{\mathrm{a}}$ & .144 & 4.114 & $\begin{array}{c}\text { Upper } \\
\text { Bound }\end{array}$ \\
1W 16A & $4.518^{\mathrm{a}}$ & .102 & 4.679 \\
2W 32A & $4.199^{\mathrm{a}}$ & .102 & 4.000 & 4.718 \\
3W 48A & $4.176^{\mathrm{a}}$ & .102 & 3.976 & 4.399 \\
4W 64A & $4.933^{\mathrm{a}}$ & .102 & 4.734 & 5.133 \\
5W 80A & $4.333^{\mathrm{a}}$ & .102 & 4.134 & 4.533 \\
\hline
\end{tabular}


a. Based on modified population marginal mean.

Pairwise Comparisons: PMCL

Dependent Variable: $b$

\begin{tabular}{|c|c|c|c|c|c|c|}
\hline & & Mean & & & $\begin{array}{r}95 \% \text { Confi } \\
\mathrm{Di}\end{array}$ & $\begin{array}{l}\text { cee Interval for } \\
\text { ence }\end{array}$ \\
\hline (I) Washir & & $\begin{array}{c}\text { Difference (I- } \\
\mathrm{J})\end{array}$ & Std. Error & Sig. ${ }^{d}$ & $\begin{array}{l}\text { Lower } \\
\text { Bound }\end{array}$ & Upper Bound \\
\hline $1 \mathrm{~W} 0 \mathrm{~A}$ & $1 \mathrm{~W} 16 \mathrm{~A}$ & $-.122^{\mathrm{a}, \mathrm{\sigma}}$ & .176 & .490 & -.467 & .224 \\
\hline & $2 W 32 A$ & $.197^{a, b}$ & .176 & .262 & -.148 & .543 \\
\hline & $3 W 48 A$ & $.221^{a, b}$ & .176 & .210 & -.125 & .566 \\
\hline & $4 W 64 A$ & $-.537^{\mathrm{a}, \mathrm{b},{ }^{*}}$ & .176 & .002 & -.882 & -.191 \\
\hline & $5 W 80 A$ & $.063^{a, b}$ & .176 & .719 & -.282 & .409 \\
\hline $1 \mathrm{~W} 16 \mathrm{~A}$ & $1 \mathrm{~W} 0 \mathrm{~A}$ & $.122^{\mathrm{a}, \mathrm{b}}$ & .176 & .490 & -.224 & .467 \\
\hline & $2 W 32 A$ & $.319^{a, b,{ }^{*}}$ & .144 & .027 & .037 & .601 \\
\hline & $3 W 48 A$ & $.342^{\mathrm{a}, \mathrm{b},{ }^{*}}$ & .144 & .017 & .060 & .625 \\
\hline & $4 W 64 A$ & $-.415^{\mathrm{a}, \mathrm{b},{ }^{*}}$ & .144 & .004 & -.697 & -.133 \\
\hline & $5 W 80 A$ & $.185^{\mathrm{a}, \mathrm{b}}$ & .144 & .198 & -.097 & .467 \\
\hline $2 W 32 A$ & $1 \mathrm{~W} 0 \mathrm{~A}$ & $-.197^{a, b}$ & .176 & .262 & -.543 & .148 \\
\hline & $1 \mathrm{~W} 16 \mathrm{~A}$ & $-.319^{a, b,{ }^{*}}$ & .144 & .027 & -.601 & -.037 \\
\hline & $3 W 48 A$ & $.023^{a, b}$ & .144 & .871 & -.259 & .305 \\
\hline & $4 W 64 A$ & $-.734^{\mathrm{a}, \mathrm{b},{ }^{\pi}}$ & .144 & .000 & -1.016 & -.452 \\
\hline & $5 W 80 A$ & $-.134^{\mathrm{a}, \mathrm{b}}$ & .144 & .351 & -.416 & .148 \\
\hline $3 W$ 48A & $1 \mathrm{~W} 0 \mathrm{~A}$ & $-.221^{a, b}$ & .176 & .210 & -.566 & .125 \\
\hline & $1 \mathrm{~W} 16 \mathrm{~A}$ & $-.342^{\mathrm{a}, \mathrm{b},{ }^{\pi}}$ & .144 & .017 & -.625 & -.060 \\
\hline & $2 W 32 A$ & $-.023^{a, b}$ & .144 & .871 & -.305 & .259 \\
\hline & $4 W 64 A$ & $-.757^{\mathrm{a}, \mathrm{b},{ }^{*}}$ & .144 & .000 & -1.039 & -.475 \\
\hline & $5 W 80 A$ & $-.157^{a, b}$ & .144 & .274 & -.440 & .125 \\
\hline $4 \mathrm{~W} 64 \mathrm{~A}$ & $1 \mathrm{~W} 0 \mathrm{~A}$ & $.537^{\mathrm{a}, \mathrm{b},{ }^{*}}$ & .176 & .002 & .191 & .882 \\
\hline & $1 \mathrm{~W} 16 \mathrm{~A}$ & $.415^{\mathrm{a}, \mathrm{b},{ }^{*}}$ & .144 & .004 & .133 & .697 \\
\hline & $2 W 32 A$ & $.734^{\mathrm{a}, \mathrm{b},{ }^{*}}$ & .144 & .000 & .452 & 1.016 \\
\hline & $3 W$ 48A & $.757^{\mathrm{a}, \mathrm{b},{ }^{\star}}$ & .144 & .000 & .475 & 1.039 \\
\hline & $5 W 80 A$ & $.600^{a, b, \pi}$ & .144 & .000 & .318 & .882 \\
\hline $5 W$ 80A & $1 \mathrm{~W} 0 \mathrm{~A}$ & $-.063^{a, b}$ & .176 & .719 & -.409 & .282 \\
\hline & $1 \mathrm{~W} 16 \mathrm{~A}$ & $-.185^{a, b}$ & .144 & .198 & -.467 & .097 \\
\hline & $2 W 32 A$ & $.134^{\mathrm{a}, \mathrm{b}}$ & .144 & .351 & -.148 & .416 \\
\hline & $3 W 48 A$ & $.157^{\mathrm{a}, \mathrm{b}}$ & .144 & .274 & -.125 & .440 \\
\hline & $4 \mathrm{~W} 64 \mathrm{~A}$ & $-.600^{\mathrm{a}, \mathrm{b},{ }^{*}}$ & .144 & .000 & -.882 & -.318 \\
\hline
\end{tabular}

Based on estimated marginal means

*. The mean difference is significant at the .05 level.

a. An estimate of the modified population marginal mean (I).

b. An estimate of the modified population marginal mean (J).

d. Adjustment for multiple comparisons: Least Significant Difference (equivalent to no adjustments).

\section{Washing Estimates: PMJX}

Dependent Variable: $b$

\begin{tabular}{|l|r|r|r|r|}
\hline & & & \multicolumn{2}{|c|}{$95 \%$ Confidence Interval } \\
\cline { 4 - 5 } washings & Mean & Std. Error & Lower Bound & Upper Bound \\
\hline 1W 0A & $4.557^{\mathrm{a}}$ & .144 & 4.275 & 4.840 \\
1W 16A & $5.419^{\mathrm{a}}$ & .102 & 5.219 & 5.618 \\
2W 32A & $5.359^{\mathrm{a}}$ & .102 & 5.160 & 5.559 \\
3W 48A & $5.319^{\mathrm{a}}$ & .102 & 5.119 & 5.519 \\
4W 64A & $5.283^{\mathrm{a}}$ & .102 & 5.083 & 5.482 \\
5W 80A & $4.629^{\mathrm{a}}$ & .102 & 4.429 & 4.829 \\
\hline
\end{tabular}


a. Based on modified population marginal mean.

Pairwise Comparisons: PMJX

Dependent Variable: $b$

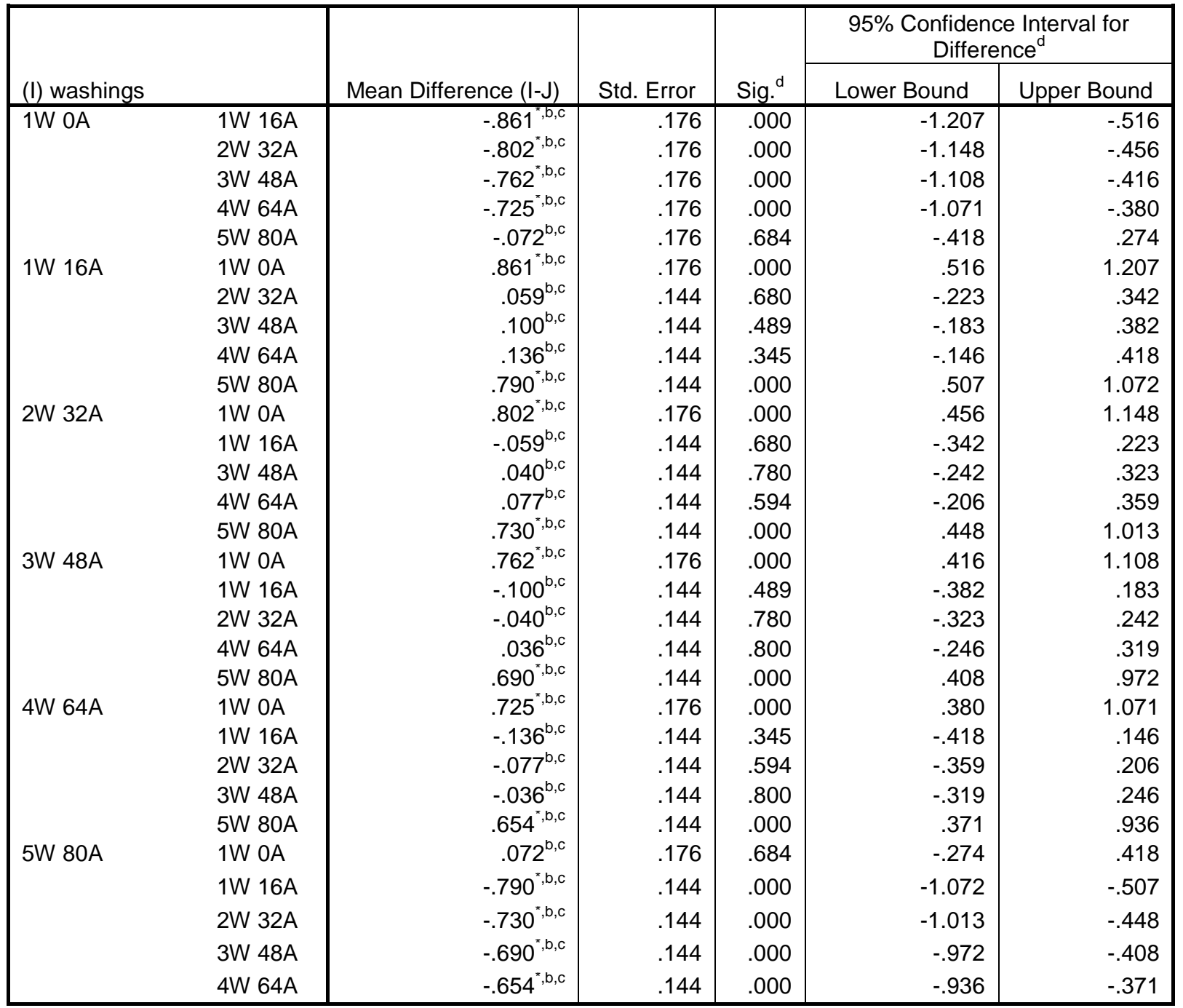

Based on estimated marginal means

*. The mean difference is significant at the .05 level.

b. An estimate of the modified population marginal mean (I).

c. An estimate of the modified population marginal mean $(\mathrm{J})$.

d. Adjustment for multiple comparisons: Least Significant Difference (equivalent to no adjustments).

Marked significant differences were observed between both reference samples. No prominent

pattern was observed between the two samples.

Foe the swatch* washing interaction below, differences in means can be observed. The mean

values are more conjugated for sample PMJX than for PMCL. Similarly to variable ' $a$ ', the

patterns for swatches 1,2 and 3 are roughly consistent between the reference samples. 
Dependent Variable: $b$

\section{Swatch * Washing: PMCL}

\begin{tabular}{|c|c|c|c|c|c|}
\hline \multirow[b]{2}{*}{ Swatch } & & \multirow[b]{2}{*}{ Mean } & \multirow[b]{2}{*}{ Std. Error } & \multicolumn{2}{|c|}{ 95\% Confidence Interval } \\
\hline & & & & Lower Bound & Upper Bound \\
\hline \multirow[t]{6}{*}{$1 \mathrm{w} /$ bleach } & $1 \mathrm{~W} 0 \mathrm{~A}$ & $3.477^{\mathrm{a}}$ & .288 & 2.913 & 4.041 \\
\hline & $1 \mathrm{~W} 16 \mathrm{~A}$ & $3.734^{\mathrm{a}}$ & .203 & 3.335 & 4.133 \\
\hline & $2 W 32 A$ & $3.121^{\mathrm{a}}$ & .203 & 2.722 & 3.520 \\
\hline & $3 W 48 A$ & $3.171^{\mathrm{a}}$ & .203 & 2.772 & 3.570 \\
\hline & $4 \mathrm{~W} 64 \mathrm{~A}$ & $4.449^{a}$ & .203 & 4.049 & 4.848 \\
\hline & $5 W 80 A$ & $3.684^{a}$ & .203 & 3.285 & 4.083 \\
\hline \multirow[t]{6}{*}{2 w/ bleach' } & $1 \mathrm{~W} 0 \mathrm{~A}$ & $5.266^{a}$ & .288 & 4.702 & 5.831 \\
\hline & $1 \mathrm{~W} 16 \mathrm{~A}$ & $5.096^{\mathrm{a}}$ & .203 & 4.697 & 5.495 \\
\hline & $2 W 32 A$ & $4.355^{\mathrm{a}}$ & .203 & 3.956 & 4.754 \\
\hline & $3 W 48 A$ & $4.402^{\mathrm{a}}$ & .203 & 4.003 & 4.801 \\
\hline & $4 \mathrm{~W} 64 \mathrm{~A}$ & $4.969^{a}$ & .203 & 4.570 & 5.368 \\
\hline & $5 W 80 A$ & $4.465^{\mathrm{a}}$ & .203 & 4.066 & 4.864 \\
\hline \multirow[t]{6}{*}{3 w/ bleach } & $1 \mathrm{~W} \mathrm{OA}$ & $4.519^{\mathrm{a}}$ & .288 & 3.954 & 5.083 \\
\hline & $1 \mathrm{~W} 16 \mathrm{~A}$ & $4.850^{\mathrm{a}}$ & .203 & 4.451 & 5.249 \\
\hline & $2 W 32 A$ & $5.170^{\mathrm{a}}$ & .203 & 4.771 & 5.569 \\
\hline & $3 W 48 A$ & $4.194^{a}$ & .203 & 3.795 & 4.593 \\
\hline & $4 W 64 A$ & $5.108^{\mathrm{a}}$ & .203 & 4.709 & 5.507 \\
\hline & $5 W 80 A$ & $3.816^{a}$ & .203 & 3.417 & 4.215 \\
\hline \multirow[t]{6}{*}{4 w/ bleach } & $1 \mathrm{~W} 0 \mathrm{~A}$ & $4.324^{a}$ & .288 & 3.760 & 4.888 \\
\hline & $1 \mathrm{~W} 16 \mathrm{~A}$ & $4.393^{a}$ & .203 & 3.994 & 4.792 \\
\hline & $2 W 32 A$ & $4.151^{\mathrm{a}}$ & .203 & 3.752 & 4.550 \\
\hline & $3 W 48 A$ & $4.936^{\mathrm{a}}$ & .203 & 4.537 & 5.336 \\
\hline & $4 W 64 A$ & $5.207^{\mathrm{a}}$ & .203 & 4.808 & 5.606 \\
\hline & $5 W 80 A$ & $5.368^{a}$ & .203 & 4.969 & 5.767 \\
\hline
\end{tabular}

a. Based on modified population marginal mean.

\section{Swatch * Washings: PMJX}

Dependent Variable: $b$

\begin{tabular}{|c|c|c|c|c|c|}
\hline \multirow[b]{2}{*}{ Swatch } & & \multirow[b]{2}{*}{ Mean } & \multirow[b]{2}{*}{ Std. Error } & \multicolumn{2}{|c|}{ 95\% Confidence Interval } \\
\hline & & & & Lower Bound & Upper Bound \\
\hline \multirow[t]{6}{*}{$1 \mathrm{w} /$ bleach } & $1 \mathrm{~W} 0 \mathrm{~A}$ & $3.849^{a}$ & .288 & 3.285 & 4.414 \\
\hline & $1 \mathrm{~W} 16 \mathrm{~A}$ & $5.137^{a}$ & .203 & 4.738 & 5.537 \\
\hline & $2 W 32 A$ & $5.307^{a}$ & .203 & 4.907 & 5.706 \\
\hline & $3 W$ 48A & $4.918^{a}$ & .203 & 4.519 & 5.318 \\
\hline & $4 W 64 A$ & $4.798^{a}$ & .203 & 4.399 & 5.197 \\
\hline & $5 W 80 A$ & $4.546^{a}$ & .203 & 4.147 & 4.945 \\
\hline \multirow[t]{6}{*}{2 w/ bleach } & $1 \mathrm{~W} O \mathrm{~A}$ & $5.328^{a}$ & .288 & 4.763 & 5.893 \\
\hline & $1 \mathrm{~W} 16 \mathrm{~A}$ & $5.884^{a}$ & .203 & 5.484 & 6.283 \\
\hline & $2 W 32 A$ & $5.448^{a}$ & .203 & 5.049 & 5.847 \\
\hline & $3 W 48 A$ & $5.863^{a}$ & .203 & 5.463 & 6.262 \\
\hline & $4 W 64 A$ & $5.142^{a}$ & .203 & 4.743 & 5.542 \\
\hline & $5 W 80 A$ & $4.894^{a}$ & .203 & 4.495 & 5.293 \\
\hline \multirow[t]{6}{*}{3 w/o bleach } & $1 \mathrm{~W} O \mathrm{~A}$ & $5.361^{a}$ & .288 & 4.797 & 5.926 \\
\hline & $1 \mathrm{~W} 16 \mathrm{~A}$ & $5.425^{a}$ & .203 & 5.026 & 5.824 \\
\hline & $2 W 32 A$ & $4.955^{a}$ & .203 & 4.556 & 5.354 \\
\hline & $3 W 48 A$ & $5.530^{\mathrm{a}}$ & .203 & 5.131 & 5.929 \\
\hline & $4 \mathrm{~W} 64 \mathrm{~A}$ & $5.590^{\mathrm{a}}$ & .203 & 5.191 & 5.990 \\
\hline & $5 W 80 A$ & $4.749^{a}$ & .203 & 4.350 & 5.149 \\
\hline \multirow[t]{6}{*}{4 w/o bleach } & $1 \mathrm{~W} O \mathrm{~A}$ & $3.691^{a}$ & .288 & 3.126 & 4.256 \\
\hline & $1 \mathrm{~W} 16 \mathrm{~A}$ & $5.229^{a}$ & .203 & 4.830 & 5.628 \\
\hline & $2 W 32 A$ & $5.728^{a}$ & .203 & 5.328 & 6.127 \\
\hline & $3 W 48 A$ & $4.966^{a}$ & .203 & 4.566 & 5.365 \\
\hline & $4 W 64 A$ & $5.600^{\mathrm{a}}$ & .203 & 5.201 & 6.000 \\
\hline & $5 W 80 A$ & $4.327^{a}$ & .203 & 3.928 & 4.726 \\
\hline
\end{tabular}

a. Based on modified population marginal mean. 

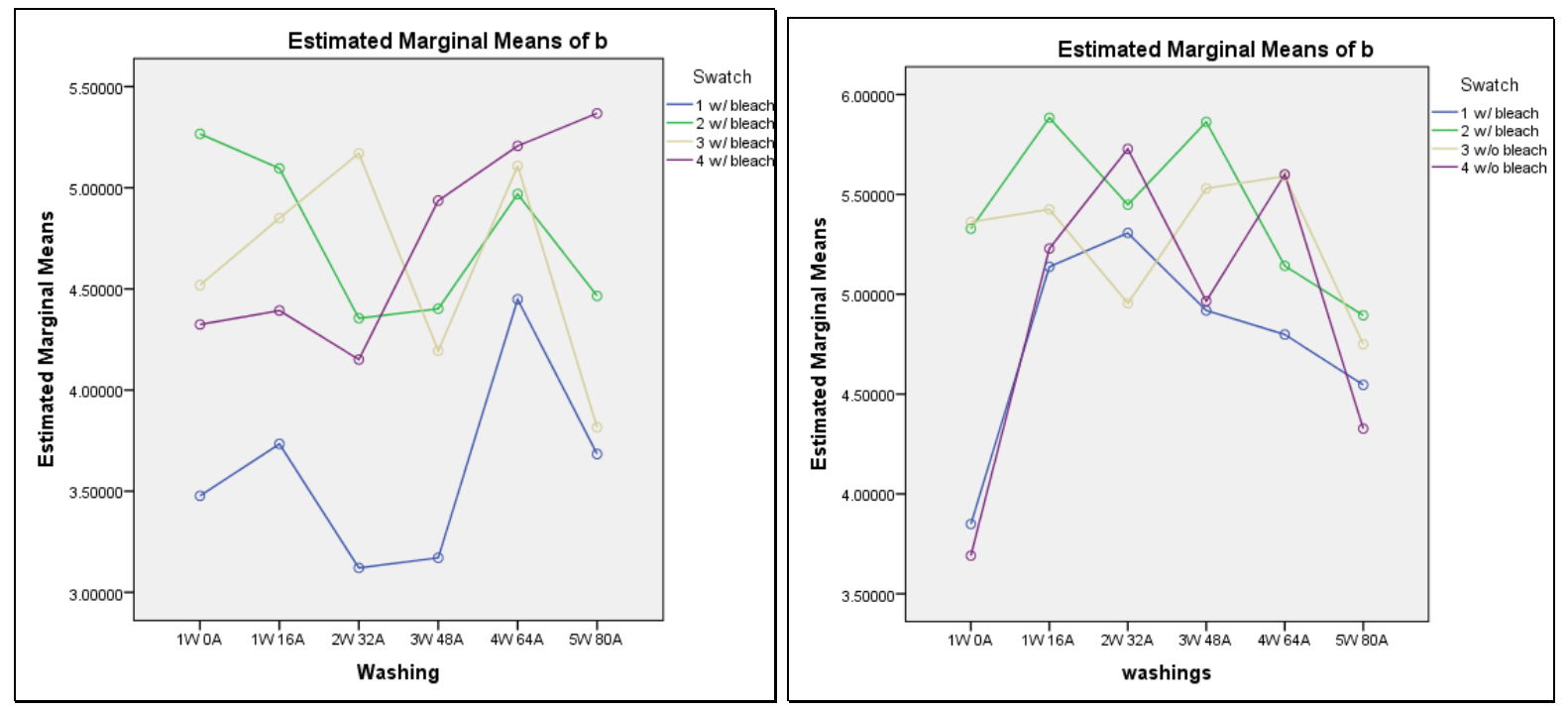

VI and VII. Samples PMP and RMP (compared simultaneously because of their close proximity on the color space for the purposes of examining if the Lab values might be similar in regards to this distance).

\section{Variable ' $L$ '}

Tests of Between-Subjects Effects: PMP

Dependent Variable: L

\begin{tabular}{|c|c|c|c|c|c|}
\hline Source & Type III Sum of Squares & $\mathrm{df}$ & Mean Square & $\mathrm{F}$ & Sig. \\
\hline Corrected Model & $11081^{\mathrm{a}}$ & 43 & 257.69 & 16.551 & .000 \\
\hline Intercept & 5739882 & 1 & 5739883 & 368672 & 0.000 \\
\hline SampleArea & 2.924 & 1 & 2.924 & .188 & .665 \\
\hline Swatch & 332.92 & 2 & 166.46 & 10.692 & .000 \\
\hline Bleached & 0.000 & 0 & & & \\
\hline Washings & 5895.7 & 5 & 1179.1 & 75.736 & .000 \\
\hline SampleArea * Swatch & 358.42 & 2 & 179.21 & 11.511 & .000 \\
\hline SampleArea * Bleached & 0.000 & 0 & & & \\
\hline SampleArea * Washings & 281.75 & 4 & 70.436 & 4.524 & .001 \\
\hline Swatch * Bleached & 0.000 & 0 & & & \\
\hline Swatch * Washings & 463.82 & 8 & 57.978 & 3.724 & .000 \\
\hline Bleached * Washings & 0.000 & 0 & & & \\
\hline SampleArea * Swatch * Bleached & 0.000 & 0 & & & \\
\hline SampleArea * Swatch * Washings & 1230.8 & 8 & 153.84 & 9.881 & .000 \\
\hline SampleArea * Bleached * Washings & 0.000 & 0 & & & \\
\hline Swatch * Bleached * Washings & 0.000 & 0 & & & \\
\hline SampleArea * Swatch * Bleached * Washings & 0.000 & 0 & & & \\
\hline Error & 13016 & 836 & 15.569 & & \\
\hline Total & 6192628 & 880 & & & \\
\hline Corrected Total & 24096 & 879 & & & \\
\hline
\end{tabular}

a. $\mathrm{R}$ Squared $=.460$ (Adjusted $\mathrm{R}$ Squared $=.432$ )

b. Computed using alpha $=.05$ 
Tests of Between-Subjects Effects: RMP

Dependent Variable: L

\begin{tabular}{|c|c|c|c|c|c|}
\hline Source & Type III Sum of Squares & df & Mean Square & $\mathrm{F}$ & Sig. \\
\hline Corrected Model & $3784.841^{\mathrm{a}}$ & 43 & 88.020 & 7.825 & .000 \\
\hline Intercept & 5867578.864 & 1 & 5867578.864 & 521618.405 & 0.000 \\
\hline SampleArea & .457 & 1 & .457 & .041 & .840 \\
\hline Swatch & 50.816 & 2 & 25.408 & 2.259 & .105 \\
\hline Bleached & 0.000 & 0 & & & \\
\hline Washings & 335.960 & 5 & 67.192 & 5.973 & .000 \\
\hline SampleArea * Swatch & 231.034 & 2 & 115.517 & 10.269 & .000 \\
\hline SampleArea * Bleached & 0.000 & 0 & & & \\
\hline SampleArea * Washings & 163.125 & 4 & 40.781 & 3.625 & .006 \\
\hline Swatch * Bleached & 0.000 & 0 & & & \\
\hline Swatch * Washings & 686.919 & 8 & 85.865 & 7.633 & .000 \\
\hline Bleached * Washings & 0.000 & 0 & & & \\
\hline SampleArea * Swatch * Bleached & 0.000 & 0 & & & \\
\hline SampleArea * Swatch * Washings & 387.426 & 8 & 48.428 & 4.305 & .000 \\
\hline SampleArea * Bleached * Washings & 0.000 & 0 & & & \\
\hline Swatch * Bleached * Washings & 0.000 & 0 & & & \\
\hline SampleArea * Swatch * Bleached * Washings & 0.000 & 0 & & & \\
\hline Error & 9403.993 & 836 & 11.249 & & \\
\hline Total & 6266988.817 & 880 & & & \\
\hline Corrected Total & 13188.834 & 879 & & & \\
\hline
\end{tabular}

a. $\mathrm{R}$ Squared $=.287$ (Adjusted R Squared $=.250$ )

b. Computed using alpha $=.05$

Insignificant results were observed for the main effects of sample area for both reference

samples, as well as for swatch for sample RMP.

\section{Swatch Estimates: PMP}

Dependent Variable: $\mathrm{L}$

\begin{tabular}{|l|r|r|r|r|}
\hline & & & \multicolumn{2}{|c|}{$95 \%$ Confidence Interval } \\
\cline { 4 - 5 } Swatch & Mean & Std. Error & Lower Bound & Upper Bound \\
\hline 1 w/ bleach & $84.240^{\mathrm{a}}$ & .266 & 83.718 & 84.762 \\
2 w/ bleach & $84.527^{\mathrm{a}}$ & .266 & 84.005 & 85.050 \\
3 w/o bleach & $84.188^{\mathrm{a}}$ & .266 & 83.666 & 84.710 \\
4 w/o bleach & $81.941^{\mathrm{a}}$ & .266 & 81.418 & 82.463 \\
\hline
\end{tabular}

a. Based on modified population marginal mean.

\section{Pairwise Comparisons}

Dependent Variable: $\mathrm{L}$

\begin{tabular}{|c|c|c|c|c|c|c|}
\hline \multirow[b]{2}{*}{ (I) Swatch } & & \multirow[b]{2}{*}{ Mean Difference (I-J) } & \multirow[b]{2}{*}{ Std. Error } & \multirow[b]{2}{*}{ Sig. ${ }^{d}$} & \multicolumn{2}{|c|}{$95 \%$ Confidence Interval for Difference ${ }^{d}$} \\
\hline & & & & & Lower Bound & Upper Bound \\
\hline \multirow[t]{3}{*}{$1 \mathrm{w} /$ bleach } & 2 w/ bleach & $-.288^{\mathrm{a}, \mathrm{b}}$ & .376 & .445 & -1.026 & .451 \\
\hline & 3 w/o bleach & $.052^{\mathrm{a}, \mathrm{b}}$ & .376 & .891 & -.687 & .790 \\
\hline & 4 w/o bleach & $2.299^{a, b,{ }^{*}}$ & .376 & .000 & 1.561 & 3.038 \\
\hline \multirow[t]{3}{*}{$2 \mathrm{w} /$ bleach } & $1 \mathrm{w} /$ bleach & $.288^{\mathrm{a}, \mathrm{b}}$ & .376 & .445 & -.451 & 1.026 \\
\hline & 3 w/o bleach & $.339^{\mathrm{a}, \mathrm{b}}$ & .376 & .367 & -.399 & 1.078 \\
\hline & 4 w/o bleach & $2.587^{\mathrm{a}, \mathrm{b},{ }^{\star}}$ & .376 & .000 & 1.848 & 3.325 \\
\hline \multirow[t]{3}{*}{3 w/o bleach } & $1 \mathrm{w} /$ bleach & $-.052^{a, b}$ & .376 & .891 & -.790 & .687 \\
\hline & 2 w/ bleach & $-.339^{a, b}$ & .376 & .367 & -1.078 & .399 \\
\hline & 4 w/o bleach & $2.248^{\mathrm{a}, \mathrm{b},{ }^{*}}$ & .376 & .000 & 1.509 & 2.986 \\
\hline \multirow[t]{3}{*}{4 w/o bleach } & $1 \mathrm{w} /$ bleach & $-2.299^{\mathrm{a}, \mathrm{b},{ }^{*}}$ & .376 & .000 & -3.038 & -1.561 \\
\hline & $2 \mathrm{w} /$ bleach & $-2.587^{\mathrm{a}, \mathrm{b},{ }^{\pi}}$ & .376 & .000 & -3.325 & -1.848 \\
\hline & 3 w/o bleach & $-2.248^{\mathrm{a}, \mathrm{b},{ }^{*}}$ & .376 & .000 & -2.986 & -1.509 \\
\hline
\end{tabular}

Based on estimated marginal means

*. The mean difference is significant at the .05 level. 
a. An estimate of the modified population marginal mean (I).

b. An estimate of the modified population marginal mean $(\mathrm{J})$.

d. Adjustment for multiple comparisons: Least Significant Difference (equivalent to no adjustments).

Significant results were only observed for sample PMP. The unbleached swatches observed

significant differences for all levels of interactions.

Dependent Variable: L

\begin{tabular}{|l|r|r|r|r|}
\hline & & & \multicolumn{2}{|c|}{$95 \%$ Confidence Interval } \\
\cline { 4 - 5 } Washings & Mean & Std. Error & Lower Bound & Upper Bound \\
\hline 1W 0A & $79.053^{\mathrm{a}}$ & .441 & 78.187 & 79.918 \\
1W 16A & $81.055^{\mathrm{a}}$ & .312 & 80.443 & 81.668 \\
2W 32A & $87.708^{\mathrm{a}}$ & .312 & 87.095 & 88.320 \\
3W 48A & $82.592^{\mathrm{a}}$ & .312 & 81.980 & 83.204 \\
4W 64A & $84.226^{\mathrm{a}}$ & .312 & 83.614 & 84.838 \\
5W 80A & $85.374^{\mathrm{a}}$ & .312 & 84.762 & 85.986 \\
\hline
\end{tabular}

a. Based on modified population marginal mean.

Pairwise Comparisons: PMP

Dependent Variable: L

\begin{tabular}{|c|c|c|c|c|c|c|}
\hline \multirow{2}{*}{\multicolumn{2}{|c|}{ (I) Washings }} & \multirow[b]{2}{*}{ Mean Difference (I-J) } & \multirow[b]{2}{*}{ Std. Error } & \multirow[b]{2}{*}{ Sig. ${ }^{d}$} & \multicolumn{2}{|c|}{ 95\% Confidence Interval for Difference ${ }^{d}$} \\
\hline & & & & & Lower Bound & Upper Bound \\
\hline \multirow[t]{5}{*}{$1 \mathrm{~W} 0 \mathrm{~A}$} & $1 \mathrm{~W} 16 \mathrm{~A}$ & $-2.003^{, 0, c}$ & .540 & .000 & -3.063 & -.942 \\
\hline & $2 W 32 A$ & $-8.655^{*}, \mathrm{~b}, \mathrm{c}$ & .540 & .000 & -9.716 & -7.595 \\
\hline & $3 W 48 A$ & $-3.539^{*}, \mathrm{~b}, \mathrm{c}$ & .540 & .000 & -4.600 & -2.479 \\
\hline & $4 \mathrm{~W} 64 \mathrm{~A}$ & $-5.174^{\pi}, \mathrm{b}, \mathrm{c}$ & .540 & .000 & -6.234 & -4.113 \\
\hline & $5 W 80 A$ & $-6.322^{x, b, c}$ & .540 & .000 & -7.382 & -5.261 \\
\hline \multirow[t]{5}{*}{ 1W 16A } & $1 \mathrm{~W} 0 \mathrm{~A}$ & $2.003^{*}, \mathrm{~b}, \mathrm{c}$ & .540 & .000 & .942 & 3.063 \\
\hline & $2 W 32 A$ & $-6.652^{x}, \mathrm{~b}, \mathrm{c}$ & .441 & .000 & -7.518 & -5.786 \\
\hline & $3 W 48 A$ & $-1.537^{\pi, b, c}$ & .441 & .001 & -2.403 & -.671 \\
\hline & $4 W 64 A$ & $-3.171^{\pi, b, c}$ & .441 & .000 & -4.037 & -2.305 \\
\hline & $5 W 80 A$ & $-4.319^{\pi, b, c}$ & .441 & .000 & -5.185 & -3.453 \\
\hline \multirow[t]{5}{*}{$2 W 32 A$} & $1 \mathrm{~W} 0 \mathrm{~A}$ & $8.655^{\pi, b, c}$ & .540 & .000 & 7.595 & 9.716 \\
\hline & $1 \mathrm{~W} 16 \mathrm{~A}$ & $6.652^{\pi, b, c}$ & .441 & .000 & 5.786 & 7.518 \\
\hline & $3 W 48 A$ & $5.116^{*}, \mathrm{~b}, \mathrm{c}$ & .441 & .000 & 4.250 & 5.981 \\
\hline & $4 W 64 A$ & $3.481^{*}, \mathrm{~b}, \mathrm{c}$ & .441 & .000 & 2.616 & 4.347 \\
\hline & $5 W 80 A$ & $2.333^{\pi, b, c}$ & .441 & .000 & 1.468 & 3.199 \\
\hline \multirow[t]{5}{*}{$3 W$ 48A } & $1 \mathrm{~W} 0 \mathrm{~A}$ & $3.539^{*}, \mathrm{~b}, \mathrm{c}$ & .540 & .000 & 2.479 & 4.600 \\
\hline & $1 \mathrm{~W} 16 \mathrm{~A}$ & $1.537^{*, \mathrm{~b}, \mathrm{c}}$ & .441 & .001 & .671 & 2.403 \\
\hline & $2 W 32 A$ & $-5.116^{\pi, b, c}$ & .441 & .000 & -5.981 & -4.250 \\
\hline & $4 W 64 A$ & $-1.634^{*}, \mathrm{~b}, \mathrm{c}$ & .441 & .000 & -2.500 & -.768 \\
\hline & $5 W 80 A$ & $-2.782^{\pi, b, c}$ & .441 & .000 & -3.648 & -1.916 \\
\hline \multirow[t]{5}{*}{$4 W 64 A$} & $1 \mathrm{~W} 0 \mathrm{~A}$ & $5.174^{\pi, b, c}$ & .540 & .000 & 4.113 & 6.234 \\
\hline & $1 \mathrm{~W} 16 \mathrm{~A}$ & $3.171^{\pi, b, c}$ & .441 & .000 & 2.305 & 4.037 \\
\hline & $2 W 32 A$ & $-3.481^{*}, \mathrm{~b}, \mathrm{c}$ & .441 & .000 & -4.347 & -2.616 \\
\hline & $3 W 48 A$ & $1.634^{\pi, \mathrm{b}, \mathrm{c}}$ & .441 & .000 & .768 & 2.500 \\
\hline & $5 W 80 A$ & $-1.148^{\mathrm{x}, \mathrm{b}, \mathrm{c}}$ & .441 & .009 & -2.014 & -.282 \\
\hline \multirow[t]{5}{*}{$5 W$ 80A } & $1 \mathrm{~W} 0 \mathrm{~A}$ & $6.322^{x, b, c}$ & .540 & .000 & 5.261 & 7.382 \\
\hline & $1 \mathrm{~W} 16 \mathrm{~A}$ & $4.319^{\pi, b, c}$ & .441 & .000 & 3.453 & 5.185 \\
\hline & $2 W 32 A$ & $-2.333^{*}, \mathrm{~b}, \mathrm{c}$ & .441 & .000 & -3.199 & -1.468 \\
\hline & $3 W 48 A$ & $2.782^{\star, b, c}$ & .441 & .000 & 1.916 & 3.648 \\
\hline & $4 W 64 A$ & $1.148^{\star, b, c}$ & .441 & .009 & .282 & 2.014 \\
\hline
\end{tabular}

Based on estimated marginal means

*. The mean difference is significant at the .05 level.

b. An estimate of the modified population marginal mean (I).

c. An estimate of the modified population marginal mean (J).

d. Adjustment for multiple comparisons: Least Significant Difference (equivalent to no adjustments). 


\section{Washing Estimates: RMP}

Dependent Variable: L

\begin{tabular}{|l|r|r|r|r|}
\hline & & & \multicolumn{2}{|c|}{ 95\% Confidence Interval } \\
\cline { 3 - 5 } Washings & Mean & Std. Error & Lower Bound & Upper Bound \\
\hline 1W 0A & $84.026^{\mathrm{a}}$ & .375 & 83.290 & 84.762 \\
1W 16A & $85.253^{\mathrm{a}}$ & .265 & 84.733 & 85.774 \\
2W 32A & $84.496^{\mathrm{a}}$ & .265 & 83.975 & 85.016 \\
3W 48A & $84.099^{\mathrm{a}}$ & .265 & 83.578 & 84.619 \\
4W 64A & $83.269^{\mathrm{a}}$ & .265 & 82.748 & 83.789 \\
5W 80A & $84.524^{\mathrm{a}}$ & .265 & 84.003 & 85.044 \\
\hline
\end{tabular}

a. Based on modified population marginal mean.

Pairwise Comparisons: RMP

Dependent Variable: L

\begin{tabular}{|c|c|c|c|c|c|c|}
\hline \multirow{2}{*}{\multicolumn{2}{|c|}{ (I) Washings }} & \multirow[b]{2}{*}{ Mean Difference (I-J) } & \multirow[b]{2}{*}{ Std. Error } & \multirow[b]{2}{*}{ Sig. ${ }^{d}$} & \multicolumn{2}{|c|}{$95 \%$ Confidence Interval for Difference } \\
\hline & & & & & Lower Bound & Upper Bound \\
\hline \multirow[t]{5}{*}{$1 \mathrm{~W} \mathrm{0A}$} & $1 \mathrm{~W} 16 \mathrm{~A}$ & $-1.227^{, b, c}$ & .459 & .008 & -2.129 & -.326 \\
\hline & $2 W 32 A$ & $-.470^{\mathrm{b}, \mathrm{c}}$ & .459 & .307 & -1.371 & .432 \\
\hline & $3 W 48 A$ & $-.073^{\mathrm{b}, \mathrm{c}}$ & .459 & .874 & -.974 & .829 \\
\hline & $4 \mathrm{~W} 64 \mathrm{~A}$ & $.757^{\mathrm{b}, \mathrm{c}}$ & .459 & .100 & -.144 & 1.659 \\
\hline & $5 W 80 A$ & $-.498^{\mathrm{b}, \mathrm{c}}$ & .459 & .279 & -1.399 & .404 \\
\hline \multirow[t]{5}{*}{$1 \mathrm{~W} 16 \mathrm{~A}$} & $1 \mathrm{~W} 0 \mathrm{~A}$ & $1.227^{\pi, \mathrm{b}, \mathrm{c}}$ & .459 & .008 & .326 & 2.129 \\
\hline & $2 W 32 A$ & $.757^{\pi, \mathrm{b}, \mathrm{c}}$ & .375 & .044 & .021 & 1.493 \\
\hline & $3 W 48 A$ & $1.154^{\pi, \mathrm{b}, \mathrm{c}}$ & .375 & .002 & .418 & 1.890 \\
\hline & $4 W 64 A$ & $1.984^{\pi, b, c}$ & .375 & .000 & 1.248 & 2.720 \\
\hline & $5 W 80 A$ & $.729^{b, c}$ & .375 & .052 & -.007 & 1.465 \\
\hline \multirow[t]{5}{*}{$2 W 32 A$} & $1 \mathrm{~W} 0 \mathrm{~A}$ & $.470^{\mathrm{b}, \mathrm{c}}$ & .459 & .307 & -.432 & 1.371 \\
\hline & $1 \mathrm{~W} 16 \mathrm{~A}$ & $-.757^{*}, \mathrm{~b}, \mathrm{c}$ & .375 & .044 & -1.493 & -.021 \\
\hline & $3 W 48 A$ & $397^{b, c}$ & .375 & .290 & -.339 & 1.133 \\
\hline & $4 W 64 A$ & $1.227^{x, b, c}$ & .375 & .001 & .491 & 1.963 \\
\hline & $5 W$ & $-.028^{b, c}$ & .375 & .940 & -.764 & .708 \\
\hline \multirow[t]{5}{*}{$3 W$ 48A } & $1 \mathrm{~W} 0 \mathrm{~A}$ & $.073^{\mathrm{b}, \mathrm{c}}$ & .459 & .874 & -.829 & .974 \\
\hline & $1 \mathrm{~W} 16 \mathrm{~A}$ & $-1.154^{\pi, b, c}$ & .375 & .002 & -1.890 & -.418 \\
\hline & $2 W 32 A$ & $-.397^{b, c}$ & .375 & .290 & -1.133 & .339 \\
\hline & $4 W 64 A$ & $.830^{\circ}, \mathrm{b}, \mathrm{c}$ & .375 & .027 & .094 & 1.566 \\
\hline & $5 W 80 A$ & $-.425^{b, c}$ & .375 & .257 & -1.161 & .311 \\
\hline \multirow[t]{5}{*}{$4 W 64 A$} & $1 \mathrm{~W} 0 \mathrm{~A}$ & $-.757^{\mathrm{b}, \mathrm{c}}$ & .459 & .100 & -1.659 & .144 \\
\hline & $1 \mathrm{~W} 16 \mathrm{~A}$ & $-1.984^{\pi, b, c}$ & .375 & .000 & -2.720 & -1.248 \\
\hline & $2 W 32 A$ & $-1.227^{\pi, b, c}$ & .375 & .001 & -1.963 & -.491 \\
\hline & $3 W$ 48A & $-.830^{\pi, b, c}$ & .375 & .027 & -1.566 & -.094 \\
\hline & $5 W$ & $-1.255^{\pi, b, c}$ & .375 & .001 & -1.991 & -.519 \\
\hline \multirow[t]{5}{*}{$5 W$ 80A } & $1 \mathrm{~W} 0 \mathrm{~A}$ & $.498^{b, c}$ & .459 & .279 & -.404 & 1.399 \\
\hline & $1 \mathrm{~W} 16 \mathrm{~A}$ & $-.729^{b, c}$ & .375 & .052 & -1.465 & .007 \\
\hline & $2 W 32 A$ & $.028^{b, c}$ & .375 & .940 & -.708 & .764 \\
\hline & $3 W$ 48A & $.425^{\mathrm{b}, \mathrm{c}}$ & .375 & .257 & -.311 & 1.161 \\
\hline & $4 W 64 A$ & $1.255^{\pi, \mathrm{b}, \mathrm{c}}$ & .375 & .001 & .519 & 1.991 \\
\hline
\end{tabular}

Based on estimated marginal means

*. The mean difference is significant at the .05 level.

b. An estimate of the modified population marginal mean (I).

c. An estimate of the modified population marginal mean $(\mathrm{J})$.

d. Adjustment for multiple comparisons: Least Significant Difference (equivalent to no adjustments).

Out of all the reference samples, sample PMP obtained significant values at all levels of pair-

wise groups. The main effects for washing were significant throughout the washed-aged process.

It can be concluded that PMP was tremendously affected by washing. Significant values were

also observed for sample RMP. 
8. Swatch * Washings: PMP

Dependent Variable: $\mathrm{L}$

\begin{tabular}{|c|c|c|c|c|c|}
\hline \multirow[b]{2}{*}{ Swatch } & & \multirow[b]{2}{*}{ Mean } & \multirow[b]{2}{*}{ Std. Error } & \multicolumn{2}{|c|}{ 95\% Confidence Interval } \\
\hline & & & & Lower Bound & Upper Bound \\
\hline \multirow[t]{6}{*}{$1 \mathrm{w} /$ bleach } & $1 \mathrm{~W} 0 \mathrm{~A}$ & $78.822^{a}$ & .882 & 77.091 & 80.554 \\
\hline & $1 \mathrm{~W} 16 \mathrm{~A}$ & $82.351^{a}$ & .624 & 81.126 & 83.575 \\
\hline & $2 W 32 A$ & $89.409^{a}$ & .624 & 88.185 & 90.634 \\
\hline & $3 W$ 48A & $82.655^{a}$ & .624 & 81.430 & 83.879 \\
\hline & $4 \mathrm{~W} 64 \mathrm{~A}$ & $83.419^{a}$ & .624 & 82.195 & 84.644 \\
\hline & $5 W 80 A$ & $86.073^{\mathrm{a}}$ & .624 & 84.848 & 87.297 \\
\hline \multirow[t]{6}{*}{2 w/ bleach } & $1 \mathrm{~W} 0 \mathrm{~A}$ & $81.441^{a}$ & .882 & 79.709 & 83.173 \\
\hline & $1 \mathrm{~W} 16 \mathrm{~A}$ & $81.244^{\mathrm{a}}$ & .624 & 80.019 & 82.468 \\
\hline & $2 W 32 A$ & $91.774^{\mathrm{a}}$ & .624 & 90.549 & 92.999 \\
\hline & $3 W 48 A$ & $82.359^{a}$ & .624 & 81.134 & 83.583 \\
\hline & $4 W 64 A$ & $84.379^{a}$ & .624 & 83.154 & 85.603 \\
\hline & $5 W$ 80A & $84.425^{\mathrm{a}}$ & .624 & 83.200 & 85.649 \\
\hline \multirow[t]{6}{*}{3 w/o bleach } & $1 \mathrm{~W} 0 \mathrm{~A}$ & $81.216^{a}$ & .882 & 79.484 & 82.948 \\
\hline & $1 \mathrm{~W} 16 \mathrm{~A}$ & $79.780^{\mathrm{a}}$ & .624 & 78.556 & 81.005 \\
\hline & $2 W 32 A$ & $85.629^{a}$ & .624 & 84.404 & 86.853 \\
\hline & $3 W 48 A$ & $84.246^{\mathrm{a}}$ & .624 & 83.021 & 85.470 \\
\hline & $4 W 64 A$ & $85.570^{a}$ & .624 & 84.345 & 86.794 \\
\hline & $5 W$ & $87.203^{a}$ & .624 & 85.978 & 88.427 \\
\hline \multirow[t]{6}{*}{4 w/o bleach } & $1 \mathrm{~W} 0 \mathrm{~A}$ & $74.731^{a}$ & .882 & 72.999 & 76.462 \\
\hline & $1 \mathrm{~W} 16 \mathrm{~A}$ & $80.846^{a}$ & .624 & 79.622 & 82.071 \\
\hline & $2 W 32 A$ & $84.018^{a}$ & .624 & 82.794 & 85.243 \\
\hline & $3 W 48 A$ & $81.109^{a}$ & .624 & 79.885 & 82.334 \\
\hline & $4 W 64 A$ & $83.537^{\mathrm{a}}$ & .624 & 82.312 & 84.762 \\
\hline & $5 W 80 A$ & $83.797^{a}$ & .624 & 82.572 & 85.021 \\
\hline
\end{tabular}

a. Based on modified population marginal mean.

Dependent Variable: $\mathrm{L}$

\section{Swatch * Washings: RMP}

\begin{tabular}{|c|c|c|c|c|c|}
\hline \multirow[b]{2}{*}{ Swatch } & & \multirow[b]{2}{*}{ Mean } & \multirow[b]{2}{*}{ Std. Error } & \multicolumn{2}{|c|}{ 95\% Confidence Interval } \\
\hline & & & & Lower Bound & Upper Bound \\
\hline \multirow[t]{6}{*}{$1 \mathrm{w} /$ bleach } & $1 \mathrm{~W} 0 \mathrm{~A}$ & $85.981^{a}$ & .750 & 84.509 & 87.453 \\
\hline & $1 \mathrm{~W} 16 \mathrm{~A}$ & $83.823^{\mathrm{a}}$ & .530 & 82.782 & 84.864 \\
\hline & $2 W 32 A$ & $84.609^{a}$ & .530 & 83.568 & 85.650 \\
\hline & $3 W 48 A$ & $84.593^{\mathrm{a}}$ & .530 & 83.552 & 85.633 \\
\hline & $4 \mathrm{~W} 64 \mathrm{~A}$ & $85.704^{a}$ & .530 & 84.664 & 86.745 \\
\hline & $5 \mathrm{~W} 80 \mathrm{~A}$ & $84.027^{a}$ & .530 & 82.986 & 85.068 \\
\hline \multirow[t]{6}{*}{2 w/ bleach } & $1 \mathrm{~W} 0 \mathrm{~A}$ & $86.187^{a}$ & .750 & 84.715 & 87.659 \\
\hline & $1 \mathrm{~W} 16 \mathrm{~A}$ & $85.663^{\mathrm{a}}$ & .530 & 84.622 & 86.704 \\
\hline & $2 W 32 A$ & $85.834^{a}$ & .530 & 84.793 & 86.875 \\
\hline & $3 W 48 A$ & $83.716^{a}$ & .530 & 82.675 & 84.757 \\
\hline & $4 W 64 A$ & $83.275^{a}$ & .530 & 82.234 & 84.316 \\
\hline & $5 W 80 A$ & $86.573^{a}$ & .530 & 85.532 & 87.614 \\
\hline \multirow[t]{6}{*}{3 w/o bleach } & $1 \mathrm{~W} 0 \mathrm{~A}$ & $83.322^{a}$ & .750 & 81.850 & 84.794 \\
\hline & $1 \mathrm{~W} 16 \mathrm{~A}$ & $86.352^{a}$ & .530 & 85.311 & 87.393 \\
\hline & $2 W 32 A$ & $84.453^{a}$ & .530 & 83.412 & 85.494 \\
\hline & $3 W 48 A$ & $84.149^{a}$ & .530 & 83.108 & 85.190 \\
\hline & $4 W 64 A$ & $80.644^{a}$ & .530 & 79.604 & 81.685 \\
\hline & $5 W 80 A$ & $85.133^{a}$ & .530 & 84.092 & 86.174 \\
\hline \multirow[t]{6}{*}{4 w/o bleach } & $1 \mathrm{~W} 0 \mathrm{~A}$ & $80.614^{a}$ & .750 & 79.141 & 82.086 \\
\hline & $1 \mathrm{~W} 16 \mathrm{~A}$ & $85.175^{a}$ & .530 & 84.134 & 86.215 \\
\hline & $2 W 32 A$ & $83.086^{a}$ & .530 & 82.046 & 84.127 \\
\hline & $3 W 48 A$ & $83.938^{a}$ & .530 & 82.897 & 84.979 \\
\hline & $4 W 64 A$ & $83.451^{a}$ & .530 & 82.410 & 84.492 \\
\hline & $5 \mathrm{~W} 80 \mathrm{~A}$ & $82.362^{a}$ & .530 & 81.321 & 83.403 \\
\hline
\end{tabular}

a. Based on modified population marginal mean. 

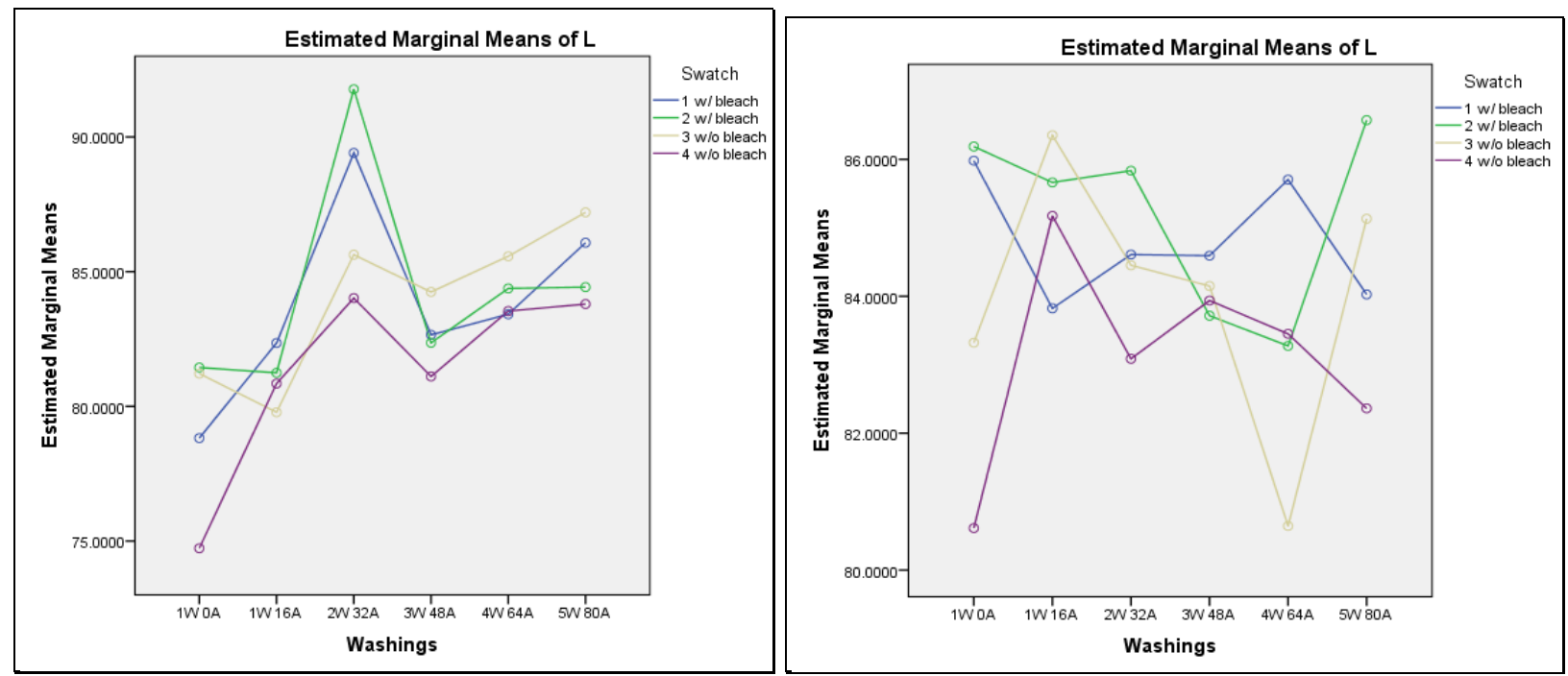

The plots above represent PMP and RMP from left to right, respectively. Unlike sample RMP which did not exhibit a particular pattern, sample PMP observed a more consistent pattern. This pattern has not been observed for any of the reference samples.

\section{Variable ' $a$ '}

Main effects were insignificant at sample area for both samples, and at swatch for sample PMP.

Dependent Variable: a

Tests of Between-Subjects Effects: PMP

\begin{tabular}{|c|c|c|c|c|c|}
\hline Source & Type III Sum of Squares & $\mathrm{df}$ & Mean Square & $\mathrm{F}$ & Sig. \\
\hline Corrected Model & $482.74^{2}$ & 43 & 11.227 & 6.762 & .000 \\
\hline SampleArea & .337 & 1 & .337 & .203 & .652 \\
\hline Bleached & 0.000 & 0 & & & \\
\hline Washings & 32.867 & 5 & 6.573 & 3.960 & .001 \\
\hline SampleArea * Swatch & 32.736 & 2 & 16.368 & 9.859 & .000 \\
\hline Swatch * Bleached & 0.000 & 0 & & & \\
\hline Swatch * Washings & 122.15 & 8 & 15.269 & 9.197 & .000 \\
\hline Bleached * Washings & 0.000 & 0 & & & \\
\hline SampleArea * Swatch * Bleached & 0.000 & 0 & & & \\
\hline SampleArea * Swatch * Washings & 83.981 & 8 & 10.498 & 6.323 & .000 \\
\hline SampleArea * Bleached * Washings & 0.000 & 0 & & & \\
\hline Corrected Total & 1870.6 & 879 & & & \\
\hline
\end{tabular}

a. R Squared $=.258$ (Adjusted R Squared $=.220$ ). b. Computed using alpha $=.05$ 
Tests of Between-Subjects Effects: RMP

Dependent Variable: a

\begin{tabular}{|c|c|c|c|c|c|}
\hline Source & Type III Sum of Squares & df & Mean Square & $\mathrm{F}$ & Sig. \\
\hline Corrected Model & $4998.0^{\mathrm{a}}$ & 43 & 116.23 & 7.043 & .000 \\
\hline Intercept & 614669 & 1 & 614669 & 37246 & 0.000 \\
\hline SampleArea & 55.971 & 1 & 55.971 & 3.392 & .066 \\
\hline Swatch & 278.62 & 2 & 139.31 & 8.441 & .000 \\
\hline Bleached & 0.000 & 0 & & & \\
\hline Washings & 308.54 & 5 & 61.709 & 3.739 & .002 \\
\hline SampleArea * Swatch & 397.01 & 2 & 198.51 & 12.029 & .000 \\
\hline SampleArea * Bleached & 0.000 & 0 & & & \\
\hline SampleArea * Washings & 832.21 & 4 & 208.05 & 12.607 & .000 \\
\hline Swatch * Bleached & 0.000 & 0 & & & \\
\hline Swatch * Washings & 1280.9 & 8 & 160.12 & 9.702 & .000 \\
\hline Bleached * Washings & 0.000 & 0 & & & \\
\hline SampleArea * Swatch * Bleached & 0.000 & 0 & & & \\
\hline SampleArea * Swatch * Washings & 713.31 & 8 & 89.164 & 5.403 & .000 \\
\hline SampleArea * Bleached * Washings & 0.000 & 0 & & & \\
\hline Swatch * Bleached * Washings & 0.000 & 0 & & & \\
\hline SampleArea * Swatch * Bleached * Washings & 0.000 & 0 & & & \\
\hline Error & 13796 & 836 & 16.503 & & \\
\hline Total & 679347 & 880 & & & \\
\hline Corrected Total & 18794 & 879 & & & \\
\hline
\end{tabular}
a. R Squared $=.266$ (Adjusted R Squared $=.228$ )
b. Computed using alpha $=.05$

2. Swatch Estimates: RMP

Dependent Variable: a

\begin{tabular}{|l|r|r|r|r|}
\hline & & & \multicolumn{2}{|c|}{$95 \%$ Confidence Interval } \\
\cline { 4 - 5 } Swatch & Mean & Std. Error & Lower Bound & Upper Bound \\
\hline 1 w/ bleach & $28.069^{\mathrm{a}}$ & .274 & 27.531 & 28.607 \\
2 w/ bleach & $26.121^{\mathrm{a}}$ & .274 & 25.583 & 26.658 \\
3 w/o bleach & $27.888^{\mathrm{a}}$ & .274 & 27.350 & 28.425 \\
4 w/o bleach & $27.513^{\mathrm{a}}$ & .274 & 26.975 & 28.050 \\
\hline
\end{tabular}

a. Based on modified population marginal mean.

Pairwise Comparisons: RMP

Dependent Variable: a

\begin{tabular}{|c|c|c|c|c|c|c|}
\hline \multirow[b]{2}{*}{ (I) Swatch } & & \multirow[b]{2}{*}{ Mean Difference (I-J) } & \multirow[b]{2}{*}{ Std. Error } & \multirow[b]{2}{*}{ Sig. ${ }^{d}$} & \multicolumn{2}{|c|}{$\begin{array}{l}\text { 95\% Confidence Interval for } \\
\text { Difference }\end{array}$} \\
\hline & & & & & Lower Bound & Upper Bound \\
\hline \multirow[t]{3}{*}{$1 \mathrm{w} /$ bleach } & $2 \mathrm{w} /$ bleach & $1.948^{\prime, b, c}$ & .387 & .000 & 1.188 & 2.708 \\
\hline & 3 w/o bleach & $.181^{\mathrm{b}, \mathrm{c}}$ & .387 & .640 & -.579 & .942 \\
\hline & 4 w/o bleach & $.556^{\mathrm{b}, \mathrm{c}}$ & .387 & .151 & -.204 & 1.317 \\
\hline \multirow[t]{3}{*}{2 w/ bleach } & 1 w/ bleach & $-1.948^{\star}, \mathrm{b}, \mathrm{c}$ & .387 & .000 & -2.708 & -1.188 \\
\hline & 3 w/o bleach & $-1.767^{*}, \mathrm{~b}, \mathrm{c}$ & .387 & .000 & -2.527 & -1.007 \\
\hline & 4 w/o bleach & $-1.392^{*}, \mathrm{~b}, \mathrm{c}$ & .387 & .000 & -2.152 & -.631 \\
\hline \multirow[t]{3}{*}{3 w/o bleach } & 1 w/ bleach & $-.181^{\mathrm{b}, \mathrm{c}}$ & .387 & .640 & -.942 & .579 \\
\hline & 2 w/ bleach & $1.767^{*}, \mathrm{~b}, \mathrm{c}$ & .387 & .000 & 1.007 & 2.527 \\
\hline & 4 w/o bleach & $.375^{\mathrm{b}, \mathrm{c}}$ & .387 & .333 & -.385 & 1.135 \\
\hline \multirow[t]{3}{*}{4 w/o bleach } & 1 w/ bleach & $-.556^{\mathrm{b}, \mathrm{c}}$ & .387 & .151 & -1.317 & .204 \\
\hline & 2 w/ bleach & $1.392^{*}, \mathrm{~b}, \mathrm{c}$ & .387 & .000 & .631 & 2.152 \\
\hline & 3 w/o bleach & $-.375^{\mathrm{b}, \mathrm{c}}$ & .387 & .333 & -1.135 & .385 \\
\hline
\end{tabular}


Based on estimated marginal means

*. The mean difference is significant at the .05 level.

b. An estimate of the modified population marginal mean (I).

c. An estimate of the modified population marginal mean $(\mathrm{J})$.

d. Adjustment for multiple comparisons: Least Significant Difference (equivalent to no adjustments).

Swatch 1 was marked as significant with swatch 2, but insignificant with swatches 3 and 4.

\section{Washing Estimates: PMP}

Dependent Variable: a

\begin{tabular}{|l|r|r|r|r|}
\hline & & & \multicolumn{2}{|c|}{$95 \%$ Confidence Interval } \\
\cline { 4 - 5 } Washings & Mean & Std. Error & Lower Bound & \multicolumn{1}{|c|}{ Upper Bound } \\
\hline 1W 0A & $6.909^{\mathrm{a}}$ & .144 & 6.626 & 7.192 \\
1W 16A & $6.311^{\mathrm{a}}$ & .102 & 6.111 & 6.511 \\
2W 32A & $6.297^{\mathrm{a}}$ & .102 & 6.097 & 6.496 \\
3W 48A & $6.433^{\mathrm{a}}$ & .102 & 6.233 & 6.633 \\
4W 64A & $6.316^{\mathrm{a}}$ & .102 & 6.116 & 6.516 \\
5W 80A & $6.327^{\mathrm{a}}$ & .102 & 6.127 & 6.527 \\
\hline
\end{tabular}

a. Based on modified population marginal mean.

Pairwise Comparisons: PMP

Dependent Variable: a

\begin{tabular}{|c|c|c|c|c|c|c|}
\hline \multirow[b]{2}{*}{ (I) Washing } & & \multirow[b]{2}{*}{ Mean Difference (I-J) } & \multirow[b]{2}{*}{ Std. Error } & \multirow[b]{2}{*}{ Sig. ${ }^{d}$} & \multicolumn{2}{|c|}{$\begin{array}{l}\text { 95\% Confidence Interval for } \\
\text { Difference }^{d}\end{array}$} \\
\hline & & & & & Lower Bound & Upper Bound \\
\hline \multirow[t]{5}{*}{$1 \mathrm{~W} 0 \mathrm{~A}$} & $1 \mathrm{~W} 16 \mathrm{~A}$ & $.597^{\circ, \mathrm{b}, \mathrm{C}}$ & .176 & .001 & .251 & .944 \\
\hline & $2 W 32 A$ & $.612^{\pi, b, c}$ & .176 & .001 & .266 & .959 \\
\hline & $3 W 48 A$ & $.476^{\pi, b, c}$ & .176 & .007 & .130 & .822 \\
\hline & $4 \mathrm{~W} 64 \mathrm{~A}$ & $.593^{\pi, b, c}$ & .176 & .001 & .247 & .939 \\
\hline & $5 W 80 A$ & $.582^{\pi, b, c}$ & .176 & .001 & .235 & .928 \\
\hline \multirow[t]{5}{*}{$1 \mathrm{~W} 16 \mathrm{~A}$} & $1 \mathrm{~W} 0 \mathrm{~A}$ & $-.597^{\pi, b, c}$ & .176 & .001 & -.944 & -.251 \\
\hline & $2 W 32 A$ & $.015^{\mathrm{b}, \mathrm{c}}$ & .144 & .918 & -.268 & .298 \\
\hline & $3 W 48 A$ & $-.121^{\mathrm{b}, \mathrm{c}}$ & .144 & .400 & -.404 & .161 \\
\hline & $4 \mathrm{~W} 64 \mathrm{~A}$ & $-.004^{b, c}$ & .144 & .975 & -.287 & .278 \\
\hline & $5 W 80 A$ & $-.016^{\mathrm{b}, \mathrm{c}}$ & .144 & .913 & -.298 & .267 \\
\hline \multirow[t]{5}{*}{$2 W 32 A$} & $1 \mathrm{~W} 0 \mathrm{~A}$ & $-.612^{x, b, c}$ & .176 & .001 & -.959 & -.266 \\
\hline & $1 \mathrm{~W} 16 \mathrm{~A}$ & $-.015^{b, c}$ & .144 & .918 & -.298 & .268 \\
\hline & $3 W 48 A$ & $-.136^{\mathrm{b}, \mathrm{c}}$ & .144 & .345 & -.419 & .146 \\
\hline & $4 \mathrm{~W} 64 \mathrm{~A}$ & $-.019^{b, c}$ & .144 & .893 & -.302 & .263 \\
\hline & $5 W 80 A$ & $-.031^{b, c}$ & .144 & .832 & -.313 & .252 \\
\hline \multirow[t]{5}{*}{$3 W$ 48A } & $1 \mathrm{~W} 0 \mathrm{~A}$ & $-.476^{\pi, b, c}$ & .176 & .007 & -.822 & -.130 \\
\hline & $1 \mathrm{~W} 16 \mathrm{~A}$ & $.121^{\mathrm{b}, \mathrm{c}}$ & .144 & .400 & -.161 & .404 \\
\hline & $2 W 32 A$ & $.136^{\mathrm{b}, \mathrm{c}}$ & .144 & .345 & -.146 & .419 \\
\hline & $4 W 64 A$ & $.117^{\mathrm{b}, \mathrm{c}}$ & .144 & .417 & -.166 & .400 \\
\hline & $5 W 80 A$ & $.106^{\mathrm{b}, \mathrm{c}}$ & .144 & .463 & -.177 & .388 \\
\hline \multirow[t]{5}{*}{$4 W 64 A$} & $1 \mathrm{~W} 0 \mathrm{~A}$ & $-.593^{x, b, c}$ & .176 & .001 & -.939 & -.247 \\
\hline & $1 \mathrm{~W} 16 \mathrm{~A}$ & $.004^{\mathrm{b}, \mathrm{c}}$ & .144 & .975 & -.278 & .287 \\
\hline & $2 W 32 A$ & $.019^{b, c}$ & .144 & .893 & -.263 & .302 \\
\hline & $3 W 48 A$ & $-.117^{\mathrm{b}, \mathrm{c}}$ & .144 & .417 & -.400 & .166 \\
\hline & $5 W$ & $-.011^{\mathrm{b}, \mathrm{c}}$ & .144 & .938 & -.294 & .271 \\
\hline \multirow[t]{5}{*}{$5 W 80 A$} & $1 \mathrm{~W} 0 \mathrm{~A}$ & $-.582^{*}, \mathrm{~b}, \mathrm{c}$ & .176 & .001 & -.928 & -.235 \\
\hline & $1 \mathrm{~W} 16 \mathrm{~A}$ & $.016^{\mathrm{b}, \mathrm{c}}$ & .144 & .913 & -.267 & .298 \\
\hline & $2 W 32 A$ & $.031^{\mathrm{b}, \mathrm{c}}$ & .144 & .832 & -.252 & .313 \\
\hline & $3 W 48 A$ & $-.106^{b, c}$ & .144 & .463 & -.388 & .177 \\
\hline & $4 W 64 A$ & $.011^{b, c}$ & .144 & .938 & -.271 & .294 \\
\hline
\end{tabular}

Based on estimated marginal means

*. The mean difference is significant at the .05 level. 
b. An estimate of the modified population marginal mean (I).

c. An estimate of the modified population marginal mean $(\mathrm{J})$.

d. Adjustment for multiple comparisons: Least Significant Difference (equivalent to no adjustments).

\section{Washing Estimates: RMP}

Dependent Variable: a

\begin{tabular}{|l|r|r|r|r|}
\hline & & & \multicolumn{2}{|c|}{$95 \%$ Confidence Interval } \\
\cline { 4 - 5 } Washings & Mean & Std. Error & Lower Bound & Upper Bound \\
\hline 1W 0A & $27.211^{\mathrm{a}}$ & .454 & 26.320 & 28.103 \\
1W 16A & $28.248^{\mathrm{a}}$ & .321 & 27.617 & 28.878 \\
2W 32A & $27.374^{\mathrm{a}}$ & .321 & 26.743 & 28.004 \\
3W 48A & $26.531^{\mathrm{a}}$ & .321 & 25.901 & 27.161 \\
4W 64A & $27.234^{\mathrm{a}}$ & .321 & 26.604 & 27.865 \\
5W 80A & $27.695^{\mathrm{a}}$ & .321 & 27.064 & 28.325 \\
\hline
\end{tabular}

a. Based on modified population marginal mean.

\section{Pairwise Comparisons: RMP}

Dependent Variable: a

\begin{tabular}{|c|c|c|c|c|c|c|}
\hline \multirow{2}{*}{\multicolumn{2}{|c|}{ (I) Washings }} & \multirow[b]{2}{*}{ Mean Difference (I-J) } & \multirow[b]{2}{*}{ Std. Error } & \multirow[b]{2}{*}{ Sig. ${ }^{d}$} & \multicolumn{2}{|c|}{$\begin{array}{l}\text { 95\% Confidence Interval for } \\
\text { Difference }^{d}\end{array}$} \\
\hline & & & & & Lower Bound & Upper Bound \\
\hline \multirow[t]{5}{*}{$1 \mathrm{~W} 0 \mathrm{~A}$} & $1 \mathrm{~W} 16 \mathrm{~A}$ & $-1.036^{\mathrm{a}, \mathrm{b}}$ & .556 & .063 & -2.128 & .055 \\
\hline & $2 W 32 A$ & $-.162^{a, b}$ & .556 & .770 & -1.254 & .929 \\
\hline & $3 W 48 A$ & $.680^{\mathrm{a}, \mathrm{b}}$ & .556 & .222 & -.412 & 1.772 \\
\hline & $4 \mathrm{~W} 64 \mathrm{~A}$ & $-.023^{\mathrm{a}, \mathrm{b}}$ & .556 & .967 & -1.115 & 1.069 \\
\hline & $5 W$ 80A & $-.483^{a, b}$ & .556 & .385 & -1.575 & .608 \\
\hline \multirow[t]{5}{*}{$1 \mathrm{~W} 16 \mathrm{~A}$} & $1 \mathrm{~W} 0 \mathrm{~A}$ & $1.036^{\mathrm{a}, \mathrm{b}}$ & .556 & .063 & -.055 & 2.128 \\
\hline & $2 W 32 A$ & $.874^{\mathrm{a}, \mathrm{b}}$ & .454 & .055 & -.018 & 1.765 \\
\hline & $3 W 48 A$ & $1.717^{\mathrm{a}, \mathrm{b},{ }^{*}}$ & .454 & .000 & .825 & 2.608 \\
\hline & $4 W 64 A$ & $1.013^{\mathrm{a}, \mathrm{b},{ }^{*}}$ & .454 & .026 & .122 & 1.905 \\
\hline & $5 W$ 80A & $.553^{\mathrm{a}, \mathrm{b}}$ & .454 & .224 & -.339 & 1.444 \\
\hline \multirow[t]{5}{*}{$2 W 32 A$} & $1 \mathrm{~W} 0 \mathrm{~A}$ & $.162^{\mathrm{a}, \mathrm{b}}$ & .556 & .770 & -.929 & 1.254 \\
\hline & $1 \mathrm{~W} 16 \mathrm{~A}$ & $-874^{\mathrm{a}, \mathrm{b}}$ & .454 & .055 & -1.765 & .018 \\
\hline & $3 W$ 48A & $.843^{\mathrm{a}, \mathrm{b}}$ & .454 & .064 & -.049 & 1.734 \\
\hline & $4 W 64 A$ & $.139^{a, b}$ & .454 & .759 & -.752 & 1.031 \\
\hline & $5 W 80 A$ & $-.321^{a, b}$ & .454 & .480 & -1.212 & .571 \\
\hline \multirow[t]{5}{*}{$3 W 48 A$} & $1 \mathrm{~W} 0 \mathrm{~A}$ & $-.680^{\mathrm{a}, \mathrm{b}}$ & .556 & .222 & -1.772 & .412 \\
\hline & $1 \mathrm{~W} 16 \mathrm{~A}$ & $-1.717^{\mathrm{a}, \mathrm{b},{ }^{*}}$ & .454 & .000 & -2.608 & -.825 \\
\hline & $2 W 32 A$ & $-.843^{\mathrm{a}, \mathrm{b}}$ & .454 & .064 & -1.734 & .049 \\
\hline & $4 W 64 A$ & $-.703^{a, b}$ & .454 & .122 & -1.595 & .188 \\
\hline & $5 W 80 A$ & $-1.164^{\mathrm{a}, \mathrm{b},{ }^{*}}$ & .454 & .011 & -2.055 & -.272 \\
\hline \multirow[t]{5}{*}{$4 W 64 A$} & $1 \mathrm{~W} 0 \mathrm{~A}$ & $.023^{a, b}$ & .556 & .967 & -1.069 & 1.115 \\
\hline & $1 \mathrm{~W} 16 \mathrm{~A}$ & $-1.013^{\mathrm{a}, \mathrm{b},{ }^{*}}$ & .454 & .026 & -1.905 & -.122 \\
\hline & $2 W 32 A$ & $-.139^{a, b}$ & .454 & .759 & -1.031 & .752 \\
\hline & $3 W$ 48A & $.703^{\mathrm{a}, \mathrm{b}}$ & .454 & .122 & -.188 & 1.595 \\
\hline & $5 W 80 A$ & $-.460^{a, b}$ & .454 & .311 & -1.352 & .431 \\
\hline \multirow[t]{5}{*}{$5 W$ 80A } & $1 \mathrm{~W} 0 \mathrm{~A}$ & $.483^{\mathrm{a}, \mathrm{b}}$ & .556 & .385 & -.608 & 1.575 \\
\hline & $1 \mathrm{~W} 16 \mathrm{~A}$ & $-.553^{\mathrm{a}, \mathrm{b}}$ & .454 & .224 & -1.444 & .339 \\
\hline & $2 W 32 A$ & $.321^{a, b}$ & .454 & .480 & -.571 & 1.212 \\
\hline & $3 W 48 A$ & $1.164^{\mathrm{a}, \mathrm{b},{ }^{\star}}$ & .454 & .011 & .272 & 2.055 \\
\hline & $4 \mathrm{~W} 64 \mathrm{~A}$ & $.460^{\mathrm{a}, \mathrm{b}}$ & .454 & .311 & -.431 & 1.352 \\
\hline
\end{tabular}

Based on estimated marginal means

*. The mean difference is significant at the .05 level.

a. An estimate of the modified population marginal mean (I).

b. An estimate of the modified population marginal mean $(\mathrm{J})$.

d. Adjustment for multiple comparisons: Least Significant Difference (equivalent to no adjustments). 
Marked significant values were observed at all levels of interactions for sample PMP at OW 0A.

However, for sample RMP, the most significant results were observed at 4W 64A.

\section{Bleached * Washings: PMP}

Dependent Variable: a

\begin{tabular}{|ll|r|r|r|r|}
\hline \multirow{2}{*}{ Bleached } & & & \multicolumn{2}{|c|}{ 95\% Confidence Interval } \\
\cline { 3 - 5 } & & Mean & Std. Error & Lower Bound & Upper Bound \\
\hline w/ bleach & 1W 0A & $6.120^{\mathrm{a}}$ & .204 & 5.720 & 6.520 \\
& 1W 16A & $6.502^{\mathrm{a}}$ & .144 & 6.219 & 6.785 \\
& 2W 32A & $6.727^{\mathrm{a}}$ & .144 & 6.444 & 7.010 \\
& 3W 48A & $6.637^{\mathrm{a}}$ & .144 & 6.354 & 6.920 \\
w/o bleach & 4W 64A & $6.110^{\mathrm{a}}$ & .144 & 5.827 & 6.393 \\
& 5W 80A & $6.556^{\mathrm{a}}$ & .144 & 6.274 & 6.839 \\
& 1W 0A & $7.698^{\mathrm{a}}$ & .204 & 7.298 & 8.098 \\
& 1W 16A & $6.121^{\mathrm{a}}$ & .144 & 5.838 & 6.404 \\
& 2W 32A & $5.866^{\mathrm{a}}$ & .144 & 5.583 & 6.149 \\
& 3W 48A & $6.229^{\mathrm{a}}$ & .144 & 5.946 & 6.511 \\
& 4W 64A & $6.522^{\mathrm{a}}$ & .144 & 6.239 & 6.805 \\
& 5W 80A & $6.098^{\mathrm{a}}$ & .144 & 5.815 & 6.381 \\
\hline
\end{tabular}

a. Based on modified population marginal mean.

\section{Bleached * Washings: RMP}

Dependent Variable: a

\begin{tabular}{|ll|r|r|r|r|}
\hline & & & \multicolumn{2}{|c|}{$95 \%$ Confidence Interval } \\
\cline { 4 - 5 } Bleached & & Mean & Std. Error & Lower Bound & Upper Bound \\
\hline w/ bleach & 1W 0A & $28.319^{\mathrm{a}}$ & .642 & 27.058 & 29.579 \\
& 1W 16A & $28.316^{\mathrm{a}}$ & .454 & 27.424 & 29.207 \\
& 2W 32A & $26.001^{\mathrm{a}}$ & .454 & 25.109 & 26.892 \\
& 3W 48A & $25.653^{\mathrm{a}}$ & .454 & 24.761 & 26.544 \\
& 4W 64A & $27.243^{\mathrm{a}}$ & .454 & 26.351 & 28.134 \\
w/o bleach & 5W 80A & $27.651^{\mathrm{a}}$ & .454 & 26.760 & 28.543 \\
& 1W 0A & $26.104^{\mathrm{a}}$ & .642 & 24.843 & 27.365 \\
& 1W 16A & $28.179^{\mathrm{a}}$ & .454 & 27.288 & 29.071 \\
& 2W 32A & $28.747^{\mathrm{a}}$ & .454 & 27.855 & 29.638 \\
& 3W 48A & $27.409^{\mathrm{a}}$ & .454 & 26.518 & 28.301 \\
& 4W 64A & $27.226^{\mathrm{a}}$ & .454 & 26.334 & 28.117 \\
& 5W 80A & $27.738^{\mathrm{a}}$ & .454 & 26.847 & 28.630 \\
\hline
\end{tabular}

a. Based on modified population marginal mean.

The plots below represent PMP and RMP from left to right, respectively. No consistent patterns are observed between these two samples at this level of interaction. Evident difference in means can be estimated at $1 \mathrm{~W} 0 \mathrm{~A}, 2 \mathrm{~W} 32$ and $3 \mathrm{~W} 48$ for both samples. 

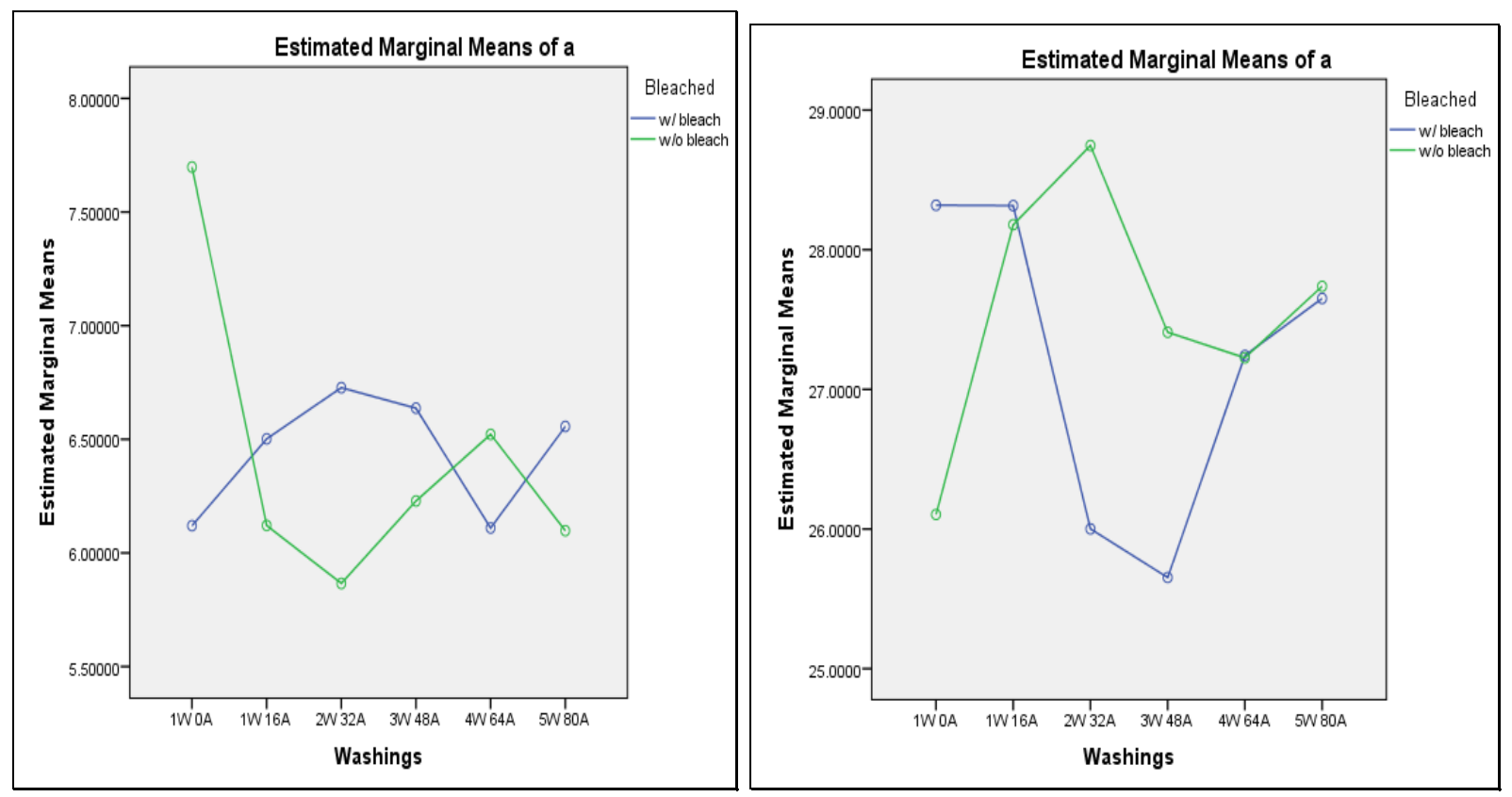

8. Swatch ${ }^{*}$ Washings: PMP

Dependent Variable: a

\begin{tabular}{|c|c|c|c|c|c|}
\hline \multirow[b]{2}{*}{ Swatch } & & \multirow[b]{2}{*}{ Mean } & \multirow[b]{2}{*}{ Std. Error } & \multicolumn{2}{|c|}{$95 \%$ Confidence Interval } \\
\hline & & & & Lower Bound & Upper Bound \\
\hline \multirow[t]{6}{*}{$1 \mathrm{w} /$ bleach } & $1 \mathrm{~W} 0 \mathrm{~A}$ & $5.449^{\mathrm{a}}$ & .288 & 4.883 & 6.014 \\
\hline & $1 \mathrm{~W} 16 \mathrm{~A}$ & $6.386^{\mathrm{a}}$ & .204 & 5.986 & 6.786 \\
\hline & $2 W 32 A$ & $7.438^{a}$ & .204 & 7.038 & 7.838 \\
\hline & $3 W 48 A$ & $6.697^{\mathrm{a}}$ & .204 & 6.297 & 7.097 \\
\hline & $4 \mathrm{~W} 64 \mathrm{~A}$ & $5.702^{a}$ & .204 & 5.302 & 6.102 \\
\hline & $5 W 80 A$ & $6.906^{a}$ & .204 & 6.506 & 7.306 \\
\hline \multirow[t]{6}{*}{2 w/ bleach } & $1 \mathrm{~W} 0 \mathrm{~A}$ & $6.791^{a}$ & .288 & 6.225 & 7.356 \\
\hline & $1 \mathrm{~W} 16 \mathrm{~A}$ & $6.618^{a}$ & .204 & 6.218 & 7.018 \\
\hline & $2 W 32 A$ & $6.016^{\mathrm{a}}$ & .204 & 5.617 & 6.416 \\
\hline & $3 W 48 A$ & $6.577^{\mathrm{a}}$ & .204 & 6.177 & 6.977 \\
\hline & $4 \mathrm{~W} 64 \mathrm{~A}$ & $6.517^{\mathrm{a}}$ & .204 & 6.117 & 6.917 \\
\hline & $5 \mathrm{~W} 80 \mathrm{~A}$ & $6.207^{\mathrm{a}}$ & .204 & 5.807 & 6.607 \\
\hline \multirow[t]{6}{*}{3 w/o bleach } & $1 \mathrm{~W} 0 \mathrm{~A}$ & $7.510^{\mathrm{a}}$ & .288 & 6.945 & 8.076 \\
\hline & $1 \mathrm{~W} 16 \mathrm{~A}$ & $5.828^{a}$ & .204 & 5.428 & 6.228 \\
\hline & $2 W 32 A$ & $6.067^{\mathrm{a}}$ & .204 & 5.667 & 6.467 \\
\hline & $3 W 48 A$ & $6.843^{a}$ & .204 & 6.443 & 7.243 \\
\hline & $4 \mathrm{~W} 64 \mathrm{~A}$ & $6.040^{\mathrm{a}}$ & .204 & 5.640 & 6.440 \\
\hline & $5 W 80 A$ & $6.364^{a}$ & .204 & 5.964 & 6.764 \\
\hline \multirow[t]{6}{*}{4 w/o bleach } & $1 \mathrm{~W} 0 \mathrm{~A}$ & $7.886^{\mathrm{a}}$ & .288 & 7.320 & 8.451 \\
\hline & $1 \mathrm{~W} 16 \mathrm{~A}$ & $6.414^{\mathrm{a}}$ & .204 & 6.014 & 6.814 \\
\hline & $2 W 32 A$ & $5.665^{a}$ & .204 & 5.265 & 6.065 \\
\hline & $3 W 48 A$ & $5.614^{a}$ & .204 & 5.215 & 6.014 \\
\hline & $4 W 64 A$ & $7.004^{a}$ & .204 & 6.604 & 7.404 \\
\hline & $5 W 80 A$ & $5.832^{a}$ & .204 & 5.432 & 6.231 \\
\hline
\end{tabular}

a. Based on modified population marginal mean. 
Dependent Variable: a

\section{Swatch * Washings: RMP}

\begin{tabular}{|l|r|r|r|}
\hline & & & \multicolumn{2}{|c|}{ 95\% Confidence Interval } \\
\cline { 3 - 5 } Swatch & & & Upper \\
Bound
\end{tabular}

a. Based on modified population marginal mean.
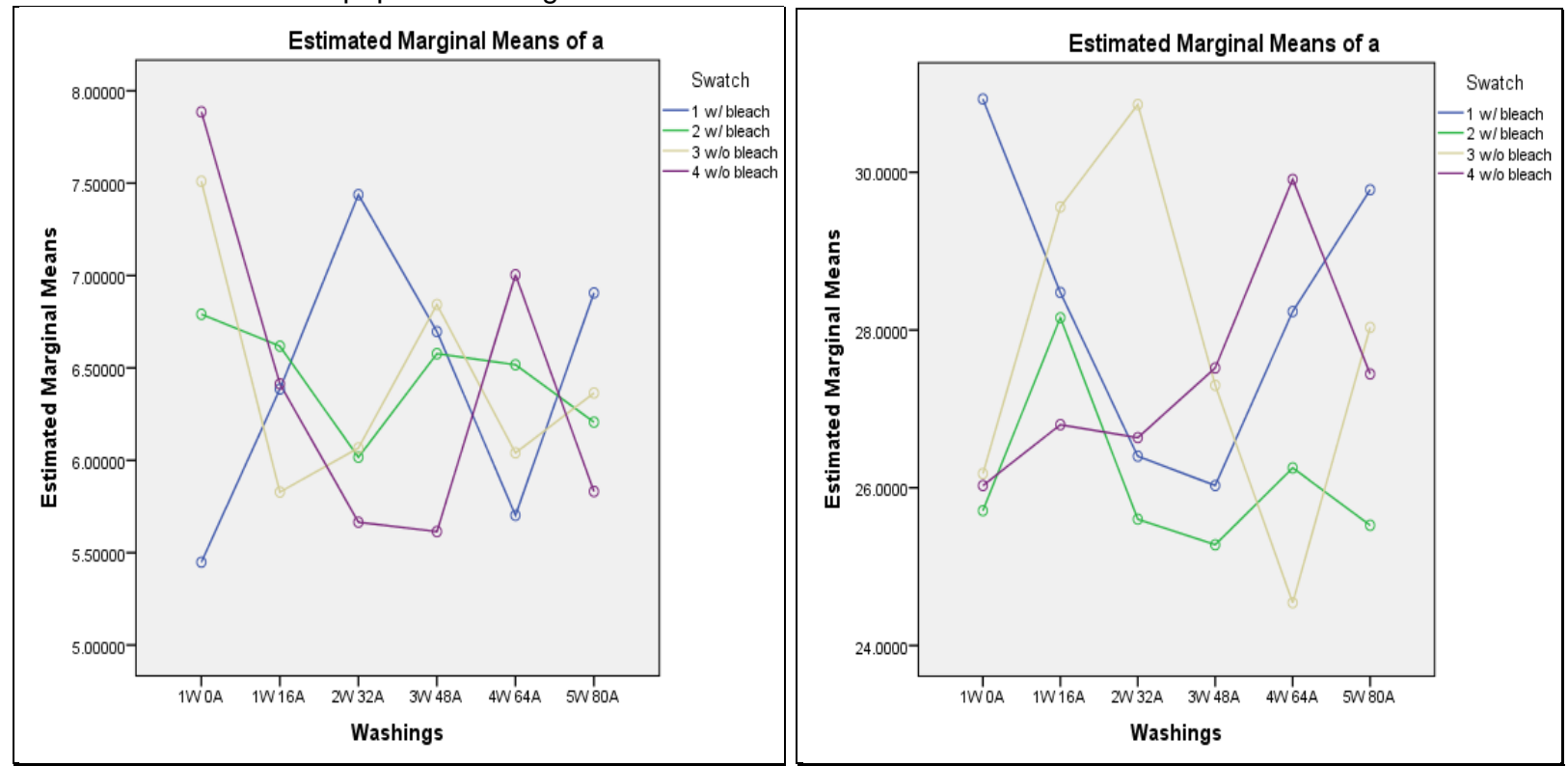

It is clear that the patterns for these two reference samples PMP (1), RMP (r) are not consistent.

In both samples, the patterns of each swatch deviate through the progression of the cycles. 
Variable ' $b$ '

Tests of Between-Subjects Effects: PMP

Dependent Variable: $b$

\begin{tabular}{|c|c|c|c|c|c|}
\hline Source & Type III Sum of Squares & $d f$ & Mean Square & $\mathrm{F}$ & Sig. \\
\hline Corrected Model & $2434.4^{\mathrm{a}}$ & 43 & 56.614 & 8.042 & .000 \\
\hline Intercept & 63800 & 1 & 63800 & 9062.2 & 0.000 \\
\hline SampleArea & 3.905 & 1 & 3.905 & .555 & .457 \\
\hline Swatch & 123.60 & 2 & 61.804 & 8.779 & .000 \\
\hline Bleached & 0.000 & 0 & & & \\
\hline Washings & 498.64 & 5 & 99.728 & 14.166 & .000 \\
\hline SampleArea * Swatch & 7.230 & 2 & 3.615 & .513 & .599 \\
\hline SampleArea * Bleached & 0.000 & 0 & & & \\
\hline SampleArea * Washings & 314.55 & 4 & 78.637 & 11.170 & .000 \\
\hline Swatch * Bleached & 0.000 & 0 & & & \\
\hline Swatch * Washings & 597.81 & 8 & 74.726 & 10.614 & .000 \\
\hline Bleached * Washings & 0.000 & 0 & & & \\
\hline SampleArea * Swatch * Bleached & 0.000 & 0 & & & \\
\hline SampleArea * Swatch * Washings & 449.98 & 8 & 56.248 & 7.990 & .000 \\
\hline SampleArea * Bleached * Washings & 0.000 & 0 & & & \\
\hline Swatch * Bleached * Washings & 0.000 & 0 & & & \\
\hline SampleArea * Swatch * Bleached * Washings & 0.000 & 0 & & & \\
\hline Error & 5885.5 & 836 & 7.040 & & \\
\hline Total & 75326 & 880 & & & \\
\hline Corrected Total & 8320.0 & 879 & & & \\
\hline
\end{tabular}

a. $\mathrm{R}$ Squared $=.293$ (Adjusted R Squared $=.256$ )

b. Computed using alpha $=.05$

Tests of Between-Subjects Effects: RMP

Dependent Variable: $b$

\begin{tabular}{|c|c|c|c|c|c|}
\hline Source & Type III Sum of Squares & df & Mean Square & $\mathrm{F}$ & Sig. \\
\hline Corrected Model & $3074.21^{a}$ & 43 & 71.493 & 6.198 & .000 \\
\hline Intercept & 203556 & 1 & 203556 & 17648 & 0.000 \\
\hline SampleArea & 17.281 & 1 & 17.281 & 1.498 & .221 \\
\hline Swatch & 106.82 & 2 & 53.409 & 4.631 & .010 \\
\hline Bleached & 0.000 & 0 & & & \\
\hline Washings & 128.04 & 5 & 25.607 & 2.220 & .050 \\
\hline SampleArea * Swatch & 271.89 & 2 & 135.944 & 11.786 & .000 \\
\hline SampleArea * Bleached & 0.000 & 0 & & & \\
\hline SampleArea * Washings & 435.65 & 4 & 108.913 & 9.443 & .000 \\
\hline Swatch * Bleached & 0.000 & 0 & & & \\
\hline Swatch * Washings & 495.07 & 8 & 61.883 & 5.365 & .000 \\
\hline Bleached * Washings & 0.000 & 0 & & & \\
\hline SampleArea * Swatch * Bleached & 0.000 & 0 & & & \\
\hline SampleArea * Swatch * Washings & 471.37 & 8 & 58.922 & 5.108 & .000 \\
\hline SampleArea * Bleached * Washings & 0.000 & 0 & & & \\
\hline Swatch * Bleached * Washings & 0.000 & 0 & & & \\
\hline SampleArea * Swatch * Bleached * Washings & 0.000 & 0 & & & \\
\hline Error & 9642.5 & 836 & 11.534 & & \\
\hline Total & 230906 & 880 & & & \\
\hline Corrected Total & 12717 & 879 & & & \\
\hline
\end{tabular}

a. R Squared $=.242$ (Adjusted R Squared $=.203$ )

b. Computed using alpha $=.05$

The main effects of sample area were observed as insignificant between both the reference

samples. The main effect for washing was also observed insignificant for sample RMP. In

addition, Sample Area* Swatch interaction was also observed to be insignificant at sample PMP. 


\section{Swatch Estimates: RMP}

Dependent Variable: $b$

\begin{tabular}{|c|c|c|c|c|}
\hline \multirow[b]{2}{*}{ Swatch } & \multirow[b]{2}{*}{ Mean } & \multirow[b]{2}{*}{ Std. Error } & \multicolumn{2}{|c|}{$95 \%$ Confidence Interval } \\
\hline & & & Lower Bound & Upper Bound \\
\hline $1 \mathrm{w} /$ bleach & $15.993^{\mathrm{a}}$ & .229 & 15.544 & 16.443 \\
\hline 2 w/ bleach & $14.658^{a}$ & .229 & 14.208 & 15.107 \\
\hline 3 w/o bleach & $16.208^{a}$ & .229 & 15.759 & 16.658 \\
\hline 4 w/o bleach & $16.125^{\mathrm{a}}$ & .229 & 15.676 & 16.575 \\
\hline
\end{tabular}

a. Based on modified population marginal mean.

Dependent Variable: $b$

Pairwise Comparisons: RMP

\begin{tabular}{|c|c|c|c|c|c|c|}
\hline \multirow[b]{2}{*}{ (I) Swatch } & & \multirow[b]{2}{*}{$\begin{array}{c}\text { Mean } \\
\text { Difference (I-J) }\end{array}$} & \multirow[b]{2}{*}{ Std. Error } & \multirow[b]{2}{*}{ Sig. ${ }^{d}$} & \multicolumn{2}{|c|}{$\begin{array}{l}95 \% \text { Confidence Interval } \\
\text { for Difference }\end{array}$} \\
\hline & & & & & $\begin{array}{l}\text { Lower } \\
\text { Bound }\end{array}$ & Upper Bound \\
\hline \multirow[t]{3}{*}{$1 \mathrm{w} /$ bleach } & 2 w/ bleach & $1.335^{, b, \mathrm{c}}$ & .324 & .000 & .700 & 1.971 \\
\hline & 3 w/o bleach & $-.215^{\mathrm{b}, \mathrm{c}}$ & .324 & .507 & -.851 & .420 \\
\hline & 4 w/o bleach & $-.132^{\mathrm{b}, \mathrm{c}}$ & .324 & .684 & -.767 & .504 \\
\hline \multirow[t]{3}{*}{$2 \mathrm{w} /$ bleach } & $1 \mathrm{w} /$ bleach & $-1.335^{\star}, \mathrm{b}, \mathrm{c}$ & .324 & .000 & -1.971 & -.700 \\
\hline & 3 w/o bleach & $-1.551^{\pi, b, c}$ & .324 & .000 & -2.186 & -.915 \\
\hline & 4 w/o bleach & $-1.467^{\pi, b, c}$ & .324 & .000 & -2.103 & -.832 \\
\hline \multirow[t]{3}{*}{3 w/o bleach } & 1 w/ bleach & $.215^{\mathrm{b}, \mathrm{c}}$ & .324 & .507 & -.420 & .851 \\
\hline & 2 w/ bleach & $1.551^{\pi}, \mathrm{b}, \mathrm{c}$ & .324 & .000 & .915 & 2.186 \\
\hline & 4 w/o bleach & $.083^{\mathrm{b}, \mathrm{c}}$ & .324 & .797 & -.552 & .719 \\
\hline \multirow[t]{3}{*}{4 w/o bleach } & $1 \mathrm{w} /$ bleach & $.132^{\mathrm{b}, \mathrm{c}}$ & .324 & .684 & -.504 & .767 \\
\hline & $2 \mathrm{w} /$ bleach & $1.467^{\pi, b, c}$ & .324 & .000 & .832 & 2.103 \\
\hline & 3 w/o bleach & $-.083^{\mathrm{b}, \mathrm{c}}$ & .324 & .797 & -.719 & .552 \\
\hline
\end{tabular}

Based on estimated marginal means

*. The mean difference is significant at the .05 level.

b. An estimate of the modified population marginal mean (I).

c. An estimate of the modified population marginal mean (J).

d. Adjustment for multiple comparisons: Least Significant Difference (equivalent to no adjustments).

A significant main effect of swatch was only observed at sample RMP. Only at swatch 2 was

significant values observed; at all other levels of interactions, no significant mean difference was

observed.

\section{Washing Estimates: PMP}

Dependent Variable: $b$

\begin{tabular}{|c|c|c|c|c|}
\hline \multirow[b]{2}{*}{ Washings } & \multirow[b]{2}{*}{ Mean } & \multirow[b]{2}{*}{ Std. Error } & \multicolumn{2}{|c|}{ 95\% Confidence Interval } \\
\hline & & & Lower Bound & Upper Bound \\
\hline $1 \mathrm{~W} 0 \mathrm{~A}$ & $-9.522^{a}$ & .297 & -10.105 & -8.940 \\
\hline $1 \mathrm{~W} 16 \mathrm{~A}$ & $-8.504^{a}$ & .210 & -8.916 & -8.092 \\
\hline $2 W 32 A$ & $-8.371^{a}$ & .210 & -8.783 & -7.959 \\
\hline $3 W$ 48A & $-9.556^{a}$ & .210 & -9.967 & -9.144 \\
\hline $4 W 64 A$ & $-9.317^{a}$ & .210 & -9.729 & -8.906 \\
\hline $5 W 80 A$ & $-7.484^{a}$ & .210 & -7.896 & -7.072 \\
\hline
\end{tabular}


a. Based on modified population marginal mean.

Pairwise Comparisons: PMP

Dependent Variable: $b$

\begin{tabular}{|c|c|c|c|c|c|c|}
\hline \multirow{2}{*}{\multicolumn{2}{|c|}{ (I) Washings }} & \multirow[b]{2}{*}{ Mean Difference (I-J) } & \multirow[b]{2}{*}{ Std. Error } & \multirow[b]{2}{*}{ Sig. ${ }^{d}$} & \multicolumn{2}{|c|}{$\begin{array}{l}\text { 95\% Confidence Interval for } \\
\text { Difference }^{d}\end{array}$} \\
\hline & & & & & Lower Bound & Upper Bound \\
\hline $1 \mathrm{~W} 0 \mathrm{~A}$ & $\begin{array}{l}1 \mathrm{~W} 16 \mathrm{~A} \\
2 \mathrm{~W} 32 \mathrm{~A} \\
3 \mathrm{~W} 48 \mathrm{~A} \\
4 \mathrm{~W} 64 \mathrm{~A} \\
5 \mathrm{~W} 80 \mathrm{~A}\end{array}$ & $\begin{array}{r}-1.018^{*, \mathrm{~b}, \mathrm{c}} \\
-1.151^{*, \mathrm{~b}, \mathrm{c}} \\
.033^{\mathrm{b}, \mathrm{c}} \\
-.205^{\mathrm{b}, \mathrm{c}} \\
-2.038^{*, \mathrm{~b}, \mathrm{c}}\end{array}$ & $\begin{array}{l}.363 \\
.363 \\
.363 \\
.363 \\
.363\end{array}$ & $\begin{array}{l}.005 \\
.002 \\
.927 \\
.573 \\
.000\end{array}$ & $\begin{array}{r}-1.731 \\
-1.865 \\
-.680 \\
-.918 \\
-2.751\end{array}$ & $\begin{array}{r}-.305 \\
-.438 \\
.747 \\
.508 \\
-1.325\end{array}$ \\
\hline \multirow[t]{3}{*}{$1 \mathrm{~W} 16 \mathrm{~A}$} & $\begin{array}{l}1 \mathrm{~W} 0 \mathrm{~A} \\
2 \mathrm{~W} 32 \mathrm{~A}\end{array}$ & $\begin{array}{r}1.018^{\star, b, c} \\
-.133^{\mathrm{b}, \mathrm{c}} \\
1052^{\pi, \mathrm{b}, \mathrm{c}}\end{array}$ & $\begin{array}{l}.363 \\
.297 \\
297\end{array}$ & $\begin{array}{l}.005 \\
.654 \\
.050\end{array}$ & $\begin{array}{r}.305 \\
-.715 \\
469\end{array}$ & $\begin{array}{r}1.731 \\
.449 \\
1.634\end{array}$ \\
\hline & $\begin{array}{l}3 \mathrm{~W} \text { 48A } \\
4 \mathrm{~W} 64 \mathrm{~A}\end{array}$ & $.813^{*, b, c}$ & $\begin{array}{l}.291 \\
.297\end{array}$ & .000 & $\begin{array}{l}.469 \\
.231\end{array}$ & $\begin{array}{l}1.634 \\
1.396\end{array}$ \\
\hline & $5 W 80 A$ & $-1.020^{\pi, b, c}$ & .297 & .001 & -1.602 & -.438 \\
\hline \multirow[t]{4}{*}{$2 W 32 A$} & 1W OA & $1.151^{\mathrm{x}, \mathrm{b}, \mathrm{c}}$ & .363 & .002 & .438 & 1.865 \\
\hline & $\begin{array}{l}1 \mathrm{~W} 16 \mathrm{~A} \\
3 \mathrm{~W} 48 \mathrm{~A}\end{array}$ & $1.185^{\star, b, c}$ & $\begin{array}{l}.291 \\
.297\end{array}$ & $\begin{array}{l}.654 \\
.000\end{array}$ & $\begin{array}{r}-.449 \\
.603\end{array}$ & $\begin{array}{l}.715 \\
1.767\end{array}$ \\
\hline & $4 W 64 A$ & $.946^{\pi, b, c}$ & .297 & .001 & .364 & 1.529 \\
\hline & $5 \mathrm{~W} 80 \mathrm{~A}$ & $-.887^{x, b, c}$ & .297 & .003 & -1.469 & -.304 \\
\hline \multirow[t]{5}{*}{$3 W$ 48A } & $1 \mathrm{~W} 0 \mathrm{~A}$ & $-.033^{\mathrm{b}, \mathrm{c}}$ & .363 & .927 & -.747 & .680 \\
\hline & $1 \mathrm{~W} 16 \mathrm{~A}$ & $-1.052^{x, b, c}$ & .297 & .000 & -1.634 & -.469 \\
\hline & $2 W 32 A$ & $-1.185^{\circ, \mathrm{b}, \mathrm{c}}$ & .297 & .000 & -1.767 & -.603 \\
\hline & $4 \mathrm{~W} 64 \mathrm{~A}$ & $-.238^{\mathrm{b}, \mathrm{c}}$ & .297 & .422 & -.821 & .344 \\
\hline & $5 W$ 80A & $-2.071^{\star}, \mathrm{b}, \mathrm{c}$ & .297 & .000 & -2.654 & -1.489 \\
\hline \multirow[t]{5}{*}{$4 \mathrm{~W} 64 \mathrm{~A}$} & $1 \mathrm{~W} 0 \mathrm{~A}$ & $.205^{\mathrm{b}, \mathrm{c}}$ & .363 & .573 & -.508 & .918 \\
\hline & $1 \mathrm{~W} 16 \mathrm{~A}$ & $-.813^{x, \mathrm{~b}, \mathrm{c}}$ & .297 & .006 & -1.396 & -.231 \\
\hline & $2 W 32 A$ & $-.946^{x}, \mathrm{~b}, \mathrm{c}$ & .297 & .001 & -1.529 & -.364 \\
\hline & $3 W 48 A$ & $.238^{\mathrm{b}, \mathrm{c}}$ & .297 & .422 & -.344 & .821 \\
\hline & $5 W$ 80A & $-1.833^{x, b, c}$ & .297 & .000 & -2.415 & -1.251 \\
\hline \multirow[t]{5}{*}{$5 W$ 80A } & $1 \mathrm{~W} 0 \mathrm{~A}$ & $2.038^{\circ, b, c}$ & .363 & .000 & 1.325 & 2.751 \\
\hline & $1 \mathrm{~W} 16 \mathrm{~A}$ & $1.020^{\pi, b, c}$ & .297 & .001 & .438 & 1.602 \\
\hline & $2 W 32 A$ & $.887^{*, b, c}$ & .297 & .003 & .304 & 1.469 \\
\hline & $3 W 48 A$ & $2.071^{*}, \mathrm{~b}, \mathrm{c}$ & .297 & .000 & 1.489 & 2.654 \\
\hline & $4 W 64 A$ & $1.833^{*}, \mathrm{~b}, \mathrm{c}$ & .297 & .000 & 1.251 & 2.415 \\
\hline
\end{tabular}

Based on estimated marginal means

*. The mean difference is significant at the .05 level.

b. An estimate of the modified population marginal mean (I).

c. An estimate of the modified population marginal mean (J).

d. Adjustment for multiple comparisons: Least Significant Difference (equivalent to no adjustments).

\section{Washing Estimates: RMP}

Dependent Variable: b

\begin{tabular}{|c|c|c|c|c|}
\hline \multirow[b]{2}{*}{ Washings } & \multirow[b]{2}{*}{ Mean } & \multirow[b]{2}{*}{ Std. Error } & \multicolumn{2}{|c|}{$95 \%$ Confidence Interval } \\
\hline & & & Lower Bound & Upper Bound \\
\hline $1 \mathrm{~W} \mathrm{0A}$ & $16.132^{\mathrm{a}}$ & .380 & 15.387 & 16.878 \\
\hline $1 \mathrm{~W} 16 \mathrm{~A}$ & $16.218^{a}$ & .268 & 15.691 & 16.745 \\
\hline $2 W 32 A$ & $15.737^{\mathrm{a}}$ & .268 & 15.210 & 16.264 \\
\hline $3 W 48 A$ & $15.093^{\mathrm{a}}$ & .268 & 14.566 & 15.620 \\
\hline $4 W 64 A$ & $15.466^{\mathrm{a}}$ & .268 & 14.939 & 15.993 \\
\hline $5 W 80 A$ & $16.023^{a}$ & .268 & 15.496 & 16.550 \\
\hline
\end{tabular}

a. Based on modified population marginal mean. 
Dependent Variable: $b$

Pairwise Comparisons: RMP

\begin{tabular}{|c|c|c|c|c|c|c|}
\hline \multirow{2}{*}{\multicolumn{2}{|c|}{ (I) Washings }} & \multirow[b]{2}{*}{ Mean Difference (I-J) } & \multirow[b]{2}{*}{ Std. Error } & \multirow[b]{2}{*}{ Sig. ${ }^{d}$} & \multicolumn{2}{|c|}{$\begin{array}{c}\text { 95\% Confidence Interval for } \\
\text { Difference }^{d}\end{array}$} \\
\hline & & & & & Lower Bound & Upper Bound \\
\hline \multirow[t]{5}{*}{$1 \mathrm{~W} 0 \mathrm{~A}$} & $1 \mathrm{~W} 16 \mathrm{~A}$ & $-.086^{\mathrm{a}, \mathrm{b}}$ & .465 & .854 & -.999 & .827 \\
\hline & $2 W 32 A$ & $.395^{\mathrm{a}, \mathrm{b}}$ & .465 & .396 & -.518 & 1.308 \\
\hline & $3 \mathrm{~W} 48 \mathrm{~A}$ & $1.040^{\mathrm{a}, \mathrm{b},{ }^{*}}$ & .465 & .026 & .127 & 1.952 \\
\hline & $4 \mathrm{~W} 64 \mathrm{~A}$ & $.666^{\mathrm{a}, \mathrm{b}}$ & .465 & .153 & -.247 & 1.579 \\
\hline & $5 W 80 A$ & $.110^{\mathrm{a}, \mathrm{b}}$ & .465 & .814 & -.803 & 1.023 \\
\hline \multirow[t]{5}{*}{$1 \mathrm{~W} 16 \mathrm{~A}$} & $1 \mathrm{~W} 0 \mathrm{~A}$ & $.086^{\mathrm{a}, \mathrm{b}}$ & .465 & .854 & -.827 & .999 \\
\hline & $2 W 32 A$ & $.481^{\mathrm{a}, \mathrm{b}}$ & .380 & .206 & -.264 & 1.226 \\
\hline & $3 W 48 A$ & $1.125^{\mathrm{a}, \mathrm{b},{ }^{*}}$ & .380 & .003 & .380 & 1.871 \\
\hline & $4 \mathrm{~W} 64 \mathrm{~A}$ & $.752^{\mathrm{a}, \mathrm{b},{ }^{*}}$ & .380 & .048 & .007 & 1.497 \\
\hline & $5 W 80 A$ & $.196^{\mathrm{a}, \mathrm{b}}$ & .380 & .607 & -.550 & .941 \\
\hline \multirow[t]{5}{*}{$2 W 32 A$} & $1 \mathrm{~W} 0 \mathrm{~A}$ & $-.395^{\mathrm{a}, \mathrm{b}}$ & .465 & .396 & -1.308 & .518 \\
\hline & $1 \mathrm{~W} 16 \mathrm{~A}$ & $-.481^{\mathrm{a}, \mathrm{b}}$ & .380 & .206 & -1.226 & .264 \\
\hline & $3 W 48 A$ & $.645^{\mathrm{a}, \mathrm{b}}$ & .380 & .090 & -.101 & 1.390 \\
\hline & $4 \mathrm{~W} 64 \mathrm{~A}$ & $.271^{\mathrm{a}, \mathrm{b}}$ & .380 & .476 & -.474 & 1.016 \\
\hline & $5 \mathrm{~W} 80 \mathrm{~A}$ & $-.285^{\mathrm{a}, \mathrm{b}}$ & .380 & .453 & -1.031 & .460 \\
\hline \multirow[t]{5}{*}{$3 W$ 48A } & $1 \mathrm{~W} 0 \mathrm{~A}$ & $-1.040^{\mathrm{a}, \mathrm{b},{ }^{\star}}$ & .465 & .026 & -1.952 & -.127 \\
\hline & $1 \mathrm{~W} 16 \mathrm{~A}$ & $-1.125^{\mathrm{a}, \mathrm{b},{ }^{\star}}$ & .380 & .003 & -1.871 & -.380 \\
\hline & $2 W 32 A$ & $-.645^{\mathrm{a}, \mathrm{b}}$ & .380 & .090 & -1.390 & .101 \\
\hline & $4 \mathrm{~W} 64 \mathrm{~A}$ & $-.374^{\mathrm{a}, \mathrm{b}}$ & .380 & .325 & -1.119 & .372 \\
\hline & $5 W 80 A$ & $-.930^{\mathrm{a}, \mathrm{b},{ }^{*}}$ & .380 & .015 & -1.675 & -.185 \\
\hline \multirow[t]{5}{*}{$4 \mathrm{~W} 64 \mathrm{~A}$} & $1 \mathrm{~W} 0 \mathrm{~A}$ & $-.666^{\mathrm{a}, \mathrm{b}}$ & .465 & .153 & -1.579 & .247 \\
\hline & $1 \mathrm{~W} 16 \mathrm{~A}$ & $-.752^{\mathrm{a}, \mathrm{b},{ }^{*}}$ & .380 & .048 & -1.497 & -.007 \\
\hline & $2 W 32 A$ & $-.271^{\mathrm{a}, \mathrm{b}}$ & .380 & .476 & -1.016 & .474 \\
\hline & $3 W 48 A$ & $.374^{a, b}$ & .380 & .325 & -.372 & 1.119 \\
\hline & $5 W 80 A$ & $-.556^{a, b}$ & .380 & .143 & -1.302 & .189 \\
\hline \multirow[t]{5}{*}{$5 W$} & $1 \mathrm{~W} 0 \mathrm{~A}$ & $-.110^{a, b}$ & .465 & .814 & -1.023 & .803 \\
\hline & $1 \mathrm{~W} 16 \mathrm{~A}$ & $-.196^{a, b}$ & .380 & .607 & -.941 & .550 \\
\hline & $2 W 32 A$ & $.285^{\mathrm{a}, \mathrm{b}}$ & .380 & .453 & -.460 & 1.031 \\
\hline & $3 W 48 A$ & $.930^{\mathrm{a}, \mathrm{b},{ }^{*}}$ & .380 & .015 & .185 & 1.675 \\
\hline & $4 \mathrm{~W} 64 \mathrm{~A}$ & $.556^{\mathrm{a}, \mathrm{b}}$ & .380 & .143 & -.189 & 1.302 \\
\hline
\end{tabular}

Based on estimated marginal means

*. The mean difference is significant at the .05 level.

a. An estimate of the modified population marginal mean (I).

b. An estimate of the modified population marginal mean $(\mathrm{J})$.

d. Adjustment for multiple comparisons: Least Significant Difference (equivalent to no adjustments).

A greater amount of marked significant differences was observed in sample PMP, whereas only

a few significant values were observed for sample RMP. In fact, for sample PMP, all the groups

observed significant results but not at all pair-wise groups. In contrast, no significant mean

differences were observed at 2W 32A for sample RMP. 
7. Bleached * Washings: PMP

Dependent Variable: $b$

\begin{tabular}{|ll|r|r|r|r|}
\hline & & & & \multicolumn{2}{|c|}{$95 \%$ Confidence Interval } \\
\cline { 4 - 5 } Bleached & & Mean & Std. Error & Lower Bound & Upper Bound \\
\hline w/ bleach & 1W 0A & $-8.696^{\mathrm{a}}$ & .420 & -9.519 & -7.872 \\
& 1W 16A & $-8.451^{\mathrm{a}}$ & .297 & -9.033 & -7.869 \\
& 2W 32A & $-8.533^{\mathrm{a}}$ & .297 & -9.116 & -7.951 \\
& 3W 48A & $-9.371^{\mathrm{a}}$ & .297 & -9.953 & -8.788 \\
& 4W 64A & $-9.098^{\mathrm{a}}$ & .297 & -9.680 & -8.515 \\
w/o bleach & 5W 80A & $-7.463^{\mathrm{a}}$ & .297 & -8.045 & -6.880 \\
& 1W 0A & $-10.349^{\mathrm{a}}$ & .420 & -11.172 & -9.525 \\
& 1W 16A & $-8.557^{\mathrm{a}}$ & .297 & -9.139 & -7.975 \\
& 2W 32A & $-8.208^{\mathrm{a}}$ & .297 & -8.791 & -7.626 \\
& 3W 48A & $-9.741^{\mathrm{a}}$ & .297 & -10.323 & -9.158 \\
& 4W 64A & $-9.537^{\mathrm{a}}$ & .297 & -10.119 & -8.955 \\
& 5W 80A & $-7.506^{\mathrm{a}}$ & .297 & -8.088 & -6.923 \\
\hline
\end{tabular}

a. Based on modified population marginal mean.

\section{Bleached * Washings: RMP}

Dependent Variable: $b$

\begin{tabular}{|ll|r|r|r|r|}
\hline & & & & \multicolumn{2}{|c|}{ 95\% Confidence Interval } \\
\cline { 3 - 5 } Bleached & & Mean & Std. Error & Lower Bound & Uper \\
Bound \\
\hline w/ bleach & 1W 0A & $16.650^{\mathrm{a}}$ & .537 & 15.596 & 17.704 \\
& 1W 16A & $15.831^{\mathrm{a}}$ & .380 & 15.086 & 16.576 \\
& 2W 32A & $15.022^{\mathrm{a}}$ & .380 & 14.276 & 15.767 \\
& 3W 48A & $13.999^{\mathrm{a}}$ & .380 & 13.254 & 14.745 \\
& 4W 64A & $15.541^{\mathrm{a}}$ & .380 & 14.796 & 16.286 \\
w/o bleach & 5W 80A & $15.573^{\mathrm{a}}$ & .380 & 14.828 & 16.318 \\
& 1W 0A & $15.615^{\mathrm{a}}$ & .537 & 14.561 & 16.669 \\
& 1W 16A & $16.605^{\mathrm{a}}$ & .380 & 15.860 & 17.351 \\
& 2W 32A & $16.453^{\mathrm{a}}$ & .380 & 15.708 & 17.198 \\
& 3W 48A & $16.186^{\mathrm{a}}$ & .380 & 15.441 & 16.932 \\
& 4W 64A & $15.392^{\mathrm{a}}$ & .380 & 14.647 & 16.137 \\
& 5W 80A & $16.473^{\mathrm{a}}$ & .380 & 15.727 & 17.218 \\
\hline
\end{tabular}

a. Based on modified population marginal mean.
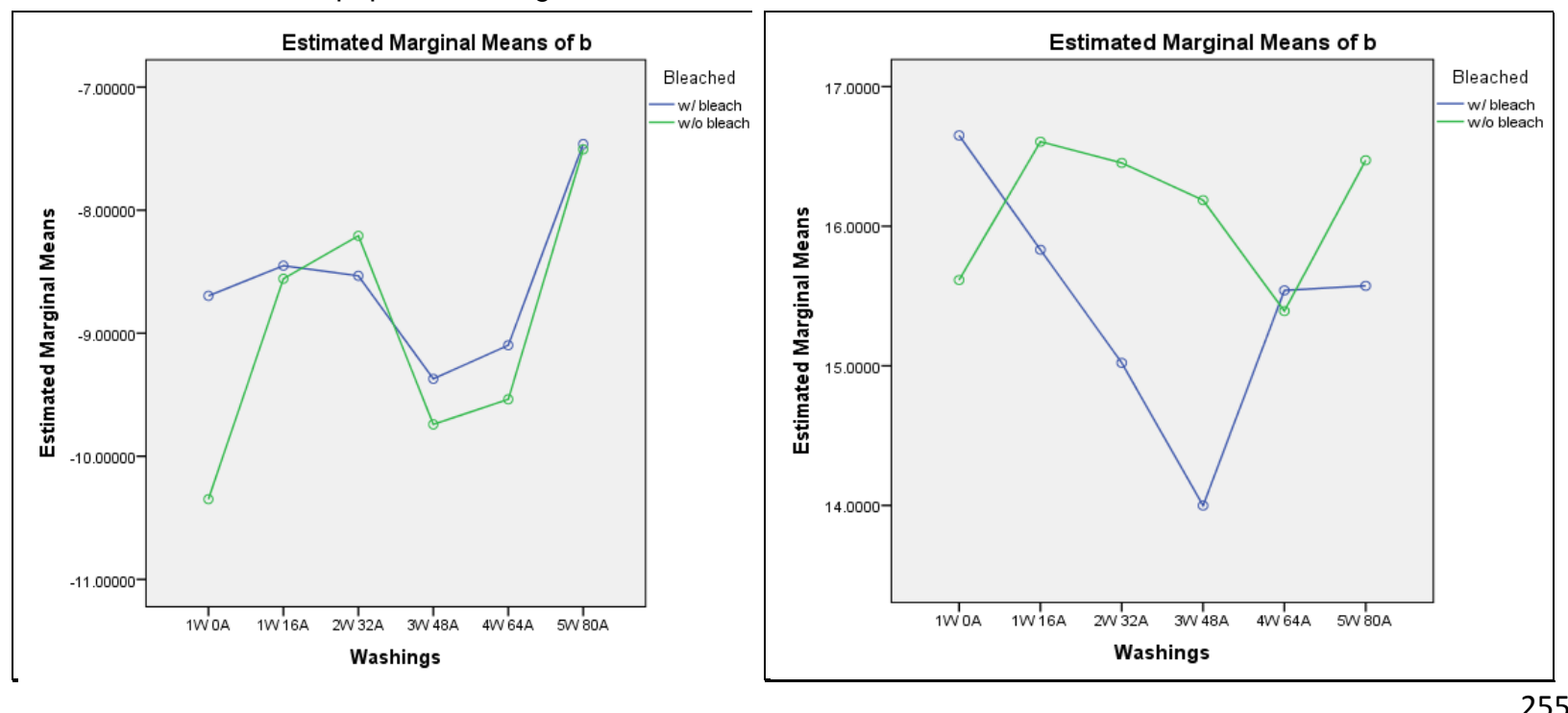
The plots above represent PMP and RMP from left to right, respectively. Overall, this interaction diagram shows a similar pattern for reference sample PMP. Apart from the untreated sample at $1 \mathrm{~W} 0 \mathrm{~A}$, the remaining pattern between the bleached and unbleached samples was consistent. It can also be observed that at $5 \mathrm{~W} 80 \mathrm{~A}$, the mean values increases for both samples.

Based on the swatch * washing interaction below, swatches 3 and 4 observed similar patterns between both reference samples.

Dependent Variable: $b$

\section{Swatch * Washings: PMP}

\begin{tabular}{|c|c|c|c|c|c|}
\hline \multirow[b]{2}{*}{ Swatch } & & \multirow[b]{2}{*}{ Mean } & \multirow[b]{2}{*}{ Std. Error } & \multicolumn{2}{|c|}{ 95\% Confidence Interval } \\
\hline & & & & Lower Bound & $\begin{array}{l}\text { Upper } \\
\text { Bound }\end{array}$ \\
\hline \multirow[t]{6}{*}{$1 \mathrm{w} /$ bleach } & $1 \mathrm{~W} 0 \mathrm{~A}$ & $-7.110^{\mathrm{a}}$ & .593 & -8.274 & -5.945 \\
\hline & $1 \mathrm{~W} 16 \mathrm{~A}$ & $-8.476^{a}$ & .420 & -9.300 & -7.653 \\
\hline & $2 W 32 A$ & $-10.544^{a}$ & .420 & -11.367 & -9.720 \\
\hline & $3 W 48 A$ & $-8.939^{a}$ & .420 & -9.762 & -8.115 \\
\hline & $4 \mathrm{~W} 64 \mathrm{~A}$ & $-7.589^{a}$ & .420 & -8.412 & -6.765 \\
\hline & $5 W 80 A$ & $-6.987^{a}$ & .420 & -7.811 & -6.164 \\
\hline \multirow[t]{6}{*}{$2 \mathrm{w} /$ bleach } & $1 \mathrm{~W} 0 \mathrm{~A}$ & $-10.282^{a}$ & .593 & -11.447 & -9.118 \\
\hline & $1 \mathrm{~W} 16 \mathrm{~A}$ & $-8.425^{a}$ & .420 & -9.249 & -7.602 \\
\hline & $2 W 32 A$ & $-6.523^{\mathrm{a}}$ & .420 & -7.346 & -5.699 \\
\hline & $3 W 48 A$ & $-9.802^{a}$ & .420 & -10.626 & -8.979 \\
\hline & $4 \mathrm{~W} 64 \mathrm{~A}$ & $-10.606^{a}$ & .420 & -11.430 & -9.783 \\
\hline & $5 W 80 A$ & $-7.938^{\mathrm{a}}$ & .420 & -8.762 & -7.115 \\
\hline \multirow[t]{6}{*}{3 w/o bleach } & $1 \mathrm{~W} \mathrm{OA}$ & $-11.383^{a}$ & .593 & -12.548 & -10.219 \\
\hline & $1 \mathrm{~W} 16 \mathrm{~A}$ & $-7.906^{a}$ & .420 & -8.729 & -7.082 \\
\hline & $2 W 32 A$ & $-7.283^{a}$ & .420 & -8.106 & -6.459 \\
\hline & $3 W 48 A$ & $-9.730^{a}$ & .420 & -10.554 & -8.907 \\
\hline & $4 W 64 A$ & $-9.357^{a}$ & .420 & -10.181 & -8.534 \\
\hline & $5 W 80 A$ & $-6.519^{a}$ & .420 & -7.343 & -5.696 \\
\hline \multirow[t]{6}{*}{4 w/o bleach } & $1 \mathrm{~W} 0 \mathrm{~A}$ & $-9.314^{a}$ & .593 & -10.478 & -8.149 \\
\hline & $1 \mathrm{~W} 16 \mathrm{~A}$ & $-9.208^{a}$ & .420 & -10.031 & -8.384 \\
\hline & $2 W 32 A$ & $-9.134^{a}$ & .420 & -9.958 & -8.311 \\
\hline & $3 W 48 A$ & $-9.751^{a}$ & .420 & -10.575 & -8.928 \\
\hline & $4 W 64 A$ & $-9.717^{a}$ & .420 & -10.540 & -8.893 \\
\hline & $5 W 80 A$ & $-8.492^{a}$ & .420 & -9.315 & -7.668 \\
\hline
\end{tabular}

a. Based on modified population marginal mean. 


\section{Swatch * Washings: RMP}

Dependent Variable: $b$

\begin{tabular}{|c|c|c|c|c|c|}
\hline \multirow[b]{2}{*}{ Swatch } & & \multirow[b]{2}{*}{ Mean } & \multirow[b]{2}{*}{ Std. Error } & \multicolumn{2}{|c|}{ 95\% Confidence Interval } \\
\hline & & & & $\begin{array}{l}\text { Lower } \\
\text { Bound }\end{array}$ & $\begin{array}{l}\text { Upper } \\
\text { Bound }\end{array}$ \\
\hline \multirow[t]{5}{*}{$1 \mathrm{w} /$ bleach } & $\begin{array}{l}1 \mathrm{~W} 0 \mathrm{~A} \\
1 \mathrm{~W} 16 \mathrm{~A}\end{array}$ & $\begin{array}{l}18.857^{\mathrm{a}} \\
16.311^{\mathrm{a}}\end{array}$ & $\begin{array}{r}.759 \\
.537\end{array}$ & $\begin{array}{l}17.367 \\
15.257\end{array}$ & $\begin{array}{l}20.348 \\
17.365\end{array}$ \\
\hline & $2 W 32 A$ & $15.477^{\mathrm{a}}$ & .537 & 14.423 & 16.531 \\
\hline & $3 W 48 A$ & $14.209^{\mathrm{a}}$ & .537 & 13.155 & 15.263 \\
\hline & $4 \mathrm{~W} 64 \mathrm{~A}$ & $15.656^{a}$ & .537 & 14.602 & 16.710 \\
\hline & $5 W 80 A$ & $16.882^{\mathrm{a}}$ & .537 & 15.828 & 17.936 \\
\hline \multirow[t]{6}{*}{2 w/ bleach } & $1 \mathrm{~W} 0 \mathrm{~A}$ & $14.443^{\mathrm{a}}$ & .759 & 12.952 & 15.933 \\
\hline & $1 \mathrm{~W} 16 \mathrm{~A}$ & $15.351^{\mathrm{a}}$ & .537 & 14.297 & 16.405 \\
\hline & $2 W 32 A$ & $14.566^{\mathrm{a}}$ & .537 & 13.512 & 15.620 \\
\hline & $3 W 48 A$ & $13.790^{\mathrm{a}}$ & .537 & 12.736 & 14.844 \\
\hline & $4 W 64 A$ & $15.426^{\mathrm{a}}$ & .537 & 14.372 & 16.480 \\
\hline & $5 W 80 A$ & $14.264^{\mathrm{a}}$ & .537 & 13.210 & 15.318 \\
\hline \multirow[t]{6}{*}{3 w/o bleach } & $1 \mathrm{~W} 0 \mathrm{~A}$ & $15.521^{\mathrm{a}}$ & .759 & 14.030 & 17.011 \\
\hline & $1 \mathrm{~W} 16 \mathrm{~A}$ & $17.141^{\mathrm{a}}$ & .537 & 16.087 & 18.195 \\
\hline & $2 W 32 A$ & $17.920^{\mathrm{a}}$ & .537 & 16.866 & 18.974 \\
\hline & $3 W 48 A$ & $16.280^{\mathrm{a}}$ & .537 & 15.226 & 17.334 \\
\hline & $4 W 64 A$ & $13.703^{a}$ & .537 & 12.649 & 14.757 \\
\hline & $5 W 80 A$ & $16.342^{\mathrm{a}}$ & .537 & 15.288 & 17.396 \\
\hline \multirow[t]{6}{*}{4 w/o bleach } & $1 \mathrm{~W} 0 \mathrm{~A}$ & $15.709^{a}$ & .759 & 14.219 & 17.200 \\
\hline & $1 \mathrm{~W} 16 \mathrm{~A}$ & $16.070^{\mathrm{a}}$ & .537 & 15.016 & 17.124 \\
\hline & $2 W 32 A$ & $14.987^{\mathrm{a}}$ & .537 & 13.933 & 16.041 \\
\hline & $3 W 48 A$ & $16.093^{a}$ & .537 & 15.039 & 17.147 \\
\hline & $4 W 64 A$ & $17.081^{\mathrm{a}}$ & .537 & 16.027 & 18.135 \\
\hline & $5 \mathrm{~W} 80 \mathrm{~A}$ & $16.603^{a}$ & .537 & 15.549 & 17.657 \\
\hline
\end{tabular}

a. Based on modified population marginal mean.
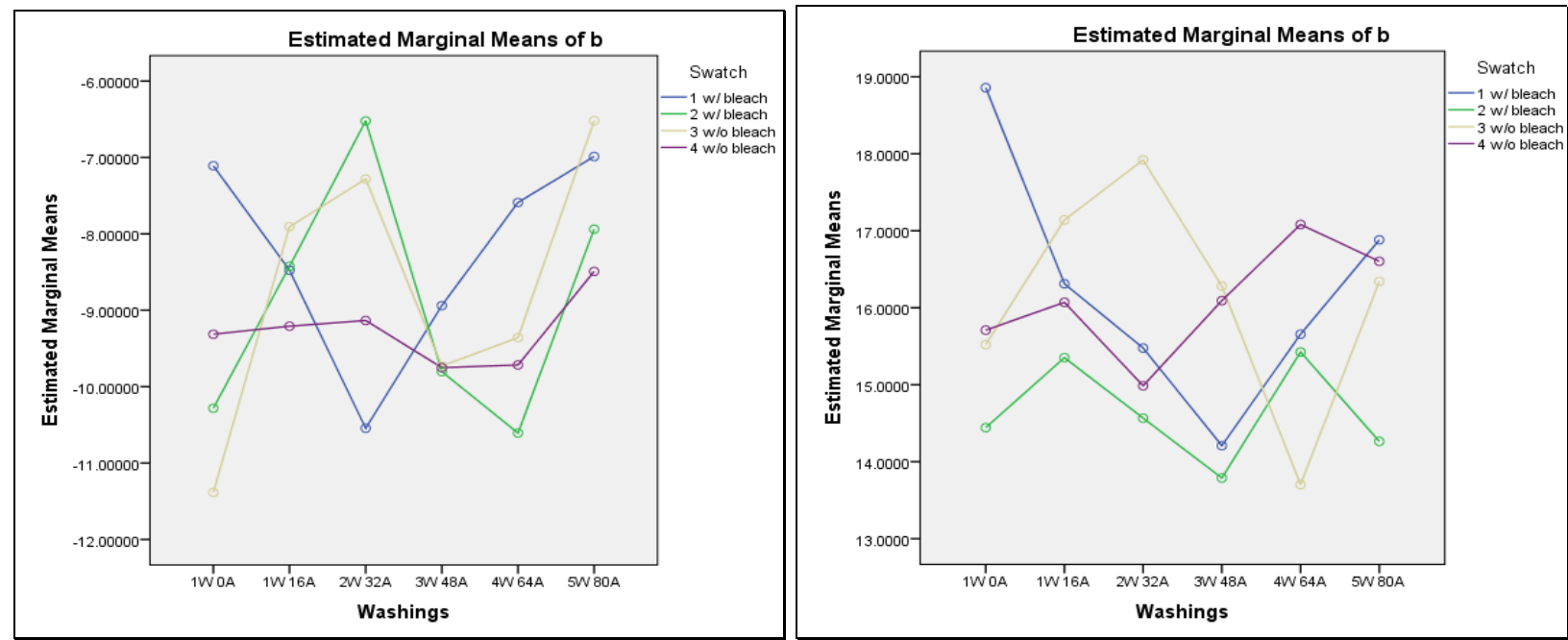

VII. Comparison of colors 
To discriminate between colors, color was chosen as an independent factor. The results of this multifactorial analysis will be discussed by dependent variables. For the interaction effects, only the interactions involving color as a factor were examined below. The Between-Subject Factors table is located in section of the appendix.

\section{Variable- L}

Dependent Variable: L

Tests of Between-Subjects Effects: all colors

\begin{tabular}{|c|c|c|c|c|c|}
\hline Source & Type III Sum of Squares & $\mathrm{df}$ & Mean Square & $\mathrm{F}$ & Sig. \\
\hline Corrected Model & $175509.408^{a}$ & 307 & 571.692 & 45.541 & .000 \\
\hline Intercept & 46512345 & 1 & 46512345.399 & 3705160.713 & .000 \\
\hline Swatch & 92.3 & 2 & 46.138 & 3.675 & .025 \\
\hline SampleArea & 43.9 & 1 & 43.935 & 3.500 & .061 \\
\hline Bleached & 0.0 & 0 & & & \\
\hline Washing & 1046.0 & 5 & 209.196 & 16.664 & .000 \\
\hline Color & 129614.6 & 6 & 21602.425 & 1720.843 & .000 \\
\hline Swatch * SampleArea & 8.8 & 2 & 4.410 & .351 & .704 \\
\hline Swatch * Bleached & 0.0 & 0 & & & \\
\hline Swatch * Washing & 519.4 & 8 & 64.919 & 5.171 & .000 \\
\hline Swatch * Color & 1124.9 & 12 & 93.742 & 7.467 & .000 \\
\hline SampleArea * Bleached & 0.0 & 0 & & & \\
\hline SampleArea * Washing & 182.9 & 4 & 45.735 & 3.643 & .006 \\
\hline SampleArea * Color & 328.6 & 6 & 54.774 & 4.363 & .000 \\
\hline Bleached * Washing & 0.0 & 0 & & & \\
\hline Bleached * Color & 0.0 & 0 & & & \\
\hline Washing * Color & 8562.2 & 30 & 285.407 & 22.735 & .000 \\
\hline Swatch * SampleArea * Bleached & 0.0 & 0 & & & \\
\hline Swatch * SampleArea * Washing & 1296.8 & 8 & 162.098 & 12.913 & .000 \\
\hline Swatch * SampleArea * Color & 1564.9 & 12 & 130.407 & 10.388 & .000 \\
\hline Swatch * Bleached * Washing & 0.0 & 0 & & & \\
\hline Swatch * Bleached * Color & 0.0 & 0 & & & \\
\hline Swatch * Washing * Color & 4761.0 & 48 & 99.188 & 7.901 & .000 \\
\hline SampleArea * Bleached * Washing & 0.0 & 0 & & & \\
\hline SampleArea * Bleached * Color & 0.0 & 0 & & & \\
\hline SampleArea * Washing * Color & 1848.4 & 24 & 77.018 & 6.135 & .000 \\
\hline Bleached * Washing * Color & 0.0 & 0 & & & \\
\hline Swatch * SampleArea * Bleached * Washing & 0.0 & 0 & & & \\
\hline Swatch * SampleArea * Bleached * Color & 0.0 & 0 & & & \\
\hline Swatch * SampleArea * Washing * Color & 5605.1 & 48 & 116.772 & 9.302 & .000 \\
\hline Swatch * Bleached * Washing * Color & 0.0 & 0 & & & \\
\hline SampleArea * Bleached * Washing * Color & 0.0 & 0 & & & \\
\hline Swatch * SampleArea * Bleached * Washing * & 0.0 & 0 & & & \\
\hline Color & & & & & \\
\hline Error & 73462.5 & 5852 & 12.553 & & \\
\hline Total & 49933424.0 & 6160 & & & \\
\hline Corrected Total & 248971.9 & 6159 & & & \\
\hline
\end{tabular}

a. $\mathrm{R}$ Squared $=.705$ (Adjusted R Squared $=.689$ )

Based on the results, the main effects of sample area and the interaction effects of Swatch *

Sample Area was not significant. 


\section{Sample Area Estimates: all colors}

Dependent Variable: L

\begin{tabular}{|l|r|r|r|r|}
\hline \multirow{2}{*}{} & & & \multicolumn{2}{|c|}{$\begin{array}{c}95 \% \text { Confidence } \\
\text { Interval }\end{array}$} \\
\cline { 4 - 5 } Sample Area & Mean & Std. Error & $\begin{array}{c}\text { Lower } \\
\text { Bound }\end{array}$ & $\begin{array}{c}\text { Upper } \\
\text { Bound }\end{array}$ \\
\hline A & $89.817^{\mathrm{a}}$ & .064 & 89.692 & 89.942 \\
B & $89.801^{\mathrm{a}}$ & .064 & 89.676 & 89.926 \\
\hline
\end{tabular}

a. Based on modified population marginal mean.

Pairwise Comparisons: all colors

Dependent Variable: L

\begin{tabular}{|c|c|c|c|c|c|c|}
\hline \multirow[b]{2}{*}{$\begin{array}{l}\text { (I) Sample } \\
\text { Area }\end{array}$} & \multirow[b]{2}{*}{$\begin{array}{l}\text { (J) Sample } \\
\text { Area }\end{array}$} & \multirow[b]{2}{*}{$\begin{array}{c}\text { Mean } \\
\text { Difference (I-J) }\end{array}$} & \multirow[b]{2}{*}{ Std. Error } & \multirow[b]{2}{*}{ Sig. ${ }^{\mathrm{C}}$} & \multicolumn{2}{|c|}{$\begin{array}{l}\text { 95\% Confidence Interval for } \\
\text { Difference }\end{array}$} \\
\hline & & & & & $\begin{array}{l}\text { Lower } \\
\text { Bound }\end{array}$ & $\begin{array}{l}\text { Upper } \\
\text { Bound }\end{array}$ \\
\hline$A$ & $\mathrm{~B}$ & $.016^{\mathrm{a}}, \mathrm{b}$ & .090 & .859 & -.161 & .193 \\
\hline$B$ & $A$ & $-.016^{a}, b$ & .090 & .859 & -.193 & .161 \\
\hline
\end{tabular}

Based on estimated marginal means

a. An estimate of the modified population marginal mean (I).

b. An estimate of the modified population marginal mean $(\mathrm{J})$.

c. Adjustment for multiple comparisons: Least Significant Difference (equivalent to no adjustments).

Based on the calculated p-values, the effects on the outcome was not dependent upon changes in

the levels of the group.

\section{Swatch Estimates: all colors}

Dependent Variable: L

\begin{tabular}{|l|r|r|r|r|}
\hline & & & \multicolumn{2}{|c|}{$95 \%$ Confidence Interval } \\
\cline { 4 - 5 } Swatch & Mean & Std. Error & Lower Bound & $\begin{array}{r}\text { Upper } \\
\text { Bound }\end{array}$ \\
\hline 1 w/ bleach & $90.172^{\mathrm{a}}$ & .090 & 89.995 & 90.349 \\
2 w/ bleach & $89.761^{\mathrm{a}}$ & .090 & 89.584 & 89.938 \\
3 w/o bleach & $89.855^{\mathrm{a}}$ & .090 & 89.678 & 90.032 \\
4 w/o bleach & $89.449^{\mathrm{a}}$ & .090 & 89.272 & 89.626 \\
\hline
\end{tabular}

a. Based on modified population marginal mean.

Pairwise Comparisons: all colors

Dependent Variable: L

\begin{tabular}{|c|c|c|c|c|c|c|}
\hline & & Mean & & & $\begin{array}{r}95 \% \text { Confid } \\
\text { for Diff }\end{array}$ & $\begin{array}{l}\text { e Interval } \\
\text { nce }^{c}\end{array}$ \\
\hline (I) Swatch & (J) Swatch & $\begin{array}{c}\text { Difference (I- } \\
\mathrm{J})\end{array}$ & Std. Error & Sig. ${ }^{c}$ & $\begin{array}{l}\text { Lower } \\
\text { Bound }\end{array}$ & $\begin{array}{l}\text { Upper } \\
\text { Bound }\end{array}$ \\
\hline $1 \mathrm{w} /$ bleach & 2 w/ bleach & $.411^{*}, a, b$ & .128 & .001 & .161 & .661 \\
\hline & 3 w/o bleach & $.317^{*}, a, b$ & .128 & .013 & .066 & .567 \\
\hline & 4 w/o bleach & $.723^{*}, a, b$ & .128 & .000 & .473 & .973 \\
\hline $2 \mathrm{w} / \mathrm{bleach}$ & $1 \mathrm{w} /$ bleach & $-.411^{*}, a, b$ & .128 & .001 & -.661 & -.161 \\
\hline & 3 w/o bleach & $-.094^{a}, \mathrm{~b}$ & .128 & .460 & -.345 & .156 \\
\hline & 4 w/o bleach & $.312^{\star}, a, b$ & .128 & .014 & .062 & .563 \\
\hline 3 w/o bleach & $1 \mathrm{w} /$ bleach & $-.317^{*}, a, b$ & .128 & .013 & -.567 & -.066 \\
\hline & 2 w/ bleach & $.094^{\mathrm{a}}, \mathrm{b}$ & .128 & .460 & -.156 & .345 \\
\hline & 4 w/o bleach & $.407^{*}, a, b$ & .128 & .001 & .156 & .657 \\
\hline 4 w/o bleach & $1 \mathrm{w} /$ bleach & $-.723^{*}, a, b$ & .128 & .000 & -.973 & -.473 \\
\hline & 2 w/ bleach & $-.312^{*}, a, b$ & .128 & .014 & -.563 & -.062 \\
\hline & 3 w/o bleach & $-.407^{*}, a, b$ & .128 & .001 & -.657 & -.156 \\
\hline
\end{tabular}

Based on estimated marginal means 
*. The mean difference is significant at the .05 level.

a. An estimate of the modified population marginal mean (I).

b. An estimate of the modified population marginal mean (J).

c. Adjustment for multiple comparisons: Least Significant Difference (equivalent to no adjustments).

Between the swatches significant values was observed for all pair-wise group except for swatch

2 and 3. This result is unpredicted as both swatches were washed using different detergents.

\section{Bleach Estimates: all colors}

Dependent Variable: L

\begin{tabular}{|l|r|r|r|r|}
\hline \multirow{2}{*}{} & & \multirow{2}{*}{} & \multicolumn{2}{|c|}{$\begin{array}{c}95 \% \text { Confidence } \\
\text { Interval }\end{array}$} \\
\cline { 3 - 5 } Bleached & Mean & Std. Error & Lower & $\begin{array}{c}\text { Upper } \\
\text { Bound }\end{array}$ \\
\hline Bleached & $89.966^{\mathrm{a}}$ & .064 & 89.841 & 90.091 \\
w/o bleached & $89.652^{\mathrm{a}}$ & .064 & 89.527 & 89.777 \\
\hline
\end{tabular}

a. Based on modified population marginal mean.

Pairwise Comparisons: all colors

Dependent Variable: L

\begin{tabular}{|c|c|c|c|c|c|c|}
\hline \multirow[b]{2}{*}{ (I) Bleached } & \multirow[b]{2}{*}{ (J) Bleached } & \multirow[b]{2}{*}{$\begin{array}{c}\text { Mean } \\
\text { Difference (I-J) }\end{array}$} & \multirow[b]{2}{*}{ Std. Error } & \multirow[b]{2}{*}{ Sig. ${ }^{\mathrm{C}}$} & \multicolumn{2}{|c|}{$\begin{array}{l}\text { 95\% Confidence Interval for } \\
\text { Difference }^{c}\end{array}$} \\
\hline & & & & & $\begin{array}{l}\text { Lower } \\
\text { Bound }\end{array}$ & Upper Bound \\
\hline bleached & w/o bleached & $.314^{*}, a, b$ & .090 & .001 & .137 & .491 \\
\hline w/o bleached & bleached & $-.314^{*}, a, b$ & .090 & .001 & -.491 & -.137 \\
\hline
\end{tabular}

Based on estimated marginal means

*. The mean difference is significant at the .05 level.

a. An estimate of the modified population marginal mean (I).

b. An estimate of the modified population marginal mean $(\mathrm{J})$.

c. Adjustment for multiple comparisons: Least Significant Difference (equivalent to no adjustments).

The calculated p- values significant; the effect of the outcome of the 'bleach' level is dependent

upon the effect of the 'unbleached' level.

For the main effect of washing below, significant values were observed for at least three pair-

wise groups for each level of factors.

\section{Washing Estimates: all color}

Dependent Variable: L

\begin{tabular}{|l|r|r|r|r|}
\hline & & \multirow{2}{*}{$\begin{array}{c}\text { Std. } \\
\text { Washing }\end{array}$} & Mean & \multicolumn{2}{|c|}{\begin{tabular}{c} 
95\% Confidence Interval \\
\cline { 4 - 5 }
\end{tabular}} & Error & Lower Bound & Upper Bound \\
\hline 1W 0A & $88.771^{\mathrm{a}}$ & .150 & 88.478 & 89.065 \\
1W 16A & $90.286^{\mathrm{a}}$ & .106 & 90.079 & 90.494 \\
2W 32A & $90.067^{\mathrm{a}}$ & .106 & 89.860 & 90.275 \\
3W 48A & $89.568^{\mathrm{a}}$ & .106 & 89.361 & 89.776 \\
4W 64A & $89.571^{\mathrm{a}}$ & .106 & 89.363 & 89.778 \\
5W 80A & $90.071^{\mathrm{a}}$ & .106 & 89.863 & 90.278 \\
\hline
\end{tabular}

a. Based on modified population marginal mean. 
Pairwise Comparisons: all colors

Dependent Variable: L

\begin{tabular}{|c|c|c|c|c|c|c|}
\hline \multirow[b]{2}{*}{ (I) Washing } & \multirow[b]{2}{*}{ (J) Washing } & \multirow[b]{2}{*}{ Mean Difference (I-J) } & \multirow[b]{2}{*}{ Std. Error } & \multirow[b]{2}{*}{ Sig. ${ }^{\mathrm{c}}$} & \multicolumn{2}{|c|}{$\begin{array}{c}\text { 95\% Confidence Interval for } \\
\text { Difference }^{c}\end{array}$} \\
\hline & & & & & Lower Bound & Upper Bound \\
\hline \multirow[t]{5}{*}{$1 \mathrm{~W} \mathrm{OA}$} & $1 \mathrm{~W} 16 \mathrm{~A}$ & $-1.515^{*}, \mathrm{a}, \mathrm{b}$ & .183 & .000 & -1.874 & -1.155 \\
\hline & $2 W 32 A$ & $-1.296^{*}, a, b$ & .183 & .000 & -1.655 & -.937 \\
\hline & $3 W 48 A$ & $-.797^{*}, a, b$ & .183 & .000 & -1.156 & -.437 \\
\hline & $4 W 64 A$ & $-.799^{*}, a, b$ & .183 & .000 & -1.159 & -.440 \\
\hline & $5 \mathrm{~W} 80 \mathrm{~A}$ & $-1.299^{*}, a, b$ & .183 & .000 & -1.659 & -.940 \\
\hline \multirow[t]{5}{*}{$1 \mathrm{~W} 16 \mathrm{~A}$} & $1 \mathrm{~W} 0 \mathrm{~A}$ & $1.515^{*}, a, b$ & .183 & .000 & 1.155 & 1.874 \\
\hline & $2 \mathrm{~W} 32 \mathrm{~A}$ & $.219^{\mathrm{a}}, \mathrm{b}$ & .150 & .144 & -.075 & .512 \\
\hline & $3 W 48 A$ & $.718^{*}, a, b$ & .150 & .000 & .424 & 1.011 \\
\hline & $4 \mathrm{~W} 64 \mathrm{~A}$ & $.716^{*}, a, b$ & .150 & .000 & .422 & 1.009 \\
\hline & $5 \mathrm{~W} 80 \mathrm{~A}$ & $.216^{\mathrm{a}}, \mathrm{b}$ & .150 & .150 & -.078 & .509 \\
\hline \multirow[t]{5}{*}{$2 \mathrm{~W} 32 \mathrm{~A}$} & $1 \mathrm{~W} 0 \mathrm{~A}$ & $1.296^{*}, a, b$ & .183 & .000 & .937 & 1.655 \\
\hline & $1 \mathrm{~W} 16 \mathrm{~A}$ & $-.219^{\mathrm{a}}, \mathrm{b}$ & .150 & .144 & -.512 & .075 \\
\hline & $3 \mathrm{~W} 48 \mathrm{~A}$ & $.499^{*}, a, b$ & .150 & .001 & .206 & .793 \\
\hline & $4 W 64 A$ & $.497^{*}, a, b$ & .150 & .001 & .203 & .790 \\
\hline & $5 \mathrm{~W} 80 \mathrm{~A}$ & $-.003^{\mathrm{a}}, \mathrm{b}$ & .150 & .982 & -.297 & .290 \\
\hline \multirow[t]{5}{*}{$3 \mathrm{~W} 48 \mathrm{~A}$} & $1 \mathrm{~W} 0 \mathrm{~A}$ & $.797^{*}, a, b$ & .183 & .000 & .437 & 1.156 \\
\hline & $1 \mathrm{~W} 16 \mathrm{~A}$ & $-.718^{*}, \mathrm{a}, \mathrm{b}$ & .150 & .000 & -1.011 & -.424 \\
\hline & $2 \mathrm{~W} 32 \mathrm{~A}$ & $-.499^{*}, a, b$ & .150 & .001 & -.793 & -.206 \\
\hline & $4 \mathrm{~W} 64 \mathrm{~A}$ & $-.002^{\mathrm{a}}, \mathrm{b}$ & .150 & .988 & -.296 & .291 \\
\hline & $5 W 80 A$ & $-.502^{*}, a, b$ & .150 & .001 & -.796 & -.209 \\
\hline \multirow[t]{5}{*}{$4 \mathrm{~W} 64 \mathrm{~A}$} & $1 \mathrm{~W} 0 \mathrm{~A}$ & $.799^{\star}, a, b$ & .183 & .000 & .440 & 1.159 \\
\hline & $1 \mathrm{~W} 16 \mathrm{~A}$ & $-.716^{\star}, \mathrm{a}, \mathrm{b}$ & .150 & .000 & -1.009 & -.422 \\
\hline & $2 \mathrm{~W} 32 \mathrm{~A}$ & $-.497^{*}, \mathrm{a}, \mathrm{b}$ & .150 & .001 & -.790 & -.203 \\
\hline & $3 W 48 A$ & $.002^{\mathrm{a}}, \mathrm{b}$ & .150 & .988 & -.291 & .296 \\
\hline & $5 \mathrm{~W} 80 \mathrm{~A}$ & $-.500^{*}, \mathrm{a}, \mathrm{b}$ & .150 & .001 & -.794 & -.207 \\
\hline \multirow[t]{5}{*}{$5 \mathrm{~W} 80 \mathrm{~A}$} & $1 \mathrm{~W} 0 \mathrm{~A}$ & $1.299^{*}, a, b$ & .183 & .000 & .940 & 1.659 \\
\hline & $1 \mathrm{~W} 16 \mathrm{~A}$ & $-.216^{\mathrm{a}}, \mathrm{b}$ & .150 & .150 & -.509 & .078 \\
\hline & $2 W 32 A$ & $.003^{a}, b$ & .150 & .982 & -.290 & .297 \\
\hline & $3 W 48 A$ & $.502^{*}, a, b$ & .150 & .001 & .209 & .796 \\
\hline & $4 W 64 A$ & $.500^{*}, a, b$ & .150 & .001 & .207 & .794 \\
\hline
\end{tabular}

Based on estimated marginal means

*. The mean difference is significant at the .05 level.

a. An estimate of the modified population marginal mean (I).

b. An estimate of the modified population marginal mean $(\mathrm{J})$.

c. Adjustment for multiple comparisons: Least Significant Difference (equivalent to no adjustments).

\section{Color Estimates: all colors}

Dependent Variable: $\mathrm{L}$

\begin{tabular}{|l|r|r|r|r|}
\hline & & & \multicolumn{2}{|c|}{ 95\% Confidence Interval } \\
\cline { 4 - 5 } Color & Mean & Std. Error & Lower Bound & \multicolumn{1}{c|}{ Upper Bound } \\
\hline Dk pink & $85.586^{\mathrm{a}}$ & .119 & 85.352 & 85.821 \\
Green & $94.339^{\mathrm{a}}$ & .119 & 94.105 & 94.573 \\
Orange & $91.323^{\mathrm{a}}$ & .119 & 91.088 & 91.557 \\
pink-C & $94.716^{\mathrm{a}}$ & .119 & 94.482 & 94.950 \\
Pink-J & $94.675^{\mathrm{a}}$ & .119 & 94.441 & 94.909 \\
Purple & $83.724^{\mathrm{a}}$ & .119 & 83.490 & 83.958 \\
Red & $84.301^{\mathrm{a}}$ & .119 & 84.066 & 84.535 \\
\hline
\end{tabular}


a. Based on modified population marginal mean.

Pairwise Comparisons: all colors

Dependent Variable: L

\begin{tabular}{|c|c|c|c|c|c|c|}
\hline \multirow[b]{2}{*}{ (I) Color } & \multirow[b]{2}{*}{ (J) Color } & \multirow[b]{2}{*}{ Mean Difference (I-J) } & \multirow[b]{2}{*}{ Std. Error } & \multirow[b]{2}{*}{ Sig. ${ }^{c}$} & \multicolumn{2}{|c|}{$\begin{array}{c}\text { 95\% Confidence Interval for } \\
\text { Difference }^{\mathrm{c}}\end{array}$} \\
\hline & & & & & Lower Bound & Upper Bound \\
\hline \multirow[t]{6}{*}{ Dk pink } & Green & $-8.752^{*}, \mathrm{a}, \mathrm{b}$ & .169 & .000 & -9.084 & -8.421 \\
\hline & Orange & $-5.736^{*}, a, b$ & .169 & .000 & -6.067 & -5.405 \\
\hline & pink-C & $-9.129^{*}, a, b$ & .169 & .000 & -9.460 & -8.798 \\
\hline & Pink-J & $-9.089^{*}, a, b$ & .169 & .000 & -9.420 & -8.757 \\
\hline & Purple & $1.863^{*}, a, b$ & .169 & .000 & 1.531 & 2.194 \\
\hline & Red & $1.286^{*}, \mathrm{a}, \mathrm{b}$ & .169 & .000 & .955 & 1.617 \\
\hline \multirow[t]{6}{*}{ Green } & dk pink & $8.752^{*}, a, b$ & .169 & .000 & 8.421 & 9.084 \\
\hline & Orange & $3.016^{*}, a, b$ & .169 & .000 & 2.685 & 3.347 \\
\hline & pink-C & $-.377^{*}, a, b$ & .169 & .026 & -.708 & -.046 \\
\hline & Pink-J & $-.336^{*}, a, b$ & .169 & .047 & -.667 & -.005 \\
\hline & Purple & $10.615^{\star}, a, b$ & .169 & .000 & 10.284 & 10.946 \\
\hline & Red & $10.038^{*}, a, b$ & .169 & .000 & 9.707 & 10.369 \\
\hline \multirow[t]{6}{*}{ Orange } & dk pink & $5.736^{*}, a, b$ & .169 & .000 & 5.405 & 6.067 \\
\hline & Green & $-3.016^{*}, a, b$ & .169 & .000 & -3.347 & -2.685 \\
\hline & pink-C & $-3.393^{*}, a, b$ & .169 & .000 & -3.724 & -3.062 \\
\hline & Pink-J & $-3.352^{*}, a, b$ & .169 & .000 & -3.684 & -3.021 \\
\hline & Purple & $7.599^{\star}, \mathrm{a}, \mathrm{b}$ & .169 & .000 & 7.267 & 7.930 \\
\hline & Red & $7.022^{*}, \mathrm{a}, \mathrm{b}$ & .169 & .000 & 6.691 & 7.353 \\
\hline \multirow[t]{6}{*}{ pink-C } & dk pink & $9.129^{*}, a, b$ & .169 & .000 & 8.798 & 9.460 \\
\hline & Green & $.377^{*}, a, b$ & .169 & .026 & .046 & .708 \\
\hline & Orange & $3.393^{*}, a, b$ & .169 & .000 & 3.062 & 3.724 \\
\hline & Pink-J & $.041^{a}, b$ & .169 & .810 & -.290 & .372 \\
\hline & Purple & $10.992^{*}, a, b$ & .169 & .000 & 10.661 & 11.323 \\
\hline & Red & $10.415^{\star}, a, b$ & .169 & .000 & 10.084 & 10.746 \\
\hline \multirow[t]{6}{*}{ Pink-J } & dk pink & $9.089^{*}, a, b$ & .169 & .000 & 8.757 & 9.420 \\
\hline & Green & $.336^{\star}, a, b$ & .169 & .047 & .005 & .667 \\
\hline & Orange & $3.352^{*}, a, b$ & .169 & .000 & 3.021 & 3.684 \\
\hline & pink-C & $-.041^{a}, b$ & .169 & .810 & -.372 & .290 \\
\hline & Purple & $10.951^{*}, a, b$ & .169 & .000 & 10.620 & 11.282 \\
\hline & Red & $10.374^{*}, a, b$ & .169 & .000 & 10.043 & 10.706 \\
\hline \multirow[t]{6}{*}{ Purple } & dk pink & $-1.863^{*}, a, b$ & .169 & .000 & -2.194 & -1.531 \\
\hline & Green & $-10.615^{\star}, a, b$ & 169 & .000 & -10.946 & -10.284 \\
\hline & Orange & $-7.599^{\star}, a, b$ & 169 & .000 & -7.930 & -7.267 \\
\hline & pink-C & $-10.992^{*}, a, b$ & 169 & .000 & -11.323 & -10.661 \\
\hline & Pink-J & $-10.951^{*}, a, b$ & .169 & .000 & -11.282 & -10.620 \\
\hline & Red & $-.577^{\star}, \mathrm{a}, \mathrm{b}$ & .169 & .001 & -.908 & -.246 \\
\hline \multirow[t]{6}{*}{ Red } & dk pink & $-1.286^{*}, a, b$ & .169 & .000 & -1.617 & -.955 \\
\hline & Green & $-10.038^{*}, a, b$ & .169 & .000 & -10.369 & -9.707 \\
\hline & Orange & $-7.022^{*}, a, b$ & .169 & .000 & -7.353 & -6.691 \\
\hline & pink-C & $-10.415^{*}, a, b$ & .169 & .000 & -10.746 & -10.084 \\
\hline & Pink-J & $-10.374^{\star}, a, b$ & .169 & .000 & -10.706 & -10.043 \\
\hline & Purple & $.577^{*}, a, b$ & .169 & .001 & .246 & .908 \\
\hline
\end{tabular}

Based on estimated marginal means

*. The mean difference is significant at the .05 level.

a. An estimate of the modified population marginal mean (I).

b. An estimate of the modified population marginal mean (J).

c. Adjustment for multiple comparisons: Least Significant Difference (equivalent to no adjustments). 
Dk pink, pink-C and pink- J refers to samples DPMP, PMCL and PMJX, respectively.

Significant values were observed for all the colors except for one particular pair-wise comparing group, pink-C and pink-J or samples PMCL and PMJX. The actual mean values are relatively similar such that at this variable, these samples cannot be differentiated. The mean differences between each pair-wise group for the remaining samples are substantial. Purple observes the lowest mean value and GMP observes the highest mean value at variable ' $L$ '.

\section{Swatch * Color}

\begin{tabular}{|c|c|c|c|c|c|}
\hline \multirow[b]{2}{*}{ Swatch } & \multirow[b]{2}{*}{ Color } & \multirow[b]{2}{*}{ Mean } & \multirow[b]{2}{*}{ Std. Error } & \multicolumn{2}{|c|}{$95 \%$ Confidence Interval } \\
\hline & & & & Lower Bound & Upper Bound \\
\hline \multirow[t]{7}{*}{$1 \mathrm{w} /$ bleach } & dk pink & $85.887^{\mathrm{a}}$ & .239 & 85.419 & 86.355 \\
\hline & Green & $93.636^{\mathrm{a}}$ & .239 & 93.168 & 94.104 \\
\hline & Orange & $92.093^{\mathrm{a}}$ & .239 & 91.625 & 92.562 \\
\hline & pink-C & $95.292^{\mathrm{a}}$ & .239 & 94.824 & 95.760 \\
\hline & Pink-J & $95.372^{a}$ & .239 & 94.904 & 95.840 \\
\hline & Purple & $84.240^{\mathrm{a}}$ & .239 & 83.771 & 84.708 \\
\hline & Red & $84.681^{\mathrm{a}}$ & .239 & 84.213 & 85.150 \\
\hline \multirow[t]{7}{*}{$2 \mathrm{w} / \mathrm{bleach}$} & dk pink & $84.908^{a}$ & .239 & 84.440 & 85.376 \\
\hline & Green & $93.596^{a}$ & .239 & 93.128 & 94.065 \\
\hline & Orange & $91.062^{\mathrm{a}}$ & .239 & 90.594 & 91.530 \\
\hline & pink-C & $95.105^{\mathrm{a}}$ & .239 & 94.637 & 95.573 \\
\hline & Pink-J & $94.008^{a}$ & .239 & 93.540 & 94.476 \\
\hline & Purple & $84.527^{\mathrm{a}}$ & .239 & 84.059 & 84.996 \\
\hline & Red & $85.119^{a}$ & .239 & 84.651 & 85.587 \\
\hline \multirow[t]{7}{*}{3 w/o bleach } & dk pink & $85.475^{a}$ & .239 & 85.007 & 85.943 \\
\hline & Green & $95.784^{a}$ & .239 & 95.316 & 96.252 \\
\hline & Orange & $90.749^{a}$ & .239 & 90.281 & 91.217 \\
\hline & pink-C & $93.817^{\mathrm{a}}$ & 239 & 93.349 & 94.285 \\
\hline & Pink-J & $94.901^{a}$ & .239 & 94.433 & 95.369 \\
\hline & Purple & $84.188^{a}$ & .239 & 83.720 & 84.656 \\
\hline & Red & $84.071^{a}$ & .239 & 83.603 & 84.540 \\
\hline \multirow[t]{7}{*}{$4 \mathrm{w} / \mathrm{o}$ bleach } & dk pink & $86.076^{a}$ & .239 & 85.607 & 86.544 \\
\hline & Green & $94.339^{a}$ & .239 & 93.871 & 94.808 \\
\hline & Orange & $91.386^{\mathrm{a}}$ & .239 & 90.917 & 91.854 \\
\hline & pink-C & $94.649^{\mathrm{a}}$ & .239 & 94.180 & 95.117 \\
\hline & Pink-J & $94.419^{a}$ & .239 & 93.951 & 94.888 \\
\hline & Purple & $81.941^{a}$ & .239 & 81.472 & 82.409 \\
\hline & Red & $83.331^{\mathrm{a}}$ & .239 & 82.862 & 83.799 \\
\hline
\end{tabular}

a. Based on modified population marginal mean. 


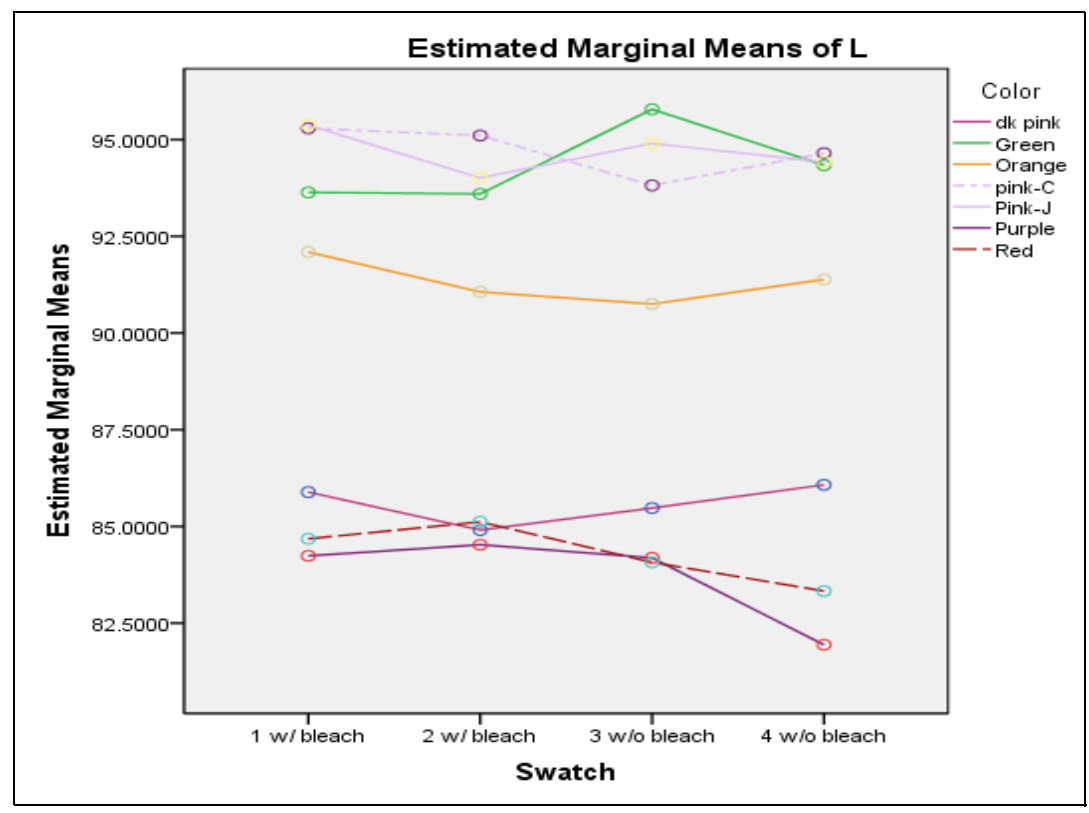

Based on the chart, DPMP, PMP and RMP are associated in one group; PMCL, PMJX and GMP are in the second group; with OSDP closely trailing the second group. Dark pink, purple and red are relatively close in hue and luminance so it is understandable that these colors would be grouped together. Although colors pink and green are on opposite ends of the CIELab space, since both are relatively light, they were also grouped together.

7. Sample Area * Color

\begin{tabular}{|ll|l|r|r|r|}
\hline \multirow{2}{*}{ Sependent Variable: $\mathrm{L}$} & & & \multicolumn{2}{|c|}{$95 \%$ Confidence Interval } \\
\cline { 4 - 5 } Sample Area & Color & Mean & Std. Error & Lower Bound & Upper Bound \\
\hline A & dk pink & $85.656^{\mathrm{a}}$ & .169 & 85.325 & 85.987 \\
& Green & $93.939^{\mathrm{a}}$ & .169 & 93.608 & 94.270 \\
& Orange & $91.373^{\mathrm{a}}$ & .169 & 91.042 & 91.704 \\
& pink-C & $94.572^{\mathrm{a}}$ & .169 & 94.241 & 94.903 \\
& Pink-J & $95.087^{\mathrm{a}}$ & .169 & 94.756 & 95.418 \\
& Purple & $83.757^{\mathrm{a}}$ & .169 & 83.426 & 84.088 \\
& Red & $84.336^{\mathrm{a}}$ & .169 & 84.005 & 84.667 \\
\hline B & $85.517^{\mathrm{a}}$ & .169 & 85.186 & 85.848 \\
& Green & $94.739^{\mathrm{a}}$ & .169 & 94.408 & 95.070 \\
& Orange & $91.272^{\mathrm{a}}$ & .169 & 90.941 & 91.603 \\
& pink-C & $94.860^{\mathrm{a}}$ & .169 & 94.529 & 95.191 \\
& Pink-J & $94.263^{\mathrm{a}}$ & .169 & 93.932 & 94.594 \\
& Purple & $83.691^{\mathrm{a}}$ & .169 & 83.360 & 84.022 \\
& Red & $84.265^{\mathrm{a}}$ & .169 & 83.934 & 84.597 \\
\hline
\end{tabular}

a. Based on modified population marginal mean. 


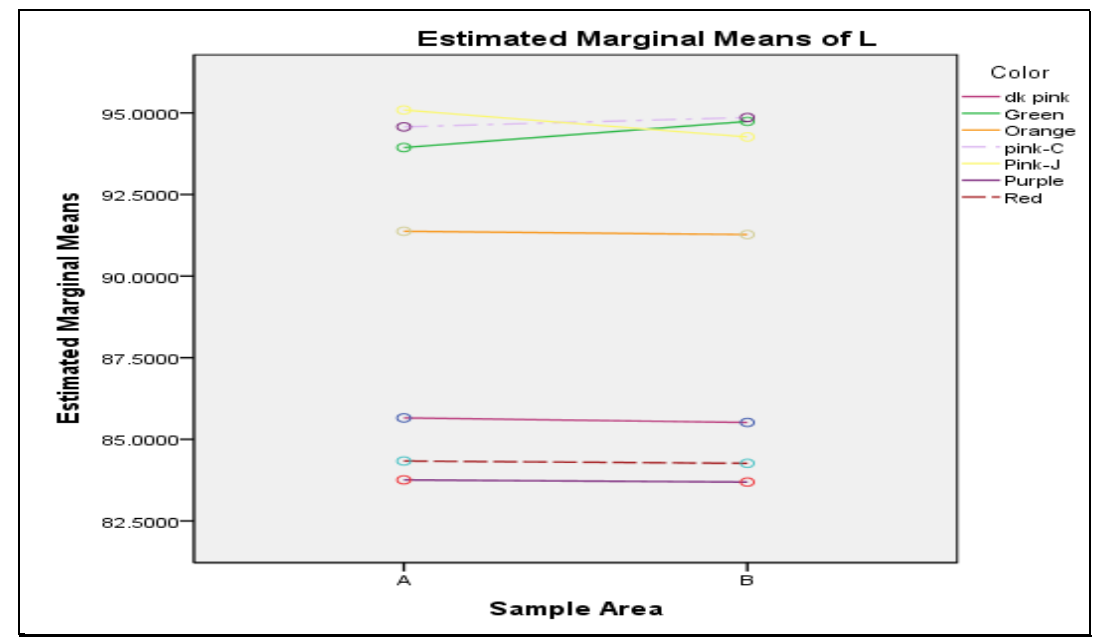

At a p- value at .704, this interaction is concluded as insignificant.

8. Bleached * Color

Dependent Variable: L

\begin{tabular}{|ll|r|r|r|r|}
\hline & & & \multicolumn{2}{|c|}{ 95\% Confidence Interval } \\
\cline { 4 - 5 } Bleached & Color & Mean & Std. Error & Lower Bound & Upper Bound \\
\hline bleached & dk pink & $85.398^{\mathrm{a}}$ & .169 & 85.066 & 85.729 \\
& Green & $93.616^{\mathrm{a}}$ & .169 & 93.285 & 93.947 \\
& Orange & $91.578^{\mathrm{a}}$ & .169 & 91.247 & 91.909 \\
& pink-C & $95.198^{\mathrm{a}}$ & .169 & 94.867 & 95.530 \\
& Pink-J & $94.690^{\mathrm{a}}$ & .169 & 94.359 & 95.021 \\
& Purple & $84.384^{\mathrm{a}}$ & .169 & 84.052 & 84.715 \\
& Red & $84.900^{\mathrm{a}}$ & .169 & 84.569 & 85.231 \\
\hline w/o bleached & dk pink & $85.775^{\mathrm{a}}$ & .169 & 85.444 & 86.106 \\
& Green & $95.062^{\mathrm{a}}$ & .169 & 94.731 & 95.393 \\
& Orange & $91.067^{\mathrm{a}}$ & .169 & 90.736 & 91.398 \\
& pink-C & $94.233^{\mathrm{a}}$ & .169 & 93.902 & 94.564 \\
& Pink-J & $94.660^{\mathrm{a}}$ & .169 & 94.329 & 94.991 \\
& Purple & $83.064^{\mathrm{a}}$ & .169 & 82.733 & 83.395 \\
& Red & $83.701^{\mathrm{a}}$ & .169 & 83.370 & 84.032 \\
\hline
\end{tabular}

a. Based on modified population marginal mean.

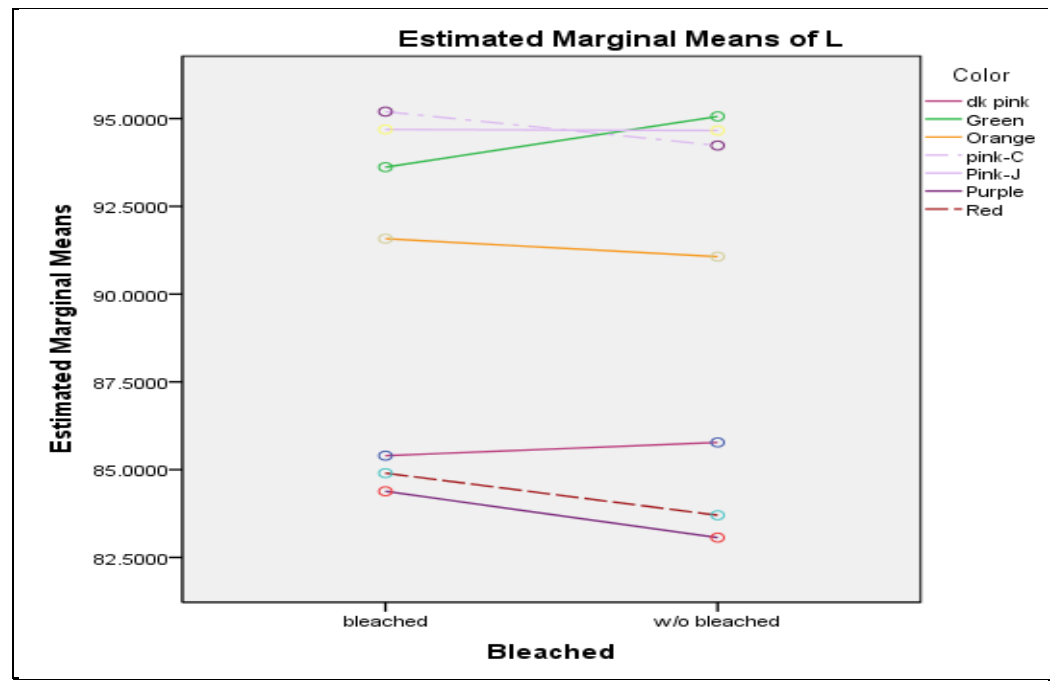


Five of the seven colors observed a decrease in mean value between the bleached and unbleached samples. A decrease in ' $L$ ' means that the samples were darker as they were washed without bleach than with bleach. This change in lightness was not evident macroscopically.

15. Washing * Color

\begin{tabular}{|c|c|c|c|c|c|}
\hline \multirow[b]{2}{*}{ Washing } & \multirow[b]{2}{*}{ Color } & \multirow[b]{2}{*}{ Mean } & \multirow[b]{2}{*}{ Std. Error } & \multicolumn{2}{|c|}{$95 \%$ Confidence Interval } \\
\hline & & & & Lower Bound & Upper Bound \\
\hline \multirow[t]{7}{*}{$1 \mathrm{~W} \mathrm{0A}$} & $\mathrm{dk}$ pink & $85.119^{a}$ & .396 & 84.342 & 85.896 \\
\hline & Green & $92.379^{a}$ & .396 & 91.602 & 93.155 \\
\hline & Orange & $92.366^{a}$ & .396 & 91.589 & 93.142 \\
\hline & pink-C & $95.802^{\mathrm{a}}$ & .396 & 95.025 & 96.579 \\
\hline & Pink-J & $92.656^{a}$ & .396 & 91.880 & 93.433 \\
\hline & Purple & $79.053^{a}$ & .396 & 78.276 & 79.829 \\
\hline & Red & $84.026^{a}$ & .396 & 83.249 & 84.803 \\
\hline \multirow[t]{7}{*}{$1 \mathrm{~W} 16 \mathrm{~A}$} & $\mathrm{dk}$ pink & $86.946^{a}$ & .280 & 86.397 & 87.495 \\
\hline & Green & $95.734^{\mathrm{a}}$ & .280 & 95.185 & 96.283 \\
\hline & Orange & $92.093^{\mathrm{a}}$ & .280 & 91.544 & 92.642 \\
\hline & pink-C & $94.625^{\mathrm{a}}$ & .280 & 94.076 & 95.174 \\
\hline & Pink-J & $96.299^{a}$ & .280 & 95.750 & 96.848 \\
\hline & Purple & $81.055^{a}$ & .280 & 80.506 & 81.604 \\
\hline & Red & $85.253^{a}$ & .280 & 84.704 & 85.802 \\
\hline \multirow[t]{7}{*}{$2 W 32 A$} & dk pink & $84.440^{a}$ & .280 & 83.891 & 84.989 \\
\hline & Green & $93.366^{a}$ & .280 & 92.817 & 93.915 \\
\hline & Orange & $90.411^{a}$ & .280 & 89.862 & 90.960 \\
\hline & pink-C & $94.739^{a}$ & .280 & 94.190 & 95.288 \\
\hline & Pink-J & $95.313^{a}$ & .280 & 94.764 & 95.862 \\
\hline & Purple & $87.708^{a}$ & .280 & 87.159 & 88.257 \\
\hline & Red & $84.496^{a}$ & .280 & 83.947 & 85.045 \\
\hline \multirow[t]{7}{*}{$3 \mathrm{~W} 48 \mathrm{~A}$} & $\mathrm{dk}$ pink & $85.383^{a}$ & .280 & 84.834 & 85.932 \\
\hline & Green & $94.085^{\mathrm{a}}$ & .280 & 93.535 & 94.634 \\
\hline & Orange & $91.907^{a}$ & .280 & 91.358 & 92.456 \\
\hline & pink-C & $93.773^{a}$ & .280 & 93.224 & 94.323 \\
\hline & Pink-J & $95.140^{\mathrm{a}}$ & .280 & 94.591 & 95.689 \\
\hline & Purple & $82.592^{a}$ & .280 & 82.043 & 83.141 \\
\hline & Red & $84.099^{a}$ & .280 & 83.550 & 84.648 \\
\hline \multirow[t]{7}{*}{$4 W 64 A$} & dk pink & $84.958^{a}$ & .280 & 84.409 & 85.508 \\
\hline & Green & $94.398^{\mathrm{a}}$ & .280 & 93.849 & 94.947 \\
\hline & Orange & $91.060^{\mathrm{a}}$ & .280 & 90.511 & 91.609 \\
\hline & pink-C & $95.591^{a}$ & .280 & 95.042 & 96.140 \\
\hline & Pink-J & $93.493^{a}$ & .280 & 92.943 & 94.042 \\
\hline & Purple & $84.226^{a}$ & .280 & 83.677 & 84.775 \\
\hline & Red & $83.269^{a}$ & .280 & 82.720 & 83.818 \\
\hline \multirow[t]{7}{*}{$5 \mathrm{~W} 80 \mathrm{~A}$} & $\mathrm{dk}$ pink & $86.439^{a}$ & .280 & 85.890 & 86.988 \\
\hline & Green & $95.092^{a}$ & .280 & 94.543 & 95.641 \\
\hline & Orange & $90.621^{a}$ & .280 & 90.072 & 91.170 \\
\hline & pink-C & $94.307^{a}$ & .280 & 93.758 & 94.856 \\
\hline & Pink-J & $94.140^{a}$ & .280 & 93.591 & 94.689 \\
\hline & Purple & $85.374^{a}$ & .280 & 84.825 & 85.923 \\
\hline & Red & $84.524^{a}$ & .280 & 83.975 & 85.073 \\
\hline
\end{tabular}

a. Based on modified population marginal mean. 


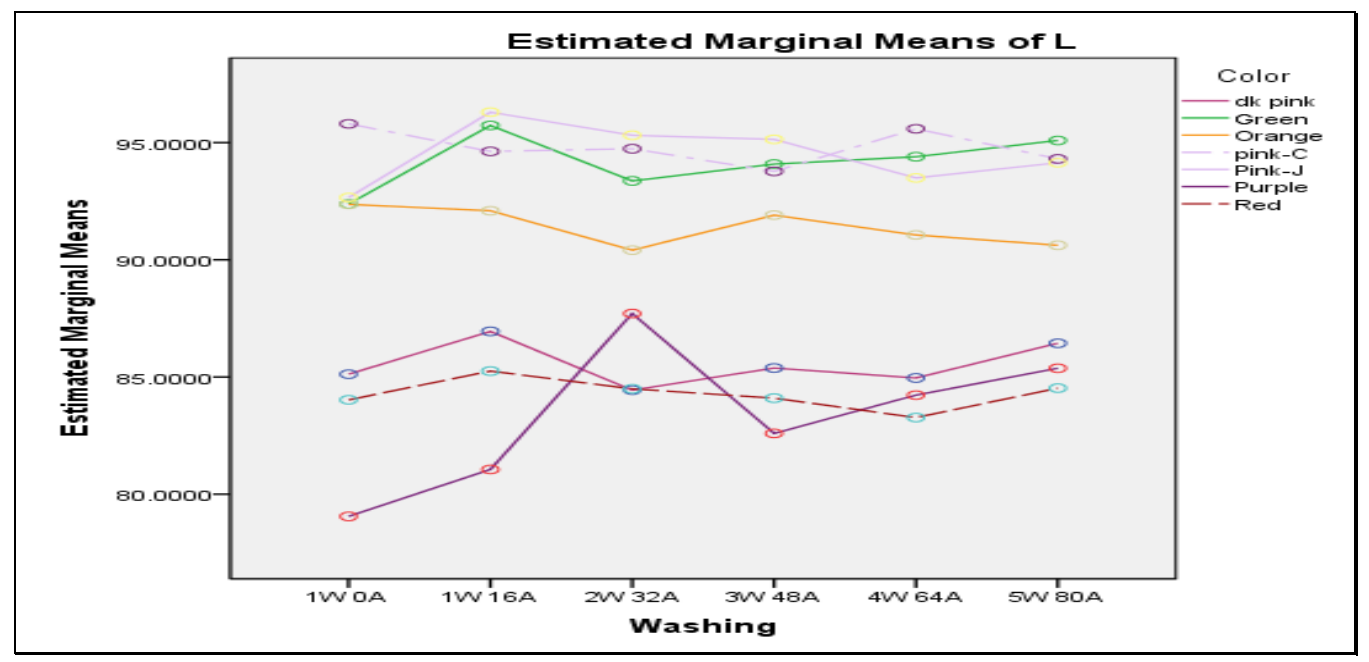

Colors red, dark pink, pink-C, orange, and green observe a similar pattern.

Variable ' $a$ '

Dependent Variable: a

Tests of Between-Subjects Effects: all colors

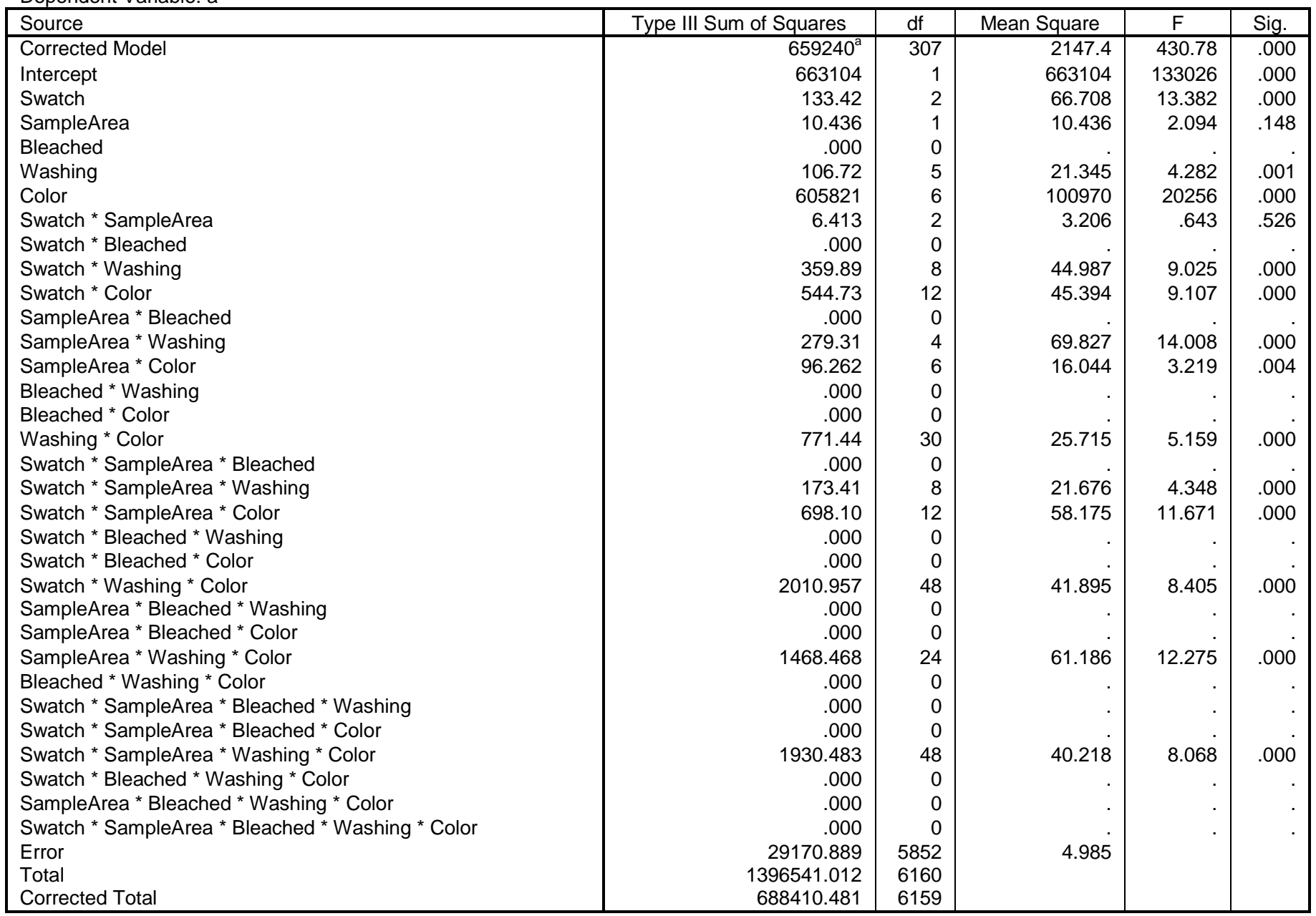


a. $\mathrm{R}$ Squared $=.958$ (Adjusted $\mathrm{R}$ Squared $=.955$ )

The main effects of the sample area and the interaction effects of Sample Area* Swatch were

both deemed insignificant.

\section{Sample Area Estimates: all colors}

Dependent Variable: a

\begin{tabular}{|c|c|c|c|c|}
\hline \multirow[b]{2}{*}{ Sample Area } & \multirow[b]{2}{*}{ Mean } & \multirow[b]{2}{*}{ Std. Error } & \multicolumn{2}{|c|}{ 95\% Confidence Interval } \\
\hline & & & Lower Bound & Upper Bound \\
\hline $\begin{array}{l}\mathrm{A} \\
\mathrm{B}\end{array}$ & $\begin{array}{l}10.787^{\mathrm{a}} \\
10.656^{\mathrm{a}}\end{array}$ & $\begin{array}{l}.040 \\
.040\end{array}$ & $\begin{array}{r}10.708 \\
10.578\end{array}$ & $\begin{array}{l}10.866 \\
10.735\end{array}$ \\
\hline
\end{tabular}

a. Based on modified population marginal mean.

Dependent Variable: a

Pairwise Comparisons: all colors

\begin{tabular}{|c|c|c|c|c|c|c|}
\hline \multirow[b]{2}{*}{ (I) Sample Area } & \multirow[b]{2}{*}{ (J) Sample Area } & \multirow[b]{2}{*}{ Mean Difference (I-J) } & \multirow[b]{2}{*}{ Std. Error } & \multirow[b]{2}{*}{ Sig. ${ }^{\mathrm{C}}$} & \multicolumn{2}{|c|}{$\begin{array}{c}\text { 95\% Confidence Interval for } \\
\text { Difference }\end{array}$} \\
\hline & & & & & Lower Bound & Upper Bound \\
\hline A & $\mathrm{B}$ & $.131^{*}, a, b$ & .057 & .022 & .019 & .242 \\
\hline $\mathrm{B}$ & $A$ & $-.131^{*}, a, b$ & .057 & .022 & -.242 & -.019 \\
\hline
\end{tabular}

Based on estimated marginal means

*. The mean difference is significant at the .05 level.

a. An estimate of the modified population marginal mean (I).

b. An estimate of the modified population marginal mean (J).

c. Adjustment for multiple comparisons: Least Significant Difference (equivalent to no adjustments).

The interactions between these two sample areas are significant. This means that the effect of the outcome on the change for either level depends on the effect of the change on the opposing level.

\section{Swatch Estimates: all color}

Dependent Variable: a

\begin{tabular}{|c|c|c|c|c|}
\hline \multirow[b]{2}{*}{ Swatch } & \multirow[b]{2}{*}{ Mean } & \multirow[b]{2}{*}{ Std. Error } & \multicolumn{2}{|c|}{ 95\% Confidence Interval } \\
\hline & & & Lower Bound & Upper Bound \\
\hline $1 \mathrm{w} /$ bleach & $10.948^{a}$ & .057 & 10.836 & 11.059 \\
\hline 2 w/ bleach & $10.483^{a}$ & .057 & 10.371 & 10.594 \\
\hline 3 w/o bleach & $10.747^{\mathrm{a}}$ & .057 & 10.635 & 10.858 \\
\hline $4 \mathrm{w} / \mathrm{o}$ bleach & $10.710^{a}$ & .057 & 10.598 & 10.821 \\
\hline
\end{tabular}

a. Based on modified population marginal mean.

Pairwise Comparisons: all color

Dependent Variable: a

\begin{tabular}{|c|c|c|c|c|c|c|}
\hline \multirow[b]{2}{*}{ (I) Swatch } & \multirow[b]{2}{*}{ (J) Swatch } & \multirow[b]{2}{*}{ Mean Difference (I-J) } & \multirow[b]{2}{*}{ Std. Error } & \multirow[b]{2}{*}{ Sig. ${ }^{\mathrm{C}}$} & \multicolumn{2}{|c|}{$95 \%$ Confidence Interval for Difference ${ }^{c}$} \\
\hline & & & & & Lower Bound & Upper Bound \\
\hline $1 \mathrm{w} /$ bleach & 3 w/o bleach & $.201^{\star}, a, b$ & .080 & .012 & .043 & .359 \\
\hline \multirow[t]{2}{*}{$2 \mathrm{w} / \mathrm{bleach}$} & $1 \mathrm{w} /$ bleach & $-.465^{\star}, a, b$ & .080 & .000 & -.623 & -.307 \\
\hline & 3 w/o bleach & $-.264^{*}, a, b$ & .080 & .001 & -.421 & -.106 \\
\hline \multirow{2}{*}{$3 \mathrm{w} / \mathrm{o}$ bleach } & $2 \mathrm{w} /$ bleach & $.264^{*}, \mathrm{a}, \mathrm{b}$ & .080 & .001 & .106 & .421 \\
\hline & 4 w/o bleach & $.037^{\mathrm{a}}$ & .080 & .648 & -.121 & .195 \\
\hline \multirow[t]{3}{*}{$4 \mathrm{w} / \mathrm{o}$ bleach } & $1 \mathrm{w} /$ bleach & $-.238^{*}, a, b$ & .080 & .003 & -.396 & -.080 \\
\hline & 2 w/ bleach & $.227^{*}, \mathrm{a,b}$ & .080 & .005 & .069 & .385 \\
\hline & 3 w/o bleach & $-.037^{a}$ & .080 & .648 & -.195 & .121 \\
\hline
\end{tabular}


Based on estimated marginal means

*. The mean difference is significant at the .05 level.

a. An estimate of the modified population marginal mean (I).

b. An estimate of the modified population marginal mean $(\mathrm{J})$.

c. Adjustment for multiple comparisons: Least Significant Difference (equivalent to no adjustments).

Significant values were observed for the bleached samples. Swatch 1 and 2 was significant at all

their pair-wise comparing groups. The pair-wise group for swatch 3 and 4 was not significant.

\section{Bleach Estimates: all colors}

Dependent Variable: a

\begin{tabular}{|l|c|r|r|r|}
\hline & & & \multicolumn{2}{|c|}{$95 \%$ Confidence Interval } \\
\cline { 4 - 5 } Bleached & Mean & Std. Error & Lower Bound & Upper Bound \\
\hline bleached & $10.715^{\mathrm{a}}$ & .040 & 10.636 & 10.794 \\
w/o bleached & $10.728^{\mathrm{a}}$ & .040 & 10.649 & 10.807 \\
\hline
\end{tabular}

a. Based on modified population marginal mean.

Pairwise Comparisons: all colors

Dependent Variable: a

\begin{tabular}{|c|c|c|c|c|c|c|}
\hline \multirow[b]{2}{*}{ (I) Bleached } & \multirow[b]{2}{*}{ (J) Bleached } & \multirow[b]{2}{*}{ Mean Difference (I-J) } & \multirow[b]{2}{*}{ Std. Error } & \multirow[b]{2}{*}{ Sig. ${ }^{\mathrm{C}}$} & \multicolumn{2}{|c|}{$\begin{array}{c}\text { 95\% Confidence Interval for } \\
\text { Difference }\end{array}$} \\
\hline & & & & & Lower Bound & Upper Bound \\
\hline bleached & w/o bleached & $-.013^{\mathrm{a}}, \mathrm{b}$ & .057 & .820 & -.124 & .099 \\
\hline w/o bleached & bleached & $.013^{\mathrm{a}}, \mathrm{b}$ & .057 & .820 & -.099 & .124 \\
\hline
\end{tabular}

Based on estimated marginal means

a. An estimate of the modified population marginal mean (I).

b. An estimate of the modified population marginal mean $(\mathrm{J})$.

c. Adjustment for multiple comparisons: Least Significant Difference (equivalent to no adjustments).

The effects of the change in the bleached sample on the outcome do not depend on the change in

the unbleached sample. This can also be interrupted inversely. In other words, while the effect of

one of the factors changes, the other factor is fixed.

For the main effect of washing below, only at the treatment cycle of 1W 16A was significant values observed.

\section{Washing Estimates: all color}

Dependent Variable: a

\begin{tabular}{|l|r|r|r|r|}
\hline & & & \multicolumn{2}{|c|}{ 95\% Confidence Interval } \\
\cline { 4 - 5 } Washing & Mean & Std. Error & Lower Bound & Upper Bound \\
\hline 1W 0A & $10.684^{\mathrm{a}}$ & .094 & 10.499 & 10.869 \\
1W 16A & $10.984^{\mathrm{a}}$ & .067 & 10.854 & 11.115 \\
2W 32A & $10.635^{\mathrm{a}}$ & .067 & 10.504 & 10.766 \\
3W 48A & $10.732^{\mathrm{a}}$ & .067 & 10.601 & 10.862 \\
4W 64A & $10.602^{\mathrm{a}}$ & .067 & 10.471 & 10.733 \\
5W 80A & $10.675^{\mathrm{a}}$ & .067 & 10.544 & 10.806 \\
\hline
\end{tabular}

a. Based on modified population marginal mean. 
Pairwise Comparisons: all color

Dependent Variable: a

\begin{tabular}{|c|c|c|c|c|c|c|}
\hline \multirow[b]{2}{*}{ (I) Washing } & \multirow[b]{2}{*}{ (J) Washing } & \multirow[b]{2}{*}{ Mean Difference (I-J) } & \multirow[b]{2}{*}{ Std. Error } & \multirow[b]{2}{*}{ Sig. ${ }^{c}$} & \multicolumn{2}{|c|}{$\begin{array}{c}\text { 95\% Confidence Interval for } \\
\text { Difference }\end{array}$} \\
\hline & & & & & Lower Bound & Upper Bound \\
\hline \multirow[t]{5}{*}{$1 \mathrm{~W} 0 \mathrm{~A}$} & $1 \mathrm{~W} 16 \mathrm{~A}$ & $-.301^{*}, a, b$ & .116 & .009 & -.527 & -.074 \\
\hline & $2 W 32 A$ & $.049^{a}, b$ & .116 & .673 & -.178 & .275 \\
\hline & $3 W 48 A$ & $-.048^{a}, b$ & .116 & .677 & -.275 & .178 \\
\hline & $4 \mathrm{~W} 64 \mathrm{~A}$ & $.082^{a}, b$ & .116 & .480 & -.145 & .308 \\
\hline & $5 \mathrm{~W} 80 \mathrm{~A}$ & $.008^{\mathrm{a}}, \mathrm{b}$ & .116 & .942 & -.218 & .235 \\
\hline \multirow[t]{5}{*}{$1 \mathrm{~W} 16 \mathrm{~A}$} & $1 \mathrm{~W} 0 \mathrm{~A}$ & $.301^{*}, a, b$ & .116 & .009 & .074 & .527 \\
\hline & $2 W 32 A$ & $.350^{*}, a, b$ & .094 & .000 & .165 & .535 \\
\hline & $3 W 48 A$ & $.253^{*}, a, b$ & .094 & .007 & .068 & .438 \\
\hline & $4 \mathrm{~W} 64 \mathrm{~A}$ & $.382^{*}, a, b$ & .094 & .000 & .197 & .567 \\
\hline & $5 \mathrm{~W} 80 \mathrm{~A}$ & $.309^{*}, a, b$ & .094 & .001 & .124 & .494 \\
\hline \multirow[t]{5}{*}{$2 W 32 A$} & $1 \mathrm{~W} 0 \mathrm{~A}$ & $-.049^{a}, b$ & .116 & .673 & -.275 & .178 \\
\hline & $1 \mathrm{~W} 16 \mathrm{~A}$ & $-.350^{*}, a, b$ & .094 & .000 & -.535 & -.165 \\
\hline & $3 W 48 A$ & $-.097^{a}, b$ & .094 & .304 & -.282 & .088 \\
\hline & $4 \mathrm{~W} 64 \mathrm{~A}$ & $.033^{a}, b$ & .094 & .728 & -.152 & .218 \\
\hline & $5 W 80 A$ & $-.040^{a}, b$ & .094 & .669 & -.225 & .145 \\
\hline \multirow[t]{5}{*}{$3 W$ 48A } & $1 \mathrm{~W} 0 \mathrm{~A}$ & $.048^{a}, b$ & .116 & .677 & -.178 & .275 \\
\hline & $1 \mathrm{~W} 16 \mathrm{~A}$ & $-.253^{*}, a, b$ & .094 & .007 & -.438 & -.068 \\
\hline & $2 W 32 A$ & $.097^{a}, b$ & .094 & .304 & -.088 & .282 \\
\hline & $4 \mathrm{~W} 64 \mathrm{~A}$ & $.130^{a}, b$ & .094 & .169 & -.055 & .315 \\
\hline & $5 \mathrm{~W} 80 \mathrm{~A}$ & $.057^{\mathrm{a}}, \mathrm{b}$ & .094 & .549 & -.128 & .242 \\
\hline \multirow[t]{5}{*}{$4 W 64 A$} & $1 \mathrm{~W} 0 \mathrm{~A}$ & $-.082^{\mathrm{a}}, \mathrm{b}$ & .116 & .480 & -.308 & .145 \\
\hline & $1 \mathrm{~W} 16 \mathrm{~A}$ & $-.382^{*}, a, b$ & .094 & .000 & -.567 & -.197 \\
\hline & $2 W 32 A$ & $-.033^{a}, b$ & .094 & .728 & -.218 & .152 \\
\hline & $3 W 48 A$ & $-.130^{a}, b$ & .094 & .169 & -.315 & .055 \\
\hline & $5 \mathrm{~W} 80 \mathrm{~A}$ & $-.073^{a}, b$ & .094 & .438 & -.258 & .112 \\
\hline \multirow[t]{5}{*}{$5 W 80 A$} & $1 \mathrm{~W} 0 \mathrm{~A}$ & $-.008^{\mathrm{a}}, \mathrm{b}$ & .116 & .942 & -.235 & .218 \\
\hline & $1 \mathrm{~W} 16 \mathrm{~A}$ & $-.309^{*}, a, b$ & .094 & .001 & -.494 & -.124 \\
\hline & $2 W 32 A$ & $.040^{a}, b$ & .094 & .669 & -.145 & .225 \\
\hline & $3 W 48 A$ & $-.057^{a}, b$ & .094 & .549 & -.242 & .128 \\
\hline & $4 W 64 A$ & $.073^{a}, b$ & .094 & .438 & -.112 & .258 \\
\hline
\end{tabular}

Based on estimated marginal means

*. The mean difference is significant at the .05 level.

a. An estimate of the modified population marginal mean (I).

b. An estimate of the modified population marginal mean $(\mathrm{J})$.

c. Adjustment for multiple comparisons: Least Significant Difference (equivalent to no adjustments).

\section{Color Estimates: all colors}

Dependent Variable: a

\begin{tabular}{|l|r|r|r|r|}
\hline & & & \multicolumn{2}{|c|}{ 95\% Confidence Interval } \\
\cline { 3 - 5 } Color & \multicolumn{1}{|c|}{ Mean } & Std. Error & Lower Bound & \multicolumn{1}{c|}{ Upper Bound } \\
\hline dk pink & $25.254^{\mathrm{a}}$ & .075 & 25.106 & 25.401 \\
Green & $.802^{\mathrm{a}}$ & .075 & .655 & .950 \\
Orange & $9.786^{\mathrm{a}}$ & .075 & 9.639 & 9.934 \\
pink-C & $2.771^{\mathrm{a}}$ & .075 & 2.623 & 2.919 \\
Pink-J & $2.653^{\mathrm{a}}$ & .075 & 2.505 & 2.801 \\
Purple & $6.389^{\mathrm{a}}$ & .075 & 6.241 & 6.536 \\
Red & $27.398^{\mathrm{a}}$ & .075 & 27.250 & 27.545 \\
\hline
\end{tabular}

a. Based on modified population marginal mean. 
Pairwise Comparisons: all colors

Dependent Variable: a

\begin{tabular}{|c|c|c|c|c|c|c|}
\hline (I) Color & (J) Color & Mean Difference (I-J) & Std. Error & Sig. ${ }^{c}$ & \multicolumn{2}{|c|}{$\begin{array}{l}\text { 95\% Confidence Interval for } \\
\text { Difference }\end{array}$} \\
\hline \multirow[t]{4}{*}{ dk pink } & Green & $24.452^{*}, \mathrm{a}, \mathrm{b}$ & .106 & .000 & 24.243 & 24.660 \\
\hline & pink-C & $22.483^{*}, a, b$ & .106 & .000 & 22.274 & 22.691 \\
\hline & Pink-J & $22.601^{*}, a, b$ & .106 & .000 & 22.392 & 22.809 \\
\hline & Purple & $18.865^{*}, a, b$ & .106 & .000 & 18.656 & 19.074 \\
\hline \multirow[t]{5}{*}{ Green } & dk pink & $-24.452^{*}, a, b$ & .106 & .000 & -24.660 & -24.243 \\
\hline & Orange & $-8.984^{*}, a, b$ & .106 & .000 & -9.193 & -8.775 \\
\hline & pink-C & $-1.969^{*}, a, b$ & .106 & .000 & -2.178 & -1.760 \\
\hline & Pink-J & $-1.851^{*}, a, b$ & .106 & .000 & -2.060 & -1.642 \\
\hline & Purple & $-5.587^{\star}, a, b$ & .106 & .000 & -5.795 & -5.378 \\
\hline \multirow{3}{*}{ Orange } & Pink-J & $7.133^{*}, a, b$ & .106 & .000 & 6.925 & 7.342 \\
\hline & Purple & $3.397^{*}, a, b$ & .106 & .000 & 3.189 & 3.606 \\
\hline & Red & $-17.611^{*}, a, b$ & .106 & .000 & -17.820 & -17.403 \\
\hline \multirow[t]{6}{*}{ pink-C } & dk pink & $-22.483^{*}, a, b$ & .106 & .000 & -22.691 & -22.274 \\
\hline & Green & $1.969^{*}, \mathrm{a}, \mathrm{b}$ & .106 & .000 & 1.760 & 2.178 \\
\hline & Orange & $-7.015^{*}, a, b$ & .106 & .000 & -7.224 & -6.807 \\
\hline & Pink-J & $.118^{\mathrm{a}}, \mathrm{b}$ & .106 & .268 & -.091 & .327 \\
\hline & Purple & $-3.618^{*}, a, b$ & .106 & .000 & -3.826 & -3.409 \\
\hline & Red & $-24.627^{*}, a, b$ & .106 & .000 & -24.835 & -24.418 \\
\hline Pink-J & dk pink & $-22.601^{*}, a, b$ & .106 & .000 & -22.809 & -22.392 \\
\hline \multirow{4}{*}{ Purple } & Orange & $-3.397^{\star}, a, b$ & .106 & .000 & -3.606 & -3.189 \\
\hline & pink-C & $3.618^{*}, a, b$ & .106 & .000 & 3.409 & 3.826 \\
\hline & Pink-J & $3.736^{*}, a, b$ & .106 & .000 & 3.527 & 3.944 \\
\hline & Red & $-21.009^{*}, a, b$ & .106 & .000 & -21.218 & -20.800 \\
\hline \multirow[t]{6}{*}{ Red } & dk pink & $2.144^{*}, \mathrm{a}, \mathrm{b}$ & .106 & .000 & 1.935 & 2.353 \\
\hline & Green & $26.595^{*}, a, b$ & .106 & .000 & 26.387 & 26.804 \\
\hline & Orange & $17.611^{*}, a, b$ & .106 & .000 & 17.403 & 17.820 \\
\hline & pink-C & $24.627^{*}, a, b$ & .106 & .000 & 24.418 & 24.835 \\
\hline & Pink-J & $24.745^{*}, a, b$ & .106 & .000 & 24.536 & 24.953 \\
\hline & Purple & $21.009^{*}, a, b$ & .106 & .000 & 20.800 & 21.218 \\
\hline
\end{tabular}

Based on estimated marginal means

*. The mean difference is significant at the .05 level.

a. An estimate of the modified population marginal mean (I).

b. An estimate of the modified population marginal mean $(\mathrm{J})$.

c. Adjustment for multiple comparisons: Least Significant Difference (equivalent to no adjustments). 
Similar to variable 'L', sample PMCL and PMJX did not produce significant results. The values between the groups are approximately equal. In this red to green region, these two samples cannot be differentiated which suggests that their ' $a$ ' values are similar. Significant mean differences were observed for the remaining colors.

\section{Swatch ${ }^{*}$ Color: all colors}

\begin{tabular}{|c|c|c|c|c|c|}
\hline \multirow[b]{2}{*}{ Swatch } & \multirow[b]{2}{*}{ Color } & \multirow[b]{2}{*}{ Mean } & \multirow[b]{2}{*}{ Std. Error } & \multicolumn{2}{|c|}{ 95\% Confidence Interval } \\
\hline & & & & Lower Bound & Upper Bound \\
\hline \multirow[t]{7}{*}{$1 \mathrm{w} /$ bleach } & dk pink & $26.438^{\mathrm{a}}$ & .151 & 26.143 & 26.733 \\
\hline & Green & $.950^{\mathrm{a}}$ & .151 & .654 & 1.245 \\
\hline & Orange & $9.835^{\mathrm{a}}$ & .151 & 9.540 & 10.130 \\
\hline & pink-C & $2.323^{a}$ & .151 & 2.028 & 2.618 \\
\hline & Pink-J & $2.500^{\mathrm{a}}$ & .151 & 2.205 & 2.795 \\
\hline & Purple & $6.519^{a}$ & .151 & 6.224 & 6.814 \\
\hline & Red & $28.069^{a}$ & .151 & 27.774 & 28.364 \\
\hline \multirow[t]{7}{*}{$2 \mathrm{w} /$ bleach } & dk pink & $24.504^{\mathrm{a}}$ & .151 & 24.209 & 24.799 \\
\hline & Green & $.826^{\mathrm{a}}$ & .151 & .531 & 1.121 \\
\hline & Orange & $9.827^{a}$ & .151 & 9.531 & 10.122 \\
\hline & pink-C & $2.892^{a}$ & .151 & 2.597 & 3.187 \\
\hline & Pink-J & $2.787^{a}$ & .151 & 2.492 & 3.082 \\
\hline & Purple & $6.424^{a}$ & .151 & 6.129 & 6.719 \\
\hline & Red & $26.121^{\mathrm{a}}$ & .151 & 25.826 & 26.416 \\
\hline \multirow[t]{7}{*}{3 w/o bleach } & dk pink & $24.733^{\mathrm{a}}$ & .151 & 24.438 & 25.028 \\
\hline & Green & $.719^{a}$ & .151 & .424 & 1.014 \\
\hline & Orange & $9.926^{a}$ & .151 & 9.631 & 10.221 \\
\hline & pink-C & $2.867^{a}$ & .151 & 2.572 & 3.162 \\
\hline & Pink-J & $2.749^{\mathrm{a}}$ & .151 & 2.454 & 3.044 \\
\hline & Purple & $6.345^{\mathrm{a}}$ & .151 & 6.050 & 6.640 \\
\hline & Red & $27.888^{a}$ & .151 & 27.593 & 28.183 \\
\hline \multirow[t]{7}{*}{4 w/o bleach } & dk pink & $25.339^{a}$ & .151 & 25.044 & 25.634 \\
\hline & Green & $.714^{a}$ & .151 & .419 & 1.009 \\
\hline & Orange & $9.557^{\mathrm{a}}$ & .151 & 9.262 & 9.852 \\
\hline & pink-C & $3.002^{a}$ & .151 & 2.707 & 3.297 \\
\hline & Pink-J & $2.577^{\mathrm{a}}$ & .151 & 2.282 & 2.872 \\
\hline & Purple & $6.267^{a}$ & .151 & 5.972 & 6.563 \\
\hline & Red & $27.513^{a}$ & .151 & 27.217 & 27.808 \\
\hline
\end{tabular}

a. Based on modified population marginal mean.

Based on the graph below, the mean values can be separated into two groups. The first group contains red and dark pink colors, and the second group contains the remaining colors. It can be observed that red and dark pink observes a consistent pattern and that between the bleached and unbleached samples, the ' $a$ ' values are affected. For the remaining colors, the mean values remain consistent among the swatches. At this level of interaction, samples PMJX and PMCL are 
indistinguishable. It can be concluded that the significant mean differences are observed between the two groups.

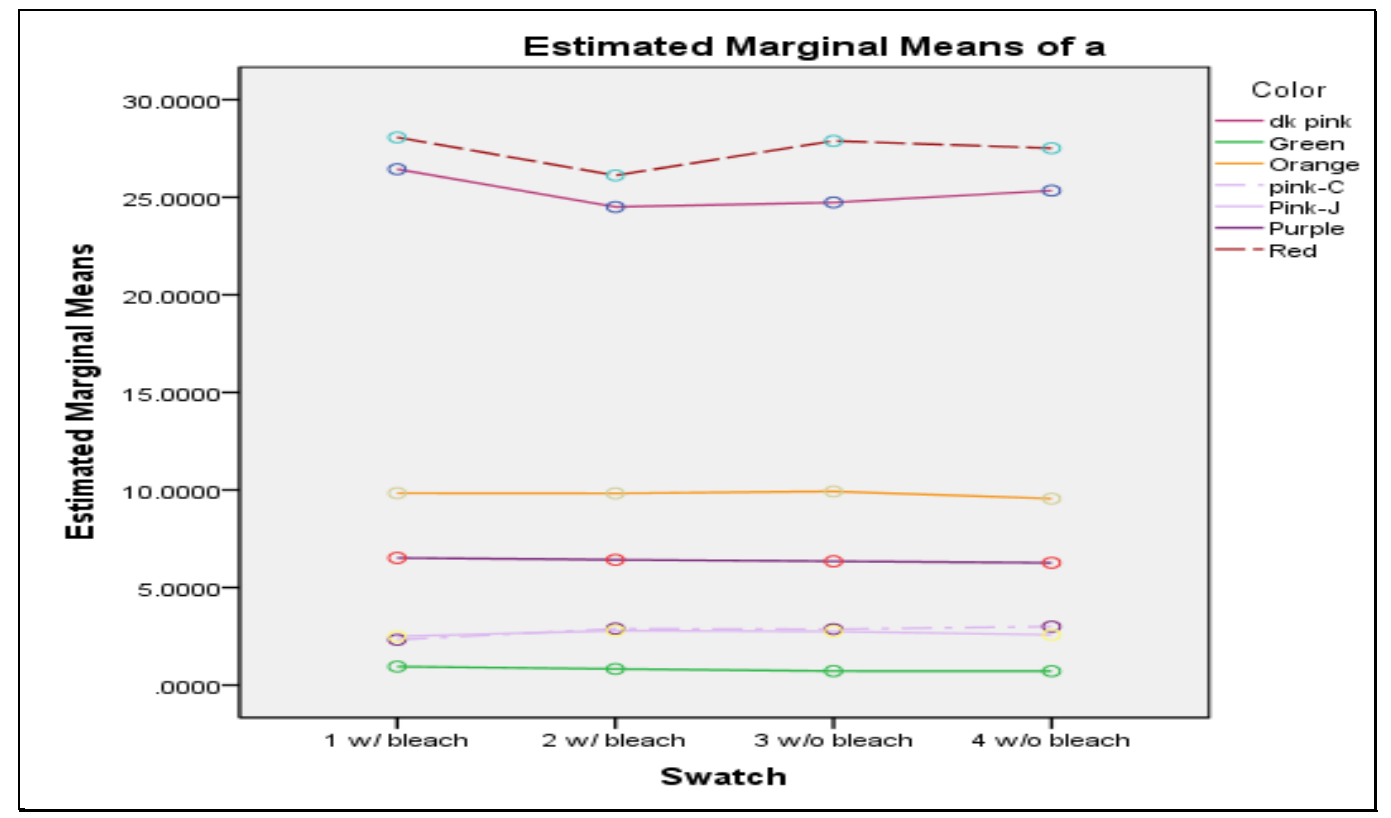

Dependent Variable: a

\begin{tabular}{|c|c|c|c|c|c|}
\hline \multirow[b]{2}{*}{ Sample Area } & \multirow[b]{2}{*}{ Color } & \multirow[b]{2}{*}{ Mean } & \multirow[b]{2}{*}{ Std. Error } & \multicolumn{2}{|c|}{ 95\% Confidence Interval } \\
\hline & & & & Lower Bound & $\begin{array}{l}\text { Upper } \\
\text { Bound }\end{array}$ \\
\hline \multirow[t]{7}{*}{$\mathrm{A}$} & dk pink & $25.538^{\mathrm{a}}$ & .106 & 25.330 & 25.747 \\
\hline & Green & $.791^{\mathrm{a}}$ & .106 & .583 & 1.000 \\
\hline & Orange & $9.710^{\mathrm{a}}$ & .106 & 9.501 & 9.919 \\
\hline & pink-C & $2.686^{\mathrm{a}}$ & .106 & 2.477 & 2.894 \\
\hline & Pink-J & $2.656^{a}$ & .106 & 2.448 & 2.865 \\
\hline & Purple & $6.368^{a}$ & .106 & 6.160 & 6.577 \\
\hline & Red & $27.760^{\mathrm{a}}$ & .106 & 27.551 & 27.969 \\
\hline \multirow[t]{7}{*}{$B$} & dk pink & $24.969^{\mathrm{a}}$ & .106 & 24.760 & 25.178 \\
\hline & Green & $.813^{\mathrm{a}}$ & .106 & .604 & 1.022 \\
\hline & Orange & $9.862^{\mathrm{a}}$ & .106 & 9.654 & 10.071 \\
\hline & pink-C & $2.856^{a}$ & .106 & 2.648 & 3.065 \\
\hline & Pink-J & $2.650^{a}$ & .106 & 2.441 & 2.859 \\
\hline & Purple & $6.409^{a}$ & .106 & 6.200 & 6.618 \\
\hline & Red & $27.035^{\mathrm{a}}$ & .106 & 26.826 & 27.244 \\
\hline
\end{tabular}

a. Based on modified population marginal mean. 


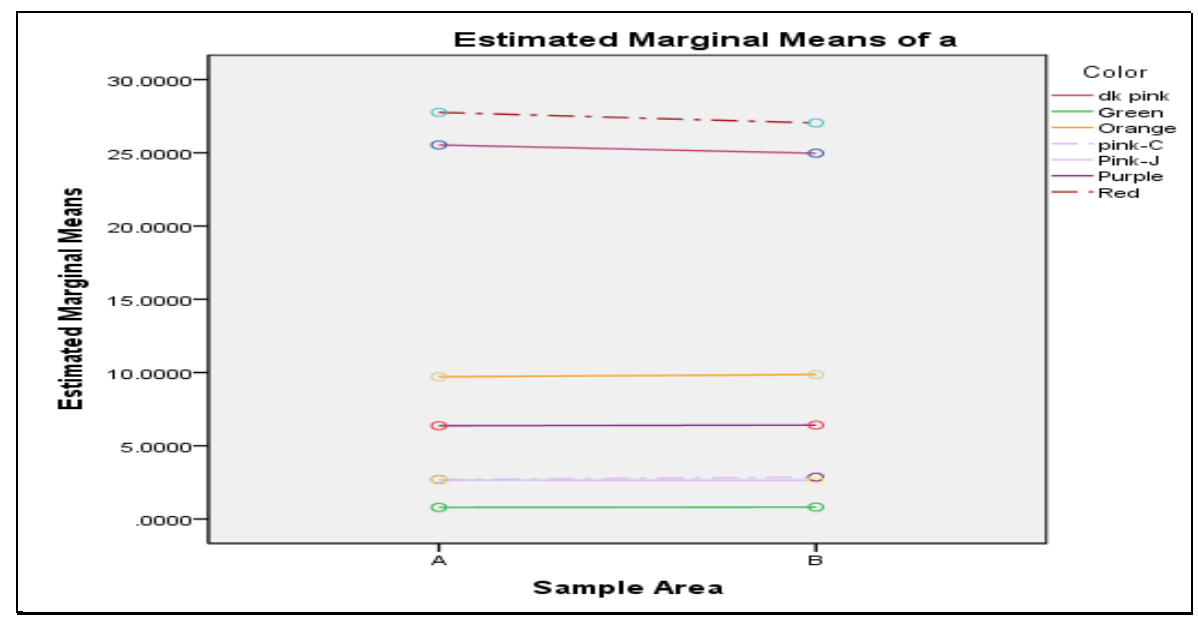

The mean values observed to be consistent between the sample areas. It can then be concluded that the significant difference observed is due to the difference in mean values between the red and dark pink samples to the remaining samples.

\section{Bleached * Color: all colors}

Dependent Variable: a

\begin{tabular}{|c|c|c|c|c|c|}
\hline \multirow[b]{2}{*}{ Bleached } & \multirow[b]{2}{*}{ Color } & \multirow[b]{2}{*}{ Mean } & \multirow[b]{2}{*}{ Std. Error } & \multicolumn{2}{|c|}{ 95\% Confidence Interval } \\
\hline & & & & Lower Bound & Upper Bound \\
\hline \multirow[t]{7}{*}{ bleached } & dk pink & $25.471^{a}$ & .106 & 25.263 & 25.680 \\
\hline & Green & $.888^{\mathrm{a}}$ & .106 & .679 & 1.097 \\
\hline & Orange & $9.831^{a}$ & .106 & 9.622 & 10.040 \\
\hline & pink-C & $2.608^{a}$ & .106 & 2.399 & 2.816 \\
\hline & Pink-J & $2.643^{\mathrm{a}}$ & .106 & 2.435 & 2.852 \\
\hline & Purple & $6.471^{\mathrm{a}}$ & .106 & 6.263 & 6.680 \\
\hline & Red & $27.095^{\mathrm{a}}$ & .106 & 26.886 & 27.304 \\
\hline \multirow[t]{7}{*}{ w/o bleached } & dk pink & $25.036^{a}$ & .106 & 24.828 & 25.245 \\
\hline & Green & $.716^{\mathrm{a}}$ & .106 & .508 & .925 \\
\hline & Orange & $9.741^{a}$ & .106 & 9.533 & 9.950 \\
\hline & pink-C & $2.934^{\mathrm{a}}$ & .106 & 2.726 & 3.143 \\
\hline & Pink-J & $2.663^{a}$ & .106 & 2.454 & 2.872 \\
\hline & Purple & $6.306^{\mathrm{a}}$ & .106 & 6.098 & 6.515 \\
\hline & Red & $27.700^{a}$ & .106 & 27.492 & 27.909 \\
\hline
\end{tabular}

a. Based on modified population marginal mean.

The mean values observed to be consistent between the bleached and unbleached. It can then be equally concluded that the significant difference observed is due to the difference in mean values between the red and dark pink samples to the remaining samples. 


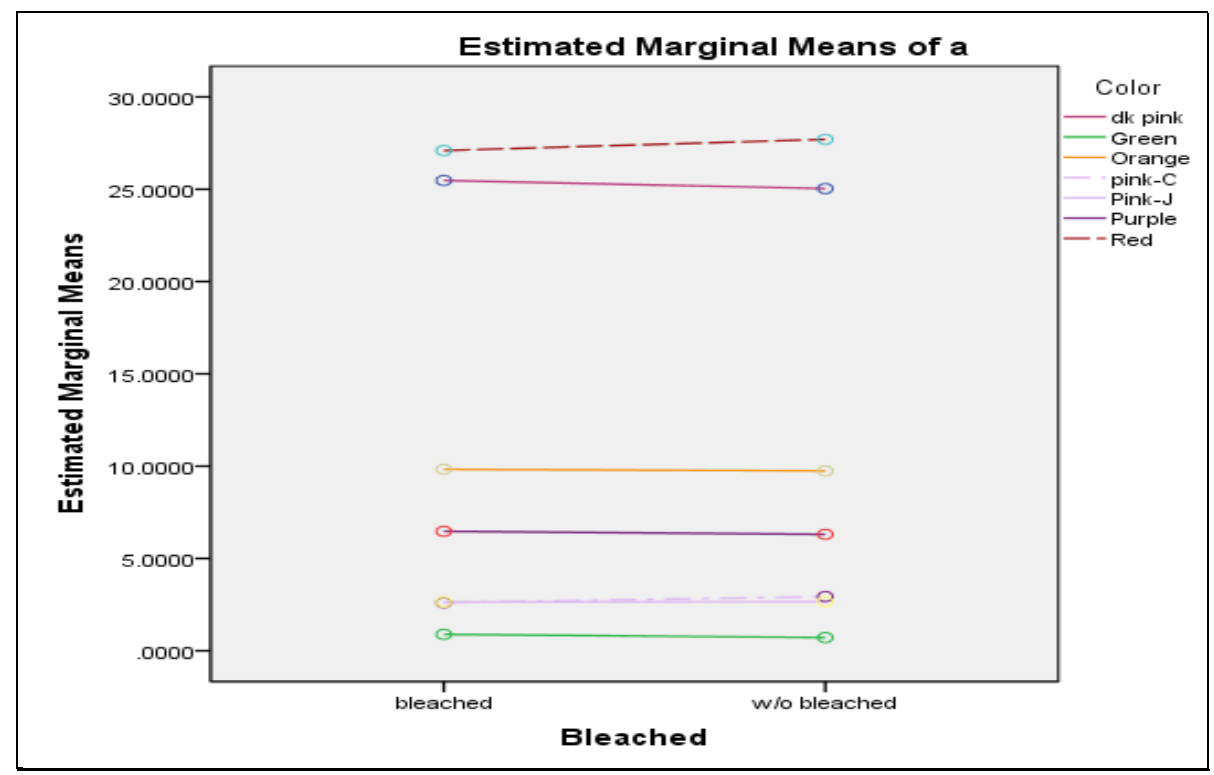

Lastly, for the interaction effects washing * color, again, it can then be equally concluded that the significant difference observed is due to the difference in mean values between the red and dark pink samples to the remaining samples. Note the corresponding table below.

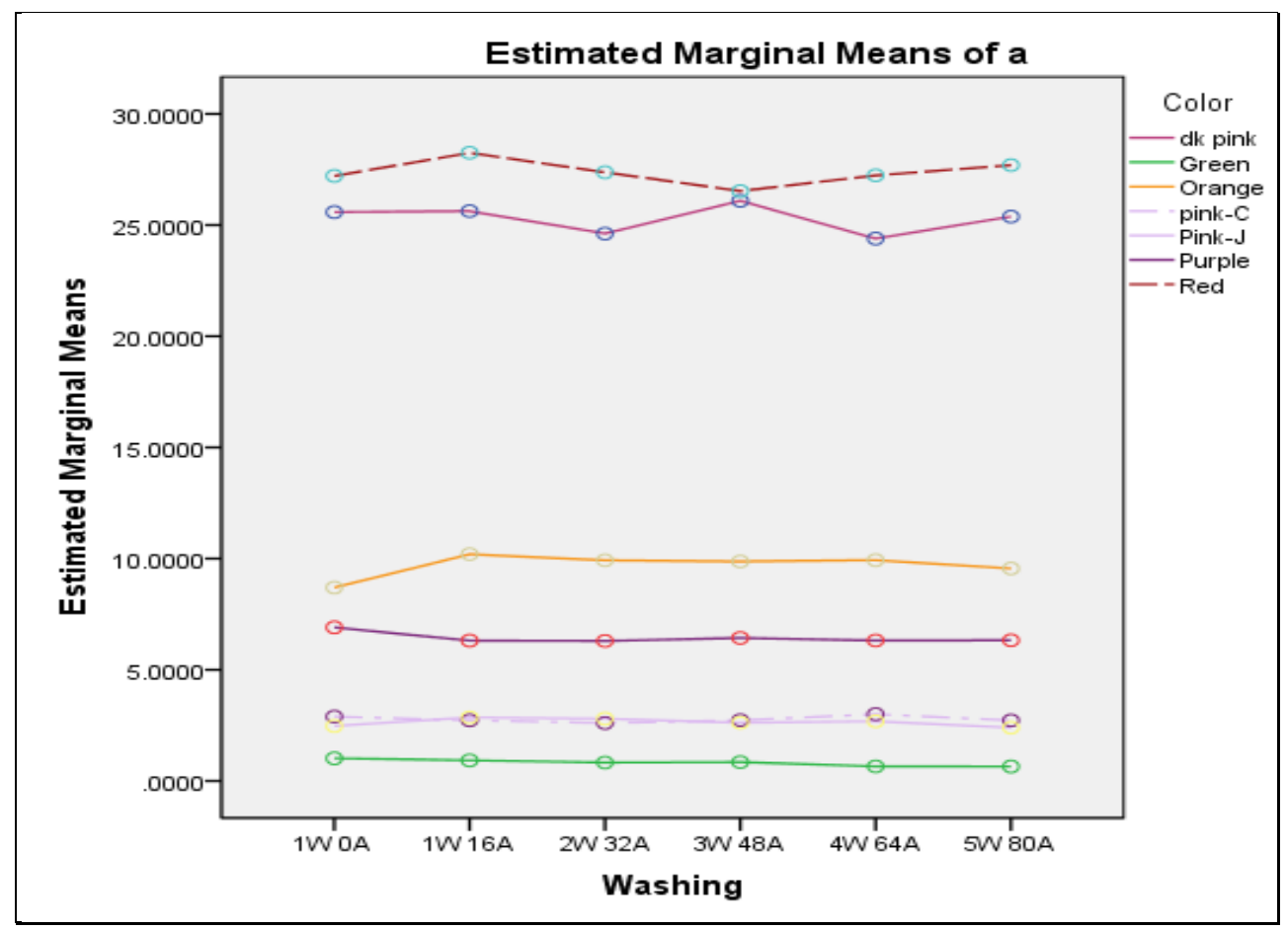




\section{Washing * Color: all color}

Dependent Variable: a

\begin{tabular}{|c|c|c|c|c|c|}
\hline \multirow[b]{2}{*}{ Washing } & \multirow[b]{2}{*}{ Color } & \multirow[b]{2}{*}{ Mean } & \multirow[b]{2}{*}{ Std. Error } & \multicolumn{2}{|c|}{ 95\% Confidence Interval } \\
\hline & & & & Lower Bound & Upper Bound \\
\hline \multirow[t]{7}{*}{$1 \mathrm{~W} 0 \mathrm{~A}$} & $\mathrm{dk}$ pink & $25.582^{\mathrm{a}}$ & .250 & 25.093 & 26.072 \\
\hline & Green & $1.018^{a}$ & .250 & .529 & 1.508 \\
\hline & Orange & $8.697^{\mathrm{a}}$ & .250 & 8.208 & 9.186 \\
\hline & pink-C & $2.896^{a}$ & .250 & 2.407 & 3.385 \\
\hline & Pink-J & $2.471^{\mathrm{a}}$ & .250 & 1.982 & 2.961 \\
\hline & Purple & $6.909^{\mathrm{a}}$ & .250 & 6.419 & 7.398 \\
\hline & Red & $27.211^{\mathrm{a}}$ & .250 & 26.722 & 27.701 \\
\hline \multirow[t]{7}{*}{$1 \mathrm{~W} 16 \mathrm{~A}$} & dk pink & $25.625^{\mathrm{a}}$ & .177 & 25.279 & 25.971 \\
\hline & Green & $.925^{\mathrm{a}}$ & .177 & .579 & 1.271 \\
\hline & Orange & $10.198^{a}$ & .177 & 9.852 & 10.544 \\
\hline & pink-C & $2.727^{\mathrm{a}}$ & .177 & 2.381 & 3.073 \\
\hline & Pink-J & $2.857^{a}$ & .177 & 2.511 & 3.203 \\
\hline & Purple & $6.311^{a}$ & .177 & 5.965 & 6.657 \\
\hline & Red & $28.248^{a}$ & .177 & 27.902 & 28.594 \\
\hline \multirow[t]{7}{*}{$2 W 32 A$} & dk pink & $24.617^{a}$ & .177 & 24.271 & 24.963 \\
\hline & Green & $.828^{\mathrm{a}}$ & .177 & .482 & 1.174 \\
\hline & Orange & $9.922^{\mathrm{a}}$ & .177 & 9.576 & 10.268 \\
\hline & pink-C & $2.606^{\mathrm{a}}$ & .177 & 2.260 & 2.952 \\
\hline & Pink-J & $2.800^{\mathrm{a}}$ & .177 & 2.454 & 3.146 \\
\hline & Purple & $6.297^{a}$ & .177 & 5.951 & 6.643 \\
\hline & Red & $27.374^{\mathrm{a}}$ & .177 & 27.028 & 27.720 \\
\hline \multirow[t]{7}{*}{$3 W$ 48A } & dk pink & $26.090^{a}$ & .177 & 25.744 & 26.436 \\
\hline & Green & $.846^{a}$ & .177 & .500 & 1.192 \\
\hline & Orange & $9.872^{a}$ & .177 & 9.526 & 10.218 \\
\hline & pink-C & $2.731^{a}$ & .177 & 2.385 & 3.077 \\
\hline & Pink-J & $2.618^{a}$ & .177 & 2.272 & 2.964 \\
\hline & Purple & $6.433^{\mathrm{a}}$ & .177 & 6.087 & 6.779 \\
\hline & Red & $26.531^{a}$ & .177 & 26.185 & 26.877 \\
\hline \multirow[t]{7}{*}{$4 W 64 A$} & dk pink & $24.392^{a}$ & .177 & 24.046 & 24.738 \\
\hline & Green & $.656^{\mathrm{a}}$ & .177 & .310 & 1.002 \\
\hline & Orange & $9.929^{\mathrm{a}}$ & .177 & 9.583 & 10.275 \\
\hline & pink-C & $3.003^{a}$ & .177 & 2.657 & 3.349 \\
\hline & Pink-J & $2.683^{a}$ & .177 & 2.337 & 3.029 \\
\hline & Purple & $6.316^{\mathrm{a}}$ & .177 & 5.970 & 6.662 \\
\hline & Red & $27.234^{a}$ & .177 & 26.888 & 27.580 \\
\hline \multirow[t]{7}{*}{$5 W 80 A$} & dk pink & $25.380^{\mathrm{a}}$ & .177 & 25.034 & 25.726 \\
\hline & Green & $.647^{\mathrm{a}}$ & .177 & .301 & .993 \\
\hline & Orange & $9.554^{a}$ & .177 & 9.208 & 9.900 \\
\hline & pink-C & $2.725^{\mathrm{a}}$ & .177 & 2.379 & 3.071 \\
\hline & Pink-J & $2.398^{a}$ & .177 & 2.052 & 2.744 \\
\hline & Purple & $6.327^{\mathrm{a}}$ & .177 & 5.981 & 6.673 \\
\hline & Red & $27.695^{\mathrm{a}}$ & .177 & 27.349 & 28.041 \\
\hline
\end{tabular}

a. Based on modified population marginal mean.

Variable ' $b$ '

The main effects of sample area and swatch, and the interaction effects of Sample Area* Swatch was determined as insignificant. 
Tests of Between-Subjects Effects: all color

Dependent Variable: $b$

\begin{tabular}{|c|c|c|c|c|c|}
\hline Source & Type III Sum of Squares & df & Mean Square & $\mathrm{F}$ & Sig. \\
\hline Corrected Model & $764478^{a}$ & 307 & 2490.2 & 379.62 & .000 \\
\hline Intercept & 492066 & 1 & 492066 & 75014 & .000 \\
\hline Swatch & 36.638 & 2 & 18.319 & 2.793 & .061 \\
\hline SampleArea & 17.385 & 1 & 17.385 & 2.650 & .104 \\
\hline Bleached & .000 & 0 & & & \\
\hline Washing & 141.53 & 5 & 28.305 & 4.315 & .001 \\
\hline Color & 711389 & 6 & 118565 & 18075 & .000 \\
\hline Swatch * SampleArea & 22.873 & 2 & 11.436 & 1.743 & .175 \\
\hline Swatch * Bleached & .000 & 0 & & & \\
\hline Swatch * Washing & 481.43 & 8 & 60.178 & 9.174 & .000 \\
\hline Swatch * Color & 377.65 & 12 & 31.471 & 4.798 & .000 \\
\hline SampleArea * Bleached & .000 & 0 & & & \\
\hline SampleArea * Washing & 288.96 & 4 & 72.239 & 11.013 & .000 \\
\hline SampleArea * Color & 151.07 & 6 & 25.179 & 3.838 & .001 \\
\hline Bleached * Washing & .000 & 0 & & & \\
\hline Bleached * Color & .000 & 0 & & & \\
\hline Washing * Color & 1411.9 & 30 & 47.063 & 7.175 & .000 \\
\hline Swatch * SampleArea * Bleached & .000 & 0 & & & \\
\hline Swatch * SampleArea * Washing & 143.14 & 8 & 17.893 & 2.728 & .005 \\
\hline Swatch * SampleArea * Color & 431.93 & 12 & 35.994 & 5.487 & .000 \\
\hline Swatch * Bleached * Washing & .000 & 0 & & & \\
\hline Swatch * Bleached * Color & .000 & 0 & & & \\
\hline Swatch * Washing * Color & 1478.6 & 48 & 30.804 & 4.696 & .000 \\
\hline SampleArea * Bleached * Washing & .000 & 0 & & & \\
\hline SampleArea * Bleached * Color & .000 & 0 & & & \\
\hline SampleArea * Washing * Color & 1586.7 & 24 & 66.112 & 10.079 & .000 \\
\hline Bleached * Washing * Color & .000 & 0 & & & \\
\hline Swatch * SampleArea * Bleached * Washing & .000 & 0 & . & . & \\
\hline Swatch * SampleArea * Bleached * Color & .000 & 0 & & & \\
\hline Swatch * SampleArea * Washing * Color & 2458.1 & 48 & 51.211 & 7.807 & .000 \\
\hline Swatch * Bleached * Washing * Color & .000 & 0 & & & \\
\hline SampleArea * Bleached * Washing * Color & .000 & 0 & & . & \\
\hline Swatch * SampleArea * Bleached * Washing * Color & .000 & 0 & & . & \\
\hline Error & 38387 & 5852 & 6.560 & & \\
\hline Total & 1326199 & 6160 & & & \\
\hline Corrected Total & 802865 & 6159 & & & \\
\hline
\end{tabular}

a. R Squared $=.952$ (Adjusted R Squared $=.950)$

\section{Sample Area Estimates: all colors}

Dependent Variable: $b$

\begin{tabular}{|l|r|r|r|r|}
\hline & & \multirow{2}{*}{ 95\% Confidence Interval } \\
\cline { 4 - 5 } Sample Area & Mean & Std. Error & Lower Bound & Upper Bound \\
\hline A & $9.212^{\mathrm{a}}$ & .046 & 9.122 & 9.302 \\
B & $9.222^{\mathrm{a}}$ & .046 & 9.132 & 9.313 \\
\hline
\end{tabular}

a. Based on modified population marginal mean.

Pairwise Comparisons: all colors

Dependent Variable: $b$

\begin{tabular}{|c|c|c|c|c|c|c|}
\hline \multirow[b]{2}{*}{ (I) Sample Area } & \multirow[b]{2}{*}{ (J) Sample Area } & \multirow[b]{2}{*}{ Mean Difference (I-J) } & \multirow[b]{2}{*}{ Std. Error } & \multirow[b]{2}{*}{ Sig. ${ }^{\mathrm{C}}$} & \multicolumn{2}{|c|}{$95 \%$ Confidence Interval for Difference ${ }^{c}$} \\
\hline & & & & & Lower Bound & Upper Bound \\
\hline A & $\mathrm{B}$ & $-.010^{\mathrm{a}}, \mathrm{b}$ & .065 & .873 & -.138 & .118 \\
\hline $\mathrm{B}$ & $A$ & $.010^{\mathrm{a}}, \mathrm{b}$ & .065 & .873 & -.118 & .138 \\
\hline
\end{tabular}

Based on estimated marginal means 
a. An estimate of the modified population marginal mean (I).

b. An estimate of the modified population marginal mean $(\mathrm{J})$.

c. Adjustment for multiple comparisons: Least Significant Difference (equivalent to no adjustments).

Between sample areas, no significant difference was observed.

Dependent Variable: $b$

\section{Swatch Estimates: all colors}

\begin{tabular}{|l|r|r|r|r|}
\hline & & & \multicolumn{2}{|c|}{$95 \%$ Confidence Interval } \\
\cline { 4 - 5 } Swatch & Mean & Std. Error & Lower Bound & Upper Bound \\
\hline 1 w/ bleach & $9.270^{\mathrm{a}}$ & .065 & 9.143 & 9.398 \\
2 w/ bleach & $9.113^{\mathrm{a}}$ & .065 & 8.985 & 9.241 \\
3 w/o bleach & $9.392^{\mathrm{a}}$ & .065 & 9.264 & 9.520 \\
4 w/o bleach & $9.093^{\mathrm{a}}$ & .065 & 8.965 & 9.221 \\
\hline
\end{tabular}

a. Based on modified population marginal mean.

Pairwise Comparisons: all colors

Dependent Variable: $b$

\begin{tabular}{|c|c|c|c|c|c|c|}
\hline \multirow[b]{2}{*}{ (I) Swatch } & \multirow[b]{2}{*}{ (J) Swatch } & \multirow[b]{2}{*}{ Mean Difference (I-J) } & \multirow[b]{2}{*}{ Std. Error } & \multirow[b]{2}{*}{ Sig. ${ }^{\mathrm{C}}$} & \multicolumn{2}{|c|}{$\begin{array}{c}95 \% \text { Confidence Interval for } \\
\text { Difference }\end{array}$} \\
\hline & & & & & Lower Bound & Upper Bound \\
\hline \multirow[t]{3}{*}{$1 \mathrm{w} /$ bleach } & $2 \mathrm{w} /$ bleach & $.157^{\mathrm{a}, \mathrm{b}}$ & .092 & .089 & -.024 & .338 \\
\hline & $3 \mathrm{w} / \mathrm{o}$ bleach & $-.121^{a}, b$ & .092 & .189 & -.302 & .060 \\
\hline & 4 w/o bleach & $.177^{a}, b$ & .092 & .055 & -.004 & .358 \\
\hline \multirow[t]{3}{*}{$2 \mathrm{w} /$ bleach } & $1 \mathrm{w} /$ bleach & $-.157^{a}, b$ & .092 & .089 & -.338 & .024 \\
\hline & 3 w/o bleach & $-.278^{a}, b,^{*}$ & .092 & .003 & -.459 & -.097 \\
\hline & 4 w/o bleach & $.020^{\mathrm{a}}, \mathrm{b}$ & .092 & .826 & -.161 & .201 \\
\hline \multirow[t]{3}{*}{$3 \mathrm{w} / \mathrm{o}$ bleach } & $1 \mathrm{w} /$ bleach & $.121^{\mathrm{a}}, \mathrm{b}$ & .092 & .189 & -.060 & .302 \\
\hline & 2 w/ bleach & $.278^{\mathrm{a}}, \mathrm{b},{ }^{*}$ & .092 & .003 & .097 & .459 \\
\hline & $4 \mathrm{w} / \mathrm{o}$ bleach & $.299^{a}, b, *$ & .092 & .001 & .118 & .480 \\
\hline \multirow[t]{3}{*}{4 w/o bleach } & $1 \mathrm{w} /$ bleach & $-.177^{a}, b$ & .092 & .055 & -.358 & .004 \\
\hline & $2 \mathrm{w} /$ bleach & $-.020^{\mathrm{a}}, \mathrm{b}$ & .092 & .826 & -.201 & .161 \\
\hline & 3 w/o bleach & $-.299^{a}, b,^{*}$ & .092 & .001 & -.480 & -.118 \\
\hline
\end{tabular}

Based on estimated marginal means

a. An estimate of the modified population marginal mean (I).

b. An estimate of the modified population marginal mean (J).

c. Adjustment for multiple comparisons: Least Significant Difference (equivalent to no adjustments).

*. The mean difference is significant at the .05 level.

Significant values were observed for all groups but not all pair-wise comparing groups. The pair-

wise groups for the bleached swatches were insignificant, while the unbleached swatches were

significant.

\section{Bleach Estimates: all colors}

Dependent Variable: $b$

\begin{tabular}{|l|r|r|r|r|}
\hline & & & \multicolumn{2}{|c|}{ 95\% Confidence Interval } \\
\cline { 4 - 5 } Bleached & Mean & Std. Error & Lower Bound & Upper Bound \\
\hline bleached & $9.192^{\mathrm{a}}$ & .046 & 9.101 & 9.282 \\
w/o bleached & $9.242^{\mathrm{a}}$ & .046 & 9.152 & 9.333 \\
\hline
\end{tabular}

a. Based on modified population marginal mean. 
Dependent Variable: $b$

Pairwise Comparisons: all colors

\begin{tabular}{|ll|r|r|r|r|r|}
\hline & & & & \multicolumn{2}{c|}{$\begin{array}{c}\text { 95\% Confidence Interval for } \\
\text { Difference }\end{array}$} \\
\cline { 3 - 8 } & & & & & \\
(I) Bleached & $(\mathrm{J})$ Bleached & Mean Difference $(\mathrm{I}-\mathrm{J})$ & Std. Error & \multicolumn{1}{c|}{ Sig. $^{\mathrm{c}}$} & Lower Bound & Upper Bound \\
\hline bleached & w/o bleached & $-.051^{\mathrm{a}}, \mathrm{b}$ & .065 & .439 & -.178 & .077 \\
\hline w/o bleached & bleached & $.051^{\mathrm{a}}, \mathrm{b}$ & .065 & .439 & -.077 & .178 \\
\hline
\end{tabular}

Based on estimated marginal means

a. An estimate of the modified population marginal mean (I).

b. An estimate of the modified population marginal mean $(\mathrm{J})$.

c. Adjustment for multiple comparisons: Least Significant Difference (equivalent to no adjustments).

Between the bleached and unbleached samples, no significant difference was observed.

Based on the main effects of washing, marked significant differences are observed for all groups

but not at all pair-wise comparing groups. No patterns were observed for this factor.

\section{Washing Estimates: all colors}

Dependent Variable: $b$

\begin{tabular}{|l|r|r|r|r|}
\hline & & & \multicolumn{2}{|c|}{ 95\% Confidence Interval } \\
\cline { 4 - 5 } Washing & Mean & Std. Error & Lower Bound & Upper Bound \\
\hline 1W 0A & $9.511^{\mathrm{a}}$ & .108 & 9.299 & 9.724 \\
1W 16A & $9.439^{\mathrm{a}}$ & .077 & 9.289 & 9.589 \\
2W 32A & $9.146^{\mathrm{a}}$ & .077 & 8.996 & 9.296 \\
3W 48A & $9.094^{\mathrm{a}}$ & .077 & 8.944 & 9.244 \\
4W 64A & $9.039^{\mathrm{a}}$ & .077 & 8.889 & 9.189 \\
5W 80A & $9.221^{\mathrm{a}}$ & .077 & 9.071 & 9.371 \\
\hline
\end{tabular}

a. Based on modified population marginal mean. 
Dependent Variable: $b$

Pairwise Comparisons: all colors

\begin{tabular}{|c|c|c|c|c|c|c|}
\hline & & & & & $\begin{array}{r}\text { 95\% Confide } \\
\text { Diff }\end{array}$ & $\begin{array}{l}\text { Interval for } \\
\text { nce }^{c}\end{array}$ \\
\hline (I) Washing & (J) Washing & Mean Difference (I-J) & Std. Error & Sig. ${ }^{\mathrm{c}}$ & Lower Bound & Upper Bound \\
\hline $1 \mathrm{~W} \mathrm{OA}$ & $1 \mathrm{~W} 16 \mathrm{~A}$ & $.073^{\mathrm{a}}, \mathrm{b}$ & .133 & .583 & -.187 & .333 \\
\hline & $2 W 32 A$ & $.365^{\mathrm{a}}, \mathrm{b},{ }^{*}$ & .133 & .006 & .106 & .625 \\
\hline & $3 W 48 A$ & $.417^{\mathrm{a}}, \mathrm{b},{ }^{*}$ & .133 & .002 & .157 & 677 \\
\hline & $4 \mathrm{~W} 64 \mathrm{~A}$ & $.472^{\mathrm{a}}, \mathrm{b},{ }^{*}$ & .133 & .000 & .212 & .732 \\
\hline & $5 \mathrm{~W} 80 \mathrm{~A}$ & $.290^{\mathrm{a}}, \mathrm{b},{ }^{*}$ & .133 & .029 & .030 & .550 \\
\hline $1 \mathrm{~W} 16 \mathrm{~A}$ & $1 \mathrm{~W} 0 \mathrm{~A}$ & $-.073^{\mathrm{a}}, \mathrm{b}$ & .133 & .583 & -.333 & .187 \\
\hline & $2 W 32 A$ & $.293^{\mathrm{a}}, \mathrm{b},{ }^{*}$ & .108 & .007 & .081 & .505 \\
\hline & $3 W 48 A$ & $.345^{\mathrm{a}}, \mathrm{b},{ }^{*}$ & .108 & .001 & .132 & .557 \\
\hline & $4 \mathrm{~W} 64 \mathrm{~A}$ & $.400^{\mathrm{a}}, \mathrm{b},{ }^{*}$ & .108 & .000 & .187 & .612 \\
\hline & $5 \mathrm{~W} 80 \mathrm{~A}$ & $.217^{\mathrm{a}}, \mathrm{b},{ }^{*}$ & .108 & .045 & .005 & .430 \\
\hline $2 W 32 A$ & $1 \mathrm{~W} 0 \mathrm{~A}$ & $-.365^{\mathrm{a}}, \mathrm{b},{ }^{*}$ & .133 & .006 & -.625 & -.106 \\
\hline & $1 \mathrm{~W} 16 \mathrm{~A}$ & $-.293^{\mathrm{a}}, \mathrm{b}, *$ & .108 & .007 & -.505 & -.081 \\
\hline & $3 W 48 A$ & $.052^{a}, b$ & .108 & .632 & -.160 & .264 \\
\hline & $4 W 64 A$ & $.107^{a}, b$ & .108 & .324 & -.105 & .319 \\
\hline & $5 W 80 A$ & $-.075^{a}, b$ & .108 & .486 & -.288 & .137 \\
\hline $3 W$ 48A & $1 \mathrm{~W} 0 \mathrm{~A}$ & $-.417^{a}, b{ }^{*}$ & .133 & .002 & -.677 & -.157 \\
\hline & $1 \mathrm{~W} 16 \mathrm{~A}$ & $-.345^{\mathrm{a}}, \mathrm{b},{ }^{*}$ & .108 & .001 & -.557 & -.132 \\
\hline & $2 W 32 A$ & $-.052^{\mathrm{a}}, \mathrm{b}$ & .108 & .632 & -.264 & .160 \\
\hline & $4 W 64 A$ & $.055^{a}, b$ & .108 & .611 & -.157 & .267 \\
\hline & $5 \mathrm{~W} 80 \mathrm{~A}$ & $-.127^{a}, b$ & .108 & .240 & -.339 & .085 \\
\hline $4 W 64 A$ & $1 \mathrm{~W} 0 \mathrm{~A}$ & $-.472^{a}, b{ }^{*}$ & .133 & .000 & -.732 & -.212 \\
\hline & $1 \mathrm{~W} 16 \mathrm{~A}$ & $-.400^{a}, b{ }^{*}$ & .108 & .000 & -.612 & -.187 \\
\hline & $2 W 32 A$ & $-.107^{a}, b$ & .108 & .324 & -.319 & .105 \\
\hline & $3 W 48 A$ & $-.055^{a}, b$ & .108 & .611 & -.267 & .157 \\
\hline & $5 \mathrm{~W} 80 \mathrm{~A}$ & $-.182^{a}, b$ & .108 & .092 & -.394 & .030 \\
\hline $5 W$ 80A & $1 \mathrm{~W} 0 \mathrm{~A}$ & $-.290^{a}, b{ }^{*}$ & .133 & .029 & -.550 & -.030 \\
\hline & $1 \mathrm{~W} 16 \mathrm{~A}$ & $-.217^{\mathrm{a}}, \mathrm{b},{ }^{*}$ & .108 & .045 & -.430 & -.005 \\
\hline & $2 W 32 A$ & $.075^{a}, b$ & .108 & .486 & -.137 & .288 \\
\hline & $3 W 48 A$ & $.127^{a}, \mathrm{~b}$ & .108 & .240 & -.085 & .339 \\
\hline & $4 W 64 A$ & $.182^{\mathrm{a}}, \mathrm{b}$ & .108 & .092 & -.030 & .394 \\
\hline
\end{tabular}

Based on estimated marginal means

a. An estimate of the modified population marginal mean (I).

b. An estimate of the modified population marginal mean (J).

c. Adjustment for multiple comparisons: Least Significant Difference (equivalent to no adjustments).

*. The mean difference is significant at the .05 level.

\section{Color Estimates: all colors}

Dependent Variable: $b$

\begin{tabular}{|l|r|r|r|r|}
\hline & & & \multicolumn{2}{|c|}{ 95\% Confidence Interval } \\
\cline { 4 - 5 } Color & Mean & Std. Error & Lower Bound & Upper Bound \\
\hline dk pink & $7.317^{\mathrm{a}}$ & .086 & 7.148 & 7.487 \\
Green & $10.325^{\mathrm{a}}$ & .086 & 10.156 & 10.494 \\
Orange & $30.286^{\mathrm{a}}$ & .086 & 30.117 & 30.455 \\
pink-C & $4.429^{\mathrm{a}}$ & .086 & 4.259 & 4.598 \\
Pink-J & $5.143^{\mathrm{a}}$ & .086 & 4.974 & 5.312 \\
Purple & $-8.726^{\mathrm{a}}$ & .086 & -8.895 & -8.557 \\
Red & $15.746^{\mathrm{a}}$ & .086 & 15.577 & 15.915 \\
\hline
\end{tabular}

a. Based on modified population marginal mean. 
Dependent Variable: $b$

Pairwise Comparisons: all colors

\begin{tabular}{|c|c|c|c|c|c|c|}
\hline \multirow{2}{*}{ (I) Color } & \multirow[b]{2}{*}{ (J) Color } & \multirow[b]{2}{*}{$\begin{array}{c}\text { Mean Difference } \\
(I-J)\end{array}$} & \multirow[b]{2}{*}{ Std. Error } & \multirow[b]{2}{*}{ Sig. ${ }^{c}$} & \multicolumn{2}{|c|}{$\begin{array}{l}95 \% \text { Confidence Interval } \\
\text { for Difference }\end{array}$} \\
\hline & & & & & $\begin{array}{l}\text { Lower } \\
\text { Bound }\end{array}$ & $\begin{array}{l}\text { Upper } \\
\text { Bound }\end{array}$ \\
\hline \multirow[t]{6}{*}{ dk pink } & Green & $-3.008^{*}, a, b$ & .122 & .000 & -3.247 & -2.768 \\
\hline & Orange & $-22.968^{*}, a, b$ & .122 & .000 & -23.208 & -22.729 \\
\hline & pink-C & $2.889^{*}, a, b$ & .122 & .000 & 2.649 & 3.128 \\
\hline & Pink-J & $2.174^{*}, a, b$ & .122 & .000 & 1.935 & 2.414 \\
\hline & Purple & $16.043^{*}, a, b$ & .122 & .000 & 15.804 & 16.283 \\
\hline & Red & $-8.429^{*}, a, b$ & .122 & .000 & -8.668 & -8.189 \\
\hline \multirow[t]{6}{*}{ Green } & dk pink & $3.008^{*}, a, b$ & .122 & .000 & 2.768 & 3.247 \\
\hline & Orange & $-19.961^{*}, a, b$ & .122 & .000 & -20.200 & -19.721 \\
\hline & pink-C & $5.896^{*}, a, b$ & .122 & .000 & 5.657 & 6.136 \\
\hline & Pink-J & $5.182^{\star}, a, b$ & .122 & .000 & 4.943 & 5.421 \\
\hline & Purple & $19.051^{*}, a, b$ & .122 & .000 & 18.812 & 19.290 \\
\hline & Red & $-5.421^{*}, a, b$ & .122 & .000 & -5.660 & -5.182 \\
\hline \multirow[t]{6}{*}{ Orange } & dk pink & $22.968^{*}, a, b$ & .122 & .000 & 22.729 & 23.208 \\
\hline & Green & $19.961^{*}, a, b$ & .122 & .000 & 19.721 & 20.200 \\
\hline & pink-C & $25.857^{*}, a, b$ & .122 & .000 & 25.618 & 26.097 \\
\hline & Pink-J & $25.143^{*}, a, b$ & .122 & .000 & 24.903 & 25.382 \\
\hline & Purple & $39.012^{*}, a, b$ & .122 & .000 & 38.772 & 39.251 \\
\hline & Red & $14.540^{*}, a, b$ & .122 & .000 & 14.300 & 14.779 \\
\hline \multirow[t]{6}{*}{ pink-C } & dk pink & $-2.889^{*}, a, b$ & .122 & .000 & -3.128 & -2.649 \\
\hline & Green & $-5.896^{\star}, a, b$ & .122 & .000 & -6.136 & -5.657 \\
\hline & Orange & $-25.857^{\star}, a, b$ & .122 & .000 & -26.097 & -25.618 \\
\hline & Pink-J & $-.715^{\star}, a, b$ & .122 & .000 & -.954 & -.475 \\
\hline & Purple & $13.155^{*}, \mathrm{a}, \mathrm{b}$ & .122 & .000 & 12.915 & 13.394 \\
\hline & Red & $-11.318^{\star}, a, b$ & .122 & .000 & -11.557 & -11.078 \\
\hline \multirow[t]{6}{*}{ Pink-J } & dk pink & $-2.174^{\star}, a, b$ & .122 & .000 & -2.414 & -1.935 \\
\hline & Green & $-5.182^{*}, a, b$ & .122 & .000 & -5.421 & -4.943 \\
\hline & Orange & $-25.143^{*}, a, b$ & .122 & .000 & -25.382 & -24.903 \\
\hline & pink-C & $.715^{*}, \mathrm{a}, \mathrm{b}$ & .122 & .000 & .475 & .954 \\
\hline & Purple & $13.869^{*}, a, b$ & .122 & .000 & 13.630 & 14.109 \\
\hline & Red & $-10.603^{*}, a, b$ & .122 & .000 & -10.842 & -10.364 \\
\hline \multirow[t]{6}{*}{ Purple } & dk pink & $-16.043^{*}, a, b$ & .122 & .000 & -16.283 & -15.804 \\
\hline & Green & $-19.051^{\star}, a, b$ & .122 & .000 & -19.290 & -18.812 \\
\hline & Orange & $-39.012^{*}, a, b$ & .122 & .000 & -39.251 & -38.772 \\
\hline & pink-C & $-13.155^{*}, a, b$ & .122 & .000 & -13.394 & -12.915 \\
\hline & Pink-J & $-13.869^{*}, a, b$ & .122 & .000 & -14.109 & -13.630 \\
\hline & Red & $-24.472^{*}, a, b$ & .122 & .000 & -24.712 & -24.233 \\
\hline \multirow[t]{6}{*}{ Red } & dk pink & $8.429^{*}, a, b$ & .122 & .000 & 8.189 & 8.668 \\
\hline & Green & $5.421^{*}, a, b$ & .122 & .000 & 5.182 & 5.660 \\
\hline & Orange & $-14.540^{*}, a, b$ & .122 & .000 & -14.779 & -14.300 \\
\hline & pink-C & $11.318^{*}, a, b$ & .122 & .000 & 11.078 & 11.557 \\
\hline & Pink-J & $10.603^{*}, a, b$ & .122 & .000 & 10.364 & 10.842 \\
\hline & Purple & $24.472^{*}, a, b$ & .122 & .000 & 24.233 & 24.712 \\
\hline
\end{tabular}

Based on estimated marginal means

*. The mean difference is significant at the .05 level.

a. An estimate of the modified population marginal mean (I).

b. An estimate of the modified population marginal mean $(\mathrm{J})$.

c. Adjustment for multiple comparisons: Least Significant Difference (equivalent to no adjustments). 
Marked significant differences were observed for all pair-wise comparison. Note that at this variable, sample PMJX and PMCL were also observed to be significantly different and thus, can be differentiated as separate colors. The values between these samples at the yellow to blue region allow discrimination between the samples.

Dependent Variable: $b$

\section{Swatch ${ }^{*}$ Color: all colors}

\begin{tabular}{|c|c|c|c|c|c|}
\hline \multirow[b]{2}{*}{ Swatch } & \multirow[b]{2}{*}{ Color } & \multirow[b]{2}{*}{ Mean } & \multirow[b]{2}{*}{ Std. Error } & \multicolumn{2}{|c|}{ 95\% Confidence Interval } \\
\hline & & & & $\begin{array}{l}\text { Lower } \\
\text { Bound }\end{array}$ & $\begin{array}{l}\text { Upper } \\
\text { Bound }\end{array}$ \\
\hline \multirow[t]{7}{*}{$1 \mathrm{w} /$ bleach } & dk pink & $7.793^{\mathrm{a}}$ & .173 & 7.455 & 8.132 \\
\hline & Green & $10.578^{a}$ & .173 & 10.239 & 10.916 \\
\hline & Orange & $30.449^{a}$ & .173 & 30.111 & 30.788 \\
\hline & pink-C & $3.618^{a}$ & .173 & 3.279 & 3.956 \\
\hline & Pink-J & $4.842^{\mathrm{a}}$ & .173 & 4.504 & 5.181 \\
\hline & Purple & $-8.380^{a}$ & .173 & -8.718 & -8.041 \\
\hline & Red & $15.993^{\mathrm{a}}$ & .173 & 15.655 & 16.332 \\
\hline \multirow[t]{7}{*}{$2 \mathrm{w} /$ bleach } & $\mathrm{dk}$ pink & $7.119^{a}$ & .173 & 6.780 & 7.457 \\
\hline & Green & $10.297^{\mathrm{a}}$ & .173 & 9.958 & 10.635 \\
\hline & Orange & $30.379^{a}$ & .173 & 30.041 & 30.718 \\
\hline & pink-C & $4.713^{a}$ & .173 & 4.374 & 5.051 \\
\hline & Pink-J & $5.435^{a}$ & .173 & 5.097 & 5.774 \\
\hline & Purple & $-8.807^{a}$ & .173 & -9.145 & -8.468 \\
\hline & Red & $14.658^{a}$ & .173 & 14.319 & 14.996 \\
\hline \multirow[t]{7}{*}{3 w/o bleach } & $\mathrm{dk}$ pink & $7.168^{\mathrm{a}}$ & .173 & 6.829 & 7.506 \\
\hline & Green & $10.384^{a}$ & .173 & 10.045 & 10.722 \\
\hline & Orange & $30.557^{\mathrm{a}}$ & .173 & 30.219 & 30.896 \\
\hline & pink-C & $4.618^{a}$ & .173 & 4.279 & 4.956 \\
\hline & Pink-J & $5.260^{\mathrm{a}}$ & .173 & 4.922 & 5.599 \\
\hline & Purple & $-8.452^{\mathrm{a}}$ & .173 & -8.791 & -8.114 \\
\hline & Red & $16.208^{a}$ & .173 & 15.870 & 16.547 \\
\hline \multirow[t]{7}{*}{$4 \mathrm{w} / \mathrm{o}$ bleach } & dk pink & $7.190^{\mathrm{a}}$ & .173 & 6.851 & 7.528 \\
\hline & Green & $10.042^{a}$ & .173 & 9.704 & 10.381 \\
\hline & Orange & $29.758^{a}$ & .173 & 29.419 & 30.096 \\
\hline & pink-C & $4.767^{a}$ & .173 & 4.428 & 5.105 \\
\hline & Pink-J & $5.035^{\mathrm{a}}$ & .173 & 4.697 & 5.374 \\
\hline & Purple & $-9.265^{a}$ & .173 & -9.604 & -8.927 \\
\hline & Red & $16.125^{\mathrm{a}}$ & .173 & 15.787 & 16.464 \\
\hline
\end{tabular}

a. Based on modified population marginal mean. 


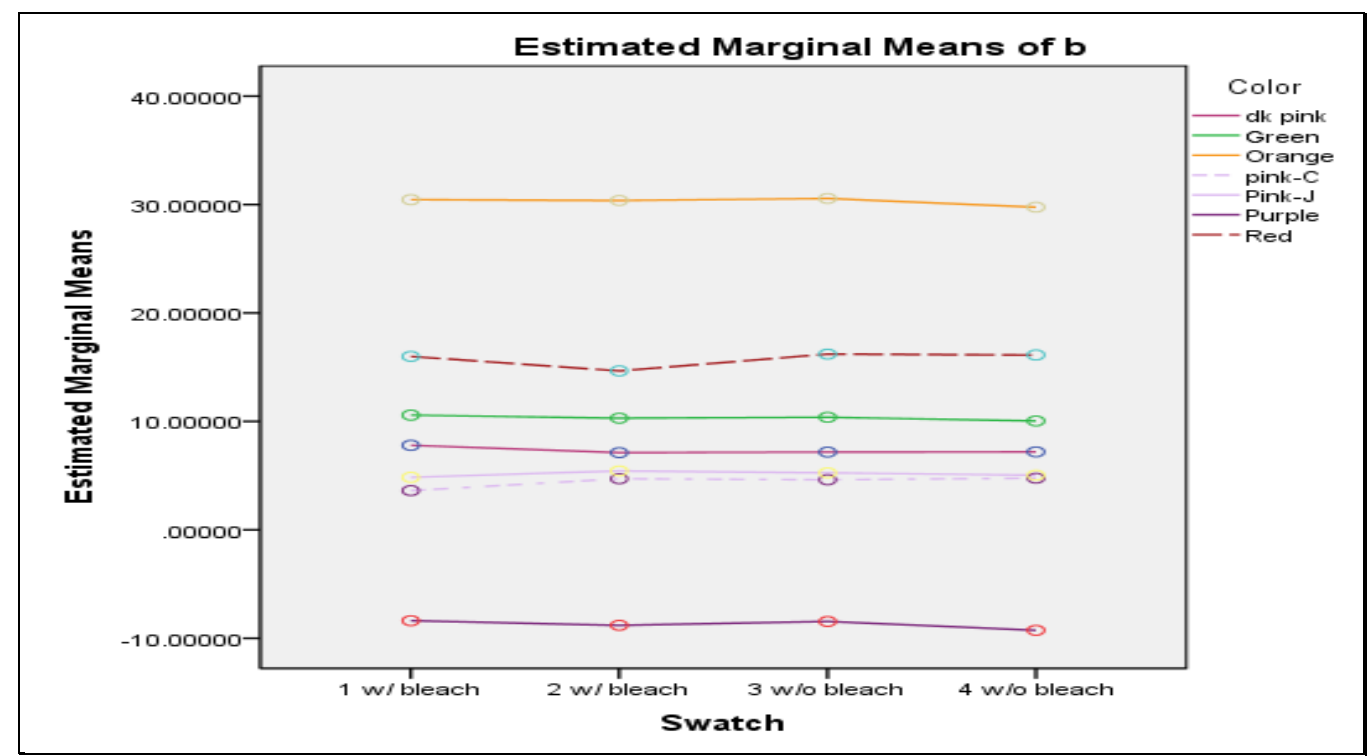

It can be observed that the bleached and unbleached swatches affect each color differently,

although these differences observe to be minimal. The significant differences are more likely due to mean difference between purple and orange.

\section{Sample Area * Color: all colors}

Dependent Variable: $b$

\begin{tabular}{|ll|r|r|r|r|}
\hline & & & & \multicolumn{2}{|c|}{$\begin{array}{c}\text { 95 Confidence } \\
\text { Interval }\end{array}$} \\
\cline { 3 - 6 } Sample Area & Color & & \multicolumn{2}{|c|}{$\begin{array}{c}\text { Lower } \\
\text { Bound }\end{array}$} & $\begin{array}{r}\text { Upper } \\
\text { Bound }\end{array}$ \\
\hline A & Mean & Std. Error & 7.249 & 7.728 \\
& Green & $7.488^{\mathrm{a}}$ & .122 & 10.337 \\
& Orange & $10.097^{\mathrm{a}}$ & .122 & 9.858 & 30.373 \\
& pink-C & $30.133^{\mathrm{a}}$ & .122 & 29.894 & 4.543 \\
& Pink-J & $4.303^{\mathrm{a}}$ & .122 & 4.064 & 5.362 \\
& Purple & $5.123^{\mathrm{a}}$ & .122 & 4.884 & -8.398 \\
& Red & $-8.637^{\mathrm{a}}$ & .122 & -8.877 & 16.215 \\
\hline B & $15.976^{\mathrm{a}}$ & .122 & 15.736 & 7.386 \\
& dk pink & $7.146^{\mathrm{a}}$ & .122 & 6.907 & 10.792 \\
& Green & $10.553^{\mathrm{a}}$ & .122 & 10.314 & 30.678 \\
& Orange & $30.438^{\mathrm{a}}$ & .122 & 30.199 & 4.793 \\
& pink-C & $4.554^{\mathrm{a}}$ & .122 & 4.315 & 5.403 \\
& Pink-J & $5.163^{\mathrm{a}}$ & .122 & 4.924 & -8.575 \\
& Purple & $-8.815^{\mathrm{a}}$ & .122 & -9.054 & 15.756 \\
\hline
\end{tabular}

a. Based on modified population marginal mean. 


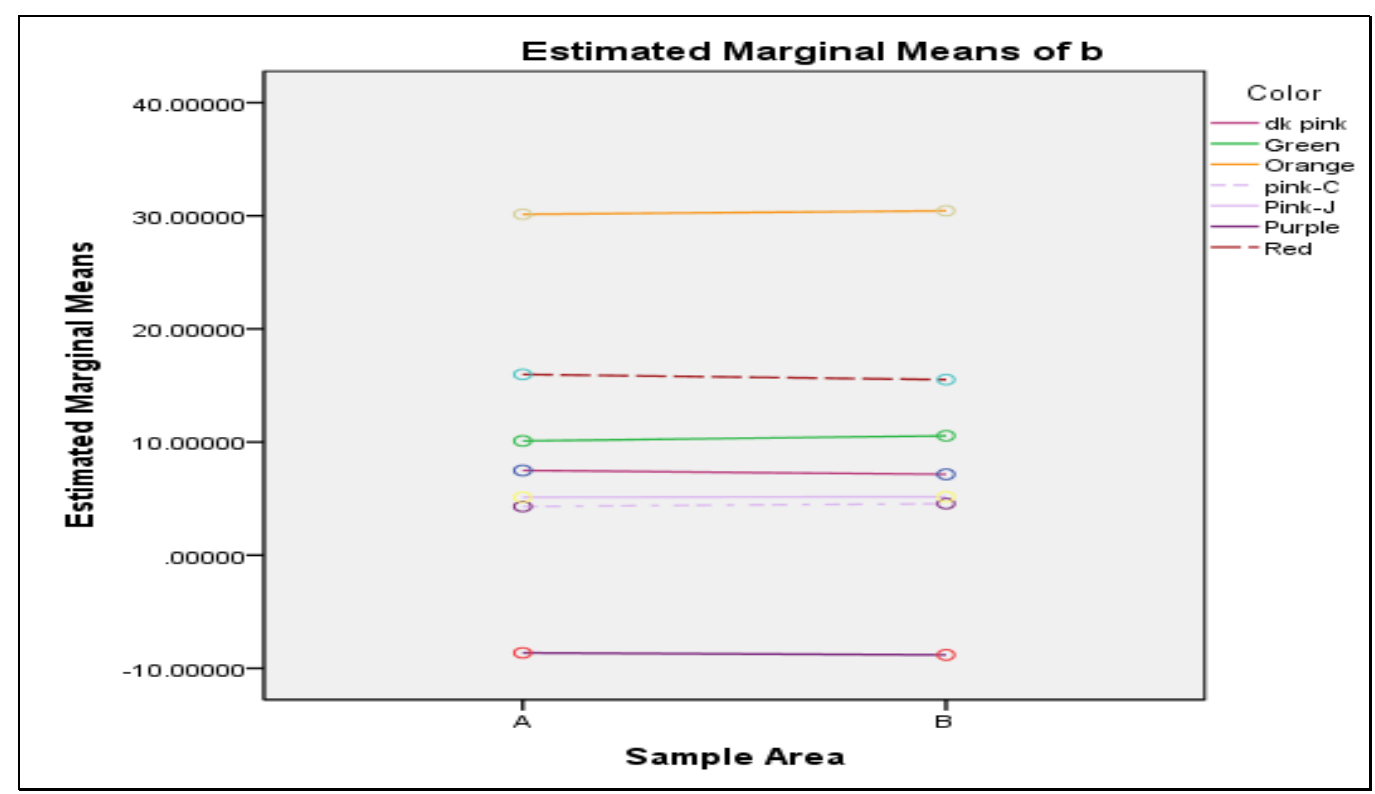

The mean values between the sample areas are minimal. However, the significant values are more likely contributed by the difference between the purple and orange values as these two samples vary the greatest in means.

Dependent Variable: $b$

8. Bleached ${ }^{*}$ Color: all colors

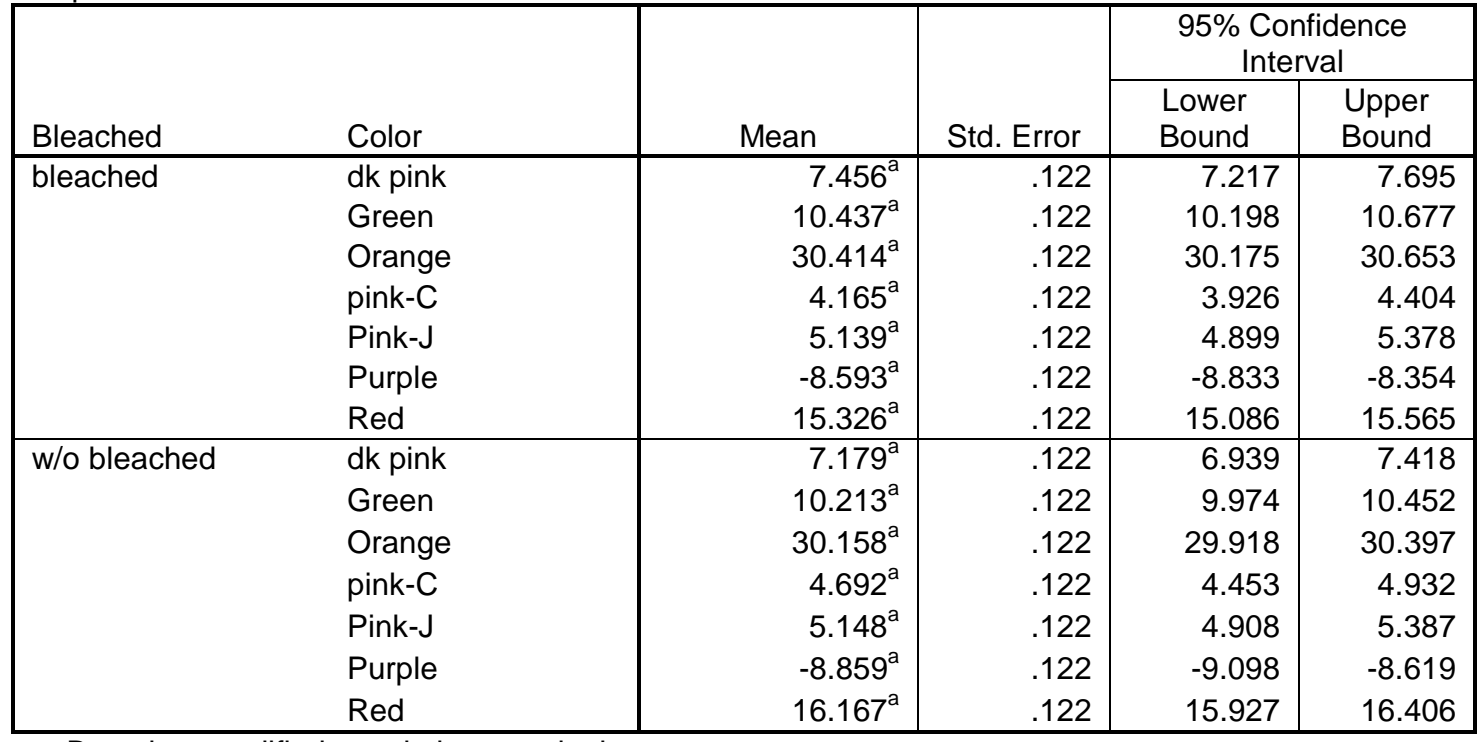

a. Based on modified population marginal mean. 


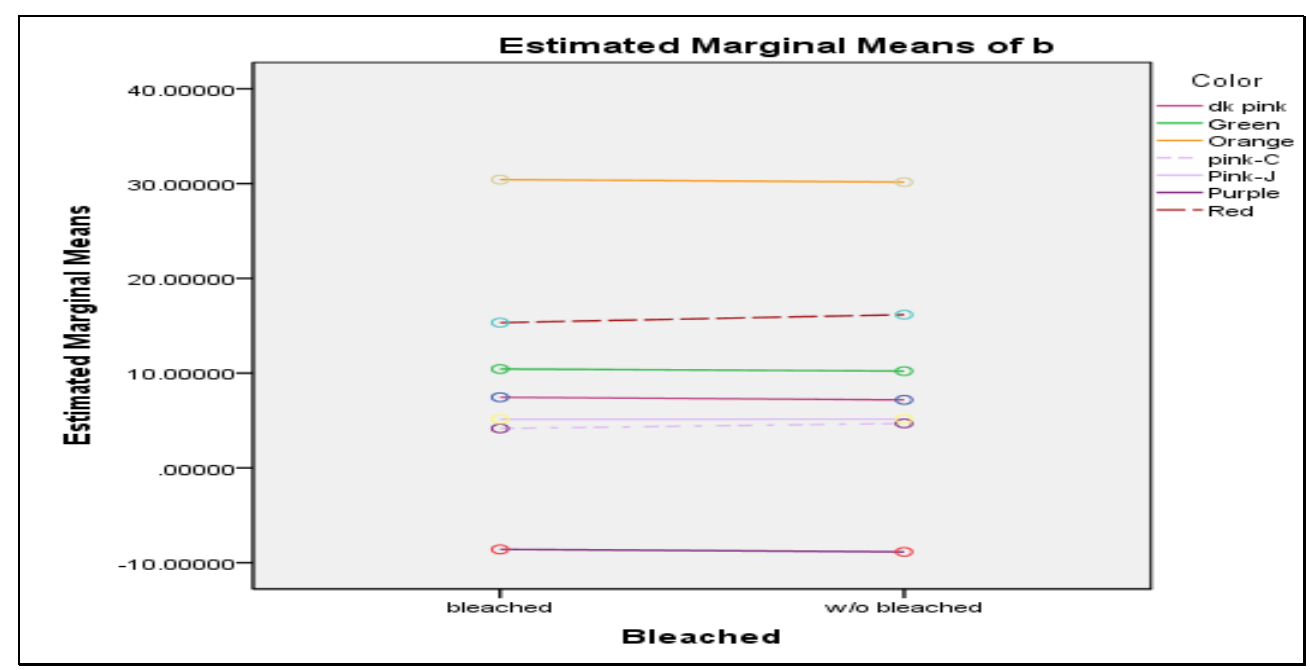

The mean values between the bleached ad unbleached samples are minimal. Similarly, the significant values are more likely contributed by the difference between the purple and orange values as these two samples vary the greatest in means.

Lastly, below is the interaction of washing * color. The differences between mean values are better observed at this variable. It is only at variable ' $b$ ', that samples PMJX and PMCL, and samples RMP (red) and DPMP (dk pink) can be differentiated suggesting that the saturation of the color was affected in the yellow/blue region.

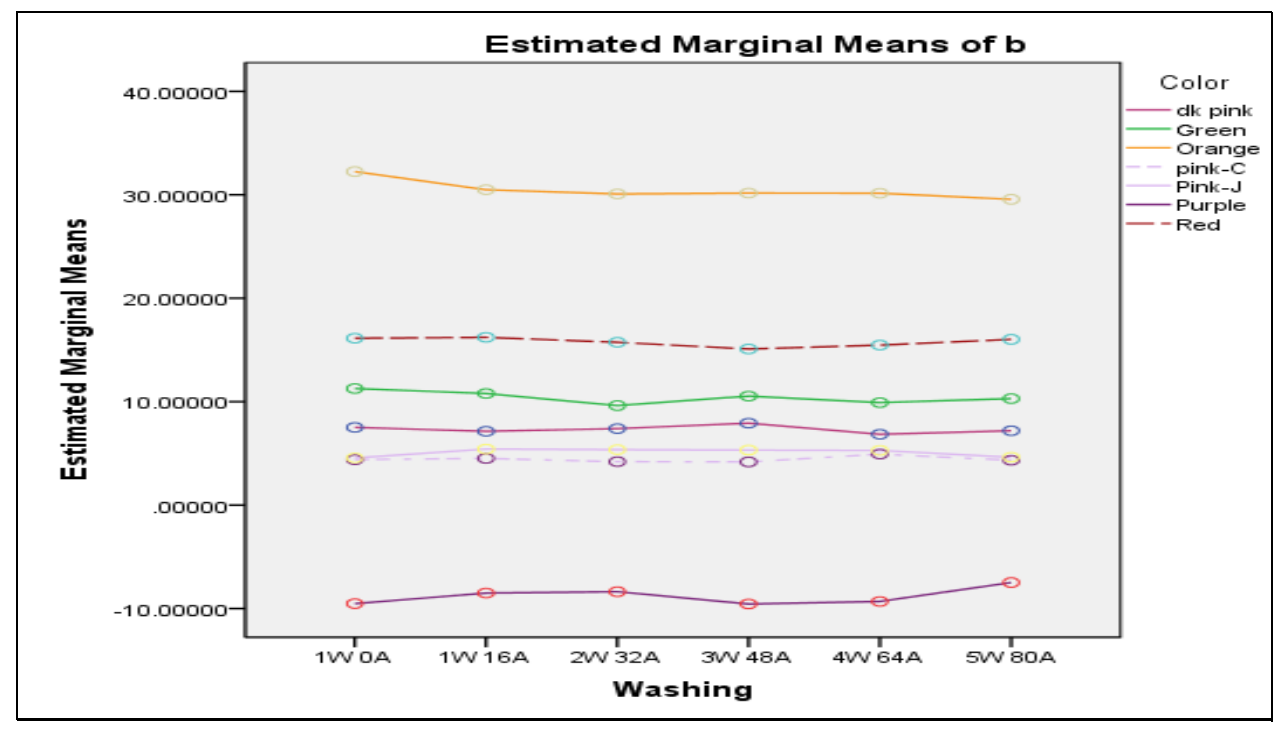


Dependent Variable: $b$

15. Washing * Color: all color

\begin{tabular}{|c|c|c|c|c|c|}
\hline \multirow[b]{2}{*}{ Washing } & \multirow[b]{2}{*}{ Color } & \multirow[b]{2}{*}{ Mean } & \multirow[b]{2}{*}{ Std. Error } & \multicolumn{2}{|c|}{$95 \%$ Confidence Interval } \\
\hline & & & & Lower Bound & Upper Bound \\
\hline \multirow[t]{7}{*}{$1 \mathrm{~W} 0 \mathrm{~A}$} & dk pink & $7.509^{\mathrm{a}}$ & .286 & 6.947 & 8.070 \\
\hline & Green & $11.265^{\mathrm{a}}$ & .286 & 10.704 & 11.826 \\
\hline & Orange & $32.242^{a}$ & .286 & 31.681 & 32.803 \\
\hline & pink-C & $4.397^{\mathrm{a}}$ & .286 & 3.835 & 4.958 \\
\hline & Pink-J & $4.557^{\mathrm{a}}$ & .286 & 3.996 & 5.119 \\
\hline & Purple & $-9.522^{a}$ & .286 & -10.084 & -8.961 \\
\hline & Red & $16.132^{a}$ & .286 & 15.571 & 16.694 \\
\hline \multirow[t]{7}{*}{$1 \mathrm{~W} 16 \mathrm{~A}$} & dk pink & $7.136^{\mathrm{a}}$ & .202 & 6.739 & 7.533 \\
\hline & Green & $10.794^{a}$ & .202 & 10.397 & 11.191 \\
\hline & Orange & $30.489^{a}$ & .202 & 30.092 & 30.886 \\
\hline & pink-C & $4.518^{a}$ & .202 & 4.121 & 4.915 \\
\hline & Pink-J & $5.419^{a}$ & .202 & 5.022 & 5.816 \\
\hline & Purple & $-8.504^{a}$ & .202 & -8.901 & -8.107 \\
\hline & Red & $16.218^{a}$ & .202 & 15.821 & 16.615 \\
\hline \multirow[t]{7}{*}{$2 W 32 A$} & dk pink & $7.394^{a}$ & .202 & 6.997 & 7.791 \\
\hline & Green & $9.621^{a}$ & .202 & 9.224 & 10.018 \\
\hline & Orange & $30.081^{a}$ & .202 & 29.684 & 30.478 \\
\hline & pink-C & $4.199^{a}$ & .202 & 3.802 & 4.596 \\
\hline & Pink-J & $5.359^{a}$ & .202 & 4.962 & 5.756 \\
\hline & Purple & $-8.371^{a}$ & .202 & -8.768 & -7.974 \\
\hline & Red & $15.737^{a}$ & .202 & 15.340 & 16.134 \\
\hline \multirow[t]{7}{*}{$3 W$ 48A } & dk pink & $7.921^{\mathrm{a}}$ & .202 & 7.524 & 8.318 \\
\hline & Green & $10.539^{a}$ & .202 & 10.142 & 10.936 \\
\hline & Orange & $30.167^{a}$ & .202 & 29.770 & 30.563 \\
\hline & pink-C & $4.176^{a}$ & .202 & 3.779 & 4.573 \\
\hline & Pink-J & $5.319^{a}$ & .202 & 4.922 & 5.716 \\
\hline & Purple & $-9.556^{a}$ & .202 & -9.953 & -9.159 \\
\hline & Red & $15.093^{a}$ & .202 & 14.696 & 15.490 \\
\hline \multirow[t]{7}{*}{$4 \mathrm{~W} 64 \mathrm{~A}$} & dk pink & $6.850^{a}$ & .202 & 6.453 & 7.247 \\
\hline & Green & $9.909^{\mathrm{a}}$ & .202 & 9.512 & 10.306 \\
\hline & Orange & $30.150^{a}$ & .202 & 29.753 & 30.547 \\
\hline & pink-C & $4.933^{a}$ & .202 & 4.536 & 5.330 \\
\hline & Pink-J & $5.283^{a}$ & .202 & 4.886 & 5.680 \\
\hline & Purple & $-9.317^{a}$ & .202 & -9.714 & -8.920 \\
\hline & Red & $15.466^{a}$ & .202 & 15.070 & 15.863 \\
\hline \multirow[t]{7}{*}{$5 W$ 80A } & dk pink & $7.191^{a}$ & .202 & 6.794 & 7.587 \\
\hline & Green & $10.293^{a}$ & .202 & 9.896 & 10.690 \\
\hline & Orange & $29.564^{a}$ & .202 & 29.168 & 29.961 \\
\hline & pink-C & $4.333^{a}$ & .202 & 3.936 & 4.730 \\
\hline & Pink-J & $4.629^{a}$ & .202 & 4.232 & 5.026 \\
\hline & Purple & $-7.484^{a}$ & .202 & -7.881 & -7.087 \\
\hline & Red & $16.023^{a}$ & .202 & 15.626 & 16.420 \\
\hline
\end{tabular}

a. Based on modified population marginal mean.

\subsection{Summary of Washed-Aged Results}

Intra-variability was not extensively studied through the washed-aged treatment as it was understood that intra-variability occurs within fibers and that this variation can influence 
analytical results. Since the sub-population being analyzed was not as intrinsic, this particular level of investigating intra-variability was not taken into consideration.

\section{Sample Area and Swatch}

In summary of the pair-wise comparisons for sample area, marked significances were observed for each color sample. Fibers extracted from relatively close distances produces mean differences, but once this distance increases, the variation becomes more significant. Sample areas and swatches both examine fiber inter-variability; sample area is a smaller subset whereas swatch is much larger. As analyses increases from sample area to swatch, the variation observes among the fibers also increases until the differences observed to be, significant. Where more samples produce insignificant results at the sample area level, they produced more significant results at the swatch level. For the two samples areas investigated, except for sample OSDP which produced insignificant values, the remaining samples observed mean significant differences. It can be concluded that the area on the swatch is being for sample OSDP is irrelevant. Between both areas, insignificant values were obtained at all three dependent variables suggest that these variables were not affected by the location of which the fiber is being analyzed. The opposite is true for sample GMP which observed significant values at all three dependent variables. The location where the fiber is analyzed plays an important role in affecting the outcome of the results. Although the sample area was performed for inter-variability purposes, at this level of analysis, caution must still be taken to ensure precise and accurate results.

The pair-wise comparisons for swatch observed to be significantly different for all the samples. Two swatches were analyzed per detergent for inter-variability purposes. At this magnified level 
of sampling, greater amounts of variation between the fibers are expected. The swatches were washed using different detergents did not play a prominent factor with the results.

Inconsistencies were observed between the swatches washed under the same parameters. It can be concluded that at this level of inter-variability between the swatches have increased the variations among them thereby affecting the results to a greater extent.

\section{Washing-Aging}

The pair-wise comparisons for bleach produced significant values for the reference samples but the results being samples varied. Sample PMJX was the only sample that produced insignificant results for bleaching at all three variables. This means that the effect between the bleached and unbleached samples was indifferent with the outcome of the results. The difference in the detergent used proved to be unimportant. Samples PMCL and RMP produced significant results at all three variables. Surprisingly, samples PMJX and PMCL differ in this level of analysis. Although both samples are not easily differentiated macroscopically, they reacted very differently to the detergent treatment. The remaining samples presented several results. Based on this treatment, it can be concluded that each sample will react differently to the type of detergent used and no one consistent pattern can be observed with the dependent variables. It is therefore possible to differentiate between garments of similar color after the aging and laundering process as this was applicable to these samples.

The pair-wise comparison for washing produced significant effects for the reference samples, although not all the pair-wise differences on levels of washing for each sample were significant. In summary of these results, the washing treatment did affect dependent variables, thereby affecting the gloss of the sample and the color saturation. 
The interaction of most concern is the Swatch* Washing. It is impervious to note how much the washing and the bleached and unbleached swatches affected the variables. For all reference samples, significances were observed. It is clear that some changes in both factors must have had an effect of the outcome, rather than this outcome due to random chance. Similar detergents used for washing did not produce similar interaction effects; at times similar patterns were observed between different treated swatches.

\section{Conclusion}

Fibers are one of the most commonly encountered yet complex types of trace evidence. One of its most discriminatory attribute, color, aids in the analysis and comparison between fibers. Spectrophotometric analysis was able to measure the minor to significant differences in textiles and fiber colors that were influenced by environmental factors. Statistical analysis was able to determine the identifying characteristics of color that changed as a result of environmental parameters.

The variability of the quantitative measurements of color was temperately observed during the validation part of the research. Due to the nature of the fiber, its process during manufacturing such as the coloring mechanism, intra- and inter-variability is observed in and between fibers of the same source. At the same time, variation that occurs for each sample is specific for each coordinate. Blue fibers are expected to vary most in the b-axis which is the blue to yellow region, green and red at the a-axis which corresponds to the red to green region. And these variations should be taken into account as the results are interpreted.

Through the treatment process of artificially exposing the fibers to UV light, the greatest amount of changes were observed for variable ' $a$ ', followed by ' $b$ ', then closely behind was ' $L$ '. This 
was determined by the summation of the marked significant differences taken from $\mathrm{G}-\mathrm{H}$ test observed for all reference samples. This concludes that both the red/green and yellow/blue regions were affected during the process of this treatment. It has been previously been reported that the use of UV light to accelerate discoloration have been reported to only affect the gloss of the samples (41). However, by using statistical methods such as ANOVA with the addition of applying post hoc comparison tests, the changes that took place for the variables could be determined.

Through the treatment process of washing and artificially aging the fibers, the greatest amount of changes were observed for variables ' $L$ ', then ' $a$ ', then ' $b$ ', which closely followed behind. These results indicate that with the combination of both these environmental factors, the gloss of the sample and the saturations of the color in the fibers were affected overtime.

Color changes are affected by environmental conditions. Through periodical washing and artificially aging, color degradation in fibers was observed. The amount of exposure to washing and aging, and the type of detergent used will play an important role in altering the gloss of the sample and the saturation of the color. The changes in color caused by the treatments were imperceptible to the observer. Overall, in the processes that were applied, color changes in the fibers were affected microscopically.

\section{Recommendations and Future Applications}

It is recommended that once the goal of a study is developed, that a statistician should be contacted to assist in the method of collection, analysis and interpretation. This will limit unsuccessful results, time wasting and resources if the study does not go as planned. 
Another recommendation that would produce additional results for gratifying results and interpretation is to perform two separate analyses when the process of washing and aging was combined. Instead of analyzing the sample after each washed-aged cycle, observe and analyze each treatment separately. By doing so, after washing, the calculation of how washing affects the color change in fiber can be accurately determined. Likewise, the calculation of how washing and aging combined affects the color changed can also be determined separately.

A future application to this study would be to continue analyzing the remaining blue, yellow and gray colors and the cotton textiles that were brought at the beginning of the experiment to observe if similarities would exist between the colors of different types of textiles.

\section{Glossary}

COX method- COX is not an acronym but rather a development of a new diagnostic single wash test protocol to identify colored cotton fabrics susceptible to oxygen bleach fading through repeated washing with domestic detergents.

Degree of freedom- is the number of population averages being compared.

Mechanical adhesion- is the process where pigments or water-insoluble dyes are made to adhere through resin binders or

Median- is the $50^{\text {th }}$ percentile which conveys information about what a typical value is.

Percentiles- are the division of the total observations into 100 equal parts. The p percentile corresponds to a value for which $\mathrm{p} \%$ of the observation lies at or below it and (100-p)\% of the observations lie at or above it. 
Quantiles- are the division of the total observation into $n$ equal parts and is a fraction of the observations at or below it.

Quartiles-are the cutpoints when a set of ordered observations is divided into four equal size sets. There are three quartiles: Q1, Q2, and Q3, corresponds to the $25^{\text {th }}, 50^{\text {th }}, 75^{\text {th }}$ percentile or 0.25 , 0.50 and 0.75 quantiles,

Skewness- measure how non-symmetric a distribution is.

Statistically significant- is when the group means are too different to have been that way by chance alone.

Variance- is a measure of dispersion; how much does each measurement (of one group) vary around the mean. 


\section{References:}

1. Paterson MD, Cook R. The Production of Color Coordinates from Microgram Quantities of Textile Fibers. 1 Forensic Science International. 1980;15(3):249-58.

2. Blackledge R. Forensic Analysis on the Cutting Edge. Hoboken, NJ: John Wiley and Sons, 2007.

3. Goodpaster JV, Liszewski EA. Forensic analysis of dyed textile fibers. Analytical and Bioanalytical Chemistry. 2009 Aug;394(8):2009-18.

4. Adolf F, Dunlop, J. Microscpectrophotometry/ Color Measurement. In: Robertson J, editor. Forensic Examination of Fibres. 2nd Edition ed. New York: CRC; 1999;251-87.

5. Palenik S. Microscopical examination of Fibers. In: Robertson J, editor. Forensic Examination of Fibres. 2 ed. New York: CRC; 1999;153-77.

6. Stoecklein $\mathrm{W}$. The role of colour and microscopic techniques for the characterisation of paint fragments. In: Caddy B, editor. Forensic Examination of Glass and Paint Analysis and Interpretation; 2001.

7. Moody V, Needles HL. Tufted carpet : textile fibers, dyes, finishes, and processes: William Andrew Publishing, 2004.

8. Neilson K, J. Interior Textiles: Fabrics, Applications, \& Historical Styles: John Wiley \& Sons, 2007.

9. Colorfastness of Cotton Textiles. Cary, NC: Cotton Incorporated; 2002 Contract No.: Document Number|.

10. Robertson J, Grieve, M. Forensic Examination of Fibers. 2 ed. New York: CRC Press, 1999.

11. Eyring M. Visible Microscopical Spectrophotometry in the Forensic Science. In: Saferstein R, editor. Forensic Science Handbook. 2 ed. Upper Saddle River, NJ: Regents/ Prentice Hall; 1993;321-87.

12. (SWGMAT) SWGFMA. Standard Guide for Microspectrophotometry and Color Measurement in Forensic Paint Analysis: Forensic Science Communications; 2007 Contract No.: Document Number|.

13. Olson LA. Color Comparison In Questioned Document Examination Using Microspectrophotometry. Journal of Forensic Sciences. 1986 Oct;31(4):1330-40.

14. Brown PK, Wald G. Visual Pigments in Single Rods and Cones of the Human Retina. Science. 1964 April 3, 1964;144(3614):45-52.

15. Stockman A, Sharpe LT. The spectral sensitivities of the middle- and long-wavelength-sensitive cones derived from measurements in observers of known genotype. Vision Research. 2000 2000;40(13):1711-37.

16. Rodrigues JD, Grossi A. Indicators and ratings for the compatibility assessment of conservation actions. Journal of Cultural Heritage. 2007 Jan-Mar;8(1):32-43.

17. Rivas T, Matias JM, Taboada J, Ordonez C. Functional experiment design for the analysis of colour changes in granite using new $\mathrm{L}^{*} \mathrm{a} * \mathrm{~b} *$ functional colour coordinates. Journal of Computational and Applied Mathematics. 2011 Jun;235(16):4701-16.

18. Laing DK, Dudley RJ, Isaacs MDJ. Colorimetric Measurements On Small Paint Fragments Using Microspectrophotometry. Forensic Science International. 1980;16(2):159-71.

19. Laing DK, Hartshorne AW, Harwood RJ. Colour measurements on single textile fibres. Forensic Science International. 1986;30(1):65-77.

20. Paterson MD, Cook R. The Production Of Color Coordinates From Microgram Quantities Of Textile Fibers .1. Forensic Science International. 1980;15(3):249-58.

21. Bell SC, Nawrocki HD, Morris KB. Forensic discrimination of glass using cathodoluminescence and CIE LAB color coordinates: A feasibility study. Forensic Science International. 2009 Aug 10;189(13):93-9. 
22. Rivas T, Matias JM, Taboada J, Ordonez C, editors. Functional ANOVA used to Detect Colour Changes in Granite Following Water-Repellent Treatment. International Conference on Computational Methods in Science and Engineering; 2009 Sep 25-30; Hersonissos, GREECE.

23. Gurgan S, Yalcin Cakir F. The effect of three different mouthrinses on the surface hardness, gloss and colour change of bleached nano composite resins. The European journal of prosthodontics and restorative dentistry. 2008 2008-Sep;16(3):104-8.

24. Martinez K, Goodall S, editors. Colour cluster analysis for pigment identification. Conference on Computer Image Analysis in the Study of Art; 2008 Jan 28-29; San Jose, CA.

25. Nori M. The Color Determination of Optically Variable Flake Pigments. In: Blackledge R, editor. Forensic Analysis on the Cutting Edge. Hoboken, NJ: John Wiley and Sons; 2007;375-98.

26. Phillips D, Duncan M, Jenkins E, Bevan G, Lloyd J, Hoffmeister J. Development of a test to predict colour fading of cotton fabrics after multi-cycle laundering with a bleach-containing domestic detergent. Journal of the Society of Dyers and Colourists. 1996;112(10):287-93.

27. Aksakal B, Alekberov V. The effect of temperature and water on the mechanical properties of wool fibres investigated with different experimental methods. Fibers and Polymers. [10.1007/s12221010-0673-9]. 2009;10(5):673-80.

28. Phillips. The effect of the bleach activator in detergents on the fastness of dyed cotton substrates. International Conference on Water and Textiles, 1999 May 12-14. 1999. p. 57.

29. Phillips D, Bevan G, Riley L, Scotney J. Effect of temperature on the correlation between the oxidative-bleach fading observed in the ISO 105 : C09 test and that after multiple domestic laundering cycles for dyed wool fabrics. Coloration Technology. [Article]. 2003;119(6):324-6.

30. McLean R. Diagnostic Test Method for Color Fading after Laundering under North American Conditions with Activated Oxygen Bleach Containing Detergents.514-20.

31. McLean RJ. Understanding interactions between detergents with bleach and textiles. Textile Chemist and Colorist \& American Dyestuff Reporter. 1999 Dec;1(4):42-5.

32. Was-Gubala J. The kinetics of colour change in textiles and fibres treated with detergent solutions: Part I--Colour perception and fluorescence microscopy analysis. Science \&amp; Justice. 2009;49(3):165-9.

33. Was-Gubala J, Grzesiak E. The kinetics of colour change in textiles and fibres treated with detergent solutions: Part II -- Spectrophotometric measurements. Science \&amp; Justice.In Press, Corrected Proof.

34. Rohwer H, Phillips DAS, Krijnen ES, Glusen B, Lodewick R, Reinhordt G, et al. Dye Fading in Laundry Washing. Comparison of Washing Machine and Linitest Results. Tenside Surfactants Deterg. [Article]. 2008 Nov-Dec;45(6):310-3.

35. Cai JY, Evans DJ, Smith SM. Bleaching of natural fibers with TAED and NOBS activated peroxide systems. AATCC Rev. [Article]. 2001 Dec;1(12):31-4.

36. Phillips D, Scotney J, Percival R, Bevan G, Lloyd J. Colour fastness to industrial laundering: an international inter-laboratory trial of the proposed ISO 105-C12 test procedure<sup $>\& \# x 2020 ;</$ sup $>$. Coloration Technology. 2002;118(6):277-80.

37. Watt $\mathrm{R}$, Roux $\mathrm{C}$, Robertson J. The population of coloured textile fibres in domestic washing machines. Science \&amp; Justice. 2005 Apr-Jun;45(2):75-83.

38. Oakes J. Formulation of colour-care and heavy-duty detergents: a review. Coloration Technology. 2005;121(1):1-6.

39. Marengo E, Robotti E, Liparota MC, Gennaro MC. Monitoring of pigmented and wooden surfaces in accelerated ageing processes by FT-Raman spectroscopy and multivariate control charts. Talanta. 2004 Jul;63(4):987-1002. 
40. Marengo E, Liparota MC, Robotti E, Bobba M, Gennaro MC. Monitoring of pigmented surfaces in accelerated ageing process by ATR-FT-IR spectroscopy and multivariate control charts. Talanta. 2005 Jun;66(5):1158-67.

41. Marengo E, Liparota MC, Robotti E, Bobba M. Monitoring of paintings under exposure to UV light by ATR-FT-IR spectroscopy and multivariate control charts. Vibrational Spectroscopy. 2006;40(2):225-34.

42. Marengo E, Liparota MC, Robotti E, Bobba M, Gennaro MC. The state of conservation of painted surfaces in the presence of accelerated ageing processes monitored by use of FT-Raman spectroscopy and multivariate control charts. Analytical and Bioanalytical Chemistry. 2005 Feb;381(4):884-95.

43. Best Laundry Detergent. Consumer Search; 2010 [updated 2010 January 2011; cited 2011 January]; 1-6]. Available from: http://www.consumersearch.com/laundry-detergent/reviews.

44. Govaerts PJ, Somers T, Offeciers FE. Box and whisker plots for graphic presentation of audiometric results of conductive hearing loss treatment. Otolaryngology - Head and Neck Surgery. 1998 June;118(6):892-5.

45. Krishnamoorthy K, Lu F, Mathew T. A parametric bootstrap approach for ANOVA with unequal variances: Fixed and random models. Computational Statistics \&amp; Data Analysis. 2007;51(12):5731-

42.

46. Fagerland MW, Sandvik L. Performance of five two-sample location tests for skewed distributions with unequal variances. Contemporary Clinical Trials. 2009;30(5):490-6.

47. Kim N. The limit distribution of a modified Shapiro-Wilk statistic for normality to Type II censored data. Journal of the Korean Statistical Society. 2011 Sep;40(3):257-66.

48. Rosenkrantz W, A. Introduction to Probability and Statistics for Science, Engineering and Finance. Boca Raton: Chapman \& Hall/CRC, 2009.

49. Tan WD, Gan FF, Chang TC. Using normal quantile plot to select an appropriate transformation to achieve normality. Computational Statistics \& Data Analysis. 2004 Apr 10;45(3):609-19.

50. Vlcek $\mathrm{O}$, Huth R. Is daily precipitation Gamma-distributed? Adverse effects of an incorrect use of the Kolmogorov-Smirnov test. Atmospheric Research. 2009 Aug;93(4):759-66.

51. Lilliefors HW. On the Kolmogorov-Smirnov test for normality with mean and variance unknown. Journal of the American Statistical Association. 1967;62:399-402.

52. Lilliefors HW. On the Kolmogorov-Smirnov test or the exponential distribution with mean unknown. Journal of the American Statistical Association. 1969;64(387-389).

53. Lilliefors HW. The Kolmogorov-Smirnov and other distance tests for the Gamma distribution and for the extreme- value distribution when parameters must be estimated. George Washington University: U.S. Department of Commerce Contract; 1973.

54. Razali NM, Wah, Yap B. Power Comparisons of Shapiro-wilk, Kolmorogov-Smirnov, Lilliefors and Anferson-Darling test. Journal of Statistical Modeling and Analysis. 2011;2(1):21-33.

55. Steinskog DJ, Tjostheim DB, Kvamsto NG. A cautionary note on the use of the KolmogorovSmirnov test for normality. Monthly Weather Review. 2007 Mar;135(3):1151-7.

56. Royston JP. An Extension Of Shapiro And Wilk-W Test For Normality To Large Samples. Applied Statistics-Journal of the Royal Statistical Society Series C. 1982;31(2):115-24.

57. Goodman SN, Berlin JA. The use of Predicted Confidence-Intervals when Planning Experiments and the Misuse of Power when Interpreting Results Annals of Internal Medicine. 1994 Aug;121(3):200-6. 58. Cuevas A, Febrero M, Fraiman R. An anova test for functional data. Computational Statistics \&amp; Data Analysis. 2004;47(1):111-22.

59. Huitson A. Analysis Of Variance. London: Charles Griffin \& Company Limited, 1966.

60. Corston RC, Andrew. A Crash Course in SPSS for Windows. 2nd ed. Malden: Blackwell Publishing, 2003. 
61. Brown AM. A new software for carrying out one-way ANOVA post hoc tests. Computer Methods and Programs in Biomedicine. 2005;79(1):89-95.

62. Furuse AY, Gordon K, Rodrigues FP, Silikas N, Watts DC. Colour-stability and gloss-retention of silorane and dimethacrylate composites with accelerated aging. Journal of Dentistry. [Article]. 2008;36(11):945-52.

63. Schaberg PG, Murakami PF, Turner MR, Heitz HK, Hawley GJ. Association of red coloration with senescence of sugar maple leaves in autumn. Trees. 2008;22:573-8.

64. Schneider LFJ, Cavalcante, L. M., Silikas, N., Watts, D. C. Degradation resistance of silorane, experimental ormocer and dimethacrylate resin-based dental composites. Journal of Oral Science. 2011;53(4):413-9.

65. Mieckowski TM. Effect of Color and Curvature on the Concentration of Morphine in Hair Analysis. Forensic Science Communication. 2002;4(4).

66. Tlusty M. Use of digital colour analysis to assess variation within individual adult American lobsters (Homarus americanus) and the process of addition of colour in white lobsters. New Zealand Journal of Marine and Freshwater. 2005;39:571-80.

67. Weber A, Van Noordwijk A. Swimming behaviour of Daphnia clones: differentiation through predator infochemicals. Journal of Plankton Research. 2002 Dec;24(12):1335-48.

68. Hsiung TH, Olejnik S. Contrast Analysis for Additive Nonorthogonal 2-Factor Designs in Unequal Variance Cases British Journal of Mathematical \& Statistical Psychology. 1994 Nov;47:337-54.

69. Sen SK, Shaykhian GA. MatLab tutorial for scientific and engineering computations: International Federation of Nonlinear Analysts (IFNA); 2008 World Congress of Nonlinear Analysts (WCNA). Nonlinear Analysis: Theory, Methods \& Applications. 2009;71(12):e1005-e20.

70. Daszykowski M, Serneels S, Kaczmarek K, Van Espen P, Croux C, Walczak B. TOMCAT: A MATLAB toolbox for multivariate calibration techniques. Chemometrics and Intelligent Laboratory Systems. 2007;85(2):269-77.

71. Verboven S, Hubert M. LIBRA: a MATLAB library for robust analysis. Chemometrics and Intelligent Laboratory Systems. 2005;75(2):127-36.

72. White PC. Surface Enhanced Resonance Raman Scattering Spectroscopy. In: Robertson J, Grieve, M., editor. Forensic Examination of Fibres. 2 ed: Taylor and Francis; 1999;337.

73. Noguchi K, Gel YR. Combination of Levene-type tests and a finite-intersection method for testing equality of variances against ordered alternatives. Journal of Nonparametric Statistics. [Article].

2010;22(7):897-913. 


\section{Appendix}

1. Validation Results

Table 1.1: Validation Result of Blue Fibers

\begin{tabular}{|c|c|c|c|c|c|c|c|c|c|c|}
\hline Date & Duplicate & blue: L & blue: a & blue: b & green: L & green: a & green: b & red: L & red: a & red: $b$ \\
\hline \multirow[t]{5}{*}{$2 / 7 / 2011$} & 1 & 85.836 & -6.436 & -13.313 & 90.518 & -1.740 & 5.021 & 77.969 & 33.685 & 15.532 \\
\hline & 2 & 80.934 & -5.467 & -10.635 & 90.515 & -1.739 & 5.011 & 77.941 & 33.699 & 15.515 \\
\hline & 3 & 81.687 & -5.521 & -10.210 & 90.839 & -1.631 & 5.298 & 77.836 & 33.771 & 15.494 \\
\hline & 4 & 81.485 & -5.729 & -11.533 & 90.770 & -1.637 & 5.197 & 77.843 & 33.792 & 15.488 \\
\hline & 5 & 81.448 & -5.722 & -11.484 & 90.770 & -1.637 & 5.197 & 77.827 & 33.770 & 15.451 \\
\hline \multirow[t]{5}{*}{$2 / 8 / 2011$} & 1 & 88.001 & -9.215 & -16.067 & 89.068 & -4.786 & 5.120 & 73.100 & 28.955 & 12.868 \\
\hline & 2 & 88.413 & -9.531 & -17.001 & 91.541 & -5.593 & 3.852 & 73.098 & 28.946 & 12.831 \\
\hline & 3 & 88.443 & -9.530 & -16.977 & 91.550 & -5.561 & 3.864 & 72.895 & 28.840 & 12.797 \\
\hline & 4 & 88.435 & -9.555 & -17.035 & 91.539 & -5.532 & 3.878 & 72.747 & 28.740 & 12.763 \\
\hline & 5 & 88.435 & -9.555 & -17.035 & 91.563 & -5.519 & 3.880 & 72.808 & 28.748 & 12.749 \\
\hline \multirow[t]{5}{*}{$2 / 9 / 2011$} & 1 & 90.112 & -4.074 & -9.994 & 87.574 & -2.625 & 4.666 & 82.219 & 28.091 & 7.577 \\
\hline & 2 & 90.114 & -4.059 & -9.964 & 87.593 & -2.613 & 4.683 & 82.219 & 28.084 & 7.584 \\
\hline & 3 & 90.089 & -4.069 & -9.988 & 87.604 & -2.593 & 4.686 & 82.222 & 28.096 & 7.613 \\
\hline & 4 & 90.045 & -4.084 & -10.004 & 87.619 & -2.583 & 4.706 & 82.219 & 28.071 & 7.569 \\
\hline & 5 & 90.005 & -4.098 & -10.013 & 87.650 & -2.576 & 4.724 & 82.168 & 28.008 & 7.496 \\
\hline \multirow[t]{5}{*}{ 2/10/2011 } & 1 & 89.687 & -4.486 & -9.186 & 88.873 & -2.059 & 5.607 & 77.355 & 28.481 & 11.539 \\
\hline & 2 & 89.843 & -4.517 & -9.248 & 88.869 & -2.064 & 5.581 & 77.476 & 28.571 & 11.557 \\
\hline & 3 & 89.960 & -4.541 & -9.288 & 88.877 & -2.065 & 5.560 & 75.711 & 26.894 & 10.774 \\
\hline & 4 & 90.093 & -4.550 & -9.291 & 88.876 & -2.067 & 5.544 & 75.713 & 26.902 & 10.773 \\
\hline & 5 & 90.056 & -4.556 & -9.297 & 88.888 & -2.066 & 5.545 & 75.730 & 26.903 & 10.788 \\
\hline \multirow[t]{5}{*}{$2 / 11 / 2011$} & 1 & 87.993 & -2.618 & -7.104 & 87.373 & -1.905 & 6.180 & 79.306 & 32.279 & 15.753 \\
\hline & 2 & 88.052 & -2.612 & -7.085 & 87.362 & -1.863 & 6.201 & 79.309 & 32.250 & 15.719 \\
\hline & 3 & 88.070 & -2.609 & -7.070 & 87.360 & -1.878 & 6.176 & 79.283 & 32.241 & 15.697 \\
\hline & 4 & 88.127 & -2.592 & -7.036 & 87.346 & -1.872 & 6.187 & 79.283 & 32.257 & 15.715 \\
\hline & 5 & 88.148 & -2.579 & -7.030 & 87.349 & -1.863 & 6.187 & 79.278 & 32.275 & 15.695 \\
\hline \multirow[t]{5}{*}{ 2/14/2011 } & 1 & 84.762 & -6.560 & -15.153 & 86.769 & -2.565 & 4.615 & 87.953 & 27.752 & 12.453 \\
\hline & 2 & 84.785 & -6.567 & -15.154 & 86.694 & -2.547 & 4.630 & 87.978 & 27.758 & 12.425 \\
\hline & 3 & 84.813 & -6.579 & -15.142 & 86.704 & -2.548 & 4.617 & 88.019 & 27.784 & 12.389 \\
\hline & 4 & 84.824 & -6.591 & -15.141 & 86.661 & -2.527 & 4.635 & 88.040 & 27.794 & 12.347 \\
\hline & 5 & 84.797 & -6.586 & -15.126 & 86.681 & -2.539 & 4.621 & 88.063 & 27.808 & 12.319 \\
\hline \multirow[t]{5}{*}{$2 / 15 / 2011$} & 1 & 89.733 & -3.468 & -8.133 & 89.708 & -1.987 & 5.314 & 81.796 & 27.344 & 10.150 \\
\hline & 2 & 89.724 & -3.456 & -8.121 & 89.726 & -1.983 & 5.312 & 81.802 & 27.325 & 10.118 \\
\hline & 3 & 89.717 & -3.449 & -8.112 & 89.741 & -1.977 & 5.316 & 81.764 & 27.288 & 10.083 \\
\hline & 4 & 89.703 & -3.448 & -8.104 & 89.733 & -1.976 & 5.309 & 81.890 & 27.318 & 10.062 \\
\hline & 5 & 89.729 & -3.441 & -8.102 & 89.746 & -1.976 & 5.302 & 82.008 & 27.344 & 10.055 \\
\hline \multirow[t]{5}{*}{$2 / 16 / 2011$} & 1 & 90.476 & -8.482 & -7.911 & 93.007 & -4.782 & 6.863 & 83.206 & 22.699 & 12.957 \\
\hline & 2 & 90.472 & -8.414 & -7.766 & 93.029 & -4.768 & 6.868 & 83.257 & 22.695 & 12.958 \\
\hline & 3 & 90.433 & -8.394 & -7.729 & 93.055 & -4.764 & 6.874 & 83.274 & 22.685 & 12.924 \\
\hline & 4 & 90.438 & -8.353 & -7.643 & 93.056 & -4.760 & 6.864 & 83.304 & 22.684 & 12.917 \\
\hline & 5 & 90.402 & -8.340 & -7.625 & 93.068 & -4.758 & 6.874 & 83.328 & 22.677 & 12.900 \\
\hline \multirow[t]{4}{*}{$2 / 17 / 2011$} & 1 & 90.230 & -14.036 & -3.436 & 93.037 & -12.295 & 9.651 & 76.304 & 16.330 & 12.618 \\
\hline & 2 & 90.211 & -14.042 & -3.455 & 93.034 & -12.286 & 9.622 & 76.299 & 16.335 & 12.613 \\
\hline & 3 & 90.217 & -14.048 & -3.453 & 93.043 & -12.279 & 9.607 & 76.329 & 16.354 & 12.633 \\
\hline & 4 & 90.206 & -14.053 & -3.467 & 93.052 & -12.279 & 9.586 & 76.316 & 16.355 & 12.608 \\
\hline
\end{tabular}




\begin{tabular}{lllllllllll} 
& 5 & 90.194 & -14.064 & -3.492 & 93.066 & -12.284 & 9.570 & 76.318 & 16.337 & 12.615 \\
\hline $2 / 18 / 2011$ & 1 & 84.942 & -5.273 & -11.492 & 88.260 & -2.206 & 4.914 & 83.768 & 23.003 & 8.921 \\
& 2 & 84.881 & -5.256 & -11.486 & 88.267 & -2.198 & 4.897 & 83.793 & 22.996 & 8.890 \\
& 3 & 84.833 & -5.251 & -11.499 & 88.259 & -2.189 & 4.873 & 83.850 & 22.995 & 8.878 \\
& 4 & 84.561 & -5.222 & -11.460 & 88.245 & -2.198 & 4.871 & 83.912 & 23.000 & 8.897 \\
& 5 & 84.522 & -5.217 & -11.458 & 88.238 & -2.188 & 4.873 & 84.039 & 23.019 & 8.937 \\
\hline
\end{tabular}

2. Descriptive Statistics from One-Way ANOVA for Aged Samples- by 'Hours'

Table 2.1 Descriptive Statistics for reference samples grouped by 'Hours'

\begin{tabular}{|c|c|c|c|c|c|c|c|c|c|}
\hline \multirow[b]{2}{*}{ DPMP } & \multirow{2}{*}{ Hours } & \multirow{2}{*}{$\mathrm{N}$} & \multirow{2}{*}{ Means } & \multirow{2}{*}{ Std. Dev } & \multirow{2}{*}{$\begin{array}{l}\text { Std. } \\
\text { Err. }\end{array}$} & \multicolumn{2}{|c|}{$\begin{array}{l}\text { 95\% Confidence } \\
\text { Interval for Means }\end{array}$} & \multirow{2}{*}{ Minimum } & \multirow{2}{*}{ Maximum } \\
\hline & & & & & & Lower Bound & Upper bound & & \\
\hline \multirow[t]{7}{*}{ L } & 0 & 45 & 84.484 & 3.922 & 0.585 & 83.306 & 85.663 & 77.915 & 89.022 \\
\hline & 16 & 60 & 86.446 & 3.638 & 0.470 & 85.506 & 87.386 & 82.267 & 94.640 \\
\hline & 32 & 60 & 83.659 & 2.694 & 0.348 & 82.963 & 84.355 & 77.552 & 86.551 \\
\hline & 48 & 60 & 83.798 & 3.064 & 0.396 & 83.006 & 84.589 & 78.376 & 88.713 \\
\hline & 64 & 60 & 86.303 & 3.465 & 0.447 & 85.408 & 87.198 & 81.939 & 94.845 \\
\hline & & 60 & 83.305 & 3.815 & 0.493 & 82.319 & 84.290 & 76.581 & 90.204 \\
\hline & All Grps & 345 & 84.674 & 3.647 & 0.196 & 84.288 & 85.060 & 76.581 & 94.845 \\
\hline \multirow[t]{7}{*}{ a } & 0 & 45 & 26.830 & 2.344 & 0.349 & 26.126 & 27.534 & 23.068 & 30.920 \\
\hline & 16 & 60 & 26.928 & 2.826 & 0.365 & 26.198 & 27.658 & 21.818 & 31.669 \\
\hline & 32 & 60 & 25.791 & 4.281 & 0.553 & 24.685 & 26.897 & 19.549 & 35.793 \\
\hline & 48 & 60 & 25.035 & 3.333 & 0.430 & 24.174 & 25.896 & 21.321 & 33.077 \\
\hline & 64 & 60 & 26.935 & 3.556 & 0.459 & 26.016 & 27.853 & 21.023 & 32.374 \\
\hline & 80 & 60 & 25.384 & 2.577 & 0.333 & 24.719 & 26.050 & 20.792 & 30.484 \\
\hline & All Grps & 345 & 26.121 & 3.323 & 0.179 & 25.769 & 26.473 & 19.549 & 35.793 \\
\hline \multirow[t]{7}{*}{ b } & 0 & 45 & 7.307 & 2.610 & 0.389 & 6.523 & 8.091 & 0.762 & 9.900 \\
\hline & 16 & 60 & 8.467 & 1.692 & 0.218 & 8.030 & 8.904 & 5.518 & 11.350 \\
\hline & 32 & 60 & 7.599 & 1.656 & 0.214 & 7.172 & 8.027 & 4.866 & 10.948 \\
\hline & 48 & 60 & 7.161 & 1.827 & 0.236 & 6.689 & 7.633 & 4.716 & 10.067 \\
\hline & 64 & 60 & 8.070 & 2.132 & 0.275 & 7.519 & 8.620 & 4.561 & 11.151 \\
\hline & 80 & 60 & 7.652 & 1.471 & 0.190 & 7.271 & 8.032 & 5.234 & 9.894 \\
\hline & All Grps & 345 & 7.727 & 1.938 & 0.104 & 7.522 & 7.932 & 0.762 & 11.350 \\
\hline
\end{tabular}




\begin{tabular}{|c|c|c|c|c|c|c|c|c|c|}
\hline \multirow[b]{2}{*}{ GMP } & \multirow{2}{*}{ Hours } & \multirow[b]{2}{*}{$\mathrm{N}$} & \multirow{2}{*}{ Means } & \multirow{2}{*}{ Std. Dev } & \multirow{2}{*}{$\begin{array}{l}\text { Std. } \\
\text { Err. }\end{array}$} & \multicolumn{2}{|c|}{$\begin{array}{c}\text { 95\% Confidence } \\
\text { Interval for Means }\end{array}$} & \multirow[b]{2}{*}{ Minimum } & \multirow{2}{*}{ Maximum } \\
\hline & & & & & & Lower Bound & Upper bound & & \\
\hline \multirow[t]{7}{*}{$\mathbf{L}$} & 0 & 45 & 95.794 & 4.521 & 0.674 & 94.436 & 97.152 & 89.960 & 103.495 \\
\hline & 16 & 60 & 94.040 & 2.459 & 0.317 & 93.405 & 94.676 & 89.593 & 97.852 \\
\hline & 32 & 60 & 92.996 & 5.132 & 0.662 & 91.671 & 94.322 & 83.374 & 100.173 \\
\hline & 48 & 60 & 93.103 & 6.201 & 0.801 & 91.501 & 94.705 & 83.389 & 105.309 \\
\hline & 64 & 60 & 95.857 & 3.756 & 0.485 & 94.886 & 96.827 & 88.712 & 101.836 \\
\hline & 80 & 60 & 95.056 & 4.035 & 0.521 & 94.014 & 96.098 & 86.814 & 100.836 \\
\hline & All Grps & 345 & 94.417 & 4.619 & 0.249 & 93.928 & 94.906 & 83.374 & 105.309 \\
\hline \multirow[t]{7}{*}{ a } & 0 & 45 & 0.961 & 1.994 & 0.297 & 0.362 & 1.560 & -0.692 & 6.522 \\
\hline & 16 & 60 & 0.954 & 0.421 & 0.054 & 0.845 & 1.063 & 0.169 & 1.846 \\
\hline & 32 & 60 & 1.143 & 0.623 & 0.080 & 0.982 & 1.303 & 0.058 & 2.486 \\
\hline & 48 & 60 & 0.830 & 0.542 & 0.070 & 0.690 & 0.970 & 0.045 & 1.897 \\
\hline & 64 & 60 & 1.263 & 0.624 & 0.081 & 1.102 & 1.424 & 0.130 & 2.298 \\
\hline & 80 & 60 & 0.711 & 0.628 & 0.081 & 0.549 & 0.874 & -0.337 & 2.351 \\
\hline & All Grps & 345 & 0.978 & 0.908 & 0.049 & 0.881 & 1.074 & -0.692 & 6.522 \\
\hline \multirow[t]{7}{*}{ b } & 0 & 45 & 11.059 & 1.584 & 0.236 & 10.583 & 11.535 & 8.570 & 13.942 \\
\hline & 16 & 60 & 10.594 & 1.982 & 0.256 & 10.082 & 11.106 & 6.737 & 14.042 \\
\hline & 32 & 60 & 11.062 & 2.198 & 0.284 & 10.494 & 11.630 & 7.959 & 15.879 \\
\hline & 48 & 60 & 10.164 & 1.846 & 0.238 & 9.687 & 10.641 & 7.201 & 13.085 \\
\hline & 64 & 60 & 10.181 & 3.175 & 0.410 & 9.361 & 11.002 & 2.443 & 15.455 \\
\hline & 80 & 60 & 10.041 & 1.902 & 0.246 & 9.549 & 10.532 & 7.953 & 14.108 \\
\hline & All Grps & 345 & 10.493 & 2.220 & 0.120 & 10.258 & 10.728 & 2.443 & 15.879 \\
\hline \multirow[b]{2}{*}{ OSDP } & \multirow{2}{*}{ Hours } & \multirow{2}{*}{$\mathrm{N}$} & \multirow{2}{*}{ Means } & \multirow{2}{*}{ Std. Dev } & \multirow{2}{*}{$\begin{array}{l}\text { Std. } \\
\text { Err. }\end{array}$} & \multicolumn{2}{|c|}{$\begin{array}{c}\text { 95\% Confidence } \\
\text { Interval for Means }\end{array}$} & \multirow{2}{*}{ Minimum } & \multirow{2}{*}{ Maximum } \\
\hline & & & & & & Lower Bound & Upper bound & & \\
\hline $\mathbf{L}$ & 0 & 45 & 92.486 & 2.607 & 0.389 & 91.703 & 93.270 & 88.425 & 96.390 \\
\hline & 16 & 60 & 92.344 & 4.559 & 0.589 & 91.166 & 93.522 & 84.527 & 98.217 \\
\hline & 32 & 60 & 92.681 & 3.938 & 0.508 & 91.664 & 93.699 & 85.111 & 100.498 \\
\hline & 48 & 60 & 93.599 & 2.797 & 0.361 & 92.877 & 94.322 & 88.053 & 98.626 \\
\hline & 64 & 60 & 91.604 & 6.352 & 0.820 & 89.963 & 93.245 & 79.250 & 103.549 \\
\hline & & 60 & 90.558 & 4.359 & 0.563 & 89.432 & 91.684 & 84.837 & 98.307 \\
\hline & All Grps & 345 & 92.200 & 4.421 & 0.238 & 91.732 & 92.668 & 79.250 & 103.549 \\
\hline$a$ & 0 & 45 & 8.994 & 0.655 & 0.098 & 8.798 & 9.191 & 7.914 & 10.016 \\
\hline & 16 & 60 & 9.769 & 1.217 & 0.157 & 9.455 & 10.084 & 7.232 & 11.365 \\
\hline & 32 & 60 & 9.780 & 2.279 & 0.294 & 9.192 & 10.369 & 4.048 & 12.144 \\
\hline & 48 & 60 & 10.462 & 1.841 & 0.238 & 9.987 & 10.938 & 5.454 & 13.428 \\
\hline & 64 & 60 & 9.917 & 1.822 & 0.235 & 9.446 & 10.387 & 6.008 & 12.405 \\
\hline & 80 & 60 & 9.549 & 1.277 & 0.165 & 9.219 & 9.879 & 7.067 & 11.516 \\
\hline & All Grps & 345 & 9.778 & 1.675 & 0.090 & 9.601 & 9.955 & 4.048 & 13.428 \\
\hline b & 0 & 45 & 36.540 & 1.743 & 0.260 & 36.017 & 37.064 & 32.915 & 38.851 \\
\hline & 16 & 60 & 32.009 & 4.207 & 0.543 & 30.922 & 33.096 & 23.392 & 37.995 \\
\hline & 32 & 60 & 31.507 & 6.424 & 0.829 & 29.848 & 33.167 & 15.639 & 38.601 \\
\hline & 48 & 60 & 34.053 & 4.761 & 0.615 & 32.824 & 35.283 & 22.190 & 42.614 \\
\hline & 64 & 60 & 32.584 & 6.129 & 0.791 & 31.000 & 34.167 & 19.993 & 41.309 \\
\hline & 80 & 60 & 30.883 & 3.600 & 0.465 & 29.953 & 31.813 & 24.022 & 36.703 \\
\hline & All Grps & 345 & 32.773 & 5.119 & 0.276 & 32.230 & 33.315 & 15.639 & 42.614 \\
\hline
\end{tabular}




\begin{tabular}{|c|c|c|c|c|c|c|c|c|c|}
\hline \multirow[b]{2}{*}{ PMCL } & \multirow[b]{2}{*}{ Hours } & \multirow[b]{2}{*}{$\mathrm{N}$} & \multirow[b]{2}{*}{ Means } & \multirow[b]{2}{*}{ Std. Dev } & \multirow{2}{*}{$\begin{array}{l}\text { Std. } \\
\text { Err. }\end{array}$} & \multicolumn{2}{|c|}{$\begin{array}{c}\text { 95\% Confidence } \\
\text { Interval for Means }\end{array}$} & \multirow[b]{2}{*}{ Minimum } & \multirow[b]{2}{*}{ Maximum } \\
\hline & & & & & & Lower Bound & Upper bound & & \\
\hline \multirow[t]{7}{*}{ L } & 0 & 45 & 97.980 & 5.550 & 0.827 & 96.312 & 99.647 & 87.129 & 106.534 \\
\hline & 16 & 60 & 94.309 & 5.876 & 0.759 & 92.791 & 95.828 & 83.570 & 105.758 \\
\hline & 32 & 60 & 94.213 & 4.330 & 0.559 & 93.094 & 95.331 & 86.034 & 100.437 \\
\hline & 48 & 60 & 93.656 & 4.182 & 0.540 & 92.576 & 94.736 & 87.407 & 102.545 \\
\hline & 64 & 60 & 94.532 & 3.596 & 0.464 & 93.603 & 95.461 & 84.559 & 98.994 \\
\hline & & 60 & 95.265 & 3.939 & 0.509 & 94.247 & 96.282 & 88.073 & 101.165 \\
\hline & All Grps & 345 & 94.862 & 4.760 & 0.256 & 94.358 & 95.367 & 83.570 & 106.534 \\
\hline \multirow[t]{7}{*}{$a$} & 0 & 45 & 3.450 & 0.765 & 0.114 & 3.220 & 3.680 & 2.107 & 4.272 \\
\hline & 16 & 60 & 2.888 & 0.622 & 0.080 & 2.727 & 3.049 & 1.717 & 3.920 \\
\hline & 32 & 60 & 3.009 & 0.511 & 0.066 & 2.877 & 3.141 & 2.037 & 3.676 \\
\hline & 48 & 60 & 3.015 & 0.965 & 0.125 & 2.765 & 3.264 & 1.601 & 5.016 \\
\hline & 64 & 60 & 2.868 & 0.770 & 0.099 & 2.669 & 3.067 & 1.542 & 3.920 \\
\hline & 80 & 60 & 2.944 & 0.635 & 0.082 & 2.780 & 3.108 & 1.481 & 4.211 \\
\hline & All Grps & 345 & 3.011 & 0.741 & 0.040 & 2.932 & 3.089 & 1.481 & 5.016 \\
\hline \multirow[t]{7}{*}{ b } & 0 & 45 & 5.436 & 1.202 & 0.179 & 5.074 & 5.797 & 2.676 & 6.678 \\
\hline & 16 & 60 & 4.310 & 0.897 & 0.116 & 4.078 & 4.542 & 2.595 & 5.819 \\
\hline & 32 & 60 & 4.899 & 0.755 & 0.097 & 4.704 & 5.094 & 3.137 & 5.883 \\
\hline & 48 & 60 & 4.713 & 1.570 & 0.203 & 4.307 & 5.119 & 2.472 & 7.086 \\
\hline & 64 & 60 & 4.729 & 1.190 & 0.154 & 4.421 & 5.036 & 2.650 & 6.581 \\
\hline & 80 & 60 & 4.716 & 1.511 & 0.195 & 4.326 & 5.106 & 1.230 & 6.727 \\
\hline & All Grps & 345 & 4.773 & 1.256 & 0.068 & 4.640 & 4.906 & 1.230 & 7.086 \\
\hline \multirow[b]{2}{*}{ PMJX } & \multirow[b]{2}{*}{ Hours } & \multirow{2}{*}{$\mathrm{N}$} & \multirow[b]{2}{*}{ Means } & \multirow[b]{2}{*}{ Std. Dev } & \multirow{2}{*}{$\begin{array}{l}\text { Std. } \\
\text { Err. }\end{array}$} & \multicolumn{2}{|c|}{$\begin{array}{l}\text { 95\% Confidence } \\
\text { Interval for Means }\end{array}$} & \multirow{2}{*}{ Minimum } & \multirow[b]{2}{*}{ Maximum } \\
\hline & & & & & & Lower Bound & Upper bound & & \\
\hline $\mathbf{L}$ & 0 & 45 & 95.837 & 5.916 & 0.882 & 94.059 & 97.614 & 85.790 & 102.507 \\
\hline & 16 & 60 & 95.223 & 3.809 & 0.492 & 94.239 & 96.207 & 86.899 & 103.330 \\
\hline & 32 & 60 & 95.596 & 4.679 & 0.604 & 94.388 & 96.805 & 89.704 & 107.488 \\
\hline & 48 & 60 & 94.815 & 3.949 & 0.510 & 93.795 & 95.835 & 88.771 & 102.092 \\
\hline & 64 & 60 & 96.427 & 3.803 & 0.491 & 95.445 & 97.410 & 91.096 & 104.538 \\
\hline & & 60 & 95.554 & 3.185 & 0.411 & 94.731 & 96.377 & 90.469 & 101.088 \\
\hline & All Grps & 345 & 95.564 & 4.228 & 0.228 & 95.116 & 96.012 & 85.790 & 107.488 \\
\hline a & 0 & 45 & 2.947 & 0.842 & 0.125 & 2.694 & 3.200 & 1.621 & 3.933 \\
\hline & 16 & 60 & 2.953 & 0.545 & 0.070 & 2.812 & 3.094 & 1.937 & 3.716 \\
\hline & 32 & 60 & 2.696 & 0.682 & 0.088 & 2.519 & 2.872 & 1.826 & 4.112 \\
\hline & 48 & 60 & 2.412 & 0.361 & 0.047 & 2.319 & 2.505 & 1.627 & 2.937 \\
\hline & 64 & 60 & 3.096 & 0.835 & 0.108 & 2.881 & 3.312 & 1.471 & 4.547 \\
\hline & 80 & 60 & 3.123 & 0.493 & 0.064 & 2.996 & 3.250 & 2.042 & 3.727 \\
\hline & All Grps & 345 & 2.868 & 0.685 & 0.037 & 2.795 & 2.940 & 1.471 & 4.547 \\
\hline b & 0 & 45 & 6.056 & 1.620 & 0.241 & 5.570 & 6.543 & 3.794 & 9.142 \\
\hline & 16 & 60 & 5.474 & 1.021 & 0.132 & 5.211 & 5.738 & 3.643 & 7.348 \\
\hline & 32 & 60 & 4.800 & 1.361 & 0.176 & 4.448 & 5.151 & 2.763 & 7.469 \\
\hline & 48 & 60 & 4.639 & 0.733 & 0.095 & 4.450 & 4.828 & 3.269 & 6.127 \\
\hline & 64 & 60 & 6.050 & 1.542 & 0.199 & 5.652 & 6.448 & 3.076 & 7.638 \\
\hline & 80 & 60 & 6.306 & 0.899 & 0.116 & 6.074 & 6.538 & 4.376 & 8.181 \\
\hline & All Grps & 345 & 5.532 & 1.375 & 0.074 & 5.387 & 5.678 & 2.763 & 9.142 \\
\hline
\end{tabular}




\begin{tabular}{|c|c|c|c|c|c|c|c|c|c|}
\hline \multirow[b]{2}{*}{ PMP } & \multirow{2}{*}{ Hours } & \multirow{2}{*}{$\mathrm{N}$} & \multirow{2}{*}{ Means } & \multirow{2}{*}{ Std. Dev } & \multirow{2}{*}{$\begin{array}{l}\text { Std. } \\
\text { Err. }\end{array}$} & \multicolumn{2}{|c|}{$\begin{array}{c}\text { 95\% Confidence } \\
\text { Interval for Means }\end{array}$} & \multirow{2}{*}{ Minimum } & \multirow{2}{*}{ Maximum } \\
\hline & & & & & & Lower Bound & Upper bound & & \\
\hline \multirow[t]{7}{*}{ L } & 0 & 45 & 83.718 & 4.444 & 0.662 & 82.383 & 85.053 & 78.487 & 90.977 \\
\hline & 16 & 60 & 80.827 & 5.272 & 0.681 & 79.465 & 82.189 & 73.407 & 87.850 \\
\hline & 32 & 60 & 79.436 & 2.717 & 0.351 & 78.734 & 80.138 & 73.864 & 83.806 \\
\hline & 48 & 60 & 79.123 & 4.784 & 0.618 & 77.887 & 80.359 & 68.977 & 85.757 \\
\hline & & 60 & 81.714 & 2.465 & 0.318 & 81.077 & 82.351 & 78.375 & 86.384 \\
\hline & 80 & 60 & 82.035 & 5.427 & 0.701 & 80.633 & 83.437 & 72.717 & 89.539 \\
\hline & All Grps & 345 & 81.030 & 4.568 & 0.246 & 80.547 & 81.514 & 68.977 & 90.977 \\
\hline \multirow[t]{7}{*}{$a$} & 0 & 45 & 6.660 & 0.741 & 0.110 & 6.437 & 6.883 & 5.748 & 8.304 \\
\hline & 16 & 60 & 7.164 & 1.345 & 0.174 & 6.816 & 7.511 & 5.461 & 9.778 \\
\hline & 32 & 60 & 6.909 & 1.729 & 0.223 & 6.463 & 7.356 & 4.592 & 9.950 \\
\hline & 48 & 60 & 6.213 & 1.719 & 0.222 & 5.769 & 6.657 & 2.791 & 9.016 \\
\hline & 64 & 60 & 6.582 & 1.322 & 0.171 & 6.241 & 6.924 & 4.535 & 8.989 \\
\hline & 80 & 60 & 5.858 & 1.384 & 0.179 & 5.500 & 6.215 & 3.311 & 7.980 \\
\hline & All Grps & 345 & 6.560 & 1.491 & 0.080 & 6.402 & 6.718 & 2.791 & 9.950 \\
\hline \multirow[t]{7}{*}{ b } & 0 & 45 & -8.771 & 2.735 & 0.408 & -9.593 & -7.950 & -12.908 & -3.225 \\
\hline & 16 & 60 & -10.024 & 2.935 & 0.379 & -10.783 & -9.266 & -15.423 & -5.169 \\
\hline & 32 & 60 & -9.887 & 2.985 & 0.385 & -10.658 & -9.116 & -16.424 & -5.809 \\
\hline & 48 & 60 & -8.914 & 2.916 & 0.376 & -9.667 & -8.161 & -14.641 & -5.039 \\
\hline & 64 & 60 & -10.913 & 3.512 & 0.453 & -11.821 & -10.006 & -16.933 & -6.347 \\
\hline & 80 & 60 & -11.226 & 3.315 & 0.428 & -12.082 & -10.370 & -16.938 & -5.530 \\
\hline & All Grps & 345 & -10.008 & 3.199 & 0.172 & -10.346 & -9.669 & -16.938 & -3.225 \\
\hline \multirow[b]{2}{*}{ RMP } & \multirow{2}{*}{ Hours } & \multirow[b]{2}{*}{$\mathrm{N}$} & \multirow{2}{*}{ Means } & \multirow{2}{*}{ Std. Dev } & \multirow{2}{*}{$\begin{array}{l}\text { Std. } \\
\text { Err. }\end{array}$} & \multicolumn{2}{|c|}{$\begin{array}{c}\text { 95\% Confidence } \\
\text { Interval for Means }\end{array}$} & \multirow{2}{*}{ Minimum } & \multirow{2}{*}{ Maximum } \\
\hline & & & & & & Lower Bound & Upper bound & & \\
\hline $\mathbf{L}$ & 0 & 45 & 80.848 & 3.737 & 0.557 & 79.725 & 81.970 & 74.676 & 87.810 \\
\hline & 16 & 60 & 81.810 & 2.698 & 0.348 & 81.113 & 82.507 & 77.150 & 87.337 \\
\hline & 32 & 60 & 82.524 & 3.774 & 0.487 & 81.549 & 83.499 & 75.446 & 90.277 \\
\hline & 48 & 60 & 82.084 & 4.313 & 0.557 & 80.970 & 83.199 & 74.870 & 90.094 \\
\hline & & 60 & 83.530 & 4.799 & 0.620 & 82.291 & 84.770 & 71.828 & 93.720 \\
\hline & & 60 & 80.481 & 3.946 & 0.509 & 79.462 & 81.501 & 71.912 & 87.660 \\
\hline & All Grps & 345 & 81.924 & 4.040 & 0.218 & 81.497 & 82.352 & 71.828 & 93.720 \\
\hline$a$ & 0 & 45 & 27.059 & 2.828 & 0.422 & 26.209 & 27.908 & 23.994 & 32.211 \\
\hline & 16 & 60 & 29.230 & 1.601 & 0.207 & 28.816 & 29.644 & 24.829 & 30.838 \\
\hline & 32 & 60 & 26.394 & 5.583 & 0.721 & 24.952 & 27.836 & 18.633 & 36.543 \\
\hline & 48 & 60 & 27.997 & 3.647 & 0.471 & 27.055 & 28.940 & 21.941 & 32.965 \\
\hline & 64 & 60 & 27.755 & 6.587 & 0.850 & 26.054 & 29.457 & 17.510 & 40.633 \\
\hline & 80 & 60 & 28.729 & 4.656 & 0.601 & 27.527 & 29.932 & 22.776 & 37.050 \\
\hline & All Grps & 345 & 27.896 & 4.601 & 0.248 & 27.408 & 28.383 & 17.510 & 40.633 \\
\hline b & 0 & 45 & 16.106 & 1.766 & 0.263 & 15.576 & 16.637 & 12.965 & 18.434 \\
\hline & 16 & 60 & 16.733 & 1.251 & 0.161 & 16.410 & 17.056 & 14.857 & 18.924 \\
\hline & 32 & 60 & 14.624 & 4.647 & 0.600 & 13.424 & 15.825 & 7.441 & 23.956 \\
\hline & 48 & 60 & 15.564 & 2.624 & 0.339 & 14.886 & 16.242 & 11.389 & 20.563 \\
\hline & 64 & 60 & 16.640 & 4.604 & 0.594 & 15.451 & 17.829 & 8.056 & 25.316 \\
\hline & 80 & 60 & 17.503 & 3.982 & 0.514 & 16.475 & 18.532 & 12.870 & 25.067 \\
\hline & All Grps & 345 & 16.199 & 3.576 & 0.193 & 15.820 & 16.578 & 7.441 & 25.316 \\
\hline
\end{tabular}


3. Test of Homogeneity of Variance- by 'Hours'

Table 3.1 Levene's test for samples grouped by 'Hours'

\begin{tabular}{|c|c|c|c|c|c|c|c|c|}
\hline \multirow[b]{2}{*}{ Variable } & \multicolumn{8}{|c|}{$\begin{array}{l}\text { Levene Test of Homogeneity of Variances (DPMP spreadsheet) } \\
\text { Marked effects are significant at } p<.05000\end{array}$} \\
\hline & \begin{tabular}{|c|} 
SS \\
Effect
\end{tabular} & $\begin{array}{c}d f \\
\text { Effect }\end{array}$ & \begin{tabular}{c|c} 
MS \\
Effect
\end{tabular} & \begin{tabular}{c|c} 
SS \\
Error
\end{tabular} & \begin{tabular}{c|c}
$\mathrm{df}$ \\
Error
\end{tabular} & \begin{tabular}{l|l} 
MS & \\
Error & \\
\end{tabular} & \begin{tabular}{l|l}
$F$ &
\end{tabular} & $p$ \\
\hline $\mathrm{L}$ & $47.6 \varepsilon$ & 5 & 9.52 & $1270 .($ & $33 \mathrm{C}$ & $3.74 \epsilon$ & $2.54 \varepsilon$ & $0.02 \varepsilon$ \\
\hline$a$ & 95.01 & 5 & $19.00 i$ & 1185. & $33 \mathrm{c}$ & $3.49 \varepsilon$ & $5.43 \varepsilon$ & $0.00 c$ \\
\hline \multirow[t]{3}{*}{ b } & $23.5<$ & 5 & $4.70 \varepsilon$ & 428.5 & $33 c$ & 1.264 & 3.725 & 0.00 \\
\hline & \multicolumn{8}{|c|}{$\begin{array}{l}\text { Levene Test of Homogeneity of Variances (GMP spreadsheet) } \\
\text { Marked effects are significant at } p<.05000\end{array}$} \\
\hline & \begin{tabular}{c|} 
SS \\
Effect
\end{tabular} & $\begin{array}{c}d f \\
\text { Effect }\end{array}$ & $\begin{array}{c}\text { MS } \\
\text { Effect }\end{array}$ & \begin{tabular}{c|} 
SS \\
Error
\end{tabular} & \begin{tabular}{|c|}
$d f$ \\
Error
\end{tabular} & \begin{tabular}{l|} 
MS \\
Error
\end{tabular} & $F$ & $p$ \\
\hline $\mathrm{L}$ & 279.81 & 5 & 55.97 & 2288. & $33 \mathrm{c}$ & $6.75 C$ & $8.29 \varepsilon$ & 0.000 \\
\hline$a$ & 36.61 & 5 & 7.32 & $124 . \mathrm{c}$ & $33 \mathrm{c}$ & $0.36 \mathrm{~s}$ & $19.86 !$ & 0.000 \\
\hline \multirow[t]{3}{*}{$\mathrm{b}$} & $44.8<$ & 5 & 8.97 & 532.5 & $33 c$ & 1.571 & $5.71 \mathrm{C}$ & 0.000 \\
\hline & \multicolumn{8}{|c|}{$\begin{array}{l}\text { Levene Test of Homogeneity of Variances (OSDP spreadsheet) } \\
\text { Marked effects are significant at } p<.05000\end{array}$} \\
\hline & \begin{tabular}{|c|} 
SS \\
Effect
\end{tabular} & $\begin{array}{c}\mathrm{df} \\
\text { Effect }\end{array}$ & $\begin{array}{c}\text { MS } \\
\text { Effect }\end{array}$ & \begin{tabular}{c|} 
SS \\
Error
\end{tabular} & \begin{tabular}{|c|}
$\mathrm{df}$ \\
Error
\end{tabular} & \begin{tabular}{l|l} 
MS \\
Error
\end{tabular} & $F$ & $p$ \\
\hline $\mathrm{L}$ & $294.7 !$ & 5 & i58.95 & 2154.4 & $33 \mathrm{c}$ & 6.355 & 9.277 & 0.000 \\
\hline$a$ & $47.0<$ & 5 & $9.40 \varepsilon$ & 351.4 & $33 c$ & 1.037 & $9.07 i$ & 0.000 \\
\hline \multirow[b]{3}{*}{ Vorioblo } & $483.0^{\prime}$ & 5 & $96.60<$ & 2915.6 & $33 c$ & $8.60 C$ & $11.23 \AA$ & 0.000 \\
\hline & \multicolumn{8}{|c|}{$\begin{array}{l}\text { Levene Test of Homogeneity of Variances (PMCL spreadsheet) } \\
\text { Marked effects are significant at } p<.05000\end{array}$} \\
\hline & \begin{tabular}{|c|} 
SS \\
Effect
\end{tabular} & \begin{tabular}{c|c}
$\mathrm{df}$ \\
Effect
\end{tabular} & \begin{tabular}{c|c} 
MS \\
Effect
\end{tabular} & \begin{tabular}{c|c|} 
SS \\
Error
\end{tabular} & $\begin{array}{c}\mathrm{df} \\
\text { Error }\end{array}$ & $\begin{array}{l}\text { MS } \\
\text { Error } \\
\end{array}$ & $\mathrm{F}$ & $p$ \\
\hline $\begin{array}{ll}L \\
\end{array}$ & $209.5 \%$ & 5 & $41.90 !$ & 2204.511 & 339 & $6.50 €$ & 6.444 & 0.00 \\
\hline a & $5.957 \varepsilon$ & 5 & $1.19 \varepsilon$ & 59.30 & $33 c$ & 0.175 & $6.81 \kappa$ & 0.00 \\
\hline \multirow[b]{3}{*}{. } & 23.907 & 5 & 4.781 & $158.51 ،$ & $33 \mathrm{C}$ & $0.46 \varepsilon$ & $10.22 t$ & 0.00 \\
\hline & \multicolumn{8}{|c|}{$\begin{array}{l}\text { Levene Test of Homogeneity of Variances (PMJX spreadsheet) } \\
\text { Marked effects are significant at } p<.05000\end{array}$} \\
\hline & \begin{tabular}{|c|} 
SS \\
Effect
\end{tabular} & $\begin{array}{c}\mathrm{df} \\
\text { Effect }\end{array}$ & $\begin{array}{c}\text { MS } \\
\text { Effect }\end{array}$ & \begin{tabular}{c|} 
SS \\
Error
\end{tabular} & \begin{tabular}{|c|}
$d f$ \\
Error
\end{tabular} & \begin{tabular}{c|c} 
MS \\
Error
\end{tabular} & $F$ & $p$ \\
\hline $\mathrm{L}$ & $174.7 t$ & 5 & $34.95 i$ & 2187.8 & $33 c$ & 6.454 & $5.41 \epsilon$ & 0.000 \\
\hline$a$ & 7.08 & 5 & $1.41 \epsilon$ & 37.85 & $33 c$ & $0.11 \epsilon$ & $12.68 t$ & 0.00 \\
\hline$b$ & $37.1 \varepsilon$ & 5 & 7.436 & $161.8 i$ & $33 c$ & $0.47 \bar{\imath}$ & $15.57 i$ & 0.00 \\
\hline
\end{tabular}


4. ANOVA Output for Aged Results- by 'Hours'

Table 4.1: ANOVA Results for reference samples

\begin{tabular}{|ll|r|r|r|r|r|}
\hline GMP & Sum of Squares & \multicolumn{1}{c|}{ df } & Mean Square & F & Sig. \\
\hline L & Between Groups & 467.35 & 5 & 93.471 & 4.611 & .000 \\
& Within Groups & 6871.4 & 339 & 20.270 & \\
& Total & 7338.7 & 344 & & \\
a & Between Groups & 12.128 & 5 & 2.426 & 3.025 & .011 \\
& Within Groups & 271.79 & 339 & .802 & \\
& Total & 283.92 & 344 & & \\
b & Between Groups & 59.055 & 5 & 11.811 & 2.447 \\
& Within Groups & 1636.5 & 339 & 4.827 & & .034 \\
& Total & 1695.6 & 344 & & & \\
\end{tabular}

\begin{tabular}{|ll|r|r|r|r|r|}
\hline OSDP & Sum of Squares & \multicolumn{1}{|c|}{$\mathrm{df}$} & Mean Square & F & Sig. \\
\hline L & Between Groups & 319.4 & 5 & 63.878 & 3.381 & .005 \\
& Within Groups & 6404.0 & 339 & 18.891 & & \\
& Total & 6723.4 & 344 & & & \\
a & Between Groups & 60.046 & 5 & 12.009 & 4.500 & .001 \\
& Within Groups & 904.6 & 339 & 2.669 & & \\
& Total & 964.7 & 344 & & & \\
b & Between Groups & 1084.6 & 5 & 216.92 & 9.272 & .000 \\
& Within Groups & 7931.0 & 339 & 23.395 & & \\
& Total & 9015.6 & 344 & & & \\
\hline
\end{tabular}

\begin{tabular}{|ll|r|r|r|r|r|}
\hline PMCL & Sum of Squares & df & Mean Square & F & Sig. \\
\hline \multirow{2}{*}{ L } & Between Groups & 584.50 & 5 & 116.90 & 5.497 & .000 \\
& Within Groups & 7208.9 & 339 & 21.27 & & \\
& Total & 7793.4 & 344 & & & \\
& Between Groups & 11.060 & 5 & 2.212 & 4.220 & .001 \\
a & Within Groups & 177.70 & 339 & .524 & & \\
& Total & 188.76 & 344 & & \\
& Between Groups & 34.090 & 5 & 6.818 & 4.545 & .001 \\
b Within Groups & 508.53 & 339 & 1.500 & & \\
& Total & 542.62 & 344 & & & \\
\hline
\end{tabular}

\begin{tabular}{|c|c|c|c|c|c|c|}
\hline \multicolumn{2}{|c|}{ PMJX } & Sum of Squares & $d f$ & Mean Square & $\mathrm{F}$ & Sig. \\
\hline \multirow[t]{3}{*}{$\bar{L}$} & Between Groups & 88.771 & 5 & 17.754 & .993 & .422 \\
\hline & Within Groups & 6060.0 & 339 & 17.876 & & \\
\hline & Total & 6148.8 & 344 & & & \\
\hline \multirow[t]{3}{*}{ a } & Between Groups & 22.007 & 5 & 4.401 & 10.708 & .000 \\
\hline & Within Groups & 139.34 & 339 & .411 & & \\
\hline & Total & 161.34 & 344 & & & \\
\hline \multirow[t]{3}{*}{ b } & Between Groups & 144.62 & 5 & 28.923 & 19.387 & .000 \\
\hline & Within Groups & 505.76 & 339 & 1.492 & & \\
\hline & Total & 650.37 & 344 & & & \\
\hline
\end{tabular}




\begin{tabular}{|ll|r|r|r|r|r|}
\hline \multirow{2}{*}{ PMP } & & Sum of Squares & df & \multicolumn{1}{c|}{$\begin{array}{c}\text { Mean } \\
\text { Square }\end{array}$} & F & Sig. \\
\hline L & Between Groups & 786.90 & 5 & 157.380 & 8.348 & .000 \\
& Within Groups & 6390.8 & 339 & 18.852 & & \\
& Total & 7177.7 & 344 & & \\
& Between Groups & 66.479 & 5 & 13.296 & 6.459 & .000 \\
& Within Groups & 697.80 & 339 & 2.058 & & \\
b & Total & 764.28 & 344 & & \\
& Between Groups & 279.74 & 5 & 55.949 & 5.852 & .000 \\
& Within Groups & 3240.8 & 339 & 9.560 & & \\
& Total & 3520.6 & 344 & & & \\
\end{tabular}

\begin{tabular}{|ll|r|r|r|r|r|}
\hline RMP & Sum of Squares & df & Mean Square & F & Sig. \\
\hline L & Between Groups & 357.07 & 5 & 71.414 & 4.604 & .000 \\
& Within Groups & 5258.0 & 339 & 15.510 & & \\
& Total & 5615.1 & 344 & & \\
a & Between Groups & 316.70 & 5 & 63.340 & 3.082 & .010 \\
& Within Groups & 6967.0 & 339 & 20.552 & & \\
& Total & 7283.7 & 344 & & \\
b & Between Groups & 308.54 & 5 & 61.708 & 5.108 & .000 \\
& Within Groups & 4095.7 & 339 & 12.082 & & \\
& Total & 4404.2 & 344 & & & \\
\end{tabular}

\section{Mean Plots for Aged Results-by 'Hours'}

Figure 5.1: Mean Plot for sample GMP

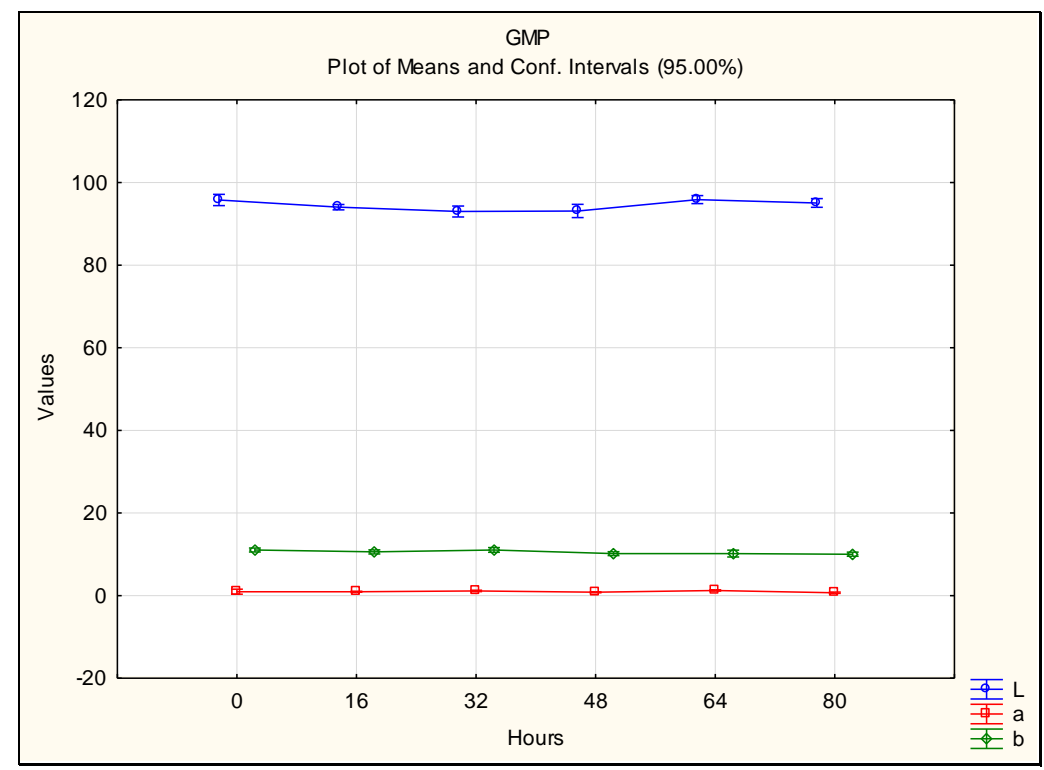


Figure 5.2: Mean Plot for sample OSDP

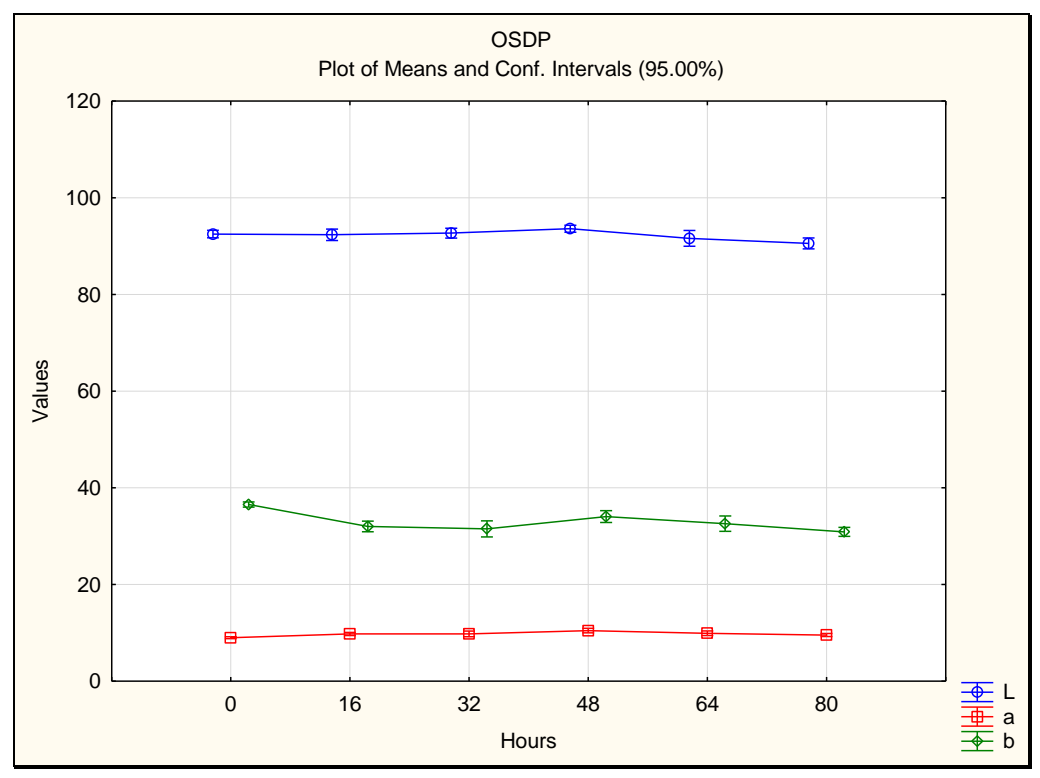

Figure 5.3: Mean Plot for sample PMCL

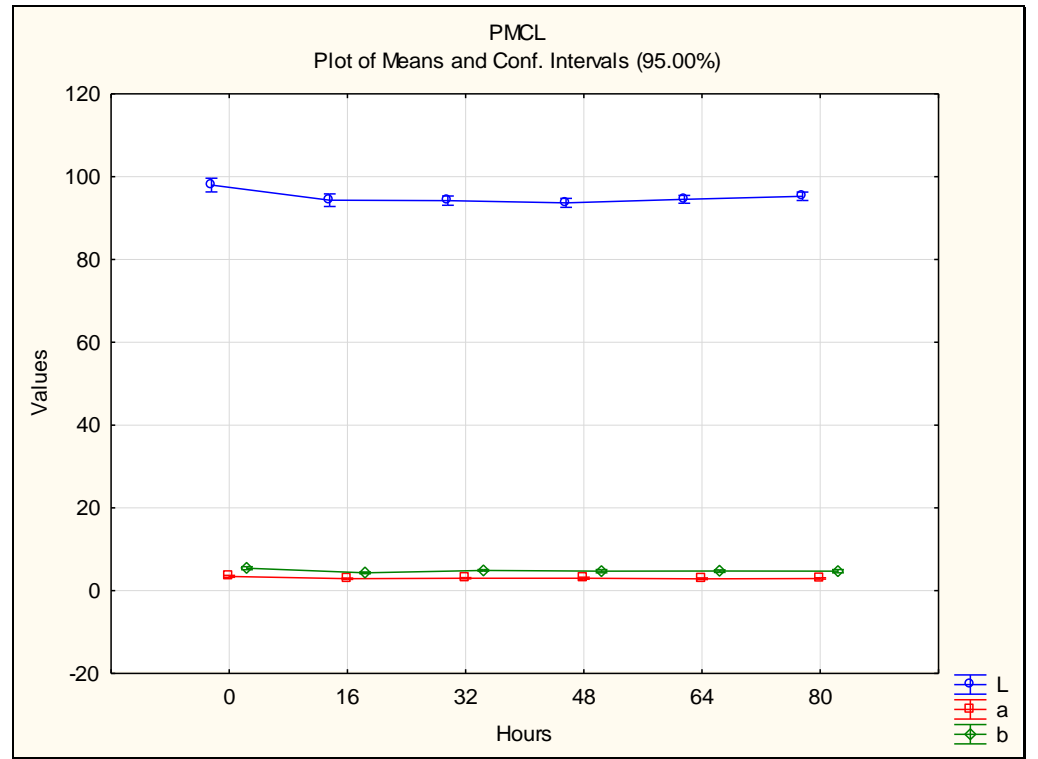


Figure 5.4: Mean Plot for sample PMJX

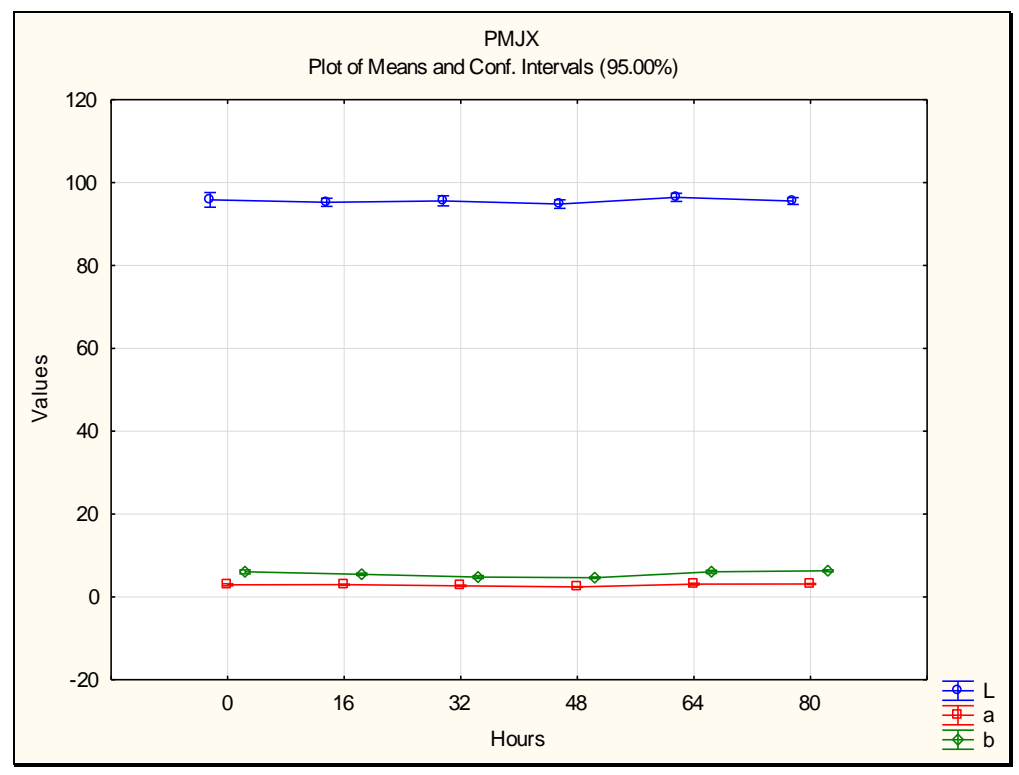

Figure 5.5: Mean Plot for sample PMP

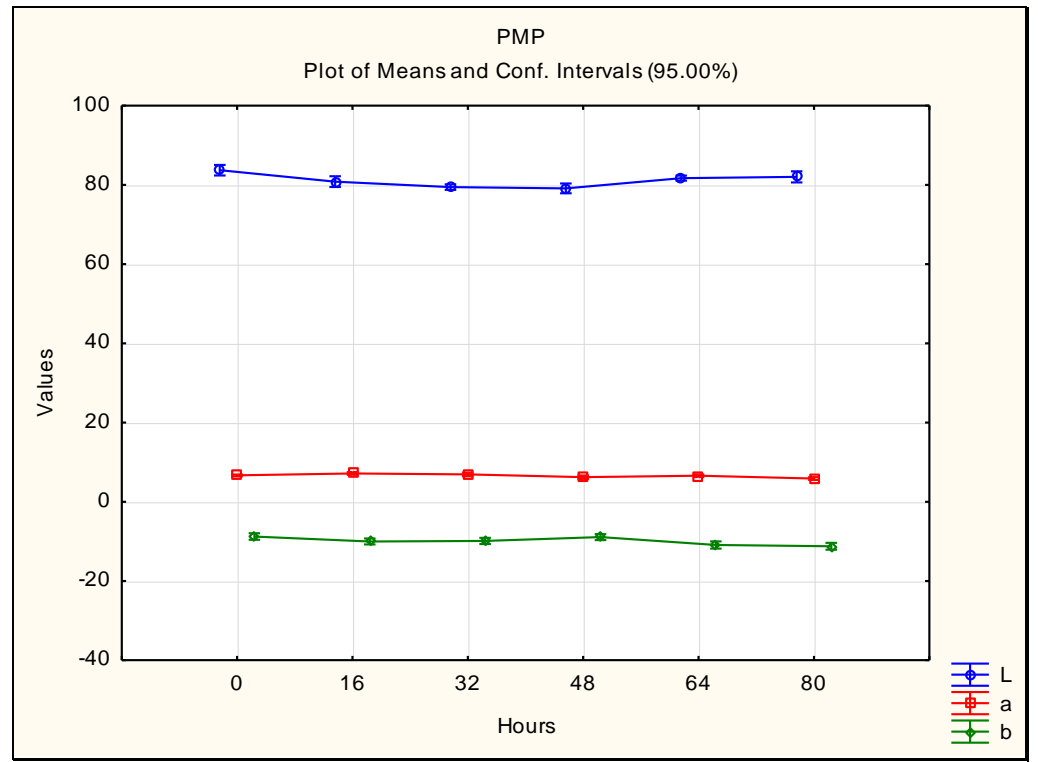


Figure 5.6: Mean Plot for sample RMP

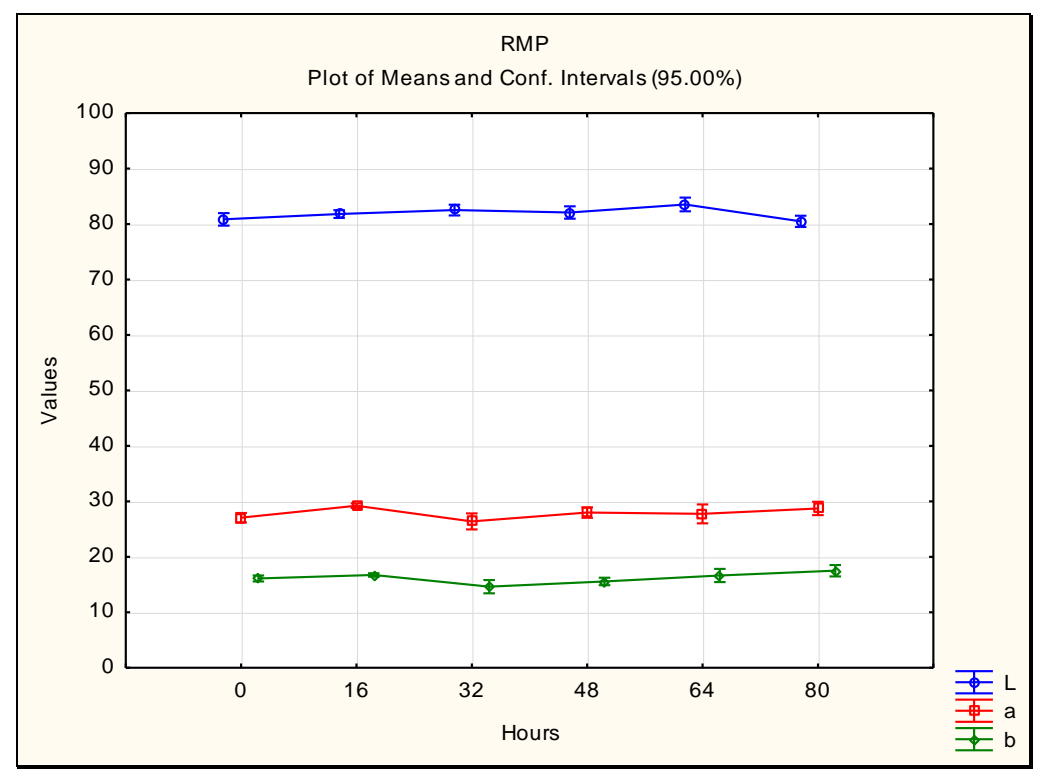

6. Descriptive Statistics from One-Way ANOVA for Aged Samples- by 'Sample Area'

Table 6.1 Descriptive Statistics for reference samples grouped by 'Sample Area'

\begin{tabular}{|c|c|c|c|c|c|c|c|c|c|}
\hline \multirow{2}{*}{\multicolumn{2}{|c|}{ DPMP }} & \multirow[b]{2}{*}{$\mathrm{N}$} & \multirow[b]{2}{*}{ Mean } & \multirow[b]{2}{*}{ Std. Deviation } & \multirow[b]{2}{*}{ Std. Error } & \multicolumn{2}{|c|}{$\begin{array}{l}\text { 95\% Confidence Interval for } \\
\text { Mean }\end{array}$} & \multirow[b]{2}{*}{ Minimum } & \multirow[b]{2}{*}{ Maximum } \\
\hline & & & & & & Lower Bound & Upper Bound & & \\
\hline \multirow[t]{4}{*}{$\mathrm{L}$} & 1 & 115 & 84.9185 & 3.7743 & 0.3520 & 84.2212 & 85.6157 & 76.5814 & 94.8448 \\
\hline & 2 & 115 & 85.0170 & 3.3014 & 0.3079 & 84.4072 & 85.6269 & 77.5515 & 89.3081 \\
\hline & 3 & 115 & 84.0858 & 3.8040 & 0.3547 & 83.3831 & 84.7885 & 77.9147 & 94.6404 \\
\hline & Total & 345 & 84.6738 & 3.6474 & 0.1964 & 84.2875 & 85.0600 & 76.5814 & 94.8448 \\
\hline \multirow[t]{4}{*}{$a$} & 1 & 115 & 27.1768 & 3.5604 & 0.3320 & 26.5190 & 27.8345 & 21.9270 & 35.7931 \\
\hline & 2 & 115 & 25.5389 & 3.2736 & 0.3053 & 24.9342 & 26.1436 & 19.5486 & 31.6690 \\
\hline & 3 & 115 & 25.6470 & 2.8704 & 0.2677 & 25.1167 & 26.1772 & 20.2630 & 30.3595 \\
\hline & Total & 345 & 26.1209 & 3.3232 & 0.1789 & 25.7690 & 26.4728 & 19.5486 & 35.7931 \\
\hline \multirow[t]{4}{*}{$\mathrm{b}$} & 1 & 115 & 8.2214 & 1.9797 & 0.1846 & 7.8557 & 8.5871 & 4.5609 & 11.3502 \\
\hline & 2 & 115 & 7.3517 & 2.1648 & 0.2019 & 6.9518 & 7.7516 & 0.7615 & 10.6772 \\
\hline & 3 & 115 & 7.6074 & 1.5231 & 0.1420 & 7.3261 & 7.8888 & 4.7164 & 10.2632 \\
\hline & Total & 345 & 7.7269 & 1.9376 & 0.1043 & 7.5217 & 7.9320 & 0.7615 & 11.3502 \\
\hline
\end{tabular}




\begin{tabular}{|c|c|c|c|c|c|c|c|c|c|}
\hline \multirow{2}{*}{\multicolumn{2}{|c|}{ GMP }} & \multirow[b]{2}{*}{$\mathbf{N}$} & \multirow[b]{2}{*}{ Mean } & \multirow[b]{2}{*}{$\begin{array}{c}\text { Std. } \\
\text { Deviation }\end{array}$} & \multirow[b]{2}{*}{ Std. Error } & \multicolumn{2}{|c|}{$\begin{array}{c}95 \% \text { Confidence Interval } \\
\text { for Mean }\end{array}$} & \multirow[b]{2}{*}{ Minimum } & \multirow[b]{2}{*}{ Maximum } \\
\hline & & & & & & $\begin{array}{l}\text { Lower } \\
\text { Bound } \\
\end{array}$ & $\begin{array}{l}\text { Upper } \\
\text { Bound } \\
\end{array}$ & & \\
\hline \multirow[t]{4}{*}{$\mathrm{L}$} & $A$ & 115 & 93.531 & 4.357 & 0.406 & 92.726 & 94.336 & 83.389 & 100.836 \\
\hline & B & 115 & 96.187 & 4.296 & 0.401 & 95.393 & 96.981 & 87.721 & 103.495 \\
\hline & C & 115 & 93.533 & 4.711 & 0.439 & 92.663 & 94.403 & 83.374 & $105.30 s$ \\
\hline & Total & 345 & 94.417 & 4.619 & 0.249 & 93.928 & 94.906 & 83.374 & $105.30 s$ \\
\hline \multirow[t]{4}{*}{ a } & A & 115 & 0.8495 & 0.8521 & 0.0795 & 0.6921 & 1.0069 & -.6918 & 2.4857 \\
\hline & B & 115 & 1.2105 & 1.2380 & 0.1154 & 0.9818 & 1.4392 & .0455 & 6.5215 \\
\hline & C & 115 & 0.8727 & 0.3866 & 0.0361 & 0.8013 & 0.9441 & -.1966 & 1.5735 \\
\hline & Total & 345 & 0.9776 & 0.9085 & 0.0489 & 0.8814 & 1.0738 & -.6918 & 6.5215 \\
\hline \multirow[t]{4}{*}{ b } & A & 115 & 10.714 & 2.791 & 0.260 & 10.198 & 11.229 & 2.443 & $15.87 \mathrm{C}$ \\
\hline & B & 115 & 10.252 & 1.992 & 0.186 & 9.884 & 10.620 & 6.737 & 13.942 \\
\hline & C & 115 & 10.514 & 1.734 & 0.162 & 10.194 & 10.834 & 7.953 & 13.876 \\
\hline & Total & 345 & 10.493 & 2.220 & 0.120 & 10.258 & 10.728 & 2.443 & 15.879 \\
\hline \multirow{2}{*}{\multicolumn{2}{|c|}{ OSDP }} & & & & & \multicolumn{2}{|c|}{$\begin{array}{l}95 \% \text { Confidence Interval } \\
\text { for Mean }\end{array}$} & & \\
\hline & & $\mathbf{N}$ & Mean & $\begin{array}{c}\text { Std. } \\
\text { Deviation }\end{array}$ & Std. Error & $\begin{array}{l}\text { Lower } \\
\text { Bound } \\
\end{array}$ & $\begin{array}{l}\text { Upper } \\
\text { Bound } \\
\end{array}$ & Minimum & Maximum \\
\hline \multirow[t]{4}{*}{$\mathrm{L}$} & A & 115 & 93.663 & 4.070 & 0.3796 & 92.9113 & 94.4151 & 88.053 & 103.549 \\
\hline & B & 115 & 90.811 & 4.527 & 0.4222 & 89.9743 & 91.6469 & 83.408 & 98.626 \\
\hline & C & 115 & 92.127 & 4.219 & 0.3934 & 91.3477 & 92.9066 & 79.250 & 100.498 \\
\hline & Total & 345 & 92.200 & 4.421 & 0.2380 & 91.7322 & 92.6685 & 79.250 & $103.54 \mathrm{~s}$ \\
\hline \multirow[t]{4}{*}{$a$} & A & 115 & 9.645 & 1.824 & .170101 & 9.30848 & 9.98242 & 4.048 & 12.405 \\
\hline & B & 115 & 9.876 & 1.808 & 168636 & 9.54190 & 10.21003 & 5.454 & 13.428 \\
\hline & C & 115 & 9.813 & 1.355 & .126345 & 9.56241 & 10.06298 & 6.008 & 12.144 \\
\hline & Total & 345 & 9.778 & 1.675 & .090158 & 9.60071 & 9.95537 & 4.048 & 13.428 \\
\hline \multirow[t]{4}{*}{$\mathrm{b}$} & A & 115 & 32.635 & 5.765 & 0.5376 & 31.5701 & 33.7001 & 15.639 & $41.30 \mathrm{~s}$ \\
\hline & B & 115 & 32.637 & 5.366 & 0.5004 & 31.6460 & 33.6287 & 22.190 & 42.614 \\
\hline & C & 115 & 33.045 & 4.115 & 0.3837 & 32.2851 & 33.8055 & 19.993 & 38.851 \\
\hline & Total & 345 & 32.773 & 5.119 & 0.2756 & 32.2305 & 33.3147 & 15.639 & 42.614 \\
\hline \multirow{2}{*}{\multicolumn{2}{|c|}{ PMCL }} & & & & & \multicolumn{2}{|c|}{$\begin{array}{l}95 \% \text { Confidence Interval } \\
\text { for Mean }\end{array}$} & & \\
\hline & & $\mathbf{N}$ & Mean & $\begin{array}{c}\text { Std. } \\
\text { Deviation }\end{array}$ & Std. Error & $\begin{array}{l}\text { Lower } \\
\text { Bound } \\
\end{array}$ & $\begin{array}{l}\text { Upper } \\
\text { Bound }\end{array}$ & Minimum & Maximum \\
\hline \multirow[t]{4}{*}{$\mathrm{L}$} & A & 115 & 94.8100 & 4.3578 & 0.4064 & 94.0050 & 95.6151 & 87.129 & 104.558 \\
\hline & B & 115 & 95.5182 & 4.8770 & 0.4548 & 94.6173 & 96.4191 & 83.570 & 106.534 \\
\hline & C & 115 & 94.2592 & 4.9783 & 0.4642 & 93.3396 & 95.1788 & 84.559 & 105.758 \\
\hline & Total & 345 & 94.8625 & 4.7597 & 0.2563 & 94.3585 & 95.3665 & 83.570 & 106.534 \\
\hline \multirow[t]{4}{*}{$a$} & A & 115 & 3.0273 & 0.8274 & 0.0772 & 2.8745 & 3.1802 & 1.4808 & 4.2719 \\
\hline & B & 115 & 3.1584 & 0.7720 & 0.0720 & 3.0158 & 3.3010 & 1.5424 & 5.0161 \\
\hline & C & 115 & 2.8461 & 0.5706 & 0.0532 & 2.7407 & 2.9515 & 1.8094 & 3.7672 \\
\hline & Total & 345 & 3.0106 & 0.7408 & 0.0399 & 2.9322 & 3.0890 & 1.4808 & 5.0161 \\
\hline \multirow[t]{4}{*}{ b } & A & 115 & 4.8037 & 1.4591 & 0.1361 & 4.5342 & 5.0733 & 1.2300 & 6.6783 \\
\hline & B & 115 & 5.1611 & 1.1794 & 0.1100 & 4.9433 & 5.3790 & 2.6503 & 7.0857 \\
\hline & C & 115 & 4.3534 & 0.9535 & 0.0889 & 4.1773 & 4.5296 & 2.5008 & 6.1958 \\
\hline & Total & 345 & 4.7728 & 1.2559 & 0.0676 & 4.6398 & 4.9058 & 1.2300 & 7.0857 \\
\hline
\end{tabular}




\begin{tabular}{|c|c|c|c|c|c|c|c|c|c|}
\hline \multirow{2}{*}{\multicolumn{2}{|c|}{ PMJX }} & \multirow[b]{2}{*}{$\mathbf{N}$} & \multirow[b]{2}{*}{ Mean } & \multirow[b]{2}{*}{$\begin{array}{c}\text { Std. } \\
\text { Deviation }\end{array}$} & \multirow[b]{2}{*}{$\begin{array}{l}\text { Std. } \\
\text { Error }\end{array}$} & \multicolumn{2}{|c|}{$\begin{array}{c}\text { 95\% Confidence Interval for } \\
\text { Mean }\end{array}$} & \multirow[b]{2}{*}{ Minimum } & \multirow[b]{2}{*}{ Maximum } \\
\hline & & & & & & Lower Bound & $\begin{array}{l}\text { Upper } \\
\text { Bound } \\
\end{array}$ & & \\
\hline \multirow[t]{4}{*}{$\bar{L}$} & $A$ & 115 & 95.4629 & 4.1315 & 0.3853 & 94.6997 & 96.2261 & 85.7904 & 103.3300 \\
\hline & B & 115 & 95.0209 & 4.3154 & 0.4024 & 94.2237 & 95.8181 & 86.0544 & 107.4880 \\
\hline & C & 115 & 96.2080 & 4.1855 & 0.3903 & 95.4348 & 96.9812 & 86.8987 & 104.5380 \\
\hline & Total & 345 & 95.5639 & 4.2278 & 0.2276 & 95.1162 & 96.0116 & 85.7904 & 107.4880 \\
\hline \multirow[t]{4}{*}{$a$} & $A$ & 115 & 2.9480 & 0.6188 & 0.0577 & 2.8337 & 3.0623 & 1.82606 & 3.89497 \\
\hline & B & 115 & 2.7231 & 0.7261 & 0.0677 & 2.5890 & 2.8573 & 1.47121 & 4.54655 \\
\hline & C & 115 & 2.9324 & 0.6880 & 0.0642 & 2.8053 & 3.0595 & 1.64672 & 4.11162 \\
\hline & Total & 345 & 2.8678 & 0.6848 & 0.0369 & 2.7953 & 2.9403 & 1.47121 & 4.54655 \\
\hline \multirow[t]{4}{*}{$\mathrm{b}$} & A & 115 & 5.4555 & 1.4026 & 0.1308 & 5.1964 & 5.7146 & 2.76309 & 8.18116 \\
\hline & B & 115 & 5.3474 & 1.3437 & 0.1253 & 5.0992 & 5.5957 & 3.07614 & 9.14167 \\
\hline & C & 115 & 5.7937 & 1.3501 & 0.1259 & 5.5443 & 6.0431 & 3.74439 & 7.55470 \\
\hline & Total & 345 & 5.5322 & 1.3750 & 0.0740 & 5.3866 & 5.6778 & 2.76309 & 9.14167 \\
\hline \multirow{2}{*}{\multicolumn{2}{|c|}{ PMP }} & \multirow[b]{2}{*}{$\mathbf{N}$} & \multirow[b]{2}{*}{ Mean } & \multirow[b]{2}{*}{$\begin{array}{c}\text { Std. } \\
\text { Deviation }\end{array}$} & \multirow[b]{2}{*}{$\begin{array}{l}\text { Std. } \\
\text { Error }\end{array}$} & \multicolumn{2}{|c|}{$\begin{array}{c}95 \% \text { Confidence Interval for } \\
\text { Mean }\end{array}$} & \multirow[b]{2}{*}{ Minimum } & \multirow[b]{2}{*}{ Maximum } \\
\hline & & & & & & $\begin{array}{l}\text { Lower } \\
\text { Bound } \\
\end{array}$ & $\begin{array}{l}\text { Upper } \\
\text { Bound }\end{array}$ & & \\
\hline \multirow[t]{4}{*}{$\mathrm{L}$} & A & 115 & 81.5562 & 4.1629 & 0.3882 & 80.7872 & 82.3252 & 73.4104 & 90.4446 \\
\hline & B & 115 & 80.8133 & 4.7655 & 0.4444 & 79.9330 & 81.6936 & 73.7668 & 90.9770 \\
\hline & C & 115 & 80.7216 & 4.7434 & 0.4423 & 79.8454 & 81.5979 & 68.9771 & 87.7592 \\
\hline & Total & 345 & 81.0304 & 4.5679 & 0.2459 & 80.5467 & 81.5141 & 68.9771 & 90.9770 \\
\hline \multirow[t]{4}{*}{ a } & A & 115 & 6.5953 & 1.3627 & 0.1271 & 6.3435 & 6.8470 & 3.9195 & 9.7779 \\
\hline & B & 115 & 6.4532 & 1.4846 & 0.1384 & 6.1790 & 6.7274 & 3.3110 & 9.5653 \\
\hline & $\mathrm{C}$ & 115 & 6.6324 & 1.6203 & 0.1511 & 6.3331 & 6.9317 & 2.7908 & 9.9504 \\
\hline & Total & 345 & 6.5603 & 1.4906 & 0.0802 & 6.4024 & 6.7181 & 2.7908 & 9.9504 \\
\hline \multirow[t]{4}{*}{$b$} & A & 115 & -9.2101 & 2.8362 & 0.2645 & -9.7341 & -8.6862 & -15.7421 & -3.2247 \\
\hline & B & 115 & -9.9362 & 3.8814 & 0.3619 & -10.6532 & -9.2192 & -16.9378 & -5.1692 \\
\hline & C & 115 & -10.8765 & 2.5228 & 0.2353 & -11.3426 & -10.4105 & -16.4240 & -6.0859 \\
\hline & Total & 345 & -10.0076 & 3.1991 & 0.1722 & -10.3464 & -9.6689 & -16.9378 & -3.2247 \\
\hline \multirow{2}{*}{\multicolumn{2}{|c|}{ RMP }} & & & & & $\begin{array}{r}\text { 95\% Confiden } \\
\mathrm{Me}\end{array}$ & nterval for & & \\
\hline & & $\mathbf{N}$ & Mean & $\begin{array}{c}\text { Std. } \\
\text { Deviation }\end{array}$ & $\begin{array}{l}\text { Std. } \\
\text { Error }\end{array}$ & $\begin{array}{l}\text { Lower } \\
\text { Bound } \\
\end{array}$ & $\begin{array}{l}\text { Upper } \\
\text { Bound }\end{array}$ & Minimum & Maximum \\
\hline $\mathrm{L}$ & A & 115 & 81.5578 & 3.62749 & .33826 & 80.8877 & 82.2279 & 71.91 & 88.12 \\
\hline & B & 115 & 81.9557 & 3.98003 & .37114 & 81.2205 & 82.6910 & 71.83 & 90.28 \\
\hline & C & 115 & 82.2636 & 4.47252 & .41706 & 81.4374 & 83.0898 & 74.68 & 93.72 \\
\hline & Total & 345 & 81.9257 & 4.04017 & 21752 & 81.4979 & 82.3535 & 71.83 & 93.72 \\
\hline a & A & 115 & 27.8357 & 4.4981 & 0.4195 & 27.0048 & 28.6667 & 18.6332 & 36.5426 \\
\hline & B & 115 & 29.3360 & 4.9071 & 0.4576 & 28.4296 & 30.2425 & 19.1991 & 40.6327 \\
\hline & $C$ & 115 & 26.5309 & 3.9502 & 0.3684 & 25.8012 & 27.2606 & 17.5098 & 32.2106 \\
\hline & Total & 345 & 27.9009 & 4.6015 & 0.2477 & 27.4136 & 28.3882 & 17.5098 & 40.6327 \\
\hline b & A & 115 & 16.4875 & 3.6896 & 0.3441 & 15.8059 & 17.1691 & 8.5244 & 23.9559 \\
\hline & B & 115 & 17.2641 & 3.9769 & 0.3708 & 16.5295 & 17.9988 & 7.4415 & 25.3159 \\
\hline & C & 115 & 14.8851 & 2.4971 & 0.2329 & 14.4238 & 15.3464 & 8.0565 & 18.6720 \\
\hline & Total & 345 & 16.2122 & 3.5781 & 0.1926 & 15.8333 & 16.5911 & 7.4415 & 25.3159 \\
\hline
\end{tabular}

7. Test of Homogeneity of Variance-by 'Sample Area'

Table 7.1 Levene's test for reference samples grouped by 'Sample Area' 


\begin{tabular}{|c|c|c|c|c|}
\hline DPMP & Levene Statistic & df1 & df2 & Sig. \\
\hline $\mathrm{L}$ & .001 & 2 & 342 & .999 \\
\hline $\mathrm{a}$ & 1.998 & 2 & 342 & .137 \\
\hline b & 6.028 & 2 & 342 & .003 \\
\hline GMP & Levene Statistic & df1 & df2 & Sig. \\
\hline $\mathrm{L}$ & .860 & 2 & 342 & .424 \\
\hline $\mathrm{a}$ & 17.590 & 2 & 342 & .000 \\
\hline b & 4.924 & 2 & 342 & .008 \\
\hline OSDP & Levene Statistic & df1 & df2 & Sig. \\
\hline $\mathrm{L}$ & 4.676 & 2 & 342 & .010 \\
\hline a & 4.312 & 2 & 342 & .014 \\
\hline $\mathrm{b}$ & 9.167 & 2 & 342 & .000 \\
\hline PMCL & Levene Statistic & df1 & df2 & Sig. \\
\hline $\mathrm{L}$ & .770 & 2 & 342 & .464 \\
\hline a & 9.031 & 2 & 342 & .000 \\
\hline b & 15.748 & 2 & 342 & .000 \\
\hline PMJX & Levene Statistic & df1 & df2 & Sig. \\
\hline $\mathrm{L}$ & .126 & 2 & 342 & .882 \\
\hline a & 2.219 & 2 & 342 & .110 \\
\hline$b$ & 3.653 & 2 & 342 & .027 \\
\hline PMP & Levene Statistic & df1 & df2 & Sig. \\
\hline $\mathrm{L}$ & .780 & 2 & 342 & .459 \\
\hline a & .626 & 2 & 342 & .535 \\
\hline b & 19.396 & 2 & 342 & .000 \\
\hline RMP & Levene Statistic & df1 & df2 & Sig. \\
\hline $\mathrm{L}$ & 2.522 & 2 & 342 & .082 \\
\hline a & 1.663 & 2 & 342 & 191 \\
\hline b & 6.934 & 2 & 342 & .001 \\
\hline
\end{tabular}

8. ANOVA Output for Aged Results- by 'Sample Area'

Table 8.1: ANOVA Output for sample DPMP

ANOVA: DPMP

\begin{tabular}{|ll|r|r|r|r|r|}
\hline & & $\begin{array}{c}\text { Sum of } \\
\text { Squares }\end{array}$ & df & $\begin{array}{c}\text { Mean } \\
\text { Square }\end{array}$ & F & Sig. \\
\hline L & Between Groups & 60.191 & 2 & 30.096 & 2.279 & .104 \\
& Within Groups & 4516.1 & 342 & 13.205 & & \\
& Total & 4576.3 & 344 & & & \\
\hline a & Between Groups & 193.0 & 2 & 96.494 & 9.151 & .000 \\
& Within Groups & 3606.1 & 342 & 10.544 & & \\
& Total & 3799.1 & 344 & & & .002 \\
& Between Groups & 45.949 & 2 & 22.974 & 6.308 & \\
& Within Groups & 1245.5 & 342 & 3.642 & & \\
& Total & 1291.5 & 344 & & & \\
& & & & & \\
\end{tabular}


Robust Tests of Equality of Means: DPMP

\begin{tabular}{|ll|r|r|r|r|}
\hline & & Statistic $^{\mathrm{a}}$ & $\mathrm{df1}$ & \multicolumn{1}{c|}{$\mathrm{df2}$} & \multicolumn{1}{c|}{ Sig. } \\
\hline $\mathrm{L}$ & Welch & 2.214 & 2 & 226.960 & .112 \\
\hline $\mathrm{a}$ & Welch & 8.258 & 2 & 226.163 & .000 \\
\hline $\mathrm{b}$ & Welch & 5.649 & 2 & 222.497 & .004 \\
\hline
\end{tabular}

a. Asymptotically $\mathrm{F}$ distributed.

Table 8.2: ANOVA Output for sample GMP

\begin{tabular}{|c|c|c|c|c|c|c|}
\hline \multicolumn{7}{|c|}{ ANOVA: GMP } \\
\hline & & $\begin{array}{l}\text { Sum of } \\
\text { Squares }\end{array}$ & df & $\begin{array}{c}\text { Mean } \\
\text { Square }\end{array}$ & $\mathrm{F}$ & Sig. \\
\hline L & $\begin{array}{l}\text { Between Groups } \\
\text { Within Groups } \\
\text { Total }\end{array}$ & $\begin{array}{r}540.494 \\
6798.2 \\
7338.7\end{array}$ & $\begin{array}{r}2 \\
342 \\
344\end{array}$ & $\begin{array}{r}270.247 \\
19.878\end{array}$ & 13.595 & .000 \\
\hline a & $\begin{array}{l}\text { Between Groups } \\
\text { Within Groups } \\
\text { Total }\end{array}$ & $\begin{array}{l}9.389 \\
274.5 \\
283.9\end{array}$ & $\begin{array}{r}2 \\
342 \\
344\end{array}$ & $\begin{array}{r}4.695 \\
.803\end{array}$ & 5.848 & .003 \\
\hline$b$ & $\begin{array}{l}\text { Between Groups } \\
\text { Within Groups } \\
\text { Total }\end{array}$ & $\begin{array}{l}12.329 \\
1683.2 \\
1695.6\end{array}$ & $\begin{array}{r}2 \\
342 \\
344\end{array}$ & $\begin{array}{l}6.165 \\
4.922\end{array}$ & 1.253 & .287 \\
\hline
\end{tabular}

Robust Tests of Equality of Means: GMP

\begin{tabular}{|ll|r|r|r|r|}
\hline & & Statistic $^{\mathrm{a}}$ & $\mathrm{df1}$ & \multicolumn{1}{c|}{ df2 } & \multicolumn{1}{c|}{ Sig. } \\
\hline $\mathrm{L}$ & Welch & 14.090 & 2 & 227.646 & .000 \\
\hline $\mathrm{a}$ & Welch & 4.074 & 2 & 187.748 & .019 \\
\hline $\mathrm{b}$ & Welch & 1.154 & 2 & 221.091 & .317 \\
\hline
\end{tabular}

a. Asymptotically F distributed.

Table 8.3: ANOVA Output for sample OSDP

ANOVA: OSDP

\begin{tabular}{|c|c|c|c|c|c|c|}
\hline & & $\begin{array}{l}\text { Sum of } \\
\text { Squares }\end{array}$ & df & Mean Square & $\mathrm{F}$ & Sig. \\
\hline \multirow[t]{3}{*}{ L } & Between Groups & 468.82 & 2 & 234.410 & 12.817 & .000 \\
\hline & Within Groups & 6254.6 & 342 & 18.288 & & \\
\hline & Total & 6723.4 & 344 & & & \\
\hline \multirow[t]{3}{*}{ a } & Between Groups & 3.263 & 2 & 1.631 & .580 & .560 \\
\hline & Within Groups & 961.4 & 342 & 2.811 & & \\
\hline & Total & 964.7 & 344 & & & \\
\hline \multirow[t]{3}{*}{$b$} & Between Groups & 12.832 & 2 & 6.416 & .244 & .784 \\
\hline & Within Groups & 9002.8 & 342 & 26.324 & & \\
\hline & Total & 9015.6 & 344 & & & \\
\hline
\end{tabular}

Robust Tests of Equality of Means: OSDP

\begin{tabular}{|ll|r|r|r|r|}
\hline & & Statistic $^{\mathrm{a}}$ & \multicolumn{1}{c|}{$\mathrm{df1}$} & \multicolumn{1}{c|}{$\mathrm{df2}$} & \multicolumn{1}{c|}{ Sig. } \\
\hline $\mathrm{L}$ & Welch & 12.732 & 2 & 227.571 & .000 \\
\hline $\mathrm{a}$ & Welch & .504 & 2 & 223.085 & .605 \\
\hline $\mathrm{b}$ & Welch & .296 & 2 & 222.729 & .744 \\
\hline
\end{tabular}

a. Asymptotically F distributed. 
Table 8.4: ANOVA Output for sample PMCL

ANOVA: PMCL

\begin{tabular}{|ll|r|r|r|r|r|}
\hline & & \multicolumn{1}{c|}{$\begin{array}{c}\text { Sum of } \\
\text { Squares }\end{array}$} & df & \multicolumn{1}{c|}{$\begin{array}{c}\text { Sean } \\
\text { Square }\end{array}$} & \multicolumn{1}{c|}{$\mathrm{F}$} & Sig. \\
\hline $\mathrm{L}$ & Between Groups & 91.620 & 2 & 45.810 & 2.034 & .132 \\
& Within Groups & 7701.74 & 342 & 22.520 & & \\
& Total & 7793.36 & 344 & & & \\
\hline $\mathrm{a}$ & Between Groups & 5.658 & 2 & 2.829 & 5.284 & .005 \\
& Within Groups & 183.10 & 342 & .535 & & \\
& Total & 188.76 & 344 & & & \\
\hline b & Between Groups & 37.680 & 2 & 18.840 & 12.760 & .000 \\
& Within Groups & 504.94 & 342 & 1.476 & & \\
& Total & 542.62 & 344 & & & \\
\hline
\end{tabular}

Robust Tests of Equality of Means: PMCL

\begin{tabular}{|ll|r|r|r|r|}
\hline & & Statistic $^{\mathrm{a}}$ & \multicolumn{1}{c|}{ df1 } & df2 & \multicolumn{1}{c|}{ Sig. } \\
\hline $\mathrm{L}$ & Welch & 1.887 & 2 & 227.169 & .154 \\
\hline $\mathrm{a}$ & Welch & 6.391 & 2 & 221.423 & .002 \\
\hline $\mathrm{b}$ & Welch & 16.628 & 2 & 221.607 & .000 \\
\hline
\end{tabular}

a. Asymptotically $\mathrm{F}$ distributed.

Table 8.5: ANOVA Output for sample PMJX

ANOVA: PMJX

\begin{tabular}{|ll|r|r|r|r|r|}
\hline & \multicolumn{1}{|c|}{$\begin{array}{c}\text { Sum of } \\
\text { Squares }\end{array}$} & df & \multicolumn{1}{c|}{$\begin{array}{c}\text { Mean } \\
\text { Square }\end{array}$} & F & Sig. \\
\hline L & Between Groups & 82.784 & 2 & 41.392 & 2.334 & .098 \\
& Within Groups & 6066.0 & 342 & 17.737 & & \\
& Total & 6148.8 & 344 & & & \\
\hline a & Between Groups & 3.625 & 2 & 1.813 & 3.931 & .021 \\
& Within Groups & 157.72 & 342 & .461 & & \\
& Total & 161.34 & 344 & & & \\
\hline b & Between Groups & 12.468 & 6.234 & 3.342 & \\
& Within Groups & 637.90 & 342 & 1.865 & & \\
& Total & 650.37 & 344 & & & \\
\hline
\end{tabular}

Robust Tests of Equality of Means: PMJX

\begin{tabular}{|ll|r|r|r|r|}
\hline & & Statistic ${ }^{2}$ & df1 & \multicolumn{1}{c|}{ df2 } & \multicolumn{1}{c|}{ Sig. } \\
\hline $\mathrm{L}$ & Welch & 2.299 & 2 & 227.925 & .103 \\
\hline $\mathrm{a}$ & Welch & 3.699 & 2 & 226.956 & .026 \\
\hline $\mathrm{b}$ & Welch & 3.407 & 2 & 227.918 & .035 \\
\hline
\end{tabular}

a. Asymptotically F distributed. 
Table 8.6: ANOVA Output for sample PMP

\begin{tabular}{|c|c|c|c|c|c|c|}
\hline \multicolumn{7}{|c|}{ ANOVA: PMP } \\
\hline & & $\begin{array}{l}\text { Sum of } \\
\text { Squares }\end{array}$ & $d f$ & Mean Square & $\mathrm{F}$ & Sig. \\
\hline $\mathrm{L}$ & $\begin{array}{l}\text { Between Groups } \\
\text { Within Groups } \\
\text { Total }\end{array}$ & $\begin{array}{r}48.174 \\
7129.48 \\
7177.65\end{array}$ & $\begin{array}{r}2 \\
342 \\
344\end{array}$ & $\begin{array}{l}24.087 \\
20.846\end{array}$ & 1.155 & .316 \\
\hline a & $\begin{array}{l}\text { Between Groups } \\
\text { Within Groups } \\
\text { Total }\end{array}$ & $\begin{array}{r}2.057 \\
762.22 \\
764.28\end{array}$ & $\begin{array}{r}2 \\
342 \\
344\end{array}$ & $\begin{array}{l}1.028 \\
2.229\end{array}$ & .461 & .631 \\
\hline$b$ & $\begin{array}{l}\text { Between Groups } \\
\text { Within Groups } \\
\text { Total }\end{array}$ & $\begin{array}{l}160.551 \\
3360.04 \\
3520.59\end{array}$ & $\begin{array}{r}2 \\
342 \\
344\end{array}$ & $\begin{array}{r}80.275 \\
9.825\end{array}$ & 8.171 & .000 \\
\hline
\end{tabular}

Robust Tests of Equality of Means: PMP

\begin{tabular}{|ll|r|r|r|r|}
\hline & & Statistic $^{\mathrm{a}}$ & $\mathrm{df1}$ & \multicolumn{1}{c|}{$\mathrm{df2}$} & \multicolumn{1}{c|}{ Sig. } \\
\hline $\mathrm{L}$ & Welch & 1.257 & 2 & 227.036 & .287 \\
\hline $\mathrm{a}$ & Welch & .449 & 2 & 226.876 & .639 \\
\hline $\mathrm{b}$ & Welch & 11.177 & 2 & 222.184 & .000 \\
\hline
\end{tabular}

a. Asymptotically $\mathrm{F}$ distributed.

Table 8.7: ANOVA Output for sample RMP

\begin{tabular}{|c|c|c|c|c|c|c|}
\hline \multicolumn{7}{|c|}{ ANOVA: RMP } \\
\hline & & Sum of Squares & df & $\begin{array}{c}\text { Mean } \\
\text { Square }\end{array}$ & $\mathrm{F}$ & Sig. \\
\hline L & $\begin{array}{l}\text { Between Groups } \\
\text { Within Groups } \\
\text { Total }\end{array}$ & $\begin{array}{r}28.794 \\
5586.3 \\
5615.1\end{array}$ & $\begin{array}{r}2 \\
342 \\
344\end{array}$ & $\begin{array}{l}14.397 \\
16.334\end{array}$ & .881 & .415 \\
\hline a & $\begin{array}{l}\text { Between Groups } \\
\text { Within Groups } \\
\text { Total }\end{array}$ & $\begin{array}{l}453.19 \\
6830.5 \\
7283.7\end{array}$ & $\begin{array}{r}2 \\
342 \\
344\end{array}$ & $\begin{array}{r}226.595 \\
19.972\end{array}$ & 11.345 & .000 \\
\hline$b$ & $\begin{array}{l}\text { Between Groups } \\
\text { Within Groups } \\
\text { Total }\end{array}$ & $\begin{array}{l}338.52 \\
4065.7 \\
4404.2\end{array}$ & $\begin{array}{r}2 \\
342 \\
344\end{array}$ & $\begin{array}{r}169.259 \\
11.888\end{array}$ & 14.238 & .000 \\
\hline
\end{tabular}

Robust Tests of Equality of Means: RMP

\begin{tabular}{|ll|r|r|r|c|}
\hline & & Statistic $^{\mathrm{a}}$ & df1 & df2 & \multicolumn{1}{c|}{ Sig. } \\
\hline $\mathrm{L}$ & Welch & .895 & 2 & 226.395 & .410 \\
\hline $\mathrm{a}$ & Welch & 11.447 & 2 & 226.151 & .000 \\
\hline $\mathrm{b}$ & Welch & 17.453 & 2 & 217.467 & .000 \\
\hline
\end{tabular}

a. Asymptotically F distributed.

9. Post Hoc Comparison test- by 'Sample Area'

Tukey or Games-Howell tests were included based on the homogeneity of variance results. Post hoc tests were not calculated for a specific variable if the ANOVA results corresponding to 
samples were deemed insignificant. For this reason, only significant values obtained for variables specific for each sample was included below.

Table 9.1: Post Hoc Comparison Results for sample DPMP

Multiple Comparisons: DPMP

\begin{tabular}{|c|c|c|c|c|c|c|c|c|}
\hline \multirow{2}{*}{\multicolumn{2}{|c|}{ Dependent Variable }} & \multirow{4}{*}{$\begin{array}{l}\text { (I) } \\
\text { Sample } \\
\text { Area } \\
1\end{array}$} & \multirow{3}{*}{$\begin{array}{l}\text { (J) } \\
\text { Sample } \\
\text { Area } \\
2\end{array}$} & \multirow{3}{*}{$\begin{array}{c}\text { Mean } \\
\text { Difference (I-J) } \\
1.63785\end{array}$} & \multirow{3}{*}{$\frac{\text { Std. Error }}{0.4282}$} & \multirow{3}{*}{$\frac{\text { Sig. }}{.000}$} & \multicolumn{2}{|c|}{$95 \%$ Confidence Interval } \\
\hline & & & & & & & \multirow{2}{*}{$\frac{\text { Lower Bound }}{0.6298}$} & \multirow{2}{*}{$\frac{\text { Upper Bound }}{2.6459}$} \\
\hline \multirow[t]{12}{*}{$\mathrm{a}$} & Tukey HSD & & & & & & & \\
\hline & & & 3 & $1.52976^{\star}$ & 0.4282 & .001 & 0.5217 & 2.5378 \\
\hline & & 2 & 1 & -1.638 & 0.4282 & .000 & -2.6459 & -0.6298 \\
\hline & & & 3 & -0.1081 & 0.4282 & .965 & -1.1161 & 0.8999 \\
\hline & & 3 & 1 & -1.5298 & 0.4282 & .001 & -2.5378 & -0.5217 \\
\hline & & & 2 & 0.1081 & 0.4282 & .965 & -0.8999 & 1.1161 \\
\hline & Games-Howell & 1 & 2 & 1.63785 & 0.4510 & .001 & 0.5738 & 2.7019 \\
\hline & & & 3 & $1.52976^{\pi}$ & 0.4265 & .001 & 0.5234 & 2.5362 \\
\hline & & 2 & 1 & -1.6378 & 0.4510 & .001 & -2.7019 & -0.5738 \\
\hline & & & 3 & -0.1081 & 0.4060 & .962 & -1.0660 & 0.8498 \\
\hline & & 3 & 1 & -1.5297 & 0.4265 & .001 & -2.5362 & -0.5234 \\
\hline & & & 2 & 0.1081 & 0.4060 & .962 & -0.8498 & 1.0660 \\
\hline \multirow[t]{12}{*}{$b$} & Tukey HSD & 1 & 2 & .86966 & 0.2517 & .002 & 0.2773 & 1.4621 \\
\hline & & & 3 & $.61396^{\star}$ & 0.2517 & .040 & 0.0215 & 1.2064 \\
\hline & & 2 & 1 & -0.8697 & 0.2517 & .002 & -1.4621 & -0.2773 \\
\hline & & & 3 & -0.2557 & 0.2517 & .567 & -0.8481 & 0.3367 \\
\hline & & 3 & 1 & -0.6140 & 0.2517 & .040 & -1.2064 & -0.0215 \\
\hline & & & 2 & 0.2557 & 0.2517 & .567 & -0.3367 & 0.8481 \\
\hline & Games-Howell & 1 & 2 & $.86966^{\star}$ & 0.2736 & .005 & 0.2243 & 1.5151 \\
\hline & & & 3 & $.61396^{*}$ & 0.2329 & .024 & 0.0642 & 1.1637 \\
\hline & & 2 & 1 & -0.8697 & 0.2736 & .005 & -1.5151 & -0.2243 \\
\hline & & & 3 & -0.2557 & 0.2468 & .555 & -0.8384 & 0.3270 \\
\hline & & 3 & 1 & -0.6140 & 0.2329 & .024 & -1.1637 & -0.0642 \\
\hline & & & 2 & 0.2557 & 0.2468 & .555 & -0.3270 & 0.8384 \\
\hline
\end{tabular}

*. The mean difference is significant at the 0.05 level. 
Homogeneous Subsets: DPMP 'a'

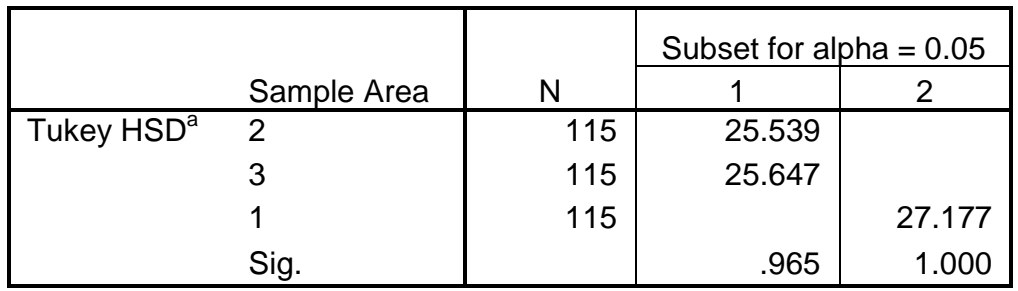

Means for groups in homogeneous subsets are displayed.

a. Uses Harmonic Mean Sample Size $=115.000$.

Table 9.2: Post Hoc Comparison Results for sample GMP

Multiple Comparisons: GMP

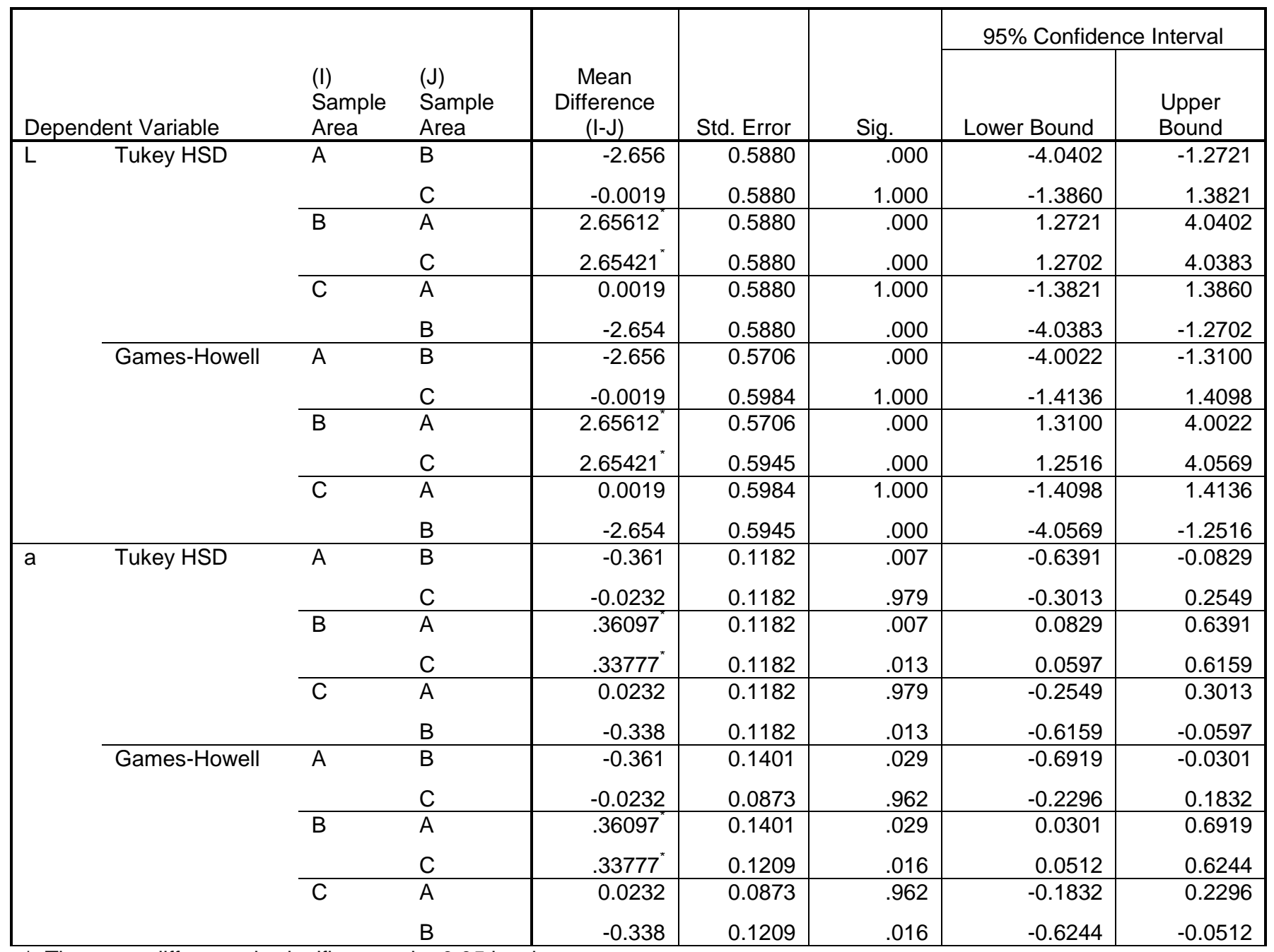

${ }^{\star}$. The mean difference is significant at the 0.05 level. 
Homogeneous Subsets: GMP 'a'

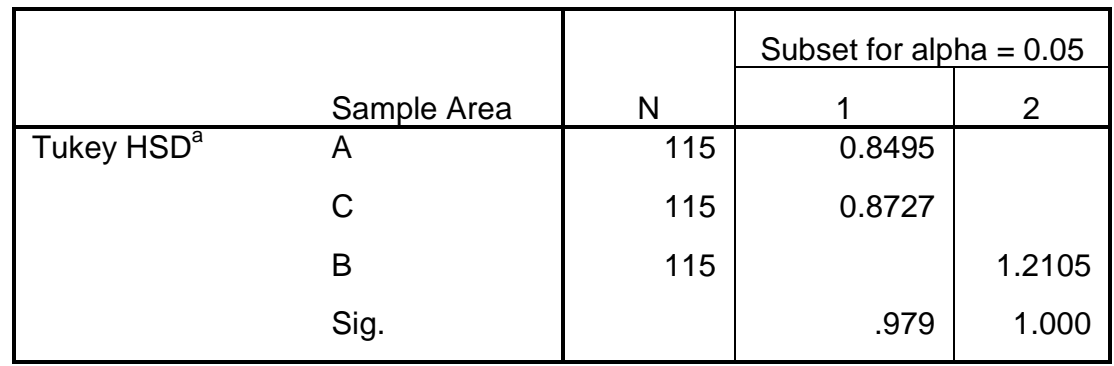

Means for groups in homogeneous subsets are displayed.

a. Uses Harmonic Mean Sample Size $=115.000$.

Table 9.3: Post Hoc Comparison Results for sample OSDP

Multiple Comparisons: OSDP

\begin{tabular}{|c|c|c|c|c|c|c|c|c|}
\hline \multirow{2}{*}{\multicolumn{2}{|c|}{$\begin{array}{l}\text { Dependent } \\
\text { Variable }\end{array}$}} & \multirow{4}{*}{$\begin{array}{l}\text { (I) Sample } \\
\text { Area } \\
\text { A }\end{array}$} & \multirow{4}{*}{$\begin{array}{l}(\mathrm{J}) \\
\text { Sample } \\
\text { Area } \\
\text { B } \\
\text { C }\end{array}$} & \multirow[b]{2}{*}{$\begin{array}{c}\text { Mean } \\
\text { Difference } \\
(\mathrm{I}-\mathrm{J})\end{array}$} & \multirow[b]{2}{*}{ Std. Error } & \multirow{3}{*}{ Sig. } & \multicolumn{2}{|c|}{ 95\% Confidence Interval } \\
\hline & & & & & & & Lower Bound & Upper Bound \\
\hline \multirow[t]{12}{*}{ L } & Tukey & & & 2.852602 & .563967 & & 1.52505 & 4.18016 \\
\hline & & & & $1.536090^{*}$ & .563967 & .019 & .20853 & 2.86365 \\
\hline & & B & $A$ & -2.852602 & .563967 & .000 & -4.18016 & -1.52505 \\
\hline & & & C & -1.316512 & .563967 & .052 & -2.64407 & .01104 \\
\hline & & C & A & -1.536090 & .563967 & .019 & -2.86365 & -.20853 \\
\hline & & & B & 1.316512 & .563967 & .052 & -.01104 & 2.64407 \\
\hline & Games- & A & B & 2.852602 & .567701 & .000 & 1.51323 & 4.19198 \\
\hline & Howell & & $\mathrm{C}$ & $1.536090^{*}$ & .546683 & .015 & .24639 & 2.82579 \\
\hline & & $B$ & A & $-2.852602^{*}$ & .567701 & .000 & -4.19198 & -1.51323 \\
\hline & & & C & -1.316512 & .577085 & .060 & -2.67797 & .04495 \\
\hline & & C & A & -1.536090 & .546683 & .015 & -2.82579 & -.24639 \\
\hline & & & B & 1.316512 & .577085 & .060 & -.04495 & 2.67797 \\
\hline
\end{tabular}


Table 9.4: Post Hoc Comparison Results for sample PMCL

Multiple Comparisons: PMCL

\begin{tabular}{|c|c|c|c|c|c|c|c|c|}
\hline \multirow{2}{*}{\multicolumn{2}{|c|}{ Dependent Variable }} & \multirow{2}{*}{$\begin{array}{l}\text { (I) } \\
\text { Sample } \\
\text { Area }\end{array}$} & \multirow{2}{*}{$\begin{array}{l}(\mathrm{J}) \\
\text { Sample } \\
\text { Area }\end{array}$} & \multirow{2}{*}{$\begin{array}{c}\text { Mean } \\
\text { Difference (I-J) }\end{array}$} & \multirow[b]{2}{*}{ Std. Error } & \multirow[b]{2}{*}{ Sig. } & \multicolumn{2}{|c|}{ 95\% Confidence Interval } \\
\hline & & & & & & & Lower Bound & Upper Bound \\
\hline \multirow[t]{12}{*}{ a } & \multirow{6}{*}{$\begin{array}{l}\text { Tukey } \\
\text { HSD }\end{array}$} & \multirow[t]{2}{*}{ A } & $\mathrm{B}$ & -0.1311 & 0.0965 & .364 & -0.3582 & 0.0961 \\
\hline & & & C & 0.1813 & 0.0965 & .147 & -0.0459 & 0.4084 \\
\hline & & \multirow[t]{2}{*}{$\mathrm{B}$} & A & 0.1311 & 0.0965 & .364 & -0.0961 & 0.3582 \\
\hline & & & $\mathrm{C}$ & $.31233^{*}$ & 0.0965 & .004 & 0.0852 & 0.5395 \\
\hline & & \multirow[t]{2}{*}{ C } & $A$ & -0.1813 & 0.0965 & .147 & -0.4084 & 0.0459 \\
\hline & & & $\mathrm{B}$ & -0.3123 & 0.0965 & .004 & -0.5395 & -0.0852 \\
\hline & \multirow{6}{*}{$\begin{array}{l}\text { Games- } \\
\text { Howell }\end{array}$} & \multirow[t]{2}{*}{ A } & B & -0.1311 & 0.1055 & .430 & -0.3800 & 0.1179 \\
\hline & & & C & 0.1813 & 0.0937 & .132 & -0.0400 & 0.4025 \\
\hline & & \multirow[t]{2}{*}{ B } & A & 0.1311 & 0.1055 & .430 & -0.1179 & 0.3800 \\
\hline & & & C & $.31233^{*}$ & 0.0895 & .002 & 0.1010 & 0.5236 \\
\hline & & \multirow[t]{2}{*}{$\mathrm{C}$} & A & -0.1813 & 0.0937 & .132 & -0.4025 & 0.0400 \\
\hline & & & $\mathrm{B}$ & -0.3123 & 0.0895 & .002 & -0.5236 & -0.1010 \\
\hline \multirow[t]{12}{*}{$b$} & \multirow{6}{*}{$\begin{array}{l}\text { Tukey } \\
\text { HSD }\end{array}$} & \multirow[t]{2}{*}{ A } & B & -0.3574 & 0.1602 & .068 & -0.7346 & 0.0198 \\
\hline & & & C & $.45031^{*}$ & 0.1602 & .014 & 0.0731 & 0.8275 \\
\hline & & \multirow[t]{2}{*}{ B } & A & 0.3574 & 0.1602 & .068 & -0.0198 & 0.7346 \\
\hline & & & C & $.80772^{\star}$ & 0.1602 & .000 & 0.4305 & 1.1849 \\
\hline & & \multirow[t]{2}{*}{$\mathrm{C}$} & A & -0.4503 & 0.1602 & .014 & -0.8275 & -0.0731 \\
\hline & & & $\mathrm{B}$ & -0.8077 & 0.1602 & .000 & -1.1849 & -0.4305 \\
\hline & \multirow{6}{*}{$\begin{array}{l}\text { Games- } \\
\text { Howell }\end{array}$} & \multirow[t]{2}{*}{ A } & B & -0.3574 & 0.1750 & .105 & -0.7703 & 0.0554 \\
\hline & & & C & $.45031^{*}$ & 0.1625 & .017 & 0.0665 & 0.8342 \\
\hline & & \multirow[t]{2}{*}{ B } & A & 0.3574 & 0.1750 & .105 & -0.0554 & 0.7703 \\
\hline & & & C & $.80772^{x}$ & 0.1414 & .000 & 0.4740 & 1.1415 \\
\hline & & \multirow[t]{2}{*}{ C } & A & -0.4503 & 0.1625 & .017 & -0.8342 & -0.0665 \\
\hline & & & $\mathrm{B}$ & -0.8077 & 0.1414 & .000 & -1.1415 & -0.4740 \\
\hline
\end{tabular}

${ }^{\star}$. The mean difference is significant at the 0.05 level.

\section{Homogeneous Subsets: PMCL 'a'}

\begin{tabular}{|ll|r|r|r|}
\hline & & \multicolumn{2}{|c|}{ Subset for alpha $=0.05$} \\
\cline { 3 - 4 } & Sample Area & $\mathrm{N}$ & 1 & \multicolumn{1}{c|}{2} \\
\hline Tukey HSD & & 115 & 2.8461 & \\
& C & 115 & 3.0273 & 3.0273 \\
B & 115 & & 3.1584 \\
Sig. & & .147 & .364 \\
\hline
\end{tabular}

Means for groups in homogeneous subsets are displayed.

a. Uses Harmonic Mean Sample Size $=115.000$. 
Homogeneous Subsets: PMCL 'b'

\begin{tabular}{|ll|r|r|r|}
\hline & & \multicolumn{2}{|c|}{ Subset for alpha $=0.05$} \\
\cline { 3 - 5 } & Sample Area & $\mathrm{N}$ & 1 & \multicolumn{1}{c|}{2} \\
\hline Tukey HSD & $\mathrm{C}$ & 115 & 4.3534 & \\
& $\mathrm{~A}$ & 115 & & 4.8037 \\
& $\mathrm{~B}$ & 115 & & 5.1611 \\
& Sig. & & 1.000 & .068 \\
\hline
\end{tabular}

Means for groups in homogeneous subsets are displayed.

a. Uses Harmonic Mean Sample Size $=115.000$.

Table 9.5: Post Hoc Comparison Results for sample PMJX

Multiple Comparisons: PMJX

\begin{tabular}{|c|c|c|c|c|c|c|c|c|}
\hline \multirow{2}{*}{\multicolumn{2}{|c|}{ Dependent Variable }} & \multirow{3}{*}{$\begin{array}{l}\text { (I) } \\
\text { Sample } \\
\text { Area } \\
\text { A }\end{array}$} & \multirow{3}{*}{$\begin{array}{l}(\mathrm{J}) \\
\text { Sample } \\
\text { Area } \\
\text { B }\end{array}$} & \multirow{3}{*}{$\begin{array}{c}\text { Mean } \\
\text { Difference } \\
(\mathrm{I}-\mathrm{J})\end{array}$} & \multirow{3}{*}{$\begin{array}{l}\begin{array}{c}\text { Std. } \\
\text { Error }\end{array} \\
0.0896\end{array}$} & \multirow{3}{*}{$\frac{\text { Sig. }}{.033}$} & \multicolumn{2}{|c|}{$95 \%$ Confidence Interval } \\
\hline & & & & & & & \multirow{2}{*}{$\begin{array}{l}\text { Lower } \\
\text { Bound } \\
0.0140\end{array}$} & \multirow{2}{*}{$\begin{array}{c}\begin{array}{c}\text { Upper } \\
\text { Bound }\end{array} \\
0.4356\end{array}$} \\
\hline$a$ & Tukey HSD & & & & & & & \\
\hline & & & C & 0.0156 & 0.0896 & .983 & -0.1952 & 0.2264 \\
\hline & & \multirow[t]{2}{*}{$\mathrm{B}$} & $\bar{A}$ & -0.2248 & 0.0896 & .033 & -0.4356 & -0.0140 \\
\hline & & & C & -0.2092 & 0.0896 & .052 & -0.4200 & 0.0016 \\
\hline & & \multirow[t]{2}{*}{ C } & A & -0.0156 & 0.0896 & .983 & -0.2264 & 0.1952 \\
\hline & & & B & 0.2092 & 0.0896 & .052 & -0.0016 & 0.4200 \\
\hline & \multirow[t]{6}{*}{ Games-Howell } & \multirow[t]{2}{*}{ A } & B & .224837 & 0.0890 & .033 & 0.0149 & 0.4347 \\
\hline & & & $\mathrm{C}$ & 0.0156 & 0.0863 & .982 & -0.1880 & 0.2192 \\
\hline & & \multirow[t]{2}{*}{ B } & A & -0.2248 & 0.0890 & .033 & -0.4347 & -0.0149 \\
\hline & & & C & -0.2092 & 0.0933 & .066 & -0.4293 & 0.0108 \\
\hline & & \multirow[t]{2}{*}{ C } & A & -0.0156 & 0.0863 & .982 & -0.2192 & 0.1880 \\
\hline & & & B & 0.2092 & 0.0933 & .066 & -0.0108 & 0.4293 \\
\hline \multirow[t]{12}{*}{$b$} & \multirow[t]{6}{*}{ Tukey HSD } & \multirow[t]{2}{*}{ A } & B & 0.1081 & 0.1801 & .820 & -0.3159 & 0.5320 \\
\hline & & & C & -0.3382 & 0.1801 & .147 & -0.7622 & 0.0857 \\
\hline & & \multirow[t]{2}{*}{ B } & A & -0.1081 & 0.1801 & .820 & -0.5320 & 0.3159 \\
\hline & & & C & -0.4463 & 0.1801 & .036 & -0.8703 & -0.0223 \\
\hline & & \multirow[t]{2}{*}{ C } & A & 0.3382 & 0.1801 & .147 & -0.0857 & 0.7622 \\
\hline & & & B & $.446300^{*}$ & 0.1801 & .036 & 0.0223 & 0.8703 \\
\hline & \multirow[t]{6}{*}{ Games-Howell } & \multirow[t]{2}{*}{ A } & B & 0.1081 & 0.1811 & .822 & -0.3192 & 0.5354 \\
\hline & & & C & -0.3382 & 0.1815 & .152 & -0.7665 & 0.0901 \\
\hline & & \multirow[t]{2}{*}{ B } & A & -0.1081 & 0.1811 & .822 & -0.5354 & 0.3192 \\
\hline & & & C & -0.4463 & 0.1776 & .034 & -0.8653 & -0.0273 \\
\hline & & \multirow[t]{2}{*}{ C } & A & 0.3382 & 0.1815 & .152 & -0.0901 & 0.7665 \\
\hline & & & B & $.446300^{*}$ & 0.1776 & .034 & 0.0273 & 0.8653 \\
\hline
\end{tabular}

${ }^{\star}$. The mean difference is significant at the 0.05 level. 
Homogeneous Subsets: PMJX ' $a$ '

\begin{tabular}{|ll|r|r|r|}
\hline & & & \multicolumn{2}{|c|}{ Subset for alpha $=0.05$} \\
\cline { 4 - 5 } & Sample Area & \multicolumn{1}{|c|}{ N } & 1 & \multicolumn{1}{c|}{2} \\
\hline Tukey & B & 115 & 2.7231360 & \\
HSD $^{2}$ & C & 115 & 2.9323758 & 2.9323758 \\
& A & 115 & & 2.9479730 \\
& Sig. & & .052 & .983 \\
\hline
\end{tabular}

Means for groups in homogeneous subsets are displayed.

a. Uses Harmonic Mean Sample Size $=115.000$.

Table 9.6: Post Hoc Comparison Results for sample PMP

Multiple Comparisons: PMP

\begin{tabular}{|c|c|c|c|c|c|c|c|c|}
\hline \multirow{2}{*}{\multicolumn{2}{|c|}{$\begin{array}{l}\text { Dependent } \\
\text { Variable }\end{array}$}} & \multirow{2}{*}{$\begin{array}{l}\text { (I) Sample } \\
\text { Area }\end{array}$} & \multirow{2}{*}{$\begin{array}{l}\text { (J) Sample } \\
\text { Area }\end{array}$} & \multirow{2}{*}{$\begin{array}{c}\text { Mean } \\
\text { Difference (I-J) }\end{array}$} & \multirow[b]{2}{*}{ Std. Error } & \multirow[b]{2}{*}{ Sig. } & \multicolumn{2}{|c|}{ 95\% Confidence Interval } \\
\hline & & & & & & & Lower Bound & Upper Bound \\
\hline \multirow[t]{12}{*}{$\mathrm{L}$} & \multirow{6}{*}{ HSD } & \multirow[t]{2}{*}{ A } & $\mathrm{B}$ & .74286 & .60212 & .434 & -0.6745 & 2.1602 \\
\hline & & & $\mathrm{C}$ & .83455 & .60212 & .349 & -0.5828 & 2.2519 \\
\hline & & \multirow[t]{2}{*}{ B } & A & -.74286 & .60212 & .434 & -2.1602 & 0.6745 \\
\hline & & & C & .09170 & .60212 & .987 & -1.3257 & 1.5091 \\
\hline & & \multirow[t]{2}{*}{ C } & A & -.83455 & .60212 & .349 & -2.2519 & 0.5828 \\
\hline & & & B & -.09170 & .60212 & .987 & -1.5091 & 1.3257 \\
\hline & \multirow{6}{*}{$\begin{array}{l}\text { Games- } \\
\text { Howell }\end{array}$} & \multirow[t]{2}{*}{ A } & B & .74286 & .59006 & .420 & -0.6493 & 2.1350 \\
\hline & & & $\mathrm{C}$ & .83455 & .58852 & .333 & -0.5540 & 2.2231 \\
\hline & & \multirow[t]{2}{*}{$B$} & A & -.74286 & .59006 & .420 & -2.1350 & 0.6493 \\
\hline & & & C & .09170 & .62700 & .988 & -1.3875 & 1.5709 \\
\hline & & \multirow[t]{2}{*}{ C } & A & -.83455 & .58852 & .333 & -2.2231 & 0.5540 \\
\hline & & & B & -.091670 & .62700 & .988 & -1.5709 & 1.3875 \\
\hline \multirow[t]{12}{*}{ a } & \multirow{6}{*}{$\begin{array}{l}\text { Tukey } \\
\text { HSD }\end{array}$} & \multirow[t]{2}{*}{ A } & $\mathrm{B}$ & 0.1420 & 0.1969 & .751 & -0.3214 & 0.6055 \\
\hline & & & $\mathrm{C}$ & -0.0371 & 0.1969 & .981 & -0.5006 & 0.4263 \\
\hline & & \multirow[t]{2}{*}{ B } & A & -0.1420 & 0.1969 & .751 & -0.6055 & 0.3214 \\
\hline & & & C & -0.1792 & 0.1969 & .634 & -0.6426 & 0.2843 \\
\hline & & \multirow[t]{2}{*}{ C } & A & 0.0371 & 0.1969 & .981 & -0.4263 & 0.5006 \\
\hline & & & B & 0.1792 & 0.1969 & .634 & -0.2843 & 0.6426 \\
\hline & \multirow{6}{*}{$\begin{array}{l}\text { Games- } \\
\text { Howell }\end{array}$} & \multirow[t]{2}{*}{$A$} & B & 0.1420 & 0.1879 & .730 & -0.3013 & 0.5854 \\
\hline & & & C & -0.0371 & 0.1974 & .981 & -0.5030 & 0.4287 \\
\hline & & \multirow[t]{2}{*}{$B$} & $\bar{A}$ & -0.1420 & 0.1879 & .730 & -0.5854 & 0.3013 \\
\hline & & & C & -0.1792 & 0.2049 & .657 & -0.6626 & 0.3043 \\
\hline & & \multirow[t]{2}{*}{ C } & A & 0.0371 & 0.1974 & .981 & -0.4287 & 0.5030 \\
\hline & & & B & 0.1792 & 0.2049 & .657 & -0.3043 & 0.6626 \\
\hline \multirow[t]{12}{*}{ b } & Tukey & A & B & 0.7260 & 0.4134 & .186 & -0.2470 & 1.6991 \\
\hline & HSD & & C & $1.6663^{*}$ & 0.4134 & .000 & 0.6934 & 2.6394 \\
\hline & & B & $\bar{A}$ & -0.7260 & 0.4134 & .186 & -1.6991 & 0.2470 \\
\hline & & & C & 0.9404 & 0.4134 & .061 & -0.0327 & 1.9134 \\
\hline & & $C$ & $A$ & -1.6663 & 0.4134 & .000 & -2.6394 & -0.6934 \\
\hline & & & B & -0.9404 & 0.4134 & .061 & -1.9134 & 0.0327 \\
\hline & Games- & A & B & 0.7260 & 0.4483 & .240 & -0.3322 & 1.7842 \\
\hline & Howell & & C & $1.6663^{*}$ & 0.3540 & .000 & 0.8313 & 2.5015 \\
\hline & & B & $A$ & -0.7260 & 0.4483 & .240 & -1.7842 & 0.3322 \\
\hline & & & C & 0.9404 & 0.4317 & .077 & -0.0791 & 1.9599 \\
\hline & & $\mathrm{C}$ & $A$ & -1.6663 & 0.3540 & .000 & -2.5015 & -0.8313 \\
\hline & & & B & -0.9404 & 0.4317 & .077 & -1.9599 & 0.0791 \\
\hline
\end{tabular}


*. The mean difference is significant at the 0.05 level.

Homogeneous Subsets: DPMP ' $a$ '

\begin{tabular}{|c|c|c|c|}
\hline & \multirow[b]{2}{*}{ Sample Area } & \multirow[b]{2}{*}{$\mathrm{N}$} & Subset for alpha $=0.05$ \\
\hline & & & 1 \\
\hline Tukey HSD & $\begin{array}{l}\text { B } \\
\text { A } \\
\text { C } \\
\text { Sig. }\end{array}$ & $\begin{array}{l}115 \\
115 \\
115\end{array}$ & $\begin{array}{r}6.4532 \\
6.5953 \\
6.6324 \\
.634\end{array}$ \\
\hline
\end{tabular}

Means for groups in homogeneous subsets are displayed.

a. Uses Harmonic Mean Sample Size $=115.000$.

Table 9.7: Post Hoc Comparison Results for sample RMP

Multiple Comparisons: RMP

\begin{tabular}{|c|c|c|c|c|c|c|c|c|}
\hline & & \multirow{2}{*}{$\begin{array}{l}\text { (I) Sample } \\
\text { Area }\end{array}$} & \multirow{2}{*}{$\begin{array}{l}\text { (J) Sample } \\
\text { Area }\end{array}$} & \multirow{2}{*}{$\begin{array}{c}\text { Mean } \\
\text { Difference (I-J) }\end{array}$} & \multirow[b]{2}{*}{ Std. Error } & \multirow[b]{2}{*}{ Sig. } & \multicolumn{2}{|c|}{ 95\% Confidence Interval } \\
\hline \multicolumn{2}{|c|}{ Dependent Variable } & & & & & & Lower Bound & Upper Bound \\
\hline \multirow[t]{12}{*}{$a$} & Tukey HSD & A & $\mathrm{B}$ & -1.5003 & 0.5894 & .030 & -2.8876 & -.1130 \\
\hline & & & $\mathrm{C}$ & 1.3048 & 0.5894 & .070 & -.0825 & 2.6922 \\
\hline & & $\mathrm{B}$ & A & 1.5003 & 0.5894 & .030 & .1130 & 2.8876 \\
\hline & & & C & $2.8051^{\star}$ & 0.5894 & .000 & 1.4178 & 4.1925 \\
\hline & & $\mathrm{C}$ & $A$ & -1.3048 & 0.5894 & .070 & -2.6922 & .0825 \\
\hline & & & $\mathrm{B}$ & -2.8051 & 0.5894 & .000 & -4.1925 & -1.4178 \\
\hline & Games- & $A$ & $\mathrm{~B}$ & -1.5003 & 0.6207 & .043 & -2.9648 & -.0358 \\
\hline & & & $\mathrm{C}$ & 1.3048 & 0.5582 & .053 & -.0123 & 2.6219 \\
\hline & & $\mathrm{B}$ & $A$ & $1.5003^{*}$ & 0.6207 & .043 & .0358 & 2.9648 \\
\hline & & & $\mathrm{C}$ & $2.8051^{*}$ & 0.5874 & .000 & 1.4189 & 4.1914 \\
\hline & & $\mathrm{C}$ & $A$ & -1.3048 & 0.5582 & .053 & -2.6219 & .0123 \\
\hline & & & $\mathrm{B}$ & -2.8051 & 0.5874 & .000 & -4.1914 & -1.4189 \\
\hline \multirow[t]{12}{*}{$b$} & Tukey HSD & $A$ & $B$ & -0.7767 & 0.4547 & .204 & -1.8470 & .2937 \\
\hline & & & C & $1.6024^{\pi}$ & 0.4547 & .001 & .5321 & 2.6728 \\
\hline & & $B$ & $A$ & 0.7767 & 0.4547 & .204 & -.2937 & 1.8470 \\
\hline & & & $\mathrm{C}$ & $2.3790^{*}$ & 0.4547 & .000 & 1.3087 & 3.4494 \\
\hline & & $\mathrm{C}$ & $A$ & -1.6024 & 0.4547 & .001 & -2.6728 & -.5321 \\
\hline & & & $\mathrm{B}$ & -2.3790 & 0.4547 & .000 & -3.4494 & -1.3087 \\
\hline & Games- & $A$ & $B$ & -0.7767 & 0.5059 & .276 & -1.9701 & .4168 \\
\hline & & & $\mathrm{C}$ & $1.6024^{*}$ & 0.4154 & .000 & .6214 & 2.5834 \\
\hline & & B & $A$ & 0.7767 & 0.5059 & .276 & -.4168 & 1.9701 \\
\hline & & & C & $2.3790^{*}$ & 0.4379 & .000 & 1.3448 & 3.4134 \\
\hline & & $\mathrm{C}$ & $A$ & -1.6024 & 0.4154 & .000 & -2.5834 & -.6214 \\
\hline & & & B & -2.3790 & 0.4379 & .000 & -3.4134 & -1.3448 \\
\hline
\end{tabular}

\footnotetext{
${ }^{*}$. The mean difference is significant at the 0.05 level.
} 


\section{Homogeneous Subsets 'a'}

\begin{tabular}{|ll|r|r|r|}
\hline & & & \multicolumn{2}{c|}{ Subset for alpha $=0.05$} \\
\cline { 4 - 5 } & Area & $\mathrm{N}$ & 1 & \multicolumn{1}{c|}{2} \\
\hline Tukey & C & 115 & 26.531 & \\
HSD $^{\mathrm{a}}$ & $\mathrm{A}$ & 115 & 27.836 & \\
& $\mathrm{~B}$ & 115 & & 29.336 \\
& Sig. & & .070 & 1.000 \\
\hline
\end{tabular}

Means for groups in homogeneous subsets are displayed.

a. Uses Harmonic Mean Sample Size $=115.000$.

10. Mean plots for Aged Results

Figure 10.1 Mean plots for sample DPMP grouped by 'Sample Area'

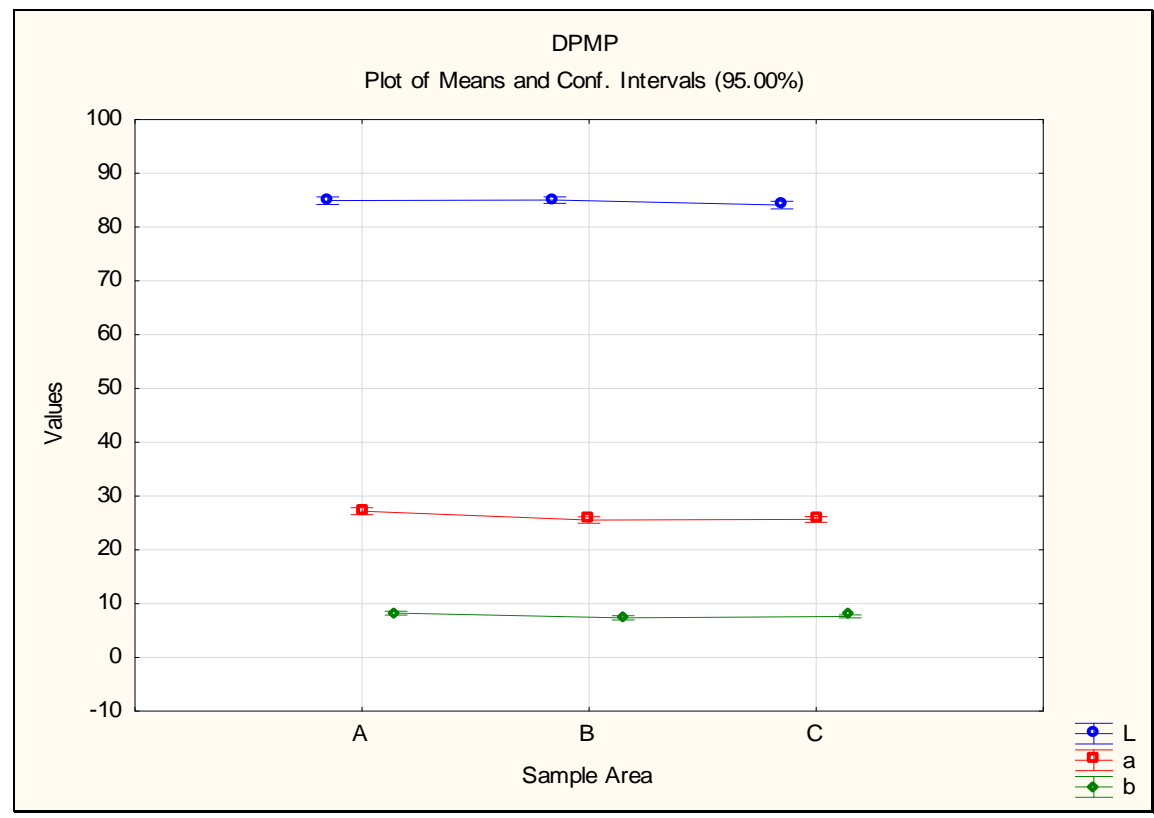



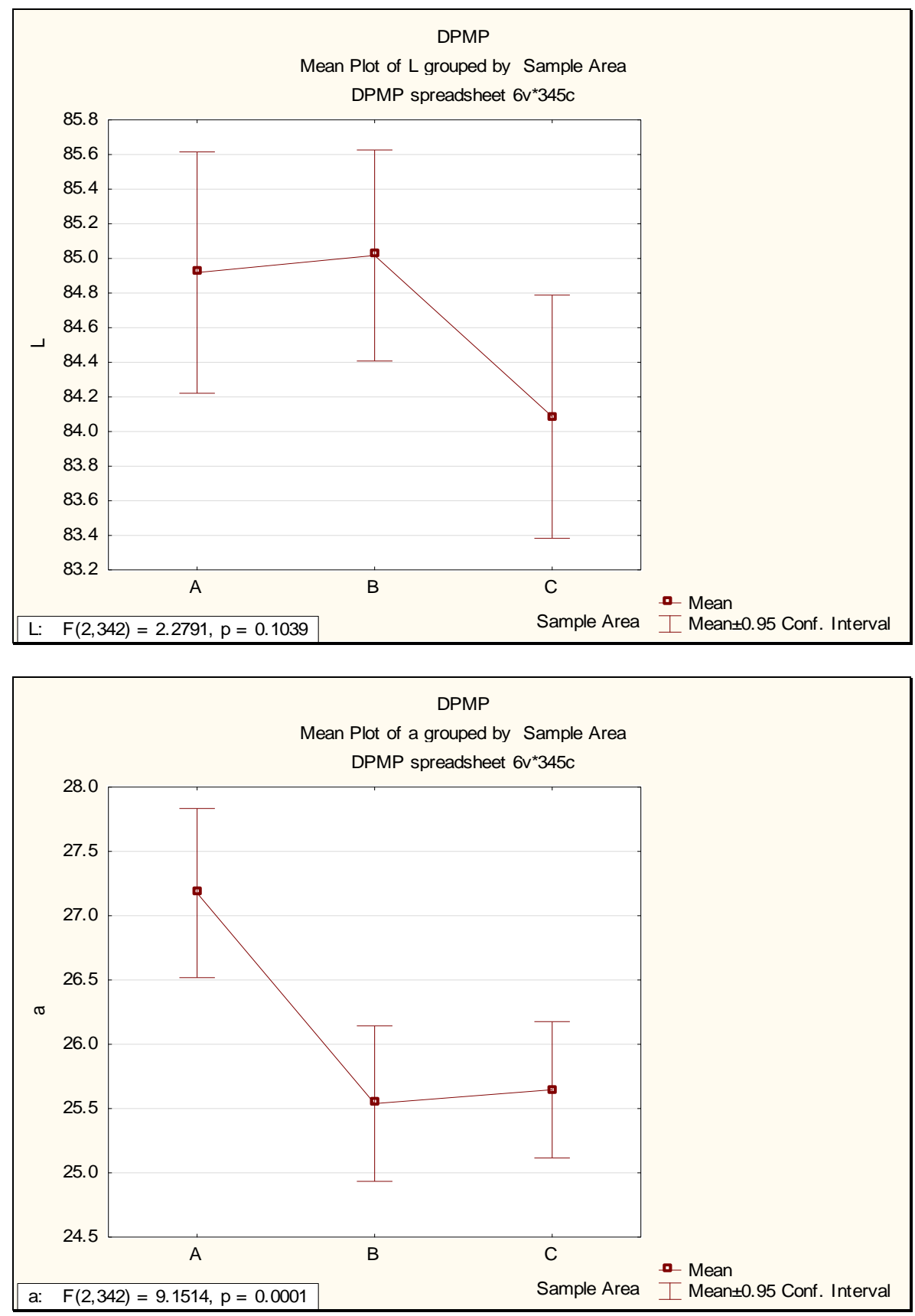
Figure 10.2 Mean plots for sample GMP grouped by 'Sample Area'
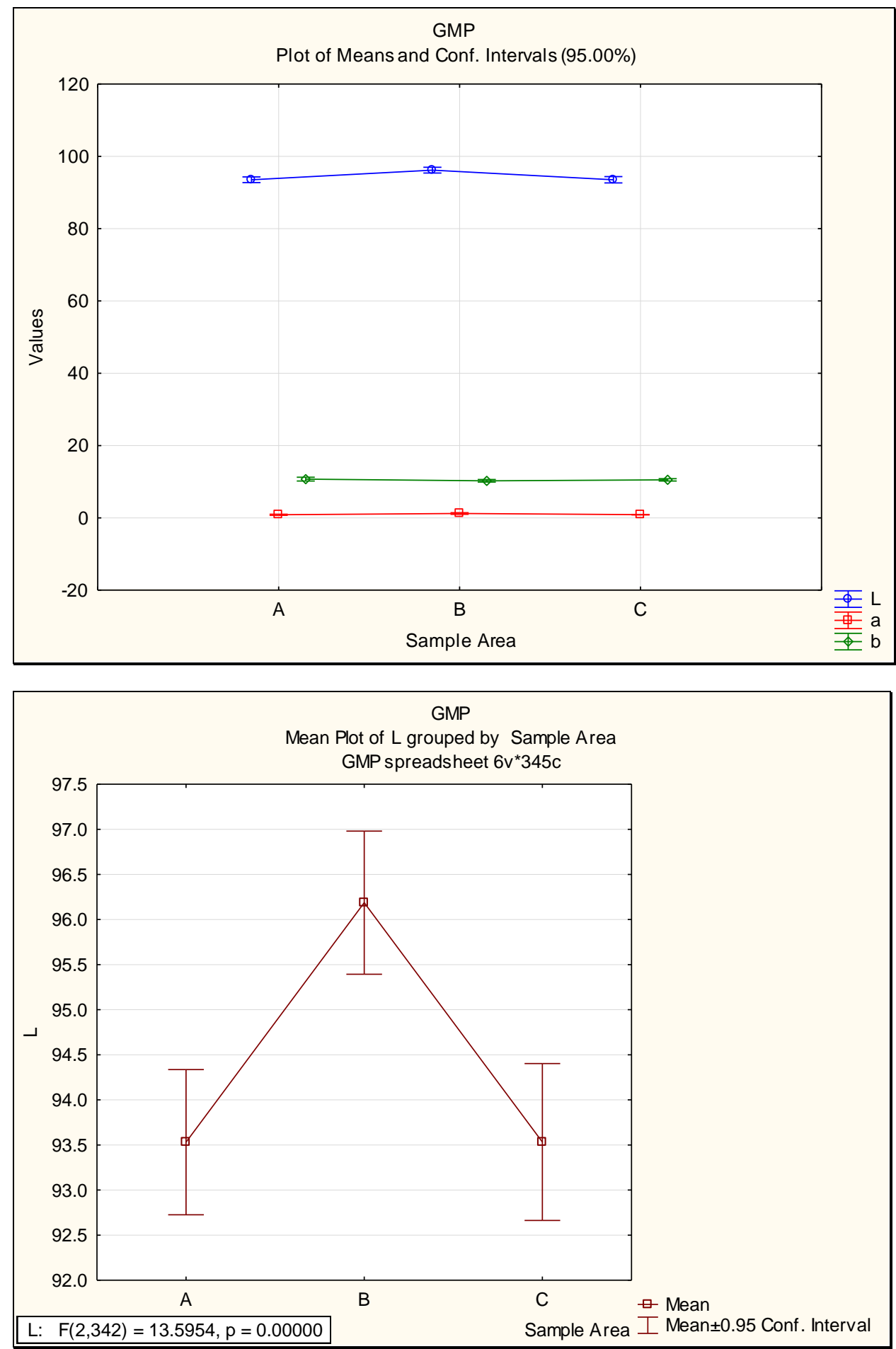

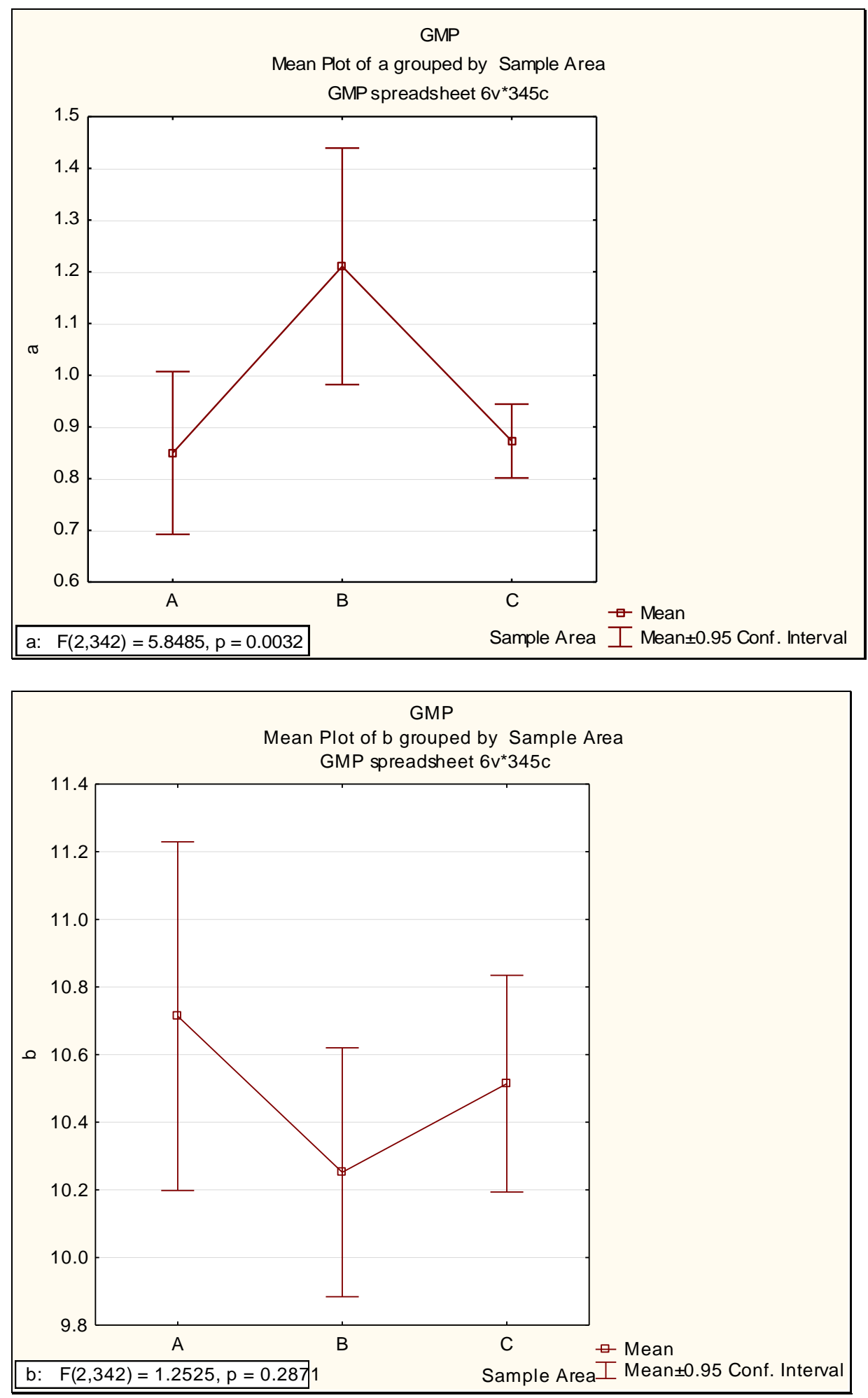
Figure 10.3 Mean plots for sample PMCL grouped by 'Sample Area'
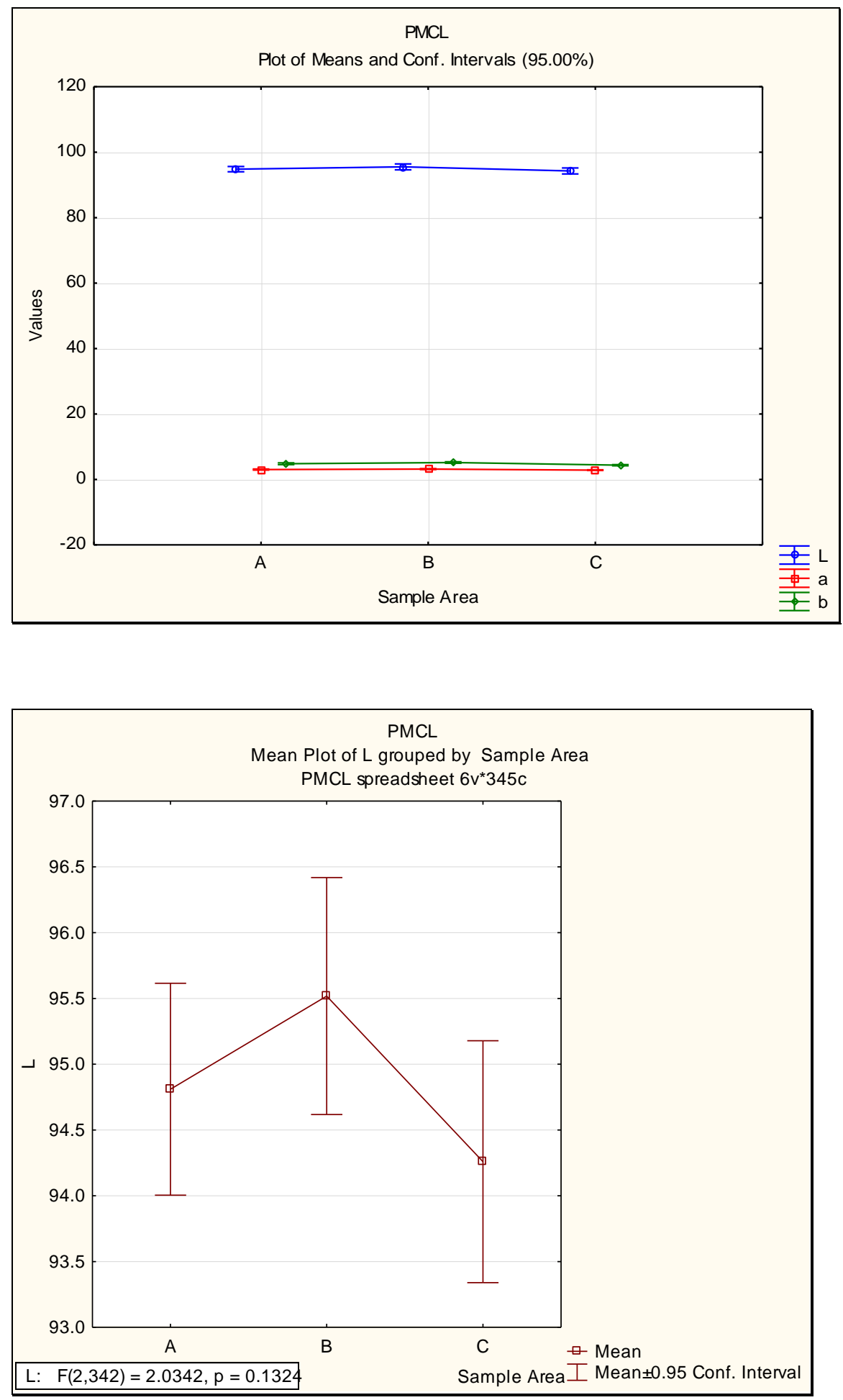

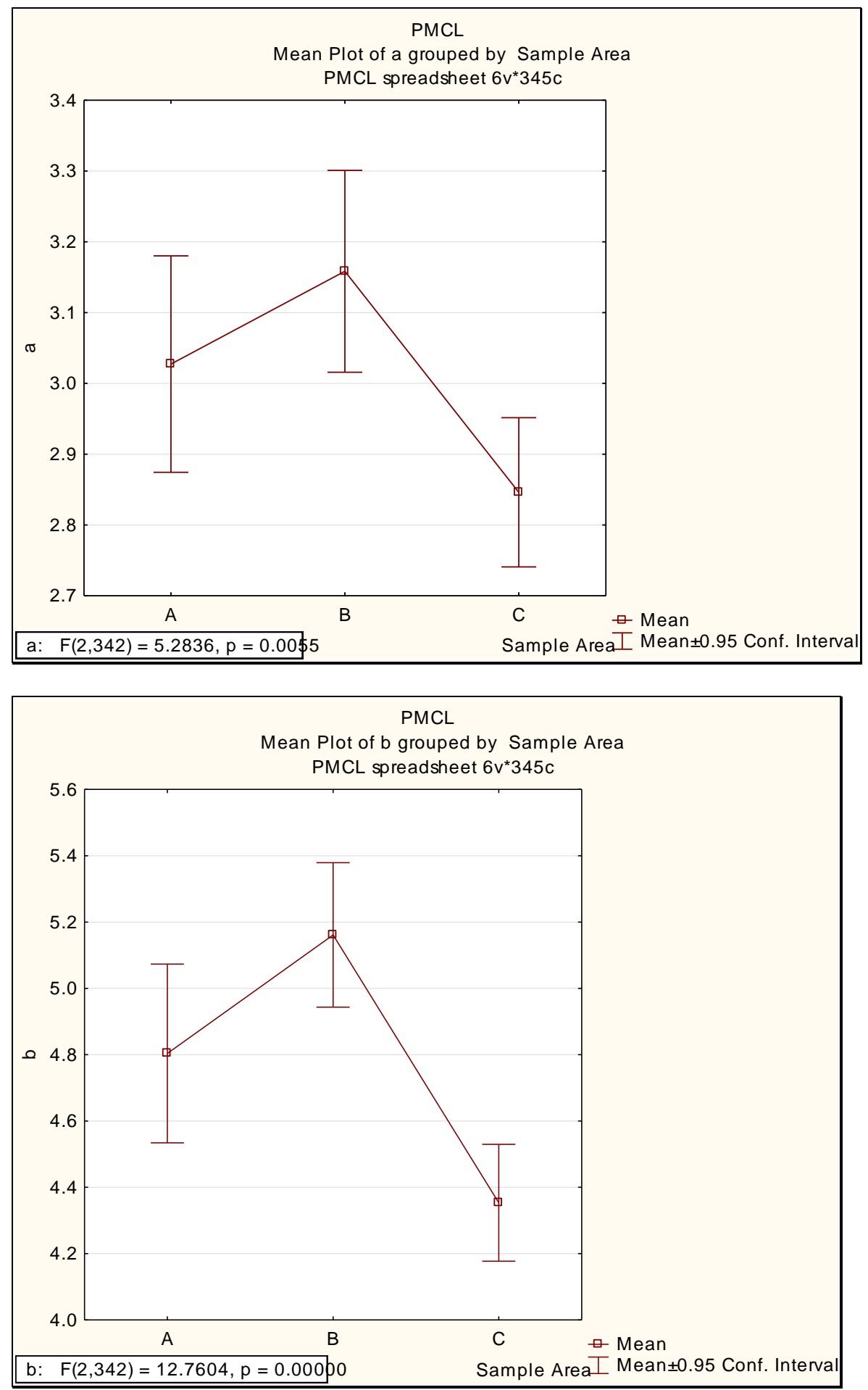
Figure 10.4 Mean plots for sample PMJX grouped by 'Sample Area'
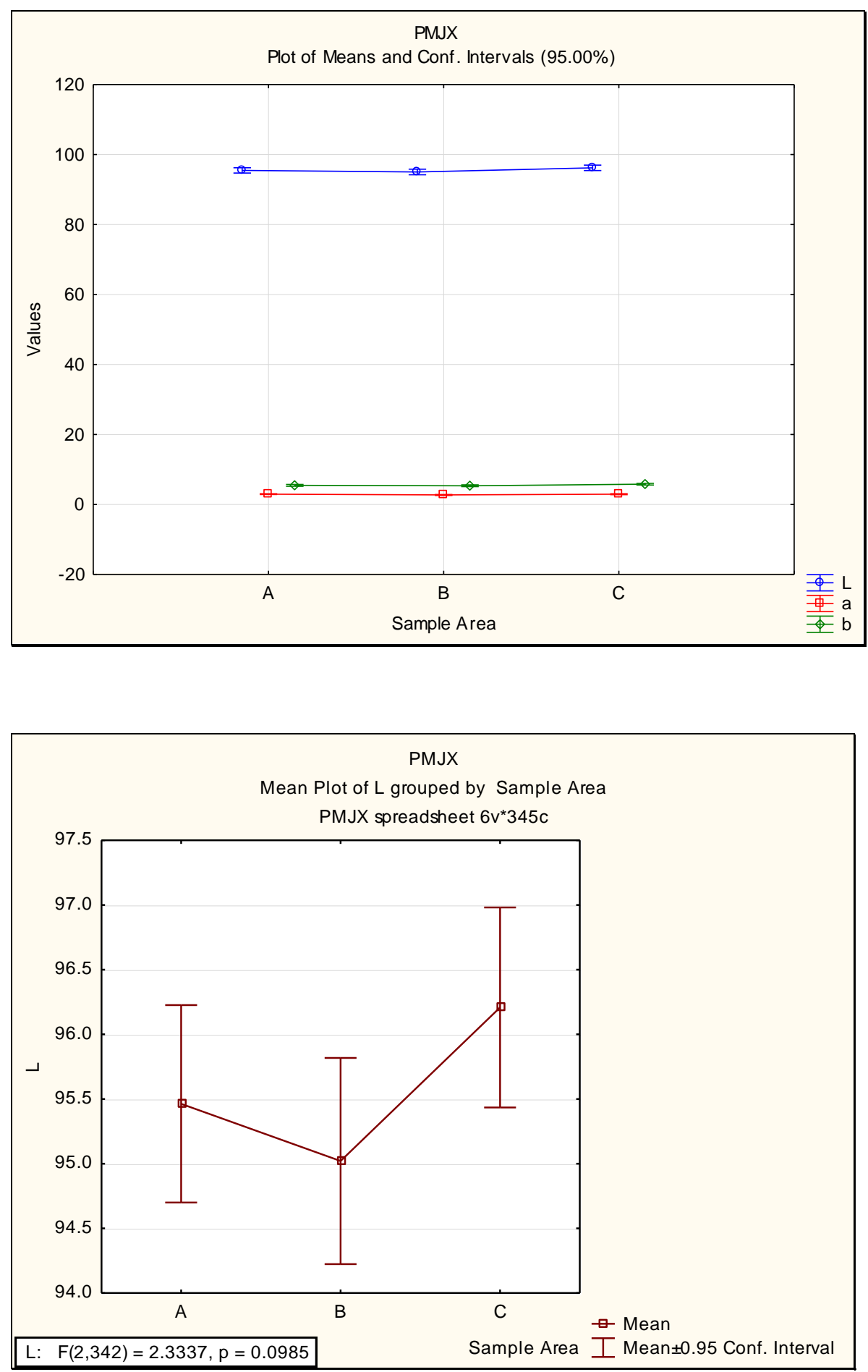


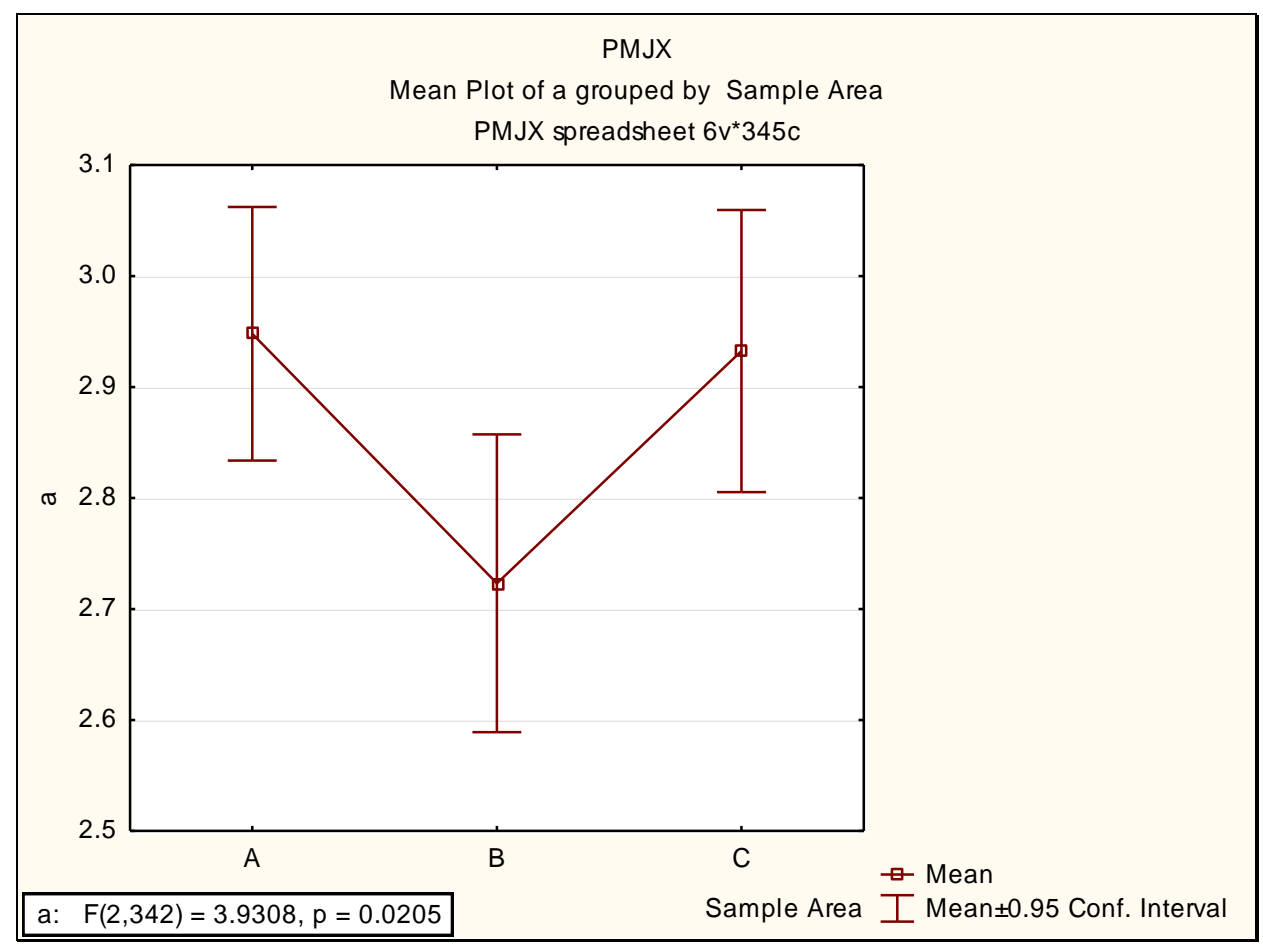

Figure 10.5 Mean plots for sample RMP grouped by 'Sample Area'

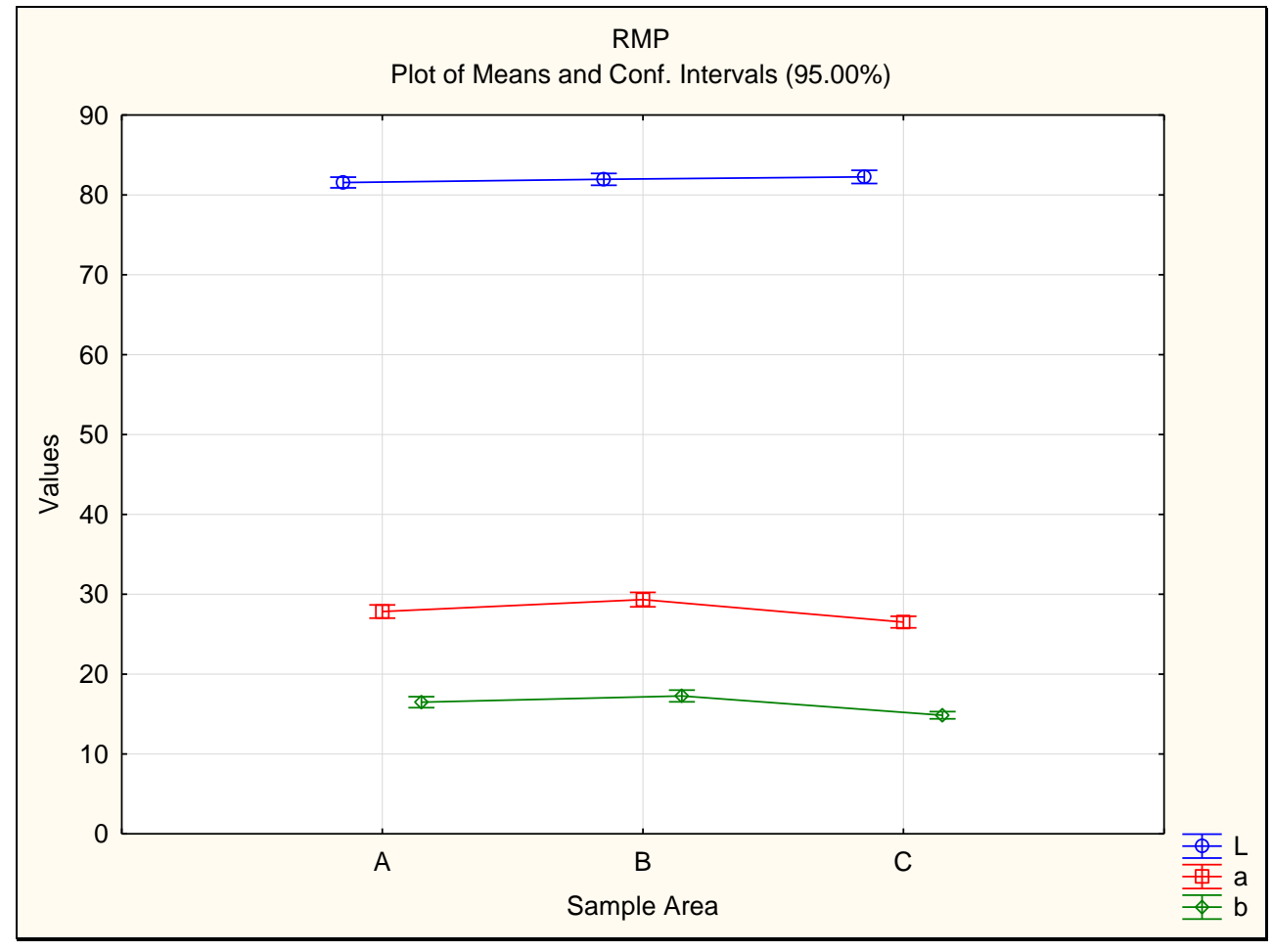



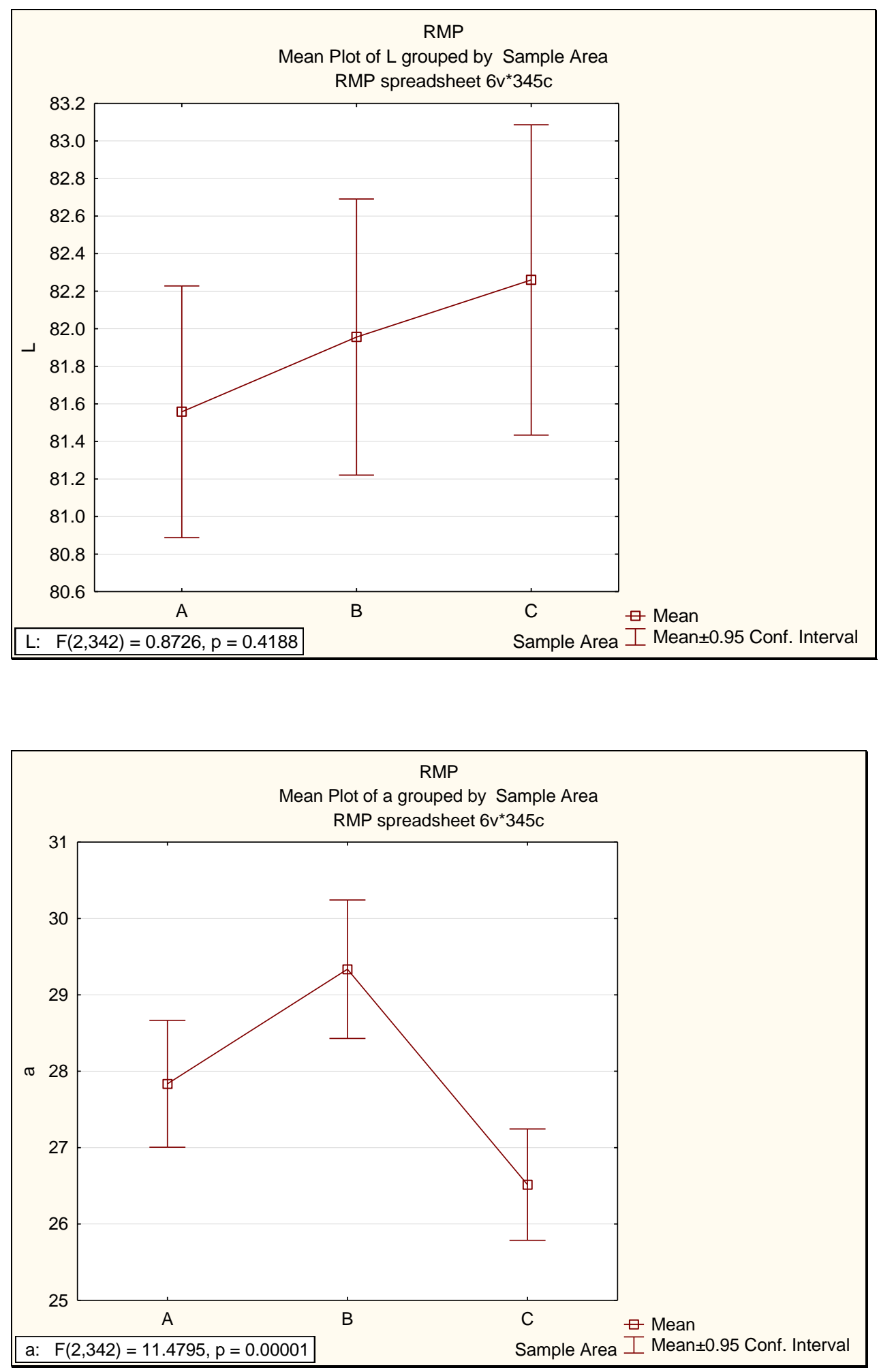
11. ANOVA Output for Aged Results- by 'Fiber'

Table 11.1 ANOVA Output for reference samples grouped by 'Fiber'

\begin{tabular}{|c|c|c|c|c|c|c|}
\hline & DPMP & Sum of Squares & df & Mean Square & $\mathbf{F}$ & Sig. \\
\hline $\bar{L}$ & Between Groups & 3729.974 & 53 & 70.377 & 24.197 & .000 \\
\hline & Within Groups & 846.357 & 291 & 2.908 & & \\
\hline & Total & 4576.331 & 344 & & & \\
\hline$a$ & Between Groups & 2590.726 & 53 & 48.882 & 11.772 & .000 \\
\hline & Within Groups & 1208.360 & 291 & 4.152 & & \\
\hline & Total & 3799.086 & 344 & & & \\
\hline$b$ & Between Groups & 964.170 & 53 & 18.192 & 16.174 & .000 \\
\hline & Within Groups & 327.302 & 291 & 1.125 & & \\
\hline & Total & 1291.472 & 344 & & & \\
\hline & GMP & Sum of Squares & df & Mean Square & $\mathbf{F}$ & Sig. \\
\hline $\mathrm{L}$ & Between Groups & 5582.6 & 53 & 105.331 & 17.454 & .000 \\
\hline & Within Groups & 1756.2 & 291 & 6.035 & & \\
\hline & Total & 7338.7 & 344 & & & \\
\hline $\mathrm{a}$ & Between Groups & 246.2 & 53 & 4.646 & 35.870 & .000 \\
\hline & Within Groups & 37.690 & 291 & .130 & & \\
\hline & Total & 283.9 & 344 & & & \\
\hline $\mathrm{b}$ & Between Groups & 1270.6 & 53 & 23.973 & 16.416 & .000 \\
\hline & Within Groups & 425.0 & 291 & 1.460 & & \\
\hline & Total & 1695.6 & 344 & & & \\
\hline & OSDP & Sum of Squares & df & Mean Square & $\mathbf{F}$ & Sig. \\
\hline$L$ & Between Groups & 5694.21 & 53 & 107.438 & 30.377 & .000 \\
\hline & Within Groups & 1029.22 & 291 & 3.537 & & \\
\hline & Total & 6723.44 & 344 & & & \\
\hline a & Between Groups & 753.18 & 53 & 14.211 & 19.552 & .000 \\
\hline & Within Groups & 211.51 & 291 & .727 & & \\
\hline & Total & 964.69 & 344 & & & \\
\hline$b$ & Between Groups & 7484.85 & 53 & 141.224 & 26.847 & .000 \\
\hline & Within Groups & 1530.76 & 291 & 5.260 & & \\
\hline & Total & 9015.61 & 344 & & & \\
\hline & PMCL & Sum of Squares & df & Mean Square & $\mathbf{F}$ & Sig. \\
\hline $\bar{L}$ & Between Groups & 5972.71 & 53 & 112.693 & 18.012 & .000 \\
\hline & Within Groups & 1820.65 & 291 & 6.257 & & \\
\hline & Total & 7793.36 & 344 & & & \\
\hline a & Between Groups & 158.45 & 53 & 2.990 & 28.705 & .000 \\
\hline & Within Groups & 30.308 & 291 & .104 & & \\
\hline & Total & 188.76 & 344 & & & \\
\hline$b$ & Between Groups & 464.20 & 53 & 8.758 & 32.502 & .000 \\
\hline & Within Groups & 78.417 & 291 & .269 & & \\
\hline & Total & 542.62 & 344 & & & \\
\hline
\end{tabular}




\begin{tabular}{|c|c|c|c|c|c|c|}
\hline & PMJX & Sum of Squares & df & Mean Square & $\mathbf{F}$ & Sig. \\
\hline $\mathrm{L}$ & Between Groups & 5621.41 & 53 & 106.064 & 58.524 & .000 \\
\hline & Within Groups & 527.385 & 291 & 1.812 & & \\
\hline & Total & 6148.79 & 344 & & & \\
\hline$a$ & Between Groups & 130.422 & 53 & 2.461 & 23.160 & .000 \\
\hline & Within Groups & 30.920 & 291 & .106 & & \\
\hline & Total & 161.342 & 344 & & & \\
\hline$b$ & Between Groups & 546.357 & 53 & 10.309 & 28.840 & .000 \\
\hline & Within Groups & 104.016 & 291 & .357 & & \\
\hline & Total & 650.373 & 344 & & & \\
\hline & PMP & Sum of Squares & df & Mean Square & $\mathbf{F}$ & Sig. \\
\hline $\mathrm{L}$ & Between Groups & 6011.57 & 53 & 113.426 & 28.306 & .000 \\
\hline & Within Groups & 1166.09 & 291 & 4.007 & & \\
\hline & Total & 7177.65 & 344 & & & \\
\hline $\bar{a}$ & Between Groups & 659.299 & 53 & 12.440 & 34.481 & .000 \\
\hline & Within Groups & 104.982 & 291 & .361 & & \\
\hline & Total & 764.281 & 344 & & & \\
\hline $\mathrm{b}$ & Between Groups & 3149.89 & 53 & 59.432 & 46.654 & .000 \\
\hline & Within Groups & 370.704 & 291 & 1.274 & & \\
\hline & Total & 3520.59 & 344 & & & \\
\hline & RMP & Sum of Squares & df & Mean Square & $\mathbf{F}$ & Sig. \\
\hline $\bar{L}$ & Between Groups & 4772.382 & 53 & 90.045 & 31.094 & .000 \\
\hline & Within Groups & 842.716 & 291 & 2.896 & & \\
\hline & Total & 5615.098 & 344 & & & \\
\hline $\bar{a}$ & Between Groups & 6101.980 & 53 & 115.132 & 28.351 & .000 \\
\hline & Within Groups & 1181.724 & 291 & 4.061 & & \\
\hline & Total & 7283.704 & 344 & & & \\
\hline $\mathrm{b}$ & Between Groups & 3966.695 & 53 & 74.843 & 49.779 & .000 \\
\hline & Within Groups & 437.522 & 291 & 1.504 & & \\
\hline & Total & 4404.217 & 344 & & & \\
\hline
\end{tabular}




\section{Mean Plots of Aged Results}

Figure 12.1: Mean plots of sample DPMP grouped by 'Fiber'
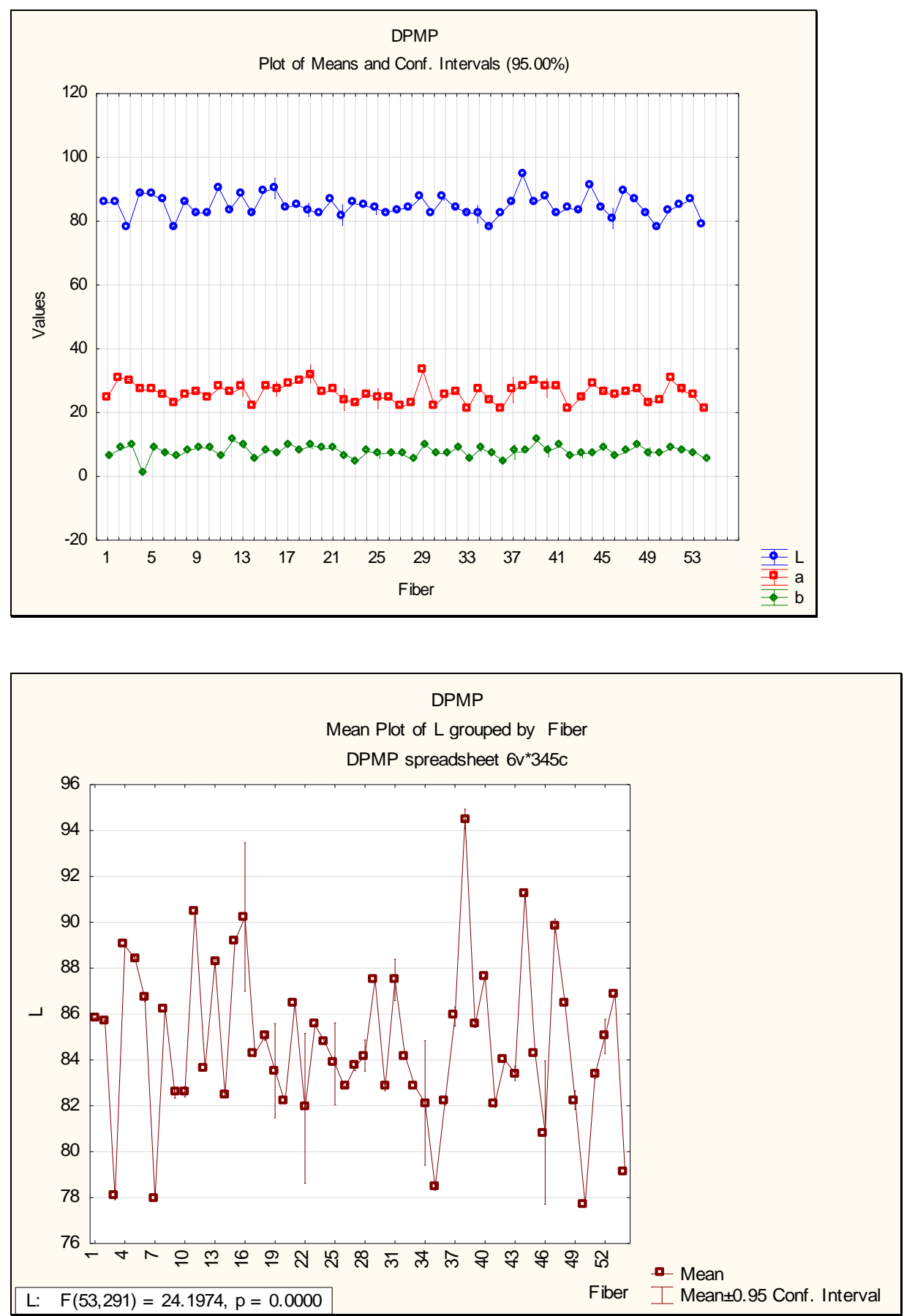

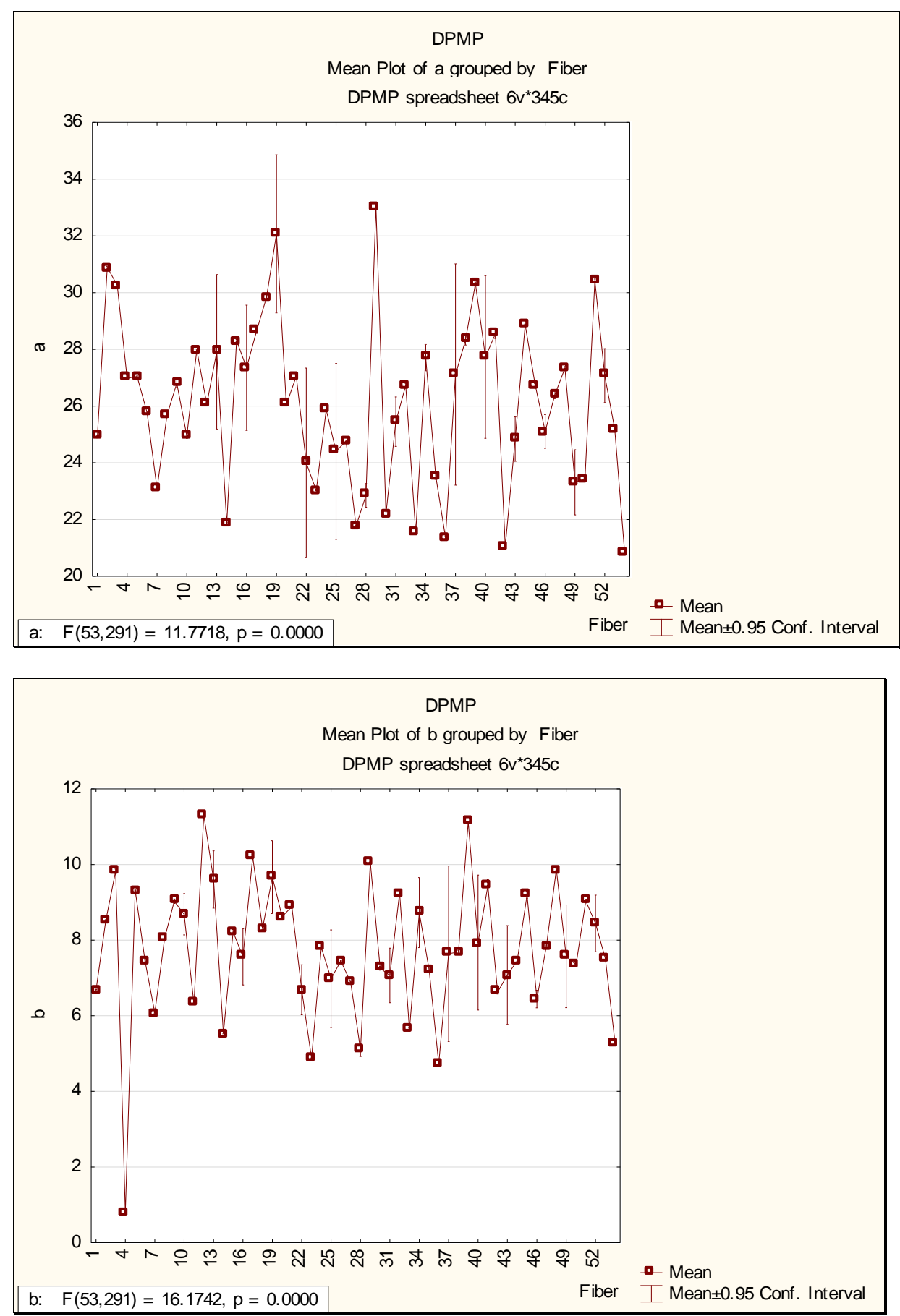
Figure 12.2: Mean plots of sample GMP grouped by 'Fiber'
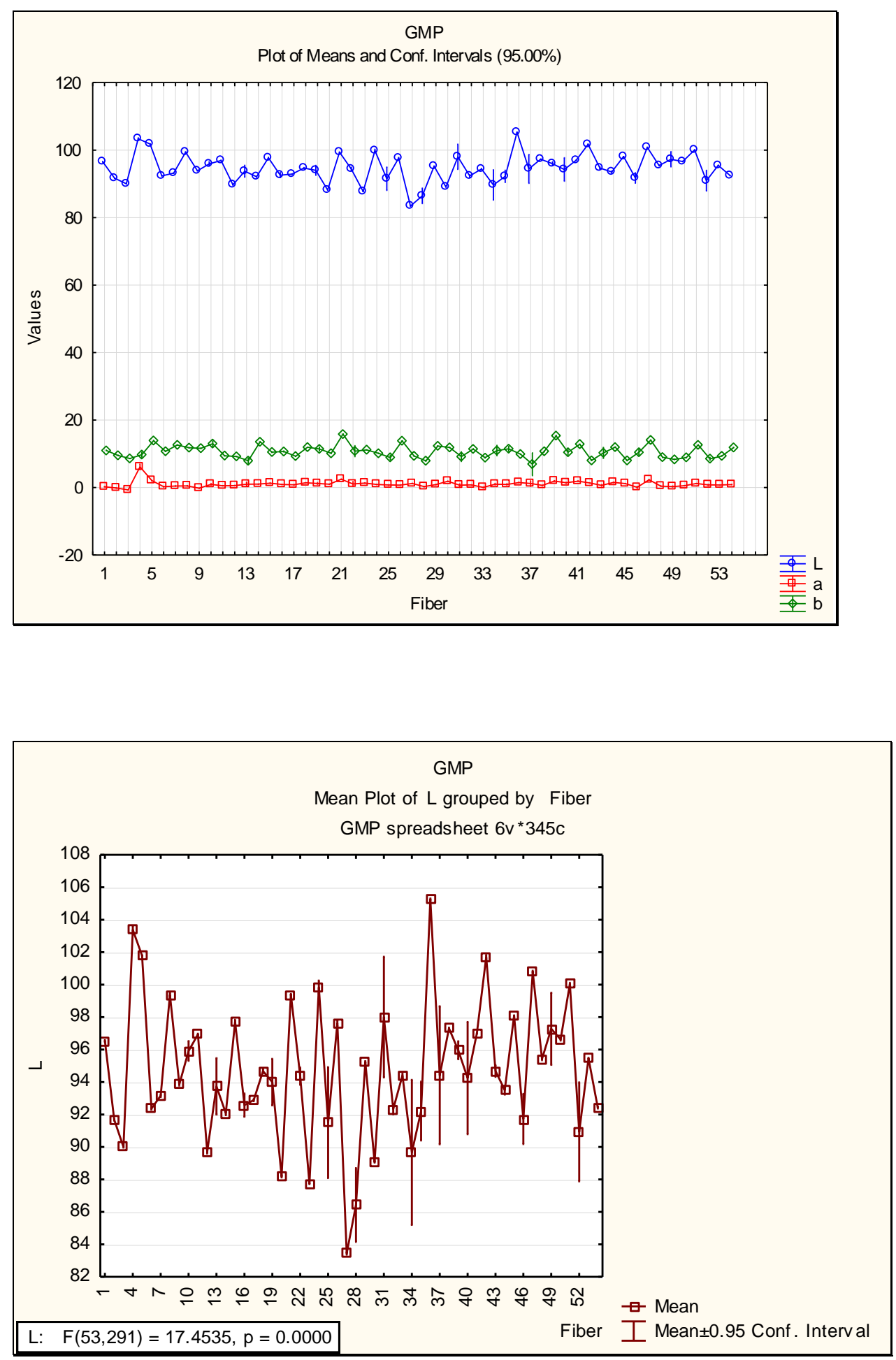

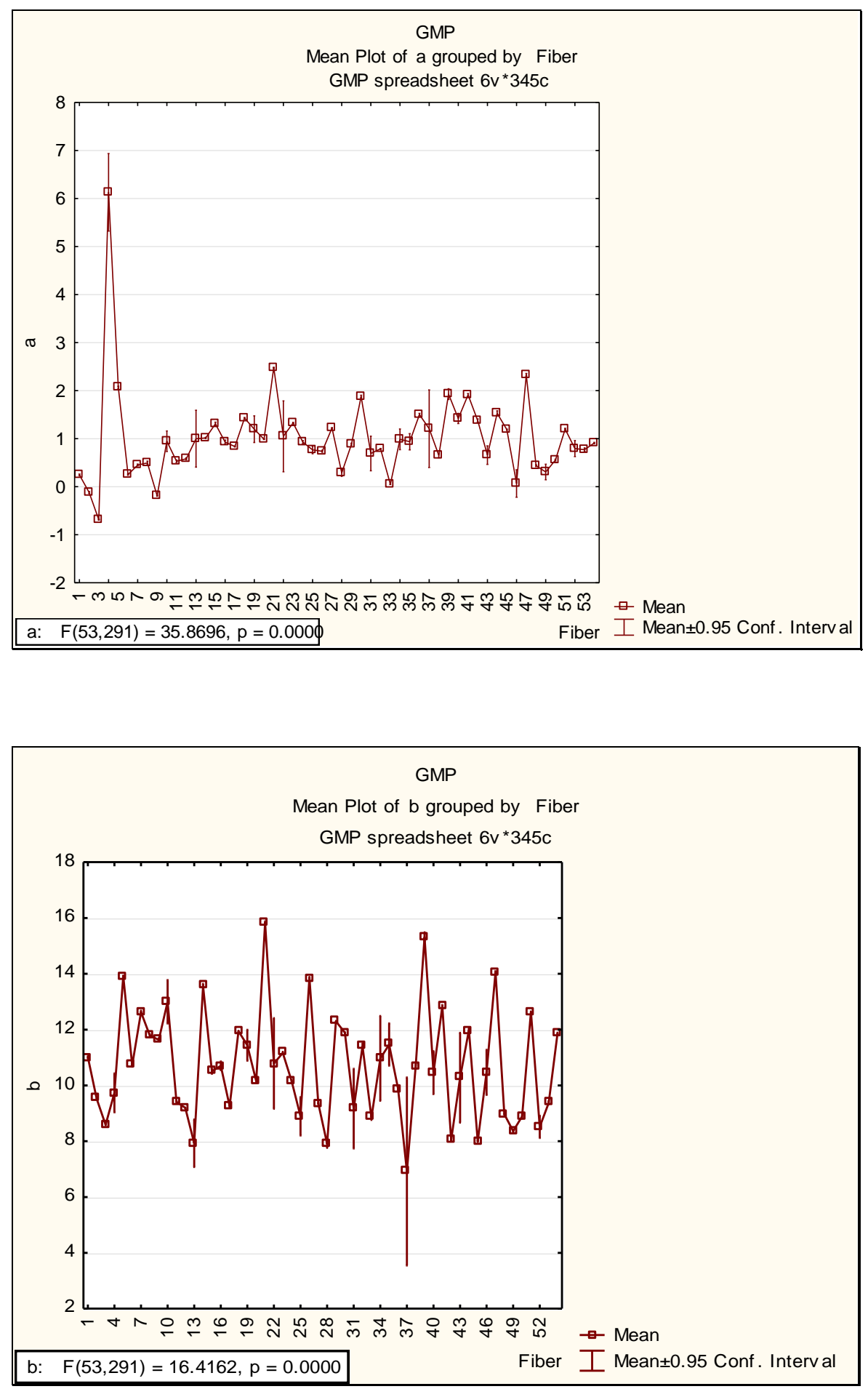
Figure 12.3: Mean plots of sample OSDP grouped by 'Fiber'
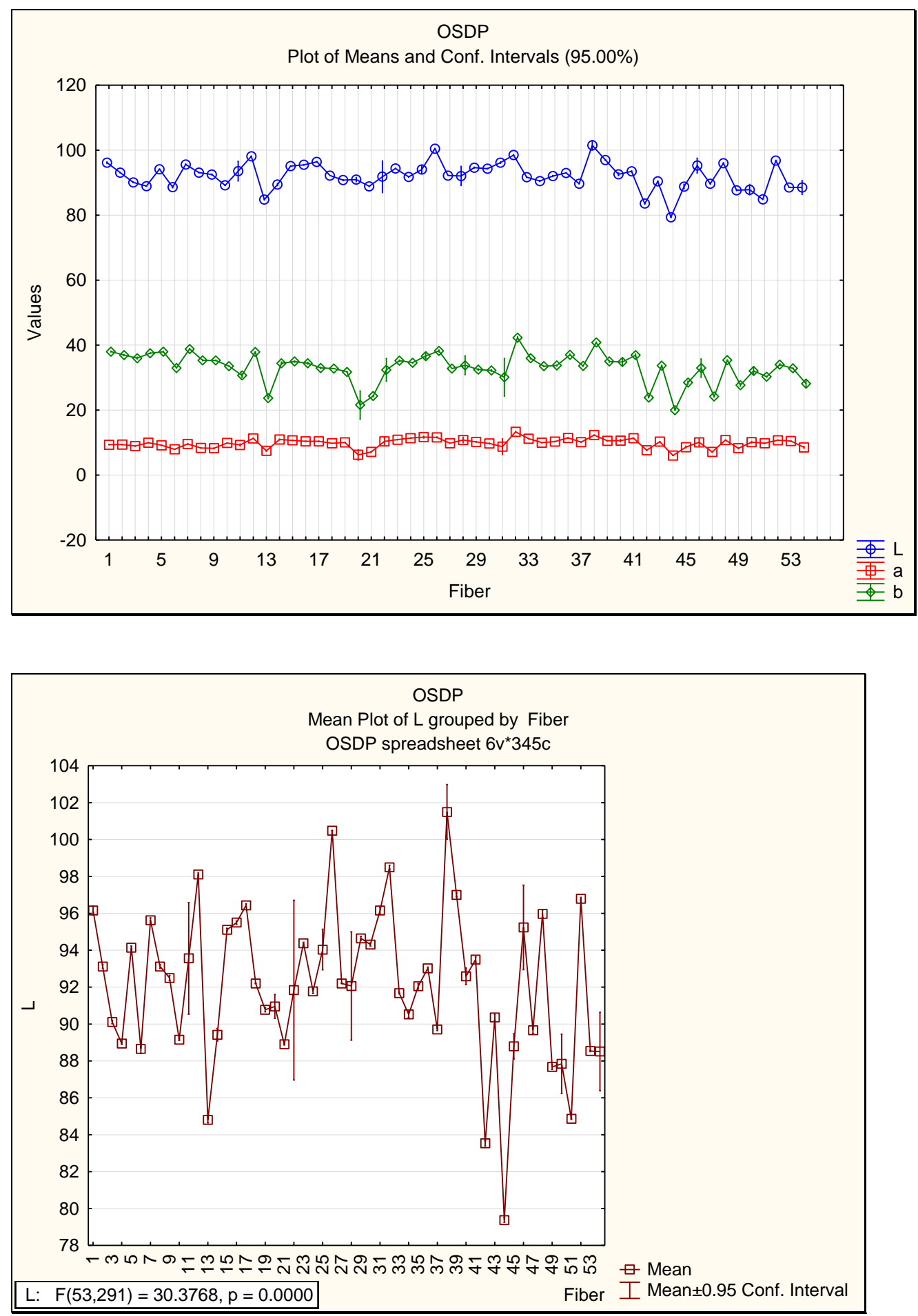

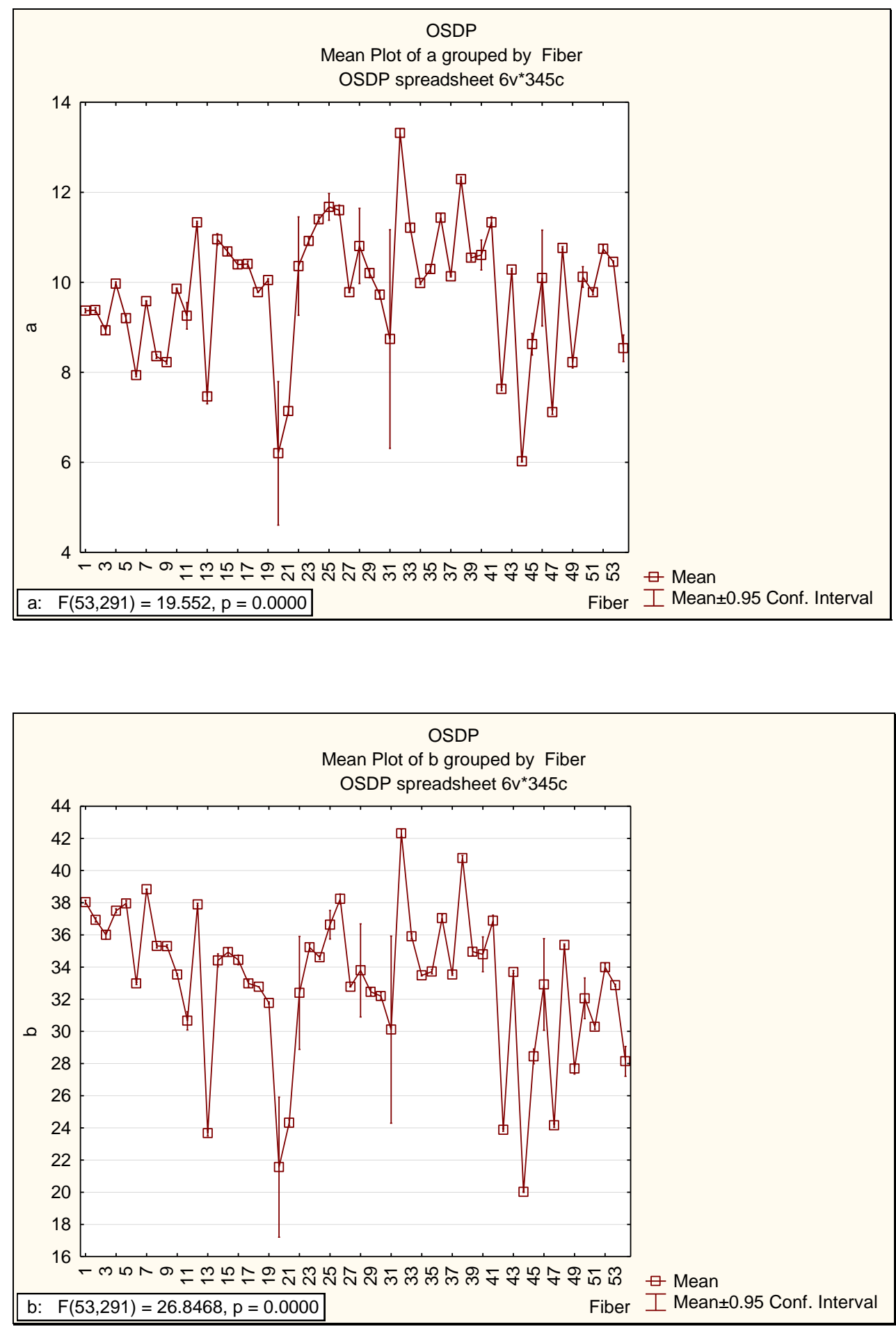
Figure 12.4: Mean plots of sample PMCL grouped by 'Fiber'
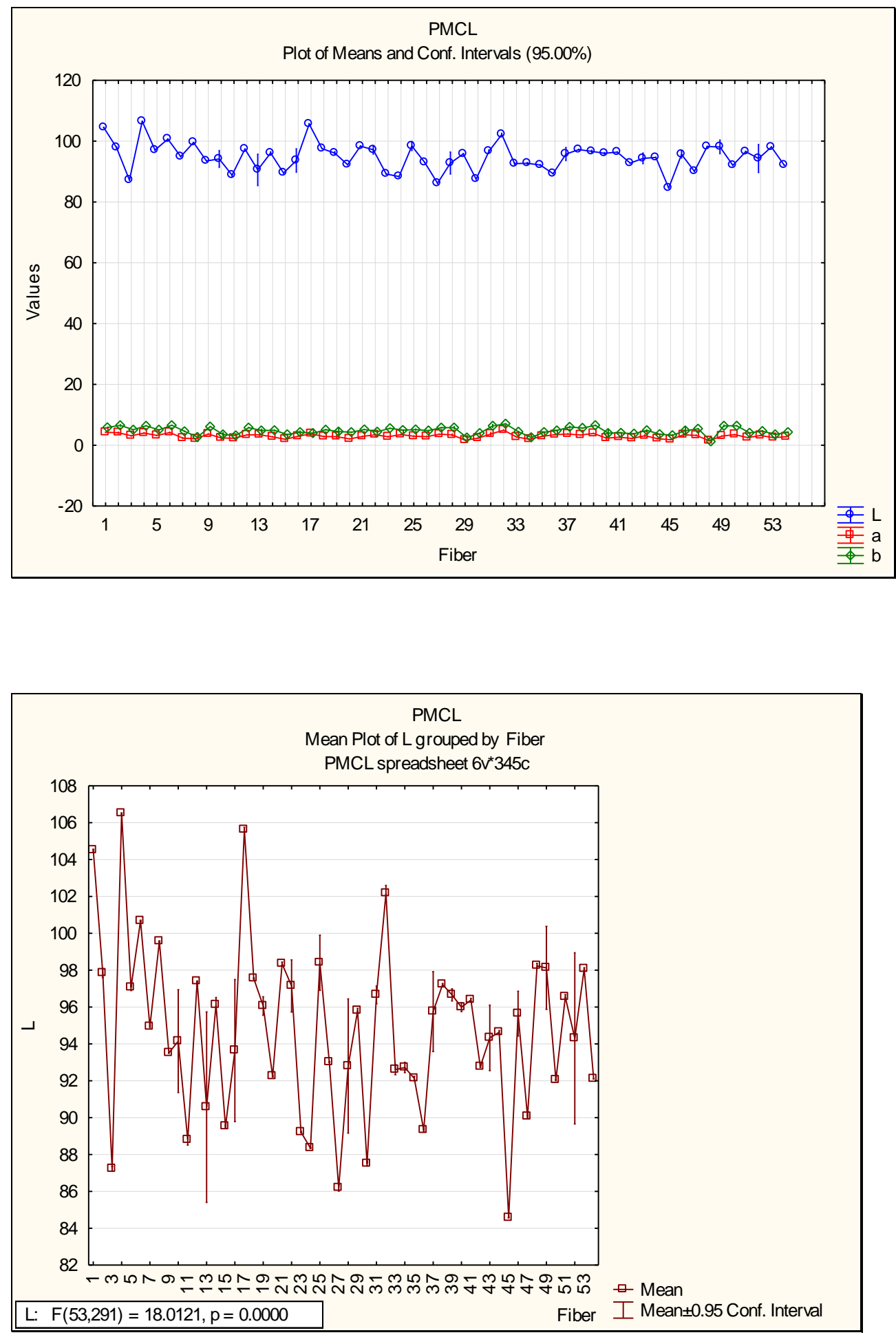

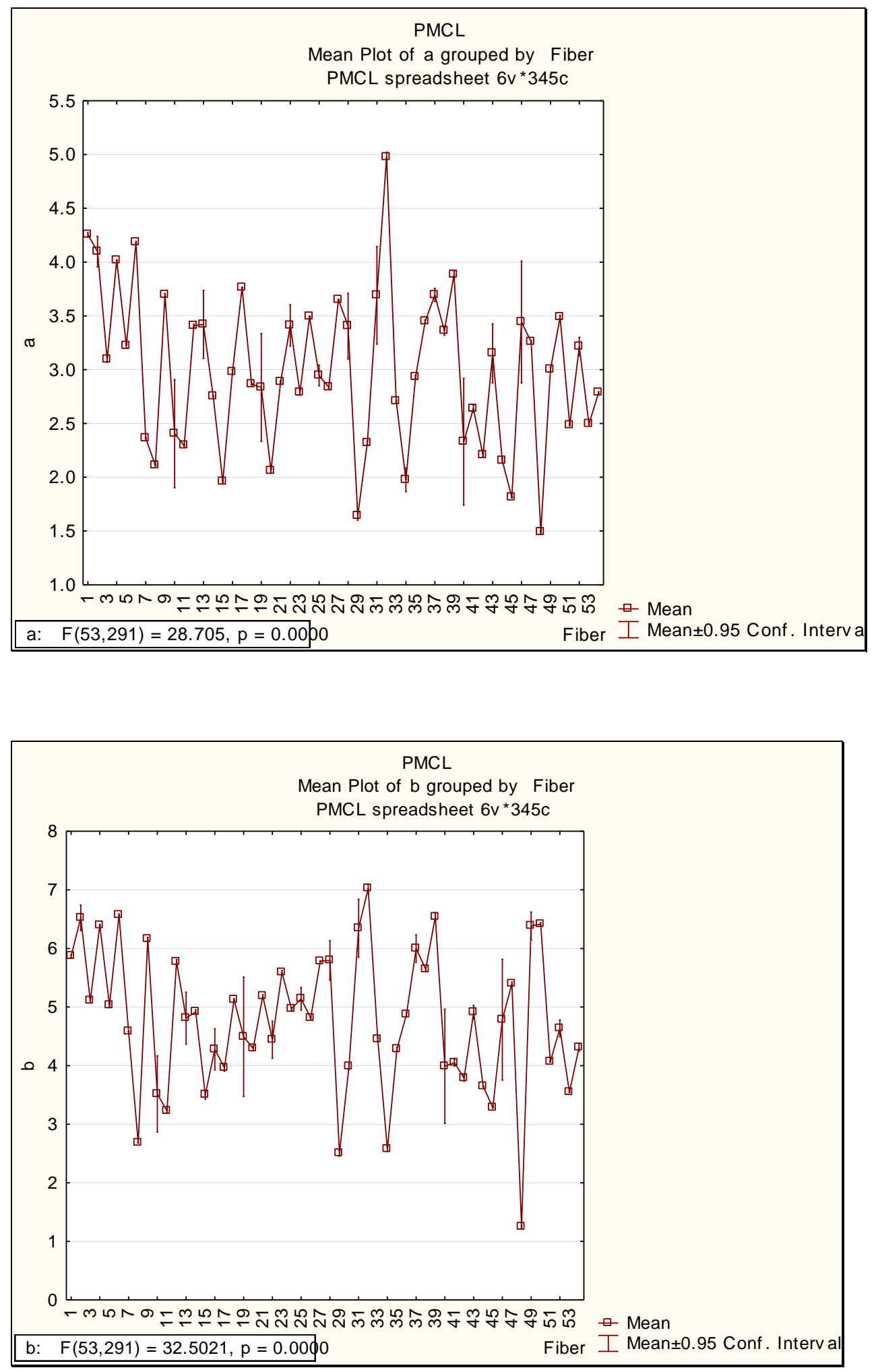
Figure 12.5: Mean plots of sample PMJX grouped by 'Fiber'
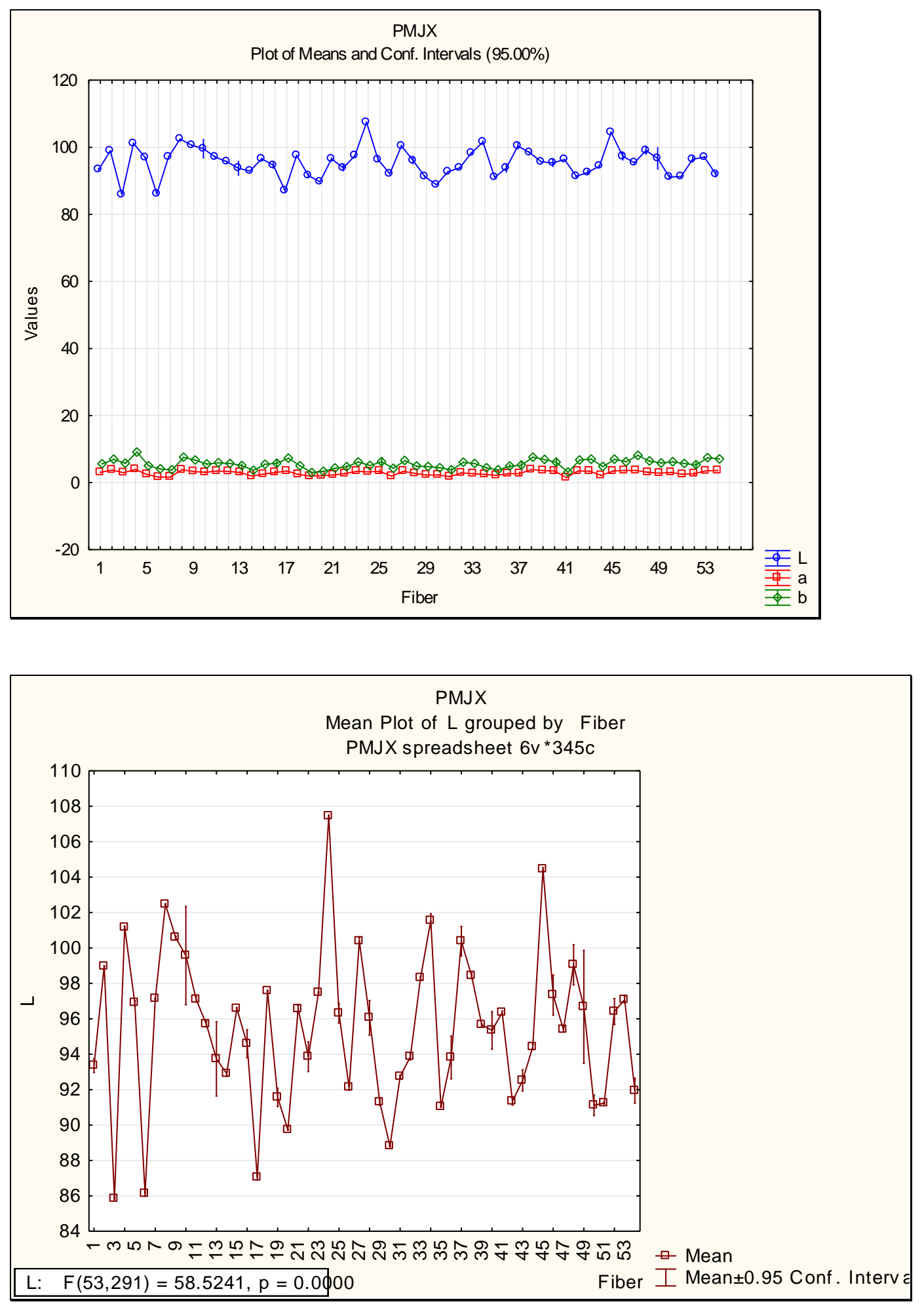


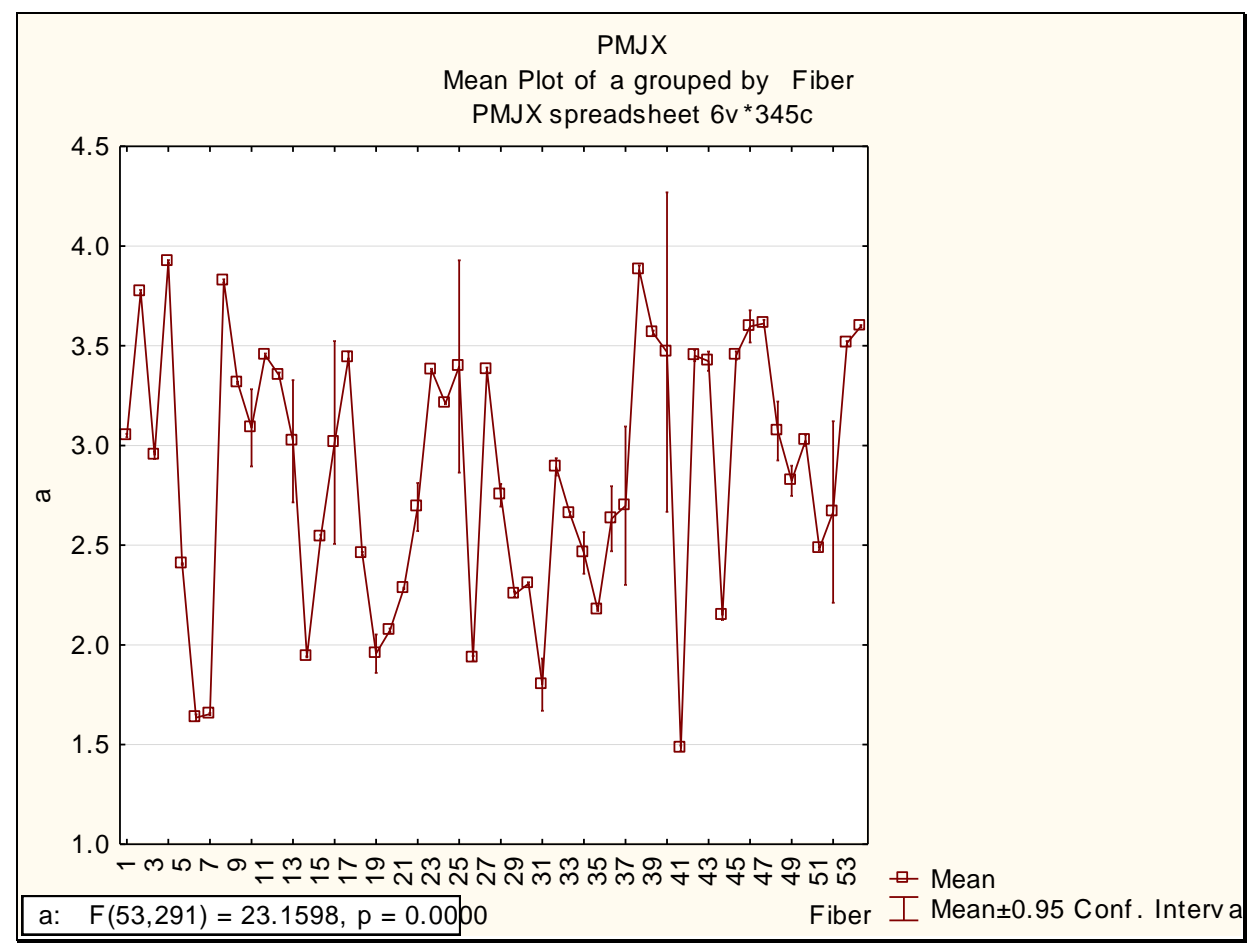

Figure 12.6: Mean plots of sample PMP grouped by 'Fiber'

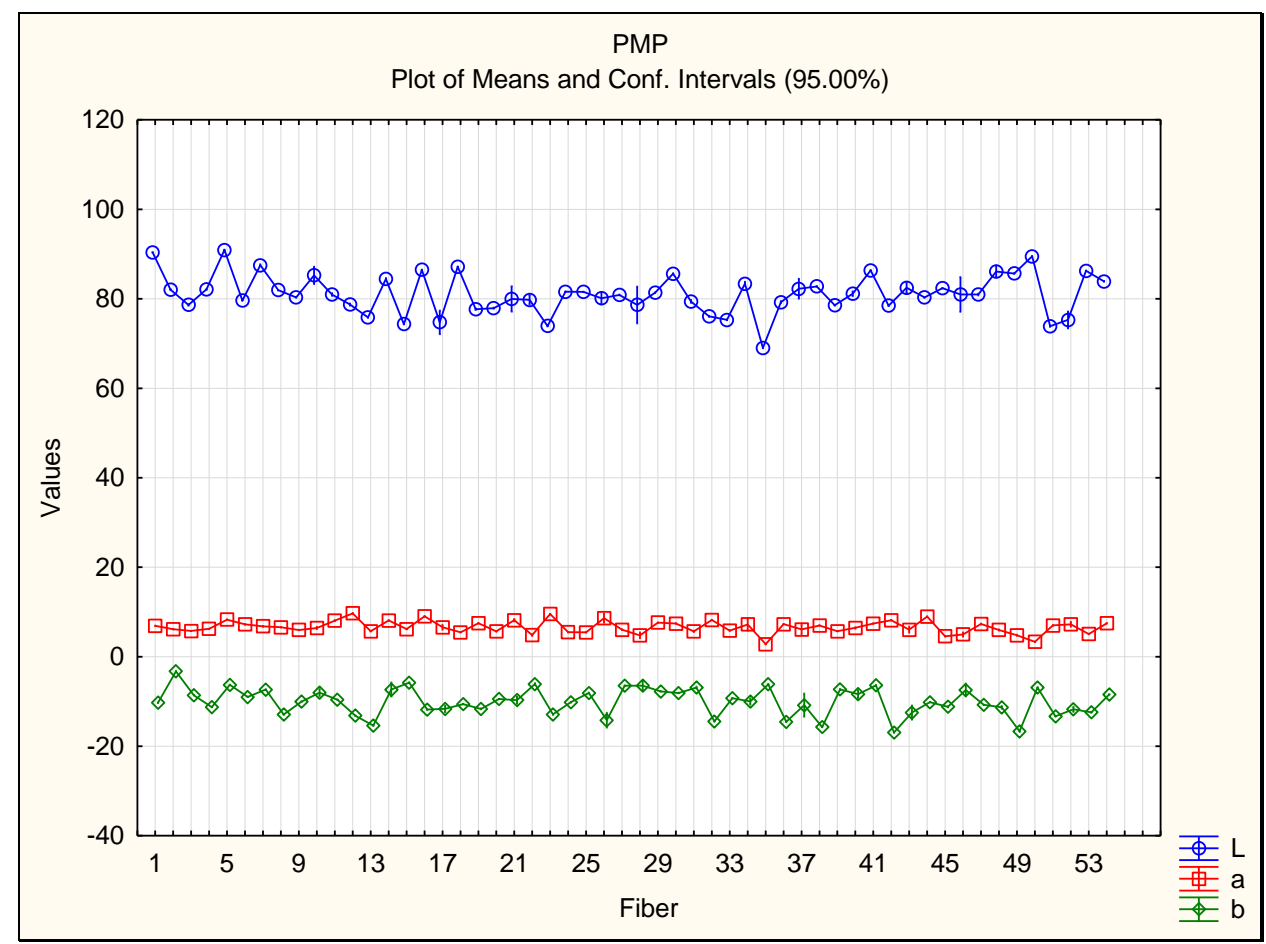



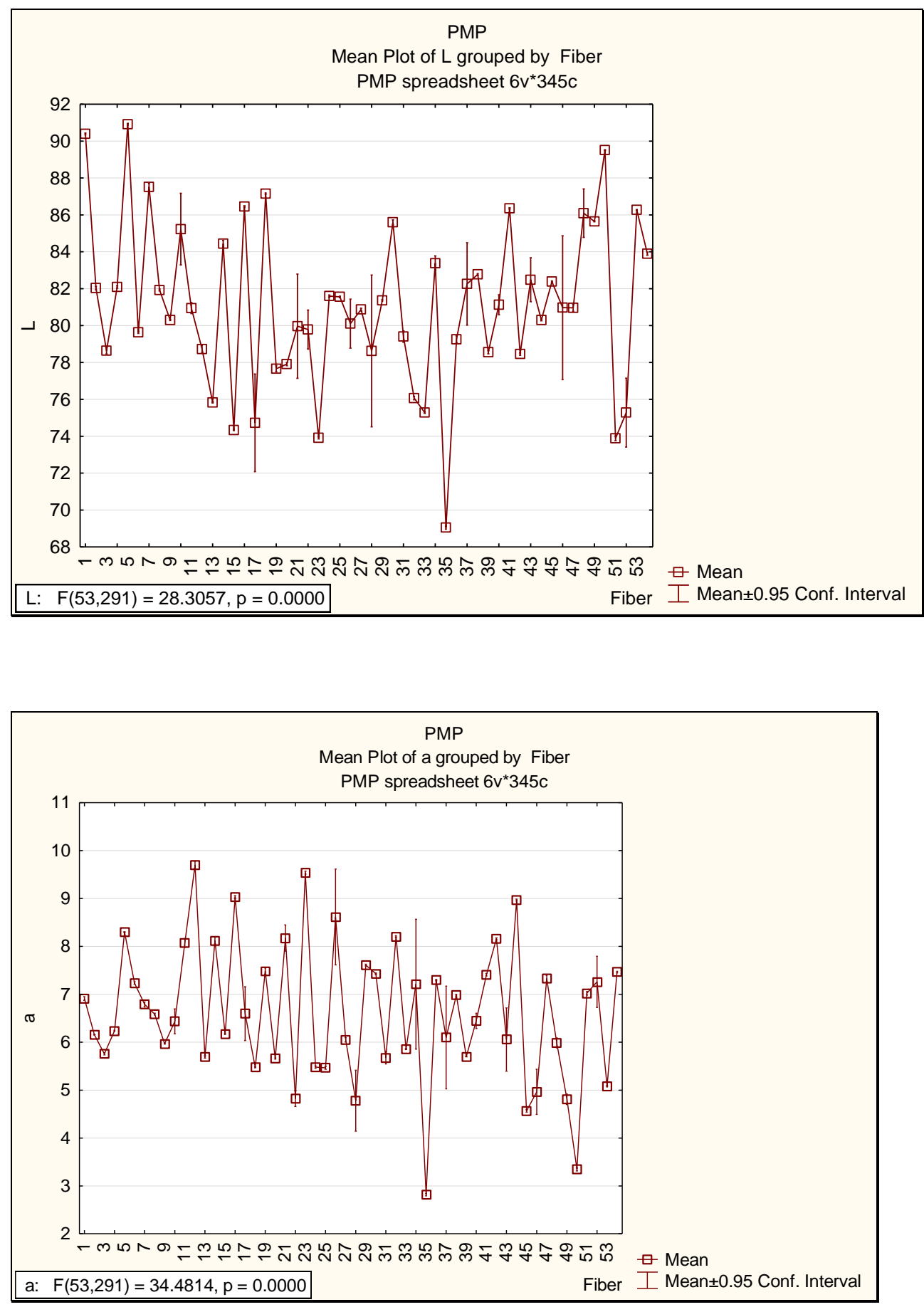


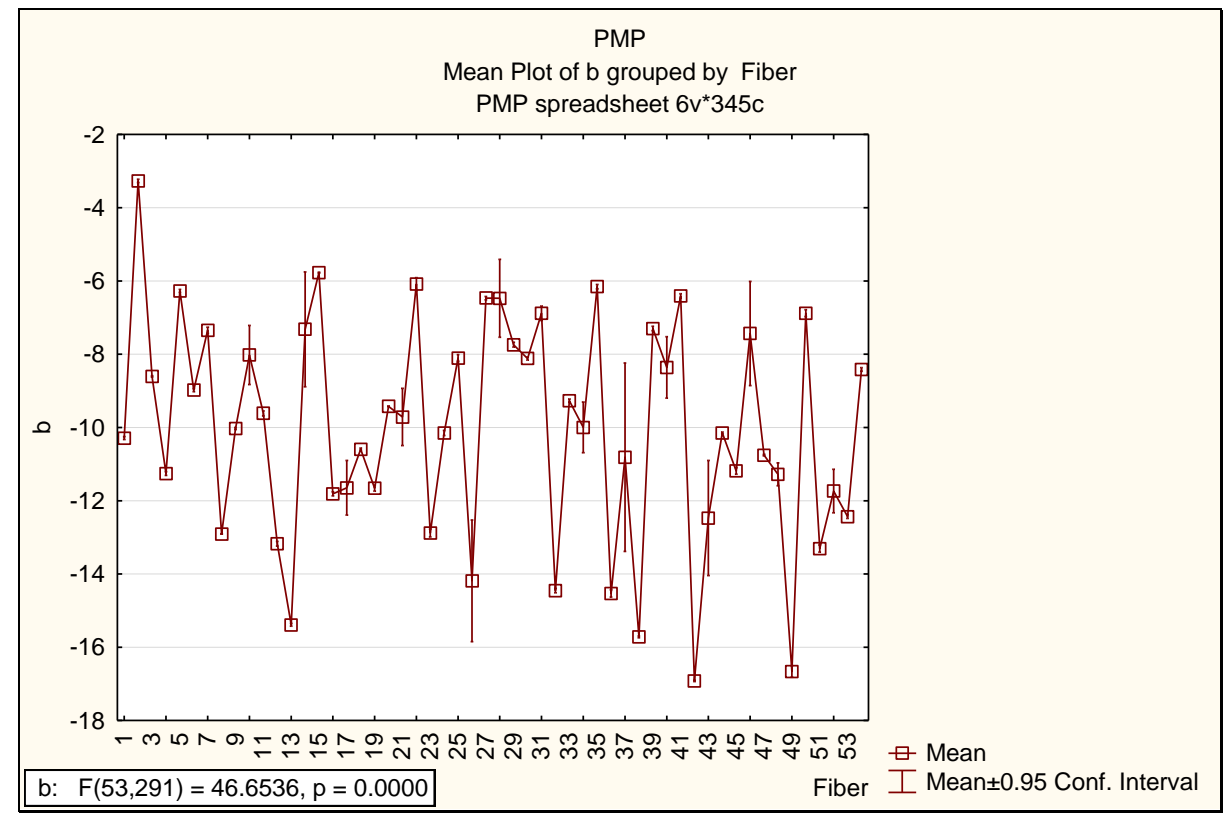

Figure 12.7: Mean plots of sample RMP grouped by 'Fiber'

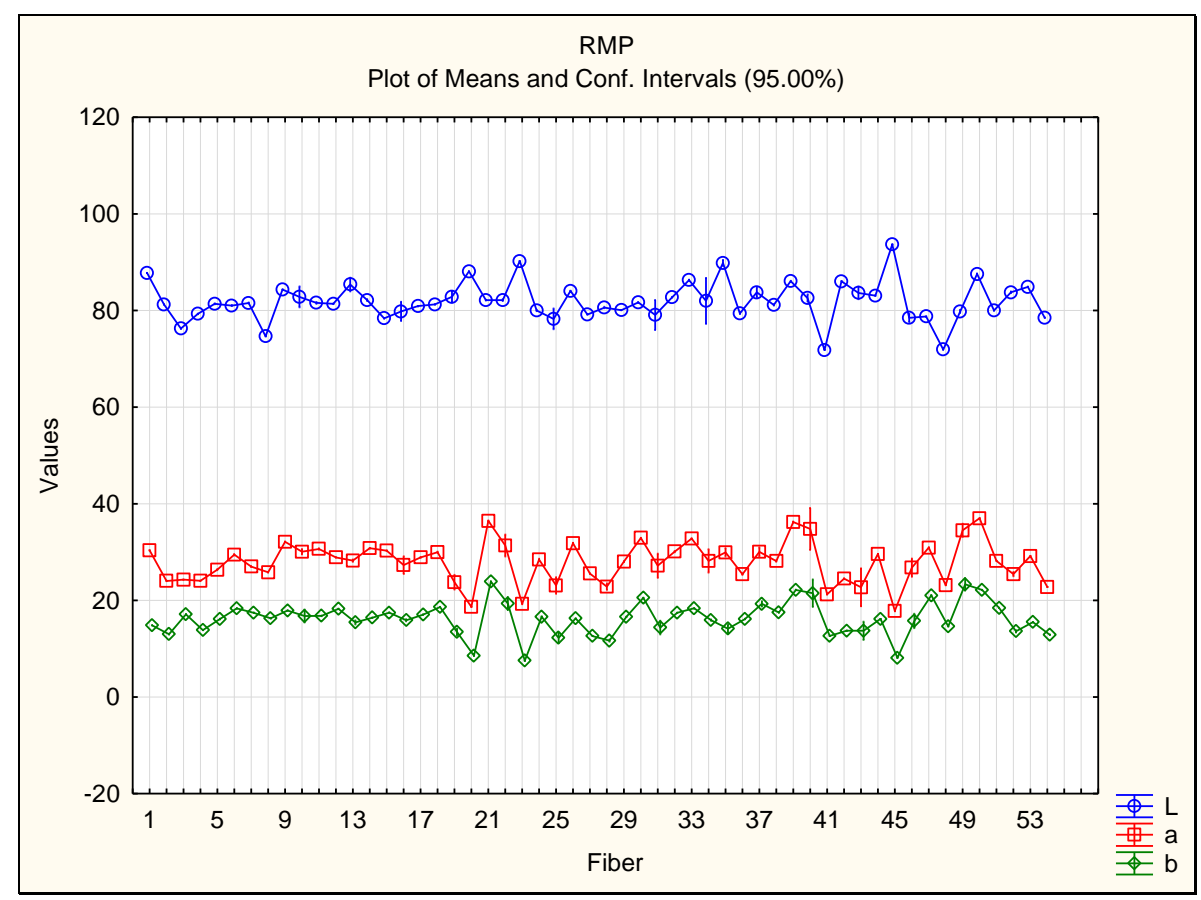



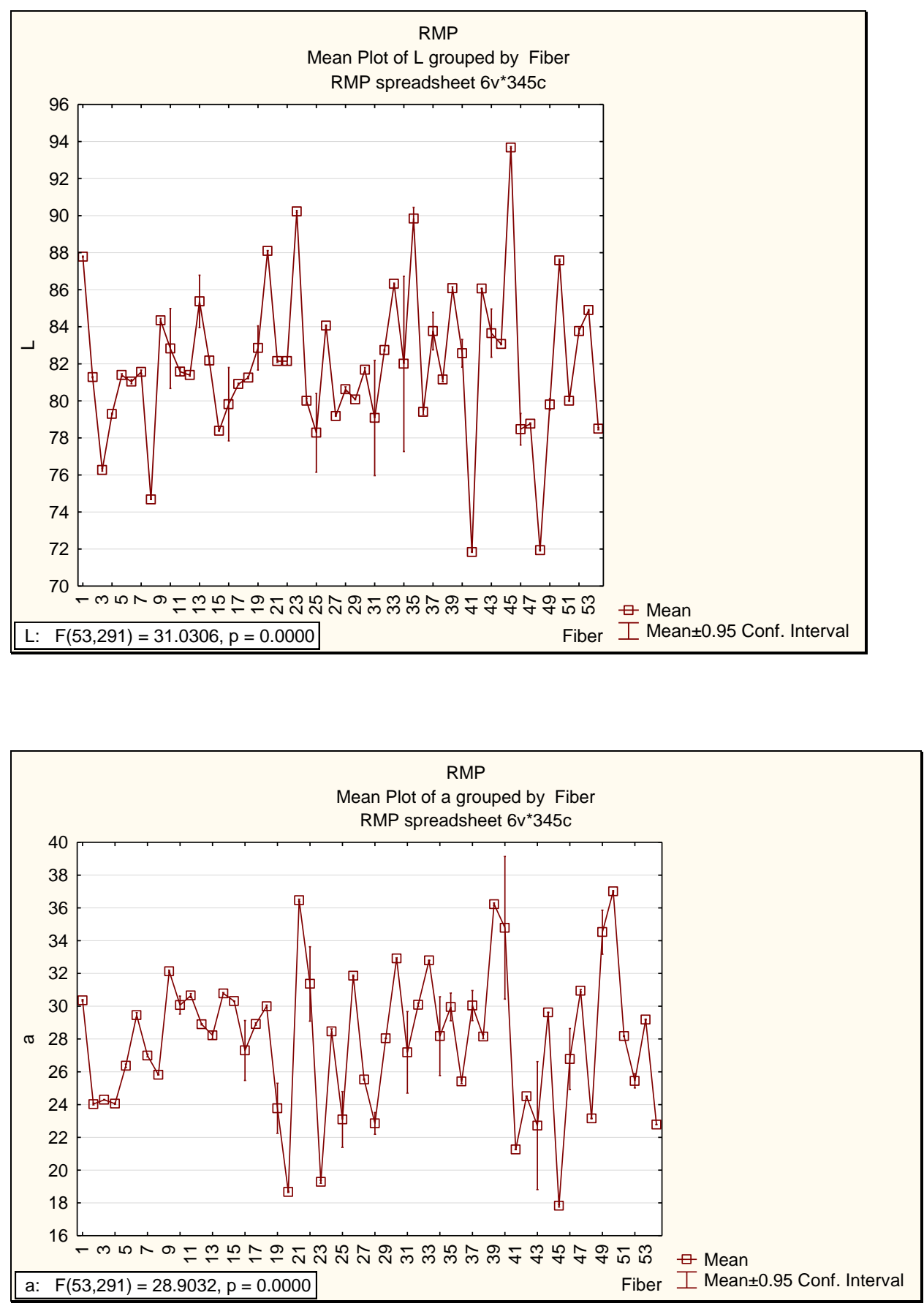


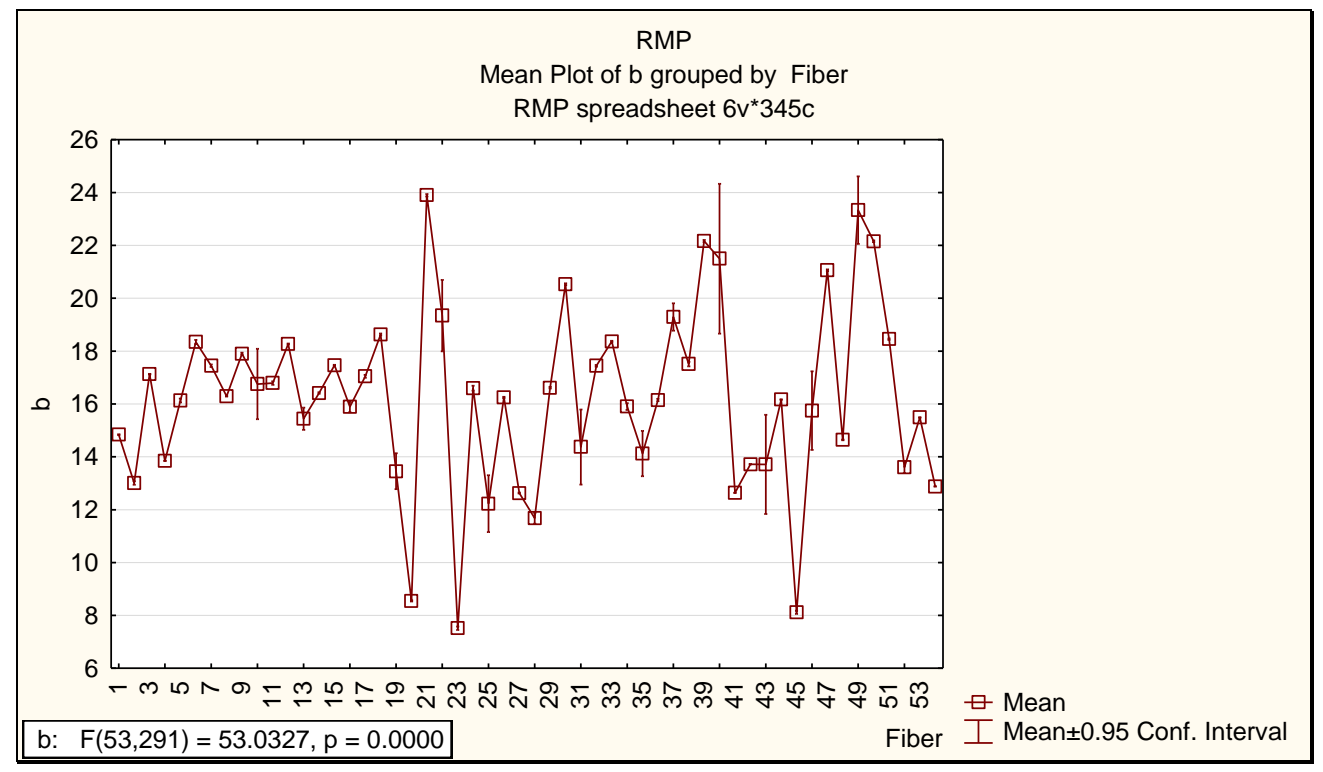

\section{ANOVA Interaction Plots}

Figure 13.1: Interaction plots of 'Hours* Sample Area' for sample DPMP at each variable

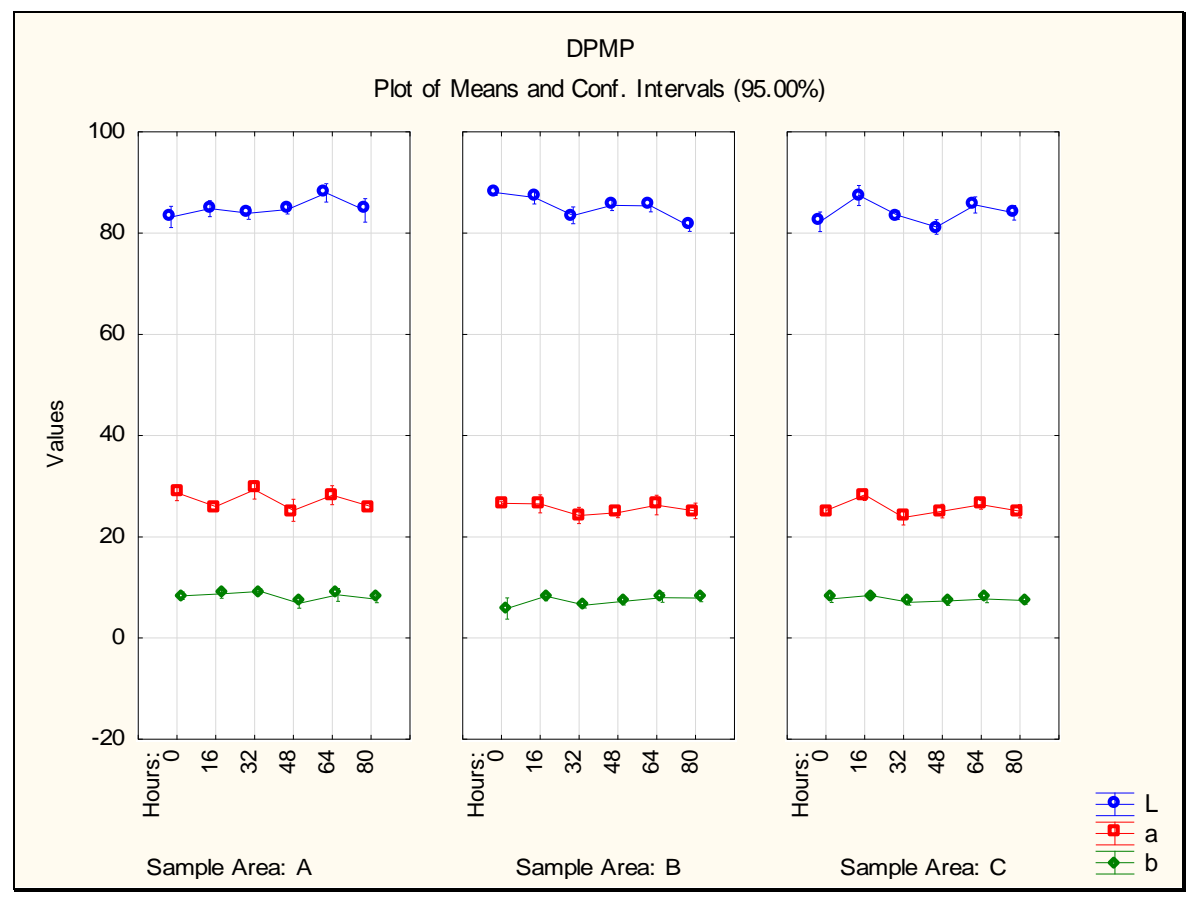



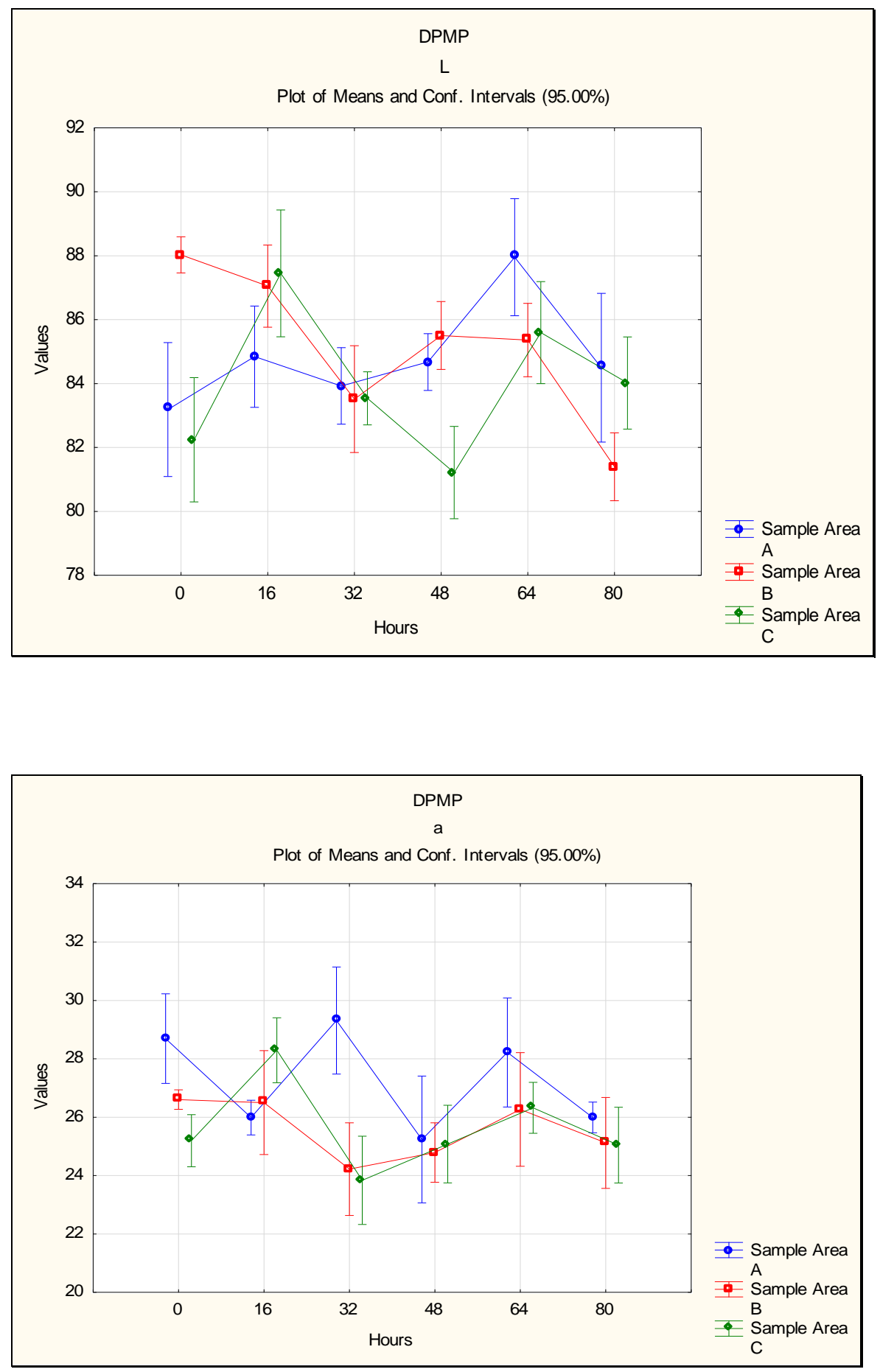


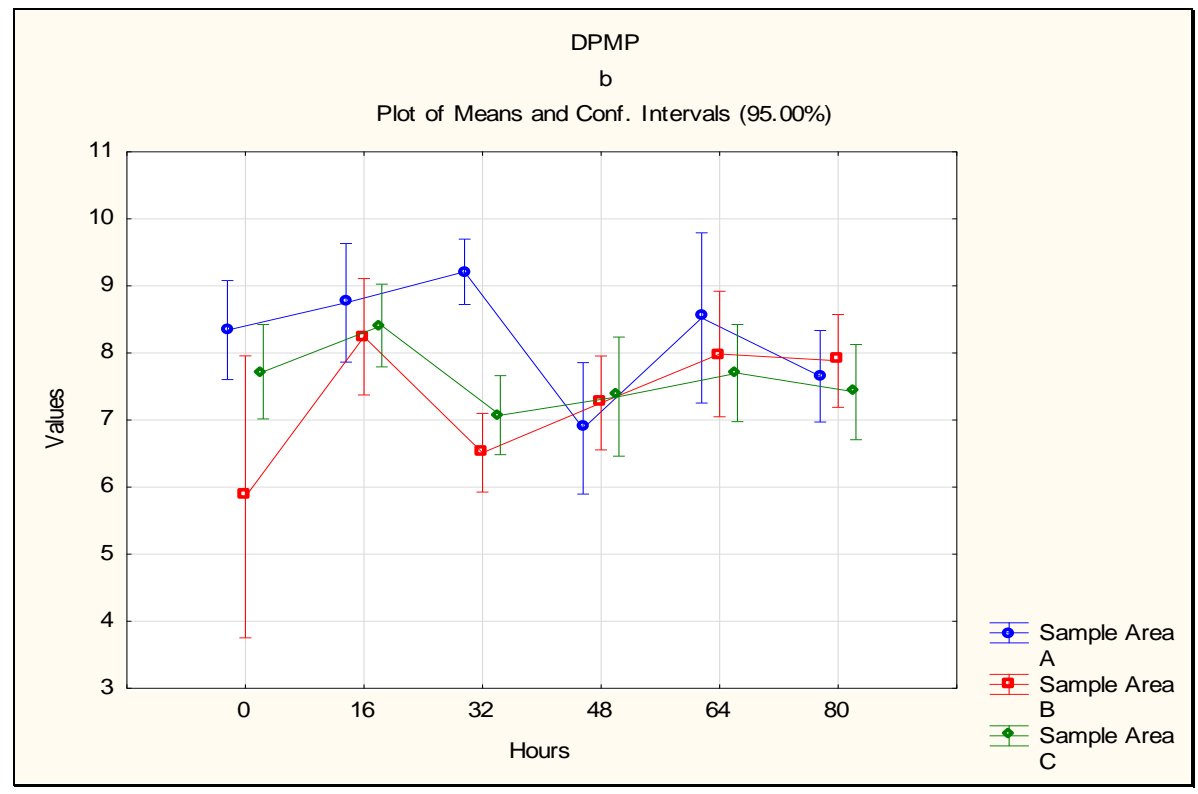

Figure 13.2: Interaction plots of 'Hours* Sample Area' for sample GMP at each variable

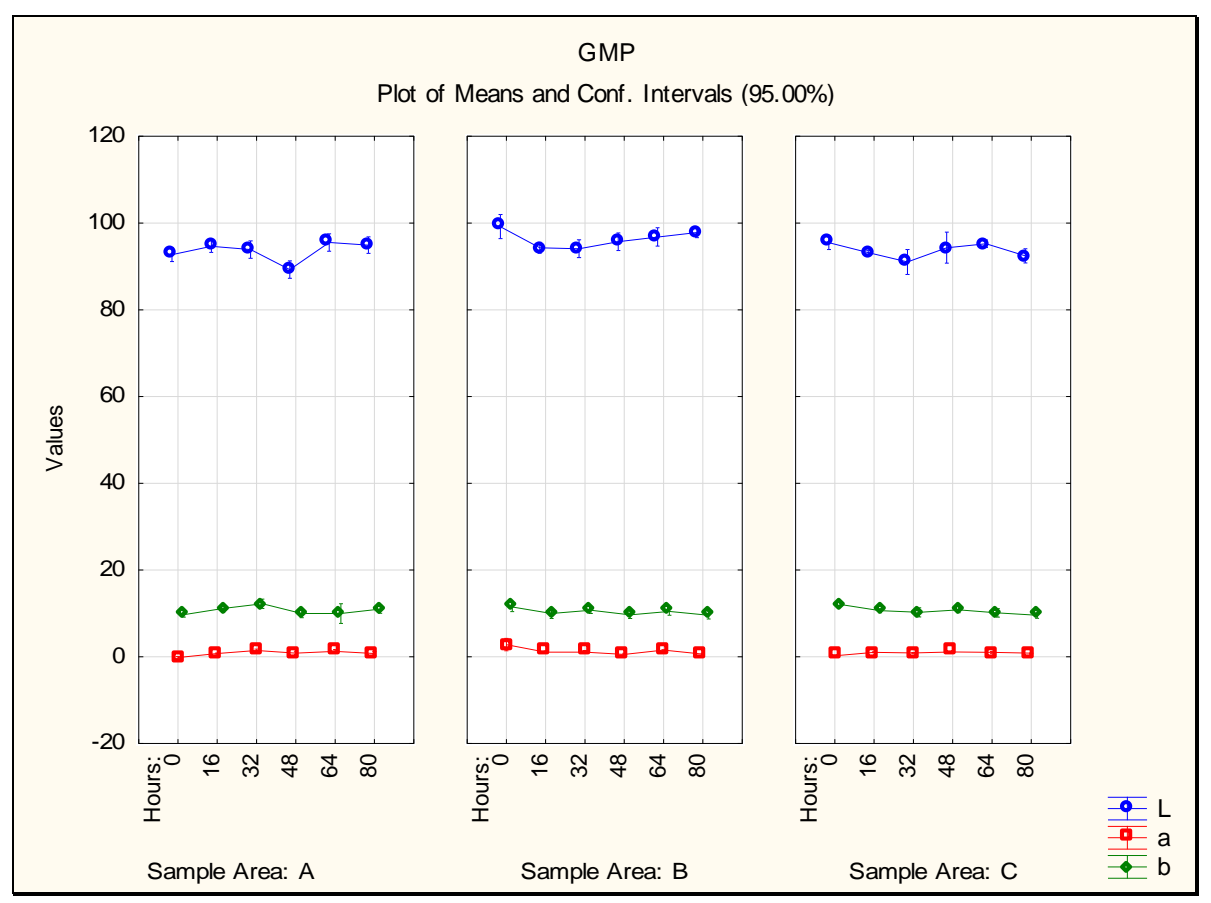



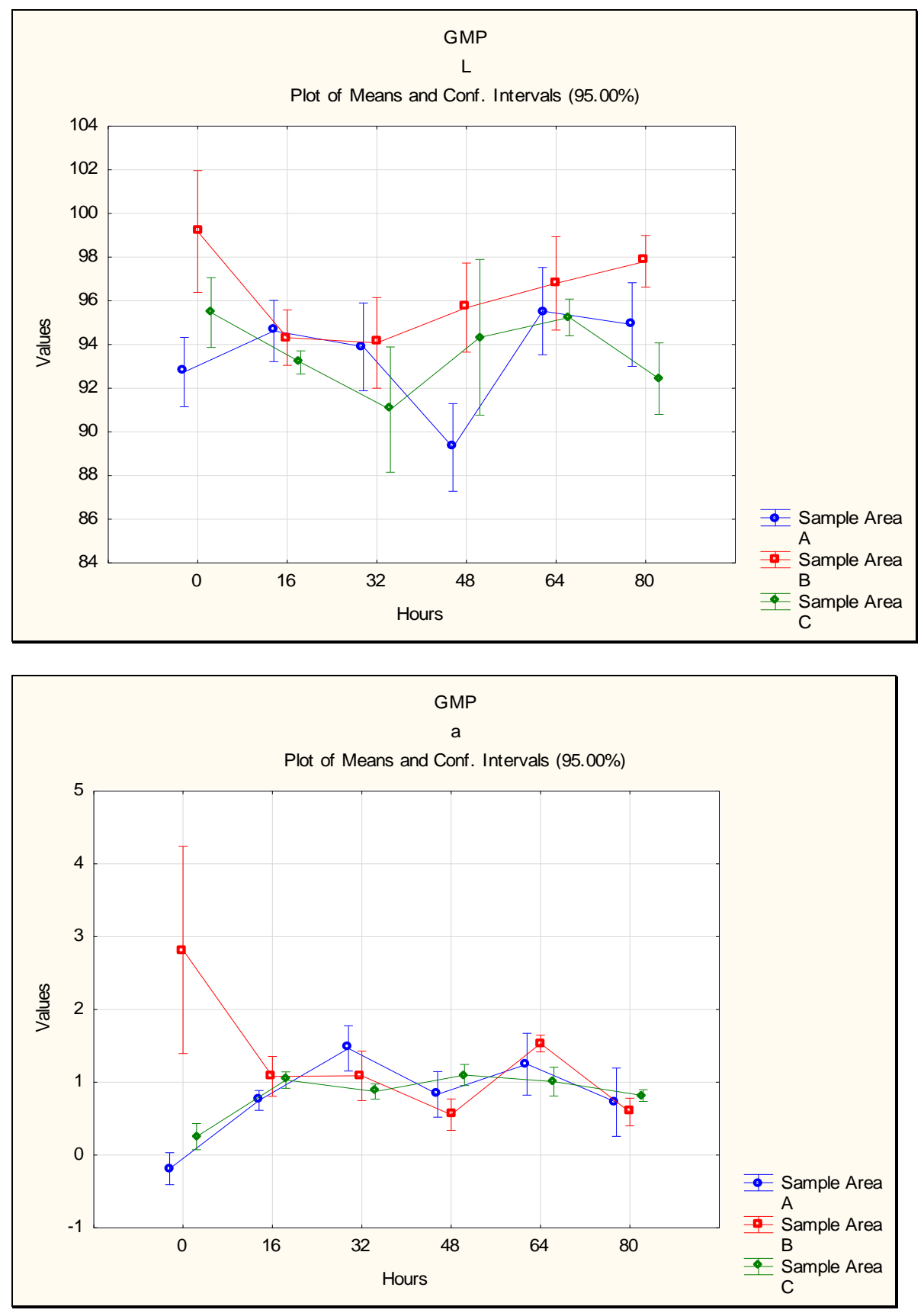


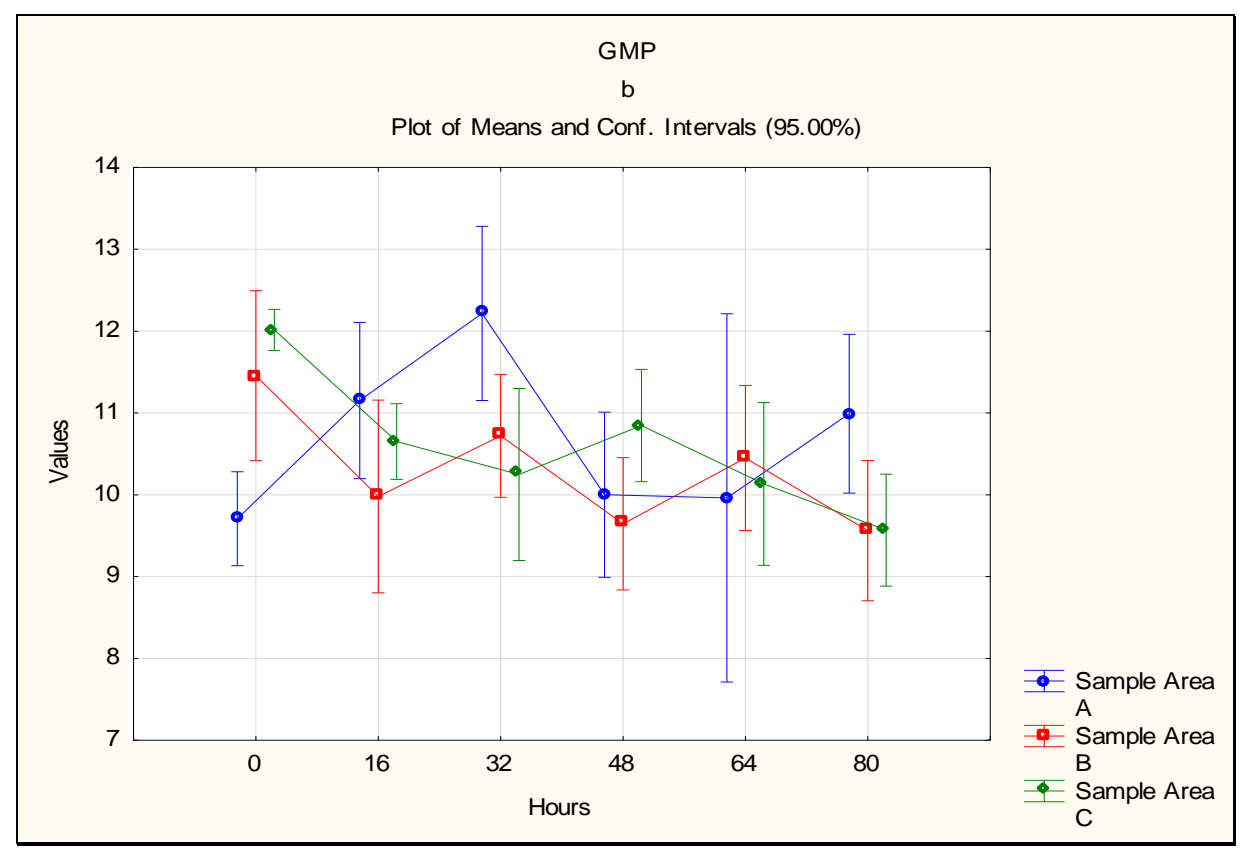

Figure 13.3: Interaction plots of 'Hours* Sample Area' for sample OSDP at each variable

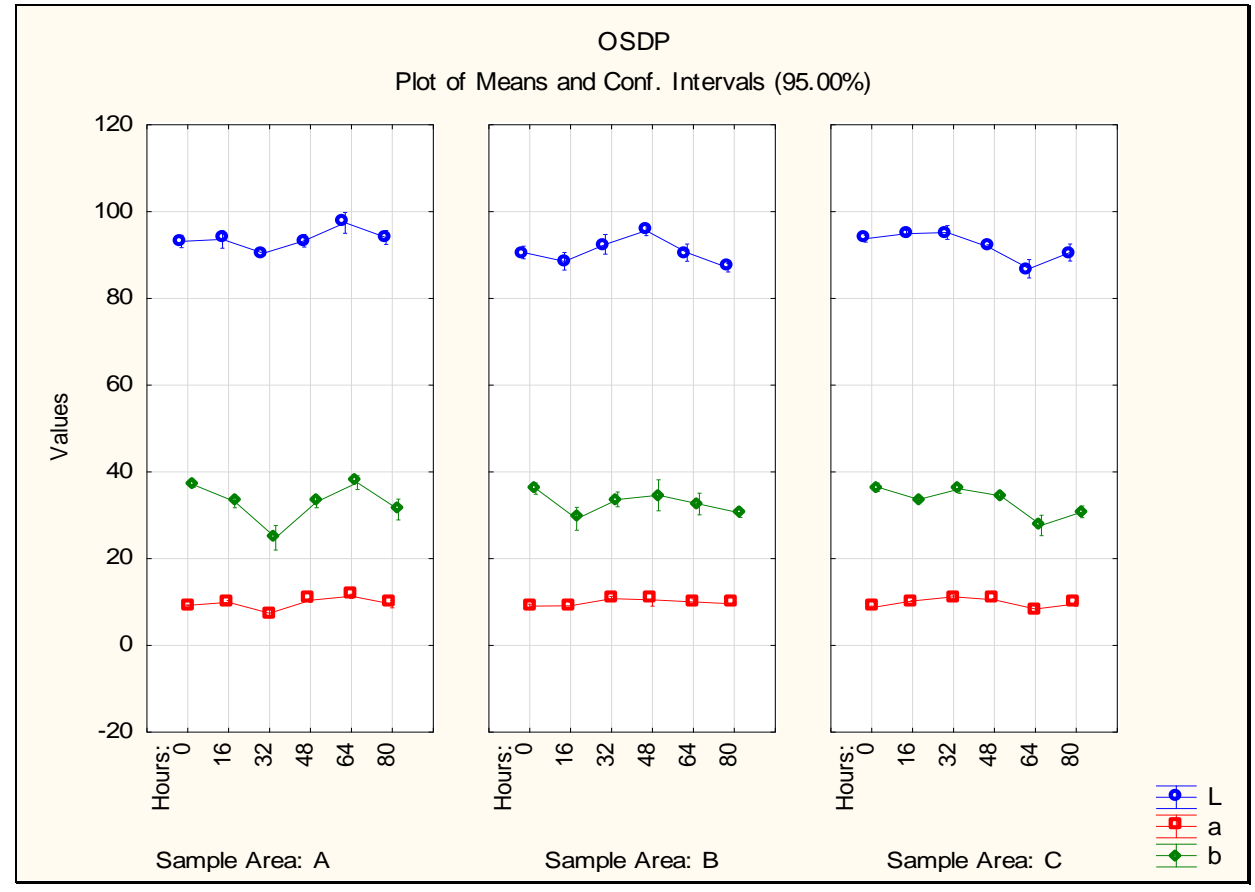



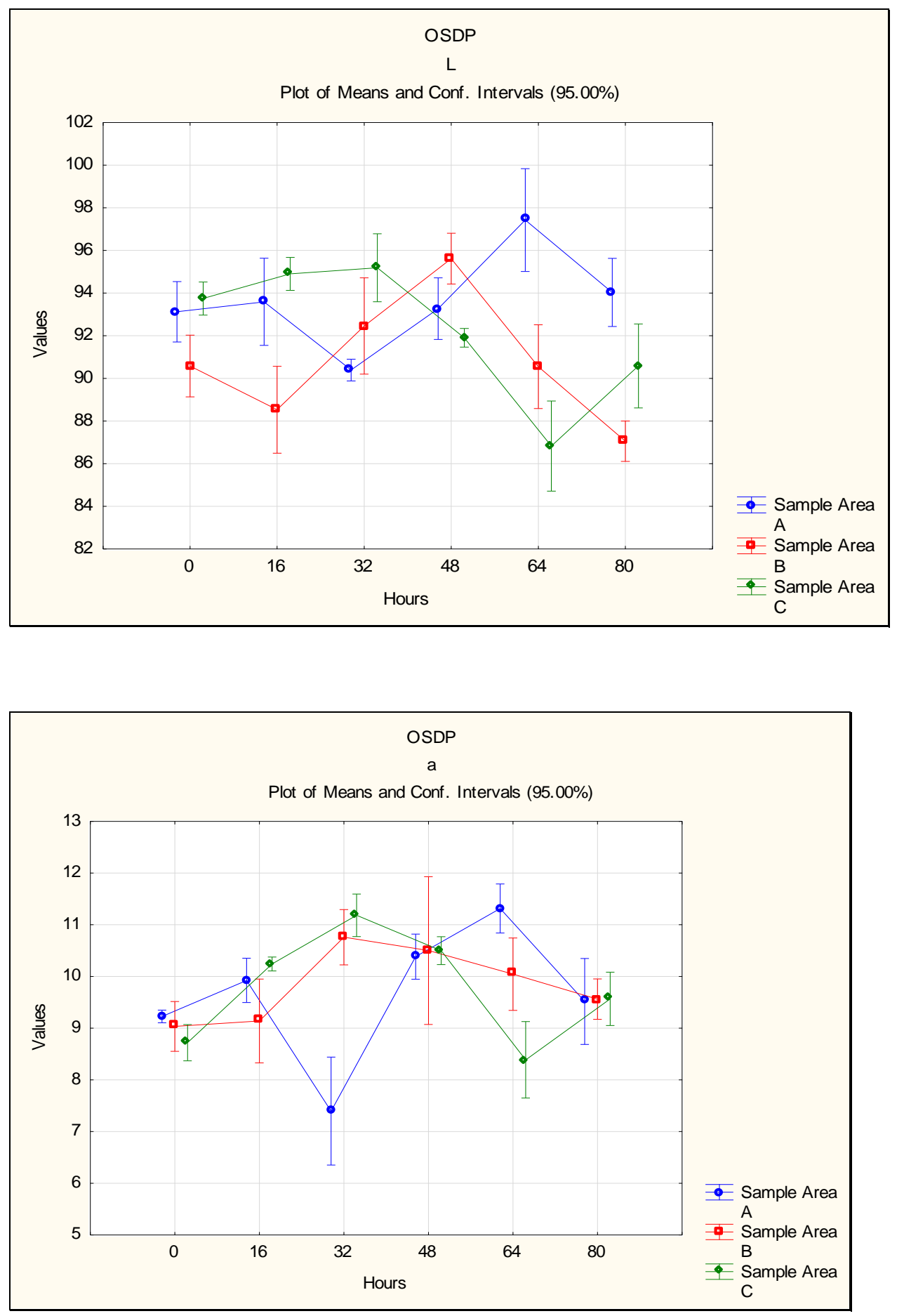


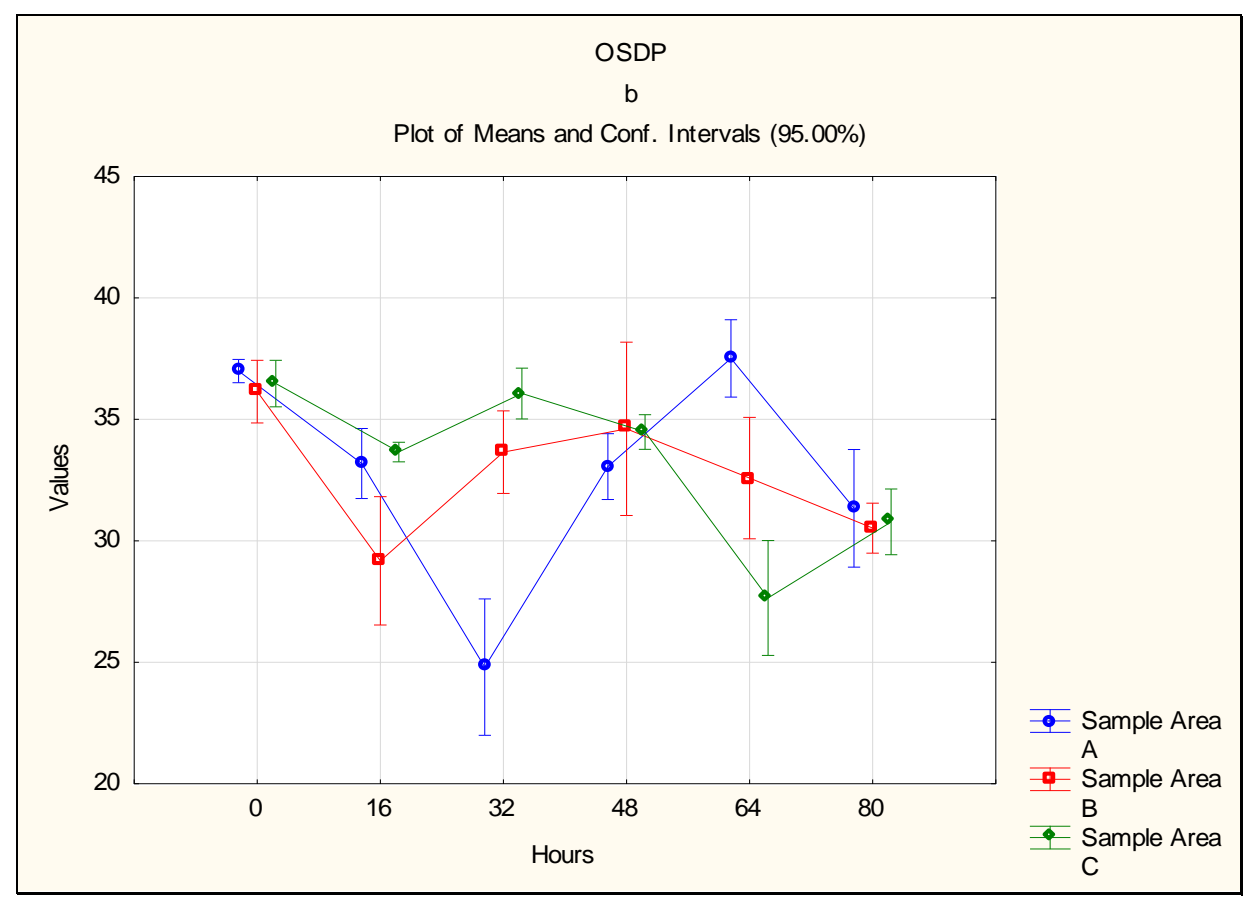

Figure 13.4: Interaction plots of 'Hours* Sample Area' for sample PMCL at each variable

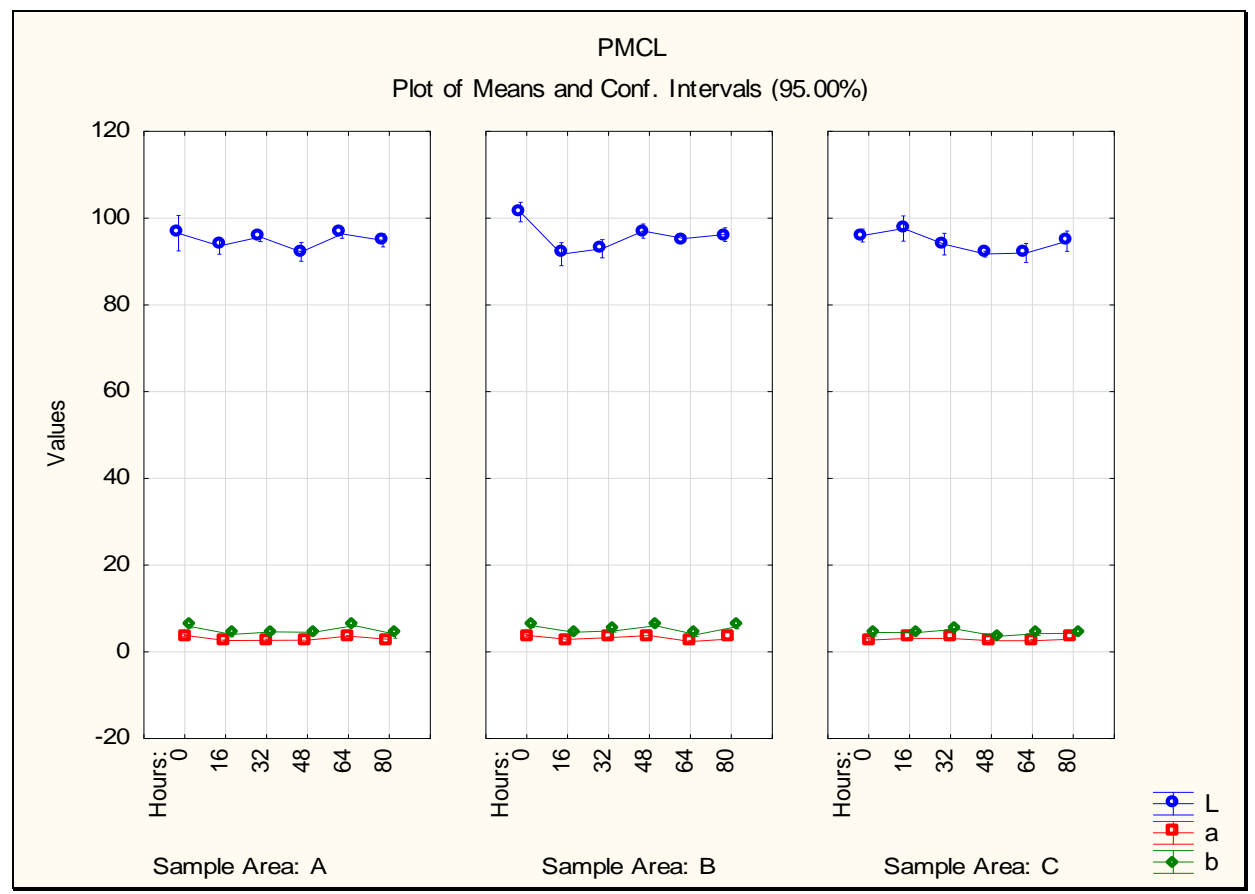



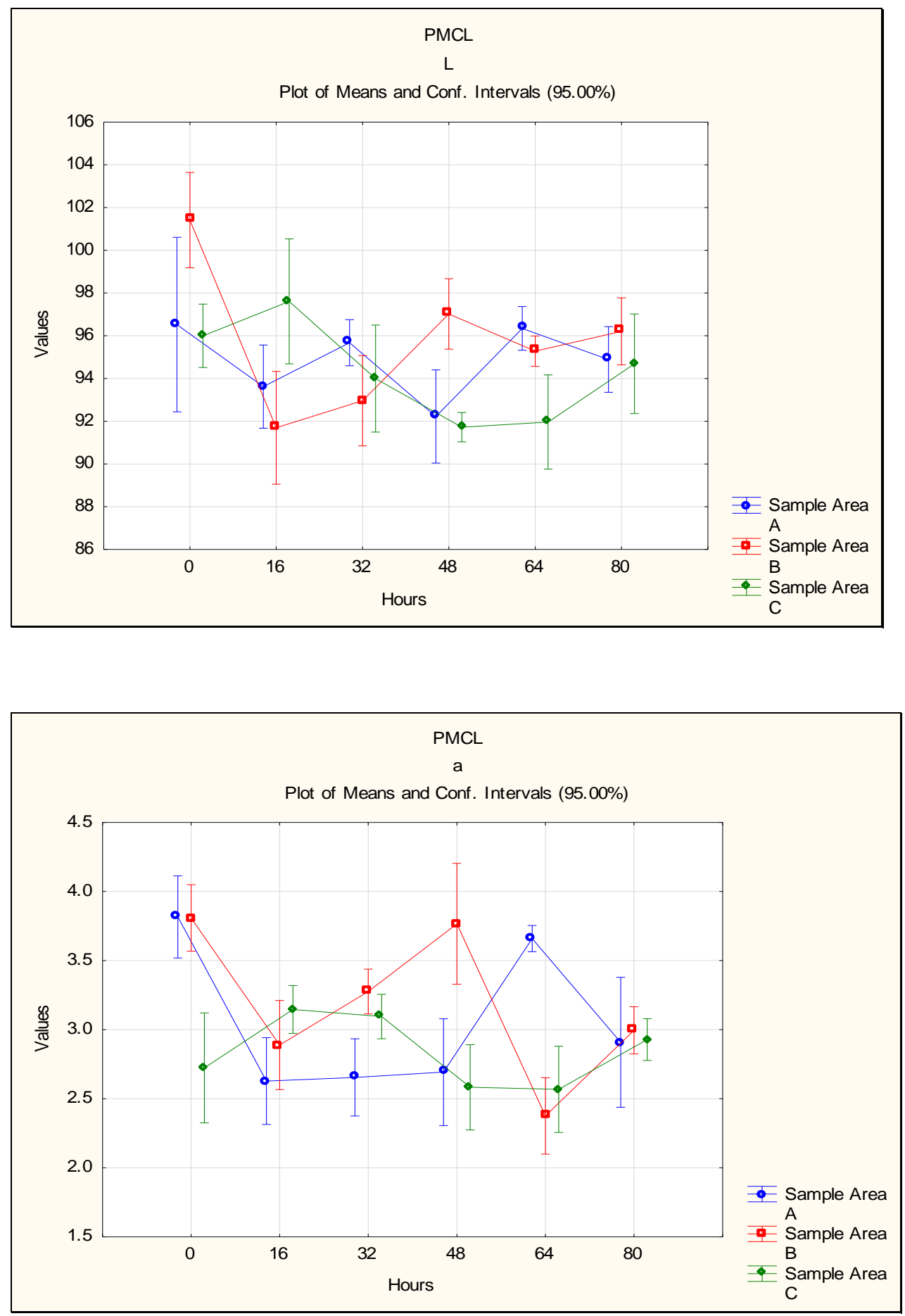


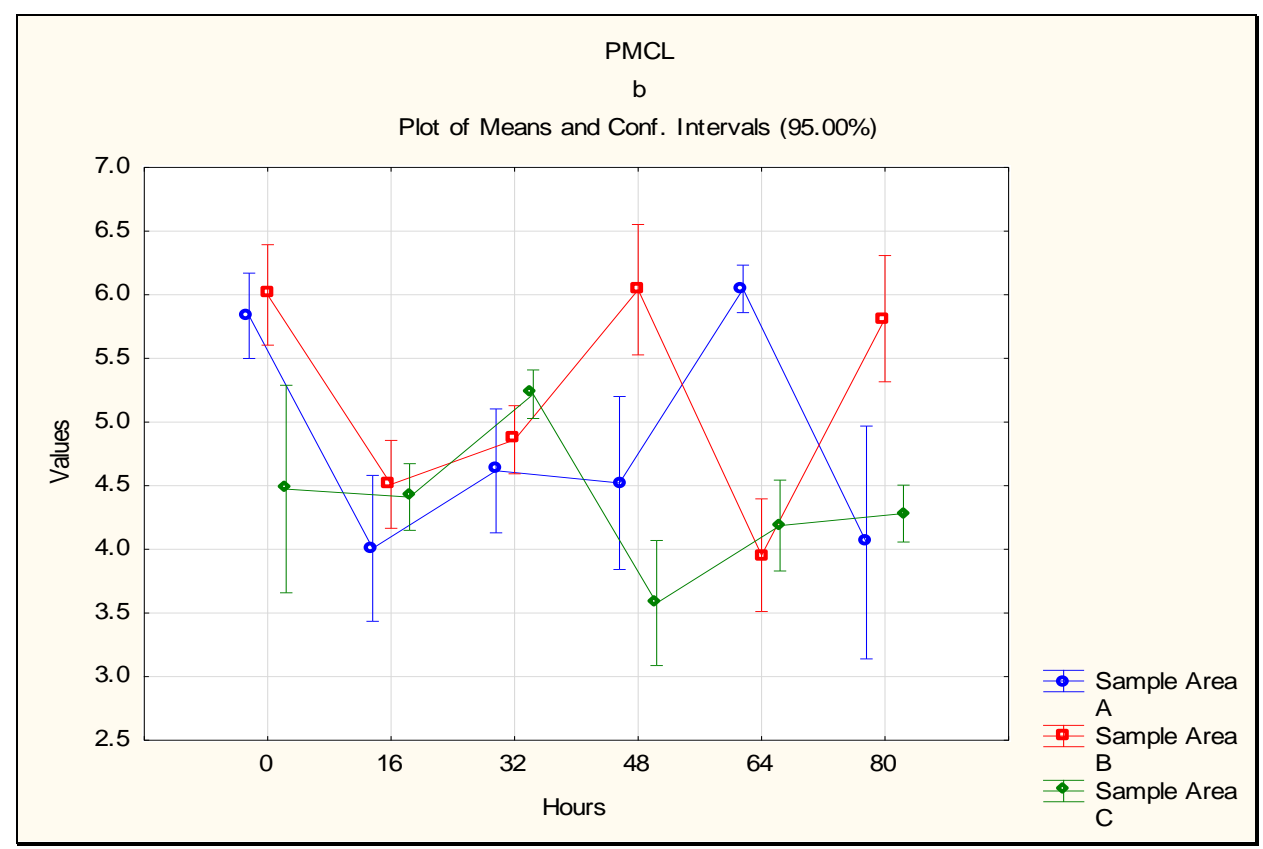

Figure 13.5: Interaction plots of 'Hours* Sample Area' for sample PMJX at each variable

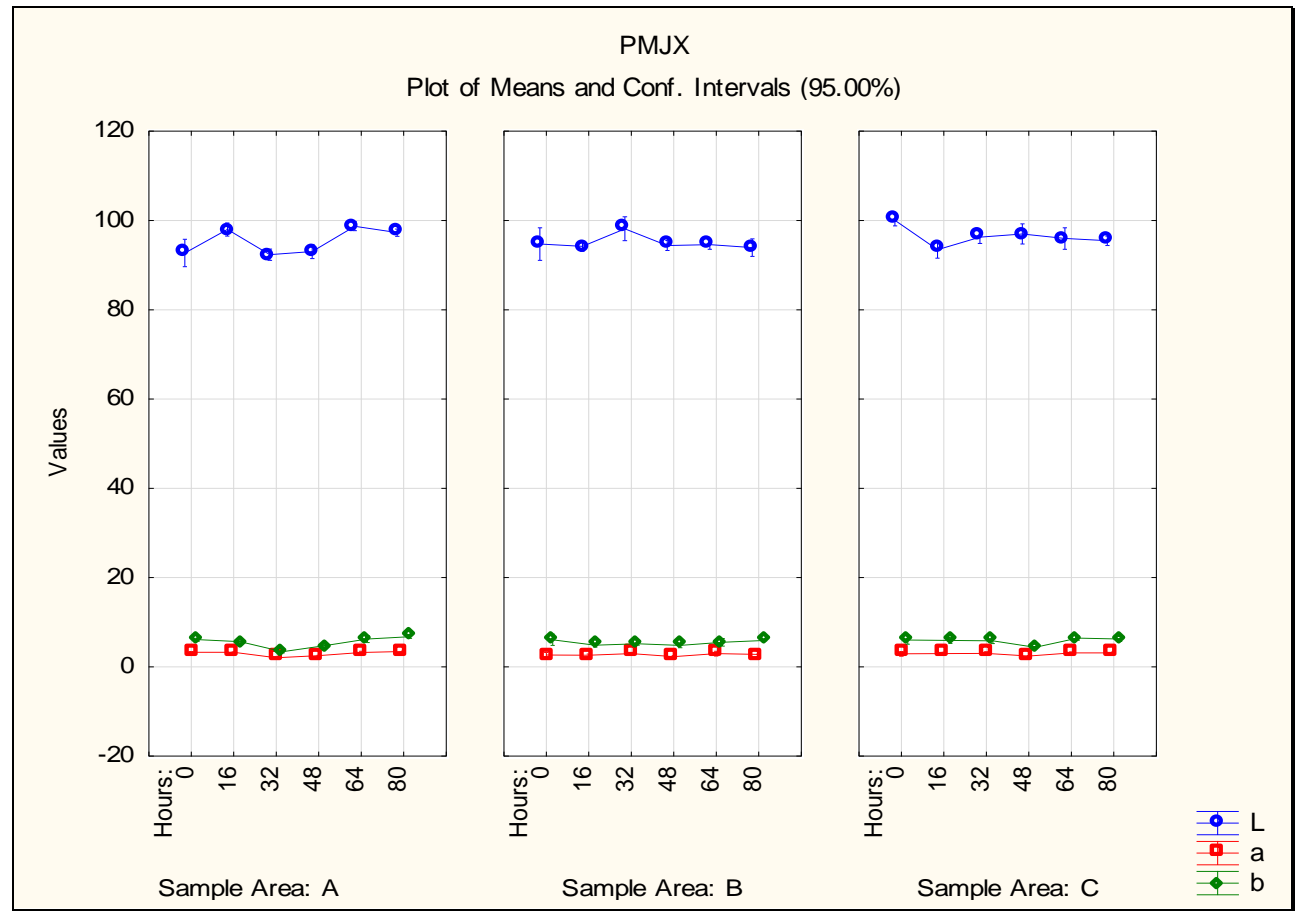



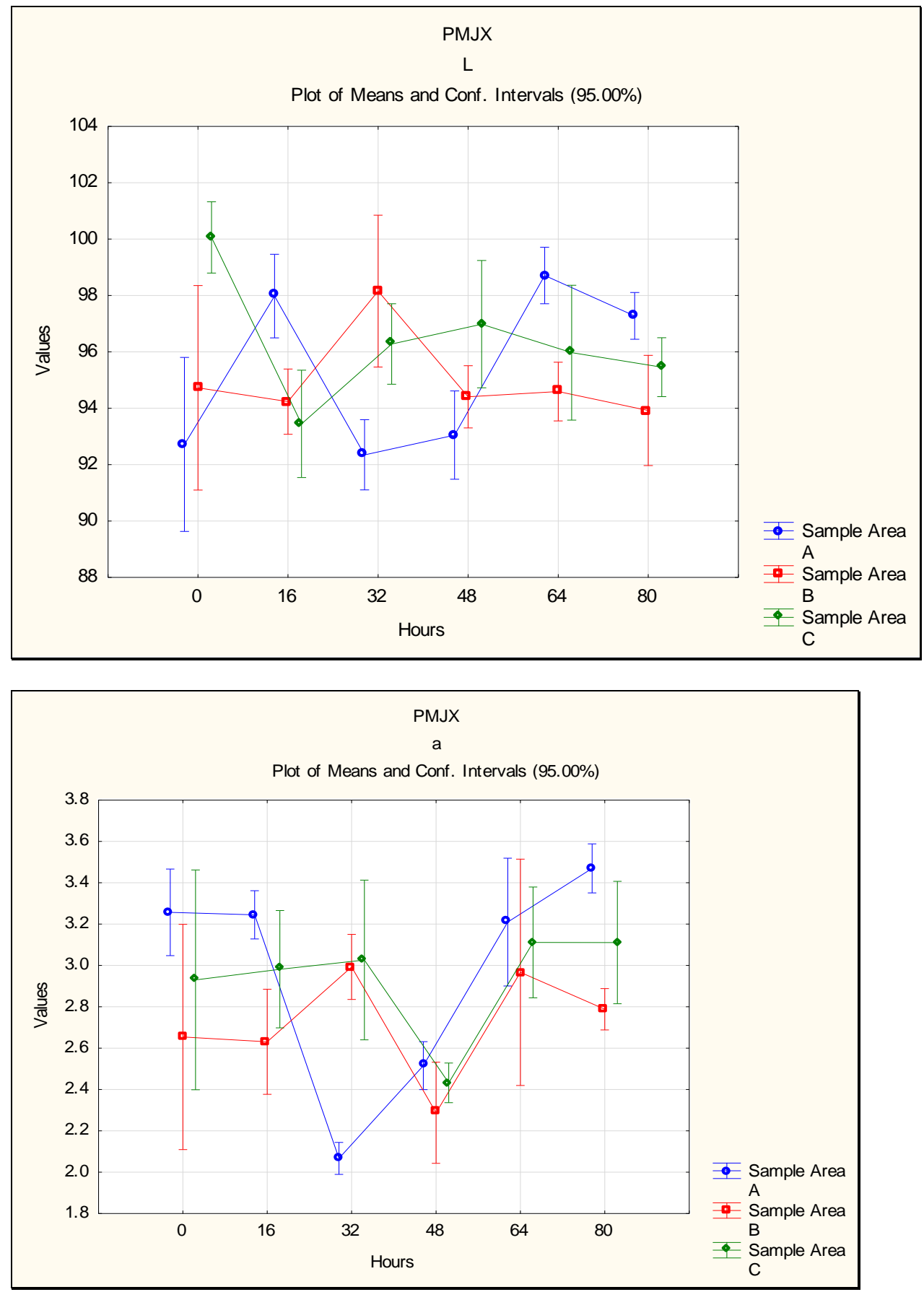
Figure 13.6: Interaction plots of 'Hours* Sample Area' for sample PMP at each variable
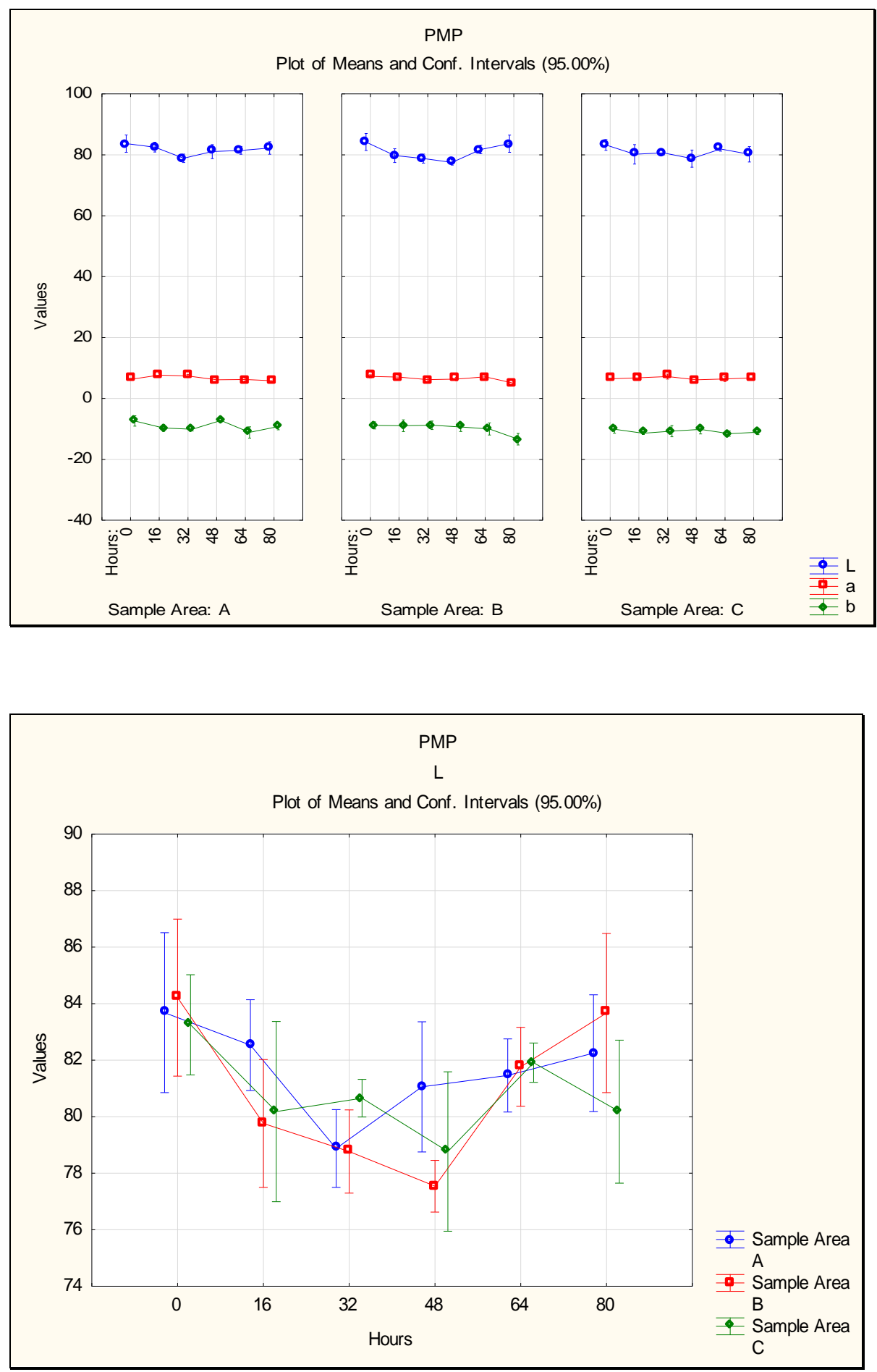

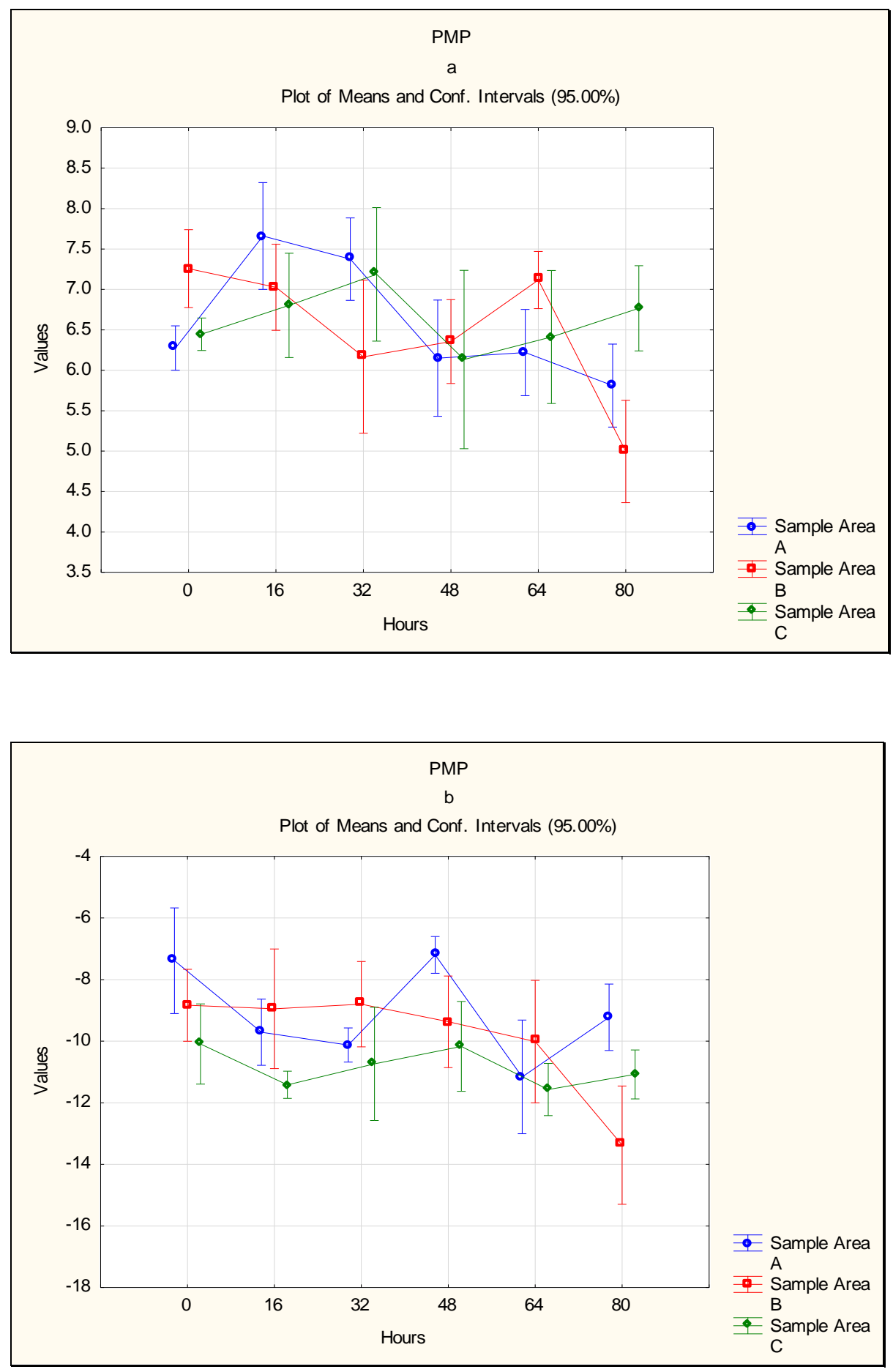
Figure 13.7: Interaction plots of 'Hours* Sample Area' for sample RMP at each variable
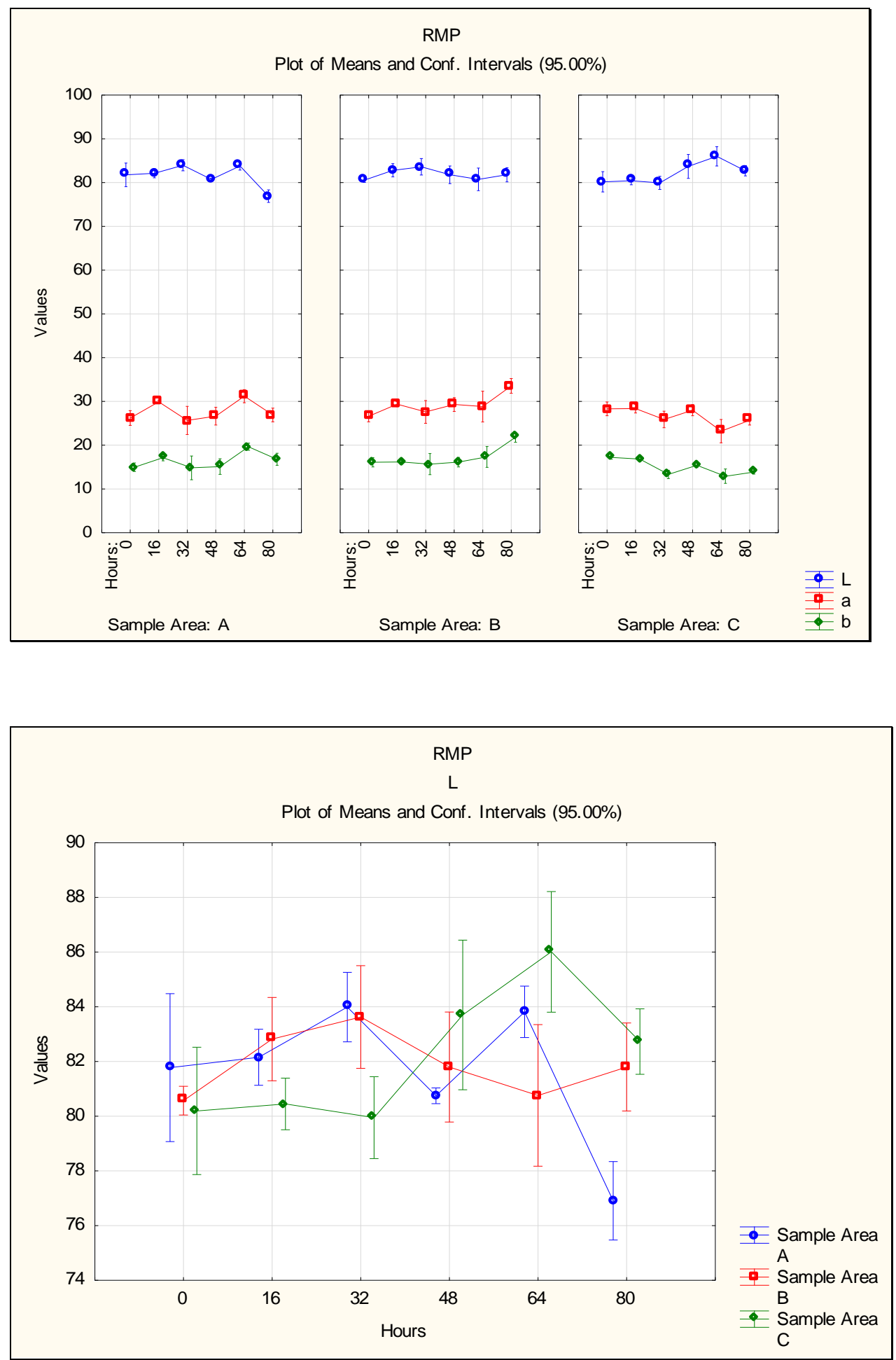

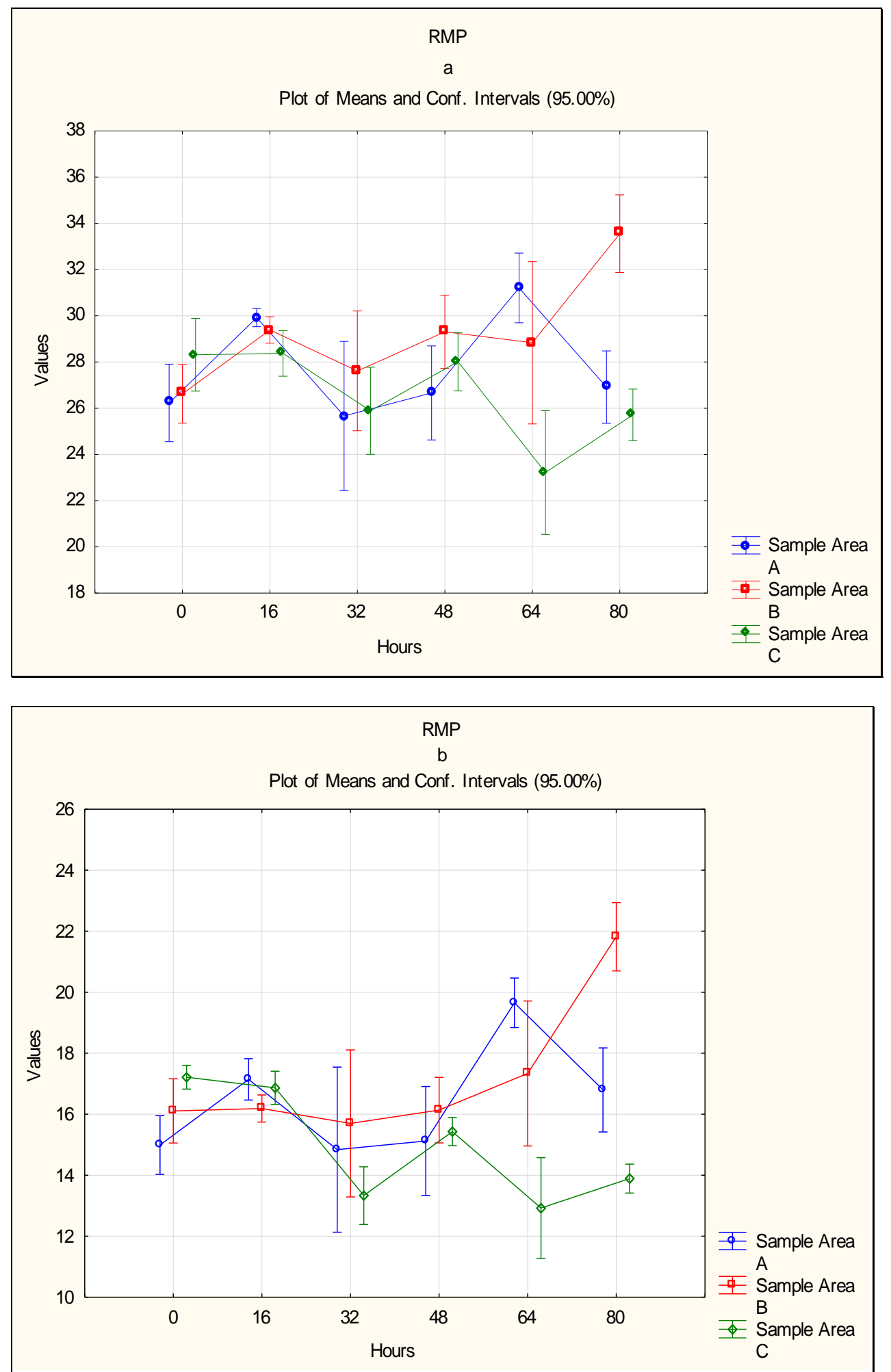
14. ANOVA Output for Washed-Aged Results

Figure 14.1 ANOVA Output for Washed-Aged Results for sample DPMP

Estimates: DPMP

Dependent Variable: a

\begin{tabular}{|l|r|r|r|r|}
\hline & & \multicolumn{2}{|c|}{$95 \%$ Confidence Interval } \\
\cline { 4 - 5 } Swatch & Mean & Std. Error & Lower Bound & Upper Bound \\
\hline 1 w/ bleach & $26.438^{\mathrm{a}}$ & 0.244 & 25.959 & 26.917 \\
2 w/ bleach & $24.504^{\mathrm{a}}$ & 0.244 & 24.025 & 24.983 \\
3 w/o bleach & $24.733^{\mathrm{a}}$ & 0.244 & 24.255 & 25.212 \\
4 w/o bleach & $25.339^{\mathrm{a}}$ & 0.244 & 24.86 & 25.818 \\
\hline
\end{tabular}

a. Based on modified population marginal mean.

\section{Pairwise Comparisons: DPMP}

Dependent Variable: a

\begin{tabular}{|c|c|c|c|c|c|c|}
\hline \multirow[b]{2}{*}{ (I) Swatch } & \multirow[b]{2}{*}{ (J) Swatch } & \multirow{2}{*}{$\begin{array}{c}\text { Mean } \\
\text { Difference (I-J) }\end{array}$} & \multirow{2}{*}{$\begin{array}{l}\text { Std. } \\
\text { Error }\end{array}$} & \multirow[b]{2}{*}{ Sig. $^{\mathrm{c}}$} & \multicolumn{2}{|c|}{$\begin{array}{c}95 \% \text { Confidence Interval for } \\
\text { Difference }\end{array}$} \\
\hline & & & & & Lower Bound & Upper Bound \\
\hline \multirow[t]{3}{*}{$1 \mathrm{w} /$ bleach } & 2 w/ bleach & $1.934^{x}, a, b$ & 0.345 & 0 & 1.257 & 2.611 \\
\hline & 3 w/o bleach & $1.705^{x, a, b}$ & 0.345 & 0 & 1.028 & 2.382 \\
\hline & 4 w/o bleach & $1.099^{\pi}, \mathrm{a}, \mathrm{b}$ & 0.345 & 0.001 & 0.422 & 1.776 \\
\hline \multirow[t]{3}{*}{$2 \mathrm{w} / \mathrm{bleach}$} & $1 \mathrm{w} /$ bleach & $-1.934^{\circ}, a, b$ & 0.345 & 0 & -2.611 & -1.257 \\
\hline & 3 w/o bleach & $-.229^{a, b}$ & 0.345 & 0.507 & -0.906 & 0.448 \\
\hline & 4 w/o bleach & $-.835^{\pi, a, b}$ & 0.345 & 0.016 & -1.512 & -0.158 \\
\hline \multirow{3}{*}{$\begin{array}{l}3 \mathrm{w} / \mathrm{o} \\
\text { bleach }\end{array}$} & $1 \mathrm{w} /$ bleach & $-1.705^{\circ, a, b}$ & 0.345 & 0 & -2.382 & $\begin{array}{l}-1.028 \\
\end{array}$ \\
\hline & 2 w/ bleach & $.229^{\mathrm{a}, \mathrm{b}}$ & 0.345 & 0.507 & -0.448 & 0.906 \\
\hline & 4 w/o bleach & $-.606^{a, b}$ & 0.345 & 0.08 & -1.283 & 0.072 \\
\hline \multirow{3}{*}{$\begin{array}{l}4 \mathrm{w} / 0 \\
\text { bleach }\end{array}$} & $1 \mathrm{w} /$ bleach & $-1.099^{\prime, a, b}$ & 0.345 & 0.001 & -1.776 & -0.422 \\
\hline & $2 \mathrm{w} / \mathrm{bleach}$ & $.835^{x, a, b}$ & 0.345 & 0.016 & 0.158 & 1.512 \\
\hline & 3 w/o bleach & $.606^{\mathrm{a}, \mathrm{b}}$ & 0.345 & 0.08 & -0.072 & 1.283 \\
\hline
\end{tabular}

Based on estimated marginal means

*. The mean difference is significant at the .05 level.

a. An estimate of the modified population marginal mean (I).

b. An estimate of the modified population marginal mean $(\mathrm{J})$.

C. Adjustment for multiple comparisons: Least Significant Difference (equivalent to no adjustments).

\section{Estimates: DPMP}

Dependent Variable: $b$

\begin{tabular}{|l|r|r|r|r|}
\hline \multirow{2}{*}{ Swatch } & & \multicolumn{2}{|c|}{$95 \%$ Confidence Interval } \\
\cline { 4 - 5 } & Mean & Std. Error & Lower Bound & Upper Bound \\
\hline 1 w/ bleach & $7.793^{\mathrm{a}}$ & 0.135 & 7.529 & 8.058 \\
2 w/ bleach & $7.119^{\mathrm{a}}$ & 0.135 & 6.854 & 7.384 \\
3 w/o bleach & $7.168^{\mathrm{a}}$ & 0.135 & 6.903 & 7.433 \\
4 w/o bleach & $7.190^{\mathrm{a}}$ & 0.135 & 6.925 & 7.454 \\
\hline
\end{tabular}

a. Based on modified population marginal mean. 
Pairwise Comparisons: DPMP

Dependent Variable: $b$

\begin{tabular}{|c|c|c|c|c|c|c|}
\hline \multirow[b]{2}{*}{ (I) Swatch } & \multirow[b]{2}{*}{ (J) Swatch } & \multirow{2}{*}{$\begin{array}{c}\text { Mean } \\
\text { Difference (I-J) }\end{array}$} & \multirow[b]{2}{*}{ Std. Error } & \multirow[b]{2}{*}{ Sig. ${ }^{c}$} & \multicolumn{2}{|c|}{$\begin{array}{c}\text { 95\% Confidence Interval for } \\
\text { Difference }\end{array}$} \\
\hline & & & & & Lower Bound & Upper Bound \\
\hline \multirow[t]{3}{*}{$1 \mathrm{w} /$ bleach } & $2 \mathrm{w} /$ bleach & $.675^{\star, a, b}$ & 0.191 & 0.000 & 0.3 & 1.049 \\
\hline & 3 w/o bleach & $.626^{x, a, b}$ & 0.191 & 0.001 & 0.251 & 1 \\
\hline & $4 \mathrm{w} / \mathrm{o}$ bleach & $.604^{\star, a, b}$ & 0.191 & 0.002 & 0.229 & 0.978 \\
\hline \multirow[t]{3}{*}{$2 \mathrm{w} / \mathrm{bleach}$} & $1 \mathrm{w} /$ bleach & $-.675^{, a, 0}$ & 0.191 & 0.000 & -1.049 & -0.3 \\
\hline & 3 w/o bleach & $-.049^{a, b}$ & 0.191 & 0.798 & -0.423 & 0.326 \\
\hline & 4 w/o bleach & $-.071^{a, b}$ & 0.191 & 0.711 & -0.445 & 0.304 \\
\hline \multirow[t]{3}{*}{3 w/o bleach } & $1 \mathrm{w} /$ bleach & $-.626, a, b$ & 0.191 & 0.001 & -1 & -0.251 \\
\hline & $2 \mathrm{w} /$ bleach & $.049^{\mathrm{a}, \mathrm{b}}$ & 0.191 & 0.798 & -0.326 & 0.423 \\
\hline & 4 w/o bleach & $-.022^{a, b}$ & 0.191 & 0.909 & -0.396 & 0.353 \\
\hline \multirow[t]{3}{*}{4 w/o bleach } & $1 \mathrm{w} /$ bleach & $-.604^{, a, b}$ & 0.191 & 0.002 & -0.978 & -0.229 \\
\hline & $2 \mathrm{w} /$ bleach & $.071^{\mathrm{a}, \mathrm{b}}$ & 0.191 & 0.711 & -0.304 & 0.445 \\
\hline & 3 w/o bleach & $.022^{\mathrm{a}, \mathrm{b}}$ & 0.191 & 0.909 & -0.353 & 0.396 \\
\hline
\end{tabular}

Based on estimated marginal means

*. The mean difference is significant at the .05 level.

a. An estimate of the modified population marginal mean (I).

b. An estimate of the modified population marginal mean $(\mathrm{J})$.

c. Adjustment for multiple comparisons: Least Significant Difference (equivalent to no adjustments).

\section{Sample Area * Swatch: DPMP}

Dependent Variable: $b$

\begin{tabular}{|ll|r|r|r|r|}
\hline & & & \multicolumn{2}{|c|}{$95 \%$ Confidence Interval } \\
\cline { 4 - 6 } Sample Area & Swatch & Mean & Std. Error & Lower Bound & Upper Bound \\
\hline A & $1 \mathrm{w} /$ bleach & $7.530^{\mathrm{a}}$ & 0.183 & 7.171 & 7.888 \\
& $2 \mathrm{w} /$ bleach & $7.493^{\mathrm{a}}$ & 0.2 & 7.1 & 7.886 \\
& $3 \mathrm{w} / \mathrm{o}$ bleach & $7.331^{\mathrm{a}}$ & 0.183 & 6.973 & 7.69 \\
& $4 \mathrm{w} / \mathrm{o}$ bleach & $7.623^{\mathrm{a}}$ & 0.2 & 7.23 & 8.015 \\
\hline B & $1 \mathrm{w} /$ bleach & $8.110^{\mathrm{a}}$ & 0.2 & 7.717 & 8.502 \\
& $2 \mathrm{w} /$ bleach & $6.807^{\mathrm{a}}$ & 0.183 & 6.449 & 7.166 \\
& $3 \mathrm{w} / \mathrm{o}$ bleach & $6.972^{\mathrm{a}}$ & 0.2 & 6.579 & 7.365 \\
& $4 \mathrm{w} / \mathrm{o}$ bleach & $6.829^{\mathrm{a}}$ & 0.183 & 6.47 & 7.187 \\
\hline
\end{tabular}

a. Based on modified population marginal mean. 
Figure 14.2 ANOVA Output for Washed-Aged Results for sample OSDP

\section{Sample Area * Swatch: OSDP}

Dependent Variable: $\mathrm{L}$

\begin{tabular}{|lr|r|r|r|r|}
\hline \multirow{2}{*}{ Sample Area } & & & \multicolumn{2}{|c|}{$95 \%$ Confidence Interval } \\
\cline { 5 - 6 } & & Mean & Std. Error & Lower Bound & \multicolumn{1}{c|}{ Upper Bound } \\
\hline A & 1 w/ bleach & $92.141^{\mathrm{a}}$ & .290 & 91.572 & 92.709 \\
& 2 w/ bleach & $90.864^{\mathrm{a}}$ & .318 & 90.241 & 91.488 \\
& 3 w/o bleach & $90.928^{\mathrm{a}}$ & .290 & 90.359 & 91.497 \\
$\mathrm{~N}$ & $91.495^{\mathrm{a}}$ & .318 & 90.872 & 92.118 \\
& 4 w/o bleach & $92.037^{\mathrm{a}}$ & .318 & 91.414 & 92.660 \\
& 1 w/ bleach & $91.227^{\mathrm{a}}$ & .290 & 90.658 & 91.796 \\
& 2 w/ bleach & 918 & 89.911 & 91.158 \\
& 3 w/o bleach & $90.534^{\mathrm{a}}$ & .318 & 90.726 & 91.863 \\
\hline
\end{tabular}

a. Based on modified population marginal mean.

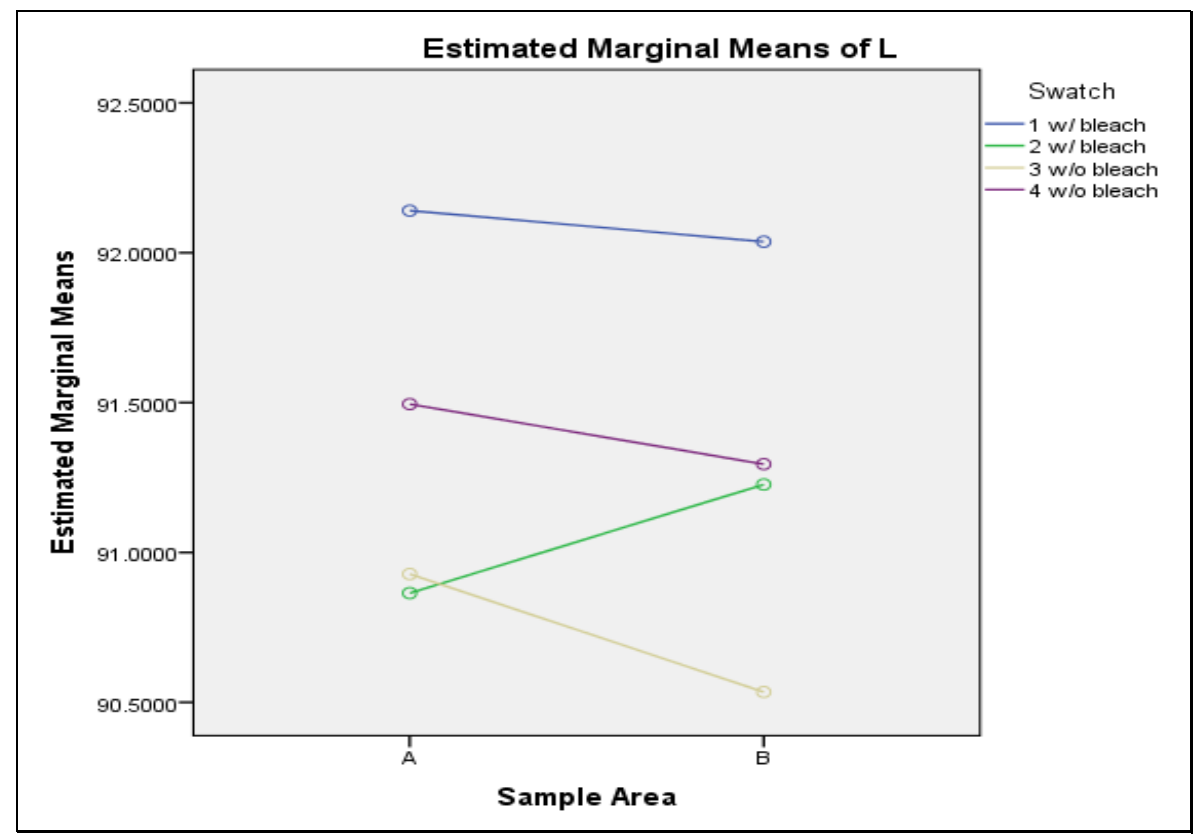

Variable ' $a$ '

\section{Sample Area * Swatch: OSDP}

Dependent Variable: a

\begin{tabular}{|c|c|c|c|c|c|}
\hline \multirow{2}{*}{\multicolumn{2}{|c|}{ Sample Area }} & \multirow[b]{2}{*}{ Mean } & \multirow[b]{2}{*}{ Std. Error } & \multicolumn{2}{|c|}{ 95\% Confidence Interval } \\
\hline & & & & Lower Bound & Upper Bound \\
\hline \multirow[t]{4}{*}{$\bar{A}$} & $1 \mathrm{w} /$ bleach & $9.481^{a}$ & .140 & 9.207 & 9.755 \\
\hline & $2 \mathrm{w} /$ bleach & $9.666^{\mathrm{a}}$ & .153 & 9.366 & 9.966 \\
\hline & 3 w/o bleach & $9.873^{\mathrm{a}}$ & .140 & 9.599 & 10.147 \\
\hline & $4 \mathrm{w} / \mathrm{o}$ bleach & $9.833^{a}$ & .153 & 9.533 & 10.133 \\
\hline \multirow[t]{4}{*}{ B } & $1 \mathrm{w} /$ bleach & $10.260^{\mathrm{a}}$ & .153 & 9.960 & 10.560 \\
\hline & $2 \mathrm{w} /$ bleach & $9.961^{\mathrm{a}}$ & .140 & 9.687 & 10.235 \\
\hline & 3 w/o bleach & $9.989^{\mathrm{a}}$ & .153 & 9.689 & 10.289 \\
\hline & 4 w/o bleach & $9.327^{\mathrm{a}}$ & .140 & 9.053 & 9.601 \\
\hline
\end{tabular}

a. Based on modified population marginal mean. 


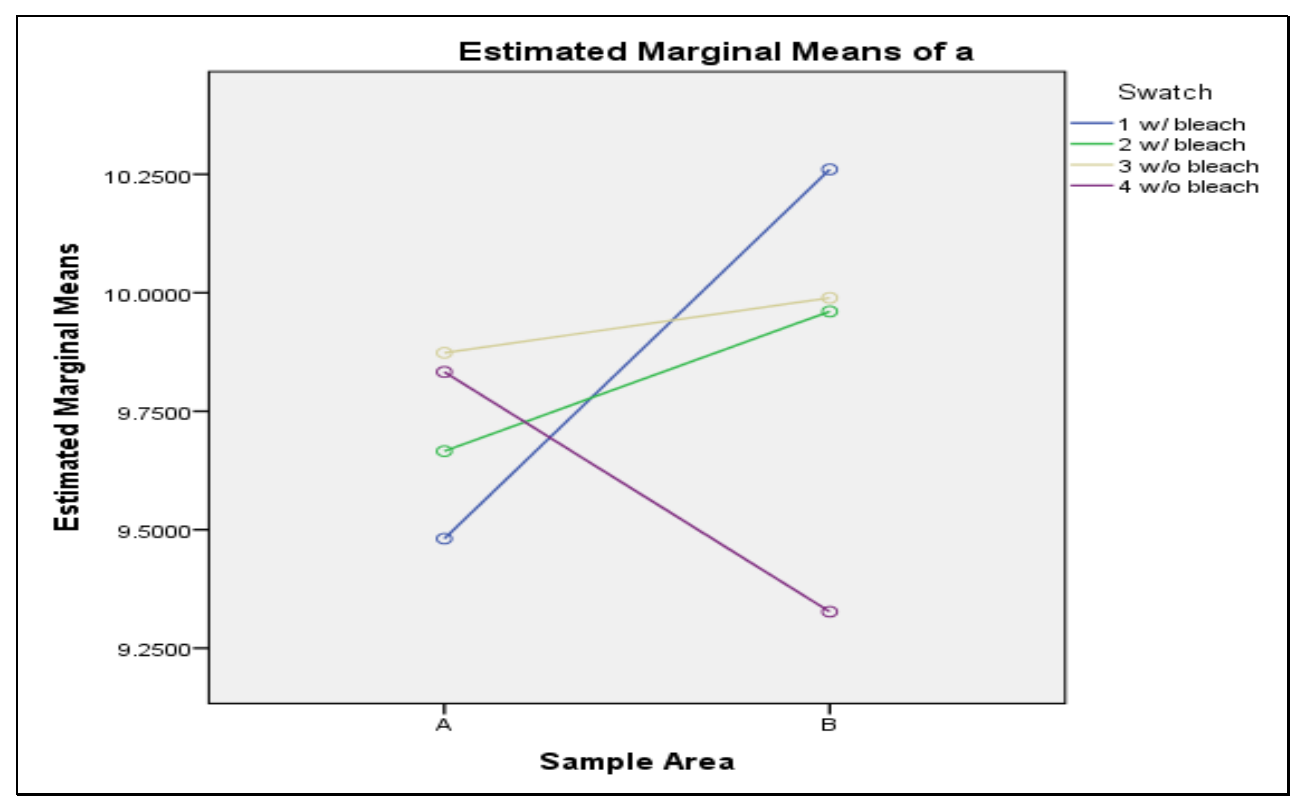

Variable ' $b$ '

\section{Sample Area * Swatch: OSDP}

Dependent Variable: $b$

\begin{tabular}{|c|c|c|c|c|c|}
\hline \multirow{2}{*}{\multicolumn{2}{|c|}{ Sample Area }} & \multirow[b]{2}{*}{ Mean } & \multirow[b]{2}{*}{ Std. Error } & \multicolumn{2}{|c|}{ 95\% Confidence Interval } \\
\hline & & & & Lower Bound & Upper Bound \\
\hline \multirow[t]{4}{*}{$\bar{A}$} & $1 \mathrm{w} /$ bleach & $30.096^{a}$ & .357 & 29.394 & 30.797 \\
\hline & 2 w/ bleach & $29.810^{a}$ & .392 & 29.042 & 30.579 \\
\hline & 3 w/o bleach & $30.577^{a}$ & .357 & 29.875 & 31.278 \\
\hline & $4 \mathrm{w} / \mathrm{o}$ bleach & $29.970^{\mathrm{a}}$ & .392 & 29.202 & 30.739 \\
\hline \multirow[t]{4}{*}{ B } & $1 \mathrm{w} /$ bleach & $30.873^{a}$ & .392 & 30.105 & 31.642 \\
\hline & $2 \mathrm{w} /$ bleach & $30.853^{a}$ & .357 & 30.151 & 31.554 \\
\hline & 3 w/o bleach & $30.534^{a}$ & .392 & 29.766 & 31.303 \\
\hline & 4 w/o bleach & $29.581^{a}$ & .357 & 28.879 & 30.282 \\
\hline
\end{tabular}

a. Based on modified population marginal mean.

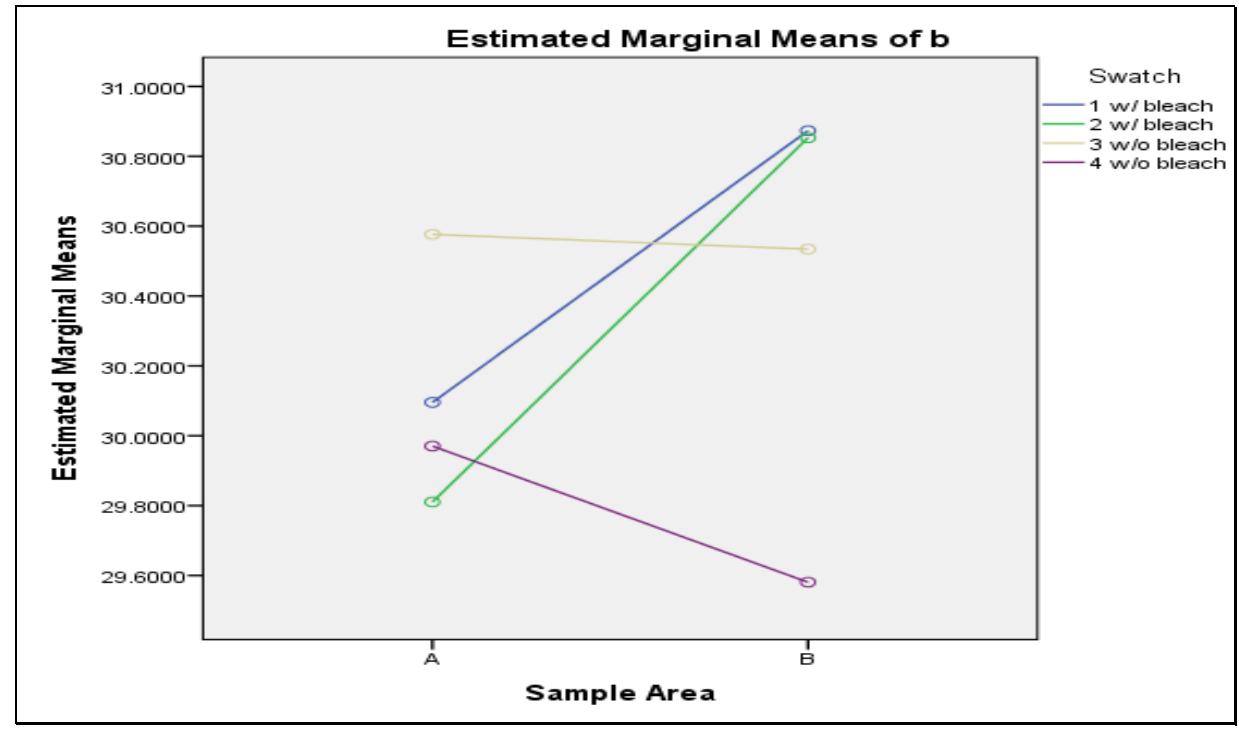


Figure 14.3 ANOVA Output for Washed-Aged Results for sample PMCL

\section{Variable ' $b$ '}

\section{Estimates: PMCL}

Dependent Variable: $b$

\begin{tabular}{|l|r|r|r|r|}
\hline & & \multicolumn{2}{|c|}{$95 \%$ Confidence Interval } \\
\cline { 4 - 5 } Sample Area & Mean & Std. Error & Lower Bound & Upper Bound \\
\hline A & $4.303^{\mathrm{a}}$ & .061 & 4.183 & 4.424 \\
B & $4.554^{\mathrm{a}}$ & .061 & 4.434 & 4.674 \\
\hline
\end{tabular}

a. Based on modified population marginal mean.

Pairwise Comparisons: PMCL

Dependent Variable: $b$

\begin{tabular}{|c|c|c|c|c|c|c|}
\hline \multirow[b]{2}{*}{ (I) Sample Area } & \multirow[b]{2}{*}{ (J) Sample Area } & \multirow[b]{2}{*}{ Mean Difference (I-J) } & \multirow[b]{2}{*}{ Std. Error } & \multirow[b]{2}{*}{ Sig. ${ }^{\mathrm{C}}$} & \multicolumn{2}{|c|}{$\begin{array}{l}\text { 95\% Confidence Interval for } \\
\text { Difference }\end{array}$} \\
\hline & & & & & Lower Bound & Upper Bound \\
\hline $\mathrm{A}$ & $\mathrm{B}$ & $-.251^{*}, \mathrm{a}, \mathrm{b}$ & .087 & .004 & -.421 & -.080 \\
\hline$B$ & $A$ & $.251^{*}, a, b$ & .087 & .004 & .080 & .421 \\
\hline
\end{tabular}

Based on estimated marginal means

*. The mean difference is significant at the .05 level.

a. An estimate of the modified population marginal mean (I).

b. An estimate of the modified population marginal mean $(\mathrm{J})$.

c. Adjustment for multiple comparisons: Least Significant Difference (equivalent to no adjustments).

\section{Swatch * Sample Area: PMCL}

Dependent Variable: $b$

\begin{tabular}{|ll|r|r|r|r|}
\hline & & & & \multicolumn{2}{|c|}{ 95\% Confidence Interval } \\
\cline { 5 - 6 } Swatch & Sample Area & Mean & Std. Error & Lower Bound & \multicolumn{1}{c|}{ Upper Bound } \\
\hline 1 w/ bleach & A & $3.820^{\mathrm{a}}$ & .117 & 3.589 & 4.050 \\
& $\mathrm{~B}$ & $3.375^{\mathrm{a}}$ & .129 & 3.122 & 3.627 \\
\hline $2 \mathrm{w} /$ bleach & $\mathrm{A}$ & $4.092^{\mathrm{a}}$ & .129 & 3.839 & 4.344 \\
& $\mathrm{~B}$ & $5.230^{\mathrm{a}}$ & .117 & 5.000 & 5.461 \\
\hline 3 w/o bleach & $\mathrm{A}$ & $4.623^{\mathrm{a}}$ & .117 & 4.393 & 4.853 \\
& $\mathrm{~B}$ & $4.611^{\mathrm{a}}$ & .129 & 4.359 & 4.864 \\
\hline 4 w/o bleach & $\mathrm{A}$ & $4.712^{\mathrm{a}}$ & .129 & 4.459 & 4.964 \\
& $\mathrm{~B}$ & $4.813^{\mathrm{a}}$ & .117 & 4.582 & 5.043 \\
\hline
\end{tabular}

a. Based on modified population marginal mean. 


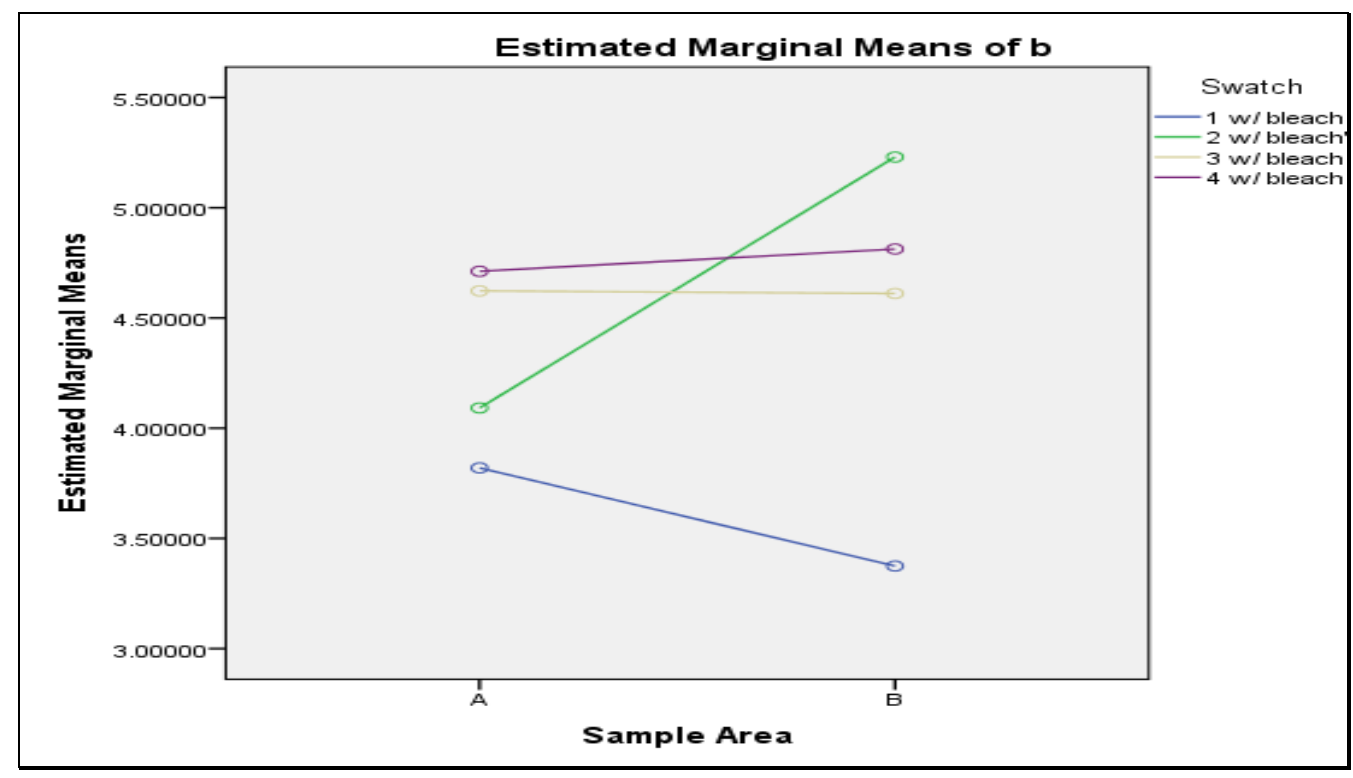

\section{Sample Area * Bleached}

Dependent Variable: $b$

\begin{tabular}{|ll|r|r|r|r|}
\hline \multirow{2}{*}{ Sample Area } & & & \multicolumn{2}{|c|}{ 95\% Confidence Interval } \\
\cline { 5 - 6 } & Bleached & Mean & Std. Error & Lower Bound & Upper Bound \\
\hline A & w/ bleach & $3.943^{\mathrm{a}}$ & .087 & 3.773 & 4.114 \\
& w/o bleach & $4.663^{\mathrm{a}}$ & .087 & 4.493 & 4.833 \\
\hline B & $4.387^{\mathrm{a}}$ & .087 & 4.217 & 4.557 \\
& w/ bleach & $4.721^{\mathrm{a}}$ & .087 & 4.551 & 4.891 \\
\hline
\end{tabular}

a. Based on modified population marginal mean.

10. Bleached * Washing: PMCL

Dependent Variable: $b$

\begin{tabular}{|ll|r|r|r|r|}
\hline \multirow{2}{*}{ Bleached } & & & \multicolumn{2}{|c|}{ 95\% Confidence Interval } \\
\cline { 4 - 6 } & Washing & Mean & Std. Error & Lower Bound & Upper Bound \\
\hline w/ bleach & 1W 0A & $4.372^{\mathrm{a}}$ & .203 & 3.973 & 4.771 \\
& 1W 16A & $4.415^{\mathrm{a}}$ & .144 & 4.133 & 4.697 \\
& 2W 32A & $3.738^{\mathrm{a}}$ & .144 & 3.456 & 4.020 \\
& 3W 48A & $3.786^{\mathrm{a}}$ & .144 & 3.504 & 4.069 \\
& 4W 64A & $4.709^{\mathrm{a}}$ & .144 & 4.427 & 4.991 \\
& 5W 80A & $4.074^{\mathrm{a}}$ & .144 & 3.792 & 4.357 \\
\hline w/0 bleach & 1W 0A & $4.421^{\mathrm{a}}$ & .203 & 4.022 & 4.820 \\
& 1W 16A & $4.621^{\mathrm{a}}$ & .144 & 4.339 & 4.904 \\
& 2W 32A & $4.660^{\mathrm{a}}$ & .144 & 4.378 & 4.942 \\
& 3W 48A & $4.565^{\mathrm{a}}$ & .144 & 4.283 & 4.847 \\
4W 64A & $5.157^{\mathrm{a}}$ & .144 & 4.875 & 5.440 \\
& 5W 80A & $4.592^{\mathrm{a}}$ & .144 & 4.310 & 4.874 \\
\hline
\end{tabular}

a. Based on modified population marginal mean. 


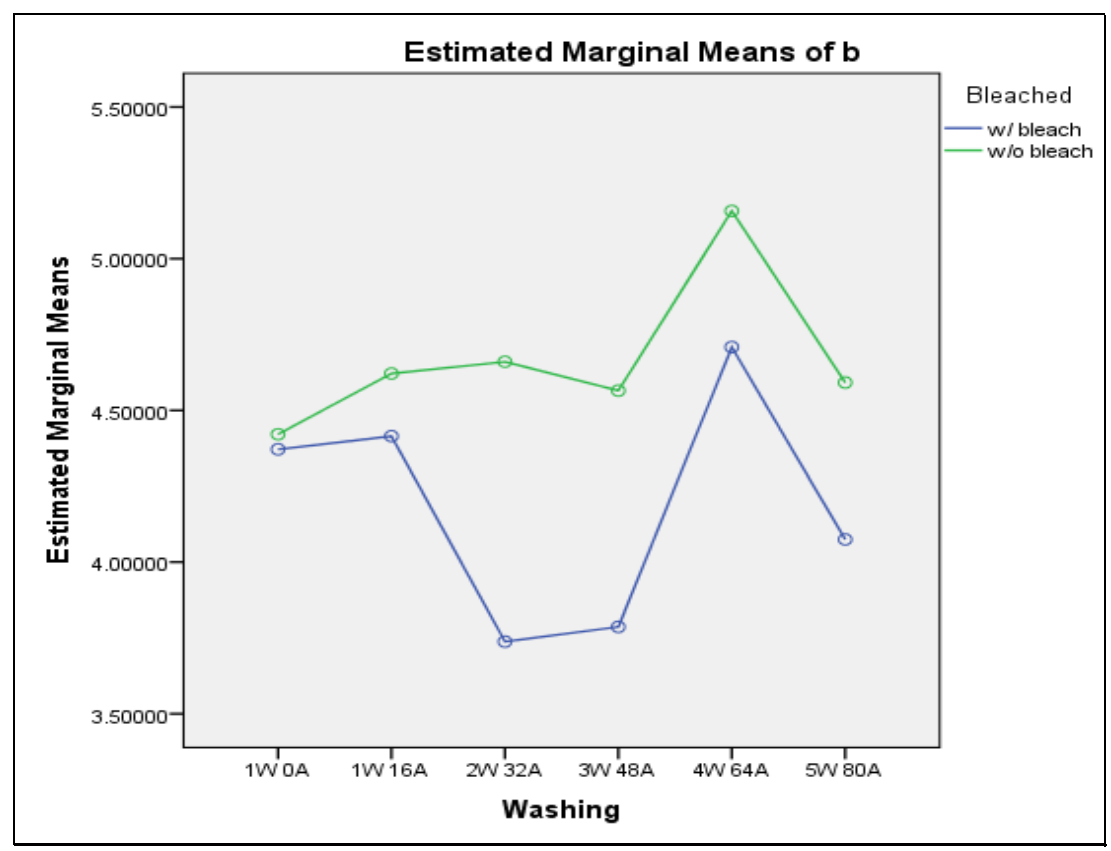

Table 14.4 ANOVA Output for Washed-Aged Results for sample PMJX

\section{Variable ' $b$ '}

\section{Estimates: PMJX}

Dependent Variable: $b$

\begin{tabular}{|l|r|r|r|r|}
\hline & & \multirow{2}{*}{ 95\% Confidence Interval } \\
\cline { 4 - 5 } Sample Area & Mean & Std. Error & Lower Bound & Upper Bound \\
\hline A & $5.123^{\mathrm{a}}$ & .061 & 5.003 & 5.243 \\
$\mathrm{~B}$ & $5.163^{\mathrm{a}}$ & .061 & 5.043 & 5.284 \\
\hline
\end{tabular}

a. Based on modified population marginal mean.

Dependent Variable: $b$

\section{Pairwise Comparisons: PMJX}

\begin{tabular}{|ll|r|r|r|r|r|}
\hline & & \multirow{2}{*}{$\begin{array}{c}\text { Mean } \\
\text { Difference } \\
\text { (I) Sample Area }\end{array}$} & & & \multicolumn{2}{|c|}{$\begin{array}{c}\text { 95\% Confidence Interval for } \\
\text { Difference }\end{array}$} \\
\cline { 5 - 8 } & & (I-J) & Std. Error & Sig. $^{\mathrm{c}}$ & Lower Bound & Upper Bound \\
\hline A & B & $-.040^{\mathrm{a}, \mathrm{b}}$ & .087 & .641 & -.211 & .130 \\
B & A & $.040^{\mathrm{a}, \mathrm{b}}$ & .087 & .641 & -.130 & .211 \\
\hline
\end{tabular}

Based on estimated marginal means

a. An estimate of the modified population marginal mean (I).

b. An estimate of the modified population marginal mean (J).

c. Adjustment for multiple comparisons: Least Significant Difference (equivalent to no adjustments). 
Dependent Variable: $b$

\section{Sample Area * Swatch: PMJX}

\begin{tabular}{|c|c|c|c|c|c|}
\hline \multirow[b]{2}{*}{ Sample Area } & & \multirow[b]{2}{*}{ Mean } & \multirow[b]{2}{*}{ Std. Error } & \multicolumn{2}{|c|}{ 95\% Confidence Interval } \\
\hline & & & & Lower Bound & Upper Bound \\
\hline \multirow[t]{4}{*}{$A$} & $1 \mathrm{w} /$ bleach & $5.059^{\mathrm{a}}$ & .117 & 4.829 & 5.290 \\
\hline & $2 \mathrm{w} /$ bleach & $5.765^{\mathrm{a}}$ & .129 & 5.512 & 6.017 \\
\hline & 3 w/o bleach & $4.883^{a}$ & .117 & 4.653 & 5.114 \\
\hline & 4 w/o bleach & $4.845^{\mathrm{a}}$ & .129 & 4.592 & 5.097 \\
\hline \multirow[t]{4}{*}{ B } & $1 \mathrm{w} /$ bleach & $4.581^{a}$ & .129 & 4.329 & 4.834 \\
\hline & $2 \mathrm{w} /$ bleach & $5.161^{a}$ & .117 & 4.930 & 5.392 \\
\hline & 3 w/o bleach & $5.712^{a}$ & .129 & 5.459 & 5.965 \\
\hline & 4 w/o bleach & $5.194^{\mathrm{a}}$ & .117 & 4.964 & 5.425 \\
\hline
\end{tabular}

a. Based on modified population marginal mean.

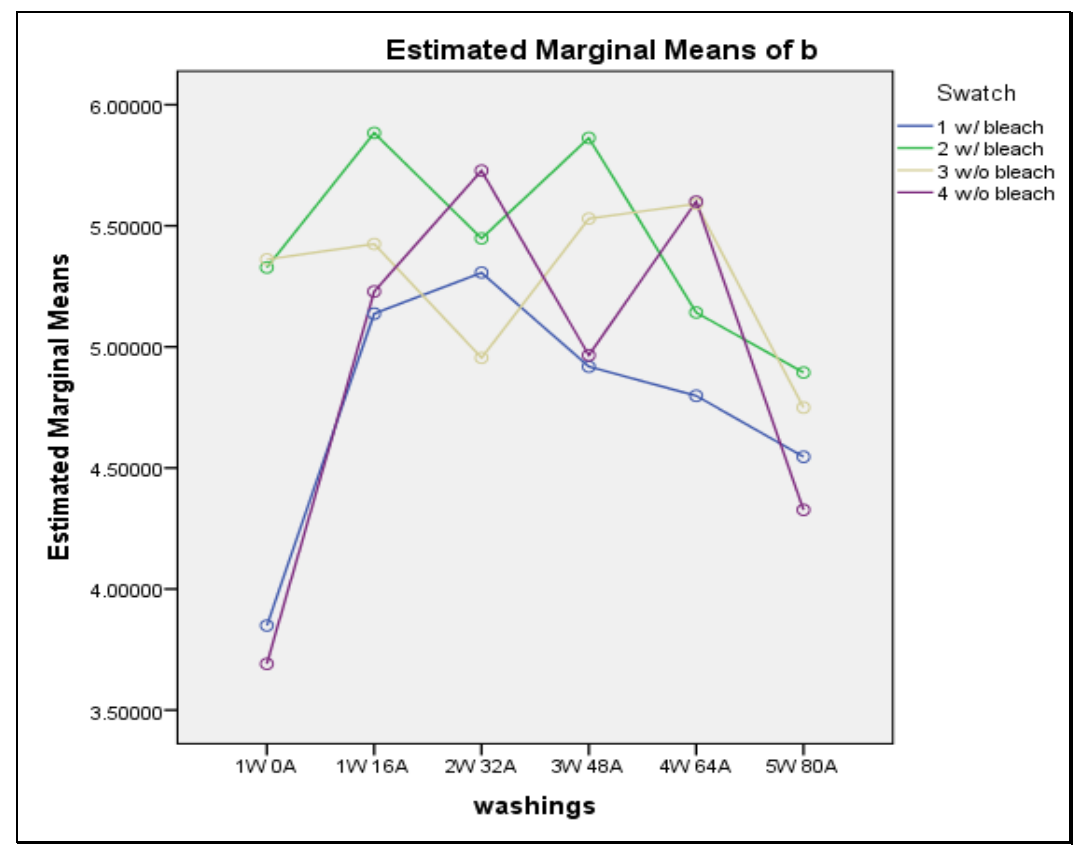

6. Sample Area * bleached: PMJX

Dependent Variable: $b$

\begin{tabular}{|c|c|c|c|c|c|}
\hline \multirow{2}{*}{\multicolumn{2}{|c|}{ Sample Area }} & \multirow[b]{2}{*}{ Mean } & \multirow[b]{2}{*}{ Std. Error } & \multicolumn{2}{|c|}{$95 \%$ Confidence Interval } \\
\hline & & & & Lower Bound & Upper Bound \\
\hline \multirow[t]{2}{*}{$\bar{A}$} & w/ bleach & $5.380^{\mathrm{a}}$ & .087 & 5.210 & 5.550 \\
\hline & w/o bleach & $4.866^{\mathrm{a}}$ & .087 & 4.696 & 5.036 \\
\hline \multirow[t]{2}{*}{ B } & w/ bleach & $4.897^{a}$ & .087 & 4.727 & 5.068 \\
\hline & w/o bleach & $5.429^{a}$ & .087 & 5.259 & 5.600 \\
\hline
\end{tabular}

a. Based on modified population marginal mean. 


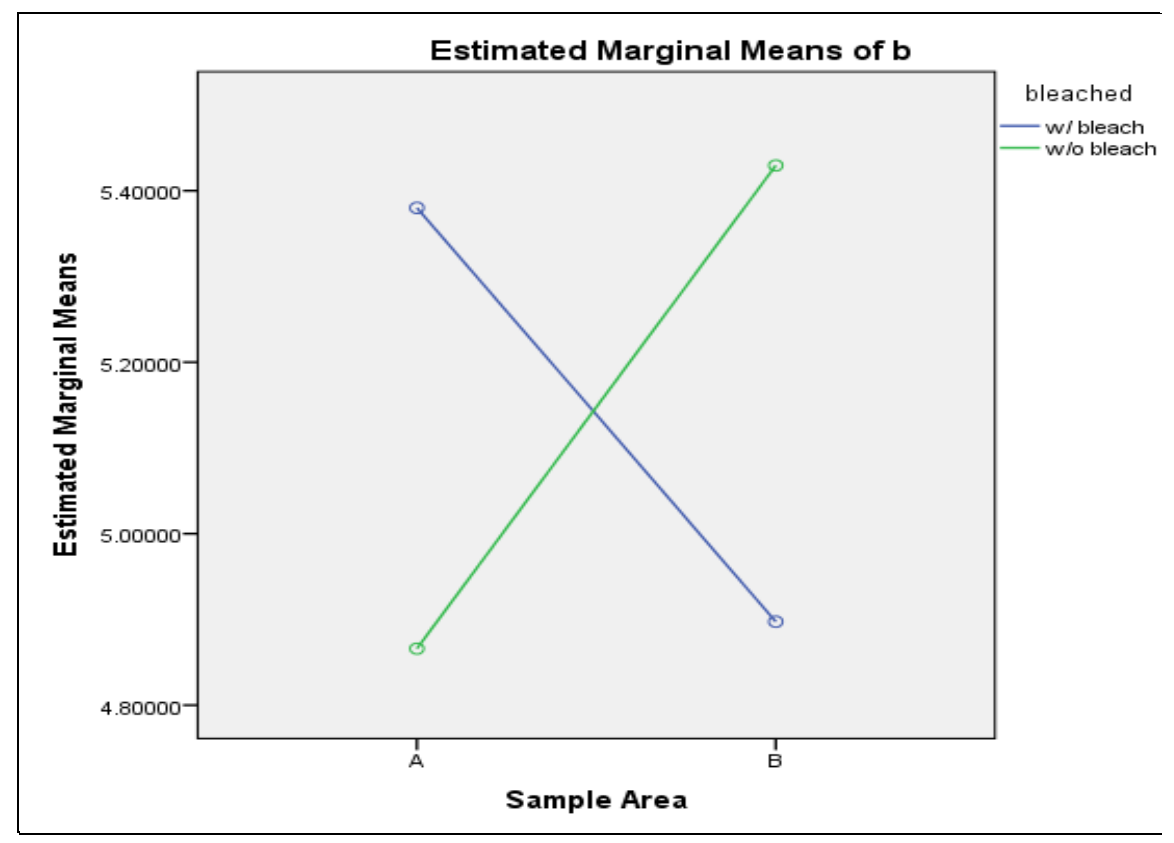

7. bleached * washings: PMJX

Dependent Variable: $b$

\begin{tabular}{|c|c|c|c|c|c|}
\hline \multirow[b]{2}{*}{ bleached } & & \multirow[b]{2}{*}{ Mean } & \multirow[b]{2}{*}{ Std. Error } & \multicolumn{2}{|c|}{ 95\% Confidence Interval } \\
\hline & & & & Lower Bound & $\begin{array}{l}\text { Upper } \\
\text { Bound }\end{array}$ \\
\hline \multirow[t]{6}{*}{ w/ bleach } & $1 \mathrm{~W} 0 \mathrm{~A}$ & $4.589^{\mathrm{a}}$ & .203 & 4.189 & 4.988 \\
\hline & $1 \mathrm{~W} 16 \mathrm{~A}$ & $5.510^{\mathrm{a}}$ & .144 & 5.228 & 5.793 \\
\hline & $2 W 32 A$ & $5.377^{\mathrm{a}}$ & .144 & 5.095 & 5.660 \\
\hline & $3 W 48 A$ & $5.391^{\mathrm{a}}$ & .144 & 5.108 & 5.673 \\
\hline & $4 \mathrm{~W} 64 \mathrm{~A}$ & $4.970^{\mathrm{a}}$ & .144 & 4.688 & 5.253 \\
\hline & $5 W 80 A$ & $4.720^{\mathrm{a}}$ & .144 & 4.438 & 5.002 \\
\hline \multirow[t]{6}{*}{ w/o bleach } & $1 \mathrm{~W} 0 \mathrm{~A}$ & $4.526^{a}$ & .203 & 4.127 & 4.925 \\
\hline & $1 \mathrm{~W} 16 \mathrm{~A}$ & $5.327^{\mathrm{a}}$ & .144 & 5.045 & 5.609 \\
\hline & $2 W 32 A$ & $5.341^{a}$ & .144 & 5.059 & 5.624 \\
\hline & $3 W 48 A$ & $5.248^{a}$ & .144 & 4.965 & 5.530 \\
\hline & $4 W 64 A$ & $5.595^{\mathrm{a}}$ & .144 & 5.313 & 5.878 \\
\hline & $5 W 80 A$ & $4.538^{\mathrm{a}}$ & .144 & 4.256 & 4.820 \\
\hline
\end{tabular}

a. Based on modified population marginal mean. 


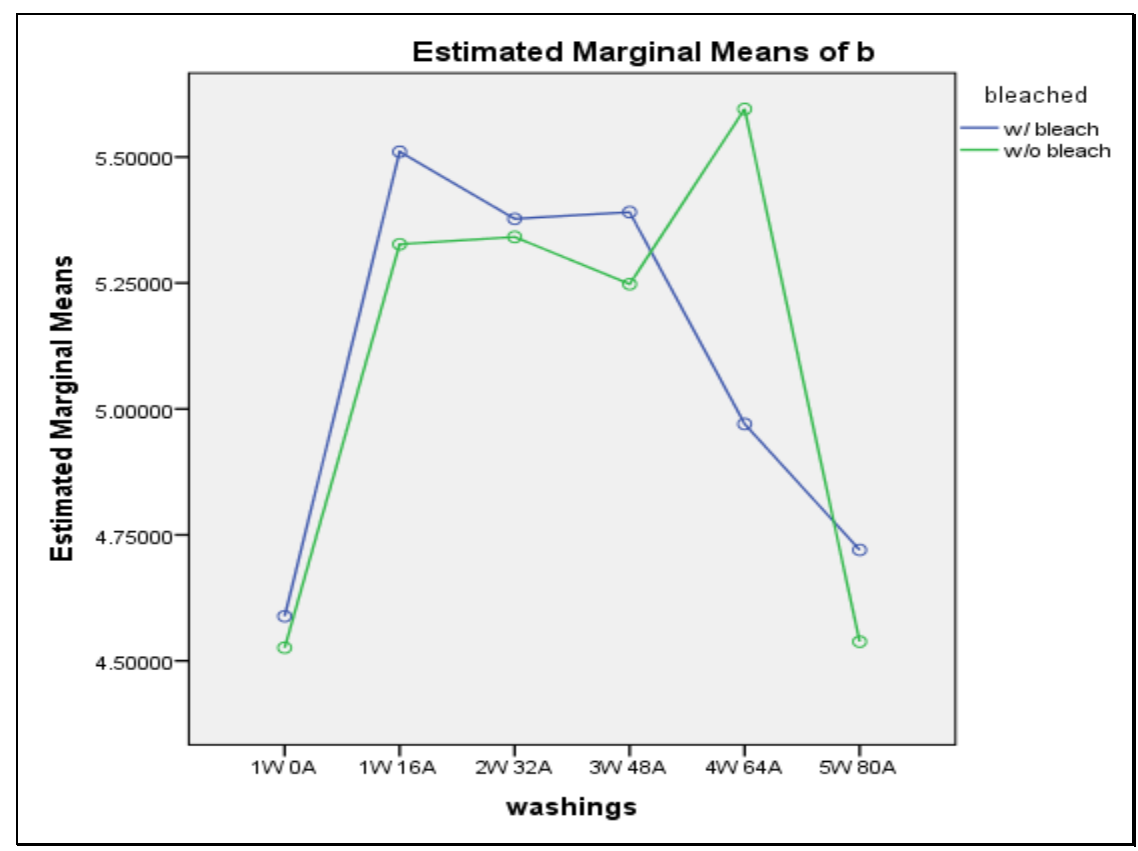

Table 14.5 ANOVA Output for Washed-Aged Results for sample PMP

Variable ' $L$ '

Dependent Variable: L

\section{Estimates: PMP}

\begin{tabular}{|c|c|c|c|c|}
\hline \multirow[b]{2}{*}{ Sample Area } & \multirow[b]{2}{*}{ Mean } & \multirow[b]{2}{*}{ Std. Error } & \multicolumn{2}{|c|}{$\begin{array}{c}\text { 95\% Confidence } \\
\text { Interval }\end{array}$} \\
\hline & & & $\begin{array}{l}\text { Lower } \\
\text { Bound } \\
\end{array}$ & $\begin{array}{l}\text { Upper } \\
\text { Bound } \\
\end{array}$ \\
\hline $\mathrm{A}$ & $83.757^{\mathrm{a}}$ & .188 & 83.388 & 84.126 \\
\hline B & $83.691^{\mathrm{a}}$ & .188 & 83.322 & 84.060 \\
\hline
\end{tabular}

a. Based on modified population marginal mean.

Pairwise Comparisons: PMP

Dependent Variable: $\mathrm{L}$

\begin{tabular}{|c|c|c|c|c|c|c|}
\hline \multirow{2}{*}{\multicolumn{2}{|c|}{ (I) Sample Area }} & \multirow{2}{*}{$\begin{array}{c}\text { Mean } \\
\text { Difference } \\
(\mathrm{I}-\mathrm{J})\end{array}$} & \multirow[b]{2}{*}{ Std. Error } & \multirow[b]{2}{*}{ Sig. ${ }^{c}$} & \multicolumn{2}{|c|}{$\begin{array}{l}\text { 95\% Confidence Interval } \\
\text { for Difference }\end{array}$} \\
\hline & & & & & $\begin{array}{l}\text { Lower } \\
\text { Bound }\end{array}$ & $\begin{array}{l}\text { Upper } \\
\text { Bound }\end{array}$ \\
\hline & $\mathrm{B}$ & $.066^{\mathrm{a}, \mathrm{o}}$ & .266 & .805 & -.456 & .588 \\
\hline B & $A$ & $-.066^{a, b}$ & .266 & .805 & -.588 & .456 \\
\hline
\end{tabular}

Based on estimated marginal means

a. An estimate of the modified population marginal mean (I).

b. An estimate of the modified population marginal mean (J).

c. Adjustment for multiple comparisons: Least Significant Difference (equivalent to no adjustments). 


\section{Estimates: PMP}

Dependent Variable: $\mathrm{L}$

\begin{tabular}{|l|r|r|r|r|}
\hline & & \multirow{2}{*}{ 95\% Confidence Interval } \\
\cline { 4 - 5 } Bleached & Mean & Std. Error & Lower Bound & Upper Bound \\
\hline w/ bleach & $84.384^{\mathrm{a}}$ & .188 & 84.014 & 84.753 \\
w/o bleach & $83.064^{\mathrm{a}}$ & .188 & 82.695 & 83.434 \\
\hline
\end{tabular}

a. Based on modified population marginal mean.

\section{Pairwise Comparisons: PMP}

Dependent Variable: L

\begin{tabular}{|c|c|c|c|c|c|c|}
\hline \multirow[b]{2}{*}{ (I) Bleached } & & \multirow{2}{*}{$\begin{array}{c}\text { Mean } \\
\text { Difference (I- } \\
\mathrm{J})\end{array}$} & \multirow[b]{2}{*}{ Std. Error } & \multirow[b]{2}{*}{ Sig. ${ }^{d}$} & \multicolumn{2}{|c|}{$\begin{array}{l}95 \% \text { Confidence Interval } \\
\text { for Difference }\end{array}$} \\
\hline & & & & & $\begin{array}{l}\text { Lower } \\
\text { Bound }\end{array}$ & Upper Bound \\
\hline $\begin{array}{l}\text { w/ bleach } \\
\text { w/o bleach }\end{array}$ & $\begin{array}{l}\text { w/o bleach } \\
\text { w/ bleach }\end{array}$ & $\begin{array}{r}1.319^{\pi, b, c} \\
-1.319^{\pi, b, c}\end{array}$ & $\begin{array}{l}.266 \\
.266\end{array}$ & $\begin{array}{l}.000 \\
.000\end{array}$ & $\begin{array}{r}.797 \\
-1.841\end{array}$ & $\begin{array}{l}1.841 \\
-.797\end{array}$ \\
\hline
\end{tabular}

Based on estimated marginal means

*. The mean difference is significant at the .05 level.

b. An estimate of the modified population marginal mean (I).

c. An estimate of the modified population marginal mean (J).

d. Adjustment for multiple comparisons: Least Significant Difference (equivalent to no adjustments).

\section{Sample Area * Swatch: PMP}

Dependent Variable: L

\begin{tabular}{|c|c|c|c|c|c|}
\hline \multirow{2}{*}{\multicolumn{2}{|c|}{ Sample Area }} & \multirow[b]{2}{*}{ Mean } & \multirow[b]{2}{*}{ Std. Error } & \multicolumn{2}{|c|}{ 95\% Confidence Interval } \\
\hline & & & & Lower Bound & Upper Bound \\
\hline \multirow[t]{4}{*}{$\bar{A}$} & $1 \mathrm{w} /$ bleach & $83.122^{\mathrm{a}}$ & .360 & 82.415 & 83.829 \\
\hline & $2 \mathrm{w} /$ bleach & $85.513^{a}$ & .395 & 84.739 & 86.288 \\
\hline & 3 w/o bleach & $84.384^{a}$ & .360 & 83.677 & 85.091 \\
\hline & 4 w/o bleach & $82.010^{a}$ & .395 & 81.235 & 82.784 \\
\hline \multirow[t]{4}{*}{ B } & $1 \mathrm{w} /$ bleach & $85.581^{a}$ & .395 & 84.807 & 86.356 \\
\hline & $2 \mathrm{w} /$ bleach & $83.706^{a}$ & .360 & 82.999 & 84.413 \\
\hline & 3 w/o bleach & $83.953^{\mathrm{a}}$ & .395 & 83.178 & 84.727 \\
\hline & 4 w/o bleach & $81.883^{a}$ & .360 & 81.176 & 82.590 \\
\hline
\end{tabular}

a. Based on modified population marginal mean. 


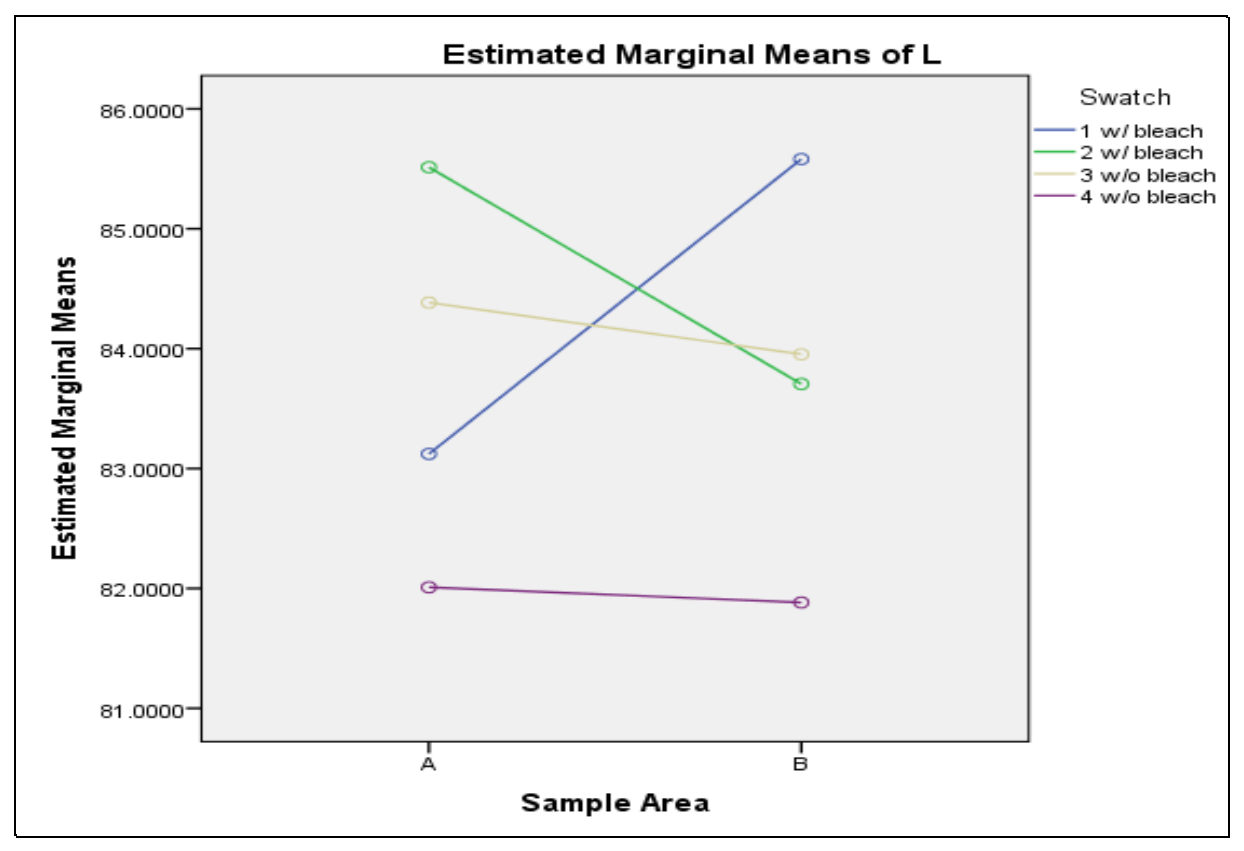

6. Sample Area * Bleached: PMP

Dependent Variable: $\mathrm{L}$

\begin{tabular}{|ll|l|r|r|r|}
\hline \multirow{2}{*}{ Sample Area } & & & \multicolumn{2}{|c|}{$95 \%$ Confidence Interval } \\
\cline { 5 - 6 } & & Mean & Std. Error & \multicolumn{1}{|c|}{ Lower Bound } & Upper Bound \\
\hline A & w/ bleach & $84.209^{\mathrm{a}}$ & .266 & 83.687 & 84.731 \\
& w/o bleach & $83.305^{\mathrm{a}}$ & .266 & 82.783 & 83.827 \\
B & w/ bleach & $84.558^{\mathrm{a}}$ & .266 & 84.036 & 85.080 \\
& w/o bleach & $82.824^{\mathrm{a}}$ & .266 & 82.302 & 83.346 \\
\hline
\end{tabular}

a. Based on modified population marginal mean.

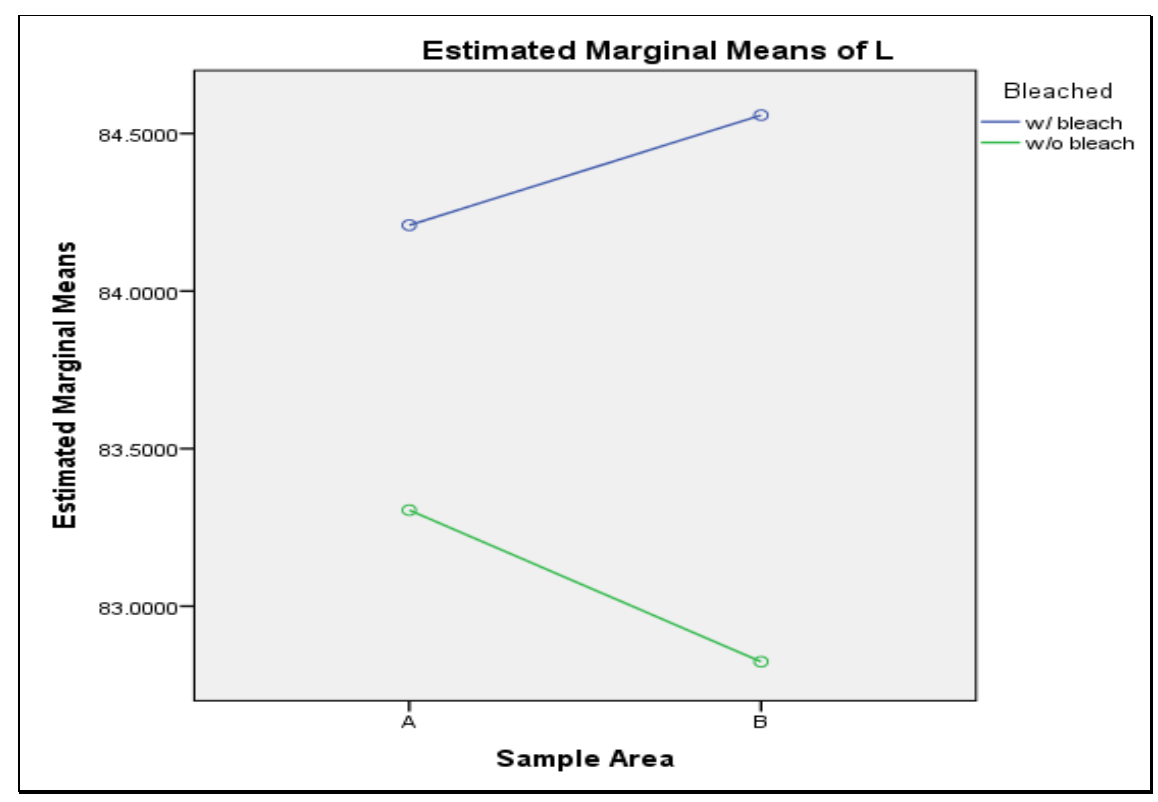




\section{Bleached ${ }^{*}$ Washings: PMP}

Dependent Variable: $\mathrm{L}$

\begin{tabular}{|ll|r|r|r|r|}
\hline & & & & \multicolumn{2}{|c|}{ 95\% Confidence Interval } \\
\cline { 4 - 6 } Bleached & & Mean & Std. Error & Lower Bound & Upper Bound \\
\hline w/ bleach & 1W 0A & $80.132^{\mathrm{a}}$ & .624 & 78.907 & 81.356 \\
& 1W 16A & $81.797^{\mathrm{a}}$ & .441 & 80.931 & 82.663 \\
& 2W 32A & $90.592^{\mathrm{a}}$ & .441 & 89.726 & 91.458 \\
& 3W 48A & $82.507^{\mathrm{a}}$ & .441 & 81.641 & 83.373 \\
& 4W 64A & $83.899^{\mathrm{a}}$ & .441 & 83.033 & 84.765 \\
w/o bleach & 5W 80A & $85.249^{\mathrm{a}}$ & .441 & 84.383 & 86.115 \\
& 1W 0A & $77.973^{\mathrm{a}}$ & .624 & 76.749 & 79.198 \\
& 1W 16A & $80.313^{\mathrm{a}}$ & .441 & 79.447 & 81.179 \\
& 2W 32A & $84.824^{\mathrm{a}}$ & .441 & 83.958 & 85.689 \\
& 3W 48A & $82.677^{\mathrm{a}}$ & .441 & 81.811 & 83.543 \\
& 4W 64A & $84.553^{\mathrm{a}}$ & .441 & 83.687 & 85.419 \\
& 5W 80A & $85.500^{\mathrm{a}}$ & .441 & 84.634 & 86.366 \\
\hline
\end{tabular}

a. Based on modified population marginal mean.

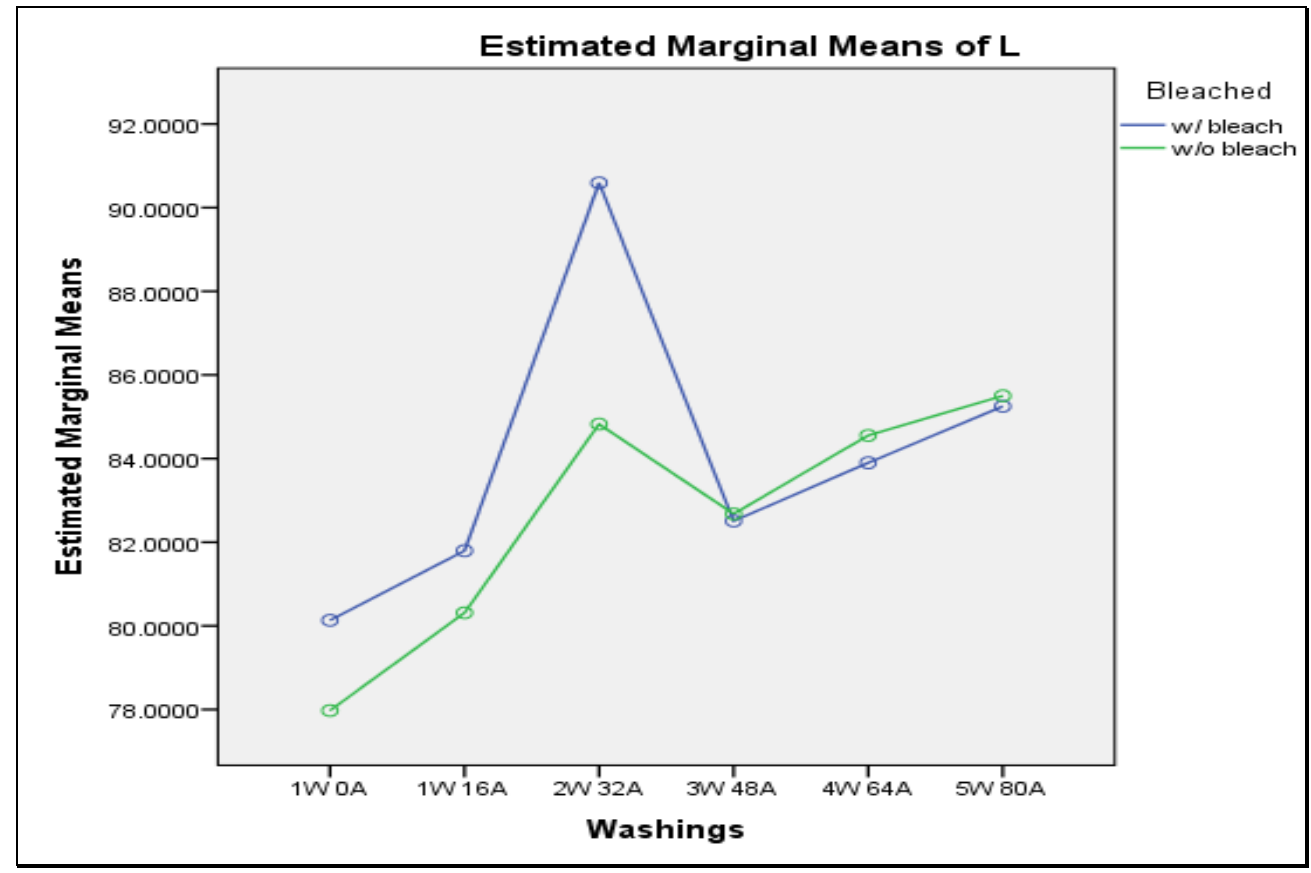

Variable ' $a$ '

Estimates: PMP

Dependent Variable: a

\begin{tabular}{|c|c|c|c|c|}
\hline \multirow[b]{2}{*}{ Swatch } & \multirow[b]{2}{*}{ Mean } & \multirow[b]{2}{*}{ Std. Error } & \multicolumn{2}{|c|}{ 95\% Confidence Interval } \\
\hline & & & Lower Bound & Upper Bound \\
\hline $1 \mathrm{w} /$ bleach & $6.519^{a}$ & .087 & 6.348 & 6.689 \\
\hline 2 w/ bleach & $6.424^{a}$ & .087 & 6.253 & 6.594 \\
\hline 3 w/o bleach & $6.345^{\mathrm{a}}$ & .087 & 6.174 & 6.515 \\
\hline 4 w/o bleach & $6.267^{\mathrm{a}}$ & .087 & 6.097 & 6.438 \\
\hline
\end{tabular}


a. Based on modified population marginal mean.

Pairwise Comparisons: PMP

Dependent Variable: a

\begin{tabular}{|c|c|c|c|c|c|c|}
\hline \multirow[b]{2}{*}{ (I) Swatch } & & \multirow[b]{2}{*}{$\begin{array}{c}\text { Mean } \\
\text { Difference (I-J) }\end{array}$} & \multirow[b]{2}{*}{ Std. Error } & \multirow[b]{2}{*}{ Sig. ${ }^{d}$} & \multicolumn{2}{|c|}{$\begin{array}{l}95 \% \text { Confidence Interval } \\
\text { for Difference }{ }^{d}\end{array}$} \\
\hline & & & & & $\begin{array}{l}\text { Lower } \\
\text { Bound }\end{array}$ & Upper Bound \\
\hline \multirow[t]{3}{*}{$1 \mathrm{w} /$ bleach } & $2 \mathrm{w} /$ bleach & $.095^{\mathrm{a}, \mathrm{b}}$ & .123 & .440 & -.146 & .336 \\
\hline & 3 w/o bleach & $.174^{\mathrm{a}, \mathrm{b}}$ & .123 & .157 & -.067 & .415 \\
\hline & 4 w/o bleach & $.251^{\mathrm{a}, \mathrm{b},{ }^{\pi}}$ & .123 & .041 & .010 & .492 \\
\hline \multirow[t]{3}{*}{2 w/ bleach } & $1 \mathrm{w} /$ bleach & $-.095^{a, b}$ & .123 & .440 & -.336 & .146 \\
\hline & 3 w/o bleach & $.079^{a, b}$ & .123 & .521 & -.162 & .320 \\
\hline & 4 w/o bleach & $.156^{\mathrm{a}, \mathrm{b}}$ & .123 & .204 & -.085 & .397 \\
\hline \multirow[t]{3}{*}{3 w/o bleach } & $1 \mathrm{w} /$ bleach & $-.174^{\mathrm{a}, \mathrm{b}}$ & .123 & .157 & -.415 & .067 \\
\hline & $2 \mathrm{w} /$ bleach & $-.079^{a, b}$ & .123 & .521 & -.320 & .162 \\
\hline & 4 w/o bleach & $.077^{\mathrm{a}, \mathrm{b}}$ & .123 & .529 & -.164 & .319 \\
\hline \multirow[t]{3}{*}{4 w/o bleach } & 1 w/ bleach & $-.251^{\mathrm{a}, \mathrm{b},{ }^{\pi}}$ & .123 & .041 & -.492 & -.010 \\
\hline & 2 w/ bleach & $-.156^{\mathrm{a}, \mathrm{b}}$ & .123 & .204 & -.397 & .085 \\
\hline & 3 w/o bleach & $-.077^{a, b}$ & .123 & .529 & -.319 & .164 \\
\hline
\end{tabular}

Based on estimated marginal means

*. The mean difference is significant at the .05 level.

a. An estimate of the modified population marginal mean (I).

b. An estimate of the modified population marginal mean (J).

d. Adjustment for multiple comparisons: Least Significant Difference (equivalent to no adjustments).

\section{Estimates: PMP}

Dependent Variable: a

\begin{tabular}{|c|c|c|c|c|}
\hline \multirow[b]{2}{*}{ Sample Area } & \multirow[b]{2}{*}{ Mean } & \multirow[b]{2}{*}{ Std. Error } & \multicolumn{2}{|c|}{$95 \%$ Confidence Interval } \\
\hline & & & Lower Bound & Upper Bound \\
\hline$A$ & $6.368^{a}$ & .061 & 6.248 & 6.489 \\
\hline B & $6.409^{\mathrm{a}}$ & .061 & 6.289 & 6.530 \\
\hline
\end{tabular}

a. Based on modified population marginal mean.

Pairwise Comparisons: PMP

Dependent Variable: a

\begin{tabular}{|c|c|c|c|c|c|c|}
\hline \multirow{2}{*}{\multicolumn{2}{|c|}{ (I) Sample Area }} & \multirow{2}{*}{$\begin{array}{c}\text { Mean } \\
\text { Difference (I-J) }\end{array}$} & \multirow[b]{2}{*}{ Std. Error } & \multirow[b]{2}{*}{ Sig. ${ }^{c}$} & \multicolumn{2}{|c|}{$\begin{array}{l}\text { 95\% Confidence Interval for } \\
\text { Difference }{ }^{c}\end{array}$} \\
\hline & & & & & Lower Bound & Upper Bound \\
\hline$A$ & $\mathrm{~B}$ & $-.041^{a, b}$ & .087 & .639 & -.211 & .130 \\
\hline$B$ & $A$ & $.041^{a, b}$ & .087 & .639 & -.130 & .211 \\
\hline
\end{tabular}

Based on estimated marginal means

a. An estimate of the modified population marginal mean (I).

b. An estimate of the modified population marginal mean (J).

c. Adjustment for multiple comparisons: Least Significant Difference (equivalent to no adjustments). 
Bleach Estimates: PMP

Dependent Variable: a

\begin{tabular}{|l|r|r|r|r|}
\hline & & & \multicolumn{2}{|c|}{$95 \%$ Confidence Interval } \\
\cline { 4 - 5 } Bleached & Mean & Std. Error & Lower Bound & Upper Bound \\
\hline w/ bleach & $6.471^{\mathrm{a}}$ & .061 & 6.351 & 6.592 \\
w/o bleach & $6.306^{\mathrm{a}}$ & .061 & 6.186 & 6.427 \\
\hline
\end{tabular}

a. Based on modified population marginal mean.

Pairwise Comparisons: PMP

Dependent Variable: a

\begin{tabular}{|ll|r|r|r|r|r|}
\hline & & & & \multicolumn{3}{|c|}{$\begin{array}{c}\text { Confidence Interval for } \\
\text { Difference }\end{array}$} \\
\cline { 5 - 7 } & & & & \\
(I) Bleached & Mean Difference (I-J) & Std. Error & Sig. $^{c}$ & Lower Bound & Upper Bound \\
\hline w/ bleach & w/o bleach & $.165^{\mathrm{a}, \mathrm{b}}$ & .087 & .058 & -.005 & .336 \\
w/o bleach & w/ bleach & $-.165^{\mathrm{a}, \mathrm{b}}$ & .087 & .058 & -.336 & .005 \\
\hline
\end{tabular}

Based on estimated marginal means

a. An estimate of the modified population marginal mean (I).

b. An estimate of the modified population marginal mean (J).

c. Adjustment for multiple comparisons: Least Significant Difference (equivalent to no adjustments).

Dependent Variable: a

\section{Sample Area * Swatch: PMP}

\begin{tabular}{|c|c|c|c|c|c|}
\hline \multirow[b]{2}{*}{ Sample Area } & & \multirow[b]{2}{*}{ Mean } & \multirow[b]{2}{*}{ Std. Error } & \multicolumn{2}{|c|}{ 95\% Confidence Interval } \\
\hline & & & & Lower Bound & Upper Bound \\
\hline \multirow[t]{4}{*}{$A$} & $1 \mathrm{w} /$ bleach & $6.630^{\mathrm{a}}$ & .118 & 6.399 & 6.861 \\
\hline & 2 w/ bleach & $6.505^{\mathrm{a}}$ & .129 & 6.252 & 6.758 \\
\hline & 3 w/o bleach & $6.094^{\mathrm{a}}$ & .118 & 5.863 & 6.325 \\
\hline & 4 w/o bleach & $6.247^{\mathrm{a}}$ & .129 & 5.994 & 6.500 \\
\hline \multirow[t]{4}{*}{ B } & $1 \mathrm{w} /$ bleach & $6.385^{\mathrm{a}}$ & .129 & 6.133 & 6.638 \\
\hline & $2 \mathrm{w} /$ bleach & $6.356^{\mathrm{a}}$ & .118 & 6.125 & 6.587 \\
\hline & 3 w/o bleach & $6.646^{\mathrm{a}}$ & .129 & 6.393 & 6.899 \\
\hline & 4 w/o bleach & $6.285^{\mathrm{a}}$ & .118 & 6.054 & 6.515 \\
\hline
\end{tabular}

a. Based on modified population marginal mean.

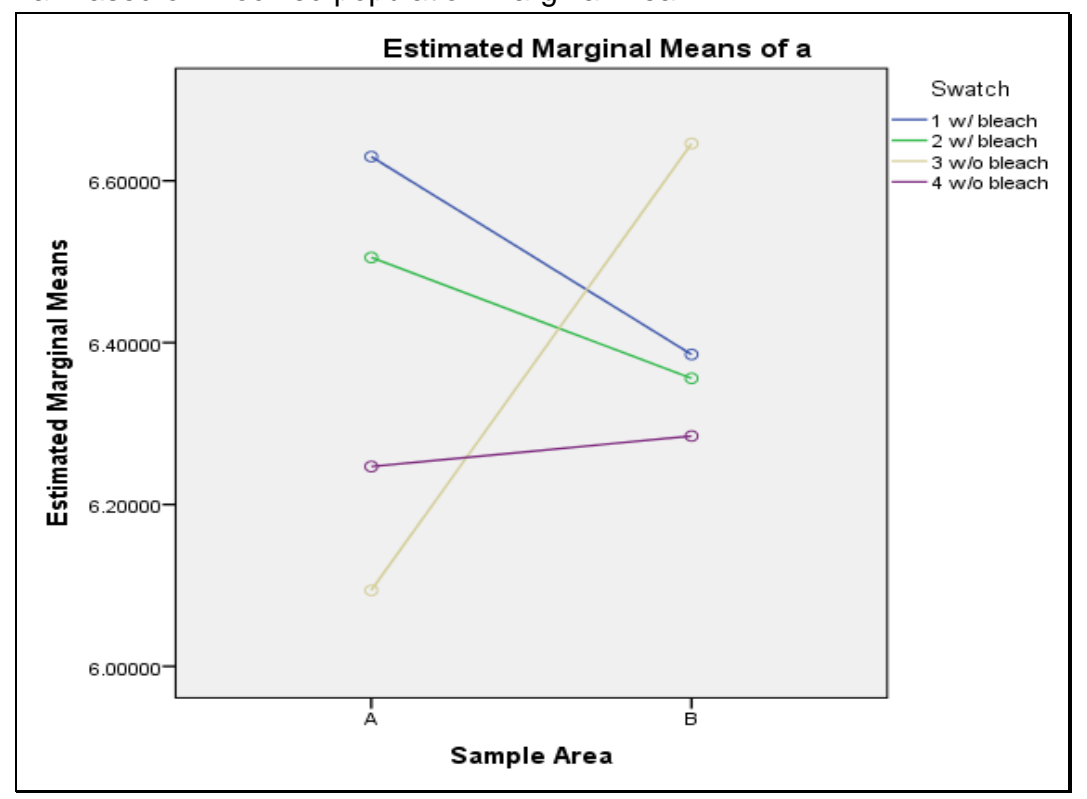


Dependent Variable: a

\section{Sample Area * Bleached: PMP}

\begin{tabular}{|c|c|c|c|c|c|}
\hline \multirow[b]{2}{*}{ Sample Area } & & \multirow[b]{2}{*}{ Mean } & \multirow[b]{2}{*}{ Std. Error } & \multicolumn{2}{|c|}{$\begin{array}{l}95 \% \text { Confidence } \\
\text { Interval }\end{array}$} \\
\hline & & & & $\begin{array}{l}\text { Lower } \\
\text { Bound }\end{array}$ & $\begin{array}{l}\text { Upper } \\
\text { Bound }\end{array}$ \\
\hline \multirow[t]{4}{*}{$\mathrm{A}$} & w/ bleach & $6.573^{\mathrm{a}}$ & .087 & 6.403 & 6.744 \\
\hline & w/o bleach & $6.164^{\mathrm{a}}$ & .087 & 5.993 & 6.334 \\
\hline & w/ bleach & $6.369^{\mathrm{a}}$ & .087 & 6.199 & 6.540 \\
\hline & w/o bleach & $6.449^{a}$ & .087 & 6.278 & 6.619 \\
\hline
\end{tabular}

a. Based on modified population marginal mean.

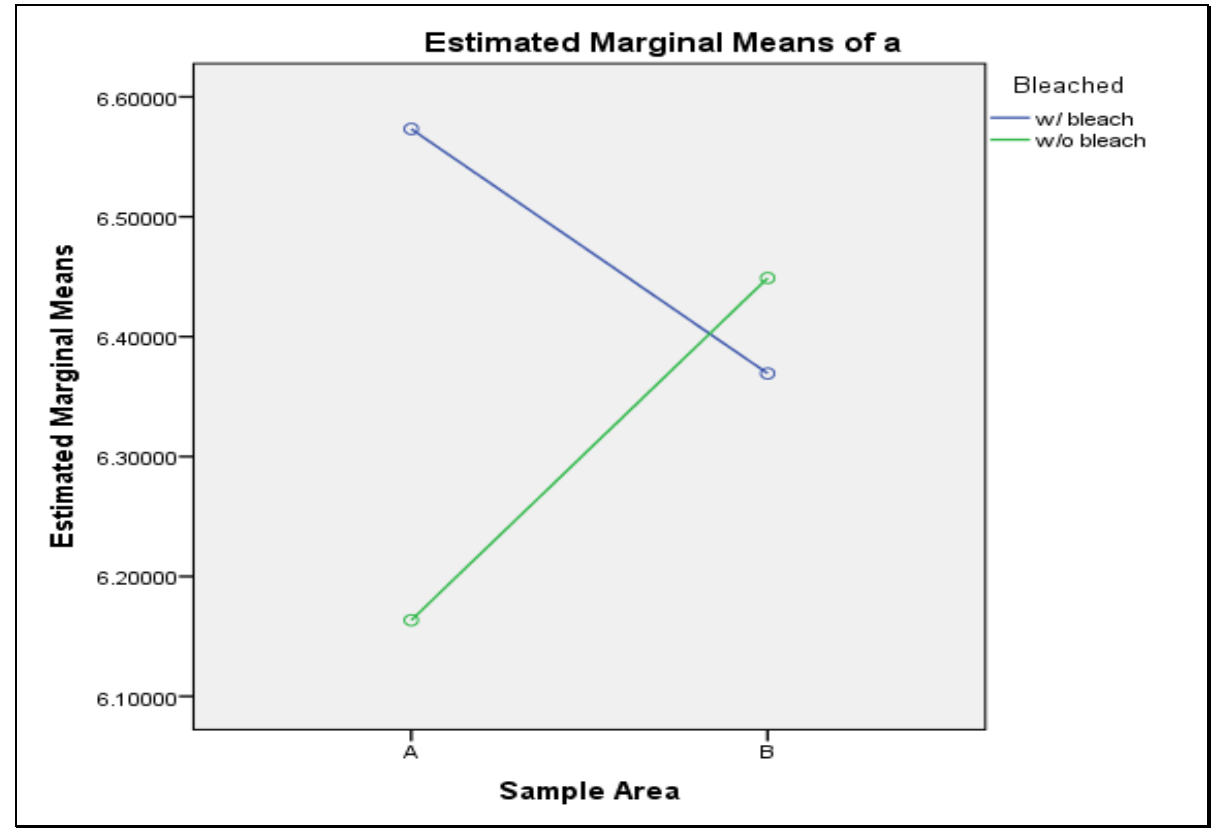

Variable ' $b$ '

Sample Area Estimates: PMP

Dependent Variable: $b$

\begin{tabular}{|l|r|r|r|r|}
\hline & & & \multicolumn{2}{|c|}{$95 \%$ Confidence Interval } \\
\cline { 4 - 5 } Sample Area & Mean & Std. Error & Lower Bound & Upper Bound \\
\hline A & $-8.637^{\mathrm{a}}$ & .126 & -8.886 & -8.389 \\
B & $-8.815^{\mathrm{a}}$ & .126 & -9.063 & -8.566 \\
\hline
\end{tabular}

a. Based on modified population marginal mean.

Pairwise Comparisons: PMP

Dependent Variable: $b$

\begin{tabular}{|ll|r|r|r|r|r|}
\hline & & & & \multicolumn{2}{|c|}{$\begin{array}{c}\text { 95\% Confidence Interval for } \\
\text { Difference }\end{array}$} \\
\cline { 5 - 7 } & & & & & \\
(I) Sample Area & & Mean Difference (I-J) & Std. Error & Sig. $^{c}$ & Lower Bound & Upper Bound \\
\hline A & B & $.177^{\mathrm{a}, \mathrm{b}}$ & .179 & .322 & -.174 & .528 \\
B & A & $-.177^{\mathrm{a}, \mathrm{b}}$ & .179 & .322 & -.528 & .174 \\
\hline
\end{tabular}

Based on estimated marginal means

a. An estimate of the modified population marginal mean (I).

b. An estimate of the modified population marginal mean (J). 
c. Adjustment for multiple comparisons: Least Significant Difference (equivalent to no adjustments).

Dependent Variable: $b$

\section{Estimates: PMP}

\begin{tabular}{|l|r|r|r|r|}
\hline & & & \multicolumn{2}{|c|}{$95 \%$ Confidence Interval } \\
\cline { 4 - 5 } Swatch & Mean & Std. Error & Lower Bound & Upper Bound \\
\hline 1 w/ bleach & $-8.380^{\mathrm{a}}$ & .179 & -8.731 & -8.029 \\
2 w/ bleach & $-8.807^{\mathrm{a}}$ & .179 & -9.158 & -8.455 \\
3 w/o bleach & $-8.452^{\mathrm{a}}$ & .179 & -8.803 & -8.101 \\
4 w/o bleach & $-9.265^{\mathrm{a}}$ & .179 & -9.616 & -8.914 \\
\hline
\end{tabular}

a. Based on modified population marginal mean.

Pairwise Comparisons: PMP

Dependent Variable: $b$

\begin{tabular}{|c|c|c|c|c|c|c|}
\hline \multirow[b]{2}{*}{ (I) Swatch } & & \multirow{2}{*}{$\begin{array}{c}\text { Mean } \\
\text { Difference (I-J) }\end{array}$} & \multirow[b]{2}{*}{ Std. Error } & \multirow[b]{2}{*}{ Sig. ${ }^{d}$} & \multicolumn{2}{|c|}{$\begin{array}{l}\text { 95\% Confidence Interval for } \\
\text { Difference }\end{array}$} \\
\hline & & & & & Lower Bound & Upper Bound \\
\hline \multirow[t]{3}{*}{$1 \mathrm{w} /$ bleach } & 2 w/ bleach & $.427^{\mathrm{a}, \mathrm{b}}$ & .253 & .092 & -.070 & .923 \\
\hline & 3 w/o bleach & $.072^{a, b}$ & .253 & .775 & -.424 & .569 \\
\hline & $4 \mathrm{w} / \mathrm{o}$ bleach & $.885^{\mathrm{a}, \mathrm{b},{ }^{*}}$ & .253 & .000 & .389 & 1.382 \\
\hline \multirow[t]{3}{*}{2 w/ bleach } & $1 \mathrm{w} /$ bleach & $-.427^{a, b}$ & .253 & .092 & -.923 & .070 \\
\hline & 3 w/o bleach & $-.354^{a, b}$ & .253 & .162 & -.851 & .142 \\
\hline & 4 w/o bleach & $.459^{a, b}$ & .253 & .070 & -.038 & .955 \\
\hline \multirow[t]{3}{*}{3 w/o bleach } & $1 \mathrm{w} /$ bleach & $-.072^{a, b}$ & .253 & .775 & -.569 & .424 \\
\hline & 2 w/ bleach & $.354^{\mathrm{a}, \mathrm{b}}$ & .253 & .162 & -.142 & .851 \\
\hline & 4 w/o bleach & $.813^{\mathrm{a}, \mathrm{b},{ }^{*}}$ & .253 & .001 & .317 & 1.310 \\
\hline \multirow[t]{3}{*}{4 w/o bleach } & $1 \mathrm{w} /$ bleach & $-.885^{\mathrm{a}, \mathrm{b},{ }^{*}}$ & .253 & .000 & -1.382 & -.389 \\
\hline & 2 w/ bleach & $-.459^{a, b}$ & .253 & .070 & -.955 & .038 \\
\hline & 3 w/o bleach & $-.813^{\mathrm{a}, \mathrm{b},{ }^{\star}}$ & .253 & .001 & -1.310 & -.317 \\
\hline
\end{tabular}

Based on estimated marginal means

${ }^{*}$. The mean difference is significant at the .05 level.

a. An estimate of the modified population marginal mean (I).

b. An estimate of the modified population marginal mean $(\mathrm{J})$.

d. Adjustment for multiple comparisons: Least Significant Difference (equivalent to no adjustments).

\section{Estimates: PMP}

Dependent Variable: $b$

\begin{tabular}{|l|r|r|r|r|}
\hline & & \multirow{2}{|c|}{ 95\% Confidence Interval } \\
\cline { 4 - 5 } Bleached & Mean & Std. Error & Lower Bound & Upper Bound \\
\hline w/ bleach & $-8.593^{\mathrm{a}}$ & .126 & -8.842 & -8.345 \\
w/o bleach & $-8.859^{\mathrm{a}}$ & .126 & -9.107 & -8.610 \\
\hline
\end{tabular}

a. Based on modified population marginal mean.

Pairwise Comparisons: PMP

Dependent Variable: $b$

\begin{tabular}{|c|c|c|c|c|c|c|}
\hline \multirow[b]{2}{*}{ (I) Bleached } & & \multirow[b]{2}{*}{ Mean Difference (I-J) } & \multirow[b]{2}{*}{ Std. Error } & \multirow[b]{2}{*}{ Sig. ${ }^{c}$} & \multicolumn{2}{|c|}{$\begin{array}{c}\text { 95\% Confidence Interval for } \\
\text { Difference }\end{array}$} \\
\hline & & & & & Lower Bound & Upper Bound \\
\hline $\begin{array}{l}\text { w/ bleach } \\
\text { w/o bleach }\end{array}$ & $\begin{array}{l}\text { w/o bleach } \\
\text { w/ bleach }\end{array}$ & $\begin{array}{r}.265^{\mathrm{a}, \mathrm{b}} \\
-.265^{\mathrm{a}, \mathrm{b}} \\
\end{array}$ & $\begin{array}{l}.179 \\
.179 \\
\end{array}$ & $\begin{array}{l}.138 \\
.138 \\
\end{array}$ & $\begin{array}{r}-.086 \\
-.617 \\
\end{array}$ & $\begin{array}{r}.617 \\
.086 \\
\end{array}$ \\
\hline
\end{tabular}

Based on estimated marginal means

a. An estimate of the modified population marginal mean (I). 
b. An estimate of the modified population marginal mean $(\mathrm{J})$.

c. Adjustment for multiple comparisons: Least Significant Difference (equivalent to no adjustments).

Dependent Variable: b

\section{Sample Area * Swatch: PMP}

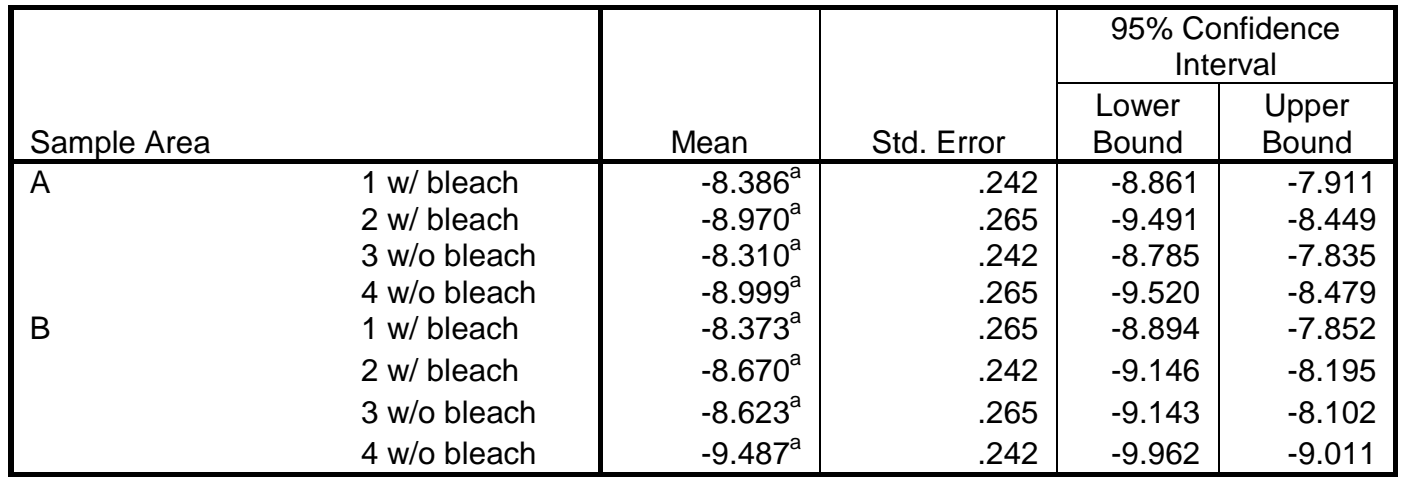

a. Based on modified population marginal mean.

\section{Sample Area * Bleached: PMP}

Dependent Variable: b

\begin{tabular}{|c|c|c|c|c|c|}
\hline \multirow[b]{2}{*}{ Sample Area } & & \multirow[b]{2}{*}{ Mean } & \multirow[b]{2}{*}{ Std. Error } & \multicolumn{2}{|c|}{$\begin{array}{l}\text { 95\% Confidence } \\
\text { Interval }\end{array}$} \\
\hline & & & & $\begin{array}{l}\text { Lower } \\
\text { Bound }\end{array}$ & $\begin{array}{l}\text { Upper } \\
\text { Bound }\end{array}$ \\
\hline \multirow[t]{2}{*}{$A$} & w/ bleach & $-8.651^{\mathrm{a}}$ & .179 & -9.003 & -8.300 \\
\hline & w/o bleach & $-8.623^{a}$ & .179 & -8.974 & -8.272 \\
\hline \multirow[t]{2}{*}{ B } & w/ bleach & $-8.535^{a}$ & .179 & -8.886 & -8.184 \\
\hline & w/o bleach & $-9.094^{a}$ & .179 & -9.445 & -8.743 \\
\hline
\end{tabular}

a. Based on modified population marginal mean.

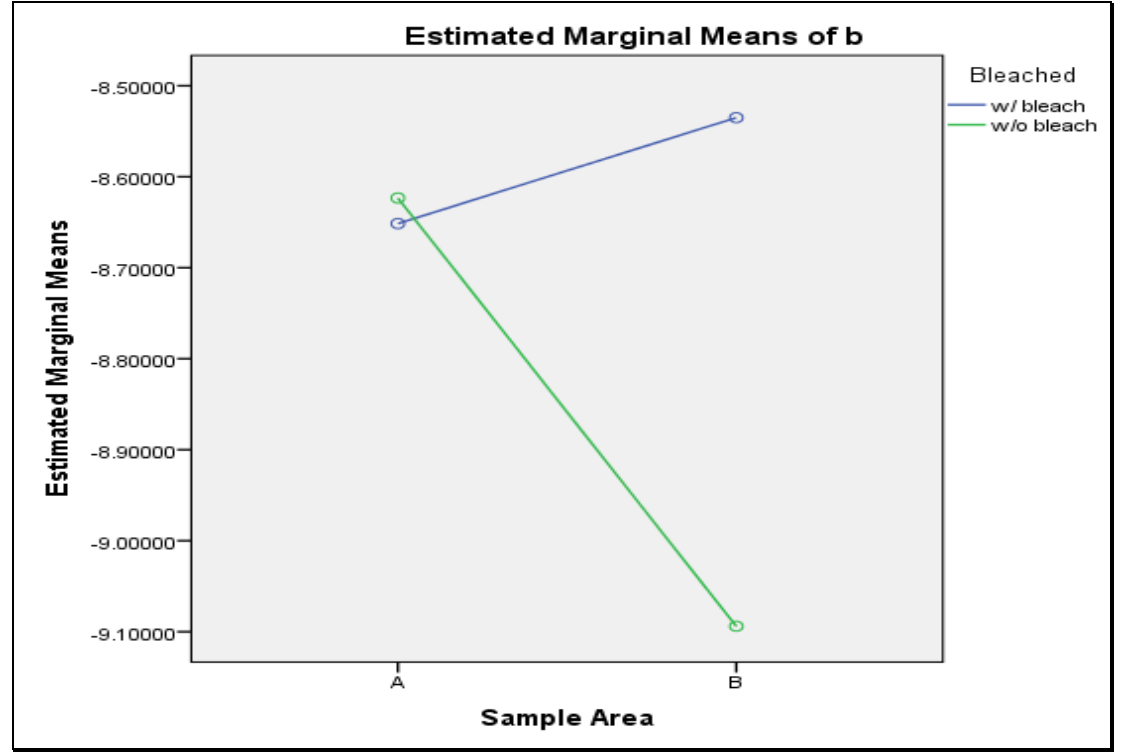


Table 14.6 ANOVA Output for Washed-Aged Results for sample RMP

\section{Variable ' $L$ '}

\begin{tabular}{|l|r|r|r|r|}
\hline & & & \multicolumn{2}{|c|}{$95 \%$ Confidence Interval } \\
\cline { 4 - 5 } Swatch & Mean & Std. Error & Lower Bound & Upper Bound \\
\hline $1 \mathrm{w} /$ bleach & $84.681^{\mathrm{a}}$ & .226 & 84.237 & 85.125 \\
$2 \mathrm{w} /$ bleach & $85.119^{\mathrm{a}}$ & .226 & 84.675 & 85.563 \\
3 w/o bleach & $84.071^{\mathrm{a}}$ & .226 & 83.627 & 84.515 \\
4 w/o bleach & $83.331^{\mathrm{a}}$ & .226 & 82.887 & 83.775 \\
\hline
\end{tabular}

a. Based on modified population marginal mean.

Pairwise Comparisons: RMP

Dependent Variable: $\mathrm{L}$

\begin{tabular}{|c|c|c|c|c|c|c|}
\hline \multirow[b]{2}{*}{ (I) Swatch } & & \multirow[b]{2}{*}{$\begin{array}{c}\text { Mean } \\
\text { Difference (I-J) }\end{array}$} & \multirow[b]{2}{*}{ Std. Error } & \multirow[b]{2}{*}{ Sig. $^{d}$} & \multicolumn{2}{|c|}{$\begin{array}{l}95 \% \text { Confidence } \\
\text { Interval for Difference }\end{array}$} \\
\hline & & & & & $\begin{array}{l}\text { Lower } \\
\text { Bound }\end{array}$ & $\begin{array}{l}\text { Upper } \\
\text { Bound }\end{array}$ \\
\hline \multirow[t]{3}{*}{$1 \mathrm{w} /$ bleach } & 2 w/ bleach & $-.438^{\mathrm{a}, \mathrm{b}}$ & .320 & .171 & -1.065 & .190 \\
\hline & 3 w/o bleach & $.610^{\mathrm{a}, \mathrm{b}}$ & .320 & .057 & -.018 & 1.238 \\
\hline & $4 \mathrm{w} / \mathrm{o}$ bleach & $1.351^{\mathrm{a}, \mathrm{b},{ }^{*}}$ & .320 & .000 & .723 & 1.978 \\
\hline \multirow[t]{3}{*}{$2 \mathrm{w} /$ bleach } & $1 \mathrm{w} /$ bleach & $.438^{\mathrm{a}, \mathrm{b}}$ & .320 & .171 & -.190 & 1.065 \\
\hline & 3 w/o bleach & $1.048^{\mathrm{a}, \mathrm{b},{ }^{*}}$ & .320 & .001 & .420 & 1.675 \\
\hline & $4 \mathrm{w} / \mathrm{o}$ bleach & $1.788^{\mathrm{a}, \mathrm{b},{ }^{*}}$ & .320 & .000 & 1.161 & 2.416 \\
\hline \multirow[t]{3}{*}{3 w/o bleach } & $1 \mathrm{w} /$ bleach & $-.610^{a, b}$ & .320 & .057 & -1.238 & .018 \\
\hline & 2 w/ bleach & $-1.048^{a, b,{ }^{*}}$ & .320 & .001 & -1.675 & -.420 \\
\hline & 4 w/o bleach & $.741^{\mathrm{a}, \mathrm{b},{ }^{\star}}$ & .320 & .021 & .113 & 1.368 \\
\hline \multirow[t]{3}{*}{$4 \mathrm{w} / \mathrm{o}$ bleach } & $1 \mathrm{w} /$ bleach & $-1.351^{a, b,{ }^{*}}$ & .320 & .000 & -1.978 & -.723 \\
\hline & $2 \mathrm{w} /$ bleach & $-1.788^{\mathrm{a}, \mathrm{b},{ }^{*}}$ & .320 & .000 & -2.416 & -1.161 \\
\hline & 3 w/o bleach & $-.741^{\mathrm{a}, \mathrm{b},{ }^{\star}}$ & .320 & .021 & -1.368 & -.113 \\
\hline
\end{tabular}

Based on estimated marginal means

*. The mean difference is significant at the .05 level.

a. An estimate of the modified population marginal mean (I).

b. An estimate of the modified population marginal mean (J).

d. Adjustment for multiple comparisons: Least Significant Difference (equivalent to no adjustments).

Sample Area Estimates: RMP

Dependent Variable: $\mathrm{L}$

\begin{tabular}{|l|r|r|r|r|}
\hline \multirow{2}{*}{ Sample Area } & & \multicolumn{2}{|c|}{$95 \%$ Confidence Interval } \\
\cline { 4 - 5 } & Mean & Std. Error & \multicolumn{2}{c|}{$\begin{array}{l}\text { Lower } \\
\text { Bound }\end{array}$} \\
\hline A & $84.336^{\mathrm{a}}$ & .160 & 84.022 & Upper \\
B & $84.265^{\mathrm{a}}$ & .160 & 83.952 & 84.650 \\
\hline
\end{tabular}

a. Based on modified population marginal mean.

Pairwise Comparisons: RMP

Dependent Variable: L 


\begin{tabular}{|c|c|c|c|c|c|c|}
\hline \multirow{2}{*}{\multicolumn{2}{|c|}{ (I) Sample Area }} & \multirow[b]{2}{*}{$\begin{array}{c}\text { Mean Difference } \\
(\mathrm{I}-J)\end{array}$} & \multirow[b]{2}{*}{ Std. Error } & \multirow[b]{2}{*}{ Sig. ${ }^{\mathrm{c}}$} & \multicolumn{2}{|c|}{$\begin{array}{l}\text { 95\% Confidence } \\
\text { Interval for Difference }\end{array}$} \\
\hline & & & & & $\begin{array}{l}\text { Lower } \\
\text { Bound }\end{array}$ & $\begin{array}{l}\text { Upper } \\
\text { Bound }\end{array}$ \\
\hline & $B$ & $.070^{\mathrm{a}, \mathrm{b}}$ & .226 & .756 & -.374 & .514 \\
\hline B & A & $-.070^{\mathrm{a}, \mathrm{b}}$ & .226 & .756 & -.514 & .374 \\
\hline
\end{tabular}

Based on estimated marginal means

a. An estimate of the modified population marginal mean (I).

b. An estimate of the modified population marginal mean $(\mathrm{J})$.

c. Adjustment for multiple comparisons: Least Significant Difference (equivalent to no adjustments).

Dependent Variable: L

Bleach Estimates: RMP

\begin{tabular}{|l|r|r|r|r|}
\hline & & & \multicolumn{2}{|c|}{$95 \%$ Confidence Interval } \\
\cline { 4 - 5 } Bleached & & & Lower & Upper \\
w/ bleach & Mean & Std. Error & Bound & Bound \\
\hline w/o bleach & $84.900^{\mathrm{a}}$ & .160 & 84.586 & 85.214 \\
\hline
\end{tabular}

a. Based on modified population marginal mean.

\section{Pairwise Comparisons: RMP}

Dependent Variable: L

\begin{tabular}{|c|c|c|c|c|c|c|}
\hline \multirow[b]{2}{*}{ (I) Bleached } & & \multirow[b]{2}{*}{$\begin{array}{c}\text { Mean } \\
\text { Difference (I-J) }\end{array}$} & \multirow[b]{2}{*}{ Std. Error } & \multirow[b]{2}{*}{ Sig. ${ }^{d}$} & \multicolumn{2}{|c|}{$\begin{array}{c}95 \% \text { Confidence } \\
\text { Interval for Difference }\end{array}$} \\
\hline & & & & & $\begin{array}{l}\text { Lower } \\
\text { Bound }\end{array}$ & $\begin{array}{l}\text { Upper } \\
\text { Bound }\end{array}$ \\
\hline $\begin{array}{l}\text { w/ bleach } \\
\text { w/o bleach }\end{array}$ & $\begin{array}{l}\text { w/o bleach } \\
\text { w/ bleach }\end{array}$ & $\begin{array}{r}1.199^{\circ, \mathrm{b}, \mathrm{c}} \\
-1.199^{\circ, \mathrm{b}, \mathrm{c}}\end{array}$ & $\begin{array}{l}.226 \\
.226\end{array}$ & $\begin{array}{l}.000 \\
.000\end{array}$ & $\begin{array}{r}.755 \\
-1.643\end{array}$ & $\begin{array}{l}1.643 \\
-.755\end{array}$ \\
\hline
\end{tabular}

Based on estimated marginal means

*. The mean difference is significant at the .05 level.

b. An estimate of the modified population marginal mean (I).

c. An estimate of the modified population marginal mean $(\mathrm{J})$.

d. Adjustment for multiple comparisons: Least Significant Difference (equivalent to no adjustments).

Dependent Variable: L

\section{Sample Area * Swatch: RMP}

\begin{tabular}{|c|c|c|c|c|c|}
\hline \multirow[b]{2}{*}{ Sample Area } & & \multirow[b]{2}{*}{ Mean } & \multirow[b]{2}{*}{ Std. Error } & \multicolumn{2}{|c|}{$95 \%$ Confidence Interval } \\
\hline & & & & Lower Bound & Upper Bound \\
\hline \multirow[t]{4}{*}{$\bar{A}$} & $1 \mathrm{w} /$ bleach & $84.262^{\mathrm{a}}$ & .306 & 83.661 & 84.863 \\
\hline & $2 \mathrm{w} /$ bleach & $84.399^{a}$ & .335 & 83.741 & 85.057 \\
\hline & 3 w/o bleach & $83.855^{\mathrm{a}}$ & .306 & 83.254 & 84.456 \\
\hline & 4 w/o bleach & $84.938^{a}$ & .335 & 84.279 & 85.596 \\
\hline \multirow[t]{4}{*}{$B$} & $1 \mathrm{w} /$ bleach & $85.184^{\mathrm{a}}$ & .335 & 84.526 & 85.842 \\
\hline & $2 \mathrm{w} /$ bleach & $85.719^{a}$ & .306 & 85.118 & 86.320 \\
\hline & 3 w/o bleach & $84.331^{a}$ & .335 & 83.673 & 84.989 \\
\hline & 4 w/o bleach & $81.992^{\mathrm{a}}$ & .306 & 81.391 & 82.593 \\
\hline
\end{tabular}

a. Based on modified population marginal mean. 


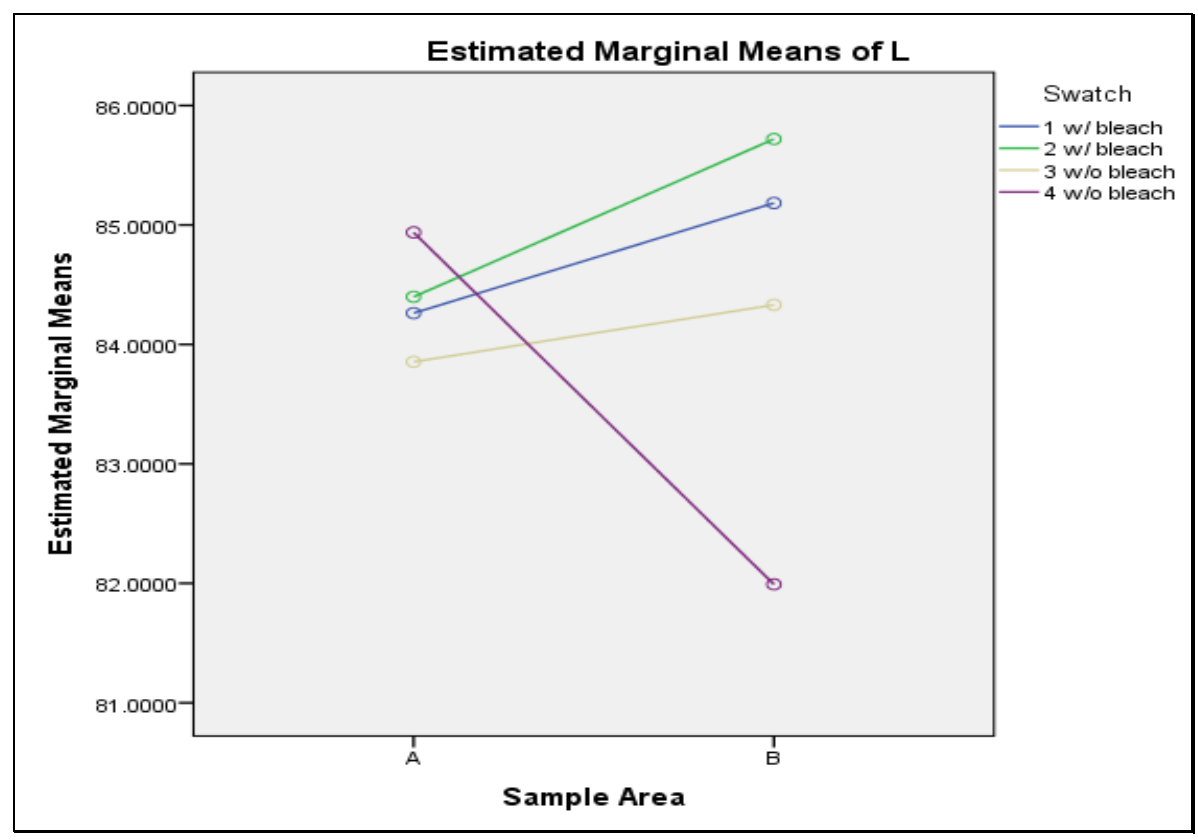

\section{Sample Area * Bleached: RMP}

Dependent Variable: L

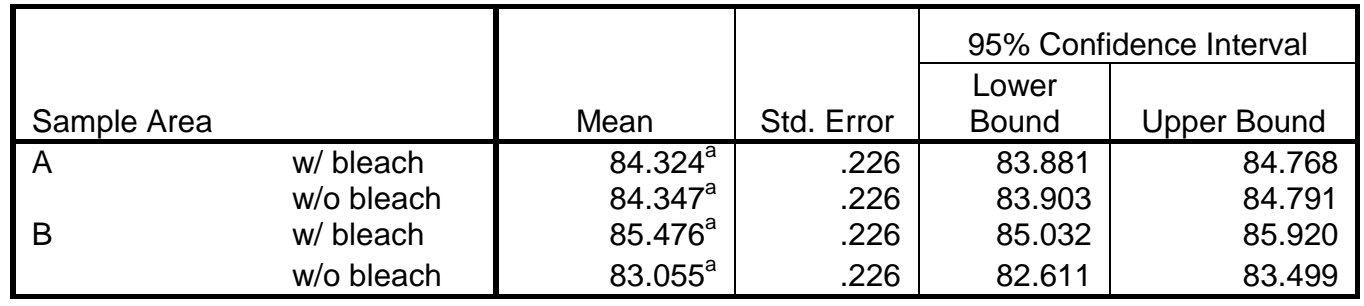

a. Based on modified population marginal mean.

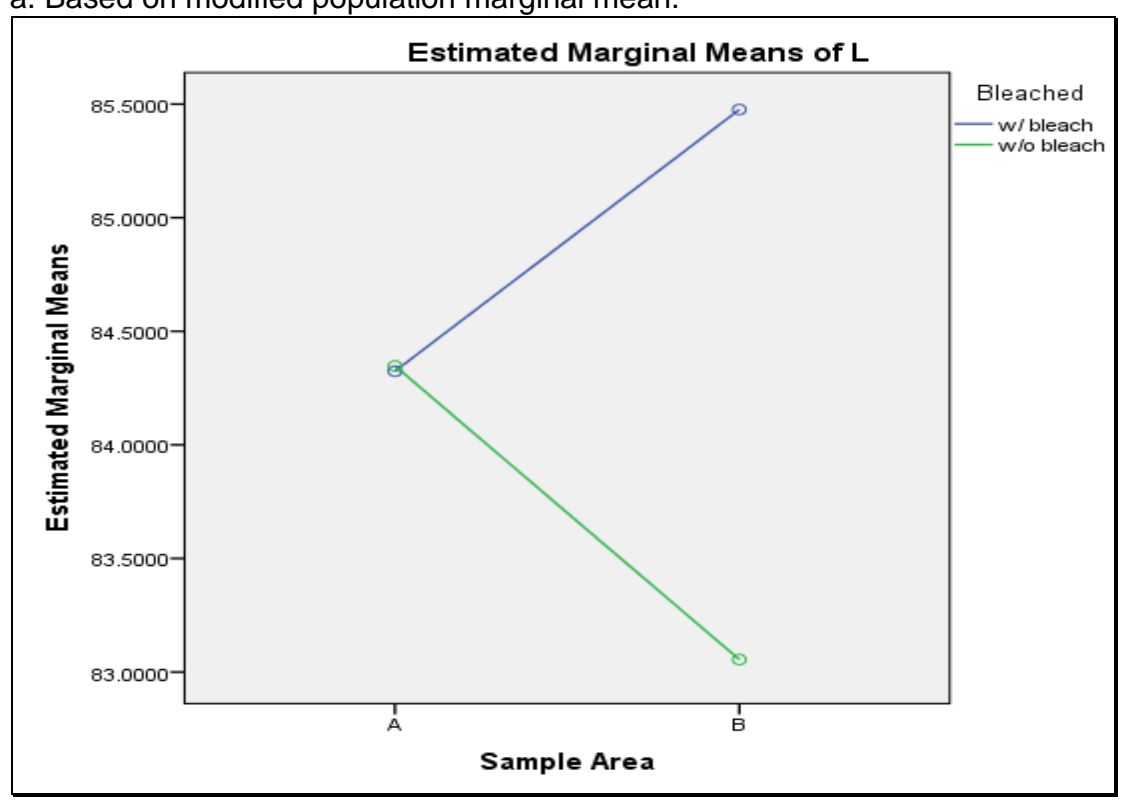




\section{Bleached * Washings: RMP}

Dependent Variable: $\mathrm{L}$

\begin{tabular}{|ll|r|r|r|r|}
\hline & & & \multicolumn{2}{|c|}{ 95\% Confidence Interval } \\
\cline { 5 - 5 } Bleached & & Mean & Std. Error & Lower Bound & Upper Bound \\
\hline w/ bleach & 1W 0A & $86.084^{\mathrm{a}}$ & .530 & 85.043 & 87.125 \\
& 1W 16A & $84.743^{\mathrm{a}}$ & .375 & 84.007 & 85.479 \\
& 2W 32A & $85.222^{\mathrm{a}}$ & .375 & 84.486 & 85.958 \\
& 3W 48A & $84.154^{\mathrm{a}}$ & .375 & 83.418 & 84.890 \\
& 4W 64A & $84.490^{\mathrm{a}}$ & .375 & 83.754 & 85.226 \\
w/o bleach & 5W 80A & $85.300^{\mathrm{a}}$ & .375 & 84.564 & 86.036 \\
& 1W 0A & $81.968^{\mathrm{a}}$ & .530 & 80.927 & 83.009 \\
& 1W 16A & $85.763^{\mathrm{a}}$ & .375 & 85.027 & 86.499 \\
& 2W 32A & $83.770^{\mathrm{a}}$ & .375 & 83.034 & 84.506 \\
& 3W 48A & $84.043^{\mathrm{a}}$ & .375 & 83.307 & 84.779 \\
& 4W 64A & $82.048^{\mathrm{a}}$ & .375 & 81.312 & 82.784 \\
& 5W 80A & $83.748^{\mathrm{a}}$ & .375 & 83.011 & 84.484 \\
\hline
\end{tabular}

a. Based on modified population marginal mean.

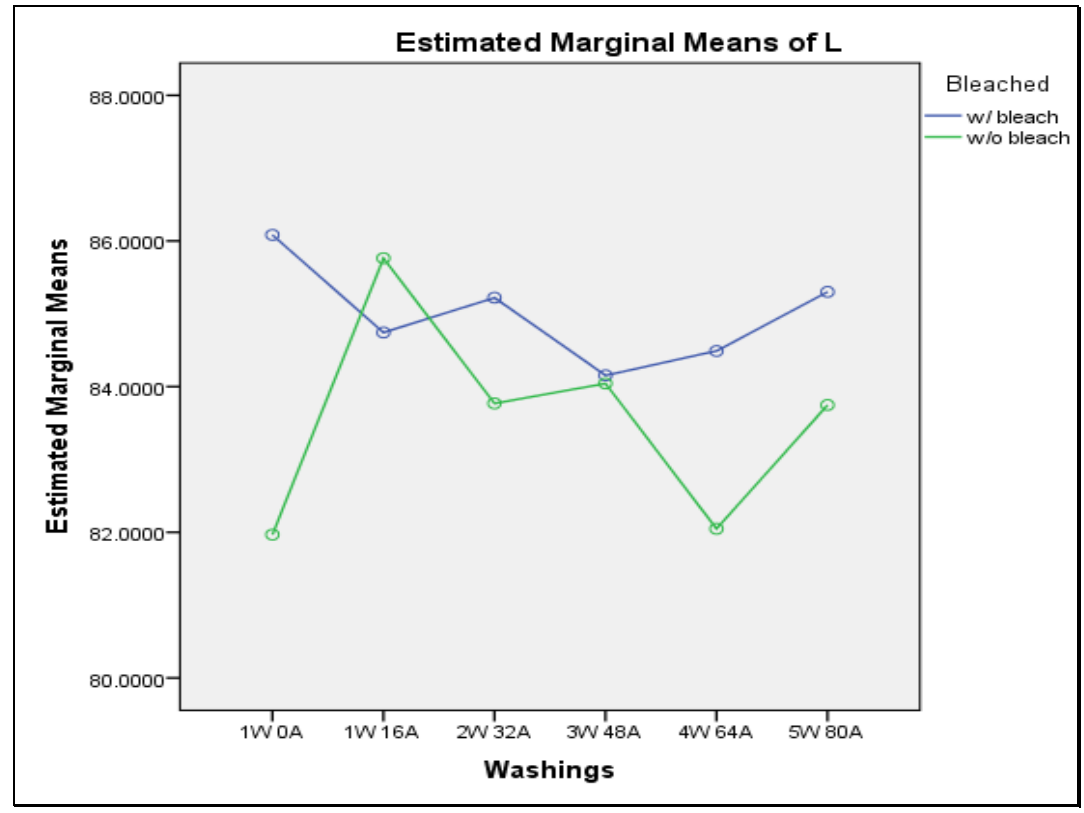

Variable ' $a$ '

Sample Area Estimates: RMP

Dependent Variable: a

\begin{tabular}{|c|c|c|c|c|}
\hline \multirow[b]{2}{*}{ Sample Area } & \multirow[b]{2}{*}{ Mean } & \multirow[b]{2}{*}{ Std. Error } & \multicolumn{2}{|c|}{ 95\% Confidence Interval } \\
\hline & & & Lower Bound & Upper Bound \\
\hline A & $27.760^{\mathrm{a}}$ & .194 & 27.380 & 28.140 \\
\hline B & $27.035^{\mathrm{a}}$ & .194 & 26.655 & 27.415 \\
\hline
\end{tabular}

a. Based on modified population marginal mean. 


\section{Pairwise Comparisons: RMP}

Dependent Variable: a

\begin{tabular}{|c|c|c|c|c|c|c|}
\hline \multirow{2}{*}{\multicolumn{2}{|c|}{ (I) Sample Area }} & \multirow[b]{2}{*}{ Mean Difference (I-J) } & \multirow[b]{2}{*}{ Std. Error } & \multirow[b]{2}{*}{ Sig. ${ }^{d}$} & \multicolumn{2}{|c|}{$\begin{array}{c}\text { 95\% Confidence Interval for } \\
\text { Difference } \\
\text { d }\end{array}$} \\
\hline & & & & & Lower Bound & Upper Bound \\
\hline & $B$ & $.725^{, b, c}$ & .274 & .008 & .187 & 1.263 \\
\hline B & A & $-.725^{\star, b, c}$ & .274 & .008 & -1.263 & -.187 \\
\hline
\end{tabular}

Based on estimated marginal means

*. The mean difference is significant at the .05 level.

b. An estimate of the modified population marginal mean (I).

c. An estimate of the modified population marginal mean (J).

d. Adjustment for multiple comparisons: Least Significant Difference (equivalent to no adjustments).

\section{Bleach Estimates: RMP}

Dependent Variable: a

\begin{tabular}{|l|c|r|r|r|}
\hline & & & \multicolumn{2}{|c|}{$95 \%$ Confidence Interval } \\
\cline { 4 - 5 } Bleached & Mean & Std. Error & Lower Bound & Upper Bound \\
\hline w/ bleach & $27.095^{\mathrm{a}}$ & .194 & 26.715 & 27.475 \\
w/o bleach & $27.700^{\mathrm{a}}$ & .194 & 27.320 & 28.080 \\
\hline
\end{tabular}

a. Based on modified population marginal mean.

Pairwise Comparisons: RMP

Dependent Variable: a

\begin{tabular}{|c|c|c|c|c|c|c|}
\hline \multirow[b]{2}{*}{ (I) Bleached } & & \multirow[b]{2}{*}{ Mean Difference (I-J) } & \multirow[b]{2}{*}{ Std. Error } & \multirow[b]{2}{*}{ Sig. $^{d}$} & \multicolumn{2}{|c|}{$\begin{array}{l}\text { 95\% Confidence Interval for } \\
\text { Difference }\end{array}$} \\
\hline & & & & & Lower Bound & Upper Bound \\
\hline $\begin{array}{l}\text { w/ bleach } \\
\text { w/o bleach }\end{array}$ & $\begin{array}{l}\text { w/o bleach } \\
\text { w/ bleach }\end{array}$ & $\begin{array}{r}-.605^{\pi, b, c} \\
.605^{\pi, b, c}\end{array}$ & $\begin{array}{l}.274 \\
.274\end{array}$ & $\begin{array}{l}.027 \\
.027\end{array}$ & $\begin{array}{r}-1.143 \\
.068\end{array}$ & $\begin{array}{r}-.068 \\
1.143\end{array}$ \\
\hline
\end{tabular}

Based on estimated marginal means

*. The mean difference is significant at the .05 level.

b. An estimate of the modified population marginal mean (I).

c. An estimate of the modified population marginal mean (J).

d. Adjustment for multiple comparisons: Least Significant Difference (equivalent to no adjustments).

Dependent Variable: a

\section{Sample Area * Swatch: RMP}

\begin{tabular}{|ll|r|r|r|r|}
\hline \multirow{2}{*}{ Sample Area } & & & \multicolumn{2}{|c|}{ 95\% Confidence Interval } \\
\cline { 4 - 6 } & & Mean & Std. Error & Lower Bound & Upper Bound \\
\hline A & $1 \mathrm{w} /$ bleach & $28.977^{\mathrm{a}}$ & .371 & 28.249 & 29.704 \\
& $2 \mathrm{w} /$ bleach & $25.724^{\mathrm{a}}$ & .406 & 24.927 & 26.521 \\
& $3 \mathrm{w} / \mathrm{o}$ bleach & $28.684^{\mathrm{a}}$ & .371 & 27.956 & 29.412 \\
$\mathrm{~B}$ & $4 \mathrm{w} / \mathrm{o}$ bleach & $27.228^{\mathrm{a}}$ & .406 & 26.431 & 28.025 \\
& $1 \mathrm{w} /$ bleach & $26.980^{\mathrm{a}}$ & .406 & 26.183 & 27.777 \\
& $2 \mathrm{w} /$ bleach & $26.452^{\mathrm{a}}$ & .371 & 25.724 & 27.179 \\
& $3 \mathrm{w} /$ o bleach & $26.933^{\mathrm{a}}$ & .406 & 26.135 & 27.730 \\
& $4 \mathrm{w} / \mathrm{o}$ bleach & $27.750^{\mathrm{a}}$ & .371 & 27.022 & 28.478 \\
\hline
\end{tabular}


a. Based on modified population marginal mean.

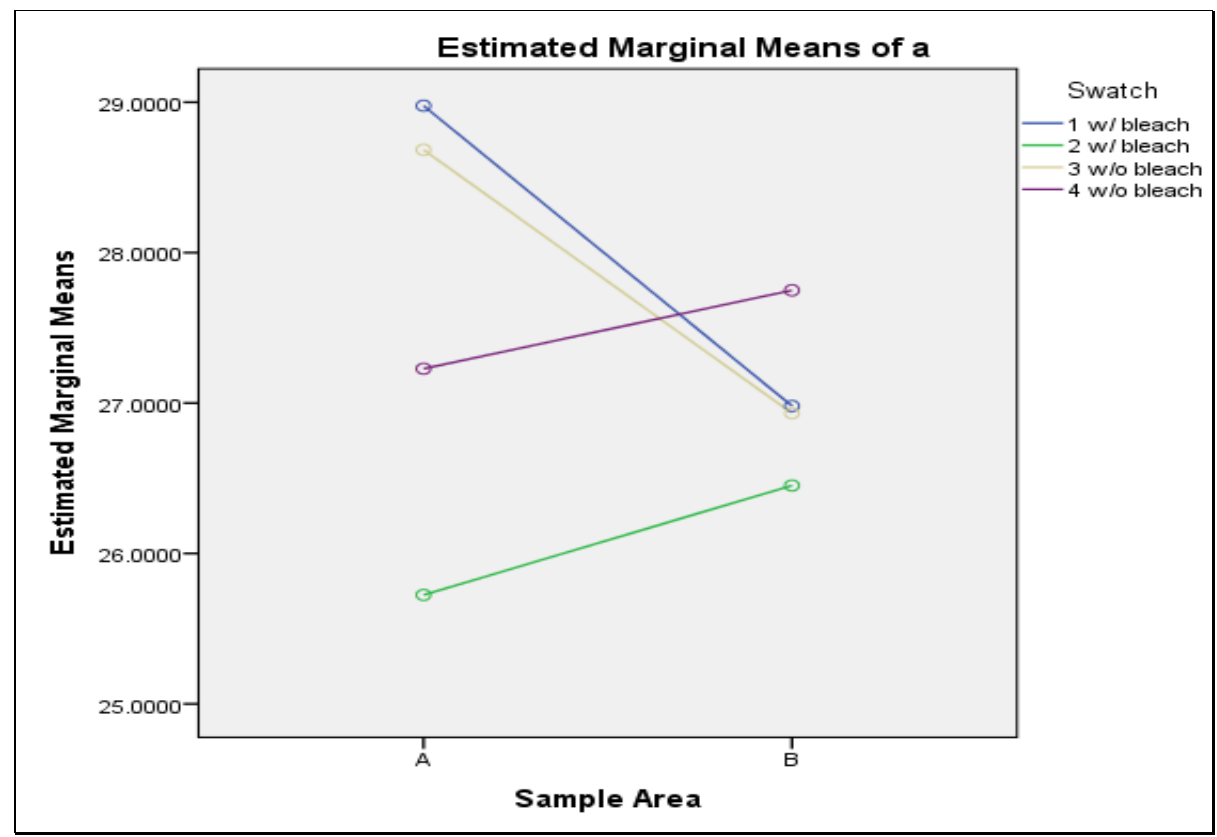

\section{Sample Area * Bleached: RMP}

Dependent Variable: a

\begin{tabular}{|ll|r|r|r|r|}
\hline \multirow{2}{*}{ Sample Area } & & \multirow{2}{*}{} & \multicolumn{2}{|c|}{ 95\% Confidence Interval } \\
\cline { 5 - 6 } & & Mean & Std. Error & Lower Bound & Upper Bound \\
\hline A & w/ bleach & $27.498^{\mathrm{a}}$ & .274 & 26.961 & 28.036 \\
& w/o bleach & $28.022^{\mathrm{a}}$ & .274 & 27.484 & 28.560 \\
B & w/ bleach & $26.692^{\mathrm{a}}$ & .274 & 26.154 & 27.229 \\
& w/o bleach & $27.378^{\mathrm{a}}$ & .274 & 26.841 & 27.916 \\
\hline
\end{tabular}

a. Based on modified population marginal mean.

\section{Variable ' $b$ '}

\section{Sample Area Estimates: RMP}

Dependent Variable: $b$

\begin{tabular}{|c|c|c|c|c|}
\hline \multirow[b]{2}{*}{ Sample Area } & \multirow[b]{2}{*}{ Mean } & \multirow[b]{2}{*}{ Std. Error } & \multicolumn{2}{|c|}{ 95\% Confidence Interval } \\
\hline & & & $\begin{array}{l}\text { Lower } \\
\text { Bound }\end{array}$ & $\begin{array}{l}\text { Upper } \\
\text { Bound }\end{array}$ \\
\hline $\begin{array}{l}A \\
B\end{array}$ & $\begin{array}{l}15.976^{\mathrm{a}} \\
15.517^{\mathrm{a}}\end{array}$ & $\begin{array}{r}.162 \\
.162\end{array}$ & $\begin{array}{l}15.658 \\
15.199\end{array}$ & $\begin{array}{l}16.294 \\
15.834\end{array}$ \\
\hline
\end{tabular}

a. Based on modified population marginal mean. 
Pairwise Comparisons: RMP

Dependent Variable: $b$

\begin{tabular}{|c|c|c|c|c|c|c|}
\hline \multirow{2}{*}{\multicolumn{2}{|c|}{ (I) Sample Area }} & \multirow[b]{2}{*}{$\begin{array}{c}\text { Mean Difference } \\
(\mathrm{I}-\mathrm{J})\end{array}$} & \multirow[b]{2}{*}{ Std. Error } & \multirow[b]{2}{*}{ Sig. $^{d}$} & \multicolumn{2}{|c|}{$\begin{array}{l}95 \% \text { Confidence Interval } \\
\text { for Difference }\end{array}$} \\
\hline & & & & & $\begin{array}{l}\text { Lower } \\
\text { Bound }\end{array}$ & $\begin{array}{l}\text { Upper } \\
\text { Bound }\end{array}$ \\
\hline A & $B$ & $.459^{\circ, \mathrm{b}, \mathrm{c}}$ & .229 & .045 & .010 & .909 \\
\hline B & A & $-.459^{*}, \mathrm{~b}, \mathrm{c}$ & .229 & .045 & -.909 & -.010 \\
\hline
\end{tabular}

Based on estimated marginal means

${ }^{*}$. The mean difference is significant at the .05 level.

b. An estimate of the modified population marginal mean (I).

c. An estimate of the modified population marginal mean $(\mathrm{J})$.

d. Adjustment for multiple comparisons: Least Significant Difference (equivalent to no adjustments).

\section{Bleach Estimates: RMP}

Dependent Variable: $b$

\begin{tabular}{|c|c|c|c|c|}
\hline \multirow[b]{2}{*}{ Bleached } & \multirow[b]{2}{*}{ Mean } & \multirow[b]{2}{*}{ Std. Error } & \multicolumn{2}{|c|}{ 95\% Confidence Interval } \\
\hline & & & $\begin{array}{l}\text { Lower } \\
\text { Bound }\end{array}$ & $\begin{array}{l}\text { Upper } \\
\text { Bound }\end{array}$ \\
\hline $\begin{array}{l}\text { w/ bleach } \\
\text { w/o bleach }\end{array}$ & $\begin{array}{l}15.326^{\mathrm{a}} \\
16.167^{\mathrm{a}}\end{array}$ & $\begin{array}{l}.162 \\
.162\end{array}$ & $\begin{array}{l}15.008 \\
15.849\end{array}$ & $\begin{array}{l}15.643 \\
16.485\end{array}$ \\
\hline
\end{tabular}

a. Based on modified population marginal mean.

Pairwise Comparisons: RMP

Dependent Variable: $b$

\begin{tabular}{|c|c|c|c|c|c|c|}
\hline \multirow[b]{2}{*}{ (I) Bleached } & & \multirow[b]{2}{*}{$\begin{array}{c}\text { Mean Difference } \\
(\mathrm{I}-\mathrm{J})\end{array}$} & \multirow[b]{2}{*}{ Std. Error } & \multirow[b]{2}{*}{ Sig. ${ }^{d}$} & \multicolumn{2}{|c|}{$\begin{array}{c}\text { 95\% Confidence } \\
\text { Interval for Difference }\end{array}$} \\
\hline & & & & & $\begin{array}{l}\text { Lower } \\
\text { Bound }\end{array}$ & $\begin{array}{l}\text { Upper } \\
\text { Bound }\end{array}$ \\
\hline $\begin{array}{l}\text { w/ bleach } \\
\text { w/o bleach }\end{array}$ & $\begin{array}{l}\text { w/o bleach } \\
\text { w/ bleach }\end{array}$ & $\begin{array}{r}-.841^{\overline{,}, \mathrm{b}, \mathrm{c}} \\
.841^{\mathrm{x}, \mathrm{b}, \mathrm{c}}\end{array}$ & $\begin{array}{l}.229 \\
.229\end{array}$ & $\begin{array}{l}.000 \\
.000\end{array}$ & $\begin{array}{r}-1.291 \\
.392\end{array}$ & $\begin{array}{r}-.392 \\
1.291\end{array}$ \\
\hline
\end{tabular}

Based on estimated marginal means

*. The mean difference is significant at the .05 level.

b. An estimate of the modified population marginal mean (I).

c. An estimate of the modified population marginal mean (J).

d. Adjustment for multiple comparisons: Least Significant Difference (equivalent to no adjustments).

Dependent Variable: $b$

\section{Sample Area * Swatch: RMP}

\begin{tabular}{|c|c|c|c|c|c|}
\hline \multirow[b]{2}{*}{ Sample Area } & & \multirow[b]{2}{*}{ Mean } & \multirow[b]{2}{*}{ Std. Error } & \multicolumn{2}{|c|}{$95 \%$ Confidence Interval } \\
\hline & & & & Lower Bound & $\begin{array}{l}\text { Upper } \\
\text { Bound }\end{array}$ \\
\hline \multirow[t]{4}{*}{$\bar{A}$} & $1 \mathrm{w} /$ bleach & $16.559^{\mathrm{a}}$ & .310 & 15.950 & 17.167 \\
\hline & 2 w/ bleach & $14.603^{a}$ & .340 & 13.936 & 15.269 \\
\hline & 3 w/o bleach & $16.923^{\mathrm{a}}$ & .310 & 16.315 & 17.532 \\
\hline & 4 w/o bleach & $15.513^{\mathrm{a}}$ & .340 & 14.846 & 16.179 \\
\hline \multirow[t]{4}{*}{ B } & $1 \mathrm{w} /$ bleach & $15.315^{a}$ & .340 & 14.648 & 15.981 \\
\hline & 2 w/ bleach & $14.704^{\mathrm{a}}$ & .310 & 14.095 & 15.312 \\
\hline & 3 w/o bleach & $15.351^{\mathrm{a}}$ & .340 & 14.684 & 16.017 \\
\hline & $4 \mathrm{w} / \mathrm{o}$ bleach & $16.636^{\mathrm{a}}$ & .310 & 16.027 & 17.244 \\
\hline
\end{tabular}

a. Based on modified population marginal mean. 


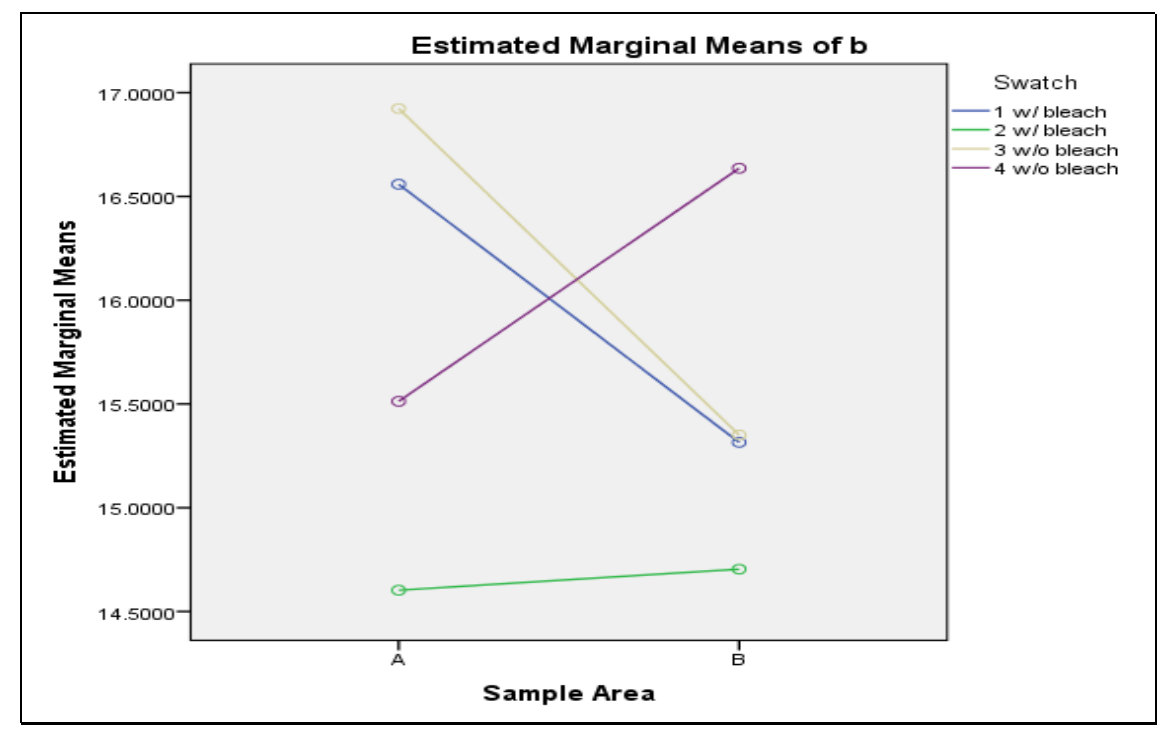

Dependent Variable: $b$

6. Sample Area * Bleached: RMP

\begin{tabular}{|ll|r|r|r|r|}
\hline & & & \multicolumn{2}{|c|}{ 95\% Confidence Interval } \\
\cline { 5 - 6 } Sample Area & & Mean & Std. Error & Lower Bound & Upper Bound \\
\hline A & w/ bleach & $15.670^{\mathrm{a}}$ & .229 & 15.220 & 16.119 \\
& w/o bleach & $16.282^{\mathrm{a}}$ & .229 & 15.833 & 16.732 \\
B & w/ bleach & $14.982^{\mathrm{a}}$ & .229 & 14.532 & 15.431 \\
& w/o bleach & $16.051^{\mathrm{a}}$ & .229 & 15.602 & 16.501 \\
\hline
\end{tabular}

a. Based on modified population marginal mean.

\section{Table 14.7 ANOVA Output Between-Subject Factors for all colors}

Between-Subjects Factors: all colors

\begin{tabular}{|c|c|c|c|}
\hline & & Value Label & $\mathrm{N}$ \\
\hline \multirow[t]{4}{*}{ Swatch } & 1 & $1 \mathrm{w} /$ bleach & 1540 \\
\hline & 2 & 2 w/ bleach & 1540 \\
\hline & 3 & 3 w/o bleach & 1540 \\
\hline & 4 & 4 w/o bleach & 1540 \\
\hline \multirow[t]{2}{*}{ Sample Area } & $A$ & A & 3080 \\
\hline & $B$ & $B$ & 3080 \\
\hline \multirow[t]{2}{*}{ Bleached } & 1 & bleached & 3080 \\
\hline & 2 & w/o bleached & 3080 \\
\hline \multirow[t]{6}{*}{ Washing } & 1 & $1 \mathrm{~W} 0 \mathrm{~A}$ & 560 \\
\hline & 2 & $1 \mathrm{~W} 16 \mathrm{~A}$ & 1120 \\
\hline & 3 & $2 W 32 A$ & 1120 \\
\hline & 4 & $3 W 48 A$ & 1120 \\
\hline & 5 & $4 W 64 A$ & 1120 \\
\hline & & $5 W$ 80A & 1120 \\
\hline \multirow[t]{7}{*}{ Color } & dk pink & & 880 \\
\hline & Green & & 880 \\
\hline & Orange & & 880 \\
\hline & pink-C & & 880 \\
\hline & Pink-J & & 880 \\
\hline & Purple & & 880 \\
\hline & Red & & 880 \\
\hline
\end{tabular}

